Supporting Information for:

\title{
Enantioselective Aminomethylamination of Conjugated Dienes with Aminals Enabled by Chiral Palladium Complex-Catalyzed C-N Bond Activation
}

\author{
Yang Liu, Yinjun Xie, Hongli Wang, and Hanmin Huang*

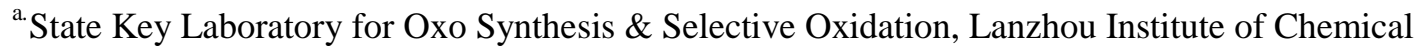 \\ Physics, Chinese Academy of Sciences, 18 Tianshui Middle Road, Lanzhou 730000, China. \\ ${ }^{\mathrm{b}}$ University of Chinese Academy of Sciences, Beijing 100049, P. R. China \\ E-mail: hmhuang@licp.cas.cn
}

\section{CONTENTS}

1 General experimental details

2 Synthesis of ligands

3 Optimization of the reaction conditions

4 General procedure for the asymmetric synthesis of chiral 1,3-diamines

5 Experimental characterization data for new products

6 Mechanism studies

7 Copies for HPLC and NMR of products 


\section{General experimental details:}

All non-aqueous reactions and manipulations were performed in nitrogen atmosphere using standard Schlenk techniques. All solvents before use were dried and degassed by standard methods and stored under nitrogen. All reactions were monitored by TLC with silica gel-coated plates. Nuclear magnetic resonance (NMR) spectra were recorded on BRUKER Avance III 400 spectrometers. Chemical shifts were reported in parts per million ( $\mathrm{ppm}$ ) down field from TMS with the solvent resonance as the internal standard. Coupling constants $(J)$ were reported in $\mathrm{Hz}$ and refered to apparent peak multiplications. High resolution mass spectra (HRMS) were recorded on Bruker micro-TOF-QII mass instrament (ESI). High performance liquid chromatography (HPLC) were recorded on Agilent 1200 instument. 1,3-dienes used here were known compounds and synthesized according to the reported methods. ${ }^{1}$ Aminals used here were known compounds and synthesized according to the reported methods. $^{2}(R)$-BINAPO (5a) was purchased from Alfa Aesar. Other chiral ligands were synthesized by ourself. 


\section{Synthesis of chiral ligands}

Starting materials 3,3-disubstituted-2,2'-dihydroxy-1,1'-binaphtyl, such as

3,3'-diphenyl-[1,1'-binaphthalene]-2,2'-diol, ${ }^{3}$

3,3'-bis(triphenylsilyl)-[1,1'-binaphthalene $]-2,2^{\prime}$-diol, ${ }^{4}$

3,3'-bis(2,4,6-triisopropylphenyl)-[1,1'-binaphthalene]-2,2'-diol, ${ }^{5}$

3,3'-di(anthracen-9-yl)-[1,1'-binaphthalene $]-2,2^{\prime}$-diol, ${ }^{6}$

and

3,3'-bis(2,4,6-triisopropylphenyl)-5,5',6,6',7,7',8,8'-octahydro-[1,1'-binaphthalene]-2,2' $-\operatorname{diol}^{7}$ were synthesized according to the known methods.

\subsection{General procedure for the synthesis of chiral ligands:}<smiles>[R]c1cc2ccccc2c(-c2c(O)c([R])cc3ccccc23)c1O</smiles>

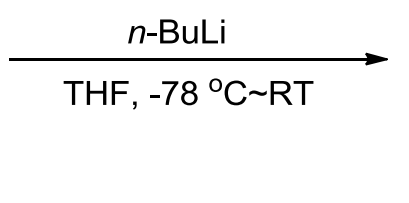<smiles>[R]c1cc2ccccc2c(-c2c(OCC)c([R])cc3ccccc23)c1OCC</smiles>

To a solution of 3,3'-disubstituted-2,2'-dihydroxy-1,1'-binaphtyl (0.2 mmol) in 20 $\mathrm{mL}$ of THF at $-78{ }^{\circ} \mathrm{C}, n$-BuLi $(240 \mu \mathrm{L}, 2.5 \mathrm{M}$ in hexane, $6 \mathrm{mmol})$ was added, then the solution was warmed to room temperature and stirred for 12 hours. After $\mathrm{Ar}_{2} \mathrm{PCl}(0.7$ mmol) was added to the reaction mixture, the resulting mixture was stirred at room temperature for another 12 hours. The solvent was removed under reduced pressure and the residue was purified by flash column chromatography on silica gel to afford the white solid (eluted with petroleum ether $/ \mathrm{CH}_{2} \mathrm{Cl}_{2}=20 / 1 \sim 5 / 1$ ).

\subsection{Experimental characterization data for ligands}

(R)-((3,3'-diphenyl-[1,1'-binaphthalene]-2,2'-diyl)bis(oxy))bis(diphenylphosphine)<smiles>c1ccc(-c2cc3ccccc3c(-c3c(-c4ccccc4)c(-c4ccccc4)cc4ccccc34)c2-c2ccccc2)cc1</smiles>

$5 b$ (5b): The title compound was prepared according to the general procedure and purified by column chromatography to give a white solid, $93 \mathrm{mg}, 56 \%$ yield. ${ }^{1} \mathrm{H}$ NMR (400 MHz, $\left.\mathrm{CDCl}_{3}\right): \delta 6.81-6.83(\mathrm{~m}, 8 \mathrm{H}), 6.93-7.05(\mathrm{~m}, 18 \mathrm{H}), 7.18-7.29$ 
$(\mathrm{m}, 10 \mathrm{H}), 7.57-7.60(\mathrm{~m}, 4 \mathrm{H}) ;{ }^{13} \mathrm{C} \mathrm{NMR}\left(100 \mathrm{MHz}, \mathrm{CDCl}_{3}\right): \delta 124.6,126.0,126.3$, $127.0,127.3,127.3,127.4,127.4,127.5,127.5,127.8,128.3,128.4,129.1,129.1$, $129.3,129.3,129.4,129.4,129.5,129.7,129.8,130.2,130.3,131.0,133.7,135.0$, 138.6, 141.9, 142.0, 142.1, 142.2, 142.3, 152.4, 152.5; ${ }^{31} \mathrm{P}$ NMR (162 MHz, $\left.\mathrm{CDCl}_{3}\right)$ : $\delta$ 112.93. HRMS (ESI) calcd for $\mathrm{C}_{56} \mathrm{H}_{41} \mathrm{O}_{2} \mathrm{P}_{2}[\mathrm{M}+\mathrm{H}]$ : 807.2576, found: 807.2574. $[\alpha]_{\mathrm{D}}^{20}=84.6\left(\mathrm{CH}_{2} \mathrm{Cl}_{2}, c 0.22\right)$.

(R)-((3,3'-bis(triphenylsilyl)-[1,1'-binaphthalene]-2,2'-diyl)bis(oxy))bis(diphenylp<smiles></smiles>

$5 c$

hosphine) (5c): The title compound was prepared according to the general procedure and purified by column chromatography to give a white solid, $72 \mathrm{mg}, 31 \%$ yield. ${ }^{1} \mathrm{H}$ NMR (400 MHz, $\left.\mathrm{CDCl}_{3}\right): \delta$ 6.13-6.18 (m, 4H), $6.44(\mathrm{t}, J=$ $7.6 \mathrm{~Hz}, 4 \mathrm{H}), 6.64-6.67(\mathrm{~m}, 2 \mathrm{H}), 6.77-6.88(\mathrm{~m}, 10 \mathrm{H})$, 6.91-7.00 (m, 6H), 7.11-7.15 (m, 2H), 7.28-7.39 (m, 18H), 7.52 (s, 2H), 7.68-7.71 (m, $12 \mathrm{H}) ;{ }^{13} \mathrm{C} \mathrm{NMR}\left(100 \mathrm{MHz}, \mathrm{CDCl}_{3}\right): \delta 118.2,123.7,125.4,125.7,126.8,126.8,127.2$, $127.3,127.6,127.9,128.1,128.2,129.0,129.2,132.0,132.2,132.3,135.3,136.1$, 136.6, 141.2, 142.1, 142.5, 159.3; ${ }^{31} \mathrm{P}$ NMR (162 $\left.\mathrm{MHz}, \mathrm{CDCl}_{3}\right): \delta 100.68 . \mathrm{HRMS}$ (ESI) calcd for $\mathrm{C}_{80} \mathrm{H}_{61} \mathrm{O}_{2} \mathrm{P}_{2} \mathrm{Si}_{2}[\mathrm{M}+\mathrm{H}]:$ 1171.3680, found: $1171.3716 .[\alpha]_{\mathrm{D}}{ }^{20}=18.1$ $\left(\mathrm{CH}_{2} \mathrm{Cl}_{2}, c 0.25\right)$.

(R)-((3,3'-dimesityl-[1,1'-binaphthalene]-2,2' -diyl)bis(oxy))bis(diphenylphosphine

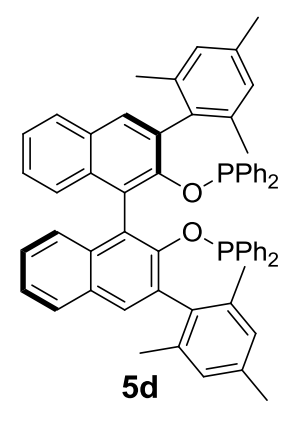

) (5d): The title compound was prepared according to the general procedure and purified by column chromatography to give a white solid, $103 \mathrm{mg}, 58 \%$ yield. ${ }^{1} \mathrm{H}$ NMR (400 $\left.\mathrm{MHz}, \mathrm{CDCl}_{3}\right): \delta 1.61(\mathrm{~s}$, $6 \mathrm{H}), 2.12(\mathrm{~s}, 6 \mathrm{H}), 2.14(\mathrm{~s}, 6 \mathrm{H}), 6.36(\mathrm{~s}, 2 \mathrm{H}), 6.53(\mathrm{~s}, 2 \mathrm{H}), 6.60(\mathrm{~s}$, 4H), 6.72-6.82 (m, 12H), 6.89-6.93 (m, 2H), 6.95-6.99 (m, 2H), 
$\left.\mathrm{CDCl}_{3}\right): \delta 21.0,21.6,124.0,124.6,125.9,126.0,127.1,127.2,127.5,127.7,127.8$ $127.9,128.0,128.0,128.2,129.4,129.6,129.7,130.8,130.8,133.6,133.6,134.8$, 136.7, 137.0, 137.1, 142.9, 143.0, 152.7; ${ }^{31} \mathrm{P} \mathrm{NMR}\left(162 \mathrm{MHz}, \mathrm{CDCl}_{3}\right): \delta 109.95$. HRMS (ESI) calcd for $\mathrm{C}_{62} \mathrm{H}_{53} \mathrm{O}_{2} \mathrm{P}_{2}[\mathrm{M}+\mathrm{H}]: 891.3515$, found: $891.3527 .[\alpha]_{\mathrm{D}}{ }^{20}=77.5$ $\left(\mathrm{CH}_{2} \mathrm{Cl}_{2}, c 0.34\right)$.

$(R)-((3,3 '$ 'bis(2,4,6-triisopropylphenyl)-[1,1'-binaphthalene]-2,2'-diyl)bis(oxy))bis

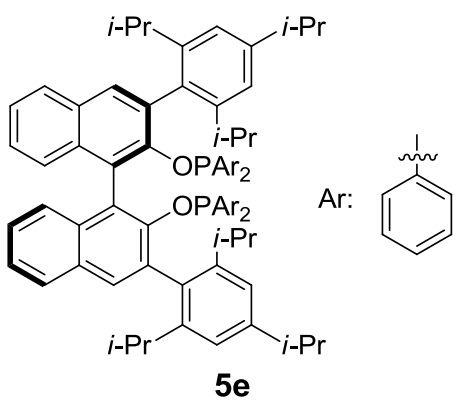
(diphenylphosphine) (5e): The title compound was prepared according to the general procedure and purified by column chromatography to give a white solid, 136 $\mathrm{mg}, 64 \%$ yield. ${ }^{1} \mathrm{H}$ NMR (400 MHz, $\left.\mathrm{CDCl}_{3}\right): \delta 0.46(\mathrm{~d}, J$ $=6.4 \mathrm{~Hz}, 6 \mathrm{H}), 0.97(\mathrm{~d}, J=6.4 \mathrm{~Hz}, 6 \mathrm{H}), 1.07(\mathrm{~d}, J=6.4$

$\mathrm{Hz}, 6 \mathrm{H}), 1.35-1.37(\mathrm{~m}, 18 \mathrm{H}), 2.72-2.75(\mathrm{~m}, 2 \mathrm{H}), 2.94-3.04(\mathrm{~m}, 2 \mathrm{H}), 6.50(\mathrm{~s}, 4 \mathrm{H})$, 6.67-6.71 (m, 8H), 6.80-6.90 (m, 8H), 7.01-7.08 (m, 4H), 7.13-7.24 (m, 6H), $7.56(\mathrm{~d}$, $J=8.0 \mathrm{~Hz}, 2 \mathrm{H}), 7.63(\mathrm{~s}, 2 \mathrm{H}) ;{ }^{13} \mathrm{C} \mathrm{NMR}\left(100 \mathrm{MHz}, \mathrm{CDCl}_{3}\right): \delta$ 21.7, 23.1, 24.3, 24.4, $25.5,26.0,30.4,31.1,34.3,120.1,121.2,124.5,125.5,125.9,127.0,127.3,127.6$, $128.0,128.1,128.2,128.5,129.8,129.9,130.0,131.7,132.6,133.6,133.9,143.3$, 146.9, 147.4, 147.9; ${ }^{31} \mathrm{P}$ NMR (162 $\left.\mathrm{MHz}, \mathrm{CDCl}_{3}\right): \delta 101.91$. HRMS (ESI) calcd for $\mathrm{C}_{74} \mathrm{H}_{77} \mathrm{O}_{2} \mathrm{P}_{2}[\mathrm{M}+\mathrm{H}]:$ 1059.5393, found: $1059.5368 .[\alpha]_{\mathrm{D}}{ }^{20}=110.2\left(\mathrm{CH}_{2} \mathrm{Cl}_{2}, c 0.30\right)$.

(R)-((3,3'-bis(2,4,6-triisopropylphenyl)-[1,1'-binaphthalene]-2,2'-diyl)bis(oxy))bis

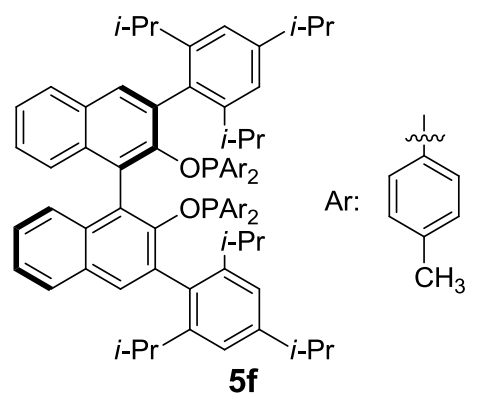
(di-p-tolylphosphine) (5f): The title compound was prepared according to the general procedure and purified by column chromatography to give a white solid, $121 \mathrm{mg}$, 54\% yield. ${ }^{1} \mathrm{H}$ NMR (400 MHz, $\left.\mathrm{CDCl}_{3}\right)$ : $\delta 0.45(\mathrm{~d}, J=6.8 \mathrm{~Hz}, 6 \mathrm{H}), 0.95(\mathrm{~d}, J=6.8 \mathrm{~Hz}, 6 \mathrm{H})$, $1.09(\mathrm{~d}, J=6.8 \mathrm{~Hz}, 6 \mathrm{H}), 1.35-1.38(\mathrm{~m}, 18 \mathrm{H}), 2.06(\mathrm{~s}, 6 \mathrm{H}), 2.16(\mathrm{~s}, 6 \mathrm{H}), 2.76-2.82(\mathrm{~m}$, 
2H), 2.94-3.05 (m, 4H), 6.36-6.39 (m, 4H), 6.49-6.57 (m, 8H), 6.65 (d, J = 7.6 Hz, 4H), $6.88(\mathrm{~d}, J=1.6 \mathrm{~Hz}, 2 \mathrm{H}), 7.06-7.10(\mathrm{~m}, 4 \mathrm{H}), 7.16-7.19(\mathrm{~m}, 4 \mathrm{H}), 7.54-7.56(\mathrm{~m}$, 2H), $7.62(\mathrm{~s}, 2 \mathrm{H}) ;{ }^{13} \mathrm{C}$ NMR $\left(100 \mathrm{MHz}, \mathrm{CDCl}_{3}\right): \delta 21.2,21.3,21.6,23.1,24.3,24.4$, $25.6,25.9,30.4,31.1,34.3,120.1,121.1,121.2,124.1,125.5,125.8,127.5,127.8$, $128.2,128.3,128.4,128.5,129.8,130.0,130.1,131.6,132.6,133.7,134.1,136.8$, 138.1, 140.0, 146.9, 147.4, 147.7, 153.1; ${ }^{31} \mathrm{P}$ NMR (162 $\left.\mathrm{MHz}, \mathrm{CDCl}_{3}\right): \delta 103.51$. HRMS (ESI) calcd for $\mathrm{C}_{78} \mathrm{H}_{85} \mathrm{O}_{2} \mathrm{P}_{2}[\mathrm{M}+\mathrm{H}]: 1115.6019$, found: 1115.6059 . $[\alpha]_{D}{ }^{20}=$ $67.9\left(\mathrm{CH}_{2} \mathrm{Cl}_{2}, c 0.30\right)$.

(R)-((3,3'-bis(2,4,6-triisopropylphenyl)-[1,1'-binaphthalene]-2,2'-diyl)bis(oxy))bis

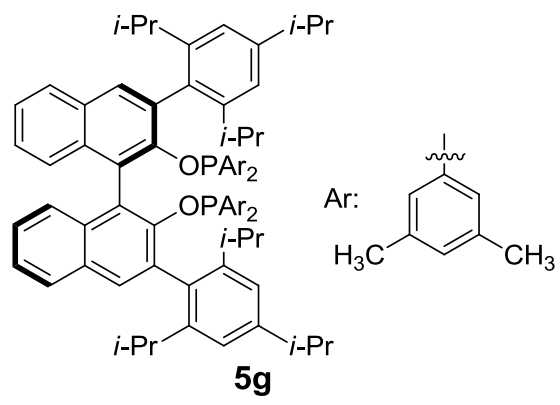
(bis(3,5-dimethylphenyl)phosphine) (5g): The title compound was prepared according to the general procedure and purified by column chromatography to give a white solid, $187 \mathrm{mg}$, $80 \%$ yield. ${ }^{1} \mathrm{H}$ NMR $\left(400 \mathrm{MHz}, \mathrm{CDCl}_{3}\right): \delta 0.58(\mathrm{~d}$, $J=6.8 \mathrm{~Hz}, 6 \mathrm{H}), 1.03-1.10(\mathrm{~m} \mathrm{12H}), 1.34(\mathrm{t}, J=7.2 \mathrm{~Hz}, 12 \mathrm{H}), 1.40(\mathrm{~d}, J=6.8 \mathrm{~Hz}$, 6H), $1.89(\mathrm{~s}, 12 \mathrm{H}), 1.96(\mathrm{~s}, 12 \mathrm{H}), 2.87-3.11(\mathrm{~m}, 6 \mathrm{H}), 6.16(\mathrm{~s}, 4 \mathrm{H}), 6.37-6.40(\mathrm{~m}$, $6 \mathrm{H}), 6.68(\mathrm{~s}, 2 \mathrm{H}), 6.99-7.24(\mathrm{~m}, 10 \mathrm{H}), 7.53(\mathrm{~d}, J=7.6 \mathrm{~Hz}, 2 \mathrm{H}), 7.60(\mathrm{~s}, 2 \mathrm{H}) ;{ }^{13} \mathrm{C}$ NMR (100 MHz, $\left.\mathrm{CDCl}_{3}\right): \delta 21.1,21.4,21.7,23.4,23.5,24.6,25.4,26.1,30.5,30.9$, $34.0,119.7,120.3,121.2,124.1,125.3,125.5,125.9,126.0,126.1,127.1,127.6$, $127.8,127.9,129.4,129.6,130.5,131.9,132.5,133.6,134.3,135.8,135.9,136.5$, 136.6, 136.6, 143.1, 143.2, 143.4, 147.0, 147.3, 153.4; ${ }^{31} \mathrm{P}$ NMR (162 MHz, $\left.\mathrm{CDCl}_{3}\right)$ : $\delta$ 100.66. HRMS (ESI) calcd for $\mathrm{C}_{82} \mathrm{H}_{93} \mathrm{O}_{2} \mathrm{P}_{2}[\mathrm{M}+\mathrm{H}]:$ 1171.6645, found: 1171.6689 . $[\alpha]_{\mathrm{D}}^{20}=18.3\left(\mathrm{CH}_{2} \mathrm{Cl}_{2}, c 0.29\right)$. 
(R)-((3,3'-bis(2,4,6-triisopropylphenyl)-[1,1'-binaphthalene]-2,2'-diyl)bis(oxy))bis

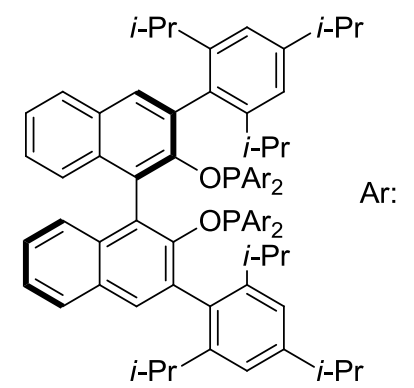

$5 \mathrm{~h}$ (bis(4-fluorophenyl)phosphine) $\quad(5 \mathrm{~h})$ : The title compound was prepared according to the general procedure and purified by column chromatography to give a white solid, $201 \mathrm{mg}, 89 \%$ yield. ${ }^{1} \mathrm{H}$ NMR $\left(400 \mathrm{MHz}, \mathrm{CDCl}_{3}\right): \delta 0.53(\mathrm{~d}, J=6.8 \mathrm{~Hz}, 6 \mathrm{H})$,

1.00-1.08 (m, 12H), 1.32-1.37 (m, 18H), 2.65-2.72 (m, 2H), 2.92-3.01 (m, 4H), 6.39-6.43 (m, 8H), 6.56-6.60 (m, 8H), 6.92-7.07 (m, 4H), 7.16-7.24 (m, 4H), 7.26-7.30 (m, 2H), 7.62-7.65 (m, 4H); $\left.{ }^{13} \mathrm{C} \mathrm{NMR} \mathrm{(100} \mathrm{MHz,} \mathrm{CDCl}_{3}\right): \delta$ 21.8, 23.0, $24.3,24.3,25.5,25.9,30.5,31.1,34.2,114.2,114.5,114.8,115.0,120.3,121.2,121.3$ $124.8,125.4,126.2,127.8,129.9,130.0,130.0,130.1,130.2,131.6,131.7,131.8$, $131.9,132.5,133.5,133.5,138.2,146.7,147.4,148.3,152.7,161.1,162.0,163.5$, 164.4; ${ }^{31} \mathrm{P}$ NMR $\left(162 \mathrm{MHz}, \mathrm{CDCl}_{3}\right): \delta 100.87 ;{ }^{19} \mathrm{~F}$ NMR $\left(376 \mathrm{MHz}, \mathrm{CDCl}_{3}\right): \delta$ -113.37, -111.93; HRMS (ESI) calcd for $\mathrm{C}_{74} \mathrm{H}_{73} \mathrm{~F}_{4} \mathrm{O}_{2} \mathrm{P}_{2}[\mathrm{M}+\mathrm{H}]$ : 1131.5016, found: 1131.5048. $[\alpha]_{\mathrm{D}}{ }^{20}=94.6\left(\mathrm{CH}_{2} \mathrm{Cl}_{2}, c 0.29\right)$.

(R)-((3,3'-bis(2,4,6-triisopropylphenyl)-[1,1'-binaphthalene]-2,2'-diyl)bis(oxy))bis

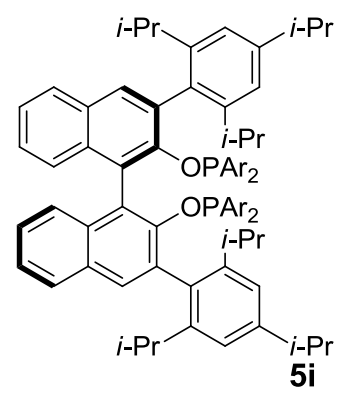
(bis(4-chlorophenyl)phosphine) (5i): The title compound was prepared according to the general procedure and purified by column chromatography to give a white solid, $179 \mathrm{mg}, 75 \%$ yield. ${ }^{1} \mathrm{H}$ NMR $\left(400 \mathrm{MHz}, \mathrm{CDCl}_{3}\right): \delta 0.54(\mathrm{~d}, J=7.2 \mathrm{~Hz}, 6 \mathrm{H}), 1.01$

$(\mathrm{d}, J=6.8 \mathrm{~Hz}, 6 \mathrm{H}), 1.05(\mathrm{~d}, J=6.8 \mathrm{~Hz}, 6 \mathrm{H}), 1.34-1.42(\mathrm{~m}, 18 \mathrm{H}), 2.62-2.69(\mathrm{~m}, 2 \mathrm{H})$, 2.91-3.00 (m, 4H), 6.37-6.41 (m, 4H), 6.53-6.57 (m, 4H), $6.68(\mathrm{~d}, J=8.0 \mathrm{~Hz}, 4 \mathrm{H})$, $6.84(\mathrm{~d}, J=8.0 \mathrm{~Hz}, 4 \mathrm{H}), 6.90(\mathrm{~d}, J=0.8 \mathrm{~Hz}, 2 \mathrm{H}), 7.05(\mathrm{~d}, J=1.2 \mathrm{~Hz}, 2 \mathrm{H}), 7.19-7.33$ $(\mathrm{m}, 6 \mathrm{H}), 7.63-7.64(\mathrm{~m}, 4 \mathrm{H}) ;{ }^{13} \mathrm{C} \mathrm{NMR}\left(100 \mathrm{MHz}, \mathrm{CDCl}_{3}\right): \delta 21.8,23.0,24.2,24.3$, 
$25.5,25.9,26.9,30.6,31.1,34.2,120.4,121.2,121.4,124.9,125.4,126.3,127.5$, $127.5,127.9,128.1,128.1,129.0,129.2,129.3,130.1,130.6,130.8,130.9,132.0$, $132.5,133.3,133.4,133.8,135.0,141.1,141.3,146.7,147.3,148.4,152.6 ;{ }^{31} \mathrm{P}$ NMR $\left(162 \mathrm{MHz}, \mathrm{CDCl}_{3}\right): \delta$ 99.94; HRMS (ESI) calcd for $\mathrm{C}_{74} \mathrm{H}_{73} \mathrm{Cl}_{4} \mathrm{O}_{2} \mathrm{P}_{2}[\mathrm{M}+\mathrm{H}]$ : 1195.3834, found: $1195.3853 .[\alpha]_{\mathrm{D}}{ }^{20}=36.8\left(\mathrm{CH}_{2} \mathrm{Cl}_{2}, c 0.30\right)$.

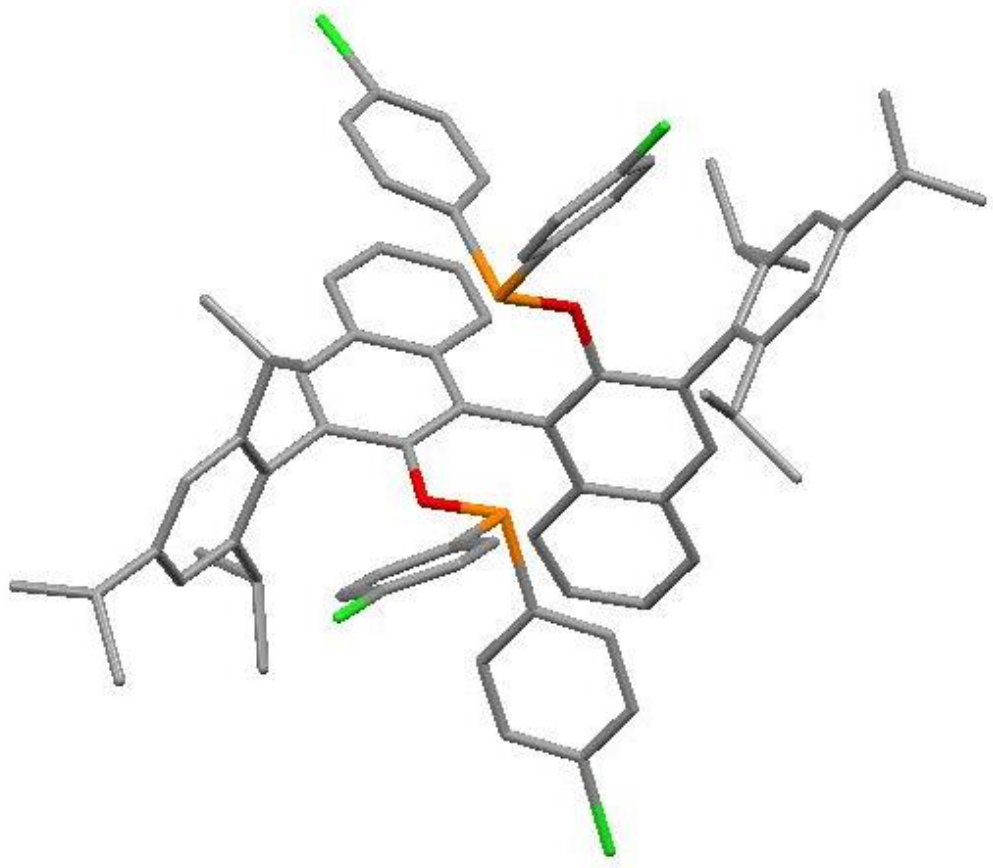

Figure S-1. X-ray structure of $\mathbf{5 i}$

(R)- ((3,3'-bis(2,4,6-triisopropylphenyl)-[1,1'-binaphthalene]-2,2'-diyl)bis(oxy)) bis(bis(4-(trifluoromethyl)phenyl)phosphine) (5j): The title compound was

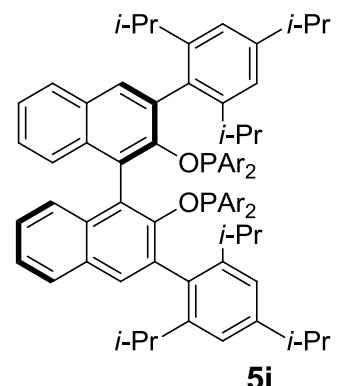

$5 \mathbf{j}$ prepared according to the general procedure and purified by column chromatography to give a white solid, $194 \mathrm{mg}, 73 \%$ yield. ${ }^{1} \mathrm{H}$ NMR $(400 \mathrm{MHz}$, $\left.\mathrm{CDCl}_{3}\right): \delta 0.46(\mathrm{~d}, J=6.8 \mathrm{~Hz}, 6 \mathrm{H}), 1.03(\mathrm{~d}, J=6.8 \mathrm{~Hz}$ $6 \mathrm{H}), 1.09(\mathrm{~d}, J=6.8 \mathrm{~Hz}, 6 \mathrm{H}), 1.36-1.38(\mathrm{~m}, 12 \mathrm{H})$, $1.44(\mathrm{~d}, J=6.8 \mathrm{~Hz}, 6 \mathrm{H}), 2.74-2.81(\mathrm{~m}, 2 \mathrm{H}), 2.96-3.03(\mathrm{~m}, 4 \mathrm{H}), 6.66-6.68(\mathrm{~m}, 4 \mathrm{H})$, 6.75-6.77 (m, 4H), $6.91(\mathrm{~s}, 2 \mathrm{H}), 6.97(\mathrm{~d}, J=8.0 \mathrm{~Hz}, 4 \mathrm{H}), 7.15-7.25(\mathrm{~m}, 12 \mathrm{H})$, 7.50-7.53 (m, 2H), $7.62(\mathrm{~s}, 2 \mathrm{H}) ;{ }^{13} \mathrm{C} \mathrm{NMR}\left(100 \mathrm{MHz}, \mathrm{CDCl}_{3}\right): \delta 21.8,23.1,24.1,24.2$, 
$25.5,25.8,30.5,31.2,34.2,120.6,120.8,121.3,122.4,124.1,124.9,125.3,126.6$ $127.8,127.9,128.0,129.3,129.4,129.5,129.6,130.2,132.1,132.4,133.2,146.5$, 147.5, 148.6, 152.2; ${ }^{31} \mathrm{P}$ NMR (162 MHz, $\left.\mathrm{CDCl}_{3}\right): \delta 95.86 ;{ }^{19} \mathrm{~F}$ NMR $(376 \mathrm{MHz}$, $\mathrm{CDCl}_{3}$ ): $\delta$-63.01, -62.99; HRMS (ESI) calcd for $\mathrm{C}_{78} \mathrm{H}_{73} \mathrm{~F}_{12} \mathrm{O}_{2} \mathrm{P}_{2}[\mathrm{M}+\mathrm{H}]$ : 1331.4889, found: 1331.4915. $[\alpha]_{\mathrm{D}}{ }^{20}=41.2\left(\mathrm{CH}_{2} \mathrm{Cl}_{2}, c 0.59\right)$.

$(R)-((3,3 '$ 'di(anthracen-9-yl)-[1,1'-binaphthalene]-2,2'-diyl)bis(oxy))bis(bis(4-fluo

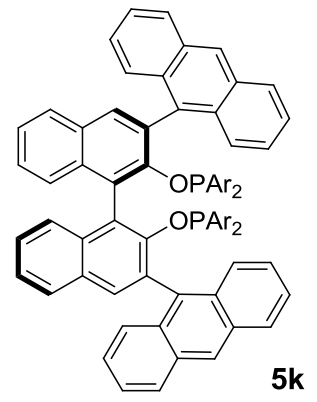
rophenyl)phosphine) (5k): The title compound was prepared according to the general procedure and purified by column chromatography to give a white solid, $86 \mathrm{mg}$, $40 \%$ yield. ${ }^{1} \mathrm{H}$ NMR (400 $\left.\mathrm{MHz}, \mathrm{CDCl}_{3}\right)$ : $\delta$ 6.02-6.10 (m, 8H), 6.31-6.32 (m, 4H), 6.43-6.48 (m, 4H), $7.08-7.12(\mathrm{~m}, 2 \mathrm{H}), 7.19-7.25(\mathrm{~m}, 2 \mathrm{H}), 7.32-7.36(\mathrm{~m}, 2 \mathrm{H}), 7.41-7.54(\mathrm{~m}, 6 \mathrm{H})$, 7.60-7.63 (m, 2H), $7.73(\mathrm{~s}, 2 \mathrm{H}), 7.79-7.86(\mathrm{~m}, 8 \mathrm{H}), 7.91(\mathrm{~d}, J=8.4 \mathrm{~Hz}, 2 \mathrm{H}), 8.11(\mathrm{~s}$, $2 \mathrm{H}) ;{ }^{13} \mathrm{C} \mathrm{NMR}\left(100 \mathrm{MHz}, \mathrm{CDCl}_{3}\right): \delta 113.9,114.1,114.4,114.6,124.2,124.8,124.8$, $25.0,125.3,125.4,126.2,126.8,126.9,127.4,127.5,128.1,128.2,128.3,129.3$, $130.6,130.9,131.0,131.1,131.2,131.6,132.5,133.2,134.0,153.7,161.3,163.8 ;{ }^{31} \mathrm{P}$ NMR (162 MHz, $\left.\mathrm{CDCl}_{3}\right): \delta 108.81 ;{ }^{19} \mathrm{~F} \mathrm{NMR}\left(376 \mathrm{MHz}, \mathrm{CDCl}_{3}\right): \delta-113.31,-112.23$; HRMS (ESI) calcd for $\mathrm{C}_{72} \mathrm{H}_{45} \mathrm{~F}_{4} \mathrm{O}_{2} \mathrm{P}_{2}[\mathrm{M}+\mathrm{H}]: 1079.2825$, found: $1079.2854 .[\alpha]_{\mathrm{D}}{ }^{20}=$ $-135.7\left(\mathrm{CH}_{2} \mathrm{Cl}_{2}, c 0.33\right)$.

\section{(R)-((3,3'-di(anthracen-9-yl)-[1,1'-binaphthalene]-2,2'-diyl)bis(oxy))bis(bis(4-fluo}

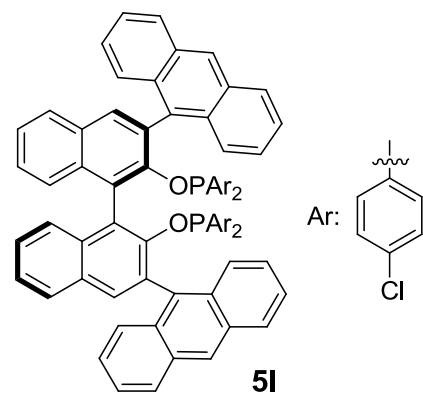

rophenyl)phosphine) (5l): The title compound was prepared according to the general procedure and purified by column chromatography to give a white solid, $135 \mathrm{mg}$, $59 \%$ yield. ${ }^{1} \mathrm{H}$ NMR $\left(400 \mathrm{MHz}, \mathrm{CDCl}_{3}\right): \delta$ 5.90-5.94 (m, 4H), 6.23-6.27 (m, 4H), $6.33(\mathrm{~d}, J=8.4 \mathrm{~Hz}, 4 \mathrm{H}), 6.72(\mathrm{~d}$, 
$J=8.0 \mathrm{~Hz}, 4 \mathrm{H}), 7.10-7.16(\mathrm{~m}, 4 \mathrm{H}), 7.33-7.37(\mathrm{~m}, 2 \mathrm{H}), 7.42-7.54(\mathrm{~m}, 4 \mathrm{H}), 7.55-7.57$ (m, 2H), 7.62-7.66 (m, 2H), $7.75(\mathrm{~s}, 2 \mathrm{H}), 7.80-7.86(\mathrm{~m}, 8 \mathrm{H}), 7.93(\mathrm{~d}, J=8.4 \mathrm{~Hz}, 2 \mathrm{H})$, $8.12(\mathrm{~s}, 2 \mathrm{H}) ;{ }^{13} \mathrm{C} \mathrm{NMR}\left(100 \mathrm{MHz}, \mathrm{CDCl}_{3}\right): \delta 124.3,124.8,124.8,125.3,125.4,125.6$, $126.1,126.7,127.0,127.1,127.1,127.6,127.6,128.2,128.2,128.4,128.5,128.6$, $128.8,129.5,129.6,129.8,131.0,131.1,131.2,131.6,132.1,133.2,133.9,133.9$, 134.5, 140.7, 153.6; ${ }^{31} \mathrm{P}$ NMR (162 MHz, $\left.\mathrm{CDCl}_{3}\right): \delta$ 107.41; HRMS (ESI) calcd for $\mathrm{C}_{72} \mathrm{H}_{45} \mathrm{Cl}_{4} \mathrm{O}_{2} \mathrm{P}_{2}[\mathrm{M}+\mathrm{H}]:$ 1143.1643, found: 1143.1637. $[\alpha]_{\mathrm{D}}{ }^{20}=-262.6\left(\mathrm{CH}_{2} \mathrm{Cl}_{2}, c\right.$ $0.29)$. 


\section{Optimization of the reaction conditions General procedure:}

The mixture of $[\mathrm{Pd}(\text { allyl }) \mathrm{Cl}]_{2}(1.8 \mathrm{mg}, 0.005 \mathrm{mmol})$, ligand $(0.011 \mathrm{mmol})$, $\mathrm{AgClO}_{4}(2.3 \mathrm{mg}, 0.011 \mathrm{mmol})$ and $\mathrm{CH}_{2} \mathrm{Cl}_{2}(0.5 \mathrm{~mL})$ was added to a Young-type tube in the glove box and stirred at $45^{\circ} \mathrm{C}$ for 30 minutes. After the mixture cooled to room temperature, aminal $(0.2 \mathrm{mmol})$ and 1,3 -diene $(0.24 \mathrm{mmol})$ were added to the mixture under nitrogen. Then the reaction mixture was degassed via the freeze-thaw method and stirred at $25{ }^{\circ} \mathrm{C}$ for 24 hours. The solvent was evaporated under reduced pressure and the residue was purified by flash column chromatography (eluted with petroleum ether/diethyl ether $=100 / 1 \sim 50 / 1)$ on a silica gel to give the desired product. The ee value was determined by HPLC with chiral column.

\subsection{Screening of ligands ${ }^{a}$}

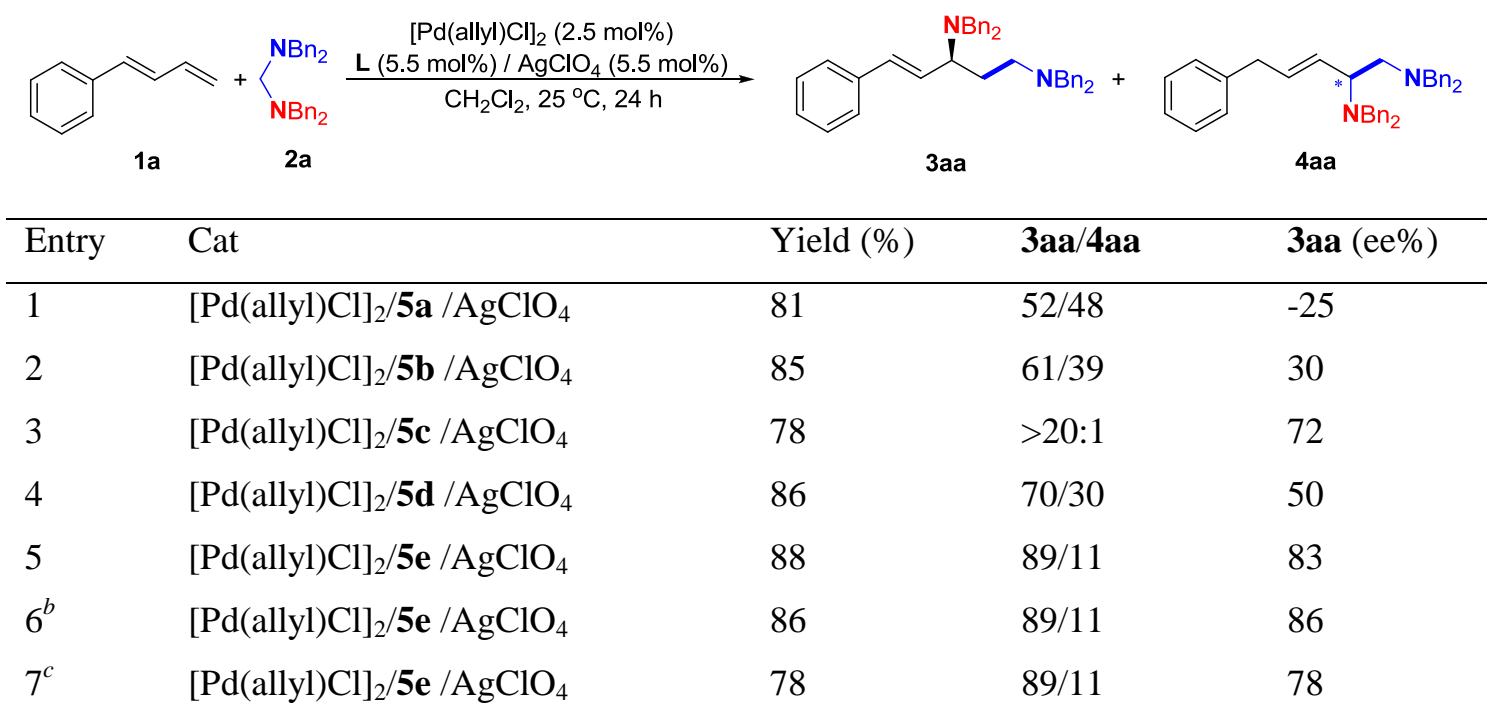

${ }^{a}$ Reaction conditions: 1a $(0.24 \mathrm{mmol}), 2 \mathrm{a}(0.2 \mathrm{mmol}),[\mathrm{Pd}(\text { allyl }) \mathrm{Cl}]_{2}(2.5 \mathrm{~mol} \%)$, ligand $(5.5$ mol\%), $\mathrm{AgClO}_{4}(5.5 \mathrm{~mol} \%), \mathrm{CH}_{2} \mathrm{Cl}_{2}(0.5 \mathrm{~mL}), 25{ }^{\circ} \mathrm{C}, 24 \mathrm{~h} .{ }^{b} 5 \mathbf{e}(7.5 \mathrm{~mol} \%) .{ }^{c} \mathbf{5 e}(10 \mathrm{~mol} \%)$. Isolated yield, the ratio of $\mathbf{3 a a} / \mathbf{4 a a}$ was determined by ${ }^{1} \mathrm{H}$ NMR, and the ee value was determined by chiral HPLC analysis. 
3.2 Screening of catalyst precursors and counter anions ${ }^{a}$

\begin{tabular}{lllll}
$\mathbf{2 a}$ & & & \\
\hline
\end{tabular}

${ }^{a}$ Reaction conditions : 1a $(0.24 \mathrm{mmol}), \mathbf{2 a}(0.2 \mathrm{mmol}),[\mathrm{Pd}(\mathrm{allyl}) \mathrm{Cl}]_{2}(2.5 \mathrm{~mol} \%), \mathbf{5 e}(5.5 \mathrm{~mol} \%)$, $\mathrm{AgX}(5.5 \mathrm{~mol} \%), \mathrm{CH}_{2} \mathrm{Cl}_{2}(0.5 \mathrm{~mL}), 25{ }^{\circ} \mathrm{C}, 24 \mathrm{~h} .{ }^{b} \mathrm{NaBARF}(5.5 \mathrm{~mol} \%) .{ }^{c} \mathrm{AgClO}_{4}$ (11 mol\%). Isolated yield, the ratio of $\mathbf{3 a a} / \mathbf{4 a a}$ was determined by ${ }^{1} \mathrm{H}$ NMR, and the ee value was determined by chiral HPLC analysis.

\subsection{Screening of solvents ${ }^{a}$}

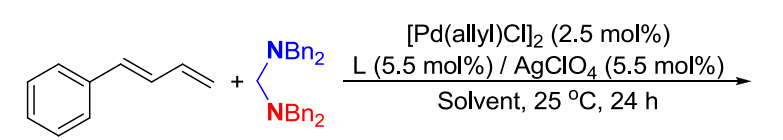

$1 \mathbf{a}$

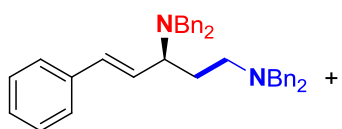

$3 a \mathbf{a}$

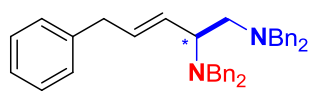

4aa

\begin{tabular}{llllll}
\hline Entry & Cat & Solvent & Yield (\%) & 3aa/4aa & 3aa (ee\%) \\
\hline 1 & {$\left[\mathrm{Pd}(\right.$ allyl $) \mathrm{Cl}_{2} / \mathbf{5 e} / \mathrm{AgClO}_{4}$} & $\mathrm{CH}_{2} \mathrm{Cl}_{2}$ & 88 & $89 / 11$ & 83 \\
2 & {$[\mathrm{Pd}(\text { allyl }) \mathrm{Cl}]_{2} / \mathbf{5 e} / \mathrm{AgClO}_{4}$} & $\mathrm{ClCH}_{2} \mathrm{CH}_{2} \mathrm{Cl}$ & 60 & $70 / 30$ & 80
\end{tabular}

${ }^{a}$ Reaction conditions : 1a $(0.24 \mathrm{mmol}), 2 \mathrm{a}(0.2 \mathrm{mmol}),[\mathrm{Pd}(\mathrm{allyl}) \mathrm{Cl}]_{2}(2.5 \mathrm{~mol} \%)$, ligand $(5.5$ mol\%), $\mathrm{AgClO}_{4}(5.5 \mathrm{~mol} \%)$, solvent $(0.5 \mathrm{~mL}), 25{ }^{\circ} \mathrm{C}, 24 \mathrm{~h}$. Isolated yield, the ratio of 3aa/4aa was determined by ${ }^{1} \mathrm{H}$ NMR, and the ee value was determined by chiral HPLC analysis. 


\subsection{Screening of ligands and temperature ${ }^{a}$}

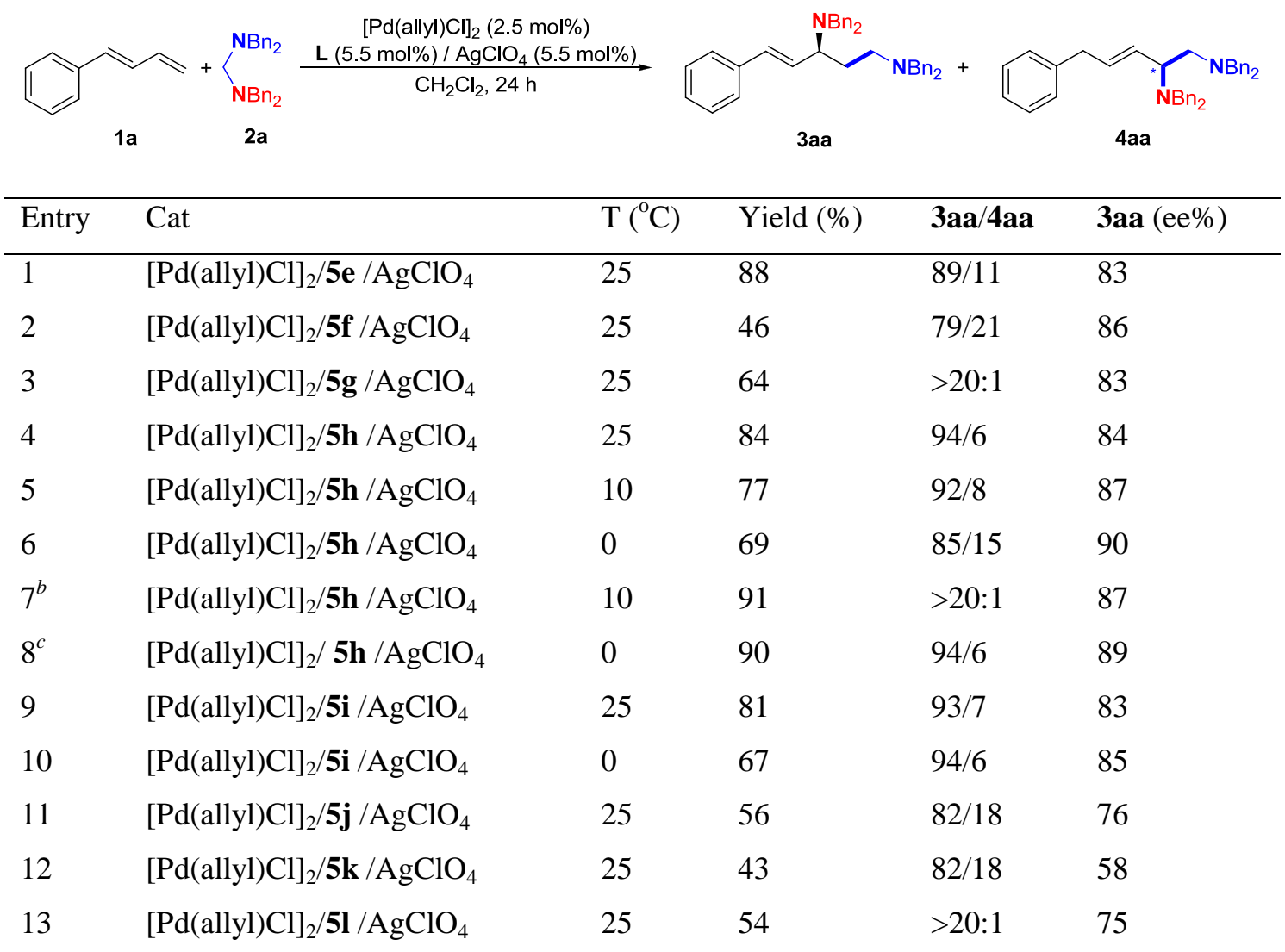

\footnotetext{
${ }^{a}$ Reaction conditions : 1a $(0.24 \mathrm{mmol}), 2 \mathrm{a}(0.2 \mathrm{mmol}),[\mathrm{Pd}(\mathrm{allyl}) \mathrm{Cl}]_{2}(2.5 \mathrm{~mol} \%)$, ligand $(5.5$ mol\%), $\mathrm{AgClO}_{4}(5.5 \mathrm{~mol} \%), \mathrm{CH}_{2} \mathrm{Cl}_{2}(0.5 \mathrm{~mL}), 24 \mathrm{~h}$. Isolated yield, the ratio of 3aa/4aa was determined by ${ }^{1} \mathrm{H}$ NMR, and the ee value was determined by chiral HPLC analysis. ${ }^{b} 48$ h. ${ }^{c}$ $[\mathrm{Pd}(\mathrm{allyl}) \mathrm{Cl}]_{2}(5 \mathrm{~mol} \%), \mathbf{5 h}(11 \mathrm{~mol} \%), \mathrm{AgClO}_{4}(11 \mathrm{~mol} \%)$.
}

\subsection{Time effect on the reactivity and selectivity ${ }^{a}$}

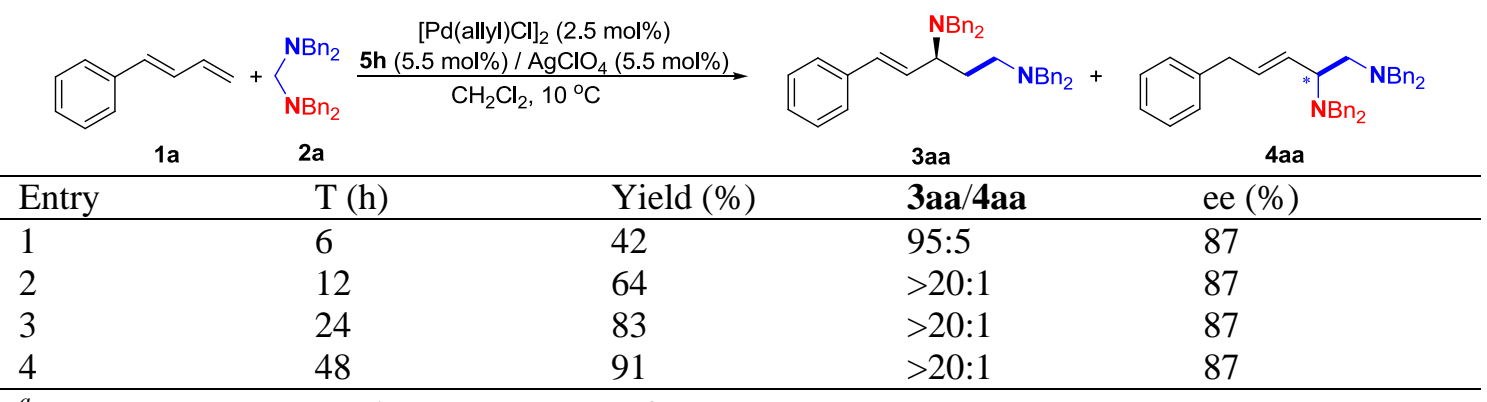

${ }^{a}$ Reaction conditions: 1a $(0.24 \mathrm{mmol}), 2 \mathrm{2a}(0.2 \mathrm{mmol}),[\mathrm{Pd}(\mathrm{allyl}) \mathrm{Cl}]_{2}(2.5 \mathrm{~mol} \%)$, ligand $(5.5$ mol\%), $\mathrm{AgClO}_{4}(5.5 \mathrm{~mol} \%), \mathrm{CH}_{2} \mathrm{Cl}_{2}(0.5 \mathrm{~mL}), 20{ }^{\circ} \mathrm{C}$. Isolated yields, the ratio of $\mathbf{3 a a} / 4 \mathbf{a a}$ was determined by ${ }^{1} \mathrm{H}$ NMR, and the ee value was for 3aa and determined by chiral HPLC analysis.

\section{General procedure for the asymmertic synthesis of chiral 1,3-diamines}




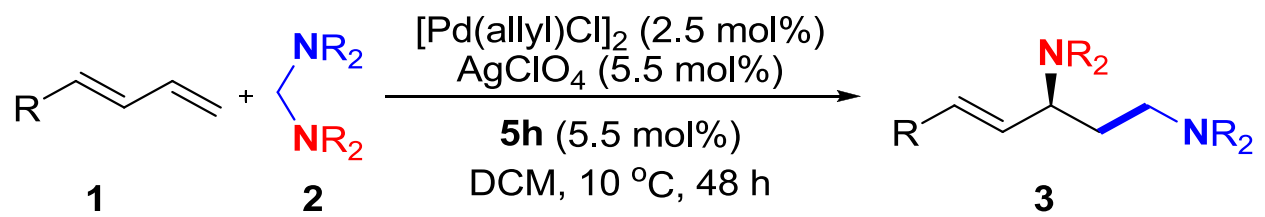

The mixture of $[\mathrm{Pd}(\text { allyl }) \mathrm{Cl}]_{2}(1.8 \mathrm{mg}, 0.005 \mathrm{mmol})$, ligand (5h) $(12.4 \mathrm{mg}, 0.011$ mmol), $\mathrm{AgClO}_{4}(2.3 \mathrm{mg}, 0.011 \mathrm{mmol})$ and $\mathrm{CH}_{2} \mathrm{Cl}_{2}(0.5 \mathrm{~mL})$ was added to a Young-type tube in the glove box and stirred at $45{ }^{\circ} \mathrm{C}$ for 30 minutes. After the mixture cooled to room temperature, aminal $(0.2 \mathrm{mmol})$ and 1,3 -diene $(0.24 \mathrm{mmol})$ were added to the mixture under nitrogen. Then the reaction mixture was degassed via the freeze-thaw method and stirred at $10{ }^{\circ} \mathrm{C}$ for 48 hours. The solvent was evaporated under reduced pressure and the residue was purified by flash column chromatography (eluted with petroleum ether/diethyl ether $=100 / 1 \sim 50 / 1$ ) on a silica gel to give the desired product. The ee value was determined by HPLC with chiral column.

\section{Experimental characterization data for new products}

$(S, E)-N^{1}, N^{1}, N^{3}, N^{3}$-tetrabenzyl-5-phenylpent-4-ene-1,3-diamine (3aa): The title compound was prepared according to the general procedure and purified by column

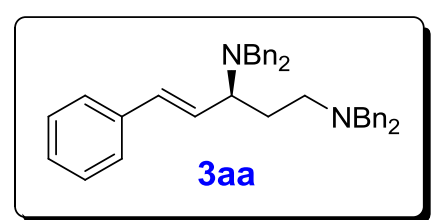

chromatography to give the colorless oil, $98 \mathrm{mg}, 91 \%$ yield (isolated yield of combination of 3aa and 4aa, 3aa:4aa > 20:1). ${ }^{1} \mathrm{H}$ NMR $\left(400 \mathrm{MHz}, \mathrm{CDCl}_{3}\right): \delta$

1.66-1.75 (m,1H), 1.97-2.06 (m, 1H), 2.27-2.33 (m, 1H), 2.59-2.66 (m, 1H), 3.20-3.25 (m, 1H), $3.28(\mathrm{~d}, J=13.6 \mathrm{~Hz}, 2 \mathrm{H}), 3.36(\mathrm{~d}, J=13.6 \mathrm{~Hz}, 2 \mathrm{H}), 3.64(\mathrm{~d}$, $J=13.6 \mathrm{~Hz}, 2 \mathrm{H}), 3.77(\mathrm{~d}, J=13.6 \mathrm{~Hz}, 2 \mathrm{H}), 5.96(\mathrm{~d}, J=16.0 \mathrm{~Hz}, 1 \mathrm{H}), 6.07(\mathrm{dd}$, $\left.J_{1}=8.8 \mathrm{~Hz}, J_{2}=16.0 \mathrm{~Hz}, 1 \mathrm{H}\right), 7.18-7.24(\mathrm{~m}, 13 \mathrm{H}), 7.28-7.31(\mathrm{~m}, 12 \mathrm{H}) ;{ }^{13} \mathrm{C}$ NMR $\left(100 \mathrm{MHz}, \mathrm{CDCl}_{3}\right) \delta 30.6,51.0,54.0,58.6,58.8,126.4,126.7,126.8$, 127.3, 128.2, 128.2, 128.5, 128.8, 129.0, 133.1, 137.3, 139.9, 140.4; HRMS (ESI) calcd for $\mathrm{C}_{39} \mathrm{H}_{41} \mathrm{~N}_{2}[\mathrm{M}+\mathrm{H}]: 537.3264$, found: 537.3253 . The ee was determined to be 
90\% ee [Determined by HPLC with a Chiralcel IA column (hexane: isopropanol= $\left.\left.99: 1,0.6 \mathrm{~mL} / \mathrm{min}, 254 \mathrm{~nm}, 25^{\circ} \mathrm{C}\right) ; \mathrm{t}_{3 \mathbf{a a} \text { (major) }}=7.5 \mathrm{~min}, \mathrm{t}_{3 \mathbf{a a}(\text { minor })}=9.9 \mathrm{~min}\right] .[\alpha]_{\mathrm{D}}{ }^{20}=$ $-65.9\left(\mathrm{CH}_{2} \mathrm{Cl}_{2}, c 0.27\right)$.

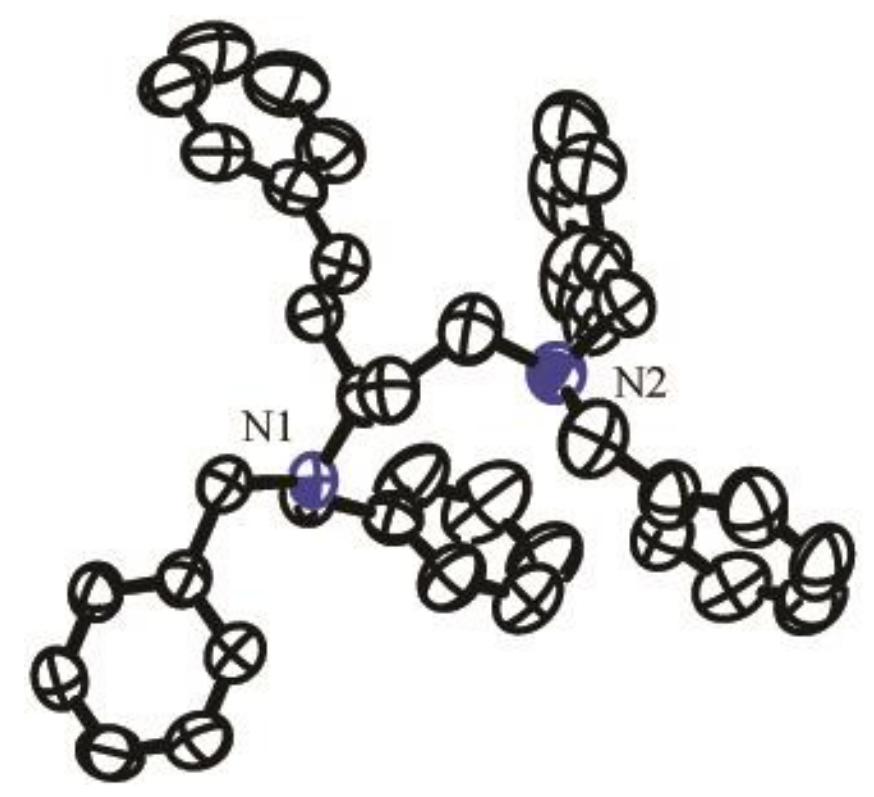

Figure S-2. X-ray structure of the product 3aa

(E)- $N^{1}, N^{1}, N^{2}, N^{2}$-tetrabenzyl-5-phenylpent-3-ene-1,2-diamine (4aa): ${ }^{1} \mathrm{H} \quad \mathrm{NMR}$

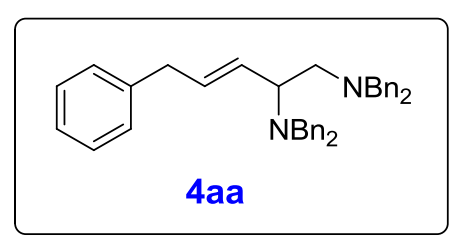
$\left(400 \mathrm{MHz}, \mathrm{CDCl}_{3}\right) \delta 2.66(\mathrm{~d}, J=7.68 \mathrm{~Hz}, 2 \mathrm{H})$, 3.00-3.02 (m, 2H), 3.48-3.63 (m, 9H), 5.49-5.56 (m, $1 \mathrm{H}), 5.66\left(\mathrm{dd}, J_{1}=7.6 \mathrm{~Hz}, J_{2}=15.6 \mathrm{~Hz}, 1 \mathrm{H}\right)$, 7.00-7.03 (m, 2H), 7.17-7.34 (m, 23H); $\left.{ }^{13} \mathrm{C} \mathrm{NMR} \mathrm{(100} \mathrm{MHz,} \mathrm{CDCl}_{3}\right) \delta 47.1$, $55.7,57.9,58.6,59.5,126.2,126.8,128.0,128.1,128.2,128.3,128.8,128.9$, 135.9, 139.6, 139.8, 143.5; HRMS (ESI) calcd for $\mathrm{C}_{39} \mathrm{H}_{41} \mathrm{~N}_{2}[\mathrm{M}+\mathrm{H}]$ : 537.3264, found: 537.3267. The structure of 4aa was further comfirmed by 2D NMR and deuteride product. 


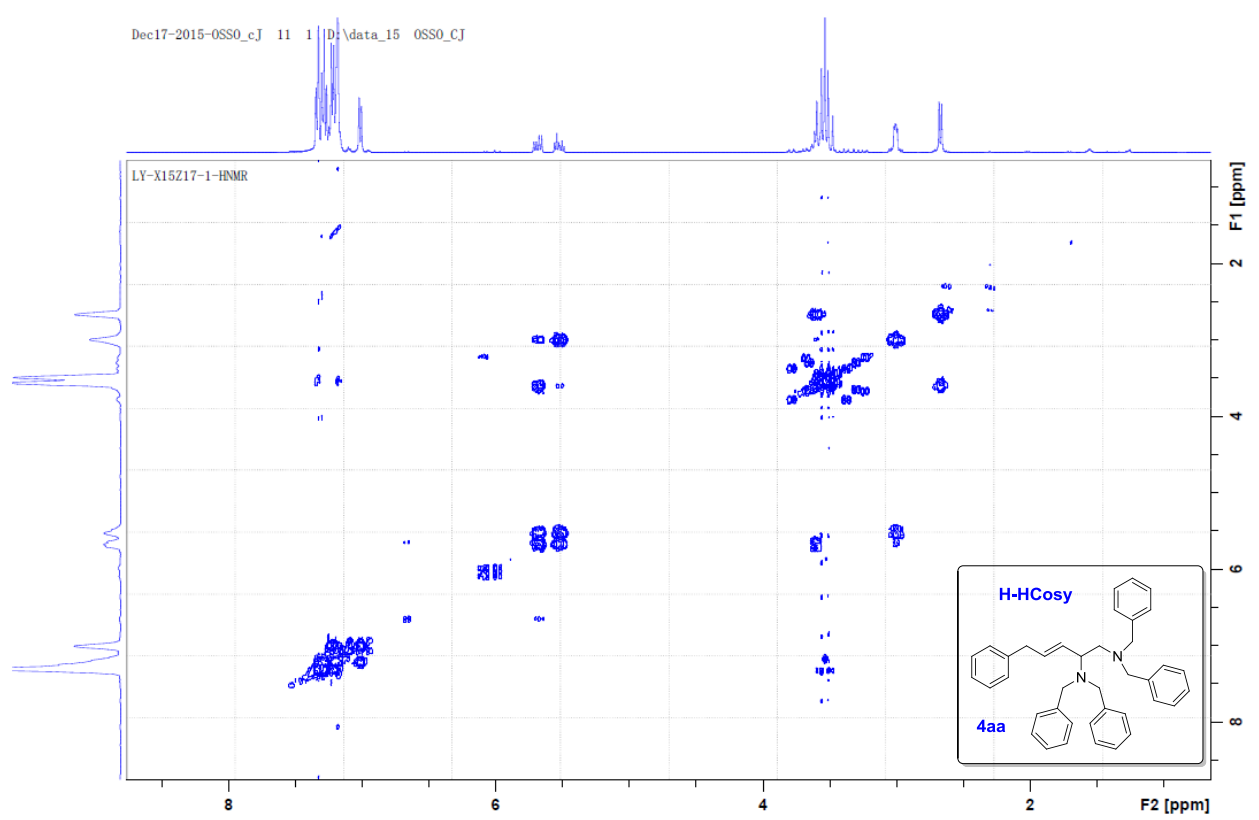

Figure S-3 H-HCOSY of 4aa

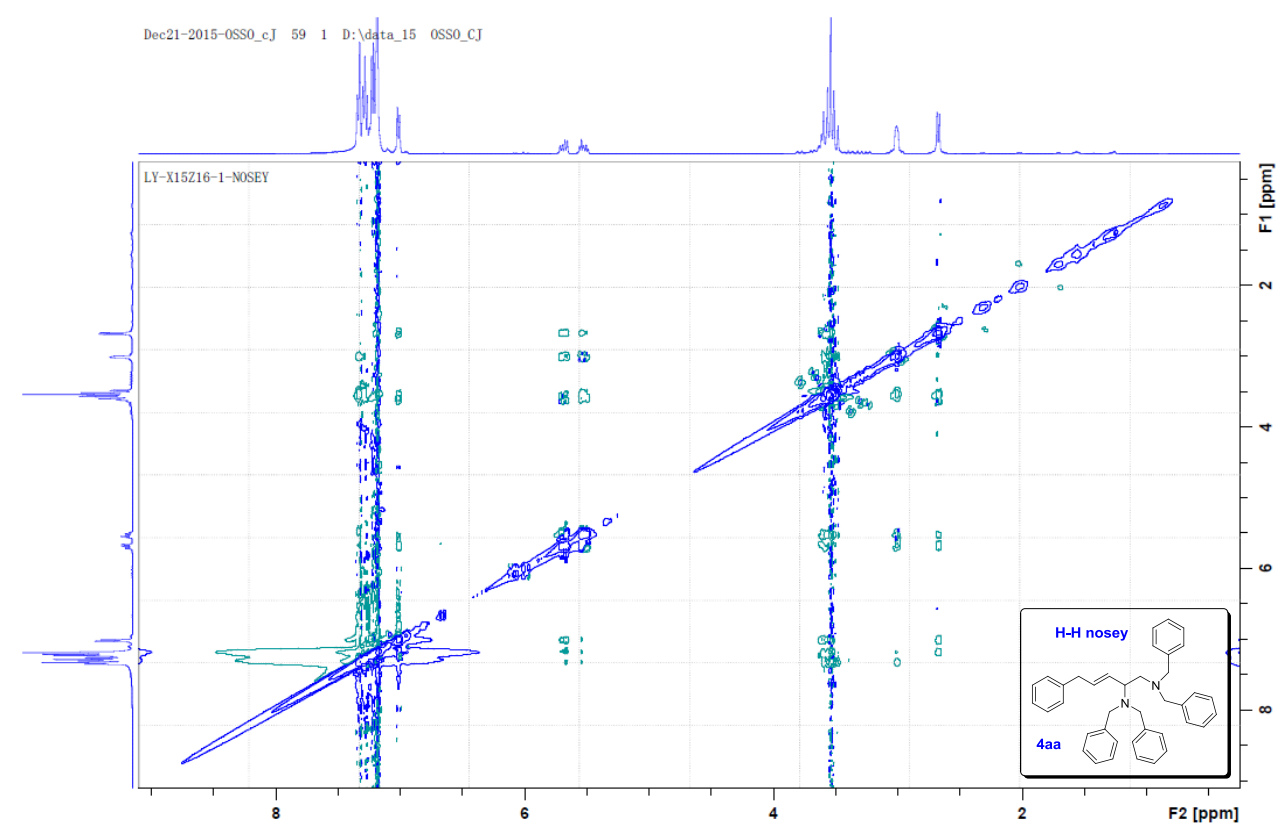

Figure S-3 H-H NOSEY of 4aa

$(E)-N^{1}, N^{1}, N^{2}, N^{2}$-tetrabenzyl-5-phenylpent-3-ene-1,2-diamine (4aa- $\left.d_{2}\right):{ }^{1} \mathrm{H}$ NMR

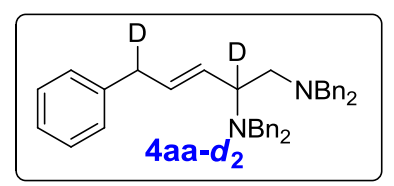

$\left(400 \mathrm{MHz}, \mathrm{CDCl}_{3}\right) \delta 2.66(\mathrm{~d}, J=7.6 \mathrm{~Hz}, 2 \mathrm{H}), 3.48-3.61$

$(\mathrm{m}, 9 \mathrm{H}), 5.50(\mathrm{~d}, J=15.6 \mathrm{~Hz}, 1 \mathrm{H}), 5.65\left(\mathrm{dd}, J_{1}=7.6 \mathrm{~Hz}\right.$,

$\left.J_{2}=15.6 \mathrm{~Hz}, 1 \mathrm{H}\right), 7.00-7.03(\mathrm{~m}, 2 \mathrm{H}), 7.18-7.26(\mathrm{~m}, 14 \mathrm{H}), 7.28-7.34(\mathrm{~m}$, 
$10 \mathrm{H}) ;{ }^{13} \mathrm{C} \mathrm{NMR}\left(100 \mathrm{MHz}, \mathrm{CDCl}_{3}\right) \delta 47.1,57.8,58.6,59.5,126.2,126.8,128.0$, $128.1,128.2,128.2,128.3,128.8,128.9,136.0,139.6,139.8,143.5$; HRMS (ESI) calcd for $\mathrm{C}_{39} \mathrm{H}_{39} \mathrm{D}_{2} \mathrm{~N}_{2}[\mathrm{M}+\mathrm{H}]: 539.3390$, found: 539.3392 .
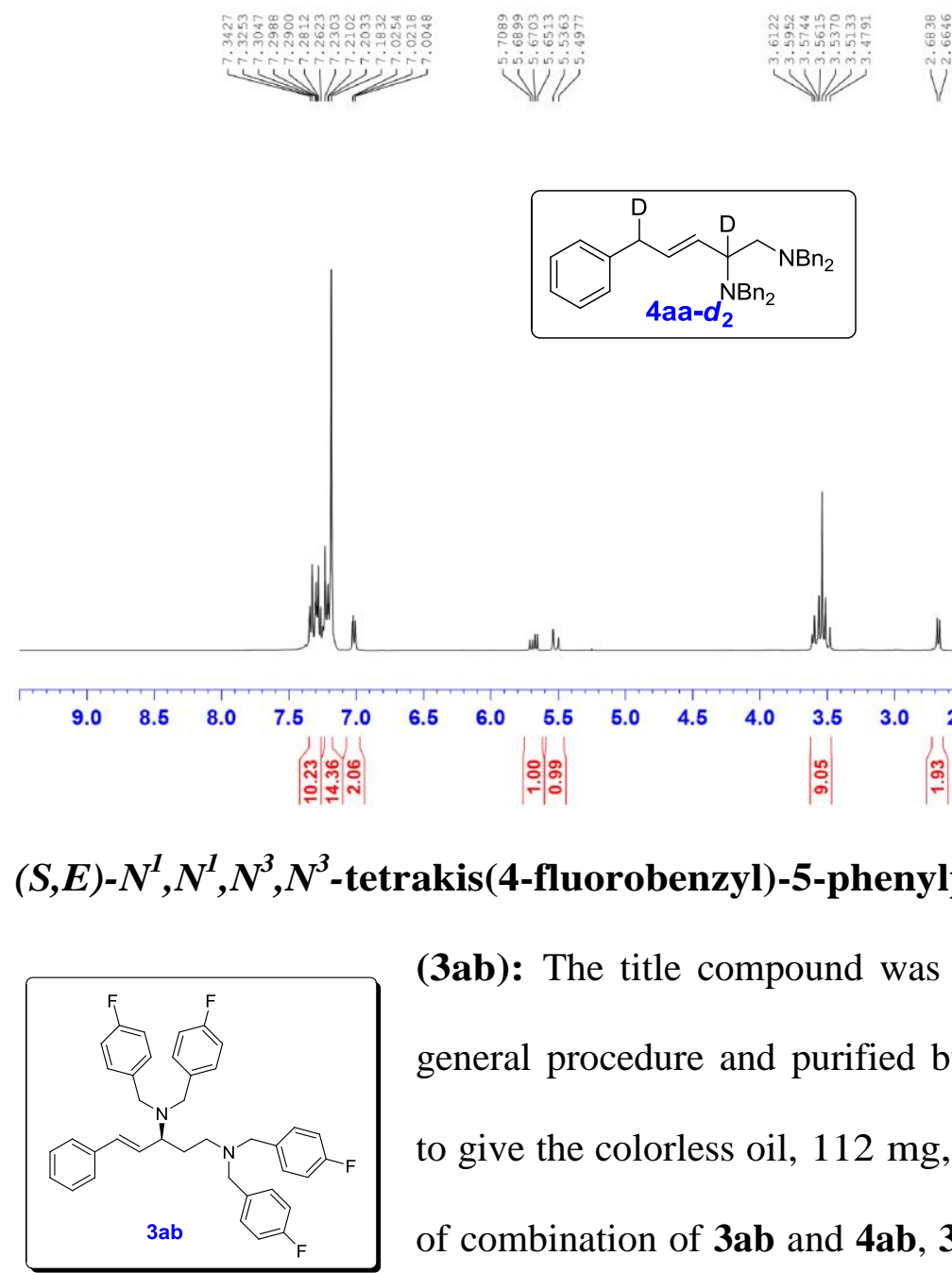

(3ab): The title compound was prepared according to the general procedure and purified by column chromatography to give the colorless oil, $112 \mathrm{mg}, 92 \%$ yield (isolated yield of combination of 3ab and 4ab, 3ab:4ab = 93:7). ${ }^{1} \mathrm{H}$ NMR $\left(400 \mathrm{MHz}, \mathrm{CDCl}_{3}\right) \delta 1.65-1.74(\mathrm{~m}, 1 \mathrm{H}), 1.89-1.98(\mathrm{~m}, 1 \mathrm{H}), 2.21-2.28(\mathrm{~m}, 1 \mathrm{H})$, 2.53-2.60 (m, 1H), 3.13-3.19 (m, 1H), $3.20(\mathrm{~d}, J=13.2 \mathrm{~Hz}, 2 \mathrm{H}), 3.30(\mathrm{~d}, J=$ $13.2 \mathrm{~Hz}, 2 \mathrm{H}), 3.56(\mathrm{~d}, J=13.2 \mathrm{~Hz}, 2 \mathrm{H}), 3.67(\mathrm{~d}, J=13.6 \mathrm{~Hz}, 2 \mathrm{H}), 5.98-6.09(\mathrm{~m}$, 2H), 6.90-6.94 (m, 4H), 6.96-7.00 (m, 4H), 7.11-7.15 (m, 4H), 7.19-7.29 (m, 5H), 7.30-7.35 (m, 4H); ${ }^{13} \mathrm{C}$ NMR (100 MHz, $\left.\mathrm{CDCl}_{3}\right) \delta 30.7,50.7,53.1,57.8$, $58.5,114.9,114.9,115.1,115.1,126.3,127.5,127.6,128.6,130.1,130.1,130.3,130.3$ 133.6, 135.3, 135.3, 135.8, 135.8, 136.8, 160.7, 163.1; ${ }^{19} \mathrm{~F}$ NMR $\left(376 \mathrm{MHz}, \mathrm{CDCl}_{3}\right)$ 
$\delta$-115.9, -116.0; HRMS (ESI) calcd for $\mathrm{C}_{39} \mathrm{H}_{37} \mathrm{~F}_{4} \mathrm{~N}_{2}[\mathrm{M}+\mathrm{H}]$ : 609.2887, found: 609.2899. The ee was determined to be $88 \%$ ee [Determined by HPLC with a Chiralcel IA column (hexane: isopropanol $=98: 2,0.5 \mathrm{~mL} / \mathrm{min}, 254 \mathrm{~nm}, 25{ }^{\circ} \mathrm{C}$ ); $\mathrm{t}_{3 \mathbf{a b}}$ $($ major $\left.)=9.8 \mathrm{~min}, \mathrm{t}_{3 \mathbf{a b} \text { (minor) }}=13.5 \mathrm{~min}\right] .[\alpha]_{\mathrm{D}}^{20}=-75.6\left(\mathrm{CH}_{2} \mathrm{Cl}_{2}, c 0.23\right)$.

$(S, E)-N^{1}, N^{1}, N^{3}, N^{3}$-tetrakis(4-fluorobenzyl)-5-phenylpent-4-ene-1,3-diamine

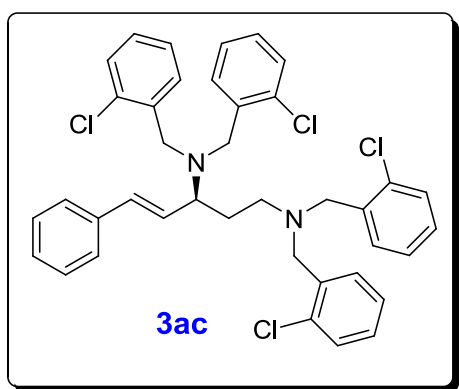

(3ac): The title compound was prepared according to the general procedure and purified by column chromatography to give colorless oil, $125 \mathrm{mg}, 93 \%$ yield (isolated yield of combination of 3ac and 4ac, 3ac:4ac > 20:1). ${ }^{1} \mathrm{H}$ NMR $\left(400 \mathrm{MHz}, \mathrm{CDCl}_{3}\right) \delta$ $1.70-1.79(\mathrm{~m}, 1 \mathrm{H}), 1.99-2.08(\mathrm{~m}, 1 \mathrm{H}), 2.34-2.42(\mathrm{~m}, 1 \mathrm{H}), 2.55-2.62(\mathrm{~m}, 1 \mathrm{H})$, 3.10-3.15 (m, 1H), 3.56-3.64 (m, 4H), 3.67-3.75 (m, 4H), $6.04\left(\mathrm{dd}, J_{1}=8.2 \mathrm{~Hz}\right.$, $\left.J_{2}=16.0 \mathrm{~Hz}, 1 \mathrm{H}\right), 6.14(\mathrm{~d}, J=16.0 \mathrm{~Hz}, 1 \mathrm{H}), 7.00-7.10(\mathrm{~m}, 8 \mathrm{H}), 7.16-7.24(\mathrm{~m}$, 9H), 7.34-7.37 (m, 2H), $7.43(\mathrm{~d}, J=7.2 \mathrm{~Hz}, 2 \mathrm{H}) ;{ }^{13} \mathrm{C} \mathrm{NMR}\left(100 \mathrm{MHz}, \mathrm{CDCl}_{3}\right)$ $\delta 30.0,51.1,51.7,55.4,60.4,126.5,126.6,126.7,127.5,127.6,127.8,127.9,128.5$, 129.3, 129.3, 130.2, 130.5, 133.8, 133.9, 134.0, 137.0, 137.3; HRMS (ESI) calcd for $\mathrm{C}_{39} \mathrm{H}_{37} \mathrm{Cl}_{4} \mathrm{~N}_{2}[\mathrm{M}+\mathrm{H}]:$ : 673.1705 , found: 673.1737 . The ee was determined to be $84 \%$ ee [Determined by HPLC with a Chiralcel OD-H column (hexane: isopropanol = $\left.\left.90: 10,0.8 \mathrm{~mL} / \min , 254 \mathrm{~nm}, 25^{\circ} \mathrm{C}\right) ; \mathrm{t}_{3 \mathbf{a c}(\text { major })}=18.6 \mathrm{~min}, \mathrm{t}_{3 \mathbf{a c}(\operatorname{minor})}=23.0 \mathrm{~min}\right] .[\alpha]_{\mathrm{D}}{ }^{20}$ $=-22.1\left(\mathrm{CH}_{2} \mathrm{Cl}_{2}, c\right.$ 1.12).

$(S, E)-N^{1}, N^{1}, N^{3}, N^{3}$-tetrakis(4-chlorobenzyl)-5-phenylpent-4-ene-1,3-diamine

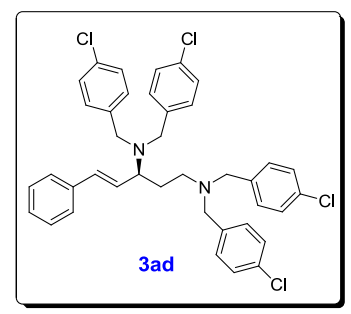
(3ad): The title compound was prepared according to the general procedure and purified by column chromatography to give colorless oil, $122 \mathrm{mg}$, 91\% yield (isolated yield of combination of $\mathbf{3 a d}$ and 4ad, 3ad:4ad > 20:1). ${ }^{1} \mathrm{H}$ NMR (400 
$\left.\mathrm{MHz}, \mathrm{CDCl}_{3}\right) \delta 1.64-1.73(\mathrm{~m}, 1 \mathrm{H}), 1.88-1.96(\mathrm{~m}, 1 \mathrm{H}), 2.22-2.29(\mathrm{~m}, 1 \mathrm{H})$, 2.51-2.59 (m, 1H), 3.11-3.17 (m, 1H), $3.20(\mathrm{~d}, J=13.6 \mathrm{~Hz}, 2 \mathrm{H}), 3.31(\mathrm{~d}, J=$ $13.6 \mathrm{~Hz}, 2 \mathrm{H}), 3.56(\mathrm{~d}, J=13.6 \mathrm{~Hz}, 2 \mathrm{H}), 3.66(\mathrm{~d}, J=13.6 \mathrm{~Hz}, 2 \mathrm{H}), 5.59-6.06(\mathrm{~m}$, 2H), $7.09(\mathrm{~d}, J=8.4 \mathrm{~Hz}, 4 \mathrm{H}), 7.18-7.22(\mathrm{~m}, 8 \mathrm{H}), 7.25-7.30(\mathrm{~m}, 7 \mathrm{H}), 7.32-7.36$ $(\mathrm{m}, 2 \mathrm{H}) ;{ }^{13} \mathrm{C}$ NMR $\left(100 \mathrm{MHz}, \mathrm{CDCl}_{3}\right) \delta 30.7,50.8,53.2,57.9,58.6,126.3$, $127.1,127.6,128.4,128.4,128.6,129.9,130.1,132.6,132.6,133.8,136.7$, 138.0, 138.5; HRMS (ESI) calcd for $\mathrm{C}_{39} \mathrm{H}_{37} \mathrm{Cl}_{4} \mathrm{~N}_{2}[\mathrm{M}+\mathrm{H}]$ : 673.1705, found: 673.1704, The ee was determined to be $93 \%$ ee [Determined by HPLC with a Chiralcel OD-H column (hexane: isopropanol=90:10, $0.8 \mathrm{~mL} / \mathrm{min}, 254 \mathrm{~nm}, 25^{\circ} \mathrm{C}$ ); $\left.\mathrm{t}_{3 \text { ad (major) }}=29.7 \mathrm{~min}, \mathrm{t}_{3 \text { ad (minor) }}=48.1 \mathrm{~min}\right] .[\alpha]_{\mathrm{D}}{ }^{20}=-55.4\left(\mathrm{CH}_{2} \mathrm{Cl}_{2}, c 1.09\right)$.

\section{$(S, E)-N^{1}, N^{1}, N^{3}, N^{3}$-tetrakis(2-bromobenzyl)-5-phenylpent-4-ene-1,3-diamin}

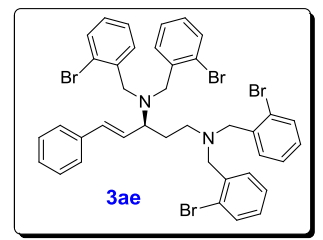

e (3ae): The title compound was prepared according to the general procedure and purified by column chromatography to give the colorless oil, $144 \mathrm{mg}, 85 \%$ yield (isolated yield of combination of 3ae and 4ae, 3ae:4ae > 20:1). ${ }^{1} \mathrm{H}$ NMR $\left(400 \mathrm{MHz}, \mathrm{CDCl}_{3}\right) \delta$ 1.79-1.88 (m, 1H), 2.08-2.17 (m, 1H), 2.45-2.52 (m, 1H), 2.64-2.71 (m, 1H), 3.17-3.23 (m, 1H), 3.64-3.71 (m, 4H), 3.74-3.81 (m, 4H), $6.12\left(\mathrm{dd}, J_{1}=8.4 \mathrm{~Hz}\right.$, $\left.J_{2}=16.0 \mathrm{~Hz}, 1 \mathrm{H}\right), 6.24(\mathrm{~d}, J=16.0 \mathrm{~Hz}, 1 \mathrm{H}), 6.98-7.05(\mathrm{~m}, 4 \mathrm{H}), 7.12-7.13(\mathrm{~m}$, 2H), 7.15-7.25 (m, 3H), 7.29-7.34 (m, 4H), 7.43-7.53 (m, 8H); ${ }^{13} \mathrm{C}$ NMR (100 $\left.\mathrm{MHz}, \mathrm{CDCl}_{3}\right) \delta 29.9,51.7,53.7,58.0,60.6,124.2,124.3,126.5,127.2,127.3,127.5$, $127.6,128.2,128.2,128.5,130.3,130.7,132.6,132.6,133.9,137.0,138.6,138.8$ HRMS (ESI) calcd for $\mathrm{C}_{39} \mathrm{H}_{37} \mathrm{Br}_{4} \mathrm{~N}_{2}[\mathrm{M}+\mathrm{H}]:$ 848.9685, found: 848.9681. The ee was determined to be $91 \%$ ee [Determined by HPLC with a Chiralcel IA column (hexane: isopropanol=99:1, $\left.0.5 \mathrm{~mL} / \mathrm{min}, 254 \mathrm{~nm}, 25{ }^{\circ} \mathrm{C}\right) ; \mathrm{t}_{3 \mathbf{a e}(\text { major })}=9.0 \mathrm{~min}, \mathrm{t}_{3 \mathbf{a e}(\text { minor })}=11.8$ $\min ] .[\alpha]_{\mathrm{D}}{ }^{20}=-48.6\left(\mathrm{CH}_{2} \mathrm{Cl}_{2}, c 1.33\right)$. 


\section{$(S, E)-N^{1}, N^{1}, N^{3}, N^{3}$-tetrakis(4-bromobenzyl)-5-phenylpent-4-ene-1,3-diamine}

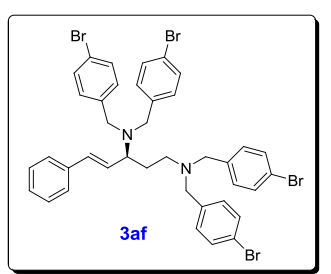

(3af): The title compound was prepared according to the general procedure and purified by column chromatography to give the colorless oil, $139 \mathrm{mg}, 82 \%$ yield (isolated yield of combination of 3af and 4af, 3af:4af > 20:1). ${ }^{1} \mathrm{H}$ NMR $\left(400 \mathrm{MHz}, \mathrm{CDCl}_{3}\right) \delta 1.64-1.72(\mathrm{~m}, 1 \mathrm{H})$, 1.87-1.95 (m, 1H), 2.23-2.29 (m, 1H), 2.51-2.58 (m, 1H), 3.12-3.17 (m, 1H), $3.19(\mathrm{~d}, J=13.6 \mathrm{~Hz}, 2 \mathrm{H}), 3.30(\mathrm{~d}, J=13.6 \mathrm{~Hz}, 2 \mathrm{H}), 3.54(\mathrm{~d}, J=13.6 \mathrm{~Hz}, 2 \mathrm{H})$, $3.64(\mathrm{~d}, J=13.6 \mathrm{~Hz}, 2 \mathrm{H}), 5.69-6.06(\mathrm{~m}, 2 \mathrm{H}), 7.04(\mathrm{~d}, J=8.4 \mathrm{~Hz}, 4 \mathrm{H}), 7.11(\mathrm{~d}$, $J=8.4 \mathrm{~Hz}, 4 \mathrm{H}), 7.24-7.30(\mathrm{~m}, 3 \mathrm{H}), 7.32-7.38(\mathrm{~m}, 6 \mathrm{H}), 7.41(\mathrm{~d}, J=8.4 \mathrm{~Hz}, 4 \mathrm{H})$;

${ }^{13} \mathrm{C}$ NMR $\left(100 \mathrm{MHz}, \mathrm{CDCl}_{3}\right) \delta 30.7,50.9,53.3,58.0,58.7,120.7,120.8,126.3$, 127.1, 127.7, 128.6, 130.3, 130.5, 131.4, 131.4, 133.8, 136.7, 138.5, 139.0; HRMS (ESI) calcd for $\mathrm{C}_{39} \mathrm{H}_{37} \mathrm{Br}_{4} \mathrm{~N}_{2}[\mathrm{M}+\mathrm{H}]$ : 848.9685, found: 848.9690. The ee was determined to be $94 \%$ ee [Determined by HPLC with a Chiralcel OD-H column (hexane: isopropanol $=90: 10,0.8 \mathrm{~mL} / \mathrm{min}, 254 \mathrm{~nm}, 25^{\circ} \mathrm{C}$ ); $\mathrm{t}_{3 \mathbf{a f}(\text { major) }}=30.2 \mathrm{~min}, \mathrm{t}_{3 \mathbf{a f}}$ (minor) $=60.3 \mathrm{~min}] .[\alpha]_{\mathrm{D}}^{20}=-31.3\left(\mathrm{CH}_{2} \mathrm{Cl}_{2}, c 0.40\right)$.

(S,E)-5-phenyl- $N^{1}, N^{1}, N^{3}, N^{3}$-tetrakis(4-(trifluoromethyl)benzyl)pent-4-ene-1,3-dia

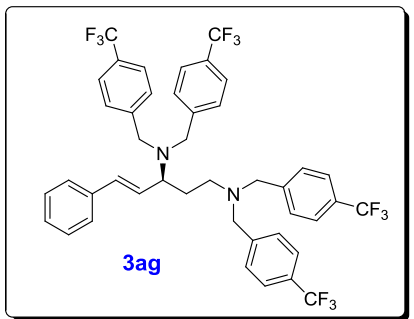

mine (3ag): The title compound was prepared according to the general procedure and purified by column chromatography to give colorless oil, $110 \mathrm{mg}, 68 \%$ yield (isolated yield of combination of 3ag and 4ag, 3ag:4ag $=$

76:24). ${ }^{1} \mathrm{H}$ NMR (400 MHz, $\left.\mathrm{CDCl}_{3}\right) \delta 1.74-1.83(\mathrm{~m}, 0.8 \mathrm{H}), 1.98-2.07$ (m, 0.8H), 2.34-2.40 (m, 0.8H), 2.59-2.73 (m, 1.2H), $3.00(\mathrm{~d}, J=6.4 \mathrm{~Hz}, 0.4 \mathrm{H}), 3.15-3.21$ $(\mathrm{m}, 0.8 \mathrm{H}), 3.37(\mathrm{~d}, J=14.0 \mathrm{~Hz}, 1.5 \mathrm{H}), 3.47-3.70(\mathrm{~m}, 5.2 \mathrm{H}), 3.77(\mathrm{~d}, J=14.0$ $\mathrm{Hz}, 1.5 \mathrm{H}), 5.47-5.72(\mathrm{~m}, 0.4 \mathrm{H}), 6.02-6.11(\mathrm{~m}, 1.6 \mathrm{H}), 6.98-7.00(\mathrm{~m}, 0.4 \mathrm{H})$, 7.21-7.38 (m, 9H), 7.40-7.49 (m, 8H), $7.54(\mathrm{~d}, J=8.0 \mathrm{~Hz}, 4 \mathrm{H}) ;{ }^{13} \mathrm{C}$ NMR $(100$ 
$\left.\mathrm{MHz} \mathrm{CDCl}_{3}\right) \delta 30.6,47.2,51.2,53.7,56.1,57.7,58.2,58.3,59.3,59.7,122.8,125.1$, $125.1,125.2,125.2,125.3,125.3,125.5,126.3,126.6,127.8,127.9,128.5,128.7$, $128.8,128.8,128.9,129.2,129.3,129.5,129.6,134.2,136.1,136.4,142.7,143.3$, 143.5, 143.6, 144.0; ${ }^{19} \mathrm{~F}$ NMR $\left(376 \mathrm{MHz}, \mathrm{CDCl}_{3}\right) \delta-62.4,-62.4,-62.4$; HRMS (ESI) calcd for $\mathrm{C}_{43} \mathrm{H}_{37} \mathrm{~F}_{12} \mathrm{~N}_{2}[\mathrm{M}+\mathrm{H}]$ : 809.2760, found: 809.2777. The ee was determined to be $96 \%$ ee [Determined by HPLC with a Chiralcel IA column (hexane: isopropanol $\left.=98: 2,0.6 \mathrm{~mL} / \mathrm{min}, 254 \mathrm{~nm}, 25^{\circ} \mathrm{C}\right) ; \mathrm{t}_{3 \mathbf{a g}(\text { major })}=11.3 \mathrm{~min}, \mathrm{t}_{3 \mathrm{ag} \text { (minor) }}=14.0$ $\min ] .[\alpha]_{\mathrm{D}}^{20}=-31.3\left(\mathrm{CH}_{2} \mathrm{Cl}_{2}, c 0.40\right)$.

$(S, E)-N^{1}, N^{1}, N^{3}, N^{3}$-tetrakis(4-methoxybenzyl)-5-phenylpent-4-ene-1,3-diami

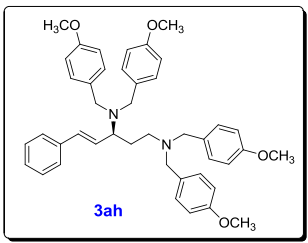
ne (3ah): The title compound was prepared according to the general procedure and purified by column chromatography to give colorless oil, $60 \mathrm{mg}, 46 \%$ yield (isolated yield of combination of 3ah and 4ah, 3ah:4ah > 20:1). ${ }^{1} \mathrm{H}$ NMR (400 $\left.\mathrm{MHz}, \mathrm{CDCl}_{3}\right) \delta$ 1.64-1.72 (m, 1H), 1.93-2.02 (m, 1H), 2.21-2.27 (m, 1H), 2.57-2.65 (m, 1H), 3.18-3.24 (m, 3H), $3.27(\mathrm{~d}, J=13.6 \mathrm{~Hz}, 2 \mathrm{H}), 3.56(\mathrm{~d}, J=13.2 \mathrm{~Hz}, 2 \mathrm{H}), 3.69(\mathrm{~d}$, $J=13.2 \mathrm{~Hz}, 2 \mathrm{H}), 3.75(\mathrm{~s}, 6 \mathrm{H}), 3.79(\mathrm{~s}, 6 \mathrm{H}), 6.00-6.12(\mathrm{~m}, 2 \mathrm{H}), 6.77(\mathrm{~d}, J=8.8$ $\mathrm{Hz}, 4 \mathrm{H}), 6.83(\mathrm{~d}, J=8.8 \mathrm{~Hz}, 4 \mathrm{H}), 7.10(\mathrm{~d}, J=8.8 \mathrm{~Hz}, 4 \mathrm{H}), 7.18-7.22(\mathrm{~m}, 5 \mathrm{H})$, 7.30-7.31 (m, 4H); ${ }^{13} \mathrm{C}$ NMR $\left(100 \mathrm{MHz}, \mathrm{CDCl}_{3}\right) \delta 30.5,50.6,53.0,55.2,55.3$, $57.7,58.2,113.5,113.6,126.3,127.3,128.4,128.5,129.8,130.0,130.1,131.9,132.5$, 133.0, 137.3, 158.5; HRMS (ESI) calcd for $\mathrm{C}_{43} \mathrm{H}_{49} \mathrm{~N}_{2} \mathrm{O}_{4}[\mathrm{M}+\mathrm{H}]$ : 657.3687, found: 657.3693. The ee was determined to be $78 \%$ ee [Determined by HPLC with a Chiralcel IA column (hexane: isopropanol=95:5, $0.6 \mathrm{~mL} / \mathrm{min}, 254 \mathrm{~nm}, 25{ }^{\circ} \mathrm{C}$ ); $\mathrm{t}_{3 a h}$ $\left(\right.$ major) $\left.=17.0 \mathrm{~min}, \mathrm{t}_{3 \mathbf{a h}(\text { minor })}=22.9 \mathrm{~min}\right] .[\alpha]_{\mathrm{D}}{ }^{20}=-58.0\left(\mathrm{CH}_{2} \mathrm{Cl}_{2}, c 0.10\right)$.

$(S, E)-N^{1}, N^{1}, N^{3}, N^{3}$-tetraethyl-5-phenylpent-4-ene-1,3-diamine (3ai): The title compound was prepared according to the general procedure and purified by column 


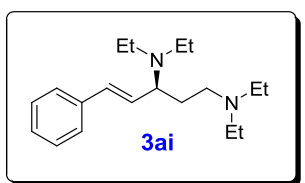

chromatography to give the colorless oil, $23 \mathrm{mg}, 40 \%$ yield (isolated yield of combination of 3ai and 4ai, 3ai:4ai > 20:1). ${ }^{1} \mathrm{H}$ NMR $\left(400 \mathrm{MHz}, \mathrm{CDCl}_{3}\right) \delta \quad 0.99-1.07 \quad(\mathrm{~m}, \quad 12 \mathrm{H})$,

1.60-1.69 (m, 1H), 1.81-1.90 (m, 1H), 2.40-2.57 (m, 8H), 2.65-2.74 (m, 2H), 3.21-3.27 (m, 1H), $6.11\left(\mathrm{dd}, J_{1}=8.8 \mathrm{~Hz}, J_{2}=16.0 \mathrm{~Hz}, 1 \mathrm{H}\right), 6.40(\mathrm{~d}, J=16.0$ $\mathrm{Hz}, 1 \mathrm{H}), 7.20-7.24(\mathrm{~m}, 1 \mathrm{H}), 7.29-7.33(\mathrm{~m}, 2 \mathrm{H}), 7.36-7.38(\mathrm{~m}, 2 \mathrm{H}) ;{ }^{13} \mathrm{C}$ NMR $\left(100 \mathrm{MHz}, \mathrm{CDCl}_{3}\right) \delta 11.8,13.7,30.1,43.7,47.0,50.4,61.1,126.2,127.2,128.5$, 130.1, 131.8, 137.3 ; HRMS (ESI) calcd for $\mathrm{C}_{19} \mathrm{H}_{33} \mathrm{~N}_{2}[\mathrm{M}+\mathrm{H}]$ : 289.2638, found: 289.2631. The ee was determined to be 55\% ee [Determined by HPLC with a Chiralcel IA column (hexane: isopropanol $=90: 10,0.6 \mathrm{~mL} / \mathrm{min}, 254 \mathrm{~nm}, 25^{\circ} \mathrm{C}$ ); $\mathrm{t}_{3 \mathrm{ai}}$ $\left(\right.$ major) $\left.=24.1 \mathrm{~min}, \mathrm{t}_{3 \mathrm{ai}(\text { minor })}=26.7 \mathrm{~min}\right] .[\alpha]_{\mathrm{D}}^{20}=-8.3\left(\mathrm{CH}_{2} \mathrm{Cl}_{2}, c 0.12\right)$.

$(S, E)-N^{I}, N^{1}, N^{3}, N^{3}$-tetrapropyl-5-phenylpent-4-ene-1,3-diamine (3aj): The title compound was prepared according to the general procedure and purified by column chromatography to give the colorless oil, $39 \mathrm{mg}, 56 \%$ yield

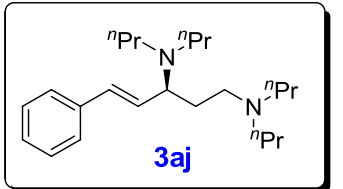
(isolated yield of combination of 3aj and 4aj, 3aj:4aj > 20:1). ${ }^{1} \mathrm{H}$ NMR $\left(400 \mathrm{MHz}, \mathrm{CDCl}_{3}\right) \delta$ 0.85-0.89 $(\mathrm{m}, 12 \mathrm{H})$, $1.40-1.50(\mathrm{~m}, 8 \mathrm{H}), 1.56-1.65(\mathrm{~m}, 1 \mathrm{H}), 1.76-1.85(\mathrm{~m}, 1 \mathrm{H}), 2.32-2.45(\mathrm{~m}, 8 \mathrm{H})$, 2.47-2.52 (m, 2H), 3.18-3.24 (m, 1H), $6.12\left(\mathrm{dd}, J_{1}=8.56 \mathrm{~Hz}, J_{2}=15.88 \mathrm{~Hz}\right.$, $1 \mathrm{H}), 6.37(\mathrm{~d}, J=15.92 \mathrm{~Hz}, 1 \mathrm{H}), 7.19-7.23(\mathrm{~m}, 1 \mathrm{H}), 7.28-7.32(\mathrm{~m}, 2 \mathrm{H}), 7.35(\mathrm{~d}$, $J=7.24 \mathrm{~Hz}, 2 \mathrm{H}) ;{ }^{13} \mathrm{C} \mathrm{NMR}\left(100 \mathrm{MHz}, \mathrm{CDCl}_{3}\right) \delta 11.9,12.0,20.3,22.0,30.2,51.8$, 52.7, 56.4, 61.3, 126.2, 127.1, 128.5, 130.0, 131.6, 137.5; HRMS (ESI) calcd for $\mathrm{C}_{23} \mathrm{H}_{41} \mathrm{~N}_{2}[\mathrm{M}+\mathrm{H}]: 345.3264$, found: 345.3255 . The ee was determined to be $56 \%$ ee [Determined by HPLC with a Chiralcel IA column (hexane: isopropanol= 95:5, 0.8

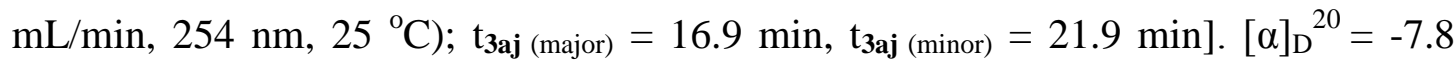
$\left(\mathrm{CH}_{2} \mathrm{Cl}_{2}, c 0.23\right)$. 
$(S, E)-N^{1}, N^{1}, N^{3}, N^{3}$-tetrabutyl-5-phenylpent-4-ene-1,3-diamine (3ak): The

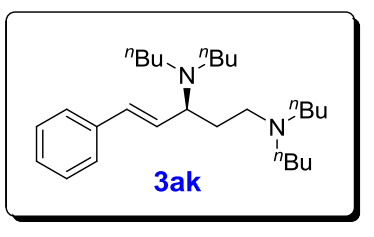
title compound was prepared according to the general procedure and purified by column chromatography to give the colorless oil, $50 \mathrm{mg}, 62 \%$ yield (isolated yield of combination of 3ak and 4ak, 3ak:4ak > 20:1). ${ }^{1} \mathrm{H}$ NMR $\left(400 \mathrm{MHz}, \mathrm{CDCl}_{3}\right) \delta$ 0.88-0.93 (m, 12H), 1.24-1.33 (m, 8H), 1.35-1.44 (m, 8H), 1.57-1.64 (m, 1H), 1.77-1.83 (m, 1H), 2.31-2.46 (m, 7H), 2.51-2.58 (m, 3H), 3.17-3.23 (m, 1H), $6.12\left(\mathrm{dd}, J_{1}=8.8 \mathrm{~Hz}, J_{2}=16.0 \mathrm{~Hz}, 1 \mathrm{H}\right), 6.38(\mathrm{~d}, J=16.0 \mathrm{~Hz}, 1 \mathrm{H}), 7.20-7.23$ $(\mathrm{m}, 1 \mathrm{H}), 7.29-7.36(\mathrm{~m}, 2 \mathrm{H}), 7.38(\mathrm{~d}, J=1.2 \mathrm{~Hz}, 2 \mathrm{H}) ;{ }^{13} \mathrm{C}$ NMR $(100 \mathrm{MHz}$, $\left.\mathrm{CDCl}_{3}\right) \delta 14.1,14.1,20.7,20.8,29.4,30.1,31.1,50.4,51.7,54.1,61.2,126.2,127.1$, 128.5, 130.0, 131.7, 137.5; HRMS (ESI) calcd for $\mathrm{C}_{27} \mathrm{H}_{49} \mathrm{~N}_{2}[\mathrm{M}+\mathrm{H}]$ : 401.3890, found: 401.3885. The ee was determined to be $59 \%$ ee [Determined by HPLC with a Chiralcel IA column (hexane: isopropanol=90:10, $0.6 \mathrm{~mL} / \mathrm{min}, 254 \mathrm{~nm}, 25^{\circ} \mathrm{C}$ ); $\mathrm{t}_{\mathbf{3 a k}}$ (major) $\left.=25.5 \mathrm{~min}, \mathrm{t}_{3 \mathbf{a k}(\text { minor })}=30.5 \mathrm{~min}\right] .[\alpha]_{\mathrm{D}}{ }^{20}=-8.6\left(\mathrm{CH}_{2} \mathrm{Cl}_{2}, c 0.36\right)$.

$(S, E)-N^{1}, N^{1}, N^{3}, N^{3}$-tetrakis(4-chlorobenzyl)-5-(m-tolyl)pent-4-ene-1,3-diami

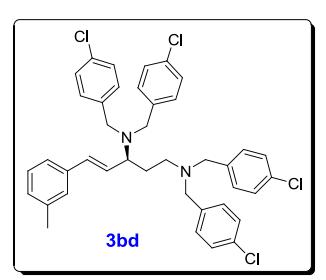
ne (3bd): The title compound was prepared according to the general procedure and purified by column chromatography to give the colorless oil, $118 \mathrm{mg}, 86 \%$ yield (isolated yield of combination of $\mathbf{3 b d}$ and $\mathbf{4 b d}$, 3bd:4bd = 93:7). ${ }^{1} \mathrm{H}$ NMR (400 $\left.\mathrm{MHz}, \mathrm{CDCl}_{3}\right) \delta 1.64-1.72(\mathrm{~m}, 1 \mathrm{H}), 1.87-1.96(\mathrm{~m}, 1 \mathrm{H}), 2.22-2.28(\mathrm{~m}, 1 \mathrm{H}), 2.38$ (s, 3H), 2.50-2.57 (m, 1H), 3.10-3.16 (m, 1H), $3.18(\mathrm{~d}, J=13.6 \mathrm{~Hz}, 2 \mathrm{H}), 3.30$ $(\mathrm{d}, J=13.6 \mathrm{~Hz}, 2 \mathrm{H}), 3.57(\mathrm{~d}, J=13.6 \mathrm{~Hz}, 2 \mathrm{H}), 3.66(\mathrm{~d}, J=13.6 \mathrm{~Hz}, 2 \mathrm{H}), 5.89$ $(\mathrm{d}, J=16.0 \mathrm{~Hz}, 1 \mathrm{H}), 5.98\left(\mathrm{dd}, J_{1}=8.8 \mathrm{~Hz}, J_{2}=16.0 \mathrm{~Hz}, 1 \mathrm{H}\right), 7.07-7.10(\mathrm{~m}$, 7H), 7.18-7.22 (m, 9H), $7.27(\mathrm{~d}, J=8.4 \mathrm{~Hz}, 4 \mathrm{H}) ;{ }^{13} \mathrm{C}$ NMR (100 MHz, $\left.\mathrm{CDCl}_{3}\right)$ $\delta 21.4,30.8,50.8,53.2,57.9,58.7,123.4,126.8,127.1,128.4,128.4,128.5$, 
130.0, 130.1, 132.6, 132.6, 133.9, 136.6, 138.0, 138.3, 138.6; HRMS (ESI) calcd for $\mathrm{C}_{40} \mathrm{H}_{39} \mathrm{Cl}_{4} \mathrm{~N}_{2}[\mathrm{M}+\mathrm{H}]:$ 687.1862, found: 687.1891 . The ee was determined to be 92\% ee [Determined by HPLC with a Chiralcel IA column (hexane: isopropanol= $\left.\left.98: 2,0.6 \mathrm{~mL} / \min , 254 \mathrm{~nm}, 25^{\circ} \mathrm{C}\right) ; \mathrm{t}_{3 \mathbf{b d}(\text { major })}=10.1 \mathrm{~min}, \mathrm{t}_{3 \mathbf{b d}(\operatorname{minor})}=12.5 \mathrm{~min}\right] .[\alpha]_{\mathrm{D}}{ }^{20}$ $=-39.6\left(\mathrm{CH}_{2} \mathrm{Cl}_{2}, c 0.53\right)$.

\section{$(S, E)-N^{1}, N^{1}, N^{3}, N^{3}$-tetrakis(4-chlorobenzyl)-5-(p-tolyl)pent-4-ene-1,3-diami}

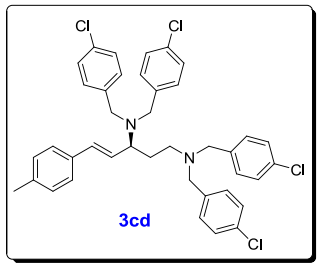

ne $(3 \mathbf{c d})$ : The title compound was prepared according to the general procedure and purified by column chromatography to give the colorless oil, $103 \mathrm{mg}, 75 \%$ yield (isolated yield of combination of 3cd and 4cd, 3cd:4cd > 20:1). ${ }^{1} \mathrm{H}$ NMR $\left(400 \mathrm{MHz}, \mathrm{CDCl}_{3}\right) \delta$ 1.56-1.64 (m, 1H), 1.79-1.88 (m, 1H), 2.14-2.20 (m, 1H), $2.28(\mathrm{~s}, 3 \mathrm{H})$, 2.44-2.51 (m, 1H), 3.02-3.07 (m, 1H), $3.12(\mathrm{~d}, J=13.6 \mathrm{~Hz}, 2 \mathrm{H}), 3.22(\mathrm{~d}, J=$ $13.6 \mathrm{~Hz}, 2 \mathrm{H}), 3.48(\mathrm{~d}, J=13.6 \mathrm{~Hz}, 2 \mathrm{H}), 3.58(\mathrm{~d}, J=13.6 \mathrm{~Hz}, 2 \mathrm{H}), 5.84-5.95(\mathrm{~m}$, 2H), 7.01-7.11 (m, 6H), 7.12-7.18 (m, 9H), 7.18-7.21 (m, 5H); ${ }^{13} \mathrm{C}$ NMR (100 $\left.\mathrm{MHz}, \mathrm{CDCl}_{3}\right) \delta 21.2,30.8,50.9,53.2,57.9,58.7,126.0,126.2,128.4,128.4,129.3$, 129.9, 130.1, 132.6, 132.7, 133.7, 133.9, 137.5, 138.0, 138.6; HRMS (ESI) calcd for $\mathrm{C}_{40} \mathrm{H}_{39} \mathrm{Cl}_{4} \mathrm{~N}_{2}[\mathrm{M}+\mathrm{H}]: 687.1862$, found:687.1871. The ee was determined to be $95 \%$ ee [Determined by HPLC with a Chiralcel IA column (hexane: isopropanol=99:1, 0.5 $\mathrm{mL} / \mathrm{min}, 254 \mathrm{~nm}, 25{ }^{\circ} \mathrm{C}$ ); $\left.\mathrm{t}_{3 \mathbf{c d}(\text { major })}=7.9 \mathrm{~min}, \mathrm{t}_{3 \mathbf{c d}(\operatorname{minor})}=9.9 \mathrm{~min}\right] .[\alpha]_{\mathrm{D}}{ }^{20}=-104.1$ $\left(\mathrm{CH}_{2} \mathrm{Cl}_{2}, c 0.24\right)$.

\section{$(S, E)-5-(4-(t e r t-b u t y l) p h e n y l)-N^{1}, N^{1}, N^{3}, N^{3}$-tetrakis(4-chlorobenzyl)pent-4-e}

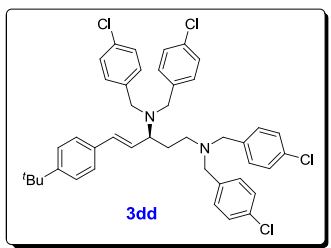

ne-1,3-diamine (3dd): The title compound was prepared according to the general procedure and purified by column chromatography to give the colorless oil, $115 \mathrm{mg}, 79 \%$ yield (isolated yield of combination of 3dd and 4dd, 3dd:4dd > 20:1). ${ }^{1} \mathrm{H}$ NMR (400 
$\left.\mathrm{MHz}, \mathrm{CDCl}_{3}\right) \delta 1.33(\mathrm{~s}, 9 \mathrm{H}), 1.64-1.72(\mathrm{~m}, 1 \mathrm{H}), 1.87-1.95(\mathrm{~m}, 1 \mathrm{H}), 2.22-2.28$ $(\mathrm{m}, 1 \mathrm{H}), 2.50-2.57(\mathrm{~m}, 1 \mathrm{H}), 3.12-3.14(\mathrm{~m}, 1 \mathrm{H}), 3.20(\mathrm{~d}, J=13.6 \mathrm{~Hz}, 2 \mathrm{H}), 3.30$ $(\mathrm{d}, J=13.6 \mathrm{~Hz}, 2 \mathrm{H}), 3.55(\mathrm{~d}, J=13.6 \mathrm{~Hz}, 2 \mathrm{H}), 3.67(\mathrm{~d}, J=13.6 \mathrm{~Hz}, 2 \mathrm{H})$, 5.94-6.03 (m, 2H), $7.09(\mathrm{~d}, J=8.4 \mathrm{~Hz}, 4 \mathrm{H}), 7.17-7.28(\mathrm{~m}, 14 \mathrm{H}), 7.36(\mathrm{~d}, J=$

$8.4 \mathrm{~Hz}, 2 \mathrm{H}) ;{ }^{13} \mathrm{C} \mathrm{NMR}\left(100 \mathrm{MHz}, \mathrm{CDCl}_{3}\right) \delta 30.8,31.3,34.6,50.8,53.2,57.9$, $58.7,125.6,126.1,126.3,128.4,128.4,129.9,130.1,132.6,132.6,133.6,133.9$, 138.0, 138.6, 150.9; HRMS (ESI) calcd for $\mathrm{C}_{43} \mathrm{H}_{45} \mathrm{Cl}_{4} \mathrm{~N}_{2}$ [M+H]: 729.2331, found: 729.2365. The ee was determined to be $88 \%$ ee [Determined by HPLC with a Chiralcel IA column (hexane: isopropanol $=98: 2,0.6 \mathrm{~mL} / \mathrm{min}, 254 \mathrm{~nm}, 25{ }^{\circ} \mathrm{C}$ ); $\mathrm{t}_{3 \mathrm{dd}}$ $\left.{ }_{(\text {major })}=8.2 \mathrm{~min}, \mathrm{t}_{3 \mathrm{dd}(\text { minor })}=11.4 \mathrm{~min}\right] .[\alpha]_{\mathrm{D}}{ }^{20}=-65.2\left(\mathrm{CH}_{2} \mathrm{Cl}_{2}, c 0.22\right)$.

$(S, E)-N^{1}, N^{1}, N^{3}, N^{3}$-tetrakis(4-chlorobenzyl)-5-(4-fluorophenyl)pent-4-ene-1,

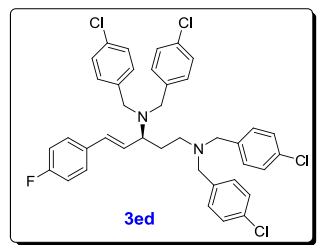

3-diamine (3ed): The title compound was prepared according to the general procedure and purified by column chromatography to give the colorless oil, $112 \mathrm{mg}, 81 \%$ yield (isolated yield of combination of 3ed and 4ed, 3ed:4ed > 20:1). ${ }^{1} \mathrm{H}$ NMR (400 $\left.\mathrm{MHz}, \mathrm{CDCl}_{3}\right) \delta$ 1.56-1.64 (m, 1H), 1.80-1.89 (m, 1H), 2.14-2.20 (m, 1H), 2.42-2.49 (m, 1H), 3.03-3.08 (m, 1H), $3.11(\mathrm{~d}, J=13.6 \mathrm{~Hz}, 2 \mathrm{H}), 3.22(\mathrm{~d}, J=13.6 \mathrm{~Hz}, 2 \mathrm{H}), 3.49(\mathrm{~d}$, $J=13.6 \mathrm{~Hz}, 2 \mathrm{H}), 3.59(\mathrm{~d}, J=13.6 \mathrm{~Hz}, 2 \mathrm{H}), 5.79-5.88(\mathrm{~m}, 2 \mathrm{H}), 6.92-6.97(\mathrm{~m}$, 2H), $7.01(\mathrm{~d}, J=8.4 \mathrm{~Hz}, 4 \mathrm{H}), 7.10-7.21(\mathrm{~m}, 14 \mathrm{H}) ;{ }^{13} \mathrm{C} \mathrm{NMR}\left(100 \mathrm{MHz}, \mathrm{CDCl}_{3}\right)$ $\delta 30.7,50.7,53.2,58.0,58.6,115.4,115.6,126.8,127.8,127.9,128.4,128.5,129.9$, $130.2,132.6,132.6,132.7,132.8,132.9,138.0,138.5,161.1,163.6 ;{ }^{19} \mathrm{~F}$ NMR (376 $\left.\mathrm{MHz}, \mathrm{CDCl}_{3}\right) \delta-114.4$; HRMS (ESI) calcd for $\mathrm{C}_{39} \mathrm{H}_{36} \mathrm{Cl}_{4} \mathrm{FN}_{2}[\mathrm{M}+\mathrm{H}]$ : 691.1611, found: 691.1642 . The ee was determined to be $88 \%$ ee [Determined by HPLC with a Chiralcel IA column (hexane: isopropanol=99:1, $0.6 \mathrm{~mL} / \mathrm{min}, 254 \mathrm{~nm}, 25{ }^{\circ} \mathrm{C}$ ); $\mathrm{t}_{3 \mathrm{ed}}$ (major) $\left.=9.5 \mathrm{~min}, \mathrm{t}_{3 \mathbf{e d} \text { (minor) }}=14.1 \mathrm{~min}\right] .[\alpha]_{\mathrm{D}}{ }^{20}=-44.1\left(\mathrm{CH}_{2} \mathrm{Cl}_{2}, c 0.30\right)$. 


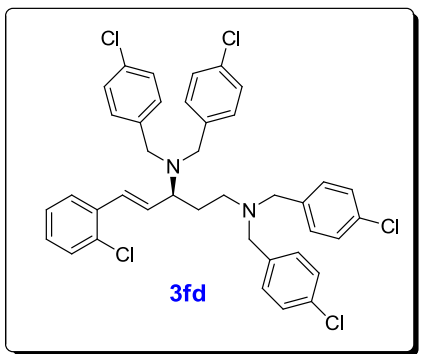

mine (3fd): The title compound was prepared according to the general procedure and purified by column chromatography to give the colorless oil, $56 \mathrm{mg}, 40 \%$ yield (isolated yield of combination of $\mathbf{3 f d}$ and $\mathbf{4 f d}$,

3fd:4fd = 76:24). ${ }^{1} \mathrm{H}$ NMR $\left(400 \mathrm{MHz}, \mathrm{CDCl}_{3}\right) \delta 1.62-1.69(\mathrm{~m}, 1 \mathrm{H}), 1.83-1.91$ (m, 1H), 2.17-2.23 (m, 1H), 2.48-2.55 (m, 1H), 3.12-3.18 (m, 3H), $3.24(\mathrm{~d}, J=$ $13.6 \mathrm{~Hz}, 2 \mathrm{H}), 3.46(\mathrm{~d}, J=13.6 \mathrm{~Hz}, 2 \mathrm{H}), 3.59(\mathrm{~d}, J=13.6 \mathrm{~Hz}, 2 \mathrm{H}), 5.78\left(\mathrm{dd}, J_{1}\right.$ $\left.=8.8 \mathrm{~Hz}, J_{2}=16.0 \mathrm{~Hz}, 1 \mathrm{H}\right), 6.44(\mathrm{~d}, J=16.0 \mathrm{~Hz}, 1 \mathrm{H}), 7.02-7.04(\mathrm{~m}, 4 \mathrm{H})$, 7.11-7.24 (m, 15H), 7.49-7.51 (m, 1H), ${ }^{13} \mathrm{C}$ NMR (100 MHz, $\left.\mathrm{CDCl}_{3}\right) \delta 30.2$, $50.8,53.1,57.8,58.3,123.5,127.1,127.6,128.4,128.5,128.9,130.0,130.0$, 130.2, 132.6, 132.7, 132.9, 133.0, 136.9, 137.8, 138.4; HRMS (ESI) calcd for $\mathrm{C}_{39} \mathrm{H}_{36} \mathrm{Cl}_{5} \mathrm{~N}_{2}[\mathrm{M}+\mathrm{H}]:$ 707.1316, found: 707.1319 . The ee was determined to be $86 \%$ ee [Determined by HPLC with a Chiralcel IA column (hexane: isopropanol= $\left.\left.98: 2,0.6 \mathrm{~mL} / \mathrm{min}, 254 \mathrm{~nm}, 25^{\circ} \mathrm{C}\right) ; \mathrm{t}_{3 \mathbf{f d}(\text { major) }}=12.9 \mathrm{~min}, \mathrm{t}_{\mathbf{3 f d}(\text { minor) }}=18.2 \mathrm{~min}\right] .[\alpha]_{\mathrm{D}}{ }^{20}=$ $-30.3\left(\mathrm{CH}_{2} \mathrm{Cl}_{2}, c 0.20\right)$.

$(S, E)-N^{1}, N^{1}, N^{3}, N^{3}$-tetrakis(4-chlorobenzyl)-5-(3-chlorophenyl)pent-4-ene-1,

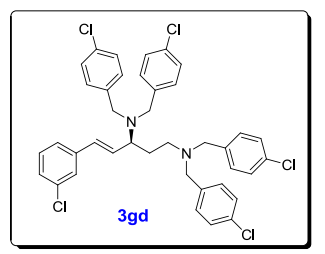

3-diamine (3gd): The title compound was prepared according to the general procedure and purified by column chromatography to give the colorless oil, $102 \mathrm{mg}, 72 \%$ yield (isolated yield of combination of 3gd and 4gd, 3gd:4gd = 92:8). ${ }^{1} \mathrm{H}$ NMR (400 $\left.\mathrm{MHz}, \mathrm{CDCl}_{3}\right) \delta 1.63-1.71(\mathrm{~m}, 1 \mathrm{H}), 1.88-1.96(\mathrm{~m}, 1 \mathrm{H}), 2.22-2.28(\mathrm{~m}, 1 \mathrm{H})$, 2.48-2.55 (m, 1H), 3.11-3.16 (m, 1H), $3.17(\mathrm{~d}, J=13.6 \mathrm{~Hz}, 2 \mathrm{H}), 3.29(\mathrm{~d}, J=$ $13.6 \mathrm{~Hz}, 2 \mathrm{H}), 3.57(\mathrm{~d}, J=13.6 \mathrm{~Hz}, 2 \mathrm{H}), 3.67(\mathrm{~d}, J=13.6 \mathrm{~Hz}, 2 \mathrm{H}), 5.83(\mathrm{~d}, J=$ $16.0 \mathrm{~Hz}, 1 \mathrm{H}), 6.00\left(\mathrm{dd}, J_{1}=8.8 \mathrm{~Hz}, J_{2}=16.0 \mathrm{~Hz}, 1 \mathrm{H}\right), 7.07-7.13(\mathrm{~m}, 5 \mathrm{H})$, 
7.18-7.24 (m, 9H), 7.26-7.29 (m, 6H); $\left.{ }^{13} \mathrm{C} \mathrm{NMR} \mathrm{(100} \mathrm{MHz,} \mathrm{CDCl}_{3}\right) \delta 30.7,50.7$, $53.2,58.0,58.5,124.7,126.1,127.6,128.4,128.5,128.8,129.8,129.9,130.1,132.4$, 132.7, 132.7, 134.6, 137.9, 138.4, 138.5; HRMS (ESI) calcd for $\mathrm{C}_{39} \mathrm{H}_{36} \mathrm{Cl}_{5} \mathrm{~N}_{2}[\mathrm{M}+\mathrm{H}]$ : 707.1316, found: 707.1303 . The ee was determined to be $83 \%$ ee [Determined by HPLC with a Chiralcel OD-H column (hexane: isopropanol=97:3, $0.6 \mathrm{~mL} / \mathrm{min}, 254$ $\left.\left.\mathrm{nm}, 25^{\circ} \mathrm{C}\right) ; \mathrm{t}_{\text {3gd (major) }}=7.2 \mathrm{~min}, \mathrm{t}_{\mathbf{3 g d}(\text { minor) }}=10.4 \mathrm{~min}\right] .[\alpha]_{\mathrm{D}}{ }^{20}=-67.7\left(\mathrm{CH}_{2} \mathrm{Cl}_{2}, c 0.25\right)$. $(S, E)-N^{1}, N^{1}, N^{3}, N^{3}$-tetrakis(4-chlorobenzyl)-5-(4-chlorophenyl)pent-4-ene-1,

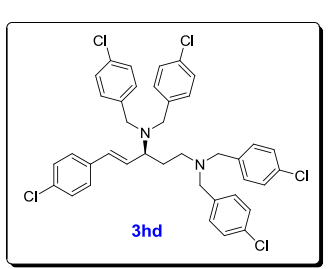
3-diamine (3hd): The title compound was prepared according to the general procedure and purified by column chromatography to give the colorless oil, $114 \mathrm{mg}, 81 \%$ (isolated yield of combination of 3hd and 4hd, 3hd:4hd > 20:1). ${ }^{1} \mathrm{H}$ NMR (400 $\left.\mathrm{MHz}, \mathrm{CDCl}_{3}\right) \delta 1.62-1.71(\mathrm{~m}, 1 \mathrm{H}), 1.88-1.96(\mathrm{~m}, 1 \mathrm{H}), 2.22-2.28(\mathrm{~m}, 1 \mathrm{H})$, 2.48-2.55 (m, 1H), 3.11-3.17 (m, 1H), $3.19(\mathrm{~d}, J=13.6 \mathrm{~Hz}, 2 \mathrm{H}), 3.30(\mathrm{~d}, J=$ $13.6 \mathrm{~Hz}, 2 \mathrm{H}), 3.56(\mathrm{~d}, J=13.6 \mathrm{~Hz}, 2 \mathrm{H}), 3.66(\mathrm{~d}, J=13.6 \mathrm{~Hz}, 2 \mathrm{H}), 5.86(\mathrm{~d}, J=$ $16.0 \mathrm{~Hz}, 1 \mathrm{H}), 5.95\left(\mathrm{dd}, J_{1}=8.8 \mathrm{~Hz}, J_{2}=16.0 \mathrm{~Hz}, 1 \mathrm{H}\right), 7.08(\mathrm{~d}, J=8.4 \mathrm{~Hz}, 4 \mathrm{H})$, 7.18-7.23 (m, 10H), 7.25-7.30 (m, 6H); $\left.{ }^{13} \mathrm{C} \mathrm{NMR} \mathrm{(100} \mathrm{MHz,} \mathrm{CDCl}_{3}\right) \delta$ 30.6, 50.7, 53.3, 58.0, 58.5, 127.5, 127.9, 128.4, 128.5, 128.7, 129.9, 130.1, 132.5, 132.6, 132.7, 133.3, 135.1, 138.0, 138.4; HRMS (ESI) calcd for $\mathrm{C}_{39} \mathrm{H}_{36} \mathrm{Cl}_{5} \mathrm{~N}_{2}[\mathrm{M}+\mathrm{H}]$ : 707.1316, found: 707.1326 . The ee was determined to be $81 \%$ ee [Determined by HPLC with a Chiralcel IA column (hexane: isopropanol= 97:3, $0.6 \mathrm{~mL} / \mathrm{min}, 254 \mathrm{~nm}$, $\left.\left.25^{\circ} \mathrm{C}\right) ; \mathrm{t}_{3 h d(\text { major })}=7.3 \mathrm{~min}, \mathrm{t}_{3 \mathrm{hd} \text { (minor) }}=10.4 \mathrm{~min}\right] .[\alpha]_{\mathrm{D}}{ }^{20}=-73.6\left(\mathrm{CH}_{2} \mathrm{Cl}_{2}, c 0.20\right)$.

(S,E)-5-(4-bromophenyl)- $N^{1}, N^{1}, N^{3}, N^{3}$-tetrakis(4-chlorobenzyl)pent-4-ene-1,

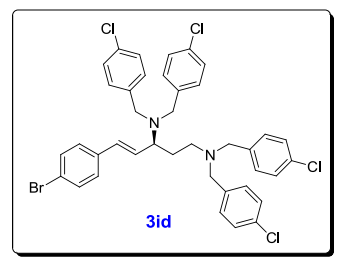

3-diamine (3id): The title compound was prepared according to the general procedure and purified by column chromatography to give the colorless oil, $126 \mathrm{mg}, 84 \%$ 
(isolated yield of combination of 3id and 4id, 3id:4id > 20:1). ${ }^{1} \mathrm{H}$ NMR (400 MHz, $\left.\mathrm{CDCl}_{3}\right) \delta$ 1.62-1.71 (m, 1H), 1.88-1.96 (m, 1H), 2.21-2.28 (m, 1H), 2.48-2.55 $(\mathrm{m}, 1 \mathrm{H}), 3.11-3.16(\mathrm{~m}, 1 \mathrm{H}), 3.18(\mathrm{~d}, J=13.6 \mathrm{~Hz}, 2 \mathrm{H}), 3.29(\mathrm{~d}, J=13.6 \mathrm{~Hz}, 2 \mathrm{H})$, $3.57(\mathrm{~d}, J=13.6 \mathrm{~Hz}, 2 \mathrm{H}), 3.66(\mathrm{~d}, J=13.6 \mathrm{~Hz}, 2 \mathrm{H}), 5.83(\mathrm{~d}, J=16.0 \mathrm{~Hz}, 1 \mathrm{H})$, $5.96\left(\mathrm{dd}, J_{1}=8.8 \mathrm{~Hz}, J_{2}=16.0 \mathrm{~Hz}, 1 \mathrm{H}\right), 7.07-7.14(\mathrm{~m}, 6 \mathrm{H}), 7.18-7.22(\mathrm{~m}, 8 \mathrm{H})$, 7.25-7.29 (m, 4H), $7.44(\mathrm{~d}, J=8.4 \mathrm{~Hz}, 2 \mathrm{H}) ;{ }^{13} \mathrm{C}$ NMR $\left(100 \mathrm{MHz}, \mathrm{CDCl}_{3}\right) \delta$ $30.6,50.7,53.2,57.9,58.5,121.4,127.8,128.0,128.4,128.5,129.9,130.1,131.7$, 132.6, 132.6, 132.7, 135.6, 138.0, 138.4; HRMS (ESI) calcd for $\mathrm{C}_{39} \mathrm{H}_{36} \mathrm{BrCl}_{4} \mathrm{~N}_{2}$ $[\mathrm{M}+\mathrm{H}]:$ 751.0810, found: 751.0833. The ee was determined to be $87 \%$ ee [Determined by HPLC with a Chiralcel IA column (hexane: isopropanol= 95:5, 0.6 $\mathrm{mL} / \mathrm{min}, 254 \mathrm{~nm}, 25{ }^{\circ} \mathrm{C}$ ); $\left.\mathrm{t}_{3 \mathbf{i d}(\text { major) }}=9.7 \mathrm{~min}, \mathrm{t}_{3 \mathbf{i d}(\text { minor })}=12.9 \mathrm{~min}\right] .[\alpha]_{\mathrm{D}}{ }^{20}=-82.1$ $\left(\mathrm{CH}_{2} \mathrm{Cl}_{2}, c 0.22\right)$.

\section{$(S, E)-N^{1}, N^{1}, N^{3}, N^{3}$-tetrakis(4-chlorobenzyl)-5-(4-(trifluoromethoxy)phenyl)}

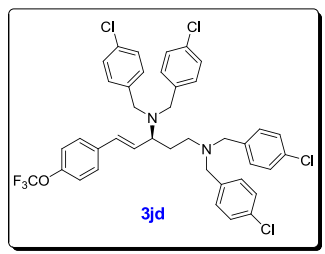
pent-4-ene-1,3-diamine (3jd): The title compound was prepared according to the general procedure and purified by column chromatography to give the colorless oil, $130 \mathrm{mg}, 86 \%$ yield (isolated yield of combination of $\mathbf{3 j d}$ and $\mathbf{4 j d}, \mathbf{3 j d} \mathbf{3} \mathbf{4 j d}=88: 12) .{ }^{1} \mathrm{H}$ NMR (400 $\left.\mathrm{MHz}, \mathrm{CDCl}_{3}\right) \delta 1.63-1.71(\mathrm{~m}, 1 \mathrm{H}), 1.89-1.97(\mathrm{~m}, 1 \mathrm{H}), 2.23-2.29(\mathrm{~m}, 1 \mathrm{H})$, 2.48-2.55 (m, 1H), 3.13-3.17 (m, 1H), $3.19(\mathrm{~d}, J=13.6 \mathrm{~Hz}, 2 \mathrm{H}), 3.30(\mathrm{~d}, J=$ $13.6 \mathrm{~Hz}, 2 \mathrm{H}), 3.57(\mathrm{~d}, J=13.6 \mathrm{~Hz}, 2 \mathrm{H}), 3.67(\mathrm{~d}, J=13.6 \mathrm{~Hz}, 2 \mathrm{H}), 5.89-6.02(\mathrm{~m}$, $2 \mathrm{H}), 7.08(\mathrm{~d}, J=8.4 \mathrm{~Hz}, 4 \mathrm{H}), 7.17-7.24(\mathrm{~m}, 10 \mathrm{H}), 7.27(\mathrm{~d}, J=8.4 \mathrm{~Hz}, 6 \mathrm{H}) ;{ }^{13} \mathrm{C}$ NMR $\left(100 \mathrm{MHz}, \mathrm{CDCl}_{3}\right) \delta 30.6,50.7,53.3,58.0,58.5,119.2,121.1,121.8$, $127.5,128.3,128.4,128.5,129.9,130.1,132.3,132.7,132.7,135.4,138.0$, 138.4, 148.5; ${ }^{19} \mathrm{~F}$ NMR $\left(376 \mathrm{MHz}, \mathrm{CDCl}_{3}\right) \delta-57.8$; HRMS (ESI) calcd for $\mathrm{C}_{40} \mathrm{H}_{36} \mathrm{Cl}_{4} \mathrm{~F}_{3} \mathrm{~N}_{2} \mathrm{O}[\mathrm{M}+\mathrm{H}]:$ 757.1528, found: 757.1531 . The ee was determined to be 
83\% ee [Determined by HPLC with a Chiralcel IA column (hexane: isopropanol= 98:2, $\left.\left.0.6 \mathrm{~mL} / \mathrm{min}, 254 \mathrm{~nm}, 25^{\circ} \mathrm{C}\right) ; \mathrm{t}_{3 \mathbf{j d}(\text { major })}=7.8 \mathrm{~min}, \mathrm{t}_{3 \mathbf{j d}(\text { minor })}=11.8 \mathrm{~min}\right] .[\alpha]_{\mathrm{D}}{ }^{20}=$ $-63.1\left(\mathrm{CH}_{2} \mathrm{Cl}_{2}, c 0.20\right)$.

$(S, E)-N^{1}, N^{1}, N^{3}, N^{3}$-tetrakis(4-chlorobenzyl)-5-(4-nitrophenyl)pent-4-ene-1,3

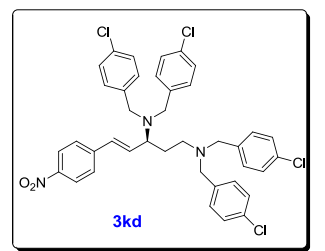

-diamine (3kd): The title compound was prepared according to the general procedure and purified by column chromatography to give the colorless oil, $118 \mathrm{mg}, 82 \%$ yield

(isolated yield of combination of $\mathbf{3 k d}$ and $\mathbf{4 k d}, \mathbf{3 k d : 4 k d}=75: 25)$. ${ }^{1} \mathrm{H}$ NMR (400 $\left.\mathrm{MHz}, \mathrm{CDCl}_{3}\right) \delta 1.65-1.73(\mathrm{~m}, 1 \mathrm{H}), 1.92-2.01(\mathrm{~m}, 1 \mathrm{H}), 2.24-2.30(\mathrm{~m}, 1 \mathrm{H})$, 2.46-2.53 (m, 1H), 3.14-3.25 (m, 3H), $3.32(\mathrm{~d}, J=13.6 \mathrm{~Hz}, 2 \mathrm{H}), 3.58(\mathrm{~d}, J=$ $13.6 \mathrm{~Hz}, 2 \mathrm{H}), 3.70(\mathrm{~d}, J=13.6 \mathrm{~Hz}, 2 \mathrm{H}), 5.91(\mathrm{~d}, J=16.0 \mathrm{~Hz}, 1 \mathrm{H}), 6.16\left(\mathrm{dd}, J_{1}\right.$ $\left.=8.8 \mathrm{~Hz}, J_{2}=16.0 \mathrm{~Hz}, 1 \mathrm{H}\right), 7.07(\mathrm{~d}, J=8.4 \mathrm{~Hz}, 4 \mathrm{H}), 7.20-7.22(\mathrm{~m}, 8 \mathrm{H})$, 7.28-7.30 (m, 4H), $7.35(\mathrm{~d}, J=8.8 \mathrm{~Hz}, 2 \mathrm{H}), 8.17(\mathrm{~d}, J=8.8 \mathrm{~Hz}, 2 \mathrm{H}) ;{ }^{13} \mathrm{C}$ NMR $\left(100 \mathrm{MHz} \mathrm{CDCl}_{3}\right) \delta 30.4,50.5,53.3,58.0,58.4,124.0,126.8,128.4,128.6,129.9$, 130.2, 131.7, 132.7, 132.7, 132.8, 137.9, 138.2, 142.9, 146.9; HRMS (ESI) calcd for $\mathrm{C}_{39} \mathrm{H}_{36} \mathrm{Cl}_{4} \mathrm{~N}_{3} \mathrm{O}_{2}[\mathrm{M}+\mathrm{H}]:$ 718.1556, found: 718.1581 . The ee was determined to be $81 \%$ ee [Determined by HPLC with a Chiralcel IA column (hexane: isopropanol= $\left.\left.95: 5,0.6 \mathrm{~mL} / \mathrm{min}, 254 \mathrm{~nm}, 25^{\circ} \mathrm{C}\right) ; \mathrm{t}_{3 \mathbf{k d}(\text { major })}=14.9 \mathrm{~min}, \mathrm{t}_{3 \mathbf{k d}(\text { minor })}=18.9 \mathrm{~min}\right] .[\alpha]_{\mathrm{D}}{ }^{20}$ $=-74.7\left(\mathrm{CH}_{2} \mathrm{Cl}_{2}, c 0.68\right)$.

$(S, E)-5-\left(\left[1,1^{\prime}\right.\right.$-biphenyl]-4-yl)- $N^{1}, N^{1}, N^{3}, N^{3}$-tetrakis(4-chlorobenzyl)pent-4-e

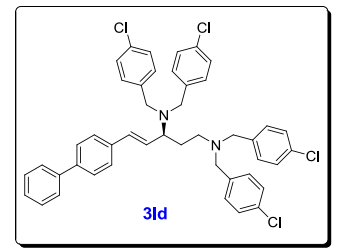
ne-1,3-diamine (3ld): The title compound was prepared according to the general procedure and purified by column chromatography to give the colorless oil, $126 \mathrm{mg}, 84 \%$ yield (isolated yield of combination of 3ld and 4ld, 3ld:4ld = 89:11). ${ }^{1} \mathrm{H}$ NMR (400 MHz, $\left.\mathrm{CDCl}_{3}\right) \delta$ 1.66-1.74 (m, 1H), 1.89-1.98 (m, 1H), 2.24-2.30 (m, 1H), 2.52-2.59 
(m, 1H), 3.14-3.18 (m, 1H), $3.20(\mathrm{~d}, J=13.6 \mathrm{~Hz}, 2 \mathrm{H}), 3.33(\mathrm{~d}, J=13.6 \mathrm{~Hz}, 2 \mathrm{H})$, $3.57(\mathrm{~d}, J=13.6 \mathrm{~Hz}, 2 \mathrm{H}), 3.68(\mathrm{~d}, J=13.6 \mathrm{~Hz}, 2 \mathrm{H}), 5.98-6.10(\mathrm{~m}, 2 \mathrm{H}), 7.09(\mathrm{~d}$, $J=8.4 \mathrm{~Hz}, 4 \mathrm{H}), 7.19-7.23(\mathrm{~m}, 8 \mathrm{H}), 7.27(\mathrm{~d}, J=8.4 \mathrm{~Hz}, 4 \mathrm{H}), 7.35(\mathrm{~d}, J=8.4 \mathrm{~Hz}$ 3H), 7.43-7.46 (m, 2H), 7.56-7.62(m, 4H); $\left.{ }^{13} \mathrm{C} \mathrm{NMR} \mathrm{(100} \mathrm{MHz,} \mathrm{CDCl}_{3}\right) \delta 30.8$, $50.8,53.3,58.0,58.7,126.8,127.0,127.3,127.3,127.4,128.4,128.5,128.8,130.0$, 130.1, 132.6, 132.7, 133.3, 135.7, 138.0, 138.6, 140.5, 140.7; HRMS (ESI) calcd for $\mathrm{C}_{45} \mathrm{H}_{41} \mathrm{Cl}_{4} \mathrm{~N}_{2}[\mathrm{M}+\mathrm{H}]:$ 749.2018, found: 749.2014 . The ee was determined to be $93 \%$ ee [Determined by HPLC with a Chiralcel OD-H column (hexane: isopropanol= 95:5, $0.8 \mathrm{~mL} / \mathrm{min}, 254 \mathrm{~nm}, 25^{\circ} \mathrm{C}$ ); $\left.\mathrm{t}_{3 \mathbf{l d}(\text { minor })}=18.4 \mathrm{~min}, \mathrm{t}_{3 \mathbf{l d}(\text { major })}=22.2 \mathrm{~min}\right] .[\alpha]_{\mathrm{D}}{ }^{20}=-99.1$ $\left(\mathrm{CH}_{2} \mathrm{Cl}_{2}, c 0.35\right)$.

$(S, E)-N^{1}, N^{1}, N^{3}, N^{3}$-tetrakis(4-chlorobenzyl)-5-(naphthalen-1-yl)pent-4-ene-1

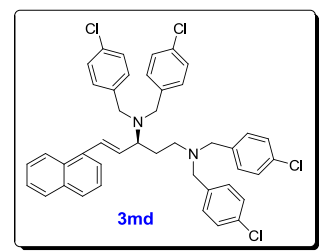
,3-diamine (3md): The title compound was prepared according to the general procedure and purified by column chromatography to give the colorless oil, $69 \mathrm{mg}, 48 \%$ yield (isolated yield of combination of $\mathbf{3 m d}$ and $\mathbf{4 m d}$, 3md:4md > 20:1). ${ }^{1} \mathrm{H}$ NMR (400 $\left.\mathrm{MHz}, \mathrm{CDCl}_{3}\right) \delta 1.67-1.75(\mathrm{~m}, 1 \mathrm{H}), 1.88-1.96(\mathrm{~m}, 1 \mathrm{H}), 2.25-2.31(\mathrm{~m}, 1 \mathrm{H})$, $2.55-2.62(\mathrm{~m}, 1 \mathrm{H}), 3.12-3.25(\mathrm{~m}, 3 \mathrm{H}), 3.32(\mathrm{~d}, J=13.6 \mathrm{~Hz}, 2 \mathrm{H}), 3.50(\mathrm{~d}, J=$ $13.6 \mathrm{~Hz}, 2 \mathrm{H}), 3.65(\mathrm{~d}, J=13.6 \mathrm{~Hz}, 2 \mathrm{H}), 5.95\left(\mathrm{dd}, J_{1}=8.8 \mathrm{~Hz}, J_{2}=15.6 \mathrm{~Hz}\right.$, $1 \mathrm{H}), 6.77(\mathrm{~d}, J=15.6 \mathrm{~Hz}, 1 \mathrm{H}), 7.06(\mathrm{~d}, J=8.4 \mathrm{~Hz}, 4 \mathrm{H}), 7.13-7.18(\mathrm{~m}, 9 \mathrm{H})$, 7.20-7.24 (m, 3H), 7.35-7.42 (m, 2H), 7.43-7.49 (m, 2H), $7.71\left(\mathrm{dd}, J_{1}=2.0 \mathrm{~Hz}\right.$, $\left.J_{2}=7.2 \mathrm{~Hz}, 1 \mathrm{H}\right), 7.78(\mathrm{~d}, J=2.0 \mathrm{~Hz}, 1 \mathrm{H}), 7.80-7.83(\mathrm{~m}, 1 \mathrm{H}) ;{ }^{13} \mathrm{C} \mathrm{NMR}(100$ $\left.\mathrm{MHz}, \mathrm{CDCl}_{3}\right) \delta 30.8,50.8,53.2,57.9,58.7,123.2,124.9,125.4,125.4,125.8$ $127.0,128.3,128.3,128.4,129.0,130.0,130.1,131.5,132.6,132.6,134.0$, 136.0, 137.8, 138.0, 138.9; HRMS (ESI) calcd for $\mathrm{C}_{43} \mathrm{H}_{39} \mathrm{Cl}_{4} \mathrm{~N}_{2}[\mathrm{M}+\mathrm{H}]$ : 723.1862, found: 723.1895 . The ee was determined to be $89 \%$ ee [Determined by HPLC with a 
Chiralcel OD-H column (hexane: isopropanol= 90:10, $0.8 \mathrm{~mL} / \mathrm{min}, 254 \mathrm{~nm}, 25^{\circ} \mathrm{C}$ ); $\left.\mathrm{t}_{3 \mathrm{md} \text { (major) }}=22.3 \mathrm{~min}, \mathrm{t}_{3 \mathrm{md}(\text { minor) }}=36.4 \mathrm{~min}\right] .[\alpha]_{\mathrm{D}}{ }^{20}=-44.7\left(\mathrm{CH}_{2} \mathrm{Cl}_{2}, c 0.11\right)$.

$(S, E)-N^{1}, N^{1}, N^{3}, N^{3}$-tetrakis(4-chlorobenzyl)-5-(naphthalen-2-yl)pent-4-ene-1

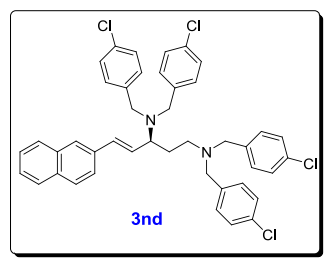
,3-diamine (3nd): The title compound was prepared according to the general procedure and purified by column chromatography to give the colorless oil, $131 \mathrm{mg}, 91 \%$ yield (isolated yield of combination of 3nd and 4nd, 3nd:4nd = 91:9). ${ }^{1} \mathrm{H}$ NMR (400 $\left.\mathrm{MHz}, \mathrm{CDCl}_{3}\right) \delta 1.60-1.68(\mathrm{~m}, 1 \mathrm{H}), 1.83-1.91(\mathrm{~m}, 1 \mathrm{H}), 2.17-2.23(\mathrm{~m}, 1 \mathrm{H})$, 2.45-2.52 (m, 1H), 3.11-3.14 (m, 3H), $3.27(\mathrm{~d}, J=13.6 \mathrm{~Hz}, 2 \mathrm{H}), 3.51(\mathrm{~d}, J=$ $13.6 \mathrm{~Hz}, 2 \mathrm{H}), 3.62(\mathrm{~d}, J=13.6 \mathrm{~Hz}, 2 \mathrm{H}), 5.94(\mathrm{~d}, J=16.0 \mathrm{~Hz}, 1 \mathrm{H}), 6.03\left(\mathrm{dd}, J_{1}\right.$ $\left.=8.8 \mathrm{~Hz}, J_{2}=16.0 \mathrm{~Hz}, 1 \mathrm{H}\right), 7.01(\mathrm{~d}, J=8.4 \mathrm{~Hz}, 4 \mathrm{H}), 7.14-7.23(\mathrm{~m}, 12 \mathrm{H})$, 7.36-7.45 (m, 3H), $7.53(\mathrm{~s}, 1 \mathrm{H}), 7.71-7.79(\mathrm{~m}, 3 \mathrm{H}) ;{ }^{13} \mathrm{C}$ NMR (100 MHz, $\left.\mathrm{CDCl}_{3}\right) \delta 30.9,50.8,53.3,58.0,58.8,123.3,125.9,126.3,126.4,127.5,127.7,128.0$, $128.2,128.4,128.5,130.0,130.2,132.6,132.7,133.0,133.7,133.9,134.1,138.0$, 138.6; HRMS (ESI) calcd for $\mathrm{C}_{43} \mathrm{H}_{39} \mathrm{Cl}_{4} \mathrm{~N}_{2}[\mathrm{M}+\mathrm{H}]$ : 723.1862 , found: 723.1878 . The ee was determined to be $92 \%$ ee [Determined by HPLC with a Chiralcel OD-H column (hexane: isopropanol $=90: 10,0.8 \mathrm{~mL} / \mathrm{min}, 254 \mathrm{~nm}, 25{ }^{\circ} \mathrm{C}$ ); $\mathrm{t}_{3 \text { nd }(\text { major })}=33.1$ $\left.\min , \mathrm{t}_{\text {3nd (minor) }}=38.6 \mathrm{~min}\right] .[\alpha]_{\mathrm{D}}{ }^{20}=-79.4\left(\mathrm{CH}_{2} \mathrm{Cl}_{2}, c 0.23\right)$.

$(S, E)-N^{1}, N^{1}, N^{3}, N^{3}$-tetrakis(4-chlorobenzyl)-5-(3,4-dimethylphenyl)pent-4-e

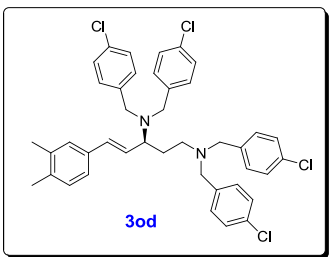
ne-1,3-diamine (3od): The title compound was prepared according to the general procedure and purified by column chromatography to give the colorless oil, $104 \mathrm{mg}, 74 \%$ yield

(isolated yield of combination of 3od and 4od, 3od:4od > 20:1). ${ }^{1} \mathrm{H}$ NMR (400 $\left.\mathrm{MHz}, \mathrm{CDCl}_{3}\right) \delta$ 1.56-1.64 (m, 1H), 1.79-1.87 (m, 1H), 2.15-2.18 (m, 1H), 2.19 (s, 3H), $2.21(\mathrm{~s}, 3 \mathrm{H}), 2.43-2.50(\mathrm{~m}, 1 \mathrm{H}), 3.01-3.07(\mathrm{~m}, 1 \mathrm{H}), 3.12(\mathrm{~d}, J=13.6 \mathrm{~Hz}$, 
$2 \mathrm{H}), 3.23(\mathrm{~d}, J=13.6 \mathrm{~Hz}, 2 \mathrm{H}), 3.49(\mathrm{~d}, J=13.6 \mathrm{~Hz}, 2 \mathrm{H}), 3.58(\mathrm{~d}, J=13.6 \mathrm{~Hz}$, 2H), 5.80-5.92 (m, 2H), 6.96-7.04 (m, 7H), 7.10-7.15 (m, 7H), 7.18-7.21 (m, 5H); ${ }^{13} \mathrm{C}$ NMR $\left(100 \mathrm{MHz}, \mathrm{CDCl}_{3}\right) \delta 19.5,19.8,30.9,50.8,53.2,57.9,58.7,123.7$, $125.8,127.6,128.4,128.4,129.9,129.9,130.1,132.5,132.6,133.8,134.3,136.2$, 136.8, 138.0, 138.6; HRMS (ESI) calcd for $\mathrm{C}_{41} \mathrm{H}_{41} \mathrm{Cl}_{4} \mathrm{~N}_{2}[\mathrm{M}+\mathrm{H}]$ : 701.2018, found: 701.2033. The ee was determined to be $96 \%$ ee [Determined by HPLC with a Chiralcel IA column (hexane: isopropanol $=99: 1,0.6 \mathrm{~mL} / \mathrm{min}, 254 \mathrm{~nm}, 25{ }^{\circ} \mathrm{C}$ ); $\mathrm{t}_{3 \mathbf{3 o d}}$ $\left(\right.$ major) $\left.=13.6 \mathrm{~min}, \mathrm{t}_{3 \mathbf{3 o d}(\operatorname{minor})}=18.3 \mathrm{~min}\right] .[\alpha]_{\mathrm{D}}{ }^{20}=-56.4\left(\mathrm{CH}_{2} \mathrm{Cl}_{2}, c 0.36\right)$.

$(S, E)-N^{1}, N^{1}, N^{3}, N^{3}$-tetrakis(4-chlorobenzyl)-5-(3,5-dimethylphenyl)pent-4-ene-1,3

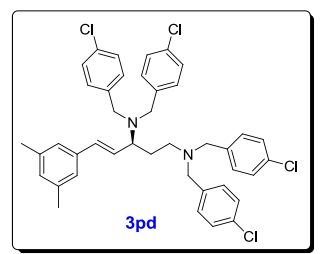
-diamine (3pd): The title compound was prepared according to the general procedure and purified by column chromatography to give the colorless oil, $106 \mathrm{mg}, 76 \%$ yield (isolated yield of combination of 3pd and 4pd, 3pd:4pd > 20:1). ${ }^{1} \mathrm{H}$ NMR (400 $\left.\mathrm{MHz}, \mathrm{CDCl}_{3}\right) \delta$ 1.63-1.72 (m, 1H), 1.86-1.95 (m, 1H), 2.22-2.29 (m, 1H), $2.33(\mathrm{~s}, 6 \mathrm{H})$, 2.49-2.56 (m, 1H), 3.09-3.15 (m, 1H), $3.18(\mathrm{~d}, J=13.6 \mathrm{~Hz}, 2 \mathrm{H}), 3.31(\mathrm{~d}, J=$ $13.6 \mathrm{~Hz}, 2 \mathrm{H}), 3.57(\mathrm{~d}, J=13.6 \mathrm{~Hz}, 2 \mathrm{H}), 3.66(\mathrm{~d}, J=13.6 \mathrm{~Hz}, 2 \mathrm{H}), 5.84(\mathrm{~d}, J=$ $16.0 \mathrm{~Hz}, 1 \mathrm{H}), 5.96\left(\mathrm{dd}, J_{1}=8.8 \mathrm{~Hz}, J_{2}=16.0 \mathrm{~Hz}, 1 \mathrm{H}\right), 6.91(\mathrm{~s}, 3 \mathrm{H}), 7.08(\mathrm{~d}, J=$ 8.4 Hz, 4H), 7.18-7.23 (m, 8H), $7.27(\mathrm{~d}, J=8.4 \mathrm{~Hz}, 4 \mathrm{H}) ;{ }^{13} \mathrm{C}$ NMR (100 MHz, $\left.\mathrm{CDCl}_{3}\right) \delta 21.3,30.9,50.8,53.2,58.0,58.7,124.2,126.6,128.4,128.4,129.4,130.0$, $130.1,132.6,132.6,134.0,136.6,138.0,138.1,138.6$; HRMS (ESI) calcd for $\mathrm{C}_{41} \mathrm{H}_{41} \mathrm{Cl}_{4} \mathrm{~N}_{2}[\mathrm{M}+\mathrm{H}]:$ 701.2018, found: 701.2040 . The ee was determined to be $88 \%$ ee [Determined by HPLC with a Chiralcel OD-H column (hexane: isopropanol= 97:3, $\left.\left.0.6 \mathrm{~mL} / \mathrm{min}, 254 \mathrm{~nm}, 25^{\circ} \mathrm{C}\right) ; \mathrm{t}_{3 \mathbf{p d} \text { (major) }}=15.1 \mathrm{~min}, \mathrm{t}_{3 \mathbf{p d}(\text { minor })}=19.5 \mathrm{~min}\right] .[\alpha]_{\mathrm{D}}{ }^{20}=$ $-69.6\left(\mathrm{CH}_{2} \mathrm{Cl}_{2}, c 0.23\right)$. 


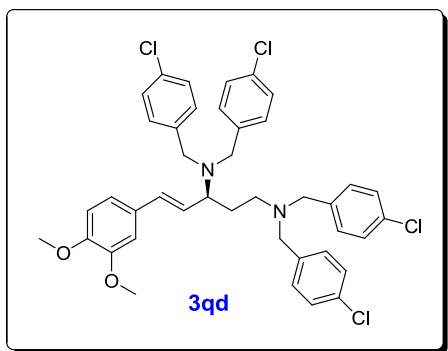

ene-1,3-diamine (3qd): The title compound was prepared according to the general procedure and purified by column chromatography to give the colorless oil, 107 $\mathrm{mg}, 73 \%$ yield (isolated yield of combination of $\mathbf{3 q d}$ and 4qd, 3qd:4qd = 48:52). ${ }^{1} \mathrm{H}$ NMR (400 MHz, $\left.\mathrm{CDCl}_{3}\right) \delta 1.64-1.73(\mathrm{~m}, 1 \mathrm{H})$, 1.88-1.96 (m, 1H), 2.23-2.30 (m, 1H), 2.51-2.58 (m, 1H), 3.11-3.12 (m, 1H), $3.21(\mathrm{~d}, J=13.6 \mathrm{~Hz}, 2 \mathrm{H}), 3.32(\mathrm{~d}, J=13.6 \mathrm{~Hz}, 2 \mathrm{H}), 3.57(\mathrm{~d}, J=13.6 \mathrm{~Hz}, 2 \mathrm{H})$, $3.67(\mathrm{~d}, J=13.6 \mathrm{~Hz}, 2 \mathrm{H}), 3.90(\mathrm{~s}, 3 \mathrm{H}), 3.93(\mathrm{~s}, 3 \mathrm{H}), 5.83-5.91(\mathrm{~m}, 2 \mathrm{H})$, 6.81-6.84 (m, 3H), $7.10(\mathrm{~d}, J=8.4 \mathrm{~Hz}, 4 \mathrm{H}), 7.18-7.22(\mathrm{~m}, 8 \mathrm{H}), 7.27(\mathrm{~d}, J=8.4$ $\mathrm{Hz}, 4 \mathrm{H}) ;{ }^{13} \mathrm{C} \mathrm{NMR}\left(100 \mathrm{MHz}, \mathrm{CDCl}_{3}\right) \delta 30.9,50.9,53.2,56.0,56.0,57.9,58.9$, $108.9,111.3,119.4,125.1,128.4,128.4,129.8,129.9,130.1,132.6,132.6,133.4$, 138.0, 138.6, 149.0, 149.2; HRMS (ESI) calcd for $\mathrm{C}_{41} \mathrm{H}_{41} \mathrm{Cl}_{4} \mathrm{~N}_{2} \mathrm{O}_{2}[\mathrm{M}+\mathrm{H}]$ : 733.1917, found: 733.1925 . The ee was determined to be $92 \%$ ee [Determined by HPLC with a Chiralcel IA column (hexane: isopropanol=99:1, $0.5 \mathrm{~mL} / \mathrm{min}, 254 \mathrm{~nm}, 25{ }^{\circ} \mathrm{C}$ ); $\mathrm{t}_{\mathbf{3 q d}}$ $\left.{ }_{(\text {major })}=11.7 \mathrm{~min}, \mathrm{t}_{3 \mathbf{q d}(\text { minor })}=15.0 \mathrm{~min}\right] .[\alpha]_{\mathrm{D}}{ }^{20}=-44.6\left(\mathrm{CH}_{2} \mathrm{Cl}_{2}, c 0.25\right)$. The structure of 3qd was confirmed by X-ray crystallographic analysis. The absolute configuration of 3qd was determined as $(S)$ by its X-ray crystallographic analysis, and the other products were tentatively assigned as $(S)$ by analogy.. 


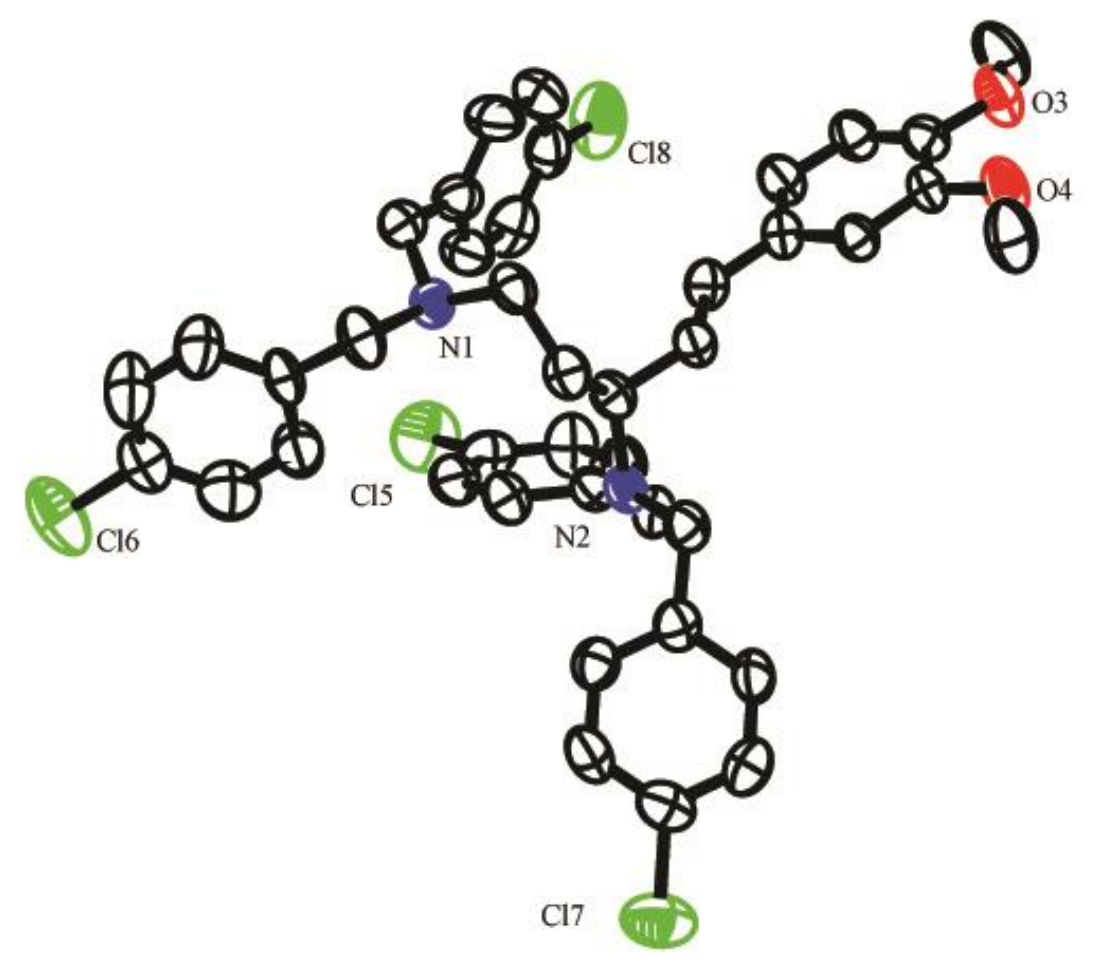

Figure S-6. X-ray structure of the product 3qd

(E)- $N^{1}, N^{1}, N^{2}, N^{2}$-tetrakis(4-chlorobenzyl)-5-(3,4-dimethoxyphenyl)pent-3-ene-1,2diamine (4qd): ${ }^{1} \mathrm{H}$ NMR $\left(400 \mathrm{MHz}, \mathrm{CDCl}_{3}\right) \delta$ 2.53-2.66 (m, 2H), $2.95(\mathrm{~d}, J=$

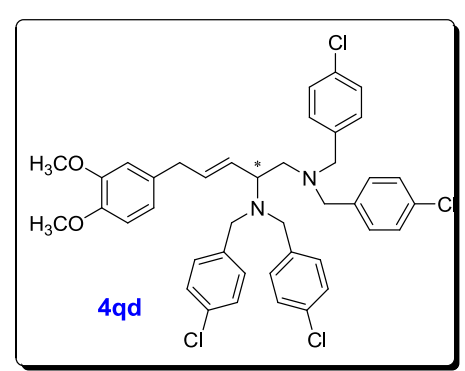
$6.4 \mathrm{~Hz}, 2 \mathrm{H}), 3.38-3.48(\mathrm{~m}, 6 \mathrm{H}), 3.51-3.56(\mathrm{~m}, 3 \mathrm{H})$, $3.70(\mathrm{~s}, 3 \mathrm{H}), 3.88(\mathrm{~s}, 3 \mathrm{H}), 5.40-5.47(\mathrm{~m}, 1 \mathrm{H}), 5.61$ $\left(\mathrm{dd}, J_{1}=7.2 \mathrm{~Hz}, J_{2}=15.6 \mathrm{~Hz}, 1 \mathrm{H}\right), 6.43(\mathrm{~d}, J=$ $2.0 \mathrm{~Hz}, 1 \mathrm{H}), 6.52-6.55(\mathrm{~m}, 1 \mathrm{H}), 6.74(\mathrm{~d}, J=8.4 \mathrm{~Hz}$ 1H), $7.05(\mathrm{~d}, J=8.4 \mathrm{~Hz}, 4 \mathrm{H}), 7.16(\mathrm{~d}, J=8.4 \mathrm{~Hz}, 4 \mathrm{H}), 7.21-7.28(\mathrm{~m}, 9 \mathrm{H}) ;{ }^{13} \mathrm{C}$ NMR $\left(100 \mathrm{MHz}, \mathrm{CDCl}_{3}\right) \delta 46.6,55.6,55.7,56.0,57.2,57.9,59.5,110.9,111.0$, $119.8,127.6,128.3,128.4,129.9,130.0,132.6,135.6,135.9,137.9,138.0$, 147.6, 148.8; HRMS (ESI) calcd for $\mathrm{C}_{41} \mathrm{H}_{41} \mathrm{Cl}_{4} \mathrm{~N}_{2} \mathrm{O}_{2}[\mathrm{M}+\mathrm{H}]$ : 733.1917, found: 733.1929. The ee was determined to be $79 \%$ ee [Determined by HPLC with a Chiralcel IA column (hexane: isopropanol= 98:2, $0.6 \mathrm{~mL} / \mathrm{min}, 218 \mathrm{~nm}, 25{ }^{\circ} \mathrm{C}$ ); $\mathrm{t}_{\mathbf{4 q d}}$ $\left.{ }_{\text {(major) }}=10.2 \mathrm{~min}, \mathrm{t}_{4 \mathbf{q d}(\text { minor) }}=13.6 \mathrm{~min}\right] .[\alpha]_{\mathrm{D}}{ }^{20}=-34.4\left(\mathrm{CH}_{2} \mathrm{Cl}_{2}, c 0.46\right)$. 


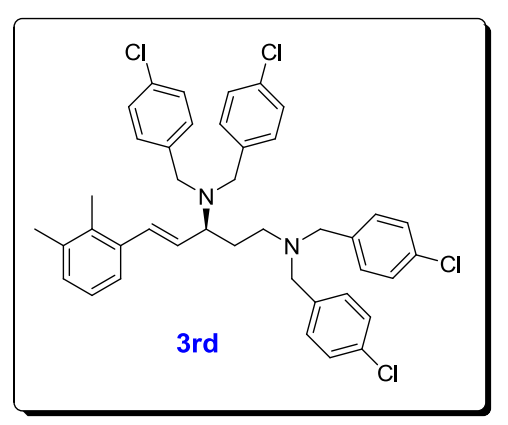

-diamine (3rd): The title compound was prepared according to the general procedure and purified by column chromatography to give the colorless oil, 36 $\mathrm{mg}, 26 \%$ yield (isolated yield of combination of $\mathbf{3 r d}$ and 4rd, 3rd:4rd = 62:38). ${ }^{1} \mathrm{H}$ NMR $(400 \mathrm{MHz}$,

$\left.\mathrm{CDCl}_{3}\right) \delta 1.67-1.76(\mathrm{~m}, 1 \mathrm{H}), 1.89-1.97(\mathrm{~m}, 1 \mathrm{H}), 2.18(\mathrm{~s}, 3 \mathrm{H}), 2.27-2.33(\mathrm{~m}, 4 \mathrm{H})$, 2.56-2.64 (m, 1H), 3.12-3.18 (m, 1H), 3.27-3.34 (m, 4H), $3.54(\mathrm{~d}, J=13.6 \mathrm{~Hz}$, $2 \mathrm{H}), 3.67(\mathrm{~d}, J=13.6 \mathrm{~Hz}, 2 \mathrm{H}), 5.78\left(\mathrm{dd}, J_{1}=9.2 \mathrm{~Hz}, J_{2}=15.6 \mathrm{~Hz}, 1 \mathrm{H}\right), 6.36(\mathrm{~d}$, $J=15.6 \mathrm{~Hz}, 1 \mathrm{H}), 7.07-7.14(\mathrm{~m}, 7 \mathrm{H}), 7.17-7.24(\mathrm{~m}, 8 \mathrm{H}), 7.27(\mathrm{~d}, J=4.80 \mathrm{~Hz}$ $4 \mathrm{H}),{ }^{13} \mathrm{C}$ NMR $\left(100 \mathrm{MHz}, \mathrm{CDCl}_{3}\right) \delta 15.6,20.6,30.6,51.0,53.2,57.8,58.9,124.2$, $125.6,128.4,128.5,128.9,129.3,129.9,130.0,132.6,132.7,132.9,133.7,136.6$, 136.9, 137.9, 138.5; HRMS (ESI) calcd for $\mathrm{C}_{41} \mathrm{H}_{41} \mathrm{Cl}_{4} \mathrm{~N}_{2}[\mathrm{M}+\mathrm{H}]$ : 701.2018, found: 701.2022; The ee was determined to be 99\% ee [Determined by HPLC with a Chiralcel OD-H column (hexane: isopropanol= 98:2, $0.6 \mathrm{~mL} / \mathrm{min}, 254 \mathrm{~nm}, 25^{\circ} \mathrm{C}$ ); $\mathrm{t}_{3 \mathrm{rd}}$ $($ major $\left.)=11.4 \mathrm{~min}, \mathrm{t}_{3 \mathbf{r d}(\operatorname{minor})}=17.6 \mathrm{~min}\right] .[\alpha]_{\mathrm{D}}{ }^{20}=-84.6\left(\mathrm{CH}_{2} \mathrm{Cl}_{2}, c 0.29\right)$.

(E)-N $N^{1}, N^{1}, N^{2}, N^{2}$-tetrakis(4-chlorobenzyl)-5-(2,3-dimethylphenyl)pent-3-ene-1,2-di

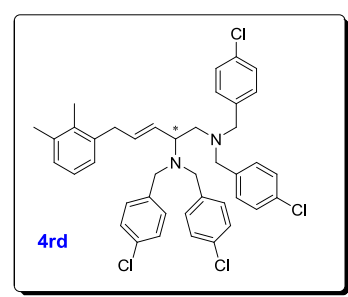

amine (4rd): ${ }^{1} \mathrm{H} \mathrm{NMR}\left(400 \mathrm{MHz}, \mathrm{CDCl}_{3}\right) \delta 2.07(\mathrm{~s}, 3 \mathrm{H})$, $2.25(\mathrm{~s}, 3 \mathrm{H}), 2.58-2.68(\mathrm{~m}, 2 \mathrm{H}), 2.94(\mathrm{~d}, J=6.4 \mathrm{~Hz}, 2 \mathrm{H})$, 3.39-3.55 (m, 8H), 3.82-3.88 (m, 1H), 5.35-5.43 (m, 1H), $5.61\left(\mathrm{dd}, J_{1}=7.2 \mathrm{~Hz}, J_{2}=15.6 \mathrm{~Hz}, 1 \mathrm{H}\right), 6.72-6.74(\mathrm{~m}$,

1H), 6.96-7.01 (m, 2H), $7.09(\mathrm{~d}, J=8.4 \mathrm{~Hz}, 4 \mathrm{H}), 7.15(\mathrm{~d}, J=8.4 \mathrm{~Hz}, 4 \mathrm{H})$, 7.20-7.26 (m, 8H); $\left.{ }^{13} \mathrm{C} \mathrm{NMR} \mathrm{(100} \mathrm{MHz,} \mathrm{CDCl}_{3}\right) \delta$ 15.0, 21.1, 42.6, 55.9, 57.2, $58.1,59.2,125.1,125.4,127.7,127.9,128.3,128.4,130.0,130.1,132.6,132.6$, 134.1, 136.2, 136.9, 137.9, 138.0, 140.7; HRMS (ESI) calcd for $\mathrm{C}_{41} \mathrm{H}_{41} \mathrm{Cl}_{4} \mathrm{~N}_{2}$ 
$[\mathrm{M}+\mathrm{H}]:$ 701.2018, found: 701.2011; The ee was determined to be $92 \%$ ee [Determined by HPLC with a Chiralcel IA column (hexane: isopropanol= 90:10, 0.6 $\left.\left.\mathrm{mL} / \mathrm{min}, 218 \mathrm{~nm}, 25{ }^{\circ} \mathrm{C}\right) ; \mathrm{t}_{4 \mathbf{r d}(\text { major })}=13.2 \mathrm{~min}, \mathrm{t}_{4 \mathbf{r d}(\operatorname{minor})}=16.9 \mathrm{~min}\right] .[\alpha]_{\mathrm{D}}{ }^{20}=-46.1$ $\left(\mathrm{CH}_{2} \mathrm{Cl}_{2}, c 0.38\right)$.

$(S, E)-N^{1}, N^{1}, N^{3}, N^{3}$-tetrakis(4-bromobenzyl)-5-(4-(tert-butyl)phenyl)pent-4-e

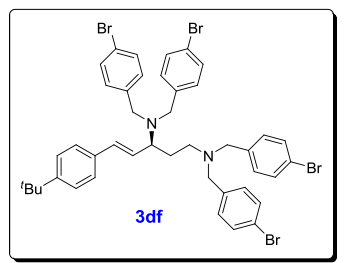
ne-1,3-diamine (3df): The title compound was prepared according to the general procedure and purified by column chromatography to give the colorless oil, $129 \mathrm{mg}, 71 \%$ yield

(isolated yield of combination of 3df and 4df, 3df:4df = 88:12). ${ }^{1} \mathrm{H}$ NMR $(400 \mathrm{MHz}$, $\left.\mathrm{CDCl}_{3}\right) \delta 1.34(\mathrm{~s}, 9 \mathrm{H}), 1.64-1.72(\mathrm{~m}, 1 \mathrm{H}), 1.86-1.95(\mathrm{~m}, 1 \mathrm{H}), 2.21-2.28(\mathrm{~m}, 1 \mathrm{H})$, 2.50-2.57 (m, 1H), 3.10-3.15 (m, 1H), $3.18(\mathrm{~d}, J=14.0 \mathrm{~Hz}, 2 \mathrm{H}), 3.28(\mathrm{~d}, J=$ $14.0 \mathrm{~Hz}, 2 \mathrm{H}), 3.54(\mathrm{~d}, J=13.6 \mathrm{~Hz}, 2 \mathrm{H}), 3.64(\mathrm{~d}, J=13.6 \mathrm{~Hz}, 2 \mathrm{H}), 5.93-6.02(\mathrm{~m}$, 2H), $7.03(\mathrm{~d}, J=8.4 \mathrm{~Hz}, 4 \mathrm{H}), 7.12(\mathrm{~d}, J=8.4 \mathrm{~Hz}, 4 \mathrm{H}), 7.24-7.26(\mathrm{~m}, 2 \mathrm{H})$, 7.35-7.38 (m, 6H), $7.41(\mathrm{~d}, J=8.4 \mathrm{~Hz}, 4 \mathrm{H}) ;{ }^{13} \mathrm{C} \mathrm{NMR}\left(100 \mathrm{MHz}, \mathrm{CDCl}_{3}\right) \delta$ $30.8,31.3,34.6,50.8,53.3,58.0,58.7,120.6,120.8,125.6,126.1,126.1,130.3$, $130.5,131.4,131.4,133.6,133.9,138.5,139.1,150.9$; HRMS (ESI) calcd for $\mathrm{C}_{43} \mathrm{H}_{45} \mathrm{Br}_{4} \mathrm{~N}_{2}[\mathrm{M}+\mathrm{H}]:$ 905.0311, found: 905.0330 . The ee was determined to be $94 \%$ ee [Determined by HPLC with a Chiralcel IA column (hexane: isopropanol= 99:1, 0.6 $\left.\left.\mathrm{mL} / \mathrm{min}, 254 \mathrm{~nm}, 25^{\circ} \mathrm{C}\right) ; \mathrm{t}_{\mathbf{3 d f}(\text { major })}=14.7 \mathrm{~min}, \mathrm{t}_{\mathbf{3 d f}(\operatorname{minor})}=19.0 \mathrm{~min}\right] .[\alpha]_{\mathrm{D}}{ }^{20}=-36.3$ $\left(\mathrm{CH}_{2} \mathrm{Cl}_{2}, c\right.$ 1.08).

$(S, E)-N^{1}, N^{1}, N^{3}, N^{3}$-tetrakis(4-bromobenzyl)-5-(4-fluorophenyl)pent-4-ene-1,3-dia

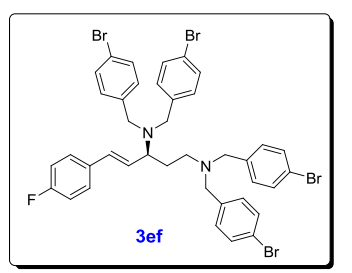
mine (3ef): The title compound was prepared according to the general procedure and purified by column chromatography to give the colorless oil, $118 \mathrm{mg}, 68 \%$ yield (isolated yield of combination of 3ef and 4ef, 3ef:4ef $=91: 9) .{ }^{1} \mathrm{H}$ NMR (400 
$\left.\mathrm{MHz}, \mathrm{CDCl}_{3}\right) \delta 1.55-1.63(\mathrm{~m}, 1 \mathrm{H}), 1.80-1.88(\mathrm{~m}, 1 \mathrm{H}), 2.15-2.21(\mathrm{~m}, 1 \mathrm{H})$, 2.42-2.49 (m, 1H), 3.03-3.07 (m, 1H), $3.11(\mathrm{~d}, J=13.6 \mathrm{~Hz}, 2 \mathrm{H}), 3.22(\mathrm{~d}, J=$ $13.6 \mathrm{~Hz}, 2 \mathrm{H}), 3.47(\mathrm{~d}, J=13.6 \mathrm{~Hz}, 2 \mathrm{H}), 3.57(\mathrm{~d}, J=13.6 \mathrm{~Hz}, 2 \mathrm{H}), 5.83-5.84(\mathrm{~m}$, 2H), 6.93-6.97 (m, 6H), $7.05(\mathrm{~d}, J=8.4 \mathrm{~Hz}, 4 \mathrm{H}), 7.15-7.18(\mathrm{~m}, 3 \mathrm{H}), 7.28(\mathrm{~d}, J$ $=8.4 \mathrm{~Hz}, 4 \mathrm{H}), 7.34(\mathrm{~d}, J=8.4 \mathrm{~Hz}, 4 \mathrm{H}) ;{ }^{13} \mathrm{C} \mathrm{NMR}\left(100 \mathrm{MHz}, \mathrm{CDCl}_{3}\right) \delta 30.7$, 50.7, 53.3, 58.0, 58.6, 115.4, 115.6, 120.7, 120.8, 126.8, 126.8, 127.8, 127.9, 130.3, $130.5,131.4,131.4,132.6,132.8,132.8,138.5,139.0,161.1,163.6 ;{ }^{19} \mathrm{~F}$ NMR $\left(376 \mathrm{MHz}, \mathrm{CDCl}_{3}\right) \quad \delta$-114.4; HRMS (ESI) calcd for $\mathrm{C}_{39} \mathrm{H}_{36} \mathrm{Br}_{4} \mathrm{FN}_{2} \quad[\mathrm{M}+\mathrm{H}]$ : 866.9591, found: 866.9562 . The ee was determined to be $92 \%$ ee [Determined by HPLC with a Chiralcel IA column (hexane: isopropanol=98:2, $0.6 \mathrm{~mL} / \mathrm{min}, 254 \mathrm{~nm}$, $\left.\left.25^{\circ} \mathrm{C}\right) ; \mathrm{t}_{3 e f(\text { major })}=15.5 \mathrm{~min}, \mathrm{t}_{3 e f(\text { minor })}=20.4 \mathrm{~min}\right] .[\alpha]_{\mathrm{D}}{ }^{20}=-23.8\left(\mathrm{CH}_{2} \mathrm{Cl}_{2}, c 0.70\right)$. $(S, E)-N^{1}, N^{1}, N^{3}, N^{3}$-tetrakis(4-bromobenzyl)-5-(3,5-dimethylphenyl)pent-4-e

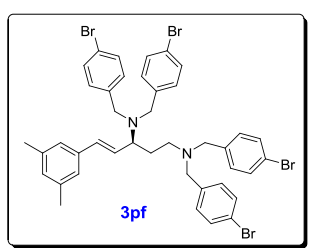
ne-1,3-diamine (3pf): The title compound was prepared according to the general procedure and purified by column chromatography to give the colorless oil, $123 \mathrm{mg}, 70 \%$ yield (isolated yield of combination of 3pf and 4pf, 3pf:4pf = 90:10). ${ }^{1} \mathrm{H}$ NMR $(400 \mathrm{MHz}$, $\left.\mathrm{CDCl}_{3}\right) \delta 1.63-1.71(\mathrm{~m}, 1 \mathrm{H}), 1.85-1.94(\mathrm{~m}, 1 \mathrm{H}), 2.21-2.27(\mathrm{~m}, 1 \mathrm{H}), 2.34(\mathrm{~s}, 6 \mathrm{H})$, 2.49-2.56 (m, 1H), 3.09-3.14 (m, 1H), $3.16(\mathrm{~d}, J=13.6 \mathrm{~Hz}, 2 \mathrm{H}), 3.29(\mathrm{~d}, J=$ $13.6 \mathrm{~Hz}, 2 \mathrm{H}), 3.55(\mathrm{~d}, J=13.6 \mathrm{~Hz}, 2 \mathrm{H}), 3.64(\mathrm{~d}, J=13.6 \mathrm{~Hz}, 2 \mathrm{H}), 5.83(\mathrm{~d}, J=$ $16.0 \mathrm{~Hz}, 1 \mathrm{H}), 5.96\left(\mathrm{dd}, J_{1}=8.8 \mathrm{~Hz}, J_{2}=16.0 \mathrm{~Hz}, 1 \mathrm{H}\right), 6.91-6.92(\mathrm{~m}, 3 \mathrm{H}), 7.02$ $(\mathrm{d}, J=8.4 \mathrm{~Hz}, 4 \mathrm{H}), 7.13(\mathrm{~d}, J=8.4 \mathrm{~Hz}, 4 \mathrm{H}), 7.36(\mathrm{~d}, J=8.4 \mathrm{~Hz}, 4 \mathrm{H}), 7.42(\mathrm{~d}, J$ $=8.4 \mathrm{~Hz}, 4 \mathrm{H}) ;{ }^{13} \mathrm{C} \mathrm{NMR}\left(100 \mathrm{MHz}, \mathrm{CDCl}_{3}\right) \delta 21.3,30.9,50.8,53.3,58.0,58.7$, $120.6,120.8,124.2,126.5,129.4,130.3,130.5,131.4,131.4,134.1,136.5,138.2$, 138.5, 139.1; HRMS (ESI) calcd for $\mathrm{C}_{41} \mathrm{H}_{41} \mathrm{Br}_{4} \mathrm{~N}_{2}$ [M+H]: 876.9998, found: 877.0014 The ee was determined to be $91 \%$ ee [Determined by HPLC with a Chiralcel IA 
column (hexane: isopropanol= 98:2, $\left.0.6 \mathrm{~mL} / \mathrm{min}, 254 \mathrm{~nm}, 25^{\circ} \mathrm{C}\right) ; \mathrm{t}_{3 \mathbf{p f}(\text { major })}=12.8 \mathrm{~min}$, $\left.\mathrm{t}_{3 \mathbf{p f} \text { (minor) }}=16.8 \mathrm{~min}\right] .[\alpha]_{\mathrm{D}}{ }^{20}=-44.0\left(\mathrm{CH}_{2} \mathrm{Cl}_{2}, c 0.51\right)$.

$(S, E)-N^{1}, N^{1}, N^{3}, N^{3}$-tetrakis(4-chlorobenzyl)-7-phenylhept-4-ene-1,3-diamine

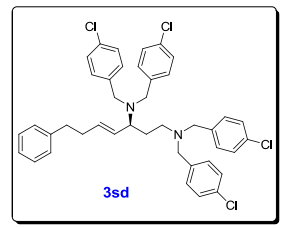

(3sd): The title compound was prepared according to the general procedure and purified by column chromatography to give the colorless oil, $46 \mathrm{mg}, 33 \%$ yield (isolated yield of combination of

3sd and 4sd, 3sd:4sd > 20:1). ${ }^{1} \mathrm{H}$ NMR (400 MHz, $\left.\mathrm{CDCl}_{3}\right) \delta 1.31-1.39(\mathrm{~m}, 1 \mathrm{H})$, $1.75-1.83(\mathrm{~m}, 1 \mathrm{H}), 2.24-2.31(\mathrm{~m}, 3 \mathrm{H}), 2.38-2.45(\mathrm{~m}, 1 \mathrm{H}), 2.54-2.61(\mathrm{~m}, 1 \mathrm{H})$, $3.00(\mathrm{~d}, J=6.4 \mathrm{~Hz}, 2 \mathrm{H}), 3.35-3.44(\mathrm{~m}, 4 \mathrm{H}), 3.48-3.57(\mathrm{~m}, 4 \mathrm{H}), 5.25\left(\mathrm{dd}, J_{1}=\right.$ $\left.9.2 \mathrm{~Hz}, J_{2}=15.6 \mathrm{~Hz}, 1 \mathrm{H}\right), 5.44-5.51(\mathrm{~m}, 1 \mathrm{H}), 7.06(\mathrm{~d}, J=7.6 \mathrm{~Hz}, 2 \mathrm{H})$, 7.14-7.20 (m, 9H), 7.22-7.26 (m, 3H), $7.29(\mathrm{~s}, 7 \mathrm{H}) ;{ }^{13} \mathrm{C}$ NMR (100 MHz, $\left.\mathrm{CDCl}_{3}\right) \delta 33.2,34.3,40.5,55.7,57.1,58.0,58.9,125.8,128.1,128.3,128.3,128.5$, 130.0, 130.1, 132.6, 132.6, 136.9, 137.9, 138.1, 142.3; HRMS (ESI) calcd for $\mathrm{C}_{41} \mathrm{H}_{41} \mathrm{Cl}_{4} \mathrm{~N}_{2}[\mathrm{M}+\mathrm{H}]:$ 701.2018, found: 701.2038 . The ee was determined to be $80 \%$ ee [Determined by HPLC with a Chiralcel OD-H column (hexane: isopropanol= 99:1, $\left.\left.0.6 \mathrm{~mL} / \mathrm{min}, 218 \mathrm{~nm}, 25^{\circ} \mathrm{C}\right) ; \mathrm{t}_{3 \text { sd }(\text { major })}=8.7 \mathrm{~min}, \mathrm{t}_{3 \text { sd }(\text { minor })}=15.9 \mathrm{~min}\right] .[\alpha]_{\mathrm{D}}{ }^{20}=-29.4$ $\left(\mathrm{CH}_{2} \mathrm{Cl}_{2}, c 0.24\right)$.

$(S, E)-N^{1}, N^{1}, N^{3}, N^{3}$-tetrakis(4-chlorobenzyl)tridec-4-ene-1,3-diamine (3td): The

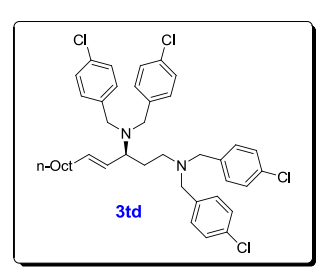
title compound was prepared according to the general procedure and purified by column chromatography to give the colorless oil, $79 \mathrm{mg}, 56 \%$ yield (isolated yield of combination of $3 \mathrm{td}$ and $\mathbf{4 t d}$,

3td:4td > 20:1). ${ }^{1} \mathrm{H}$ NMR (400 MHz, $\left.\mathrm{CDCl}_{3}\right) \delta 0.86(\mathrm{t}, 3 \mathrm{H}), 1.19-1.24(\mathrm{~m}, 14 \mathrm{H})$, 2.22-2.30 (m, 3H), $2.97(\mathrm{~d}, J=6.4 \mathrm{~Hz}, 2 \mathrm{H}), 3.36-3.50(\mathrm{~m}, 8 \mathrm{H}), 5.19\left(\mathrm{dd}, J_{1}=\right.$ $\left.8.0 \mathrm{~Hz}, J_{2}=15.6 \mathrm{~Hz}, 1 \mathrm{H}\right), 5.39-5.46(\mathrm{~m}, 1 \mathrm{H}), 7.19(\mathrm{~s}, 8 \mathrm{H}), 7.27(\mathrm{~s}, 8 \mathrm{H}) ;{ }^{13} \mathrm{C}$ NMR (100 MHz, $\left.\mathrm{CDCl}_{3}\right) \delta 14.1,22.7,27.0,29.3,29.6,29.7,31.9,32.8,41.1,55.7$, 
57.0, 58.0, 59.0, 127.3, 128.3, 128.4, 130.0, 130.0, 132.5, 132.6, 137.6, 138.1, 138.1;

HRMS (ESI) calcd for $\mathrm{C}_{41} \mathrm{H}_{49} \mathrm{Cl}_{4} \mathrm{~N}_{2}[\mathrm{M}+\mathrm{H}]$ : 709.2644 , found: 709.2669. The ee was determined to be $77 \%$ ee [Determined by HPLC with a Chiralcel OD-H column (hexane: isopropanol $\left.=98: 2,0.6 \mathrm{~mL} / \mathrm{min}, 218 \mathrm{~nm}, 25^{\circ} \mathrm{C}\right) ; \mathrm{t}_{3 \mathbf{t d}(\text { major })}=7.6 \mathrm{~min}, \mathrm{t}_{3 \mathrm{td}(\operatorname{minor})}$ $=11.9 \min ] .[\alpha]_{\mathrm{D}}^{20}=-31.6\left(\mathrm{CH}_{2} \mathrm{Cl}_{2}, c 0.34\right)$.

$(S, E)-N^{1}, N^{1}, N^{3}, N^{3}$-tetrakis(4-chlorobenzyl)-5-(furan-2-yl)pent-4-ene-1,3-diamine

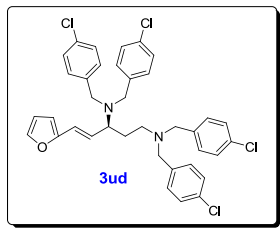

(3ud): The title compound was prepared according to the general procedure and purified by column chromatography to give the colorless oil, $86 \mathrm{mg}, 65 \%$ yield (isolated yield of combination of

3ud and 4ud, 3ud:4ud = 47:53). ${ }^{1} \mathrm{H}$ NMR $\left(400 \mathrm{MHz}, \mathrm{CDCl}_{3}\right) \delta 1.61-1.69(\mathrm{~m}, 1 \mathrm{H})$, $1.85-1.93(\mathrm{~m}, 1 \mathrm{H}), 2.19-2.25(\mathrm{~m}, 1 \mathrm{H}), 2.48-2.55(\mathrm{~m}, 1 \mathrm{H}), 3.05-3.11(\mathrm{~m}, 1 \mathrm{H})$, $3.15(\mathrm{~d}, J=13.6 \mathrm{~Hz}, 2 \mathrm{H}), 3.29(\mathrm{~d}, J=13.6 \mathrm{~Hz}, 2 \mathrm{H}), 3.56(\mathrm{~d}, J=13.6 \mathrm{~Hz}, 2 \mathrm{H})$, $3.64(\mathrm{~d}, J=13.6 \mathrm{~Hz}, 2 \mathrm{H}), 5.61(\mathrm{~d}, J=15.6 \mathrm{~Hz}, 1 \mathrm{H}), 5.92\left(\mathrm{dd}, J_{1}=9.2 \mathrm{~Hz}, J_{2}=\right.$ $16.0 \mathrm{~Hz}, 1 \mathrm{H}), 6.17(\mathrm{~d}, J=3.2 \mathrm{~Hz}, 1 \mathrm{H}), 6.39\left(\mathrm{dd}, J_{1}=2.0 \mathrm{~Hz}, J_{2}=3.2 \mathrm{~Hz}, 1 \mathrm{H}\right)$, $7.06(\mathrm{~d}, J=8.4 \mathrm{~Hz}, 4 \mathrm{H}), 7.18-7.23(\mathrm{~m}, 8 \mathrm{H}), 7.26-7.28(\mathrm{~m}, 4 \mathrm{H}), 7.34(\mathrm{~d}, J=1.2$ $\mathrm{Hz}, 1 \mathrm{H}) ;{ }^{13} \mathrm{C} \mathrm{NMR}\left(100 \mathrm{MHz}, \mathrm{CDCl}_{3}\right) \delta 30.8,50.6,53.2,57.9,58.4,107.9,111.4$, $122.0,125.7,128.4,128.4,129.9,130.2,132.6,132.6,138.0,138.6,141.8,152.2$. HRMS (ESI) calcd for $\mathrm{C}_{37} \mathrm{H}_{35} \mathrm{Cl}_{4} \mathrm{~N}_{2} \mathrm{O}[\mathrm{M}+\mathrm{H}]$ : 663.1498, found: 663.1495. The ee was determined to be $96 \%$ ee [Determined by HPLC with a Chiralcel OD-H column (hexane: isopropanol $=98: 2,0.8 \mathrm{~mL} / \mathrm{min}, 254 \mathrm{~nm}, 25^{\circ} \mathrm{C}$ ); $\mathrm{t}_{\text {3ud (major) }}=7.4 \mathrm{~min}$, $\left.\mathrm{t}_{3 \mathbf{u d}(\text { minor) }}=12.4 \mathrm{~min}\right] .[\alpha]_{\mathrm{D}}{ }^{20}=-59.4\left(\mathrm{CH}_{2} \mathrm{Cl}_{2}, c 0.41\right)$.

(E)- $N^{1}, N^{1}, N^{2}, N^{2}$-tetrakis(4-chlorobenzyl)-5-(furan-2-yl)pent-3-ene-1,2-diamine

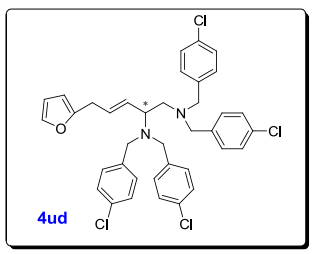
(4ud): ${ }^{1} \mathrm{H}$ NMR $\left(400 \mathrm{MHz}, \mathrm{CDCl}_{3}\right) \delta 2.55\left(\mathrm{dd}, J_{1}=7.6 \mathrm{~Hz}\right.$, $\left.J_{2}=12.8 \mathrm{~Hz}, 1 \mathrm{H}\right), 2.73\left(\mathrm{dd}, J_{1}=7.6 \mathrm{~Hz}, J_{2}=12.8 \mathrm{~Hz}, 1 \mathrm{H}\right)$, $2.97(\mathrm{~d}, J=5.6 \mathrm{~Hz}, 2 \mathrm{H}), 3.43-3.51(\mathrm{~m}, 8 \mathrm{H}), 3.63-3.68(\mathrm{~m}$, 
$1 \mathrm{H}), 5.44-5.58(\mathrm{~m}, 2 \mathrm{H}), 5.92(\mathrm{~d}, J=3.2 \mathrm{~Hz}, 1 \mathrm{H}), 6.28\left(\mathrm{dd}, J_{1}=1.6 \mathrm{~Hz}, J_{2}=3.2\right.$

$\mathrm{Hz}, 1 \mathrm{H}), 7.11(\mathrm{~d}, J=8.4 \mathrm{~Hz}, 4 \mathrm{H}), 7.17(\mathrm{~d}, J=8.4 \mathrm{~Hz}, 4 \mathrm{H}), 7.22-7.28(\mathrm{~m}, 9 \mathrm{H})$;

${ }^{13} \mathrm{C}$ NMR $\left(100 \mathrm{MHz}, \mathrm{CDCl}_{3}\right) \delta 41.0,55.5,57.2,57.3,57.8,105.5,110.2,128.3$, 128.4, 128.9, 130.0, 132.6, 132.9, 137.8, 138.0, 141.1, 155.8; HRMS (ESI) calcd for $\mathrm{C}_{37} \mathrm{H}_{35} \mathrm{Cl}_{4} \mathrm{~N}_{2} \mathrm{O}[\mathrm{M}+\mathrm{H}]:$ 663.1498, found: 663.1495 . The ee was determined to be 85\% ee [Determined by HPLC with a Chiralcel OD-H column (hexane: isopropanol= $\left.\left.99: 1,0.5 \mathrm{~mL} / \mathrm{min}, 218 \mathrm{~nm}, 25{ }^{\circ} \mathrm{C}\right) ; \mathrm{t}_{4 \mathbf{u d}(\text { major) }}=9.0 \mathrm{~min}, \mathrm{t}_{4 \mathbf{u d}(\operatorname{minor})}=15.0 \mathrm{~min}\right] .[\alpha]_{\mathrm{D}}{ }^{20}=$ $-41.3\left(\mathrm{CH}_{2} \mathrm{Cl}_{2}, c 0.56\right)$.

\section{Mechanism studies.}

\subsection{Synthesis of $\mathrm{Pd}(5 \mathrm{e})(\mathrm{allyl})\left(\mathrm{ClO}_{4}\right)$}
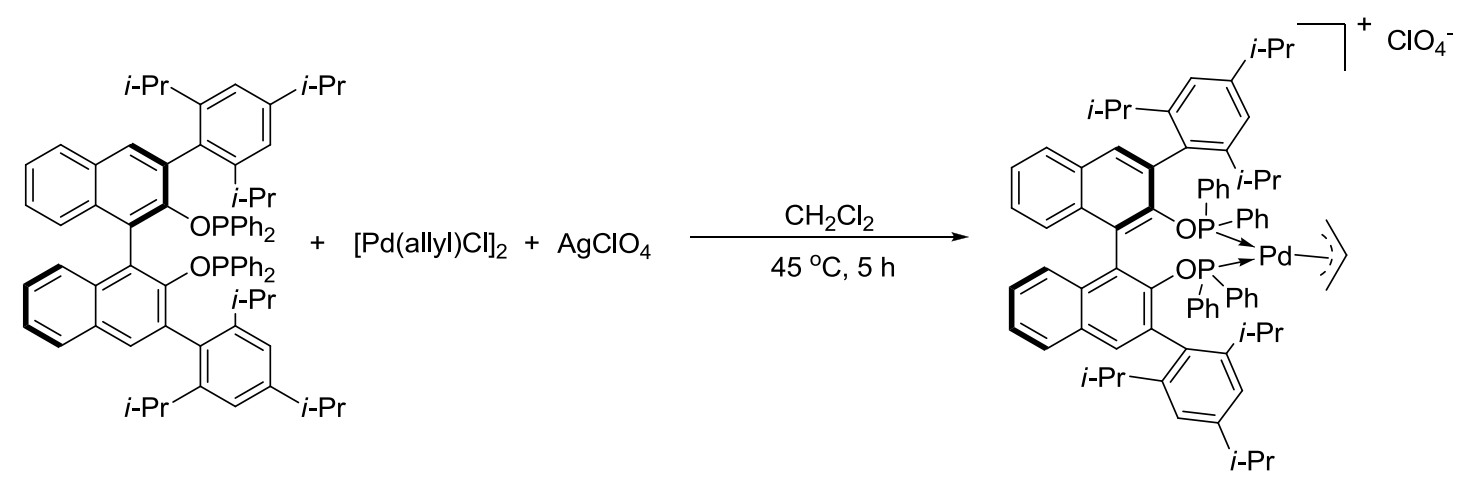

Under nitrogen, the mixture of $[\mathrm{Pd}(\mathrm{allyl}) \mathrm{Cl}]_{2}(18.2 \mathrm{mg}, 0.05 \mathrm{mmol})$, ligand (116.5 mg, $0.11 \mathrm{mmol}), \mathrm{AgClO}_{4}(23 \mathrm{mg}, 0.11 \mathrm{mmol})$ and $\mathrm{CH}_{2} \mathrm{Cl}_{2}(4 \mathrm{~mL})$ was added to a Young-type tube and stirred at $45{ }^{\circ} \mathrm{C}$ for 5 hours. After the mixture cooled to the room temperature, $\mathrm{AgCl}$ was removed via fitration. A yellow complex was afforded when the solvent was removed under vacumm. The struture of the complex was analyzed by NMR, HRMS and X-ray. ${ }^{1} \mathrm{H}$ NMR (400 MHz, $\left.\mathrm{CDCl}_{3}\right): \delta 0.86-0.90(\mathrm{~m}, 6 \mathrm{H}), 1.03(\mathrm{~d}$, $J=6.8 \mathrm{~Hz}, 3 \mathrm{H}), 1.11(\mathrm{~d}, J=6.8 \mathrm{~Hz}, 3 \mathrm{H}), 1.23(\mathrm{~d}, J=6.8 \mathrm{~Hz}, 3 \mathrm{H}), 1.28-1.39(\mathrm{~m}, 18 \mathrm{H})$, $1.46(\mathrm{~d}, J=6.8 \mathrm{~Hz}, 3 \mathrm{H}), 2.65-2.71(\mathrm{~m}, 1 \mathrm{H}), 2.80-2.84(\mathrm{~m}, 2 \mathrm{H}), 2.90-3.01(\mathrm{~m}, 4 \mathrm{H})$, $3.21(\mathrm{~s}, 1 \mathrm{H}), 3.65-3.71(\mathrm{~m}, 1 \mathrm{H}), 3.86-3.89(\mathrm{~m}, 1 \mathrm{H})$, 5.58-5.71 (m, 1H), 6.20-6.24 (m, $2 \mathrm{H}), 6.27-6.33(\mathrm{~m}, 2 \mathrm{H}), 6.74-6.80(\mathrm{~m}, 3 \mathrm{H}), 6.86-6.97(\mathrm{~m}, 10 \mathrm{H}), 7.00-7.11(\mathrm{~m}, 6 \mathrm{H})$, 
7.18-7.19 (m, 2H), 7.23-7.32 (m, 3H), 7.39 (s, 1H), 7.41-7.55 (m, 5H); ${ }^{13} \mathrm{C}$ NMR $(100$ $\left.\mathrm{MHz}, \mathrm{CDCl}_{3}\right): \delta 22.7,23.1,23.9,23.9,24.2,24.5,24.8,25.0,25.1,26.0,26.3,30.4$, $30.5,30.8,30.9,34.0,34.1,121.2,121.4,122.1,122.2,125.3,126.0,126.9,127.0$ $127.8,127.9,128.0,128.1,128.2,128.8,129.1,129.3,129.5,129.6,130.2,130.9$, $131.1,131.3,132.1,132.3,134.1,134.5,146.3,147.5,147.7,149.0,149.1,151.8 ;{ }^{31} \mathrm{P}$ NMR (162 MHz, $\left.\mathrm{CDCl}_{3}\right): \delta 129.40,129.66,132.26,132.51$; HRMS (ESI) calcd for $\mathrm{C}_{77} \mathrm{H}_{81} \mathrm{P}_{2} \mathrm{O}_{2} \mathrm{Pd}\left[\mathrm{M}-\mathrm{ClO}_{4}\right.$ ]: 1205.4765 , found: 1205.4665 . The structure of confirmed by X-ray crytallographic analysis.

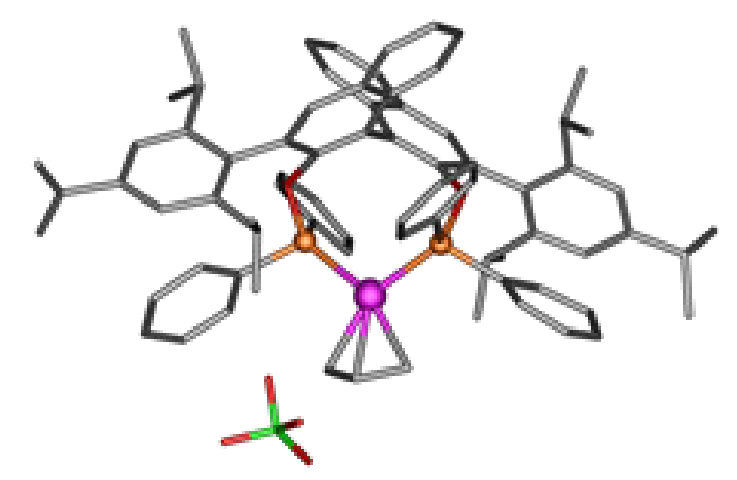

Figure S-7. X-ray structure of the complex $\mathrm{Pd}(\mathbf{5 e})($ allyl $)\left(\mathrm{ClO}_{4}\right)$

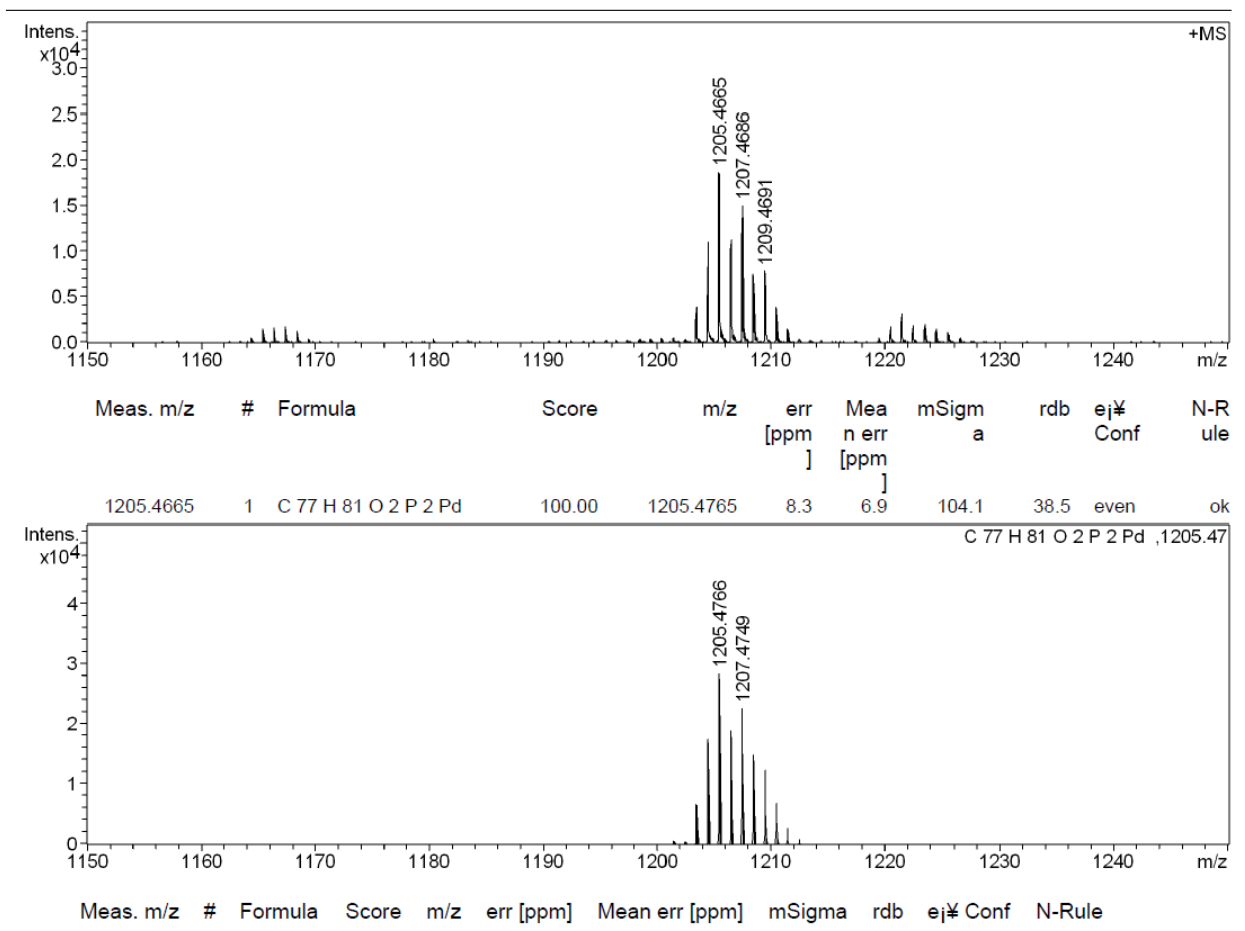

Figure S-8. HRMS of the complex $\mathrm{Pd}(\mathbf{5 e})(\operatorname{allyl})\left(\mathrm{ClO}_{4}\right)$ 


\subsection{Experimental studies on isotope effect}
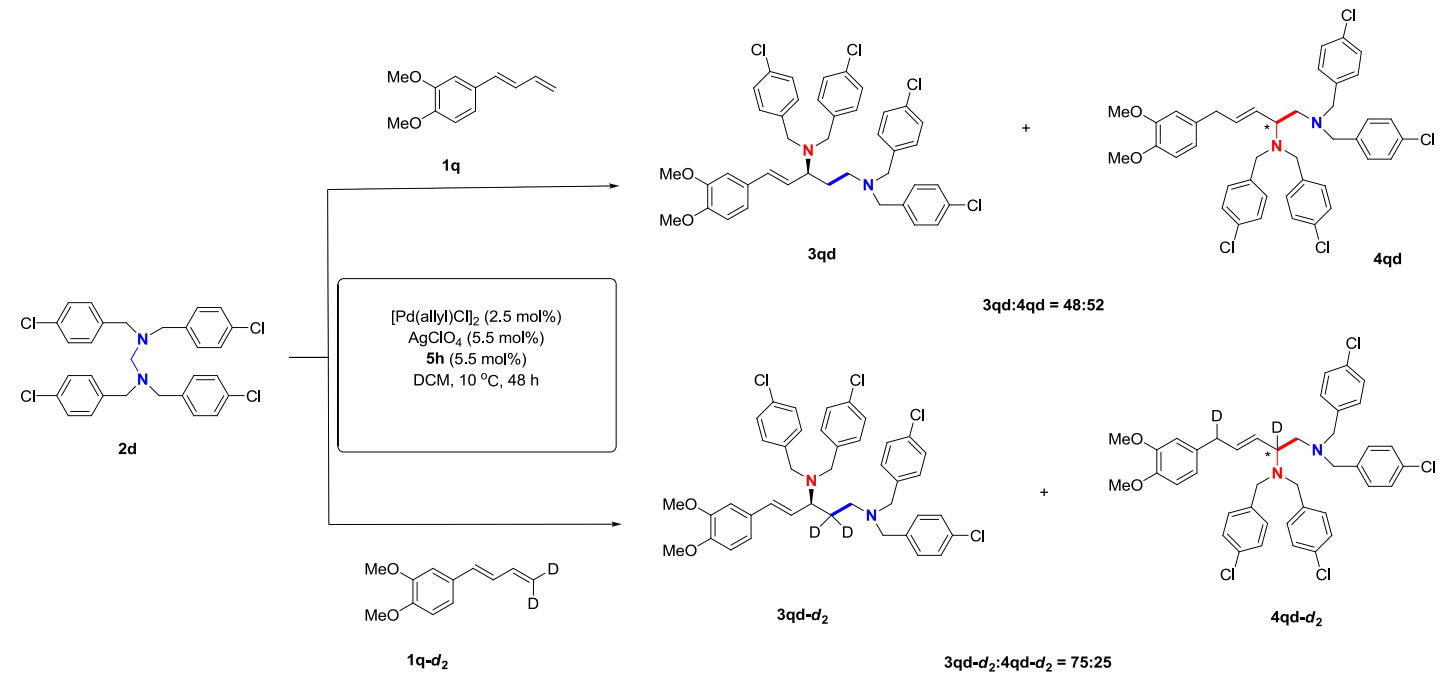

3qd:4qd $=48: 52$
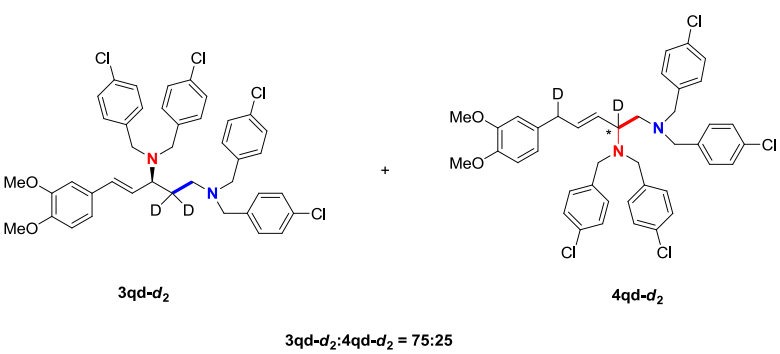

The mixture of $[\mathrm{Pd}(\mathrm{allyl}) \mathrm{Cl}]_{2}(1.8 \mathrm{mg}, 0.005 \mathrm{mmol}), \mathbf{5 h}(12.8 \mathrm{mg}, 0.011 \mathrm{mmol})$, $\mathrm{AgClO}_{4}(2.4 \mathrm{mg}, 0.011 \mathrm{mmol})$ and $\mathrm{CH}_{2} \mathrm{Cl}_{2}(0.5 \mathrm{~mL})$ was added to a Young-type tube in the glove box and stirred at $45{ }^{\circ} \mathrm{C}$ for 30 minutes. After the mixture cooled to room temperature, $N, N, N^{\prime}, N^{\prime}$-tetrakis(4-chlorobenzyl)methanediamine (2d) $(0.2 \mathrm{mmol})$ and $\mathbf{1 q}-\boldsymbol{d}_{\mathbf{2}}(0.24 \mathrm{mmol})$ were added under nitrogen. The reaction mixture was degassed via the freeze-thaw method and stirred at $10{ }^{\circ} \mathrm{C}$ for 48 hours. The solvent was evaporated under reduced pressure and the residue was purified by flash column chromatography (eluted with petroleum ether/diethyl ether $=100 / 1 \sim 50 / 1$ ) on a silica gel to give the desired product, $68 \%$ yield [(isolated yield of combination of $3 q \mathbf{q}-\boldsymbol{d}_{\mathbf{2}}$ and $\mathbf{4 q d}-\boldsymbol{d}_{\mathbf{2}}$ ),

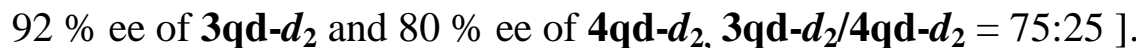

\section{$(S, E)-N^{1}, N^{1}, N^{3}, N^{3}$-tetrakis(4-chlorobenzyl)-5-(3,4-dimethoxyphenyl)pent-4-}

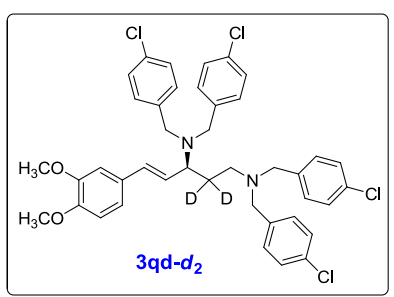

ene-1,3-diamine (3qd- $\left.\boldsymbol{d}_{2}\right)$ : ${ }^{1} \mathrm{H}$ NMR $\left(400 \mathrm{MHz}, \mathrm{CDCl}_{3}\right)$

$\delta 2.23(\mathrm{~d}, J=12.8 \mathrm{~Hz}, 1 \mathrm{H}), 2.51(\mathrm{~d}, J=12.8 \mathrm{~Hz}, 1 \mathrm{H})$, 3.11-3.12 (m, 1H), $3.20(\mathrm{~d}, J=13.6 \mathrm{~Hz}, 2 \mathrm{H}), 3.32(\mathrm{~d}, J$ $=13.6 \mathrm{~Hz}, 2 \mathrm{H}), 3.57(\mathrm{~d}, J=13.6 \mathrm{~Hz}, 2 \mathrm{H}), 3.66(\mathrm{~d}, J=$

$13.6 \mathrm{~Hz}, 2 \mathrm{H}), 3.90(\mathrm{~s}, 3 \mathrm{H}), 3.94(\mathrm{~s}, 3 \mathrm{H}), 5.87-5.88(\mathrm{~m}, 2 \mathrm{H}), 6.81-6.84(\mathrm{~m}, 3 \mathrm{H})$,

$7.09(\mathrm{~d}, J=8.4 \mathrm{~Hz}, 4 \mathrm{H}), 7.18-7.22(\mathrm{~m}, 8 \mathrm{H}), 7.27(\mathrm{~d}, J=8.4 \mathrm{~Hz}, 4 \mathrm{H}),{ }^{13} \mathrm{C} \mathrm{NMR}$ 
$\left(100 \mathrm{MHz}, \mathrm{CDCl}_{3}\right) \delta 50.7,50.8,53.2,56.0,56.0,57.9,58.7,58.8,108.8,111.2$ $119.4,125.1,128.4,128.4,129.8,129.8,129.9,130.1,132.5,132.6,133.4$, 138.0, 138.6, 148.9, 149.1; HRMS (ESI) calcd for $\mathrm{C}_{41} \mathrm{H}_{39} \mathrm{D}_{2} \mathrm{Cl}_{4} \mathrm{~N}_{2} \mathrm{O}_{2}[\mathrm{M}+\mathrm{H}]$ : 735.2042, found: 735.2005 . The ee was determined to be $92 \%$ ee [Determined by HPLC with a Chiralcel IA column (hexane: isopropanol=99:1, $0.5 \mathrm{~mL} / \mathrm{min}, 254 \mathrm{~nm}$, $\left.25{ }^{\circ} \mathrm{C}\right) ; \mathrm{t}_{3 \mathrm{qd}-d 2}$ (major) $\left.=11.5 \min , \mathrm{t}_{3 \mathrm{qd}-d 2 \text { (minor) }}=14.9 \min \right] .[\alpha]_{\mathrm{D}}{ }^{20}=-42.5\left(\mathrm{CH}_{2} \mathrm{Cl}_{2}, c\right.$ $0.36)$.

(E)- $N^{1}, N^{1}, N^{2}, N^{2}$-tetrakis(4-chlorobenzyl)-5-(3,4-dimethoxyphenyl)pent-3-ene-1,2-

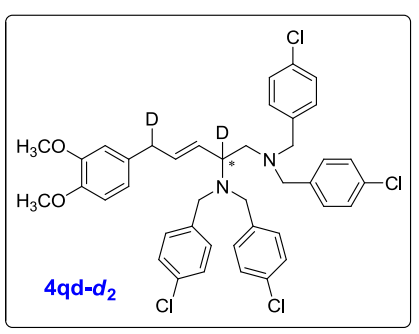
diamine $\left(\mathbf{4 q d}-\boldsymbol{d}_{2}\right):{ }^{1} \mathrm{H} \quad \mathrm{NMR}\left(400 \mathrm{MHz}, \mathrm{CDCl}_{3}\right) \quad \delta$ 2.54-2.67 (m, 2H), 3.38-3.56 (m, 9H), $3.70(\mathrm{~s}, 3 \mathrm{H})$, $3.88(\mathrm{~s}, 3 \mathrm{H}), 5.41(\mathrm{~d}, J=15.6 \mathrm{~Hz}, 1 \mathrm{H}), 5.60\left(\mathrm{dd}, J_{1}=\right.$ $\left.7.6 \mathrm{~Hz}, J_{2}=15.6 \mathrm{~Hz}, 1 \mathrm{H}\right), 6.43(\mathrm{~d}, J=1.6 \mathrm{~Hz}, 1 \mathrm{H})$, 6.52-6.55 (m, 1H), $6.74(\mathrm{~d}, J=8.4 \mathrm{~Hz}, 1 \mathrm{H}), 7.05(\mathrm{~d}, J=8.4 \mathrm{~Hz}, 4 \mathrm{H}), 7.16(\mathrm{~d}, J$ $=8.4 \mathrm{~Hz}, 4 \mathrm{H}), 7.21-7.27(\mathrm{~m}, 8 \mathrm{H}),{ }^{13} \mathrm{C} \mathrm{NMR}\left(100 \mathrm{MHz}, \mathrm{CDCl}_{3}\right) \delta 46.6,55.7$, $56.0,57.2,57.2,57.9,59.5,110.0,110.0,119.8,127.4,127.5,128.3,128.4$, $129.9,130.0,132.6,135.6,136.0,136.0,137.9,138.0,147.6,148.8 ;$ HRMS (ESI) calcd for $\mathrm{C}_{41} \mathrm{H}_{39} \mathrm{D}_{2} \mathrm{Cl}_{4} \mathrm{~N}_{2} \mathrm{O}_{2}[\mathrm{M}+\mathrm{H}]$ : 735.2042, found: 735.1997. The ee was determined to be $80 \%$ ee [Determined by HPLC with a Chiralcel IA column (hexane: isopropanol $=98: 2,0.6 \mathrm{~mL} / \mathrm{min}, 218 \mathrm{~nm}, 25^{\circ} \mathrm{C}$ ); $\mathbf{t}_{\mathbf{4 q d}-\mathbf{d} 2 \text { (major) }}=9.7 \mathrm{~min}$, $\left.\mathrm{t}_{4 \mathbf{q d}-\boldsymbol{d} \mathbf{2} \text { (minor) }}=12.5 \mathrm{~min}\right] .[\alpha]_{\mathrm{D}}{ }^{20}=-36.2\left(\mathrm{CH}_{2} \mathrm{Cl}_{2}, c 0.30\right)$. 


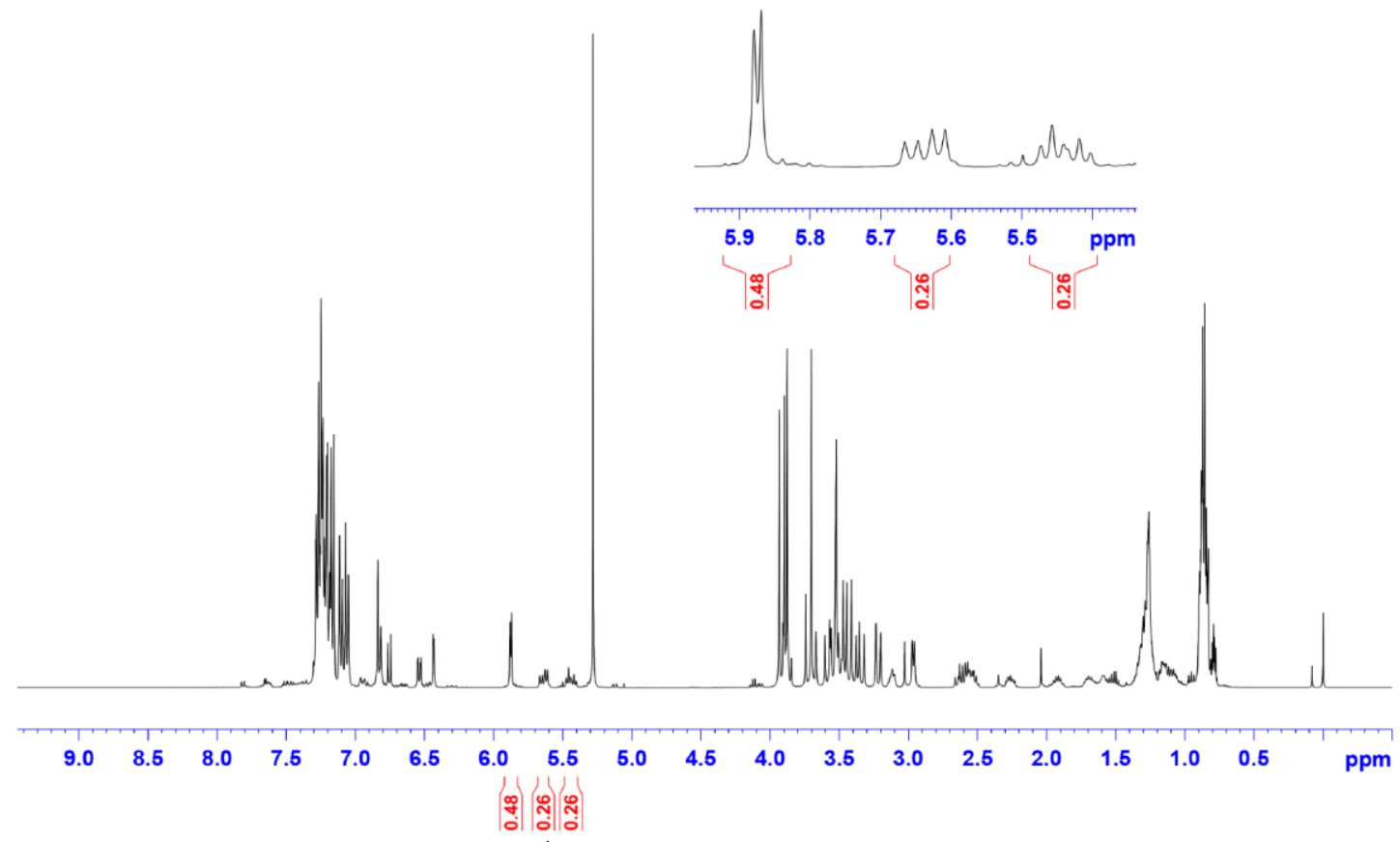

Figure S-9. The raw ${ }^{1} \mathrm{H}-\mathrm{NMR}$ spectra for the reaction of $\mathbf{1 q}$ with $\mathbf{2 d}$

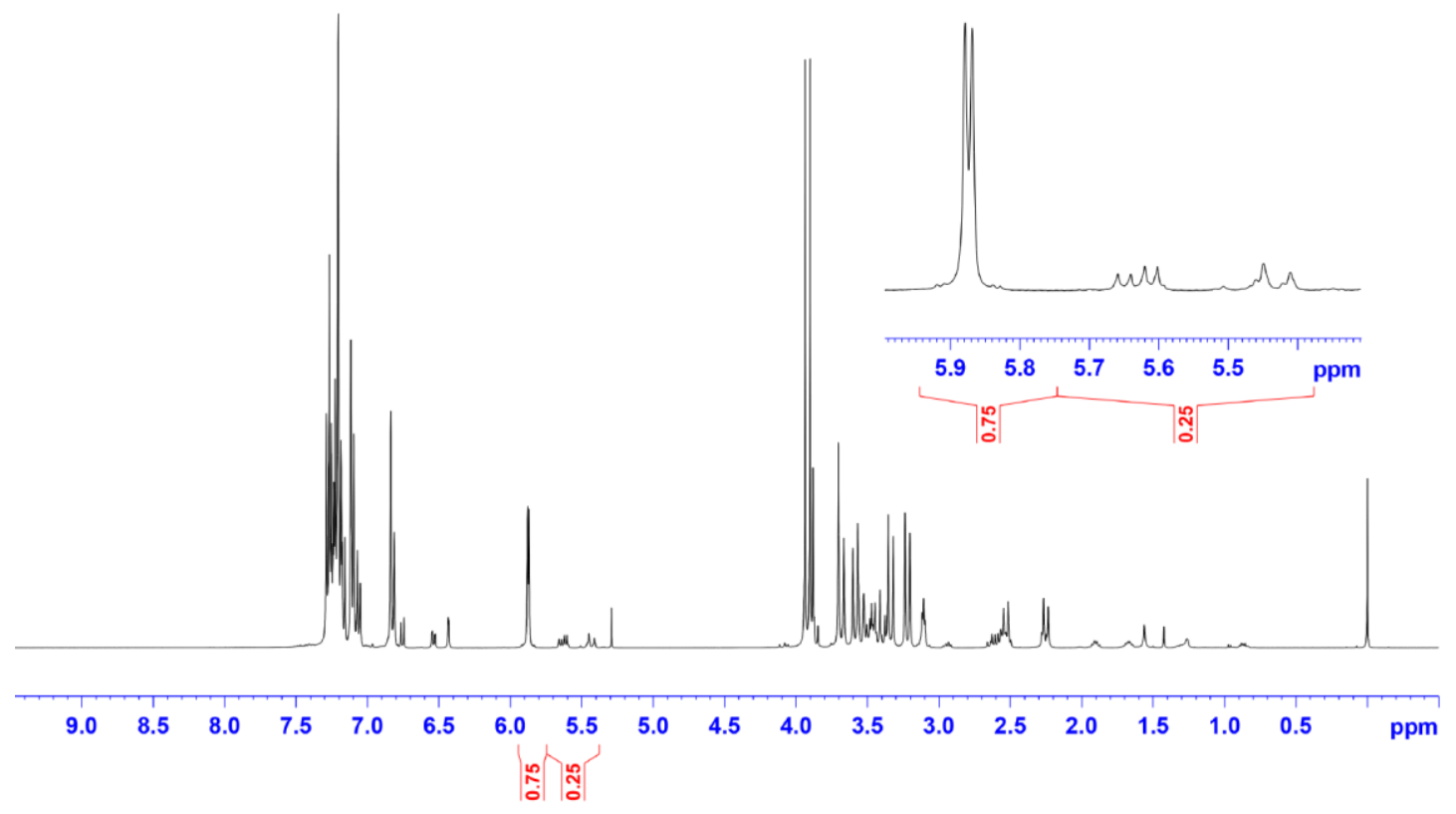

Figure S-10. The raw ${ }^{1} \mathrm{H}-\mathrm{NMR}$ spectra for the reaction of $\mathbf{1 q}-\boldsymbol{d}_{\mathbf{2}}$ with $\mathbf{2 d}$ 


\subsection{Synthesis of D-label 3aa and 4aa}

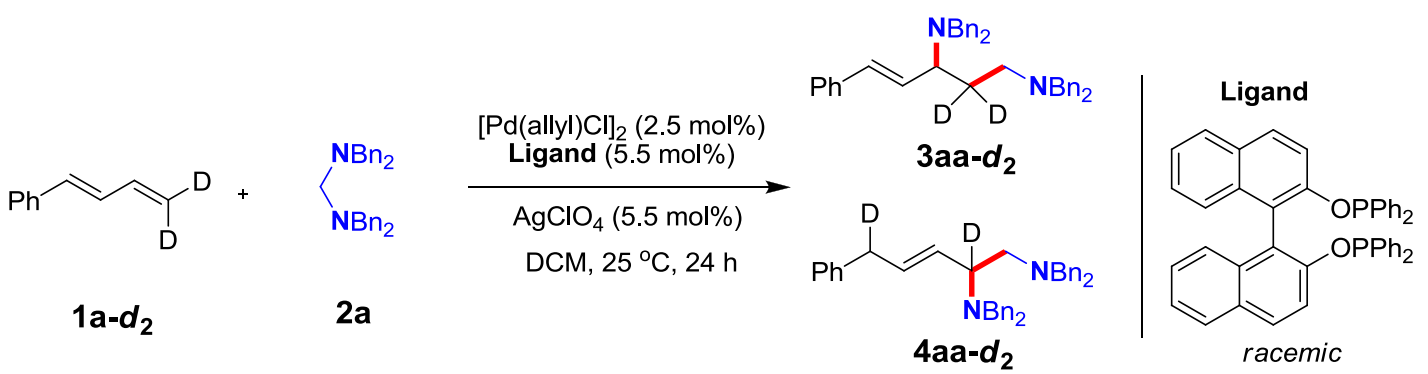

The mixture of $[\mathrm{Pd}(\mathrm{allyl}) \mathrm{Cl}]_{2}(5.7 \mathrm{mg}, 0.016 \mathrm{mmol})$, ligand $(22.5 \mathrm{mg}, 0.035 \mathrm{mmol})$, $\mathrm{AgClO}_{4}(7.2 \mathrm{mg}, 0.035 \mathrm{mmol})$ and $\mathrm{CH}_{2} \mathrm{Cl}_{2}(1.5 \mathrm{~mL})$ was added to a Young-type tube in the glove box and stirred at $45{ }^{\circ} \mathrm{C}$ for 30 minutes. After the mixture cooled to room temperature, then $N, N, N^{\prime}, N^{\prime}$-tetrakis(4-chlorobenzyl)methanediamine (2d) $\quad(0.625$ mmol $)$ and $1 \mathbf{a}-\boldsymbol{d}_{\mathbf{2}}(0.75 \mathrm{mmol})$ were added under nitrogen. The reaction mixture was degassed via the freeze-thaw method and stirred at $25{ }^{\circ} \mathrm{C}$ for 24 hours. The solvent was evaporated under reduced pressure and the residue was purified by flash column chromatography (eluted with petroleum ether/diethyl ether $=100 / 1 \sim 50 / 1$ ) on a silica gel to give the desired product, 58\% yield (isolated yield of combination of 3aa- $\boldsymbol{d}_{\mathbf{2}}$ and $\mathbf{4 a a -} \boldsymbol{d}_{\mathbf{2}}$ ).

$(E)-N^{1}, N^{1}, N^{3}, N^{3}$-tetrabenzyl-5-phenylpent-4-ene-1,3-diamine (3aa- $\left.d_{2}\right):{ }^{1} \mathrm{H}$ NMR $\left(400 \mathrm{MHz}, \mathrm{CDCl}_{3}\right): \delta 2.20(\mathrm{~d}, J=12.8 \mathrm{~Hz}, 1 \mathrm{H}), 2.51(\mathrm{~d}, J=12.8 \mathrm{~Hz}, 1 \mathrm{H}), 3.13$

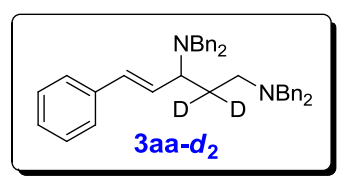

$(\mathrm{d}, J=8.4 \mathrm{~Hz}, 1 \mathrm{H}), 3.20(\mathrm{~d}, J=13.6 \mathrm{~Hz}, 2 \mathrm{H}), 3.28(\mathrm{~d}, J=$ $13.6 \mathrm{~Hz}, 2 \mathrm{H}), 3.55(\mathrm{~d}, J=13.6 \mathrm{~Hz}, 2 \mathrm{H}), 3.68(\mathrm{~d}, J=13.6$ $\mathrm{Hz}, 2 \mathrm{H}), 5.89(\mathrm{~d}, J=13.6 \mathrm{~Hz}, 1 \mathrm{H}), 5.98\left(\mathrm{dd}, J_{l}=8.4 \mathrm{~Hz}, J_{2}=16.0 \mathrm{~Hz}, 1 \mathrm{H}\right)$, 7.10-7.16 (m, 13H), 7.18-7.24 (m, 12H); $\left.{ }^{13} \mathrm{C} \mathrm{NMR} \mathrm{(100} \mathrm{MHz,} \mathrm{CDCl}_{3}\right) \delta 50.7$, 53.2, 57.9, 58.5, 126.3, 127.1, 127.6, 128.4, 128.4, 128.6, 129.9, 130.1, 132.6, 132.6, 133.8, 136.7, 138.0, 138.5; HRMS (ESI) calcd for $\mathrm{C}_{39} \mathrm{H}_{39} \mathrm{D}_{2} \mathrm{~N}_{2}[\mathrm{M}+\mathrm{H}]$ : 539.3390, found: 539.3394 . 
6.4 Stoichiometric reaction of $\mathrm{Pd}(\mathrm{Xantphos})\left(\mathrm{CH}_{2} \mathrm{NBn}_{2}\right)(\mathrm{OTf})$ with $1 \mathrm{a}$ and $\operatorname{LiNBn}_{2}$

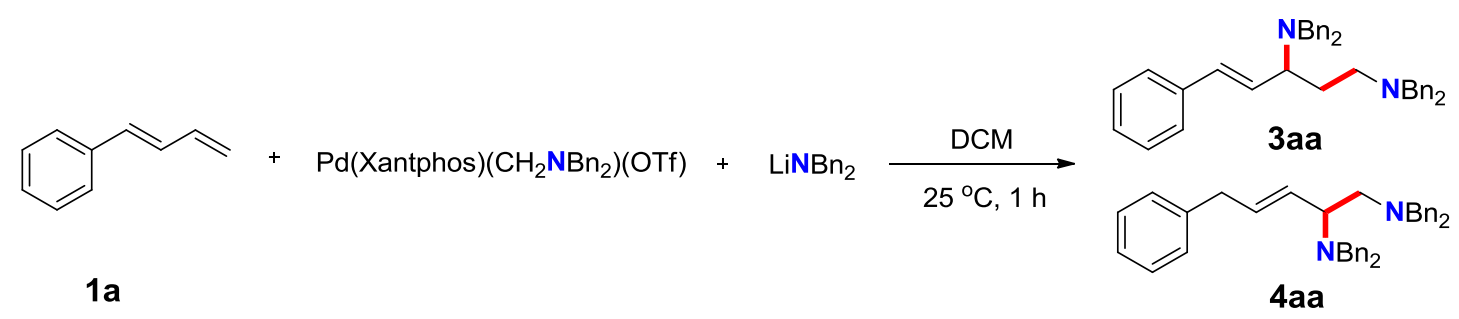

Under nitrogen, the mixture of (E)-buta-1,3-dien-1-ylbenzene (1a) (15.6 mg, 0.12 mmol), Pd(Xantphos) $\left(\mathrm{CH}_{2} \mathrm{NBn}_{2}\right)$ OTf $(104.4 \mathrm{mg}, 0.1 \mathrm{mmol}), \mathrm{DCM}(1.0 \mathrm{~mL})$ and $\operatorname{LiNBn}_{2}(0.1 \mathrm{mmol}, 0.22 \mathrm{~mL})$ was added to a Young-type tube and stirred at room temperature for one hour. The solvent was evaporated under reduced pressure and the residue was purified by flash column chromatography (eluted with petroleum ether/diethyl ether $=100 / 1 \sim 50 / 1)$ on a silica gel to give the desired product, $22 \%$ yield (isolated yield of combination of 3aa and 4aa, 3aa/4aa $=27: 73$ ).

6.5 Synthesis of 1-(buta-1,3-dien-1-yl)-3-methylbenzene ${ }^{1}$

\subsubsection{Synthesis of $(E)-1$-(buta-1,3-dien-1-yl)-3-methylbenzene}

Step 1:

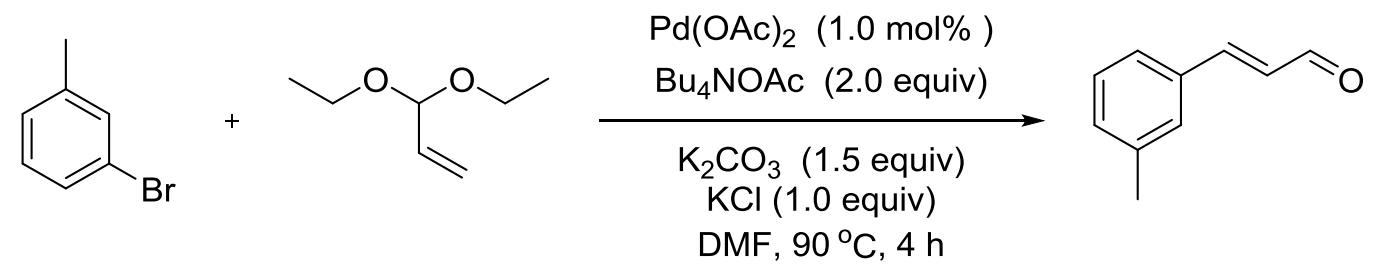

To a stirred solution of 1-bromo-3-methylbenzene (4.28 g, $25 \mathrm{mmol})$ in $40 \mathrm{~mL}$ of DMF was added acrolein diethyl acetal $(11.5 \mathrm{~mL}, 75 \mathrm{mmol}),{ }^{n} \mathrm{Bu}_{4} \mathrm{NOAc}(15.1 \mathrm{~g}, 50$ mmol) and stirred at $90{ }^{\circ} \mathrm{C}$ for 4 hours. After the mixture cooled to room temperature, $2 \mathrm{M} \mathrm{HCl}$ was slowly added to the reaction mixture and stirred at room temperature for another 10 minutes. Then the mixture was extracted with $\mathrm{Et}_{2} \mathrm{O}(30 \mathrm{~mL}$ x 2$)$ and dried with anhydrous $\mathrm{Na}_{2} \mathrm{SO}_{4}$. The solvent was evaporated under reduced pressure and the 
residue was purified by flash column chromatography (eluted with petroleum ether/ethyl acetate $=50 / 1 \sim 20 / 1)$ on a silica gel to give the desired product (490 mg, $13 \%)$

\section{Step 2 :}

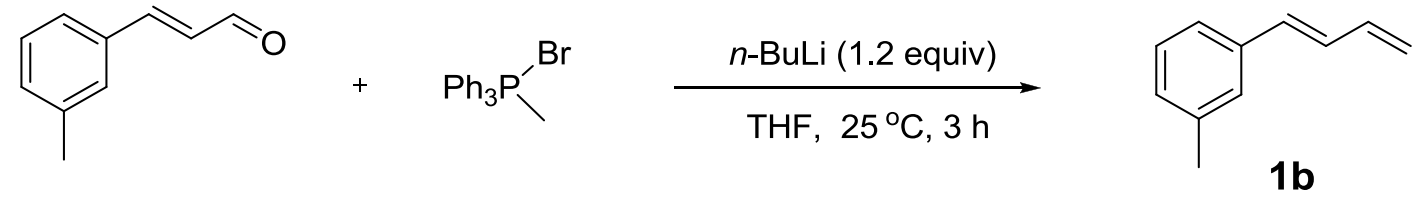

To a suspension of methyltriphenylphosphonium bromide $(1.42 \mathrm{~g}, 4.0 \mathrm{mmol})$ in THF (20 mL) was added dropwise n-butyllithium $(2.5 \mathrm{M}$ in hexane, $1.6 \mathrm{~mL}, 4.0$ mmol) at $0{ }^{\circ} \mathrm{C}$. Then the reaction mixture was stirred for 2 hours and the solvent of (E)-3-( $m$-tolyl)acrylaldehyde (490 mg, $3.36 \mathrm{mmol})$ in THF $(10 \mathrm{~mL})$ was added. After stirring 30 minutes, the mixture was warmed to room temperature and stirred for additional 3 hours. Then the mixture was quenched with saturated solution of $\mathrm{NH}_{4} \mathrm{Cl}$ $(20 \mathrm{~mL})$, extracted with $\mathrm{Et}_{2} \mathrm{O}(3 \times 10 \mathrm{~mL})$ and dried over $\mathrm{Na}_{2} \mathrm{SO}_{4}$. The solvent was evaporated under reduced pressure and the residue was purified by flash column chromatography (eluted with low-boiling petroleum) on a silica gel to give the desired product (386 mg, $80 \%) .{ }^{1} \mathrm{H} \mathrm{NMR}\left(400 \mathrm{MHz}, \mathrm{CDCl}_{3}\right): \delta 2.33(\mathrm{~s}, 3 \mathrm{H}), 5.14(\mathrm{~d}, J=9.6$ $\mathrm{Hz}, 1 \mathrm{H}), 5.29-5.33(\mathrm{~m}, 1 \mathrm{H}), 6.44-6.54(\mathrm{~m}, 2 \mathrm{H}), 6.73\left(\mathrm{dd}, J_{1}=10.8 \mathrm{~Hz}, J_{2}=15.6 \mathrm{~Hz}\right.$, 1H), 7.03-7.21 (m, 4H); ${ }^{13} \mathrm{C}$ NMR $\left(100 \mathrm{MHz}, \mathrm{CDCl}_{3}\right): \delta 21.4,117.4,123.7,127.2$, $128.5,128.6,129.5,133.0,137.1,137.3,138.2$.

\subsubsection{Synthesis of (E/Z)-1-(buta-1,3-dien-1-yl)-3-methylbenzene}

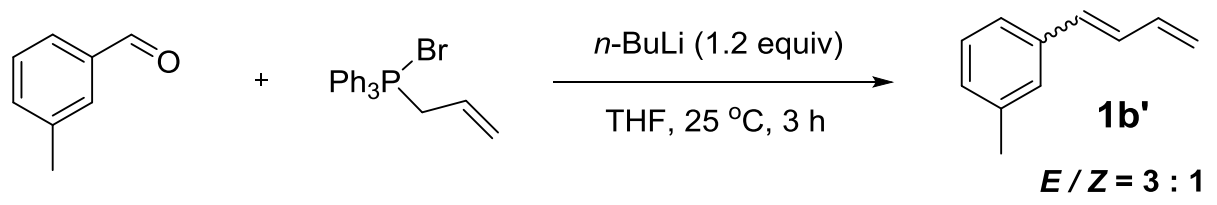

To a suspension of allyltriphenylphosphonium bromide (11.49 g, $25 \mathrm{mmol})$ in THF $(80 \mathrm{ml})$ was added dropwise $n$-butyllithium $(2.5 \mathrm{M}$ in hexane, $12 \mathrm{~mL}, 30 \mathrm{mmol})$ at 
$0{ }^{\circ} \mathrm{C}$. Then the mixture was stirred for 2 hours and the solvent of 3-methylbenzaldehyde (3.0 g, $25 \mathrm{mmol})$ in THF (20 mL) was added. After stirring 30 minutes, the solution was warmed to room temperature and stirred for additional 3 hours. Then the mixture was quenched with saturated solution of $\mathrm{NH}_{4} \mathrm{Cl}(20 \mathrm{~mL})$, extracted with $\mathrm{Et}_{2} \mathrm{O}(3 \times 10 \mathrm{~mL})$ and dried over $\mathrm{Na}_{2} \mathrm{SO}_{4}$. The solvent was evaporated under reduced pressure and the residue was purified by flash column chromatography (eluted with low-boiling petroleum) on a silica gel to give the desired product ( $2.14 \mathrm{~g}$, $59 \%$ ). ${ }^{1} \mathrm{H}$ NMR (400 MHz, $\left.\mathrm{CDCl}_{3}\right): \delta 2.34(\mathrm{~s}, 0.76 \mathrm{H}), 2.35(\mathrm{~s}, 2.28 \mathrm{H}), 5.15\left(\mathrm{dd}, J_{1}=\right.$ $\left.9.2 \mathrm{~Hz}, J_{2}=21.2 \mathrm{~Hz}, 1.00 \mathrm{H}\right), 5.30-5.39(\mathrm{~m}, 1.03 \mathrm{H}), 6.21-6.27(\mathrm{~m}, 0.70 \mathrm{H}), 6.42-6.55$ $(\mathrm{m}, 1.25 \mathrm{H}), 6.75-6.94(\mathrm{~m}, 0.97 \mathrm{H}), 7.05-7.25(\mathrm{~m}, 4.12 \mathrm{H}) ;{ }^{13} \mathrm{C}$ NMR $(100 \mathrm{MHz}$, $\left.\mathrm{CDCl}_{3}\right): \delta 21.5,117.4,119.5,123.7,126.1,127.2,127.9,128.2,128.5,128.5,129.5$ $129.7,130.6,130.7,133.0,133.4,137.1,137.3,137.3,137.8,138.2$.

\subsection{Effect of configuration of 1,3-diene}

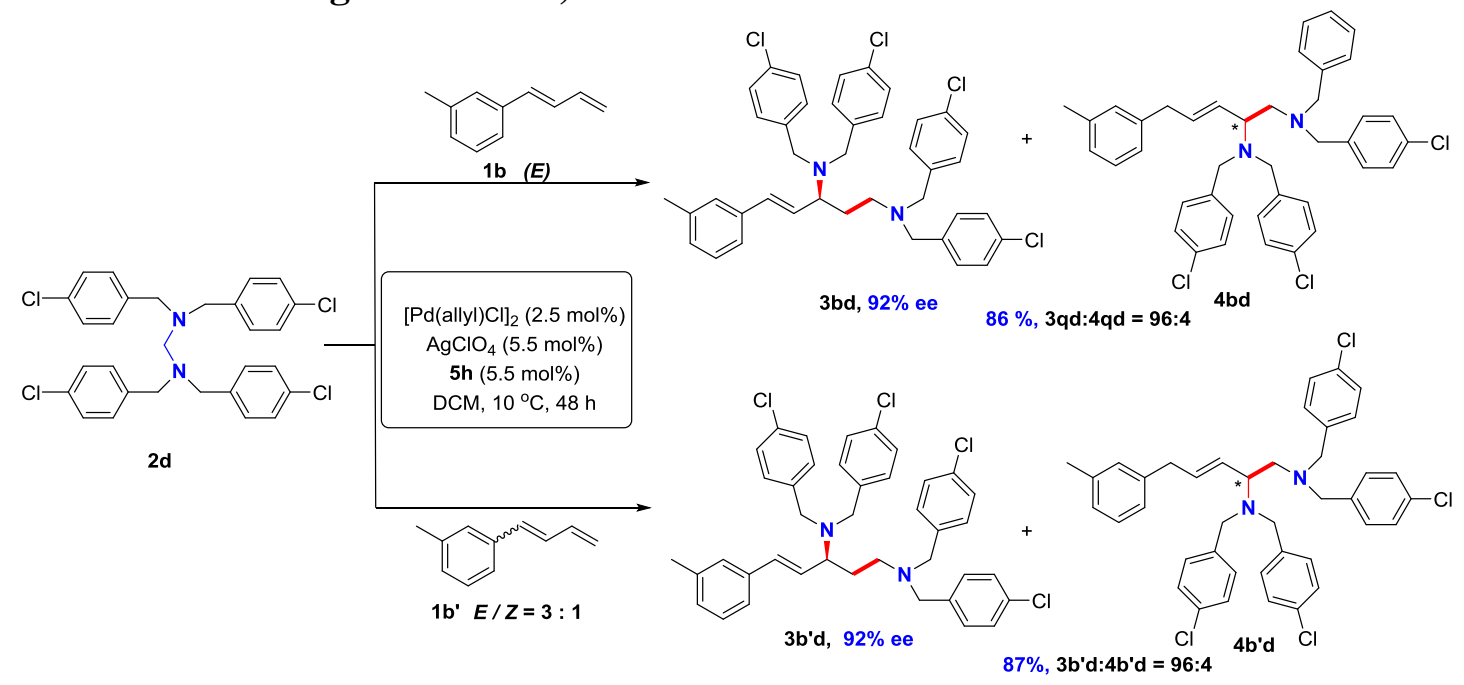

\section{Synthesis of 3b'd}

In a glove box, the mixture of $[\mathrm{Pd}(\text { allyl }) \mathrm{Cl}]_{2}(1.8 \mathrm{mg}, 0.005 \mathrm{mmol})$, ligand $(\mathbf{5 h})$ (12.4 mg, $0.011 \mathrm{mmol}), \mathrm{AgClO}_{4}(2.3 \mathrm{mg}, 0.011 \mathrm{mmol})$ and $\mathrm{CH}_{2} \mathrm{Cl}_{2}(0.5 \mathrm{~mL})$ was added to a Young-type tube and stirred at $45{ }^{\circ} \mathrm{C}$ for 30 minutes. After the mixture cooled to room temperature, then $N, N, N^{\prime}, N^{\prime}$-tetrakis(4-chlorobenzyl)methanediamine 
(2d) $(0.2 \mathrm{mmol})$ and $\mathbf{1} \mathbf{b}^{\prime}(0.24 \mathrm{mmol})$ were added under nitrogen. The reaction mixture was degassed via the freeze-thaw method and stirred at $10{ }^{\circ} \mathrm{C}$ for 48 hours. The solvent was evaporated under reduced pressure and the residue was purified by flash column chromatography (eluted with petroleum ether/diethyl ether $=100 / 1 \sim$ $50 / 1$ ) on a silica gel to give the desired product, $87 \%$ yield [(isolated yield of combination of 3b'd and 4b'd), $92 \%$ ee of $\left.\mathbf{3 b} \mathbf{d} \mathbf{d}, \mathbf{3 b} \mathbf{d} \mathbf{d}: \mathbf{4} \mathbf{b}^{\mathbf{\prime}} \mathbf{d}=96: 4\right]$.

\subsection{Gram scale reactions for synthesis of 3aa and 3ad.}

\section{Synthesis of 3aa in gram scale}

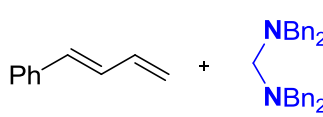

$1 \mathbf{a}$

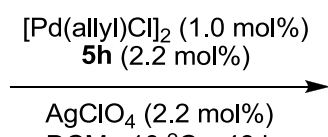

$\mathrm{DCM}, 10^{\circ} \mathrm{C}, 48 \mathrm{~h}$

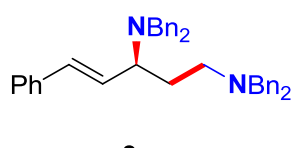

3aa

In the glove box, the mixture of $[\mathrm{Pd}(\text { allyl }) \mathrm{Cl}]_{2}(36.5 \mathrm{mg}, 1.0 \mathrm{~mol} \%), \mathbf{5 h}(248 \mathrm{mg}$, $2.2 \mathrm{~mol} \%), \mathrm{AgClO}_{4}(45 \mathrm{mg}, 2.2 \mathrm{~mol} \%)$ and $\mathrm{CH}_{2} \mathrm{Cl}_{2}(10 \mathrm{~mL})$ was added to a Young-type tube and stirred at $45{ }^{\circ} \mathrm{C}$ for 30 minutes. After the mixture cooled to room temperature, $\quad N, N, N^{\prime}, N^{\prime}$-tetrabenzylmethanediamine $\quad(\mathbf{2 a}) \quad\left(\begin{array}{lll}10 & \mathrm{mmol}\end{array}\right) \quad$ and (E)-buta-1,3-dien-1-ylbenzene 1a $(12 \mathrm{mmol})$ were added under nitrogen. The reaction mixture was stirred at $10{ }^{\circ} \mathrm{C}$ for 48 hours. The solvent was evaporated under reduced pressure and the residue was purified by flash column chromatography (eluted with petroleum ether/diethyl ether $=100 / 1 \sim 50 / 1)$ on a silica gel to give the desired product 3aa, $4.56 \mathrm{~g}, 84 \%$ ee, and $85 \%$ yield (3aa:4aa > 20:1).

\section{Synthesis of 3ad in gram scale.}
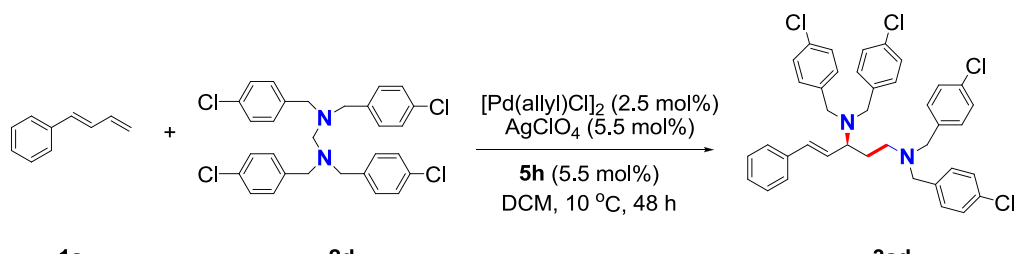

In the glove box, the mixture of $[\mathrm{Pd}(\text { allyl }) \mathrm{Cl}]_{2}(45 \mathrm{mg}, 0.12 \mathrm{mmol}), \mathbf{5 h}(305 \mathrm{mg}$, $0.27 \mathrm{mmol}), \mathrm{AgClO}_{4}(56 \mathrm{mg}, 0.27 \mathrm{mmol})$ and $\mathrm{CH}_{2} \mathrm{Cl}_{2}(5.0 \mathrm{~mL})$ was added to a 
Young-type tube and stirred at $45^{\circ} \mathrm{C}$ for 30 minutes. After the mixture cooled to room temperature, $N, N, N^{\prime}, N^{\prime}$-tetrakis(4-chlorobenzyl)methanediamine (2d) $(5.0 \mathrm{mmol})$ and (E)-buta-1,3-dien-1-ylbenzene $\mathbf{1 a}(6.0 \mathrm{mmol})$ were added under nitrogen. The reaction mixture was degassed via the freeze-thaw method and stirred at $10{ }^{\circ} \mathrm{C}$ for 48 hours. The solvent was evaporated under reduced pressure and the residue was purified by flash column chromatography (eluted with petroleum ether/diethyl ether $=$ 100/1 50/1) on a silica gel to give the desired product 3ad, $2.03 \mathrm{~g}, 92 \%$ ee and $89 \%$ yield (3ad:4ad > 20:1).

\section{References}

1. a) Battistuzzi, G.; Cacchi, S.; Fabrizi, G. Org. Lett., 2003, 5, 777; b) Lishchynskyi, A.; Muniz, K. Chem. Eur. J. 2012, 18, 2212.

2. a) Heaney, H.; Papageorgiou, G.; Wilkins, R. F. Tetrahedron., 1997, 53, 2941; b) T. Rosenau, A. Potthast and P. Kosma, Tetrahedron., 2004, 60, 301.

3. Wu, T. R.; Shen, L.; Chong, J. M. Org. Lett., 2004, 6, 2701.

4. Storer, R. I.; Carrera, D. E.; Ni, Y.; MacMillan, D. W. C. J. Am. Chem. Soc. 2006, 128,84 .

5. a) Li, C.-J.; Wang, D.; Slaven IV, W. T. Tetrahedron Letters, 1996, 37, 4459; b) Zhu, S. S.; Cefalo, D. R.; La, D. S.; Jamieson, J. Y.; Davis, W. M.; Hoveyda, A. H.; Schrock, R. R. J. Am. Chem. Soc. 1999, 121, 8251.

6. Crouch, I. T.; Neff, R. K.; Frantz, D. E. J. Am. Chem. Soc. 2013, 135, 4970.

7. Romanov-Michailidis, F.; Guenee, L.; Alexakis, A. Angew. Chem. Int. Ed. 2013, 52,9266 


\section{Copies for HPLC and NMR of products}

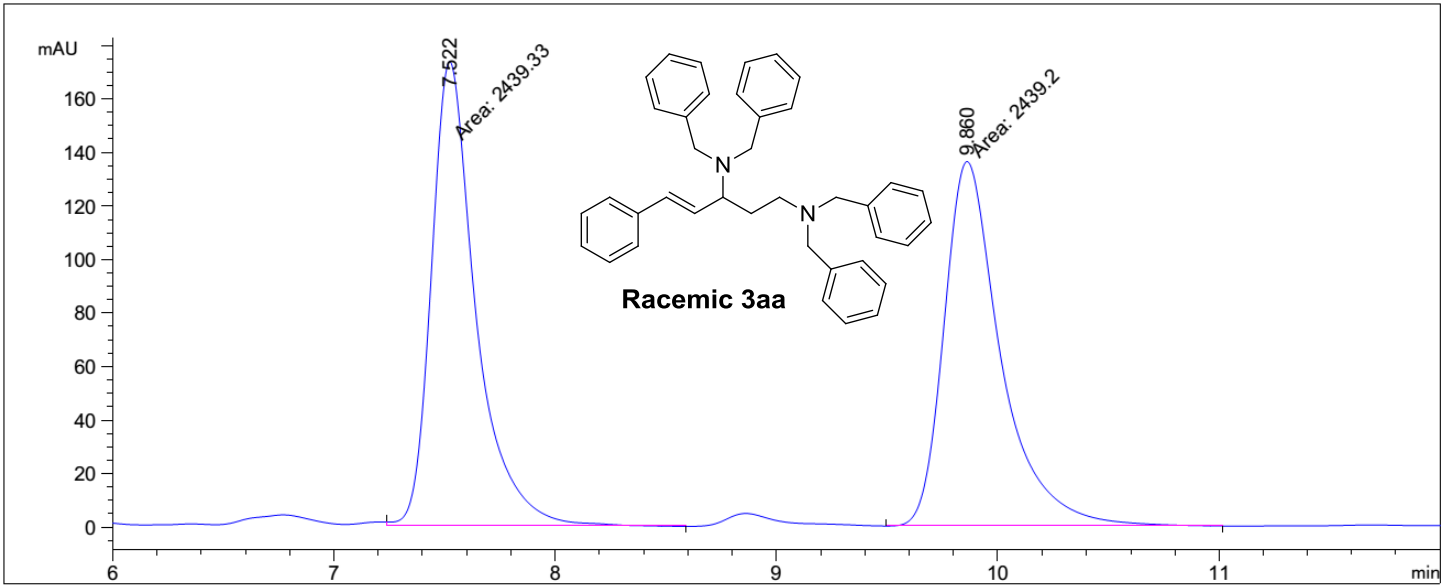

\begin{tabular}{|c|c|c|c|c|c|c|c|c|}
\hline \multirow{2}{*}{$\begin{array}{c}\text { Peak } \\
\#\end{array}$} & \multirow{2}{*}{$\begin{array}{c}\text { RetTime } \\
\text { [min] }\end{array}$} & \multirow{2}{*}{ Type } & \multirow{2}{*}{$\begin{array}{l}\text { Width } \\
\text { [min] }\end{array}$} & \multicolumn{2}{|c|}{ Area } & \multicolumn{2}{|c|}{ Height } & \multirow{2}{*}{$\begin{array}{c}\text { Area } \\
\quad \%\end{array}$} \\
\hline & & & & $\mathrm{mAU}$ & ${ }^{\star} \mathrm{S}$ & {$[\mathrm{mAU}$} & ] & \\
\hline 1 & & & & & 7 & & & \\
\hline 2 & 9.860 & MM & 0.2984 & 243 & 19995 & 13 & 25639 & 49.9987 \\
\hline
\end{tabular}

Totals :

$4878.52832 \quad 310.00006$

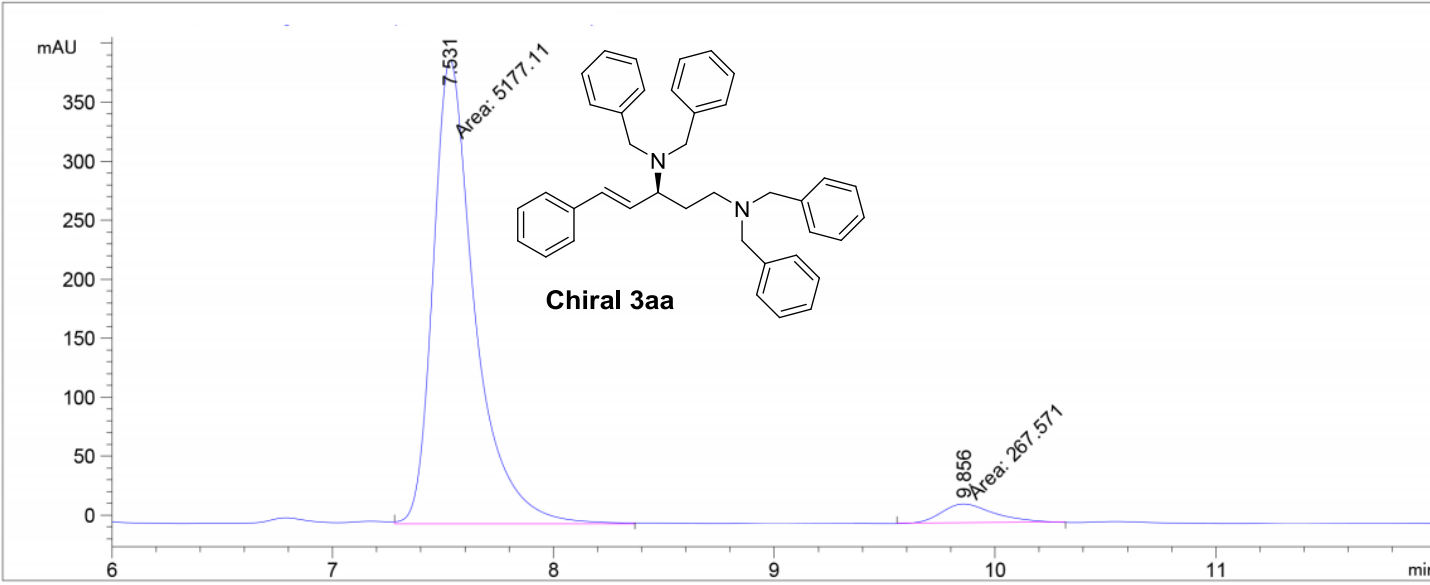

\begin{tabular}{|c|c|c|c|c|c|c|c|c|}
\hline Peak & RetTime & Type & Width & & & $\mathrm{He}$ & ht & Area \\
\hline$\#$ & {$[\mathrm{~min}]$} & & {$[\mathrm{min}]$} & $\mathrm{mAU}$ & ${ }^{\star} \mathrm{s}$ & {$[\mathrm{mAU}$} & ] & 8 \\
\hline & & & & $\mid--$ & -- & -- & -- & -- \\
\hline 1 & 7 & $\mathbb{M}$ & 5 & 517 & 10791 & 39 & 61 & 356 \\
\hline 2 & 856 & $\mathrm{MM}$ & .2779 & 267 & 57111 & & 4671 & 4.9144 \\
\hline
\end{tabular}

Totals :

$5444.67902 \quad 409.19833$ 

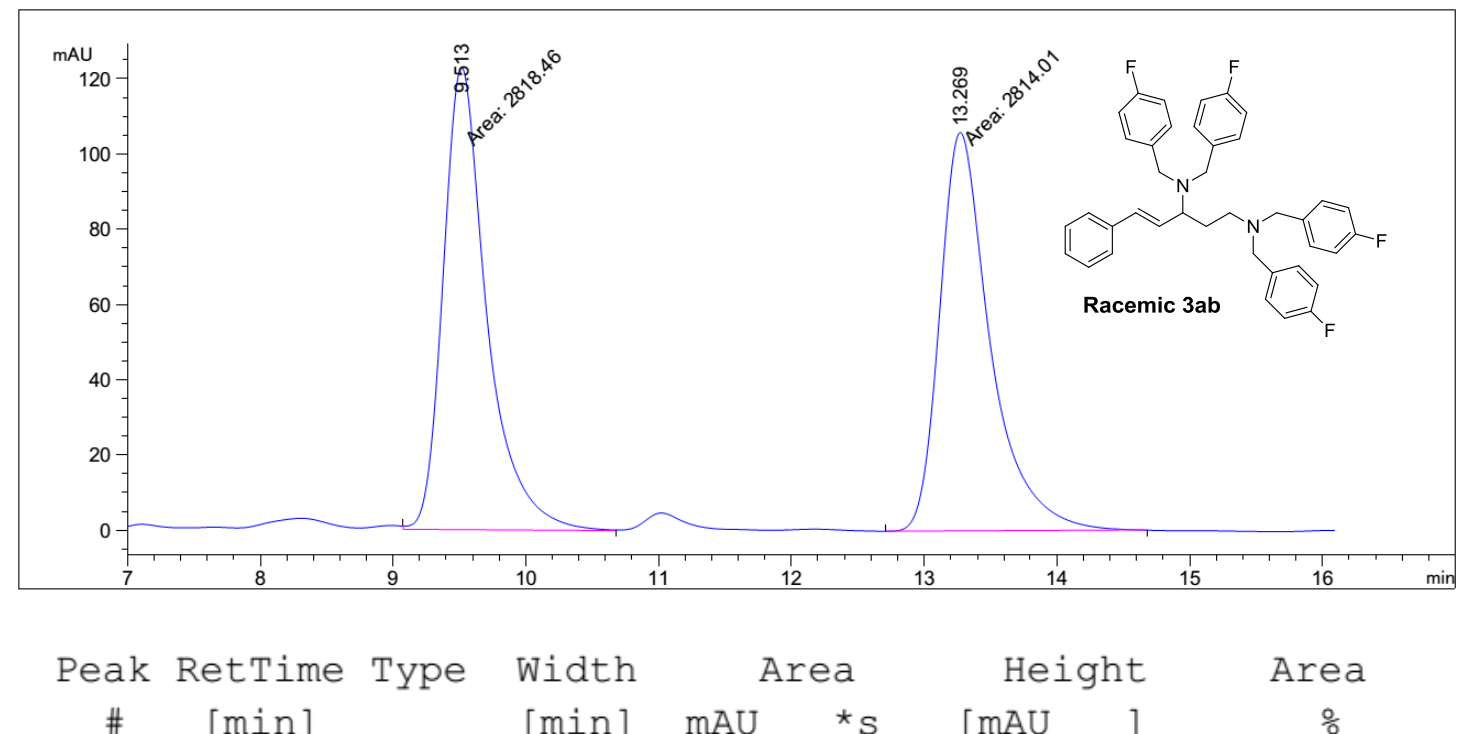

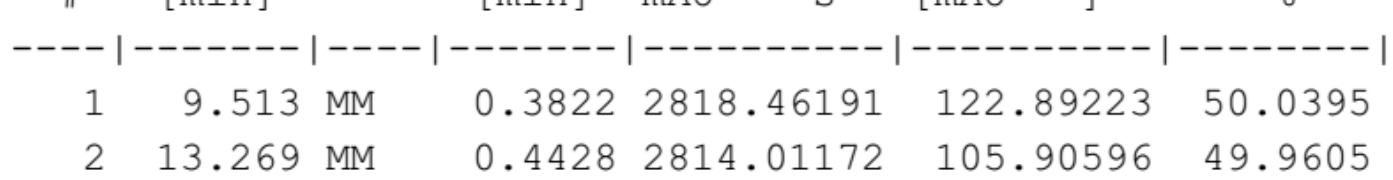

Totals :

$5632.47363 \quad 228.79819$

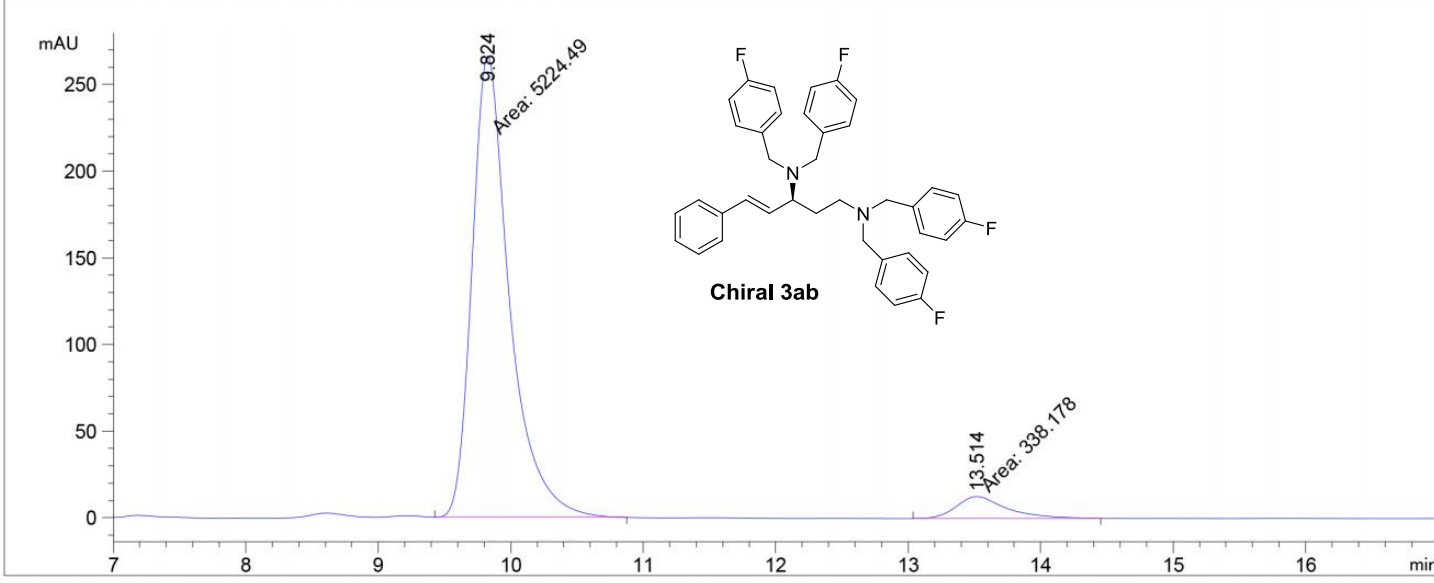

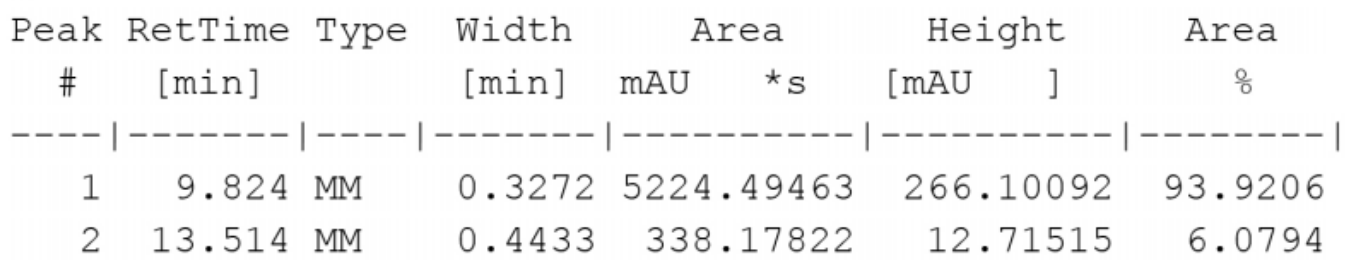

Totals :

$5562.67285 \quad 278.81607$ 

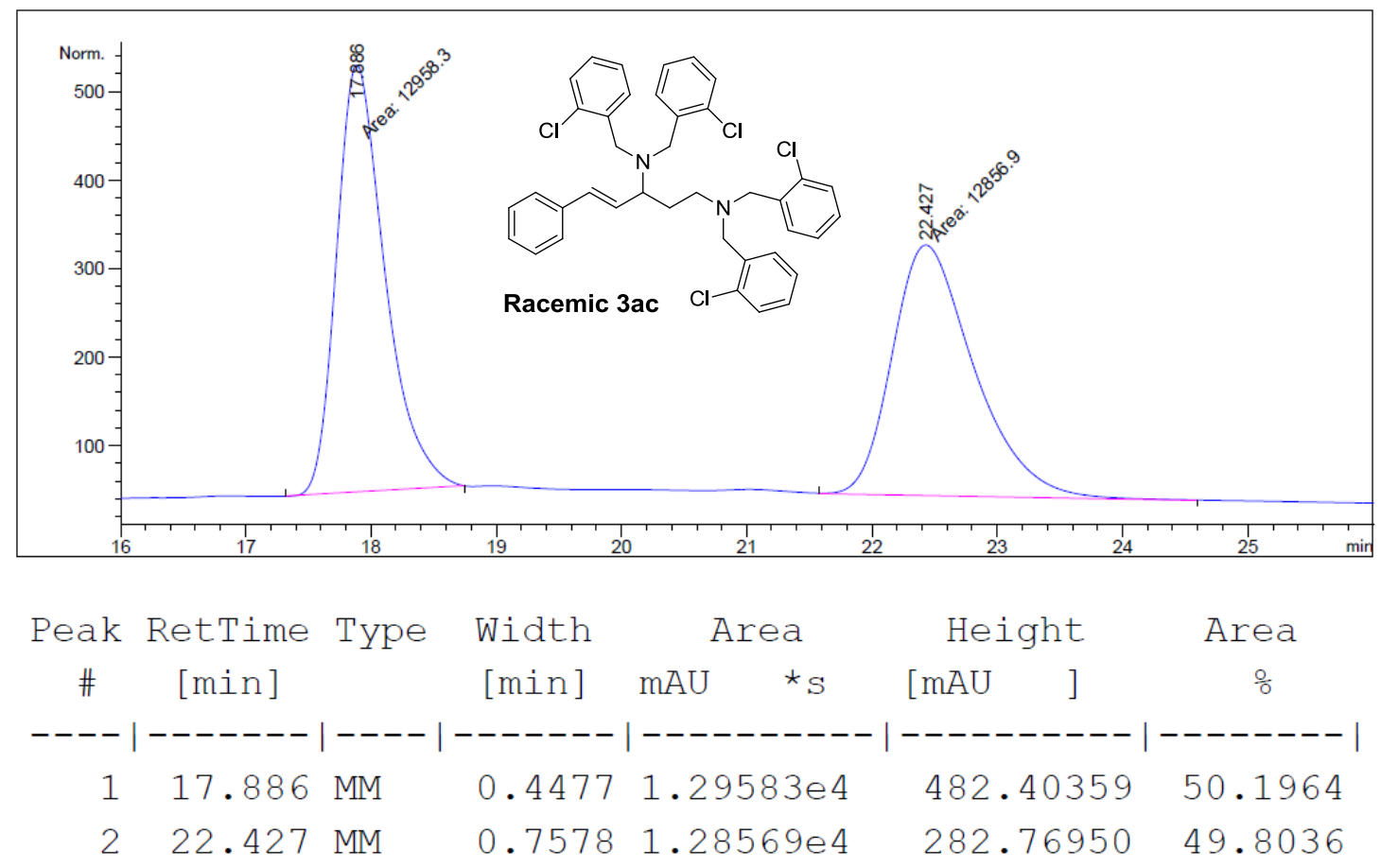

Totals : $\quad 2.58152 e 4 \quad 765.17310$
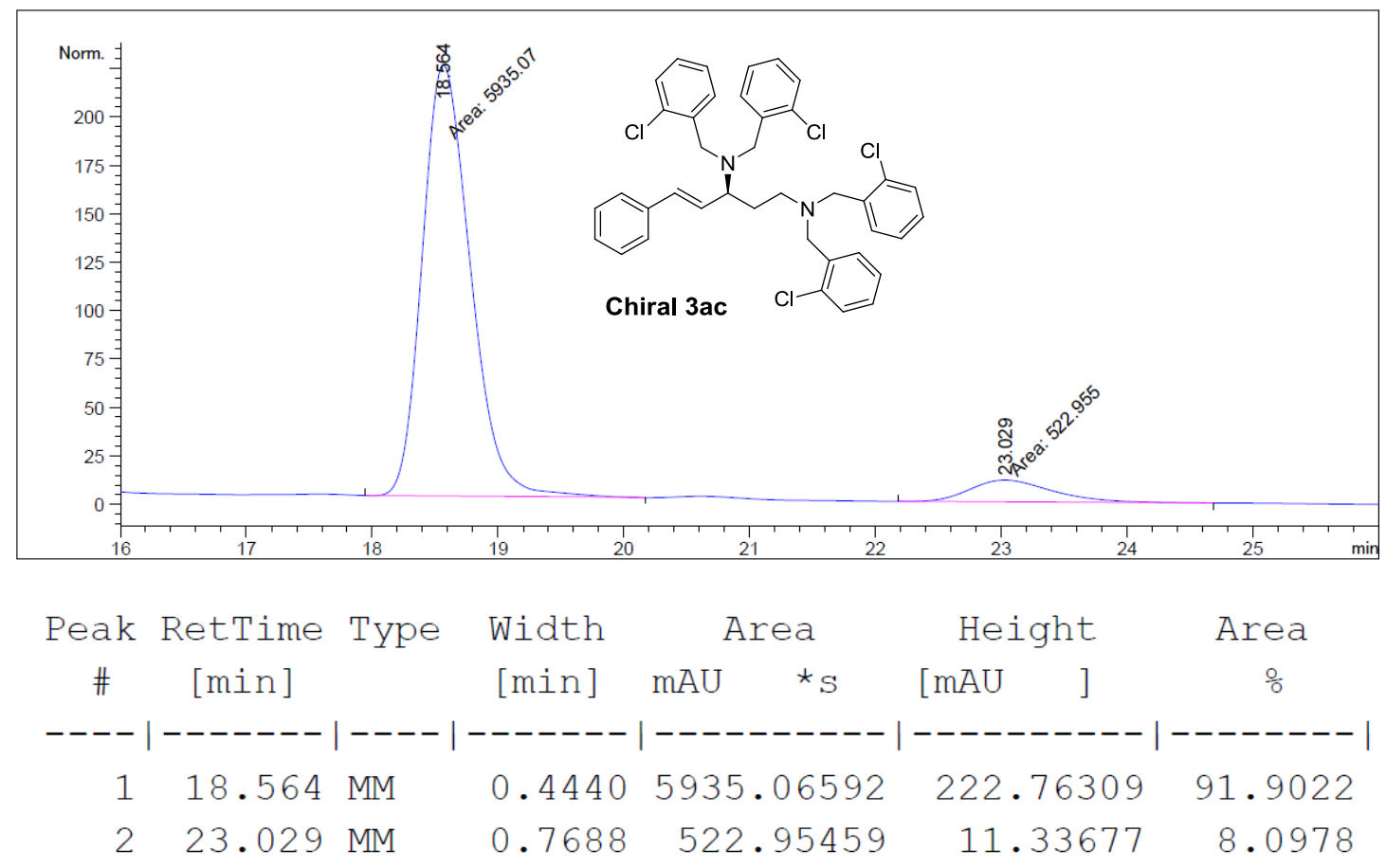

Totals :

$6458.02051 \quad 234.09986$ 


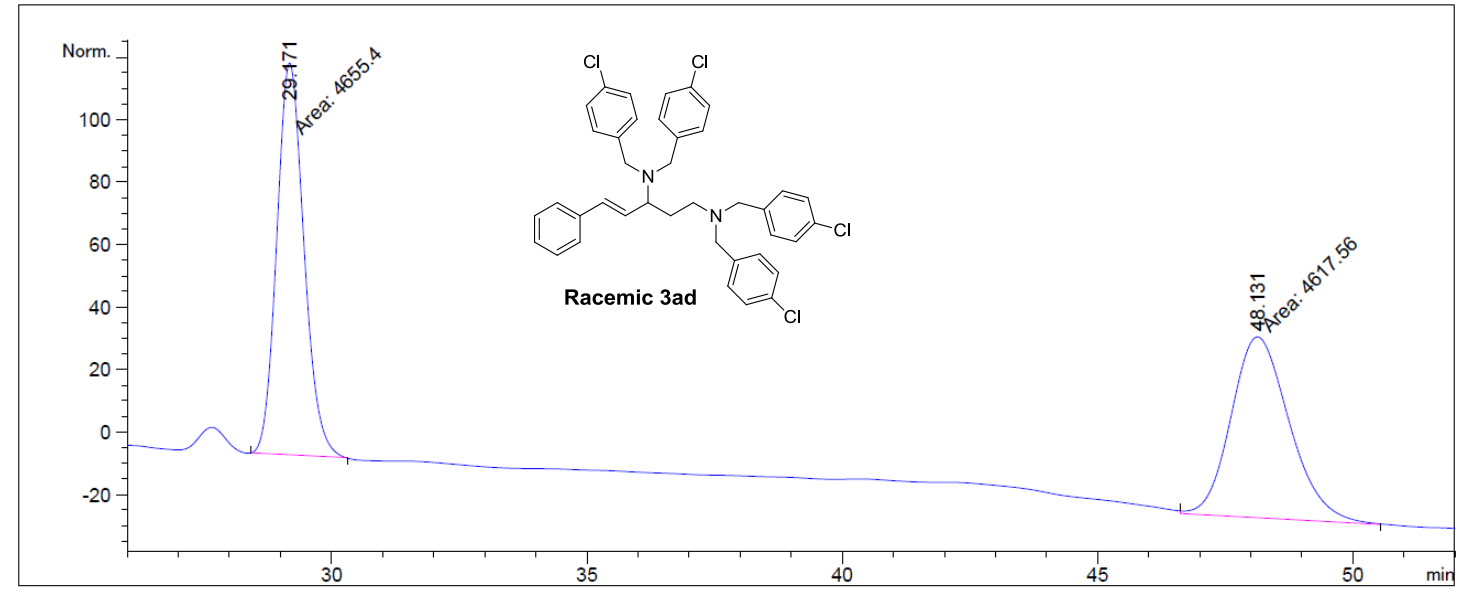

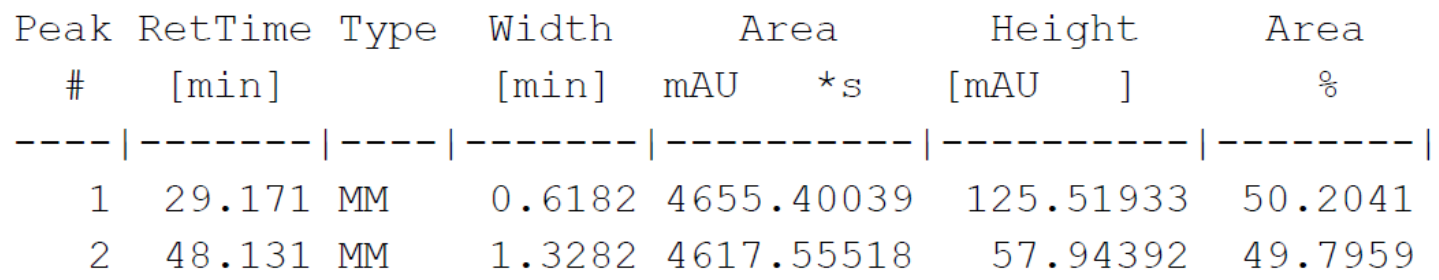

Totals :

$9272.95557 \quad 183.46324$

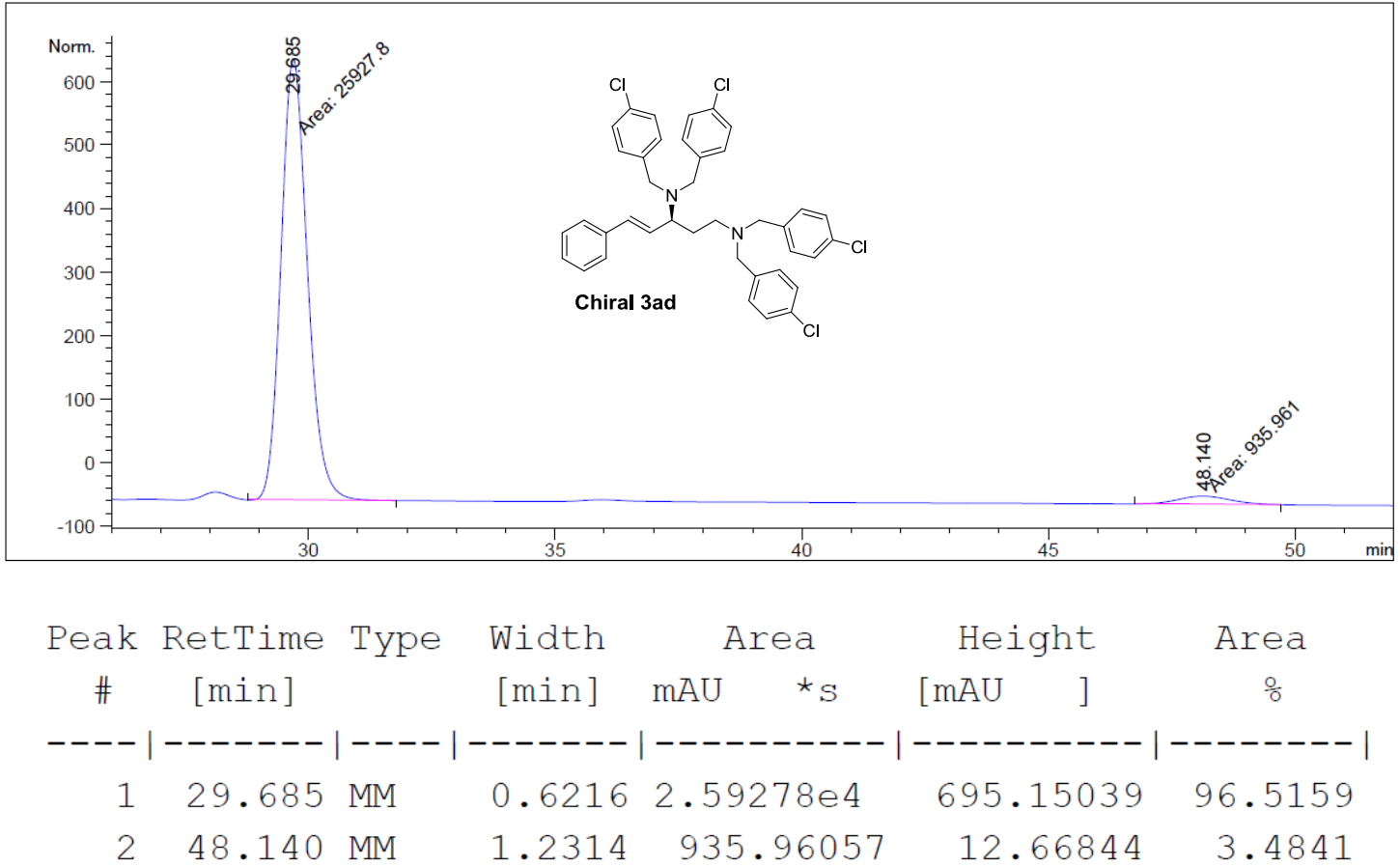

Totals :

$2.68638 e 4 \quad 707.81883$ 

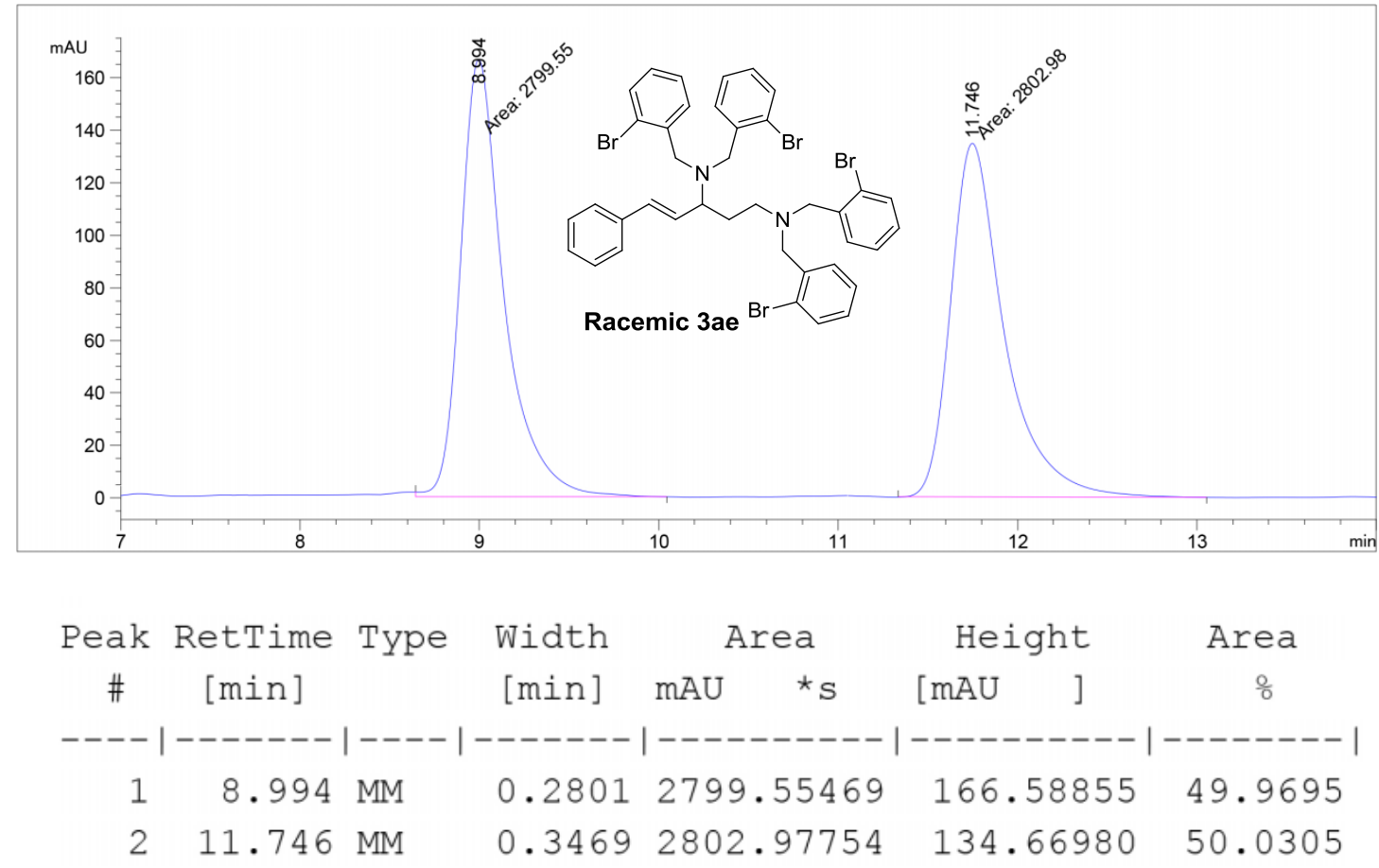

Totals :

$5602.53223 \quad 301.25835$
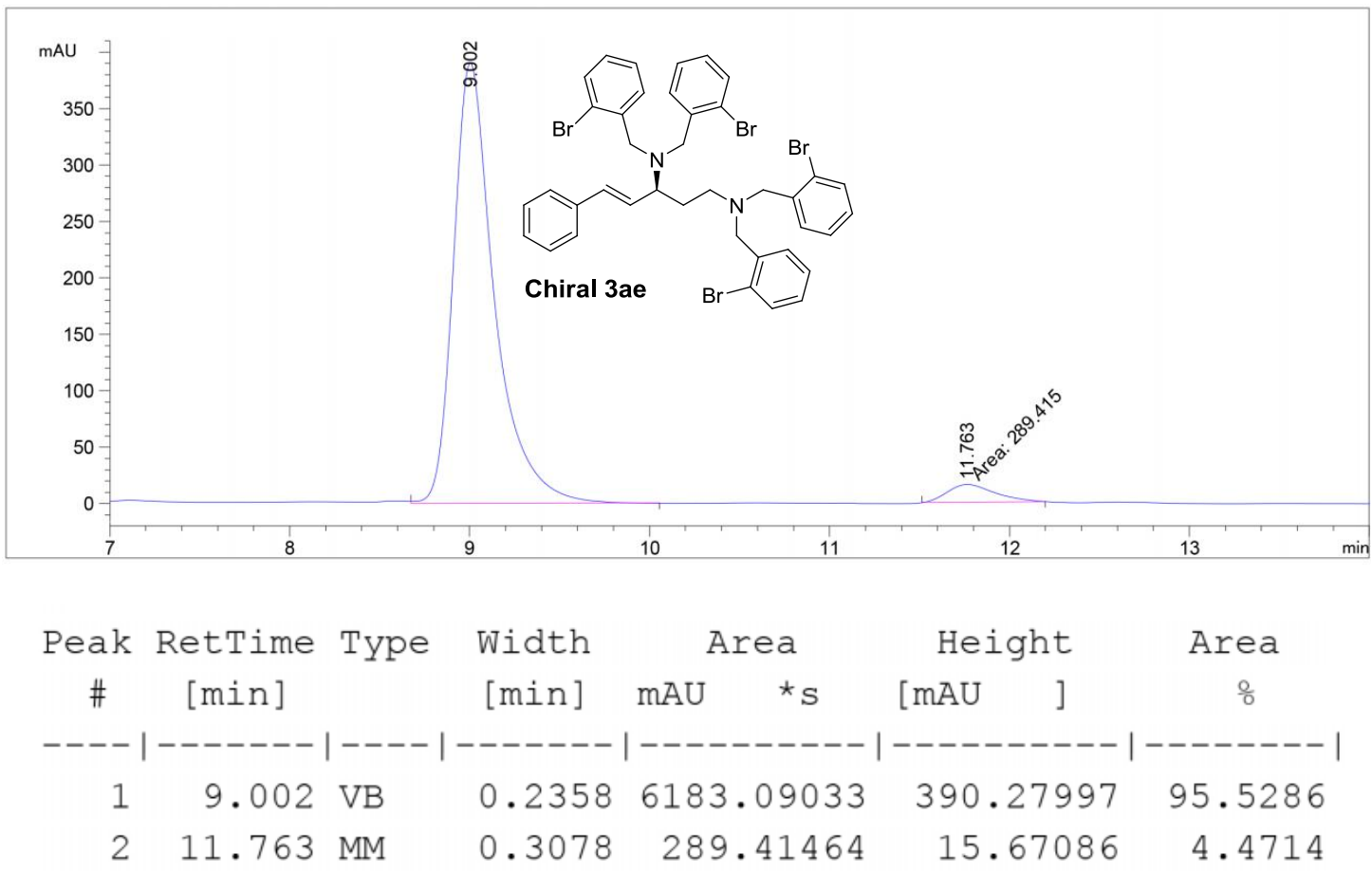

Totals :

$6472.50497 \quad 405.95083$ 


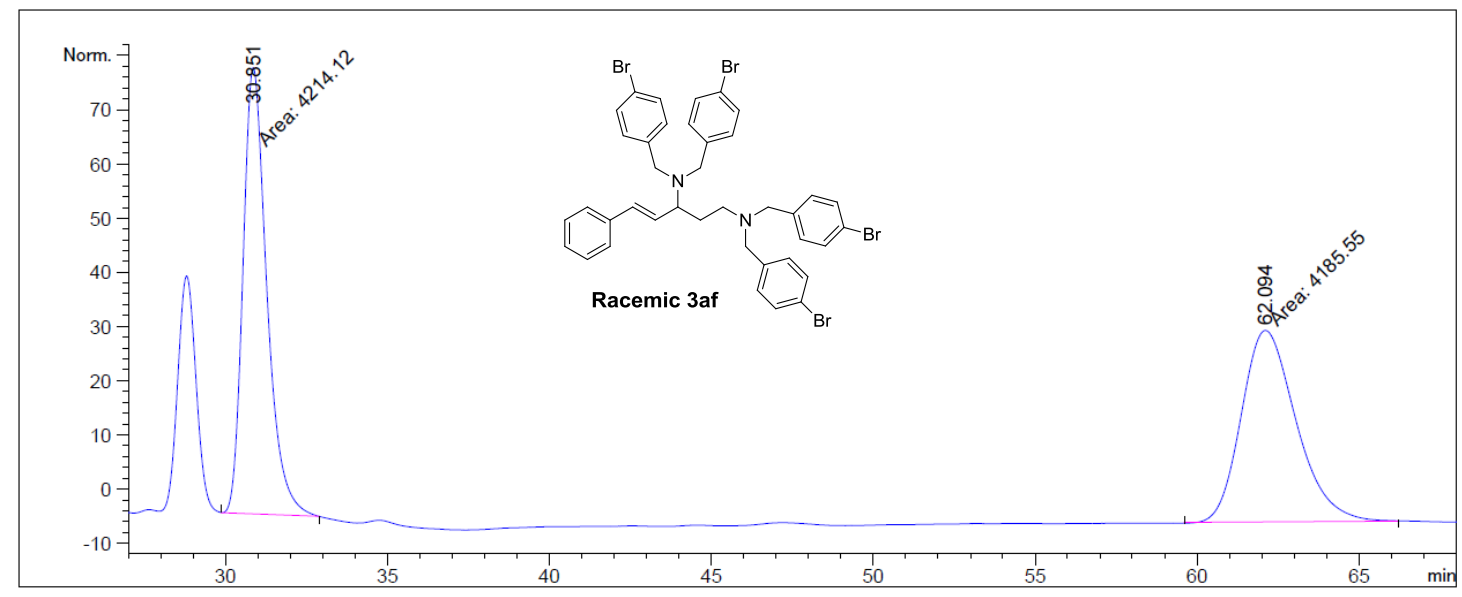

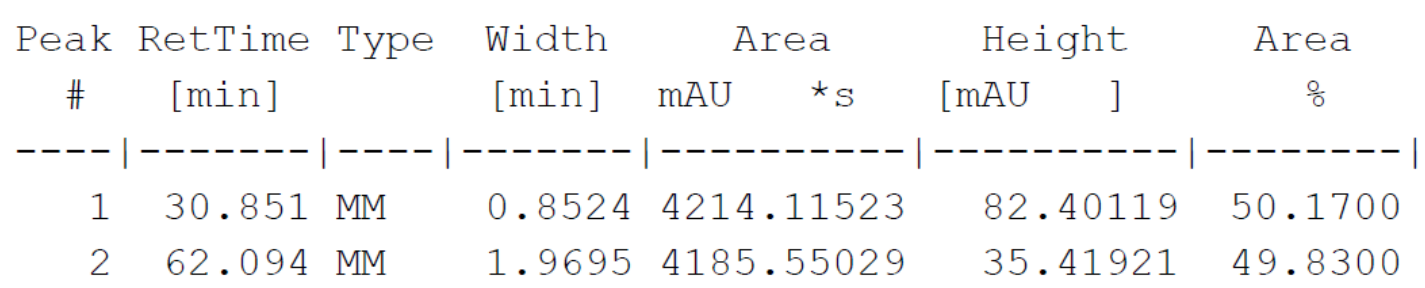

Totals :

$8399.66553 \quad 117.82040$

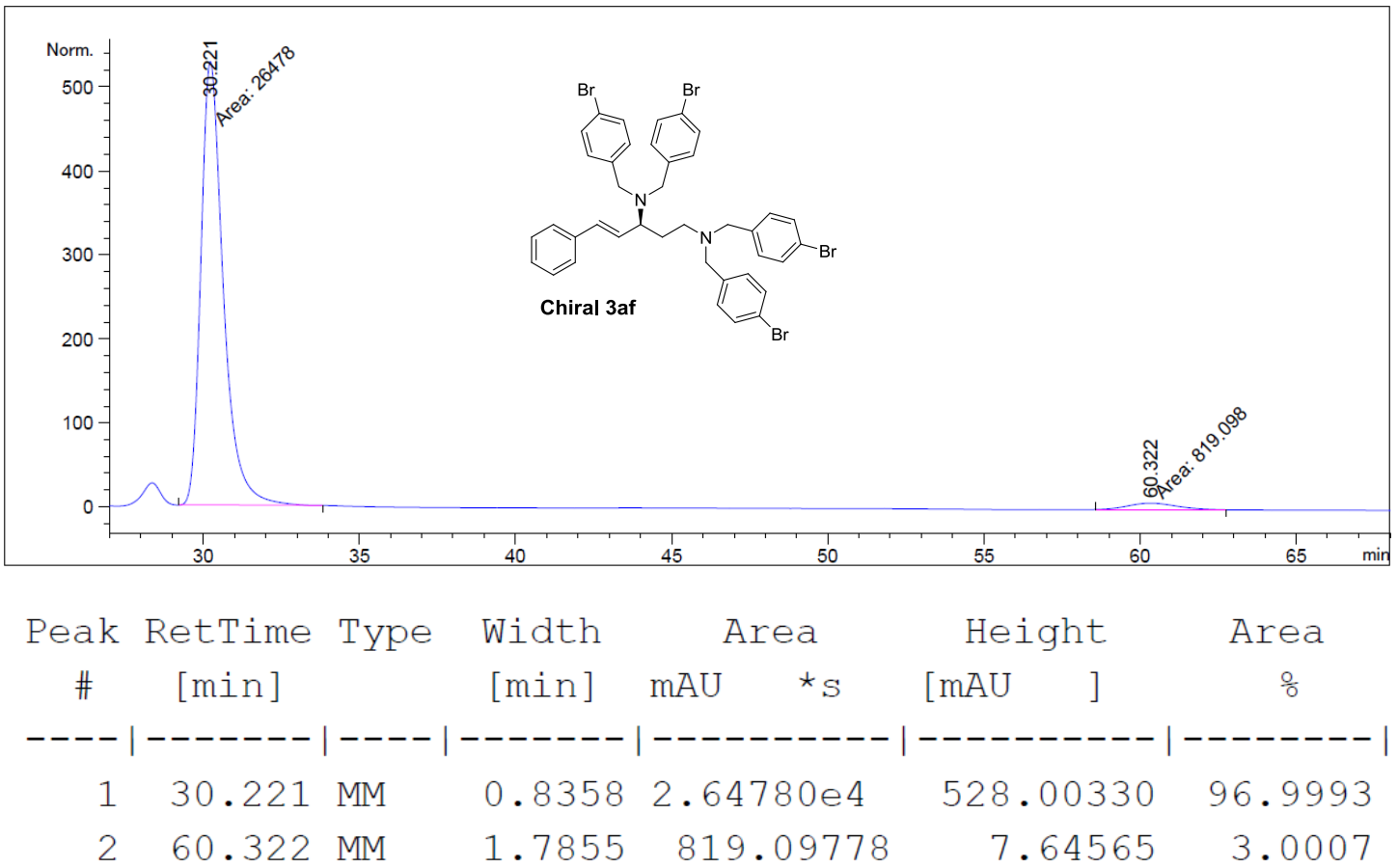

Totals :

2.72971 e4 535.64895 


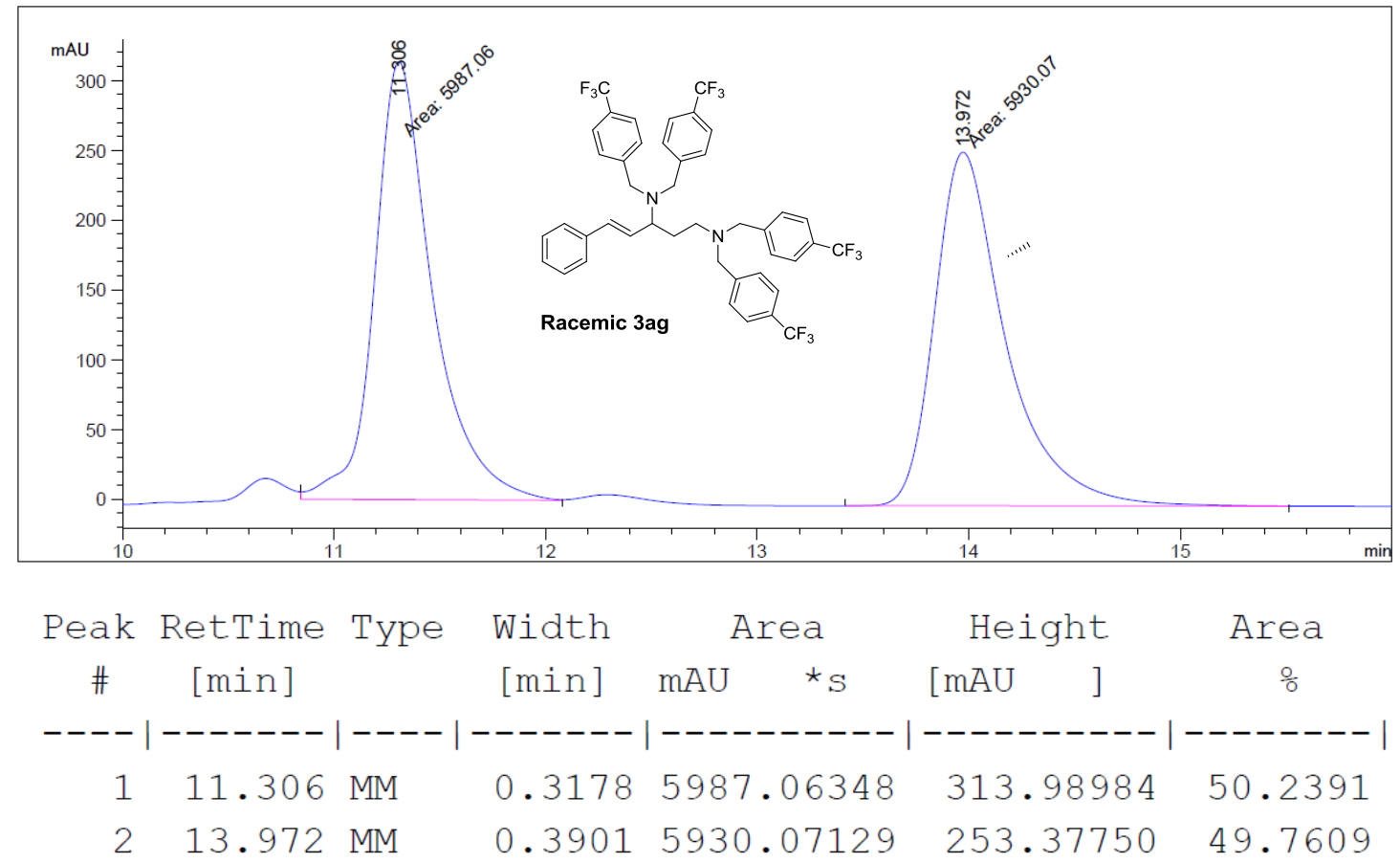

Totals :

$1.19171 \mathrm{e} 4 \quad 567.36734$

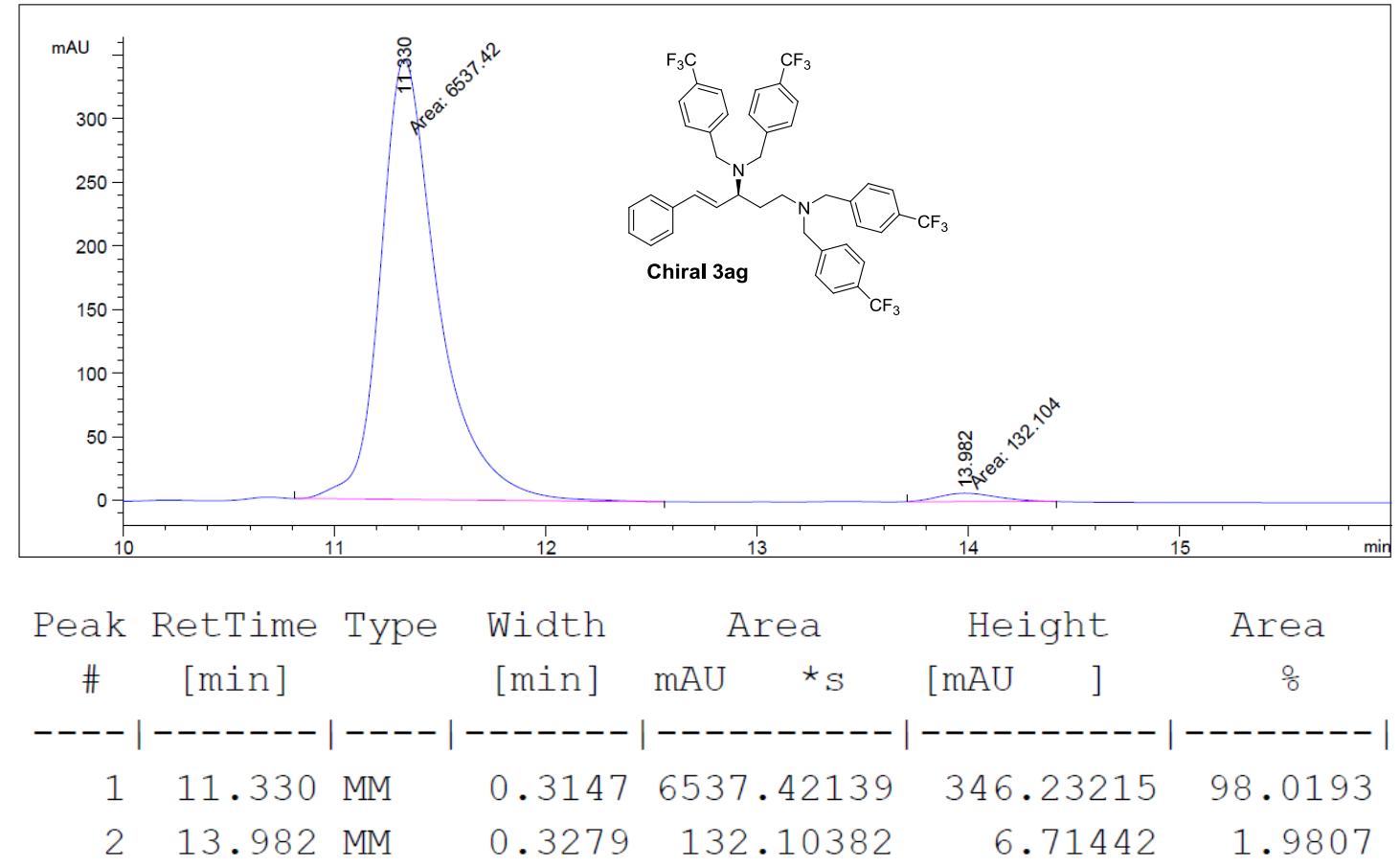

Totals :

$6669.52521 \quad 352.94657$ 

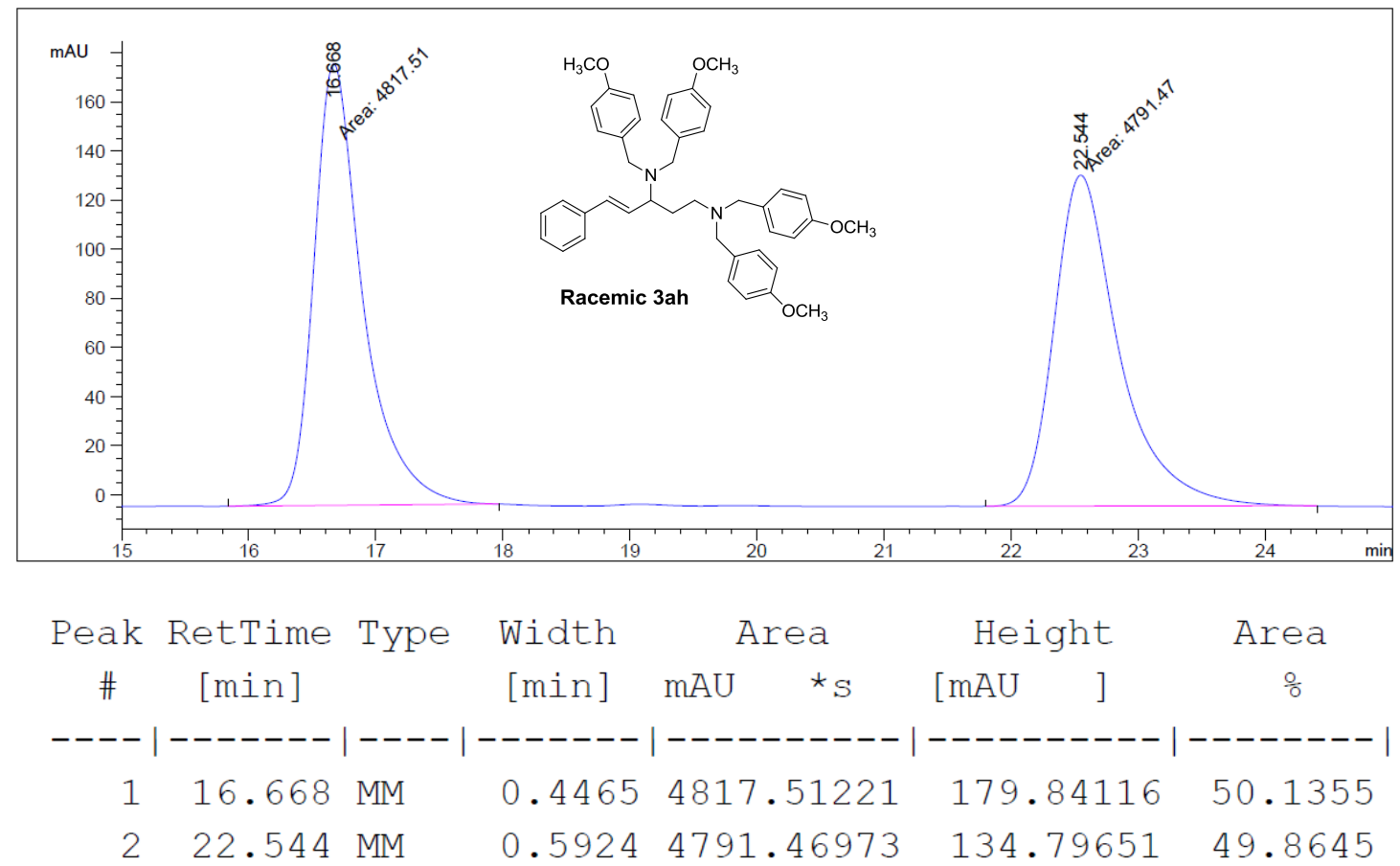

Totals :

$9608.98193 \quad 314.63766$
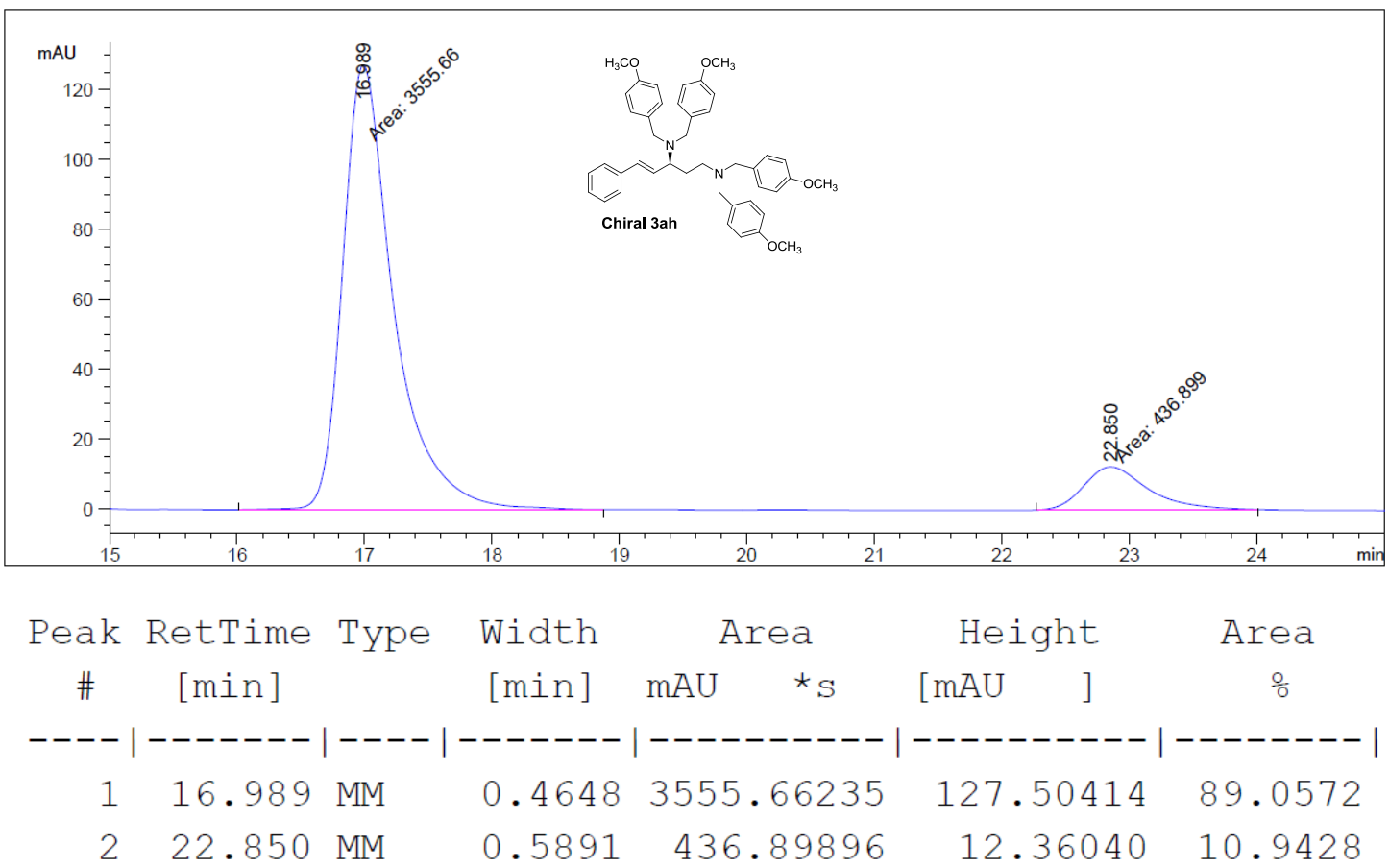

Totals :

$3992.56131 \quad 139.86454$ 

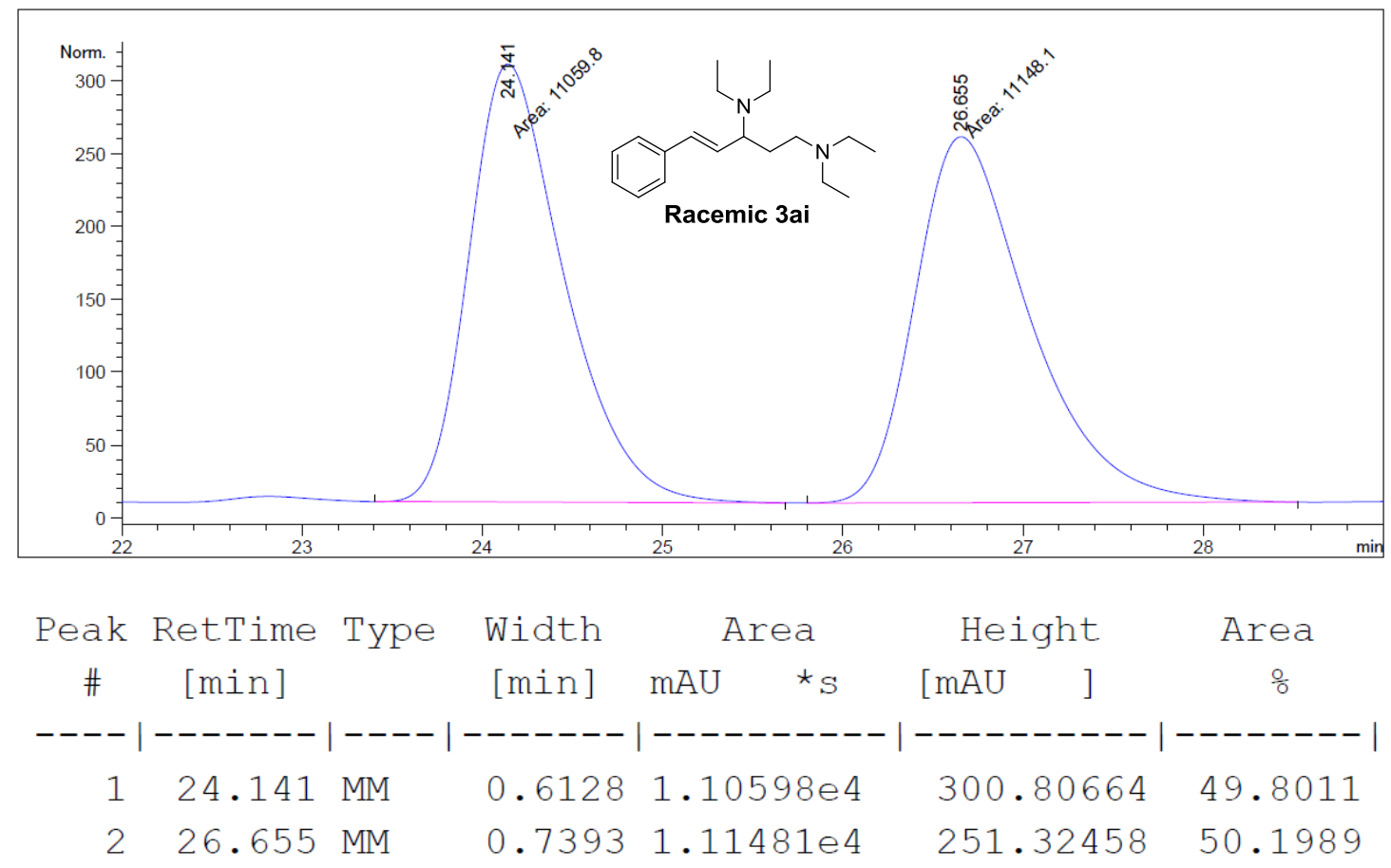

Totals :

$2.22078 e 4 \quad 552.13123$

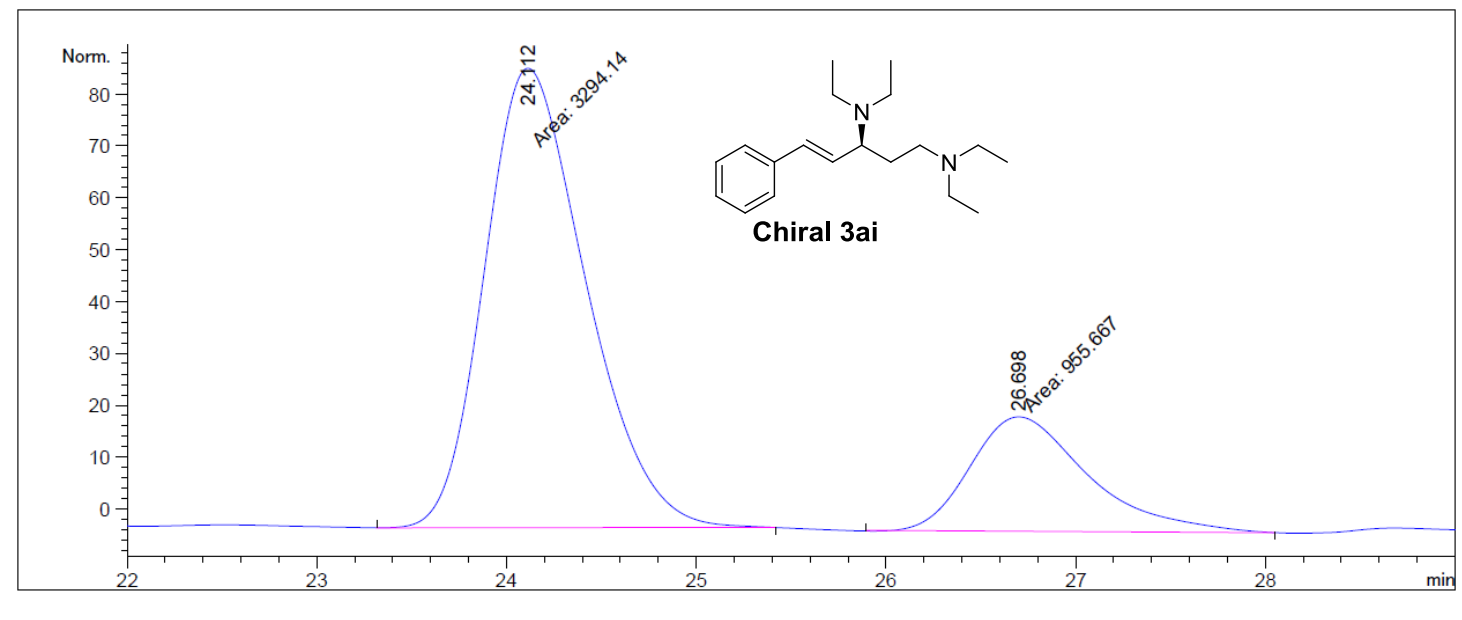

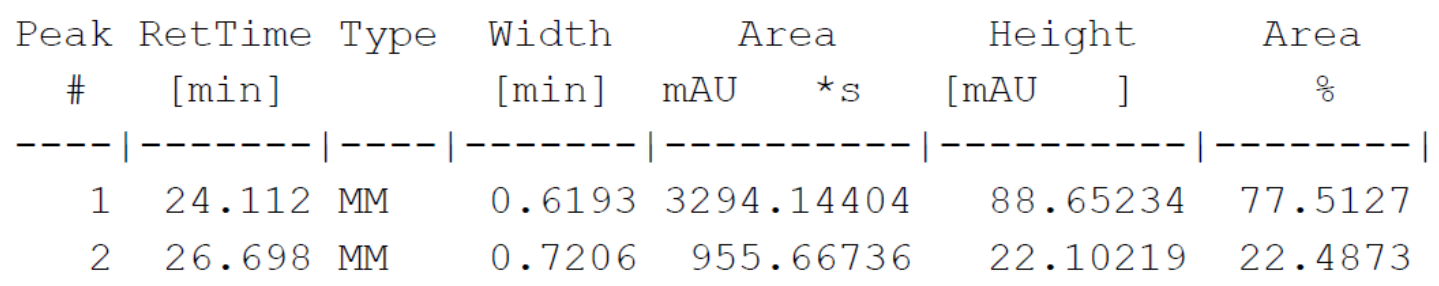

Totals :

$4249.81140 \quad 110.75452$ 


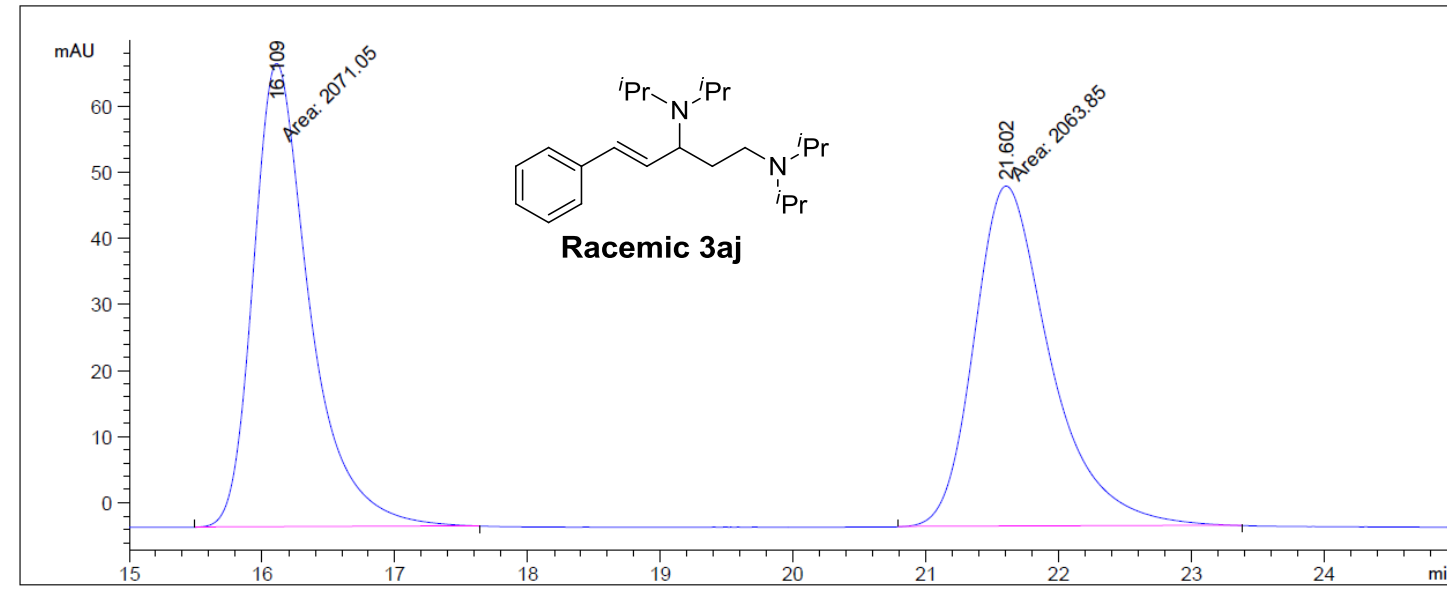

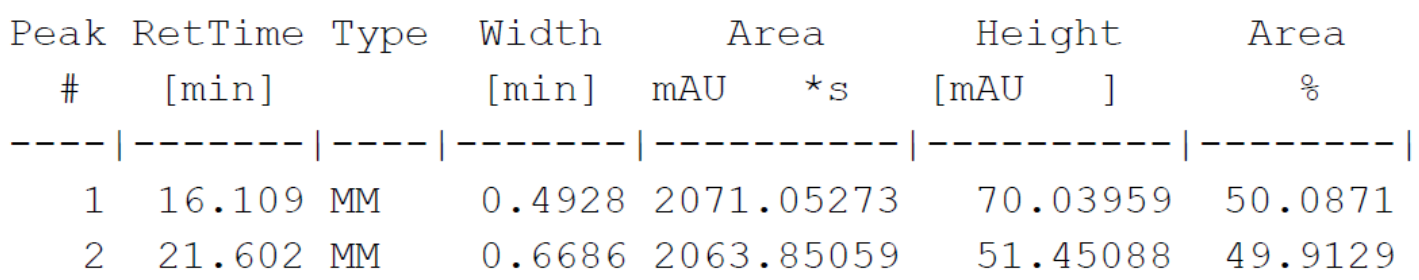

Totals :

$4134.90332 \quad 121.49047$

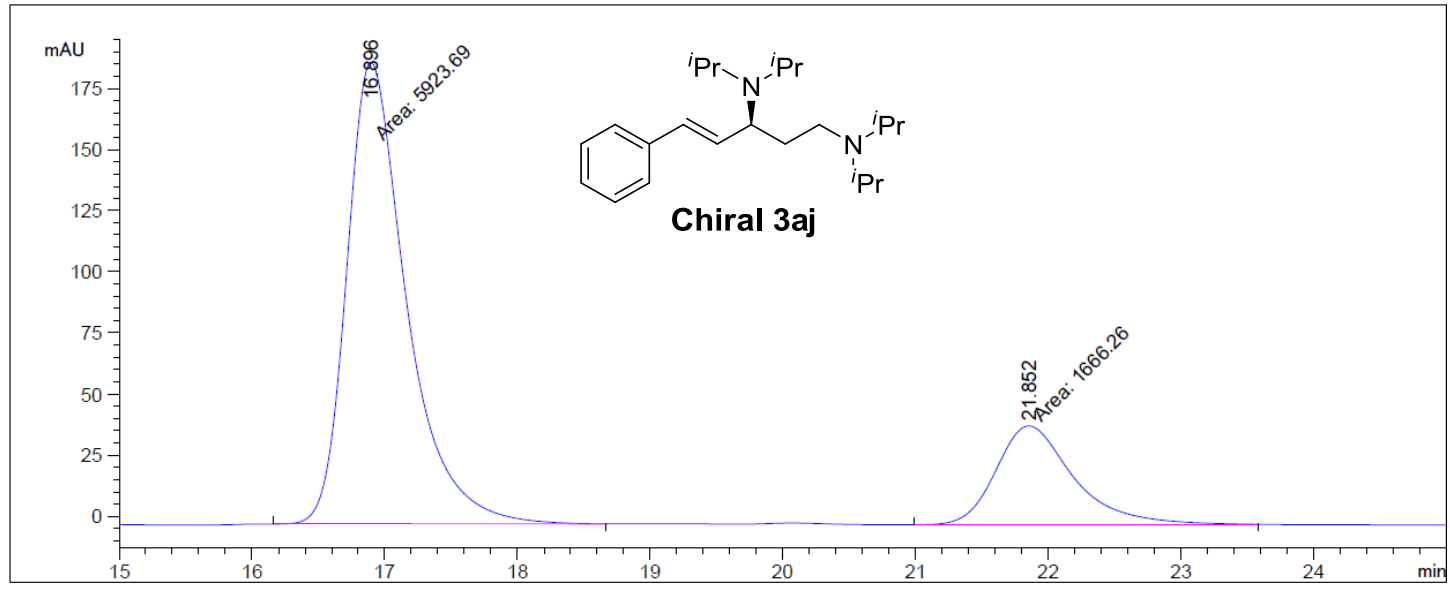

\begin{tabular}{|c|c|c|c|c|c|c|c|}
\hline \multirow{2}{*}{$\begin{array}{c}\text { Peak } \\
\#\end{array}$} & \multirow{2}{*}{$\begin{array}{c}\text { RetTime } \\
\text { [min] }\end{array}$} & \multirow[t]{2}{*}{ Type } & \multirow{2}{*}{$\begin{array}{l}\text { Width } \\
\text { [min] }\end{array}$} & \multicolumn{2}{|c|}{ Area } & Height & \multirow{2}{*}{$\begin{array}{c}\text { Area } \\
\frac{\circ}{0}\end{array}$} \\
\hline & & & & $\mathrm{mAU}$ & $\star_{S}$ & {$[\mathrm{mAU}$} & \\
\hline & & & & & & -- & \\
\hline 1 & & $\mathbb{I M}$ & & 592 & 69434 & 18 & 65 \\
\hline 2 & 52 & & 53 & 166 & 25891 & 40.52660 & 535 \\
\hline
\end{tabular}

Totals :

$7589.95325 \quad 229.46466$ 


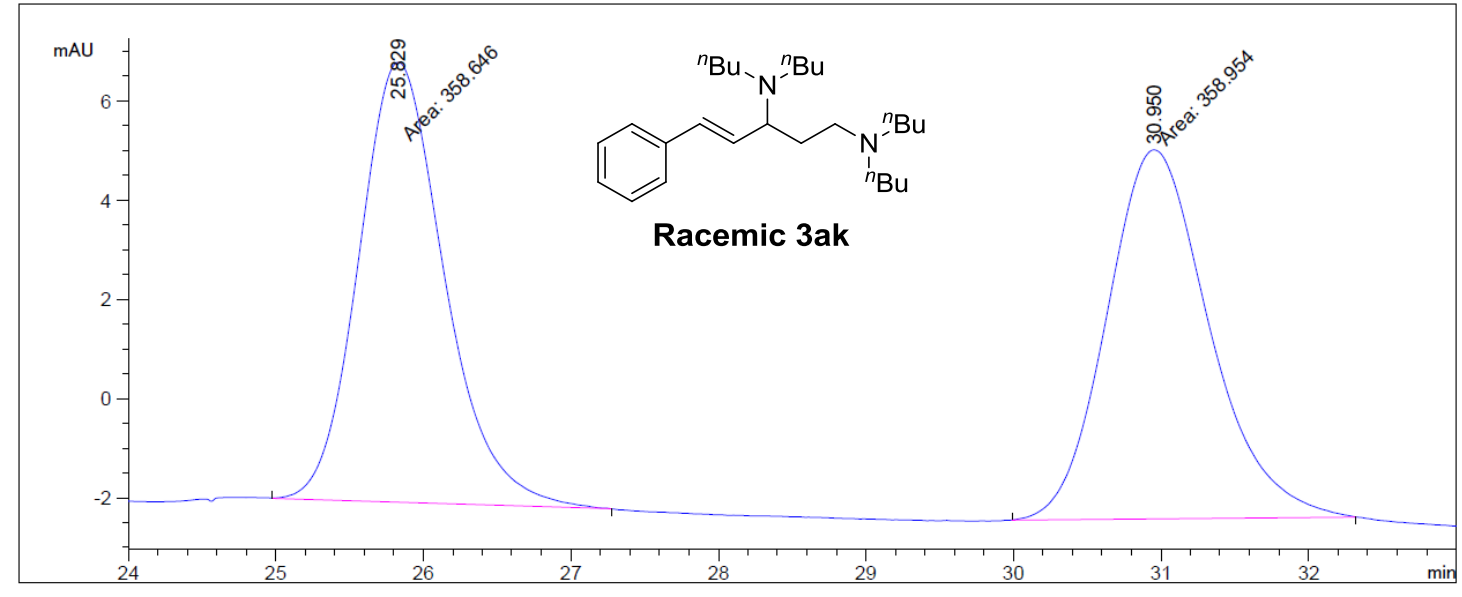

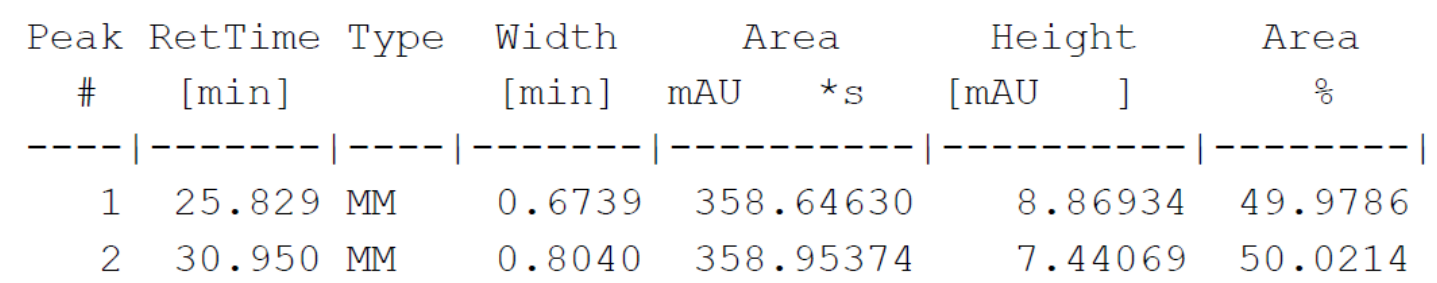

Totals :

$717.60004 \quad 16.31003$

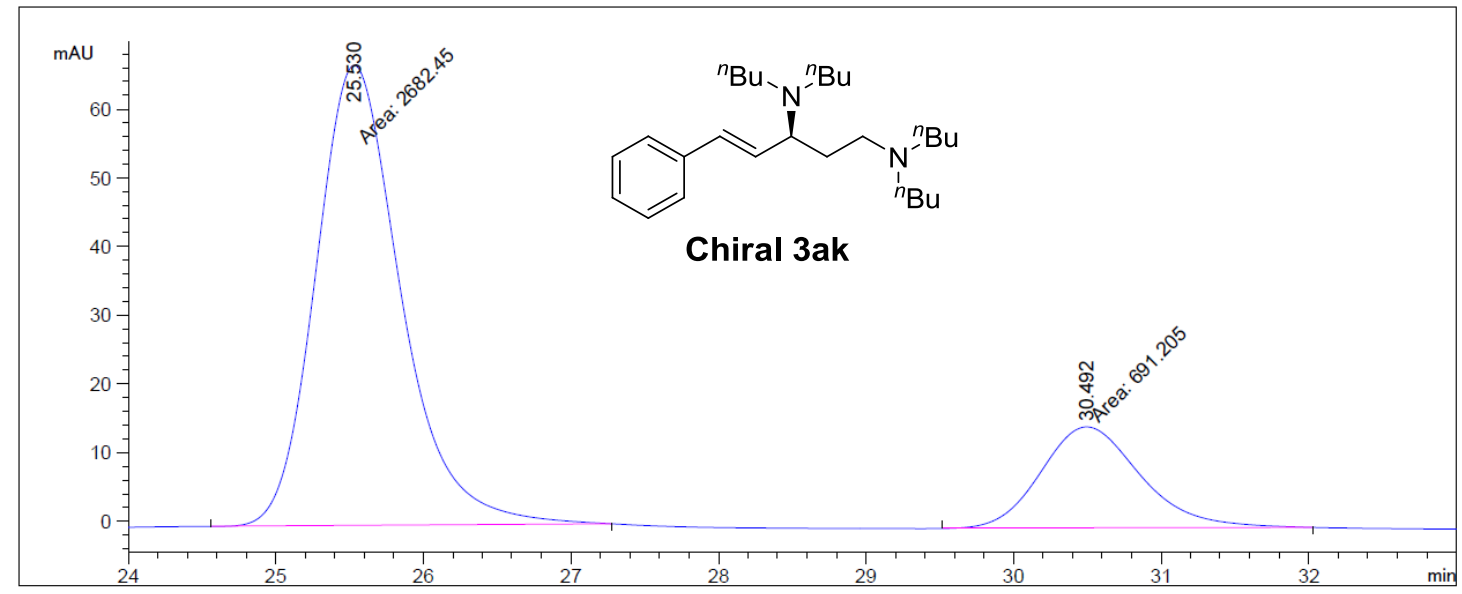

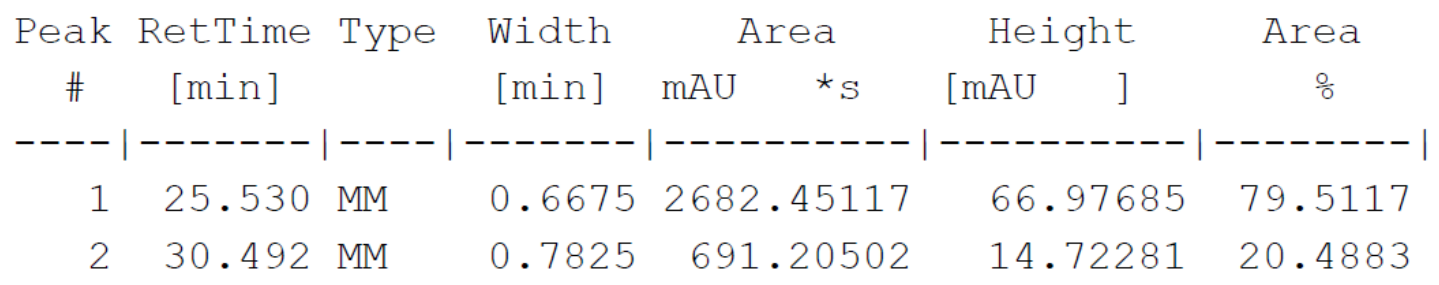

Totals :

$3373.65619 \quad 81.69967$ 


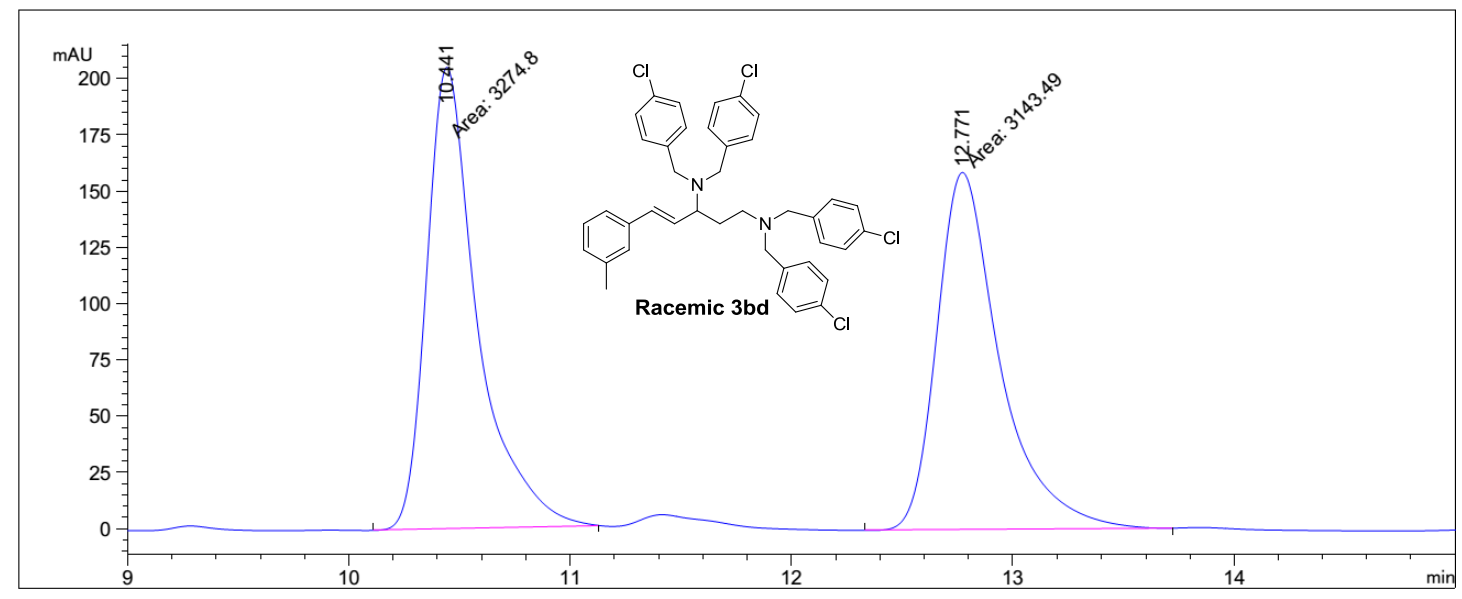

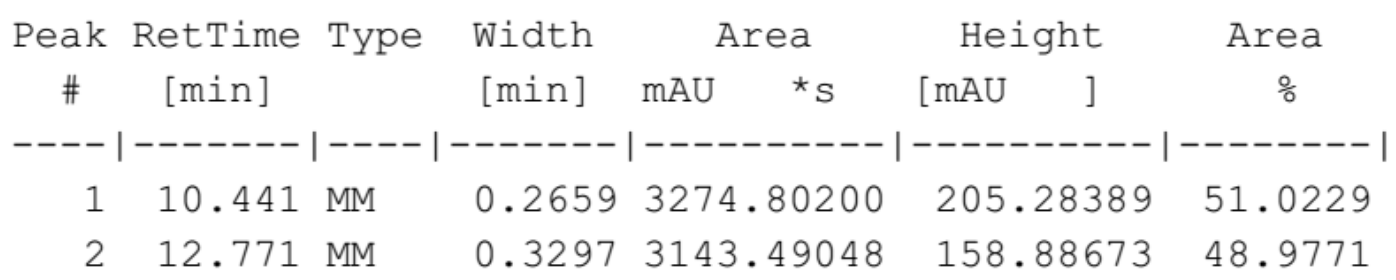

Totals :

$6418.29248 \quad 364.17062$

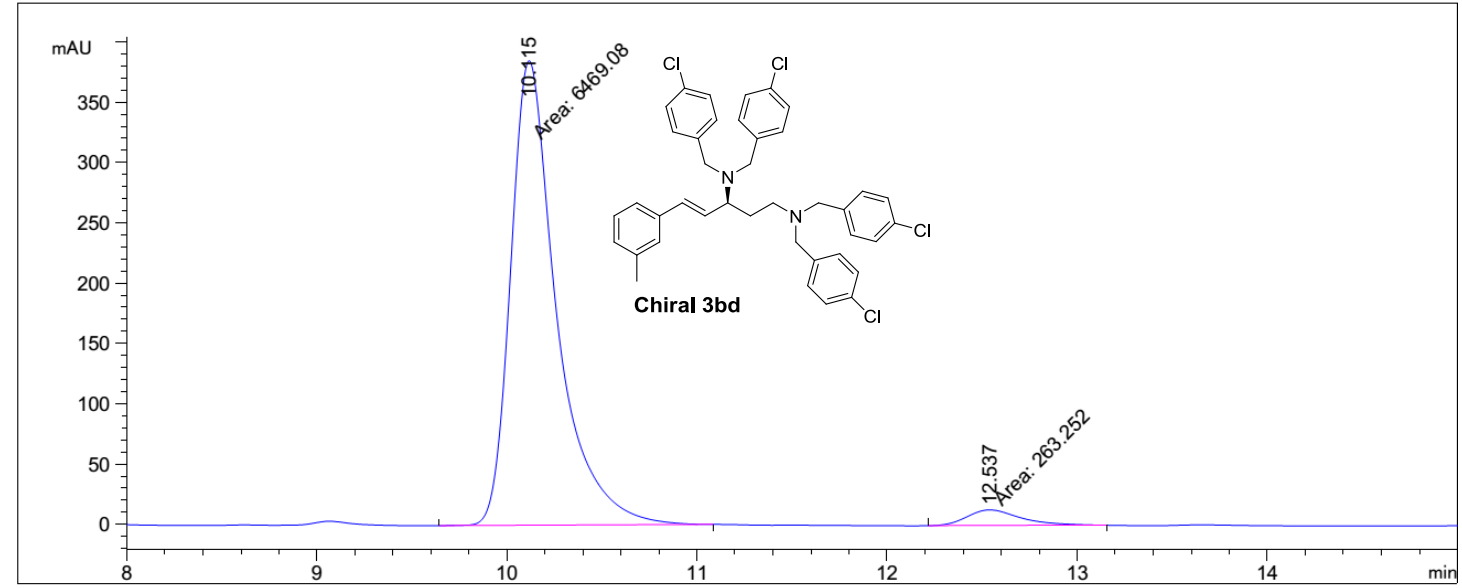

\begin{tabular}{|c|c|c|c|c|c|c|c|}
\hline \multirow{2}{*}{$\begin{array}{c}\text { Peak } \\
\quad \#\end{array}$} & \multirow{2}{*}{\multicolumn{2}{|c|}{$\begin{array}{l}\text { RetTime Type } \\
\text { [min] }\end{array}$}} & \multirow{2}{*}{$\begin{array}{l}\text { Width } \\
\text { [min] }\end{array}$} & \multicolumn{2}{|c|}{ Area } & Height & \multirow{2}{*}{$\begin{array}{c}\text { Area } \\
\quad \%\end{array}$} \\
\hline & & & & mAU & ${ }^{\star} \mathrm{S}$ & {$[\mathrm{mAU} \quad]$} & \\
\hline & & & & & & - & --1 \\
\hline 1 & & $\mathbb{M}$ & & 64 & 8301 & 75 & 897 \\
\hline 2 & 12 & $\mathrm{M}$ & 0. & 26 & 5186 & 13.13950 & 3.9103 \\
\hline
\end{tabular}

Totals :

$6732.33487 \quad 398.57825$ 


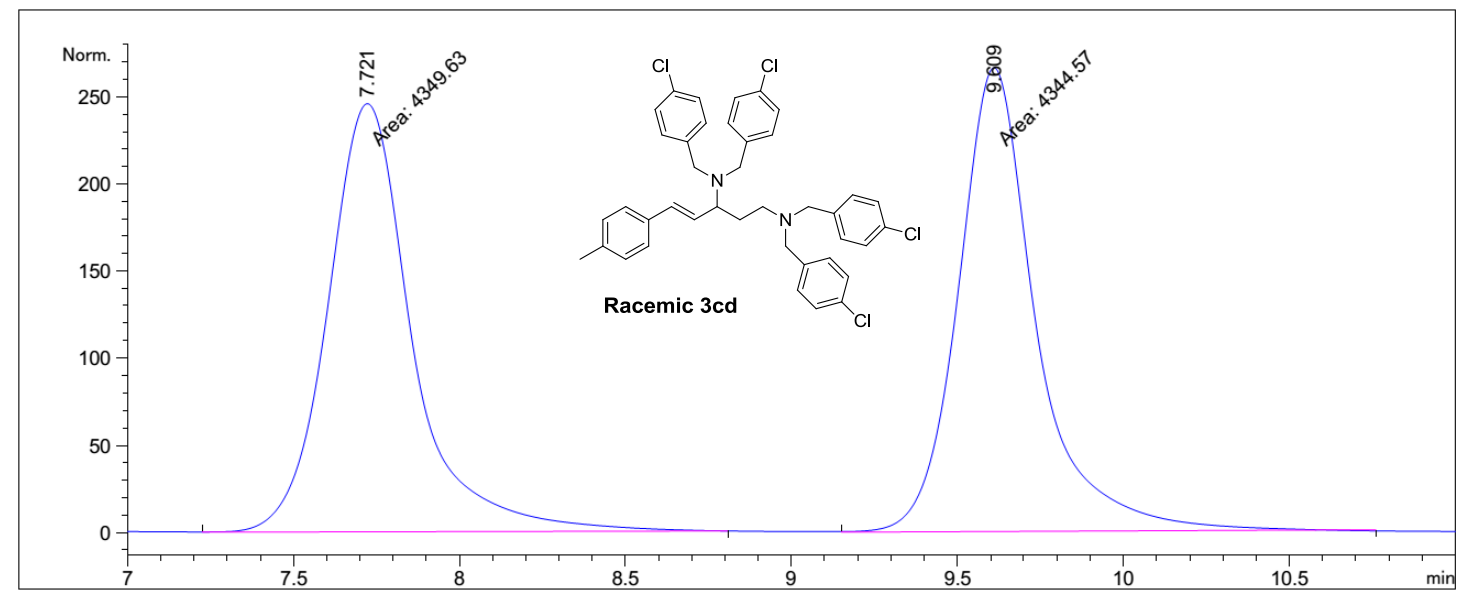

Peak RetTime Type Width Area Height Area

\begin{tabular}{ccccccc}
$\#$ & {$[\mathrm{~min}]$} & {$[\mathrm{min}]$} & $\mathrm{mAU}$ & ${ }_{\mathrm{S}}$ & {$[\mathrm{mAU}]$} & $\%$ \\
\hline 1 & $7.721 \mathrm{MM}$ & 0.2952 & 4349.63184 & 245.61685 & 50.0291 \\
2 & $9.609 \mathrm{MM}$ & 0.2722 & 4344.56934 & 266.05762 & 49.9709
\end{tabular}

Totals :

$8694.20117 \quad 511.67447$
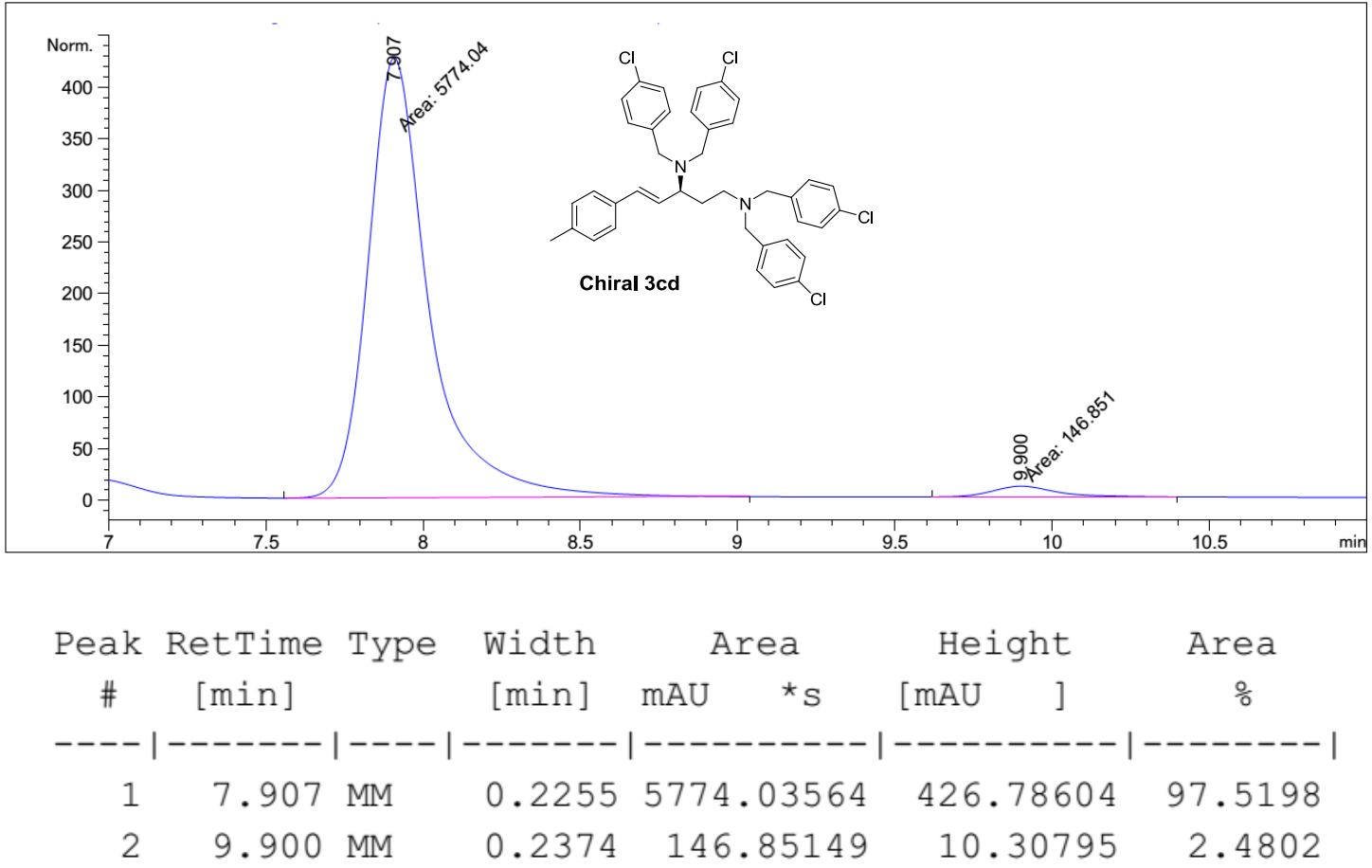

Totals :

$5920.88713 \quad 437.09399$ 


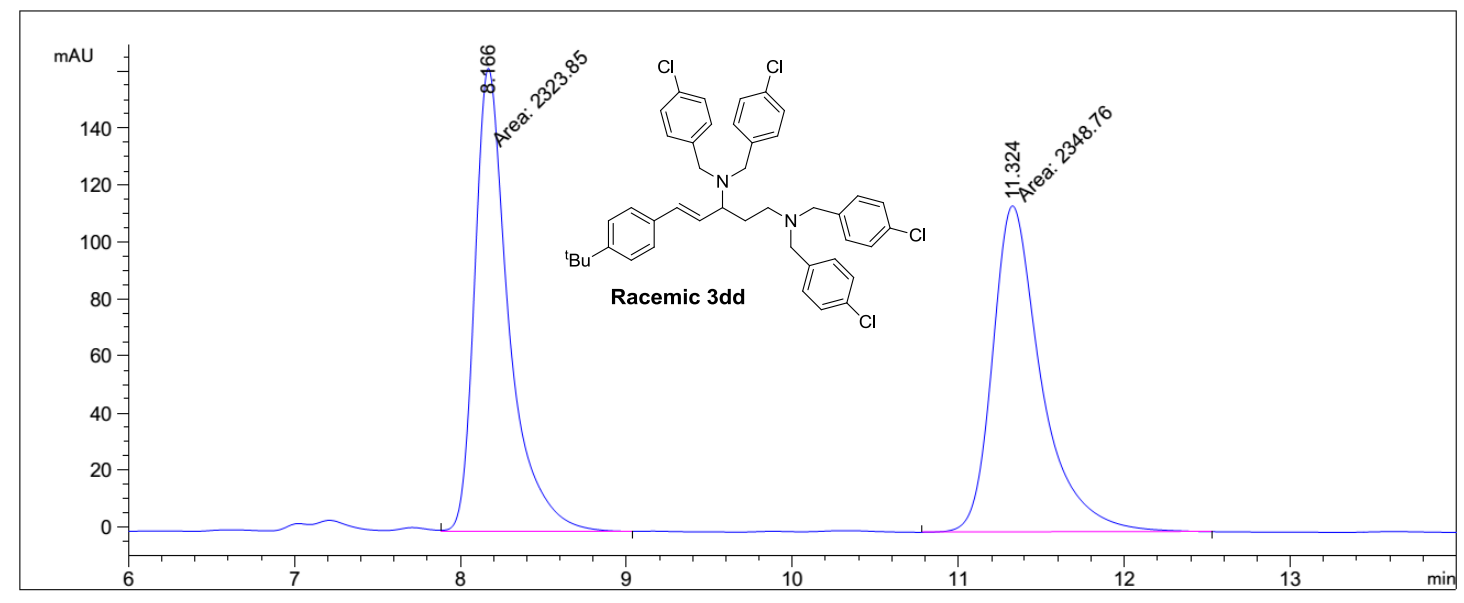

\begin{tabular}{|c|c|c|c|c|c|c|c|}
\hline \multirow{2}{*}{$\begin{array}{c}\text { Peak } \\
\quad \#\end{array}$} & \multirow{2}{*}{$\begin{array}{c}\text { RetTime } \\
\text { [min] }\end{array}$} & \multirow[t]{2}{*}{ Type } & \multirow{2}{*}{$\begin{array}{l}\text { Width } \\
\text { [min] }\end{array}$} & \multicolumn{2}{|c|}{ Area } & Height & \multirow{2}{*}{$\begin{array}{c}\text { Area } \\
\quad \%\end{array}$} \\
\hline & & & & $\mathrm{mAU}$ & ${ }^{\star} \mathrm{S}$ & {$[\mathrm{mAU}$} & \\
\hline & & & & & & & \\
\hline 1 & & & & ? & & & \\
\hline 2 & 24 & . & & 234 & 5757 & 53858 & 2665 \\
\hline
\end{tabular}

Totals :

$4672.60791 \quad 277.14436$
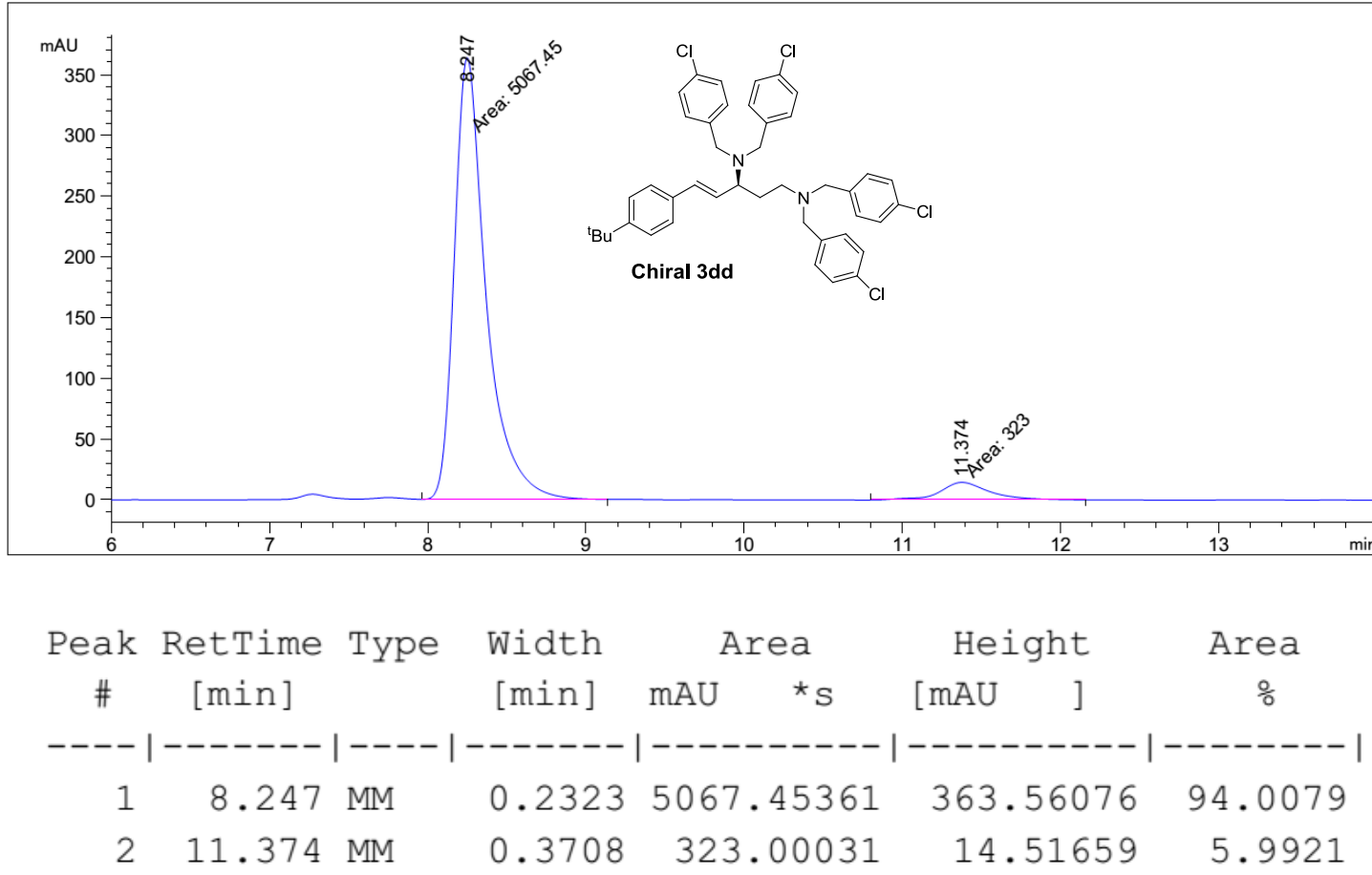

Totals :

$5390.45392 \quad 378.07736$ 

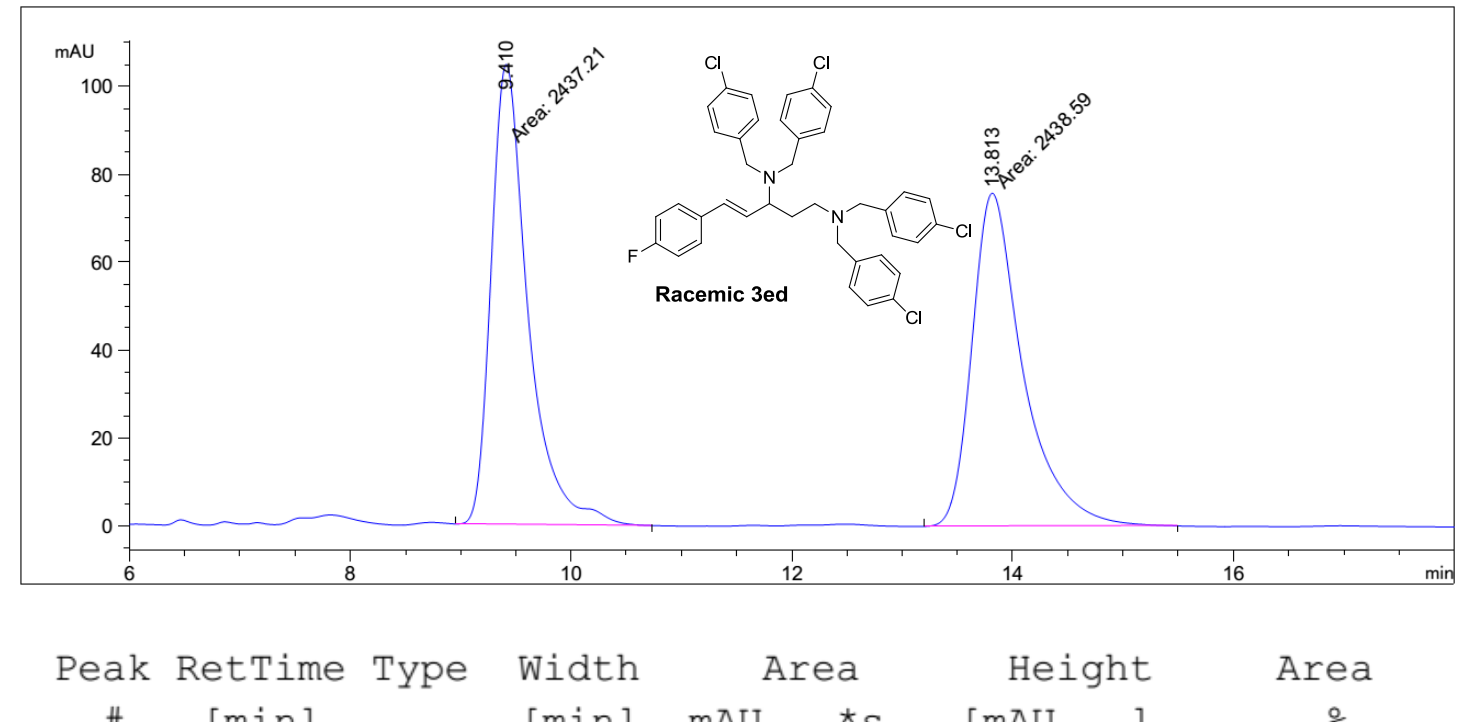

$\begin{array}{crrrrrr}\# & {[\mathrm{~min}]} & {[\mathrm{min}]} & \mathrm{mAU} & * \mathrm{~S} & {[\mathrm{mAU}]} & \% \\ -1 & 9.410 & \mathrm{MM} & 0.3879 & 2437.20923 & 104.72191 & 49.9859 \\ 2 & 13.813 & \mathrm{MM} & 0.5360 & 2438.58521 & 75.83119 & 50.0141\end{array}$

Totals :

$4875.79443 \quad 180.55310$

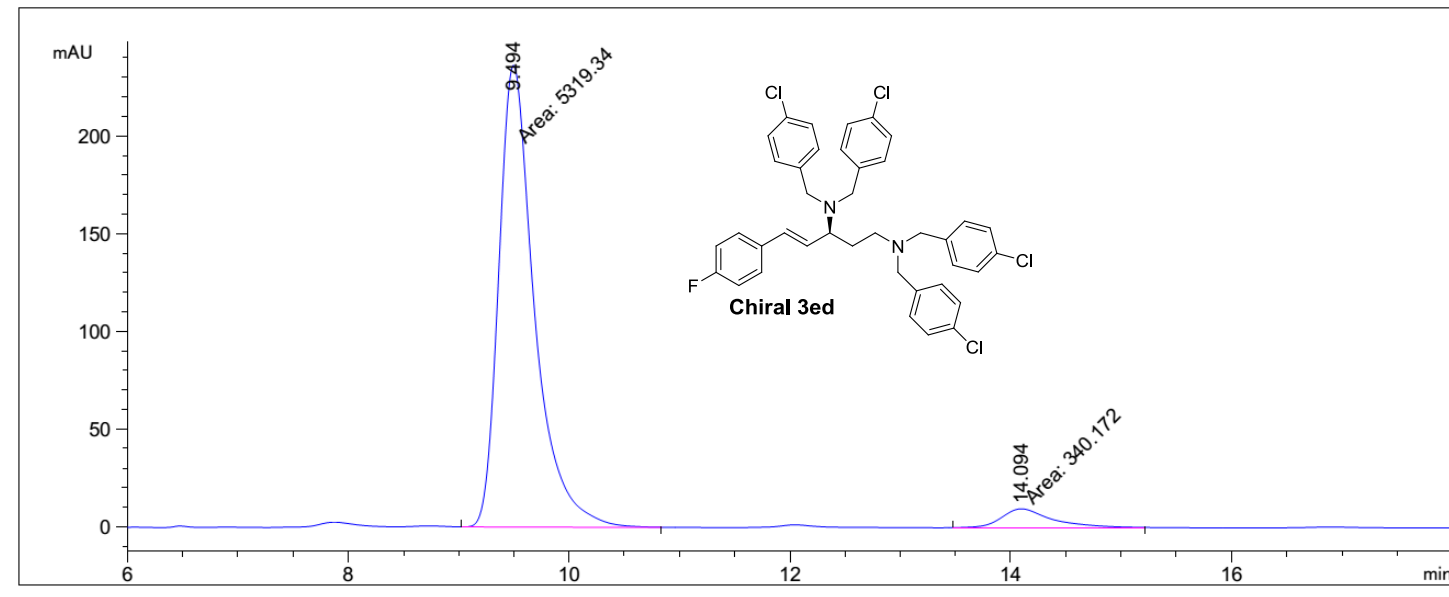

\begin{tabular}{|c|c|c|c|c|c|c|c|}
\hline ak & RetTime & Type & Width & & ea & Height & Area \\
\hline \# & [min] & & {$[\mathrm{min}]$} & mAU & ${ }^{\star} \mathrm{S}$ & {$[\mathrm{mAU}$} & $\%$ \\
\hline & & & & & --- & --- & -- \\
\hline 1 & 94 & MM & 50 & 531 & 3643 & 236.41 & 93 \\
\hline 2 & 4.094 & $\mathbb{M}$ & 708 & 34 & 17227 & 9.93306 & 6.0106 \\
\hline
\end{tabular}

Totals :

$5659.50870 \quad 246.35124$ 

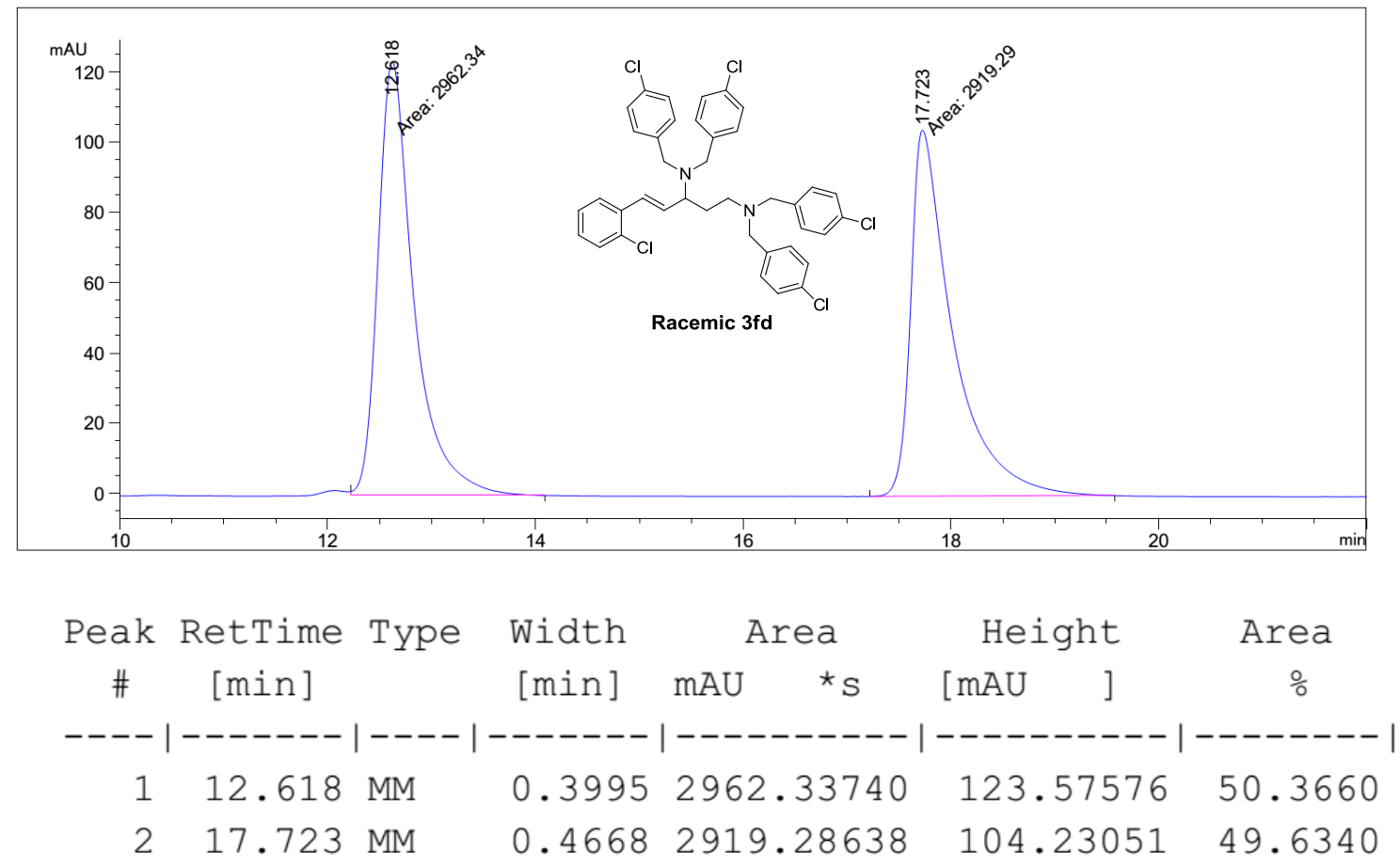

Totals :

$5881.62378 \quad 227.80627$
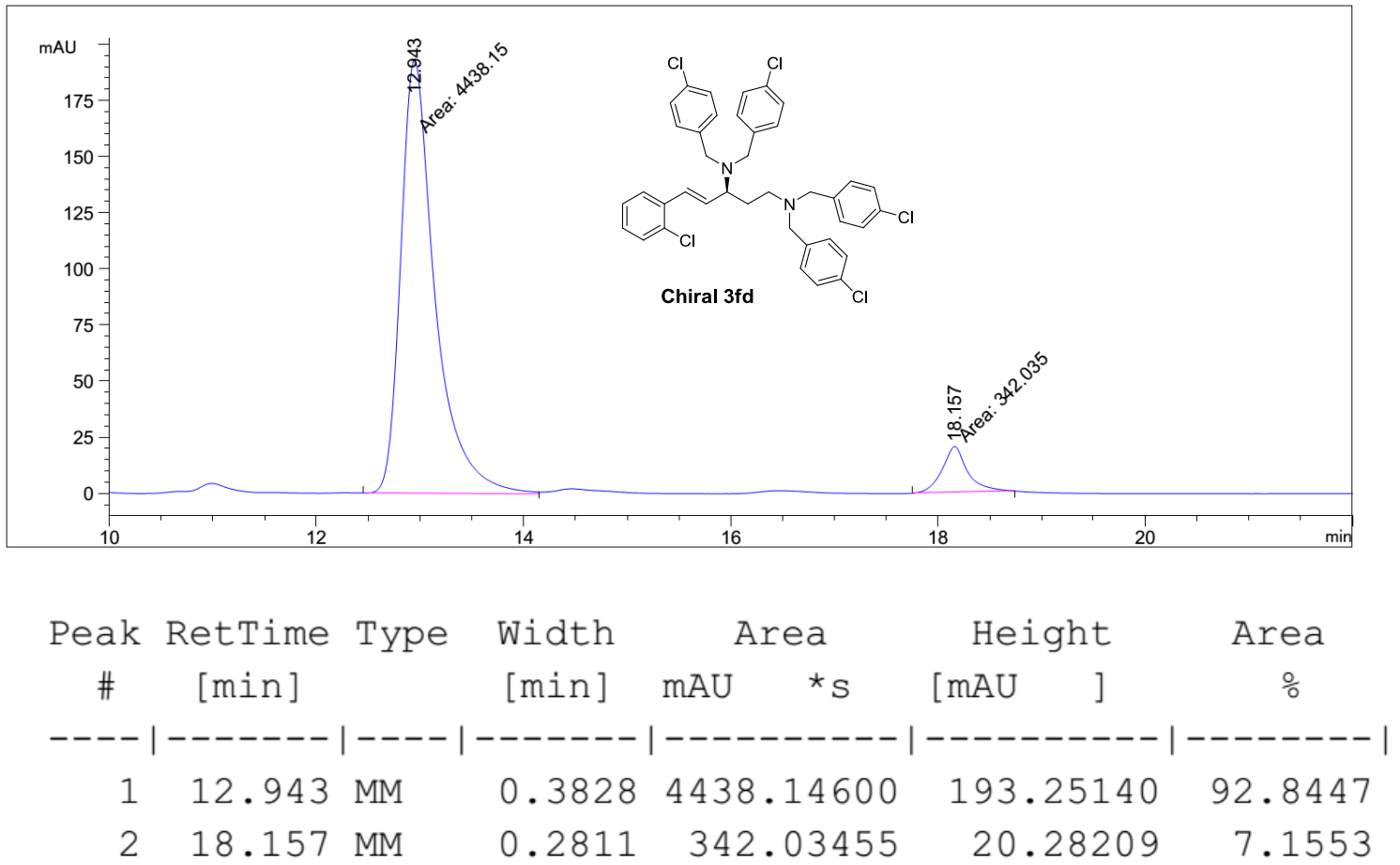

Totals :

$4780.18054 \quad 213.53349$ 

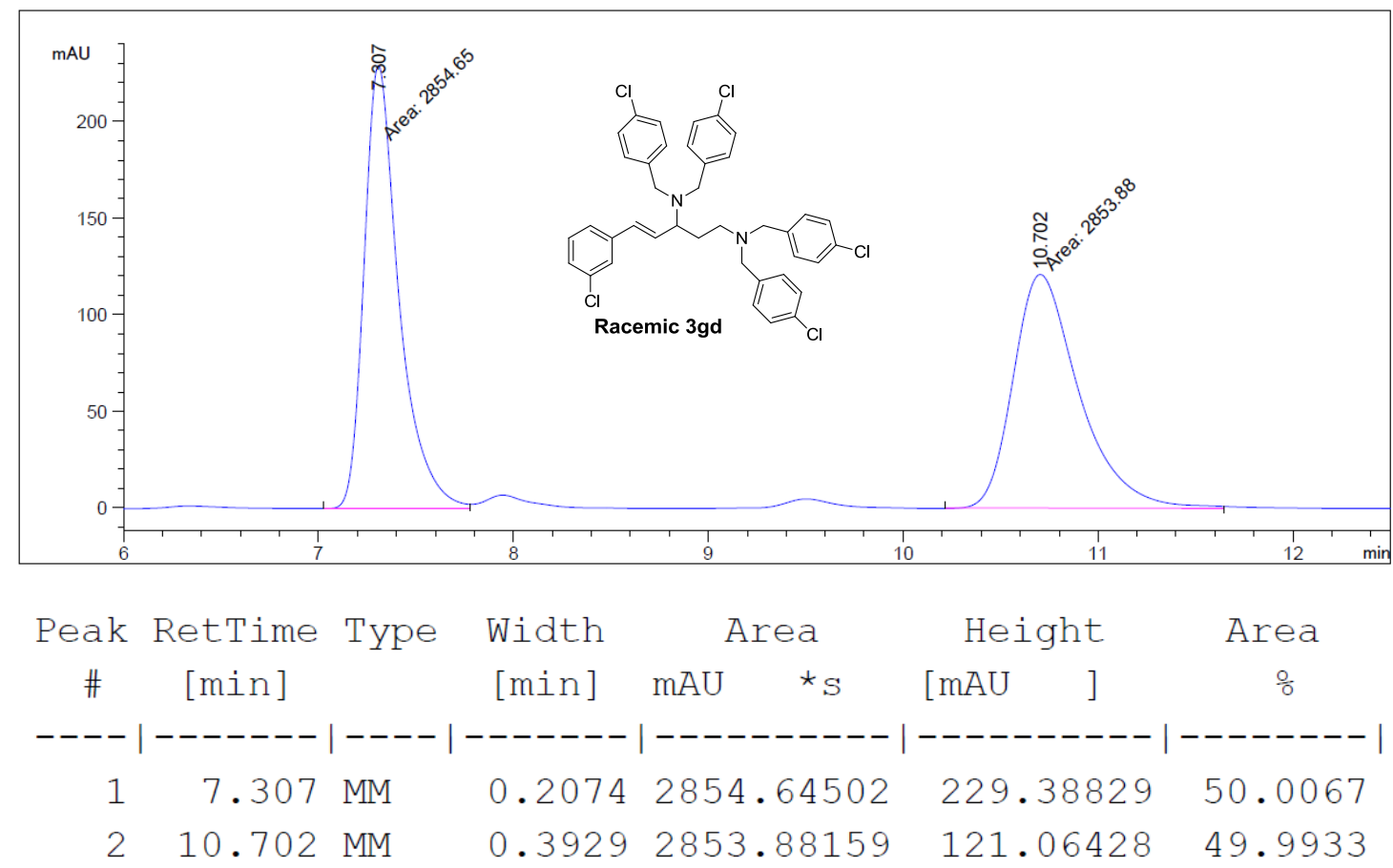

Totals :

$5708.52661 \quad 350.45257$

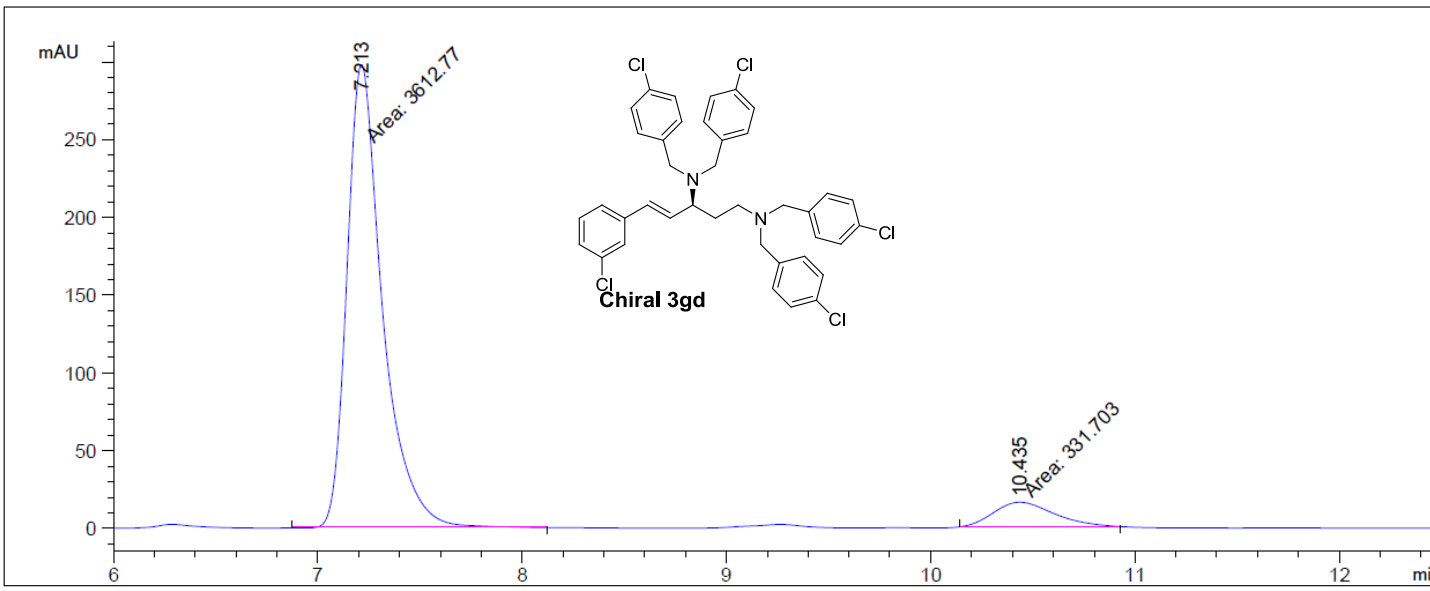

\begin{tabular}{|c|c|c|c|c|c|c|c|}
\hline \multirow{2}{*}{$\begin{array}{c}\text { Peak } \\
\#\end{array}$} & \multirow{2}{*}{$\begin{array}{c}\text { RetTime } \\
\text { [min] }\end{array}$} & \multirow{2}{*}{ Type } & \multirow{2}{*}{$\begin{array}{l}\text { Width } \\
\text { [min] }\end{array}$} & \multicolumn{2}{|c|}{ Area } & Height & \multirow{2}{*}{$\begin{array}{c}\text { Area } \\
\frac{\circ}{0}\end{array}$} \\
\hline & & & & mAU & $\star_{\mathrm{S}}$ & {$[\mathrm{mAU}$} & \\
\hline & & & & & & & \\
\hline 1 & & & & 361 & 026 & 75 & 91 \\
\hline 2 & 10.435 & MM & 77 & 33 & 70328 & 15.90100 & 4093 \\
\hline
\end{tabular}

Totals :

$3944.47354 \quad 313.80975$ 


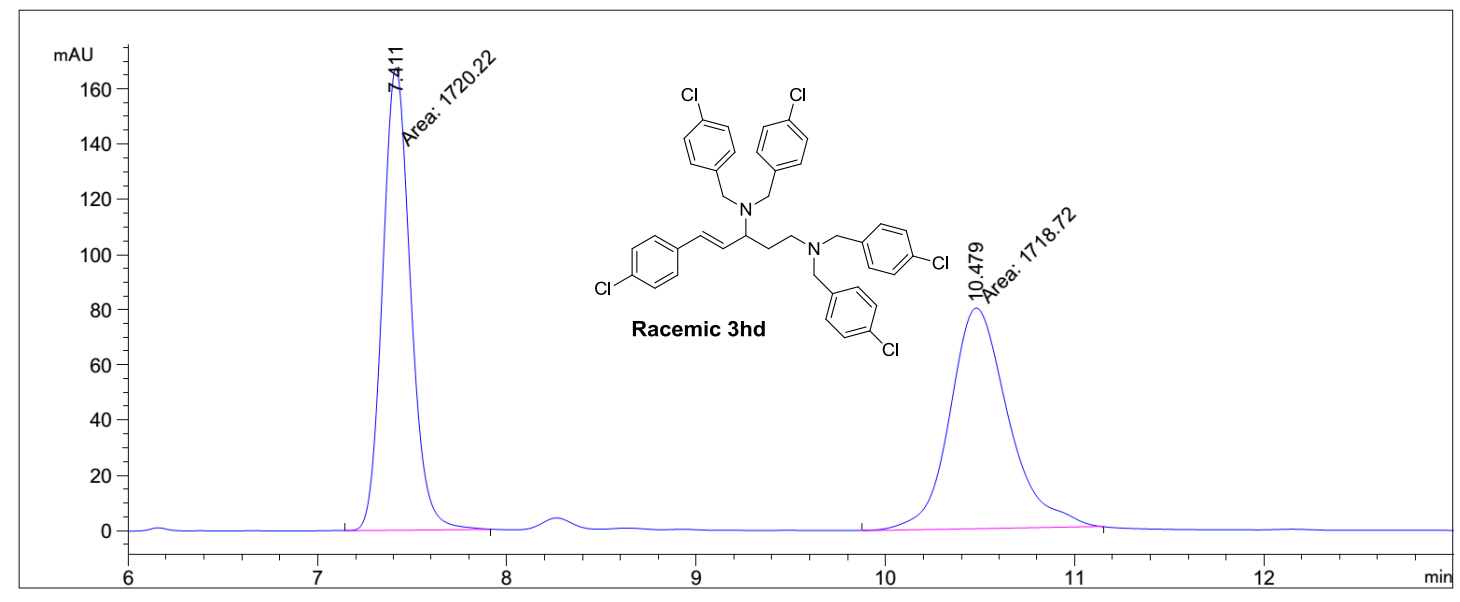

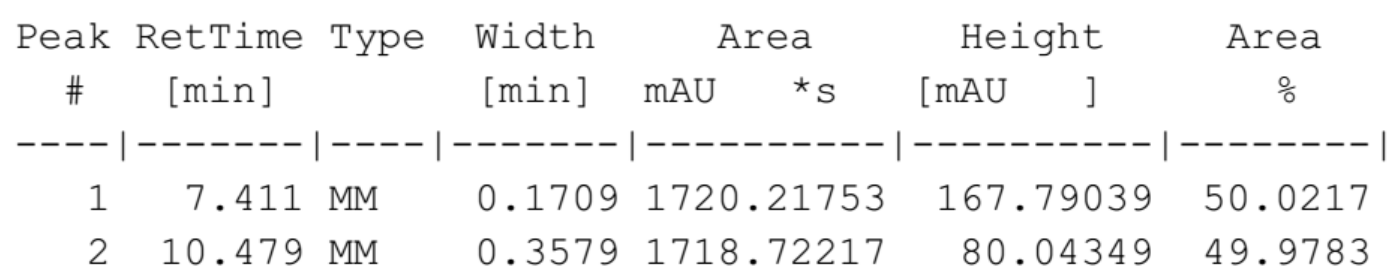

Totals :

$3438.93970 \quad 247.83388$
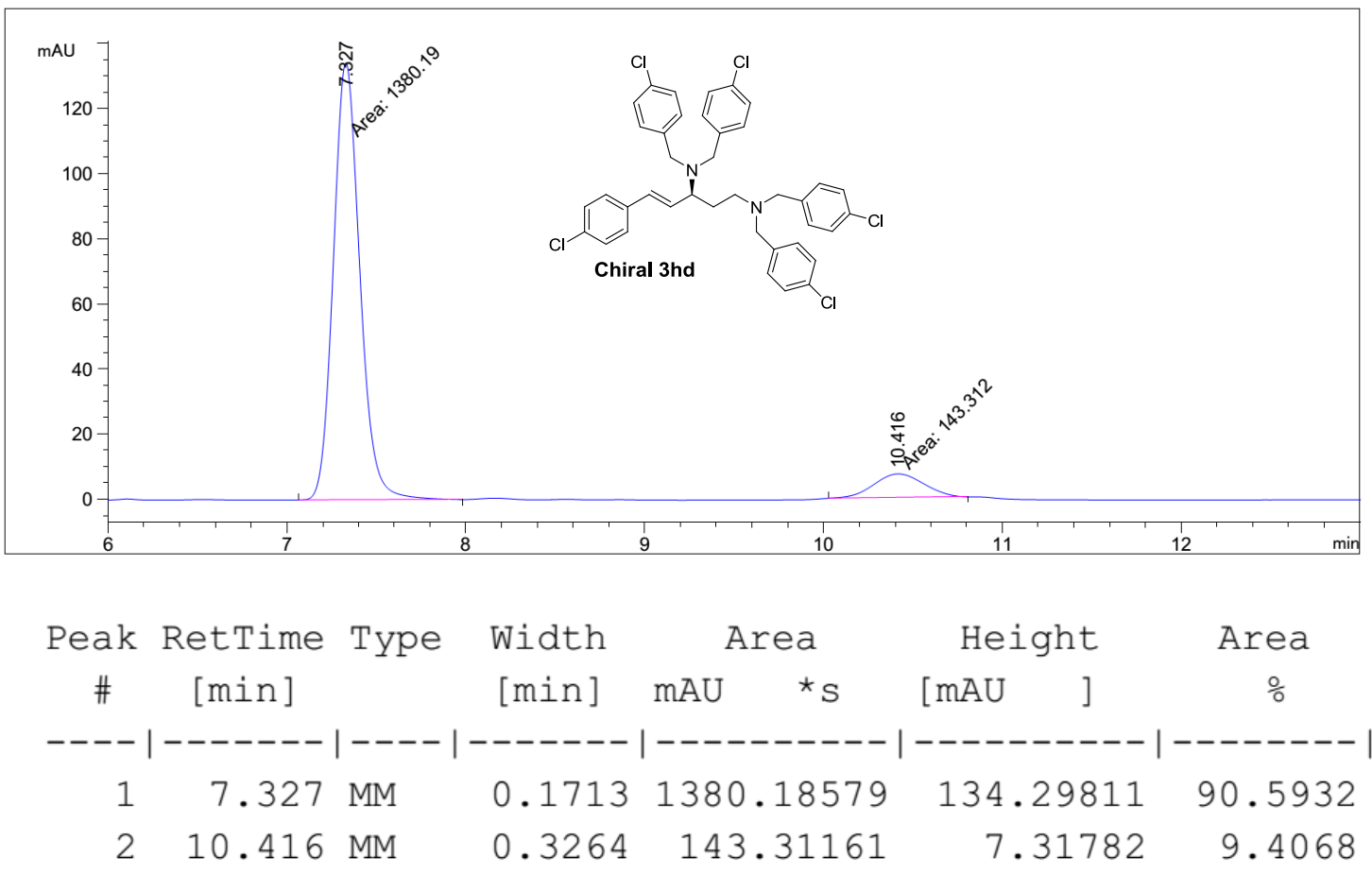

Totals :

$1523.49741 \quad 141.61593$ 

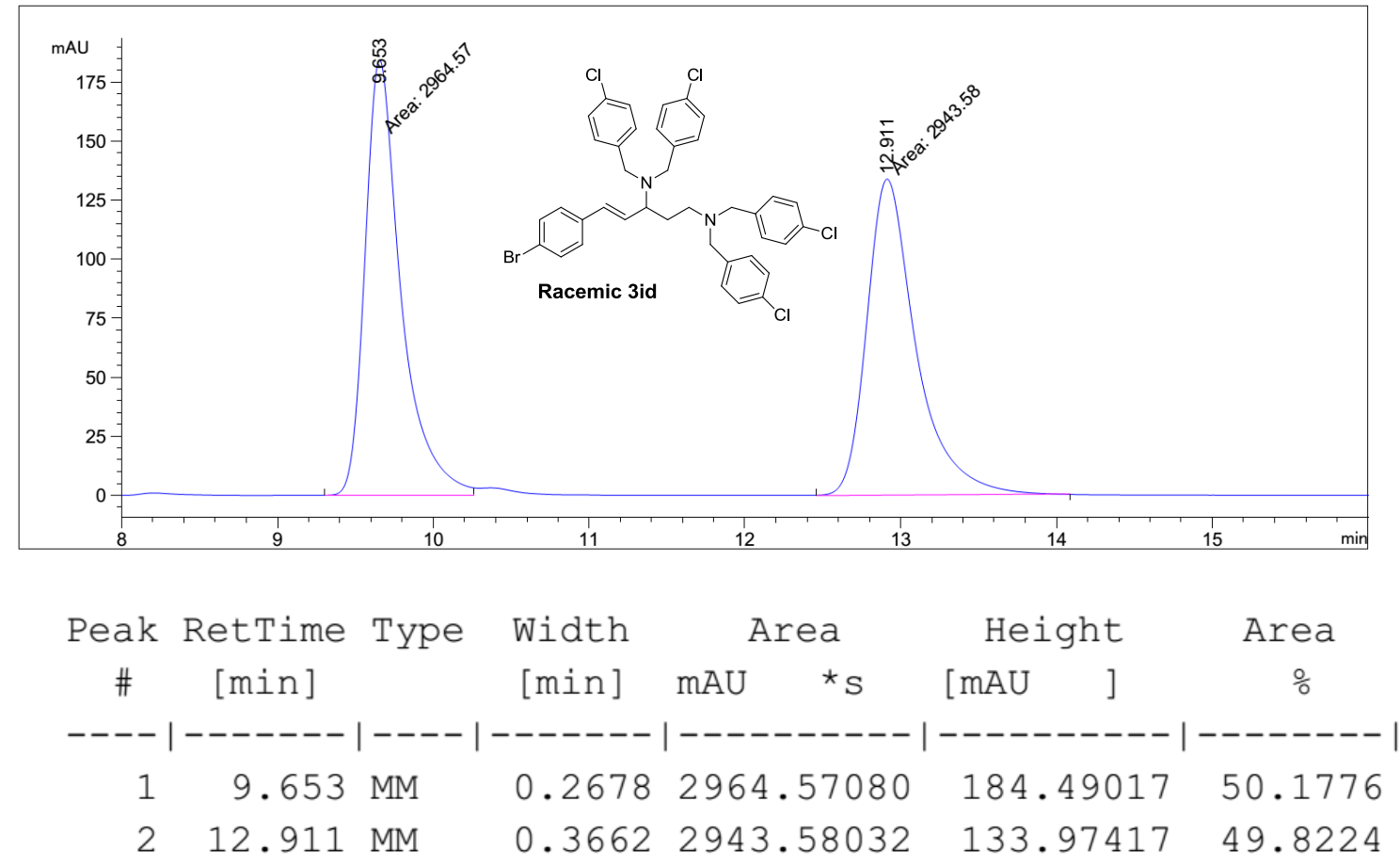

Totals :

$5908.15112 \quad 318.46434$

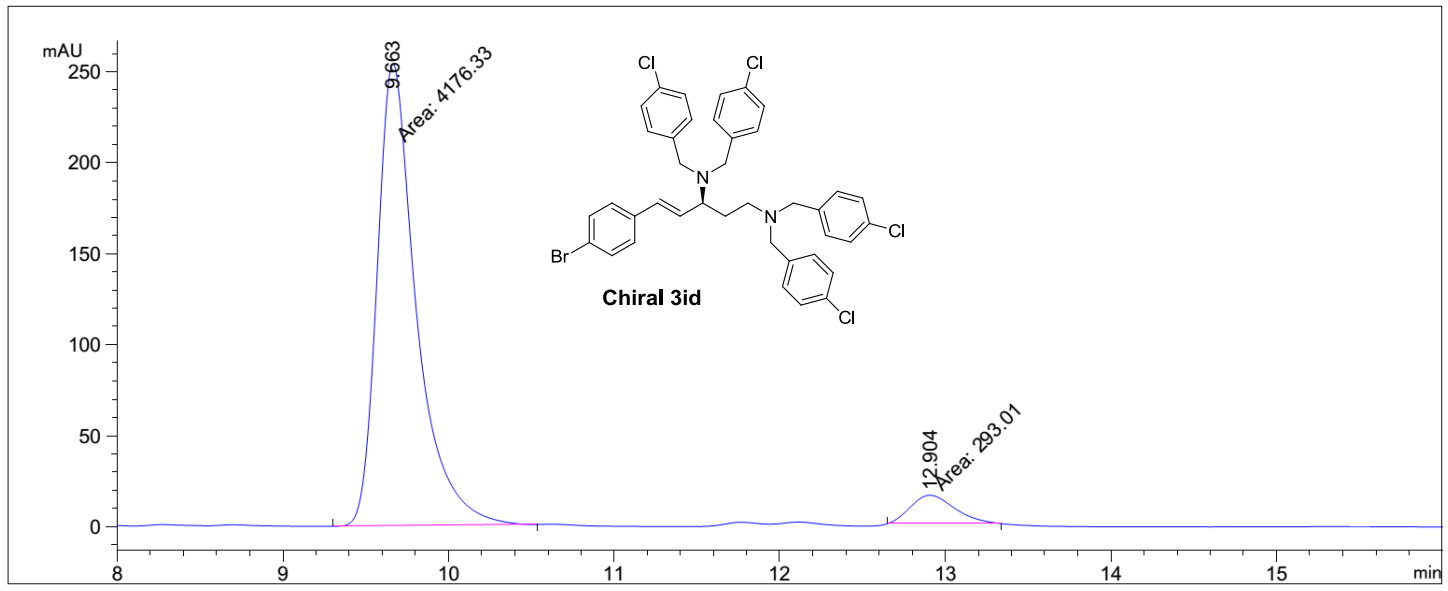

\begin{tabular}{|c|c|c|c|c|c|c|c|}
\hline \multirow{2}{*}{$\begin{array}{c}\text { Peak } \\
\#\end{array}$} & \multirow{2}{*}{$\begin{array}{c}\text { RetTime } \\
\text { [min] }\end{array}$} & \multirow{2}{*}{ Type } & \multirow{2}{*}{$\begin{array}{l}\text { Width } \\
\text { [min] }\end{array}$} & \multicolumn{2}{|c|}{ Area } & Height & \multirow{2}{*}{$\begin{array}{c}\text { Area } \\
\%\end{array}$} \\
\hline & & & & mAU & ${ }^{\star} \mathrm{S}$ & {$[\mathrm{mAU}$} & \\
\hline & & & & & --- & -0 & 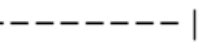 \\
\hline 1 & & & & 417 & 2861 & & 40 \\
\hline 2 & 904 & $M$ & 319 & 29 & 01004 & & 5560 \\
\hline
\end{tabular}

Totals :

$4469.33865 \quad 269.81194$ 

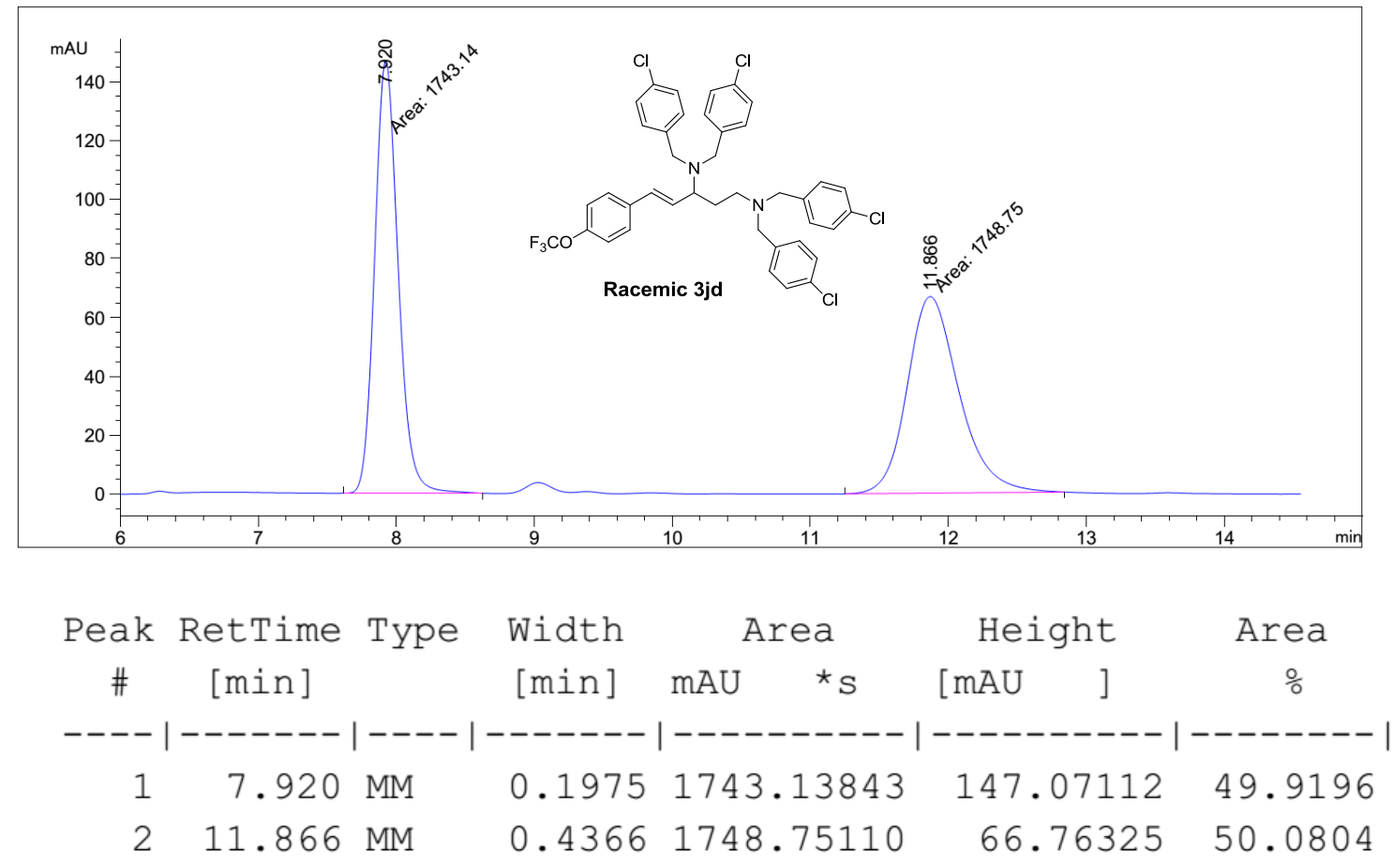

Totals :

$3491.88953 \quad 213.83437$
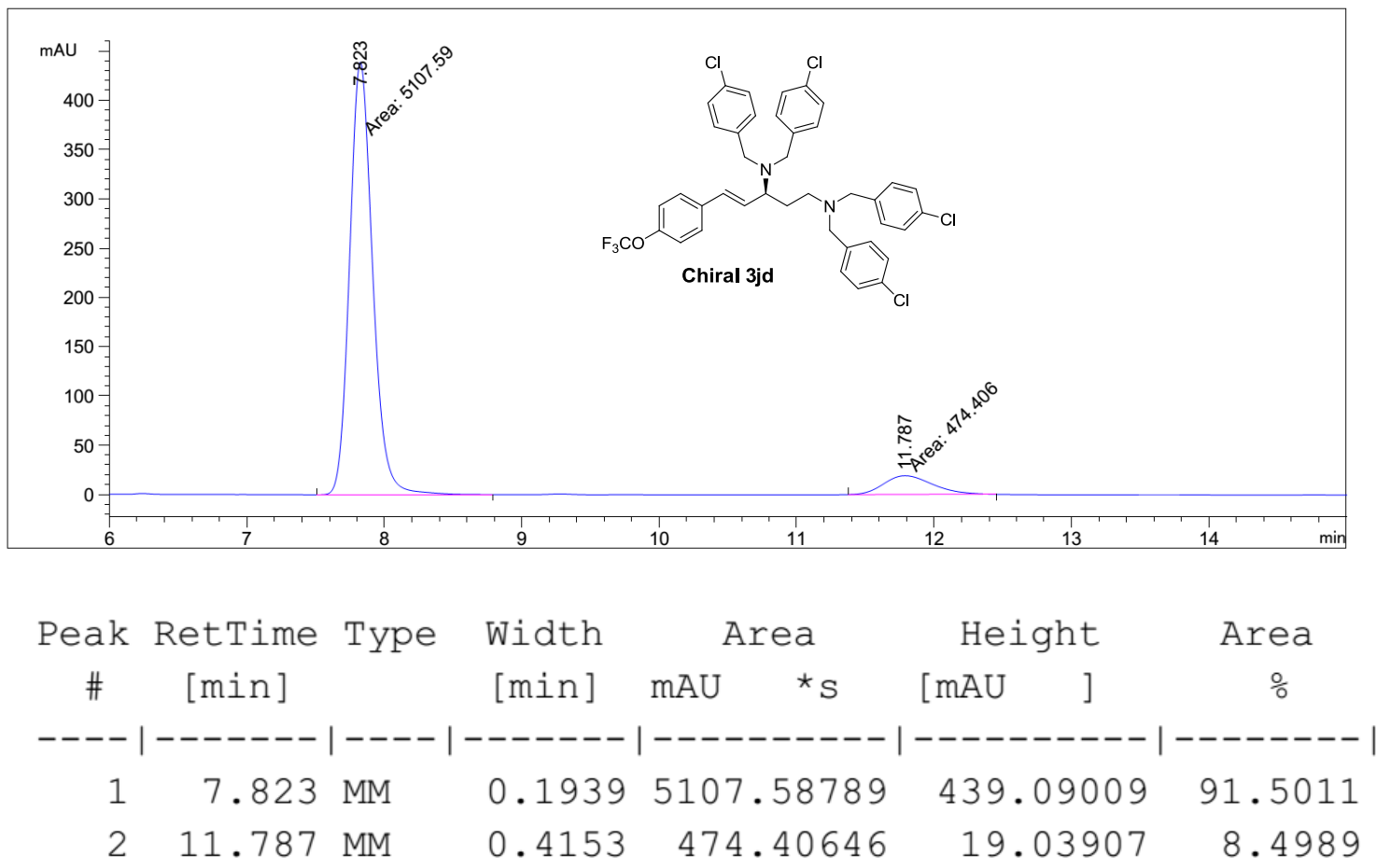

Totals :

$5581.99435 \quad 458.12916$ 


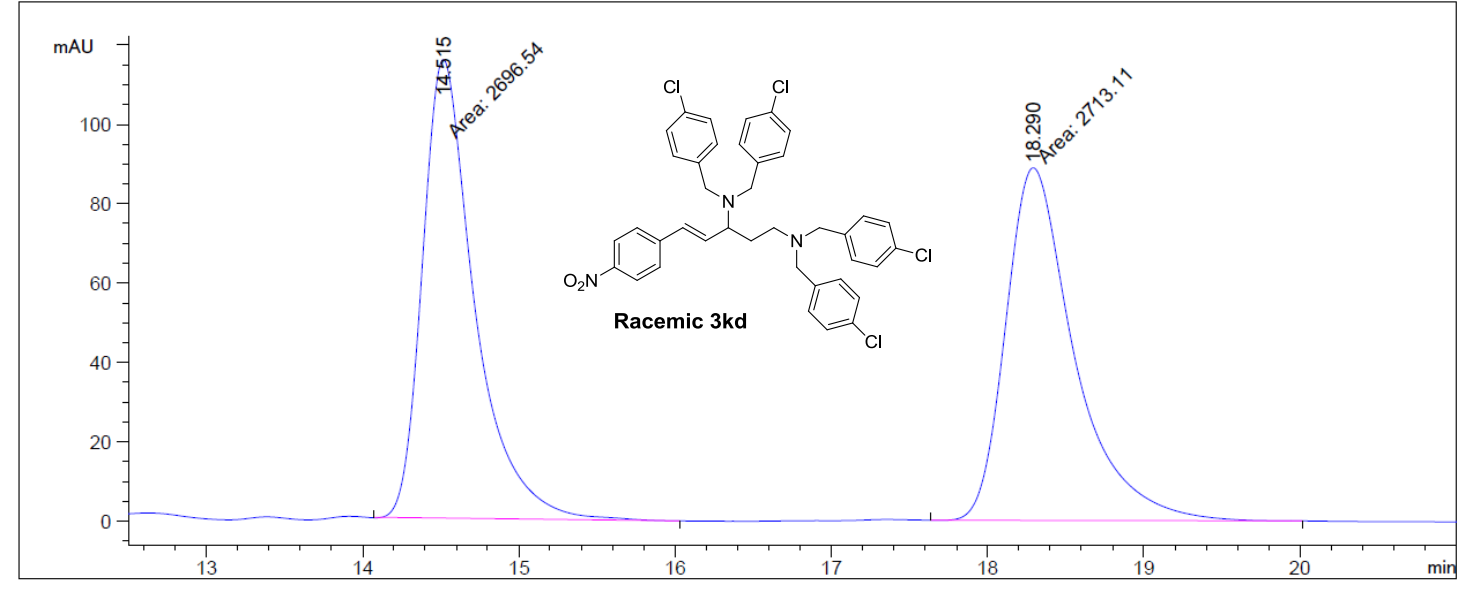

$\begin{array}{cccccc}\text { Peak RetTime Type } & \text { Width } & \text { Area } & \text { Height } & \text { Area } \\ \# & {[\mathrm{~min}]} & {[\mathrm{min}]} & \mathrm{mAU} \quad{ }_{\mathrm{S}} & {[\mathrm{mAU}]} & \frac{\circ}{\circ}\end{array}$

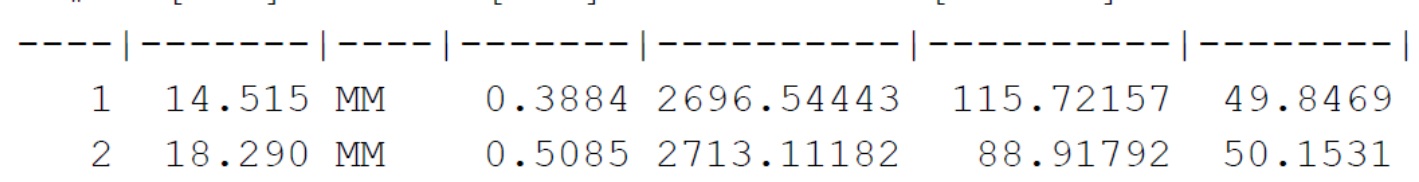

Totals :

$5409.65625 \quad 204.63950$
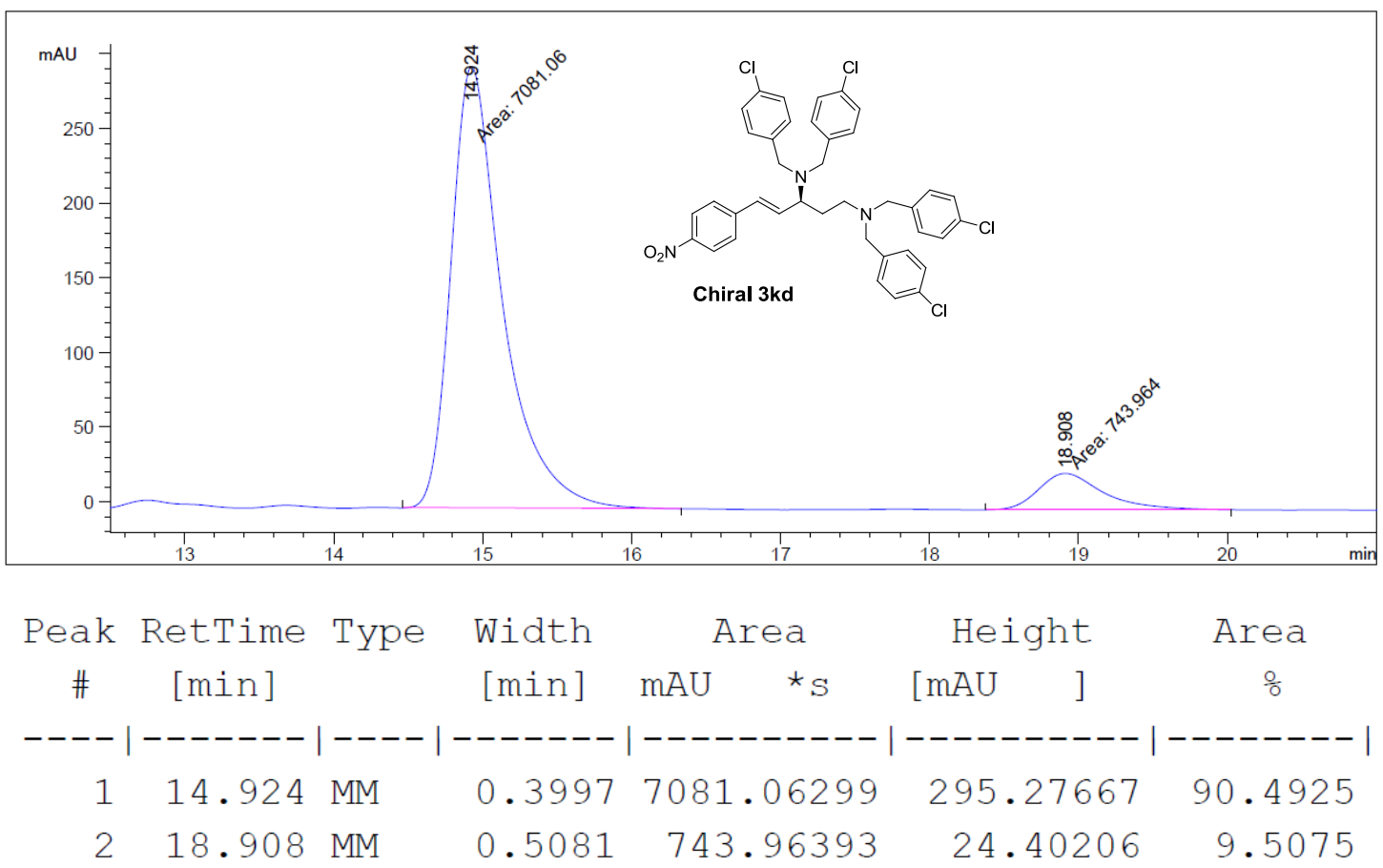

Totals :

$7825.02692 \quad 319.67873$ 

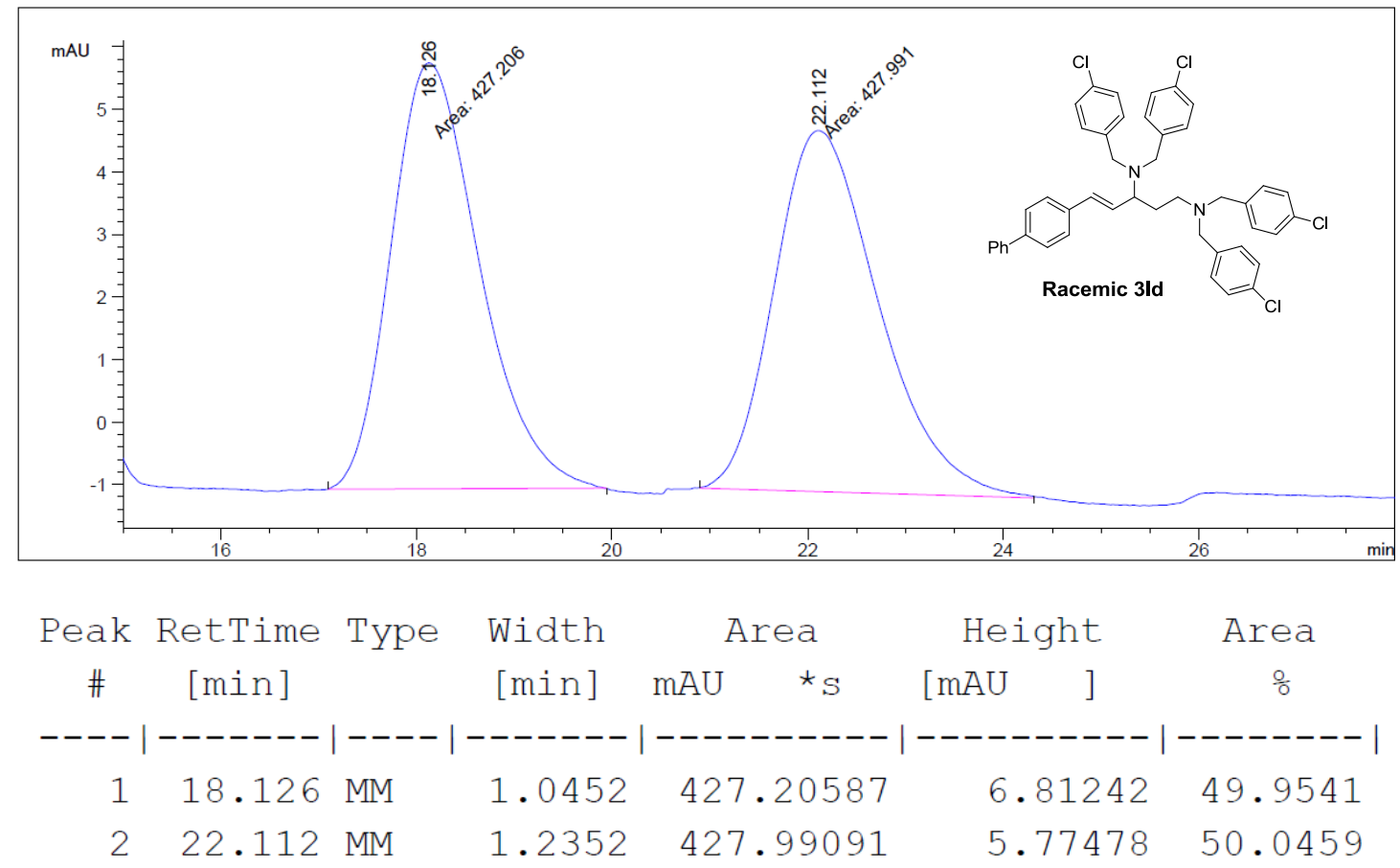

Totals :

$855.19678 \quad 12.58720$
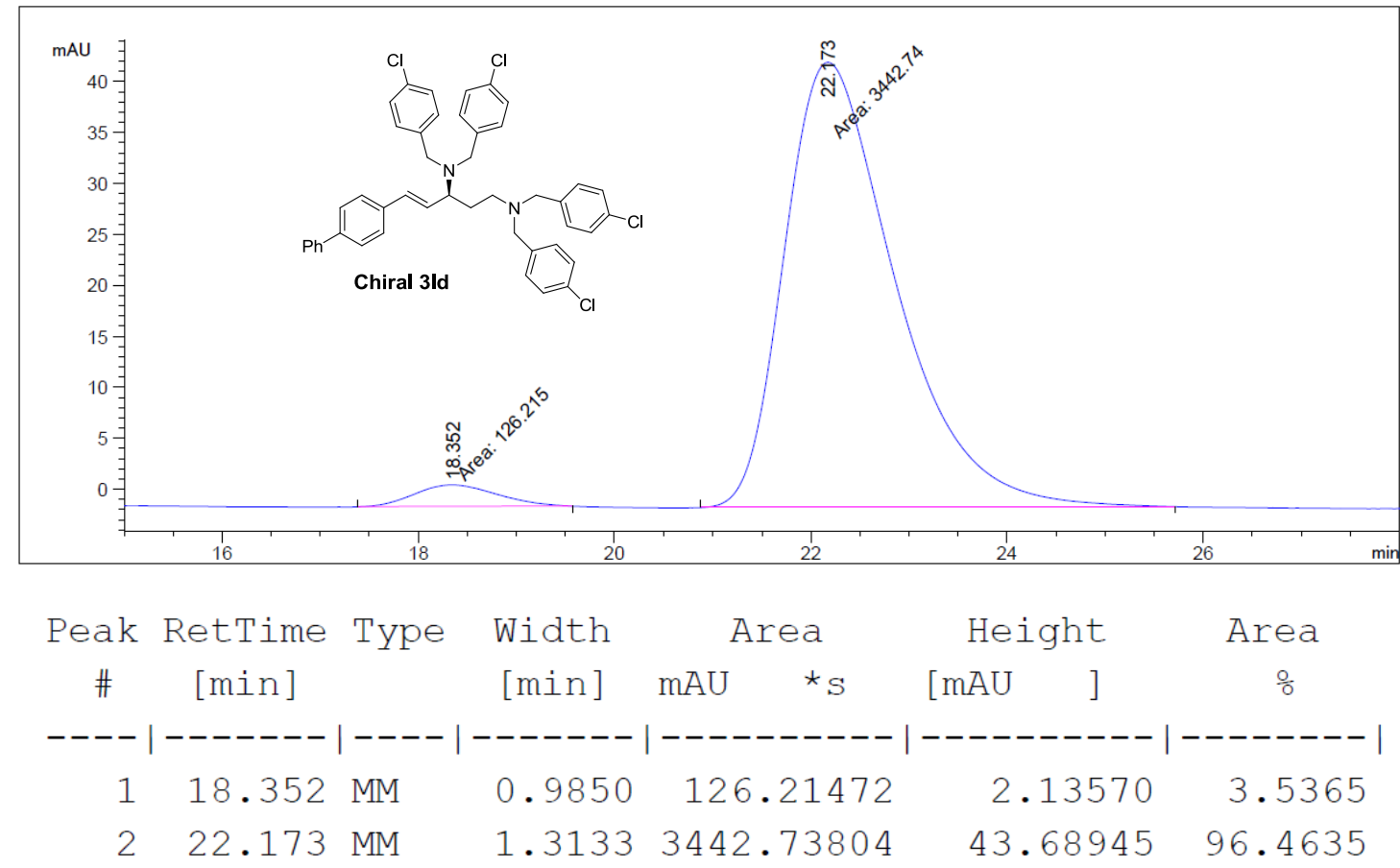

Totals :

$3568.95276 \quad 45.82515$ 


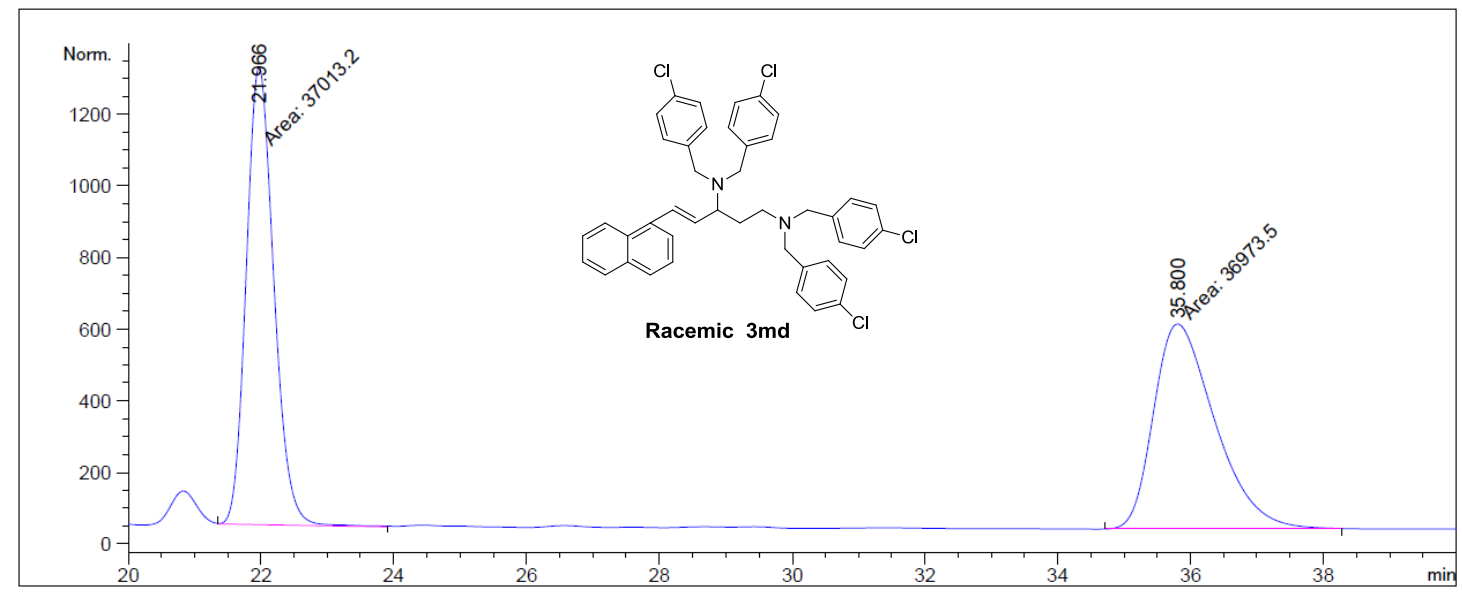

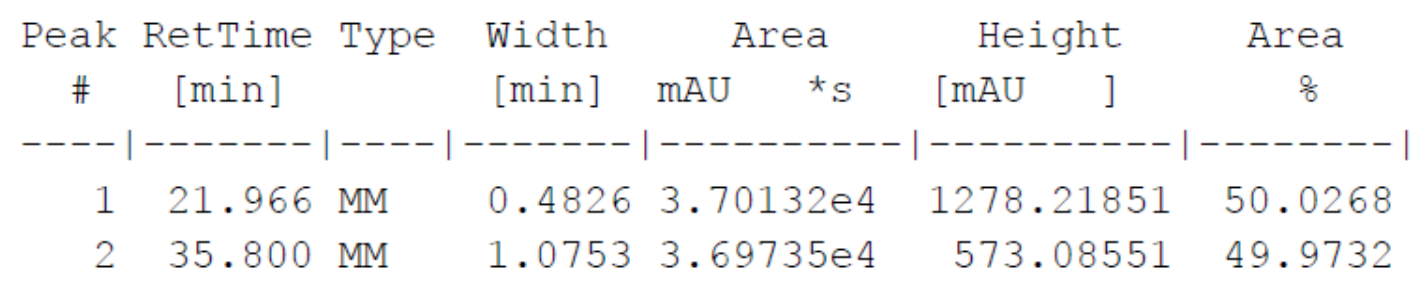

Totals :

7.39867e4 1851.30402

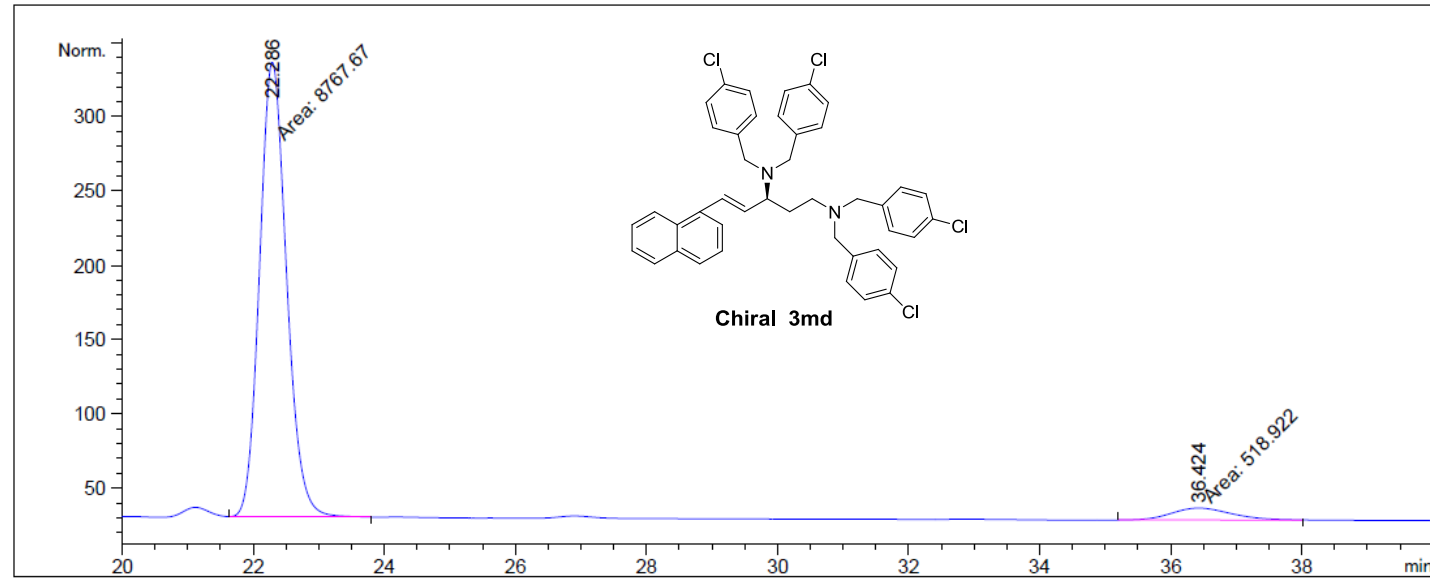

\begin{tabular}{|c|c|c|c|c|c|c|c|}
\hline \multirow{2}{*}{$\begin{array}{c}\text { Peak } \\
\#\end{array}$} & \multirow{2}{*}{$\begin{array}{c}\text { RetTime } \\
\text { [min] }\end{array}$} & \multirow{2}{*}{ Type } & \multirow{2}{*}{$\begin{array}{l}\text { Width } \\
{[\mathrm{min}]}\end{array}$} & \multicolumn{2}{|c|}{ Area } & Height & \multirow{2}{*}{$\begin{array}{c}\text { Area } \\
\frac{\circ}{0}\end{array}$} \\
\hline & & & & $\mathrm{mAU}$ & ${ }^{*} \mathrm{~s}$ & {$[\mathrm{mAU}$} & \\
\hline & & & & & & & \\
\hline 1 & 22 . & MM & & 876 & 92 & 306.19 & 21 \\
\hline 2 & 36.424 & MM & 0760 & 51 & 92230 & 8.03811 & 5.5879 \\
\hline
\end{tabular}

Totals :

$9286.59222 \quad 314.23516$ 


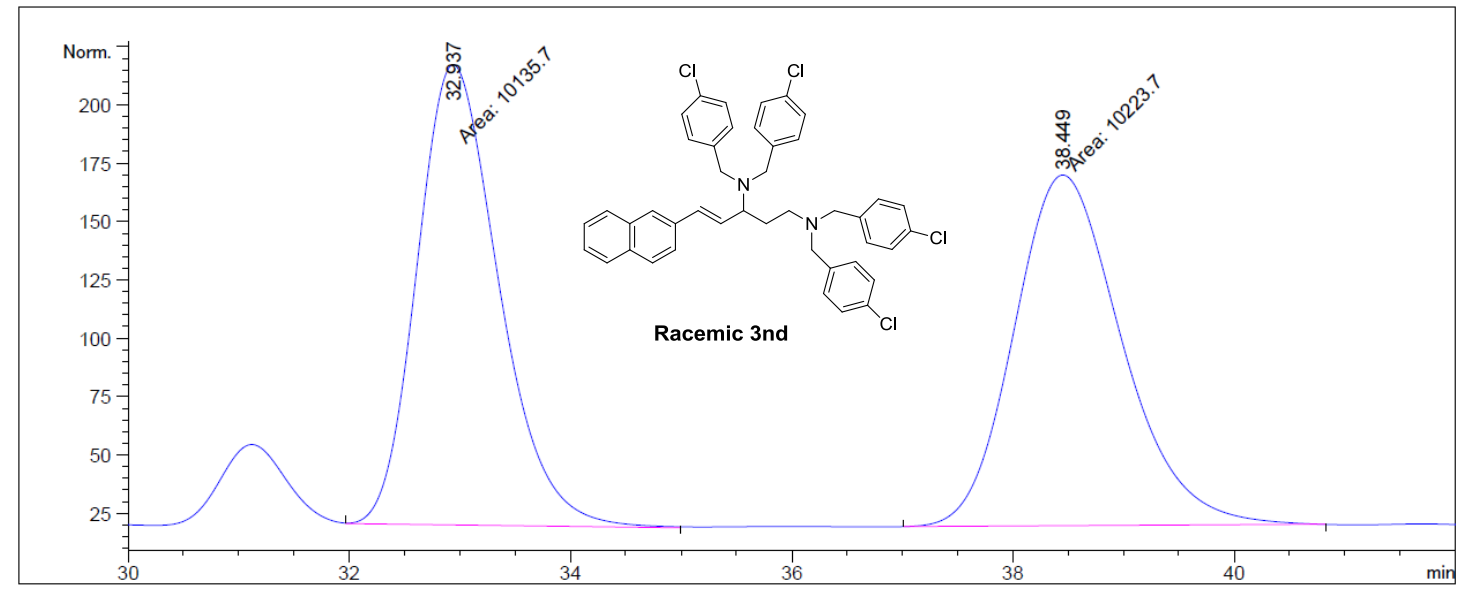

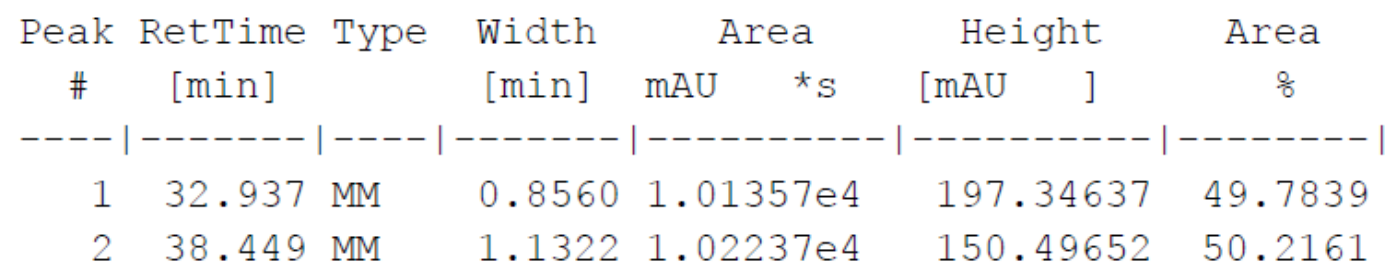

Totals : $\quad 2.03594 \mathrm{e} 4 \quad 347.84290$

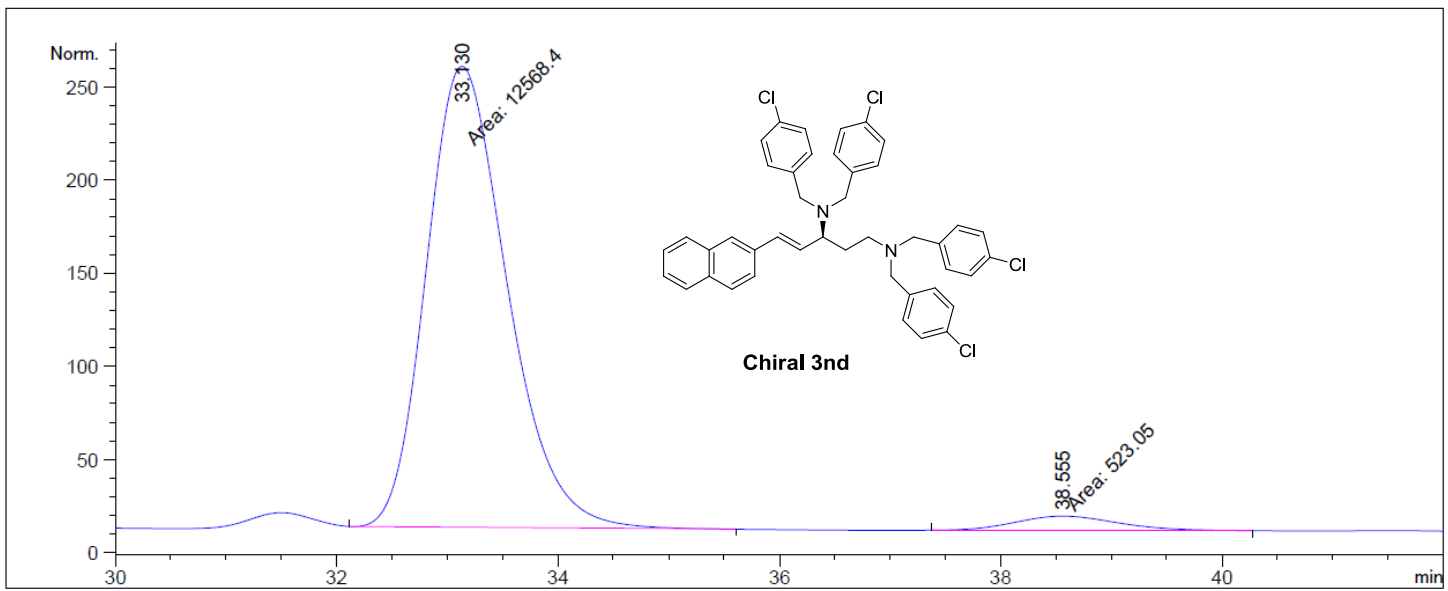

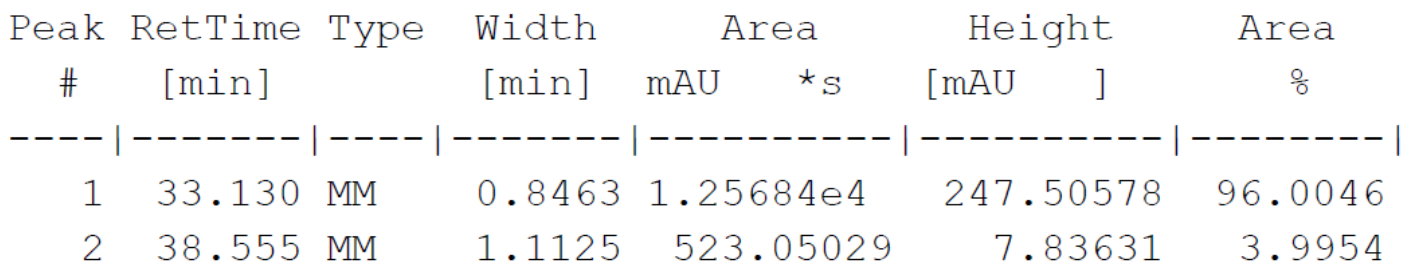

Totals :

1.30914 e4 255.34209 

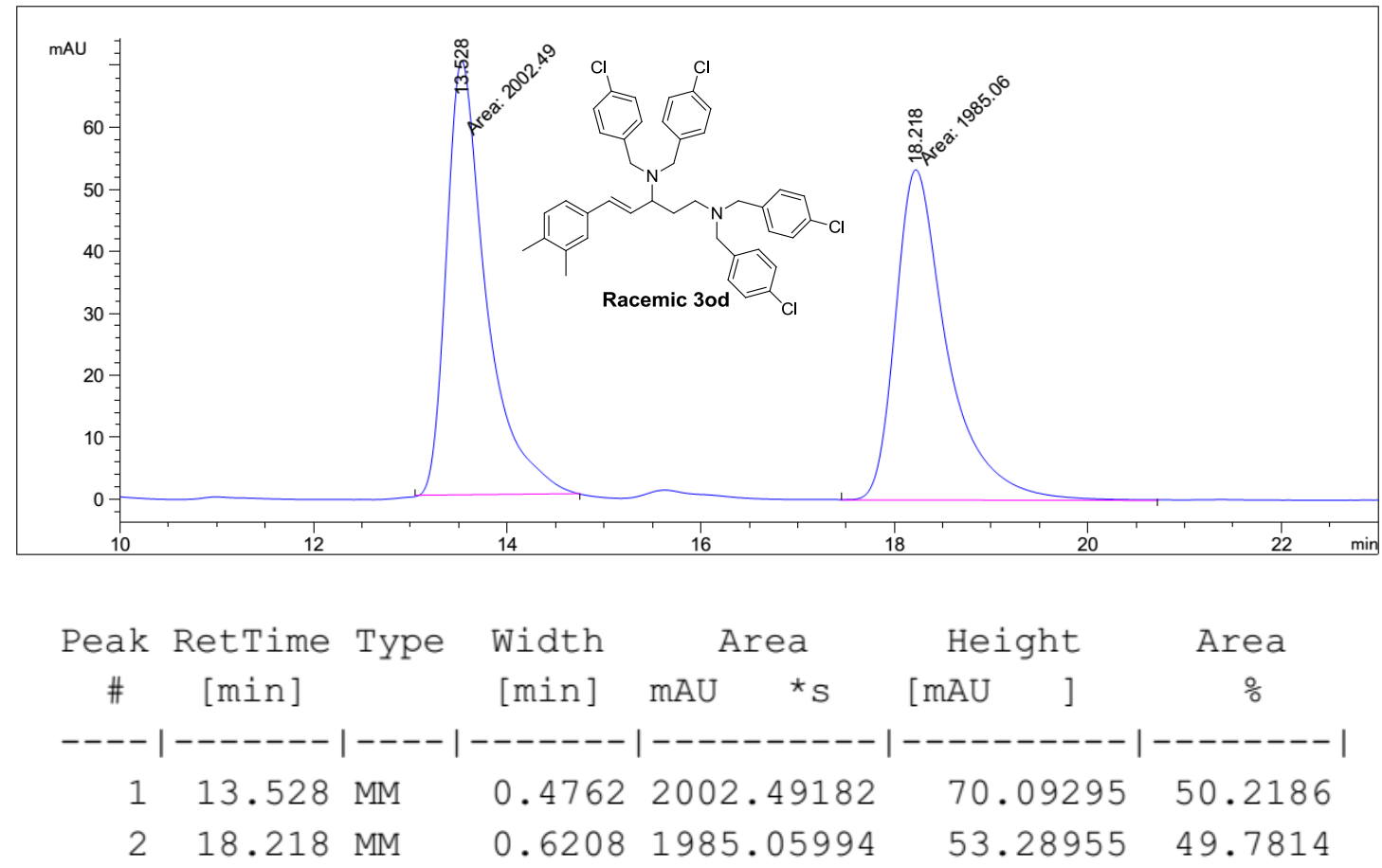

Totals : $\quad 3987.55176 \quad 123.38250$
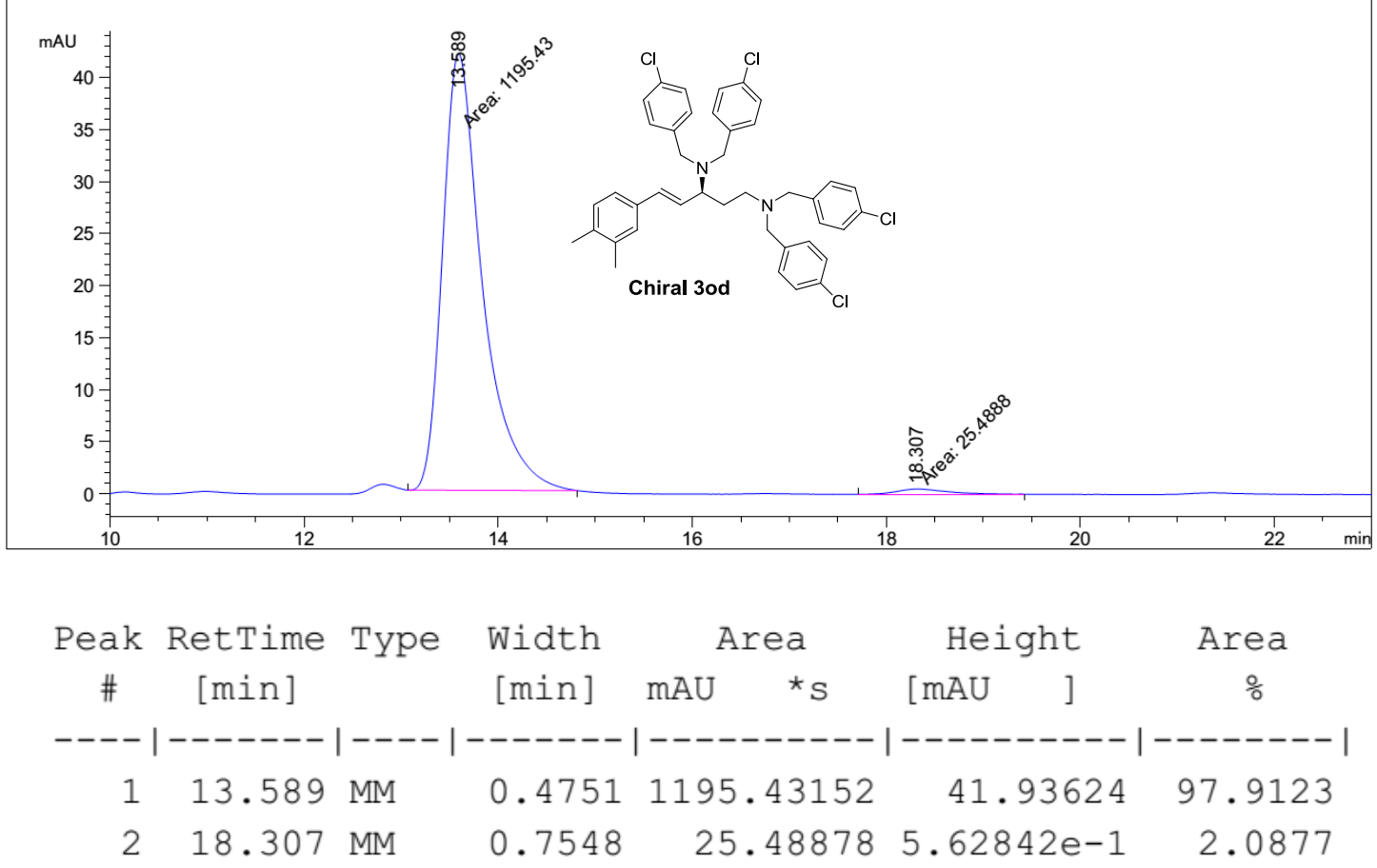

Totals :

$1220.92030 \quad 42.49909$ 

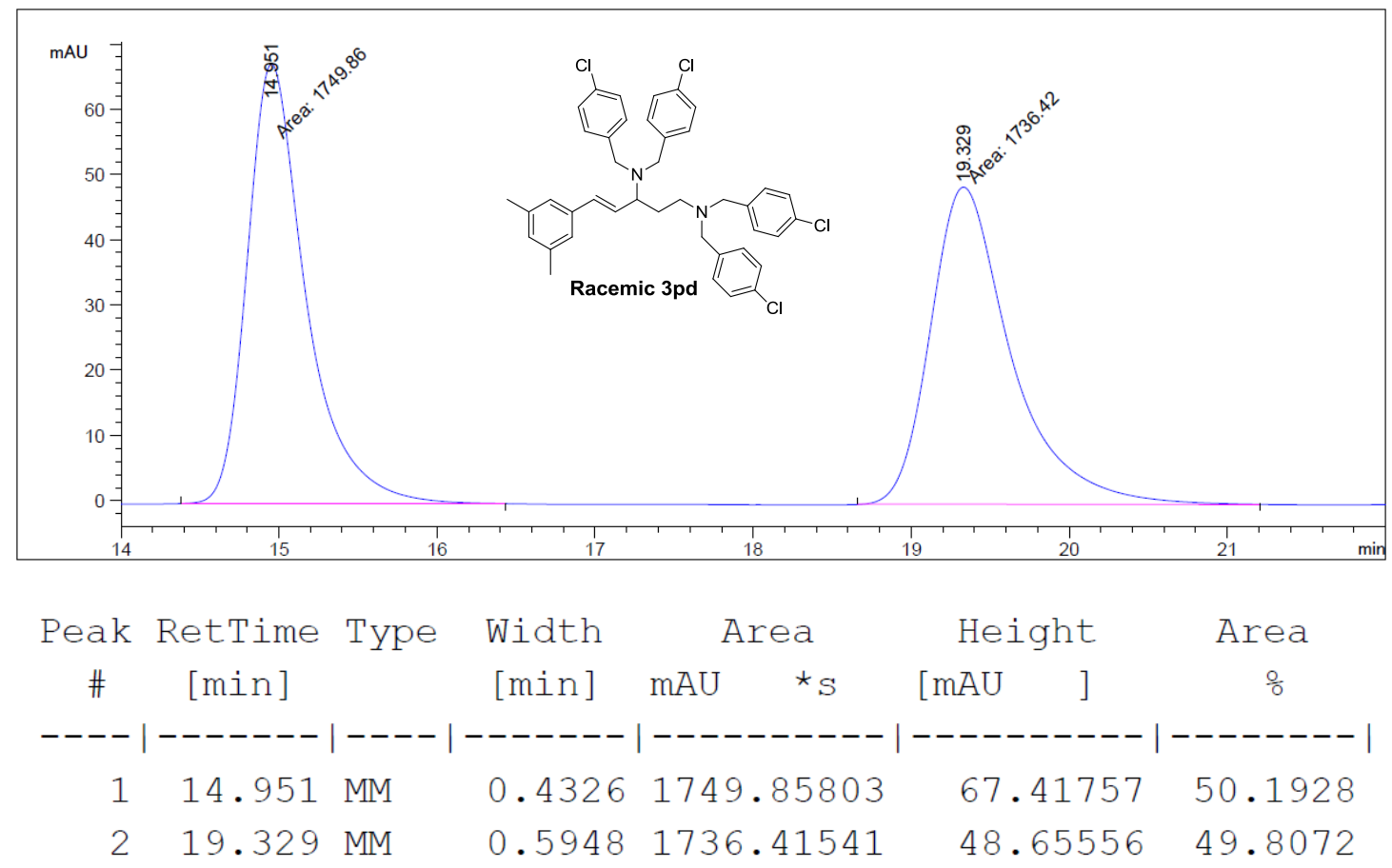

Totals :

$3486.27344 \quad 116.07314$

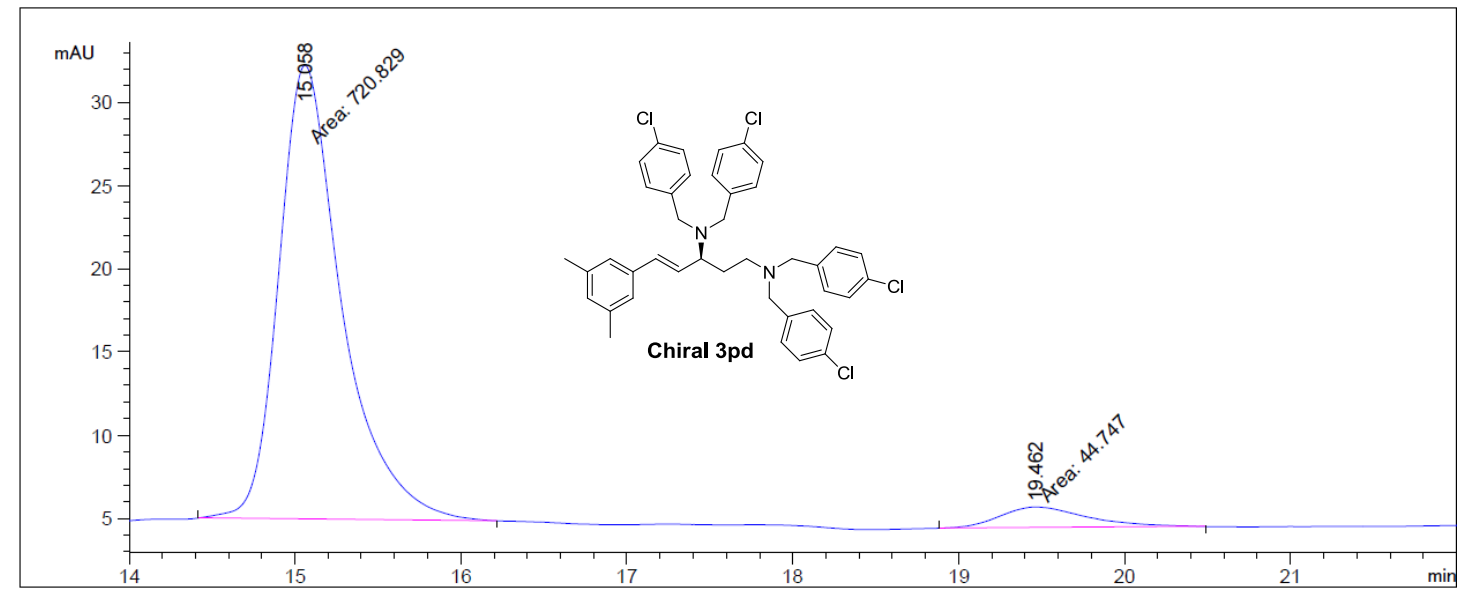

\begin{tabular}{|c|c|c|c|c|c|c|c|}
\hline \multirow{2}{*}{$\begin{array}{c}\text { Peak } \\
\#\end{array}$} & \multirow{2}{*}{$\begin{array}{c}\text { RetTime } \\
\text { [min] }\end{array}$} & \multirow[t]{2}{*}{ Type } & \multirow{2}{*}{$\begin{array}{l}\text { Width } \\
{[\min ]}\end{array}$} & \multicolumn{2}{|c|}{ Area } & Height & \multirow{2}{*}{$\begin{array}{c}\text { Area } \\
\quad \frac{\circ}{0}\end{array}$} \\
\hline & & & & $\mathrm{mAU}$ & $\star_{\mathrm{S}}$ & {$[\mathrm{mAU}$} & \\
\hline & & & & & -1 & -- & \\
\hline 1 & & $\mathrm{MM}$ & & 72 & 867 & 80 & 51 \\
\hline 2 & 19 & MM & 51 & & 74700 & 1.23258 & 8449 \\
\hline
\end{tabular}

Totals :

$765.57567 \quad 28.48038$ 

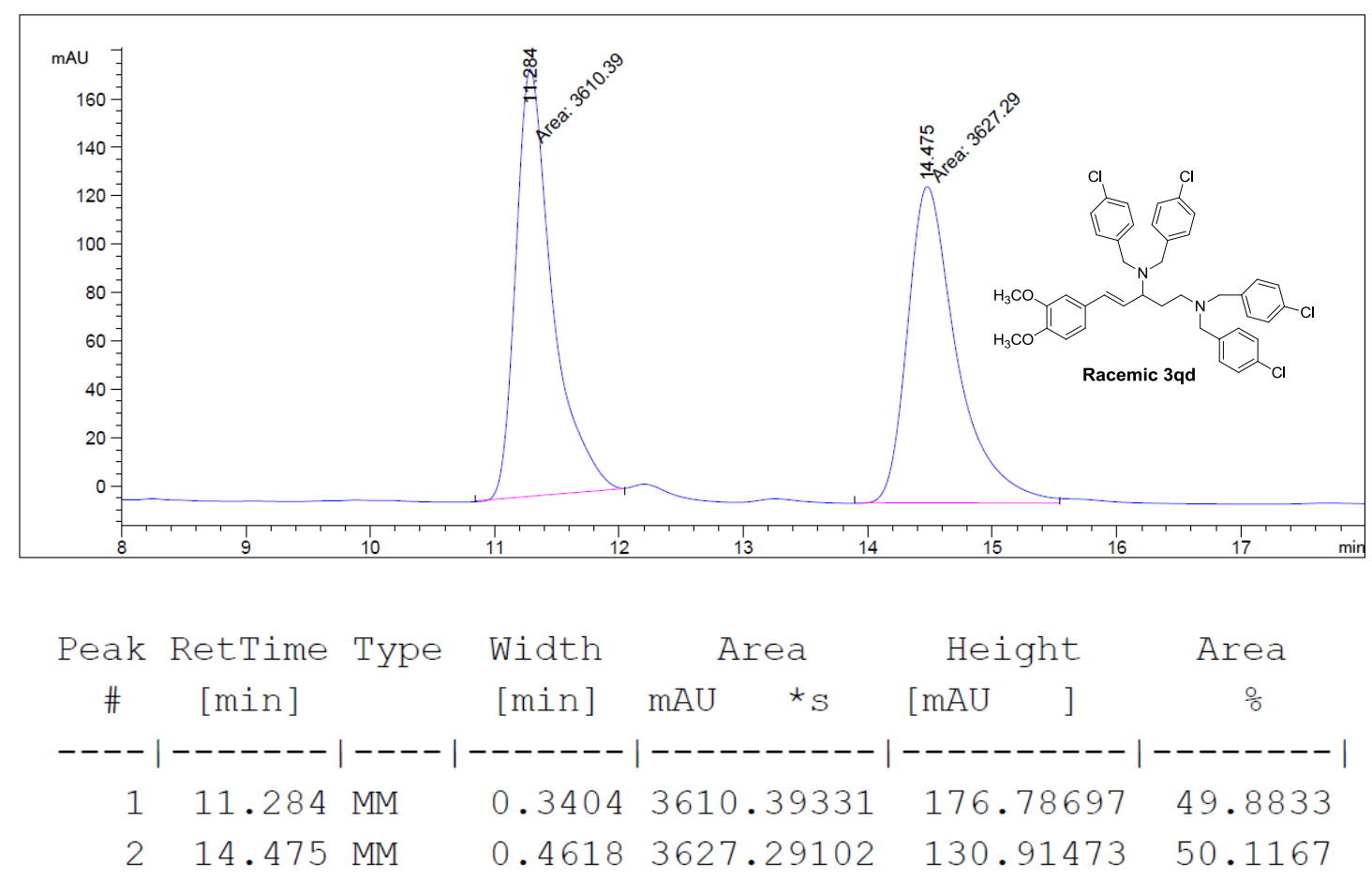

Totals :

$7237.68433 \quad 307.70171$

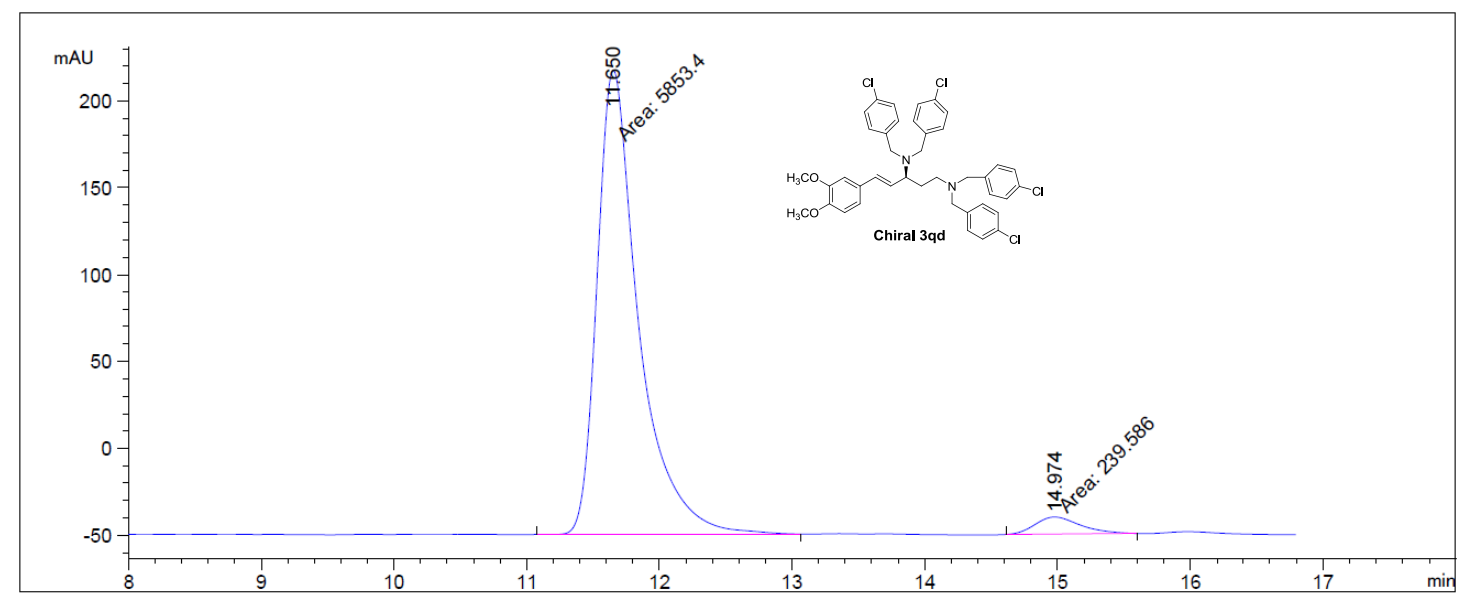

Peak RetTime Type Width Area Height Area

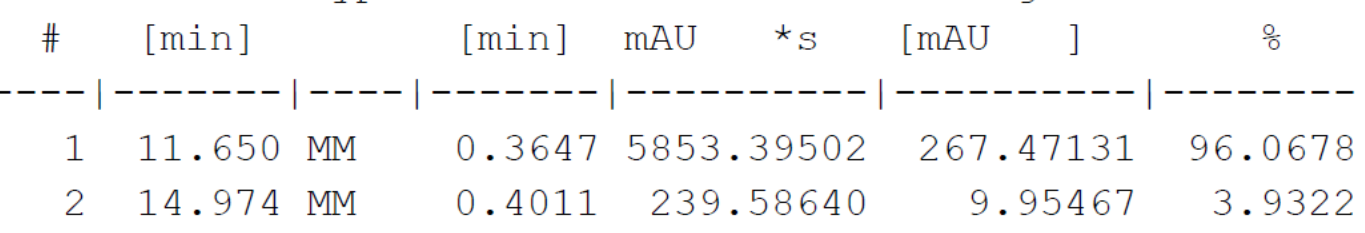

Totals :

$6092.98141 \quad 277.42598$ 

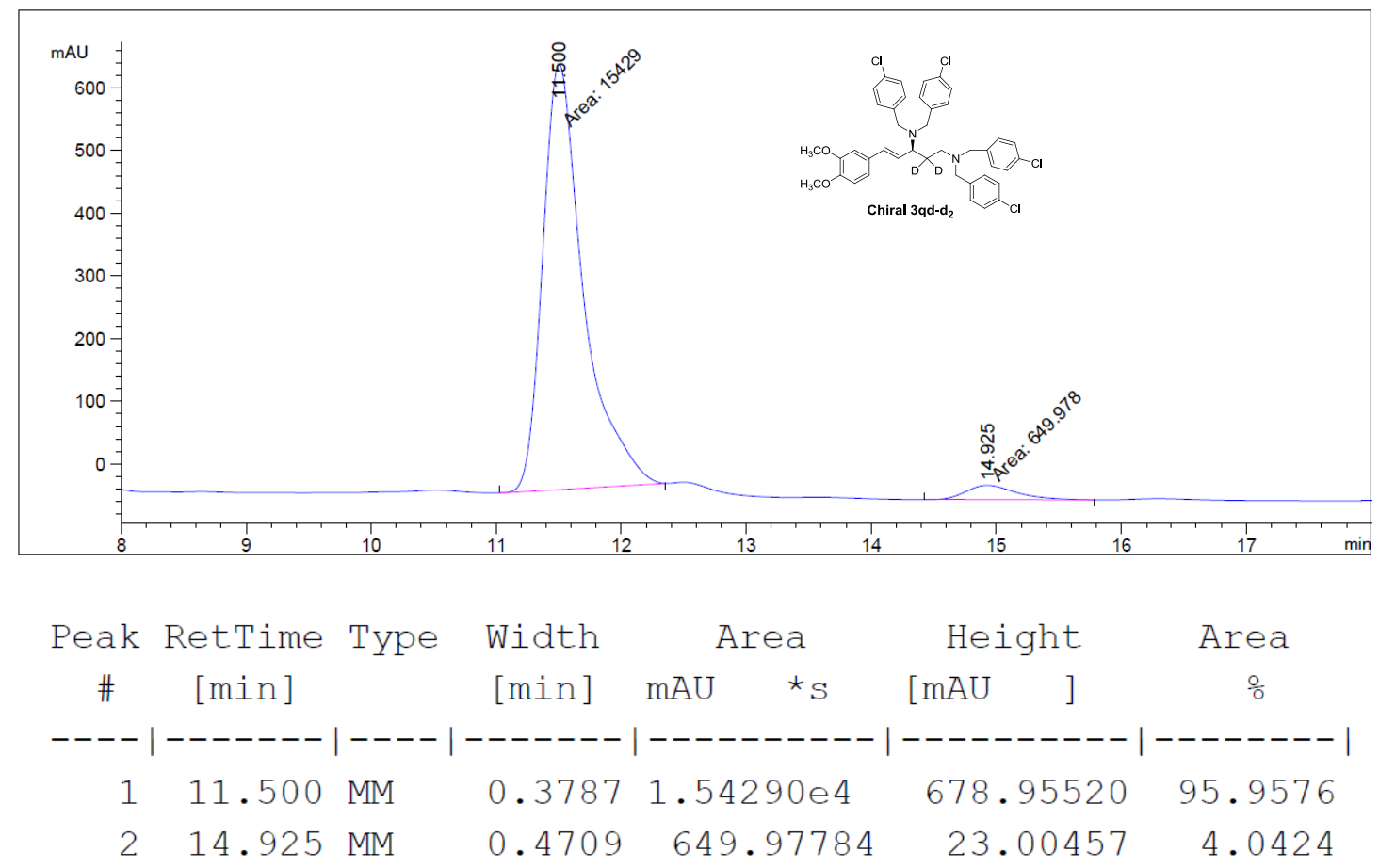

Totals :

$1.60790 e 4 \quad 701.95977$
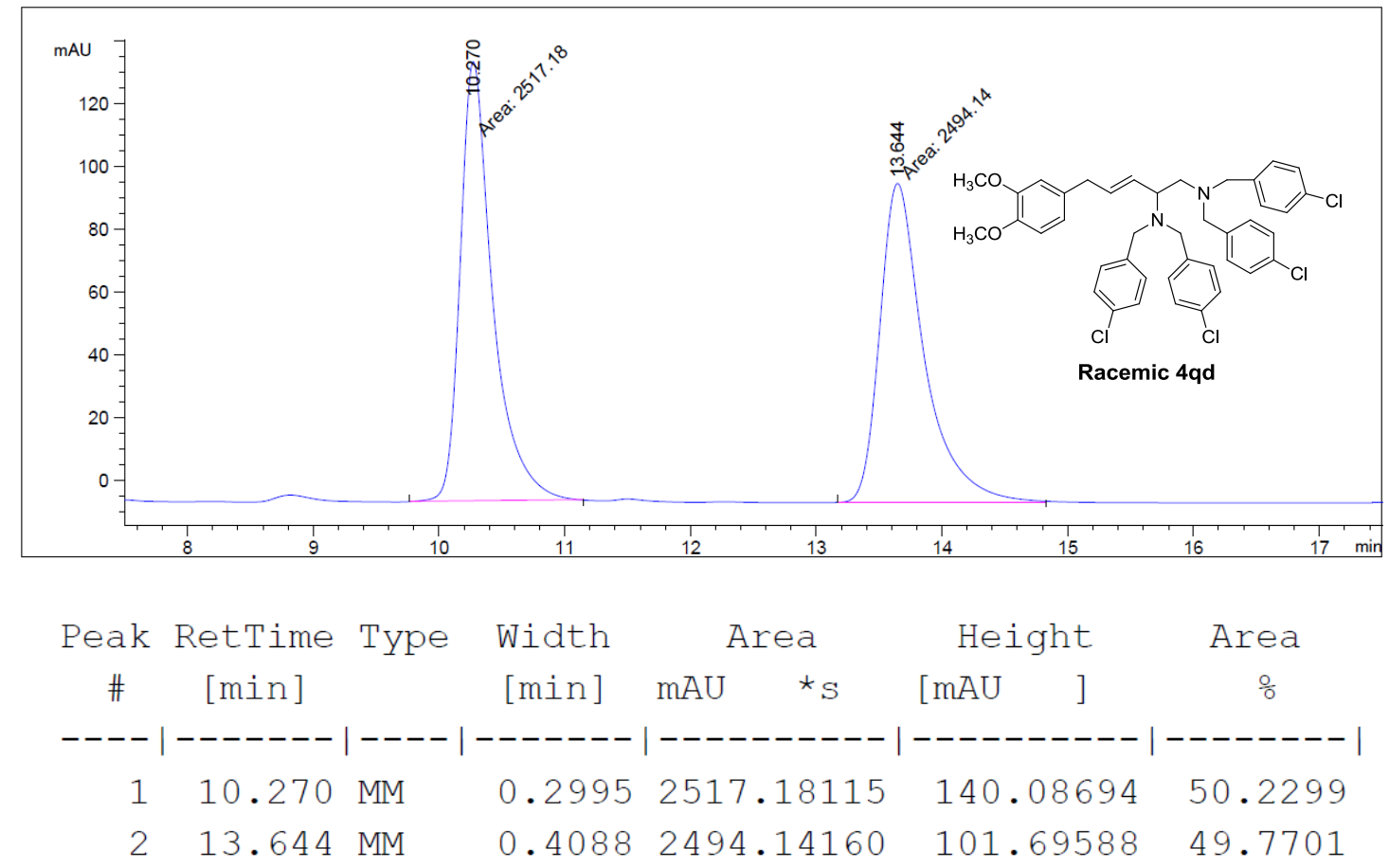

Totals :

$5011.32275 \quad 241.78283$ 

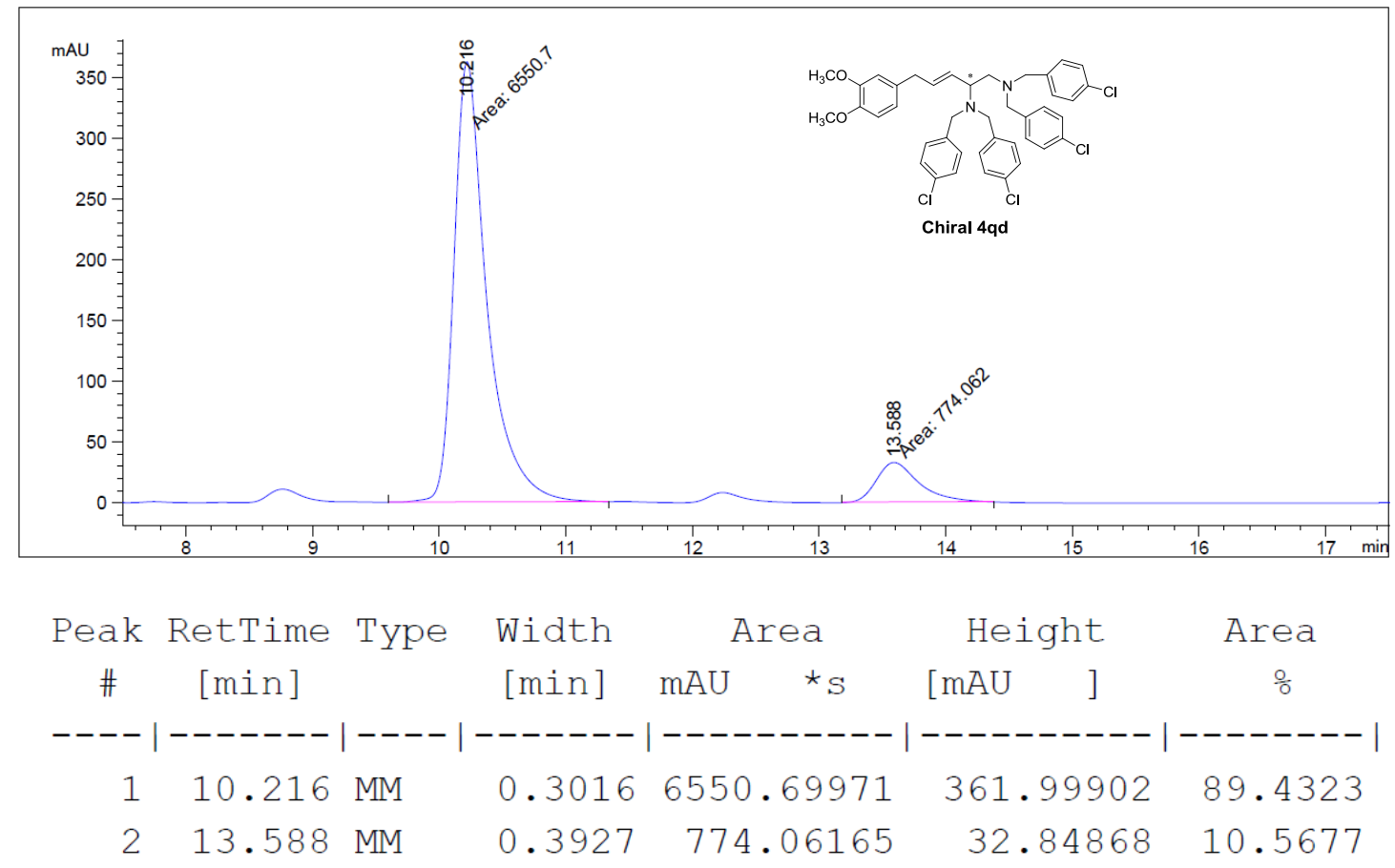

Totals :

$7324.76135 \quad 394.84771$
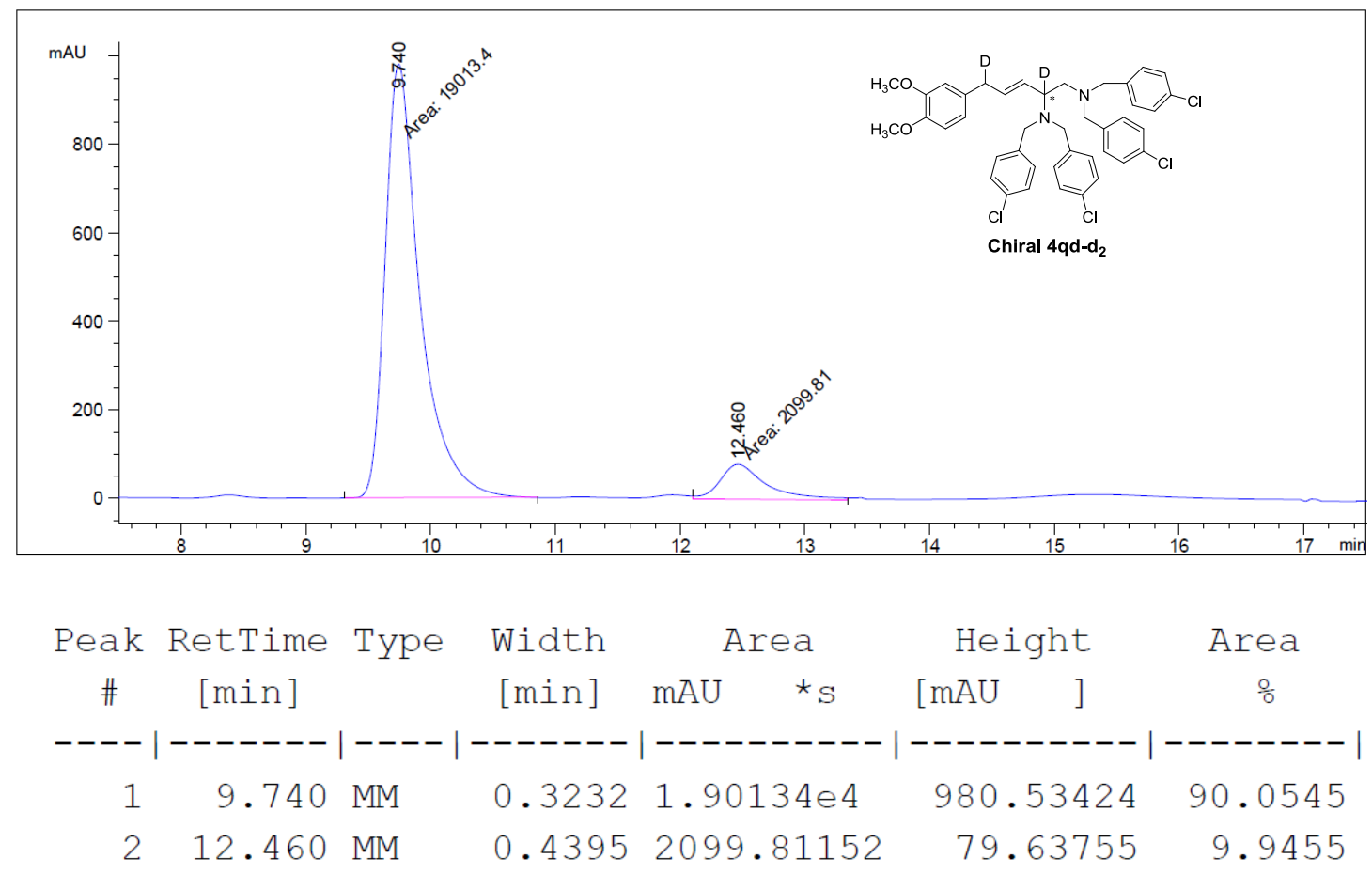

Totals :

$2.11132 e 4 \quad 1060.17179$ 

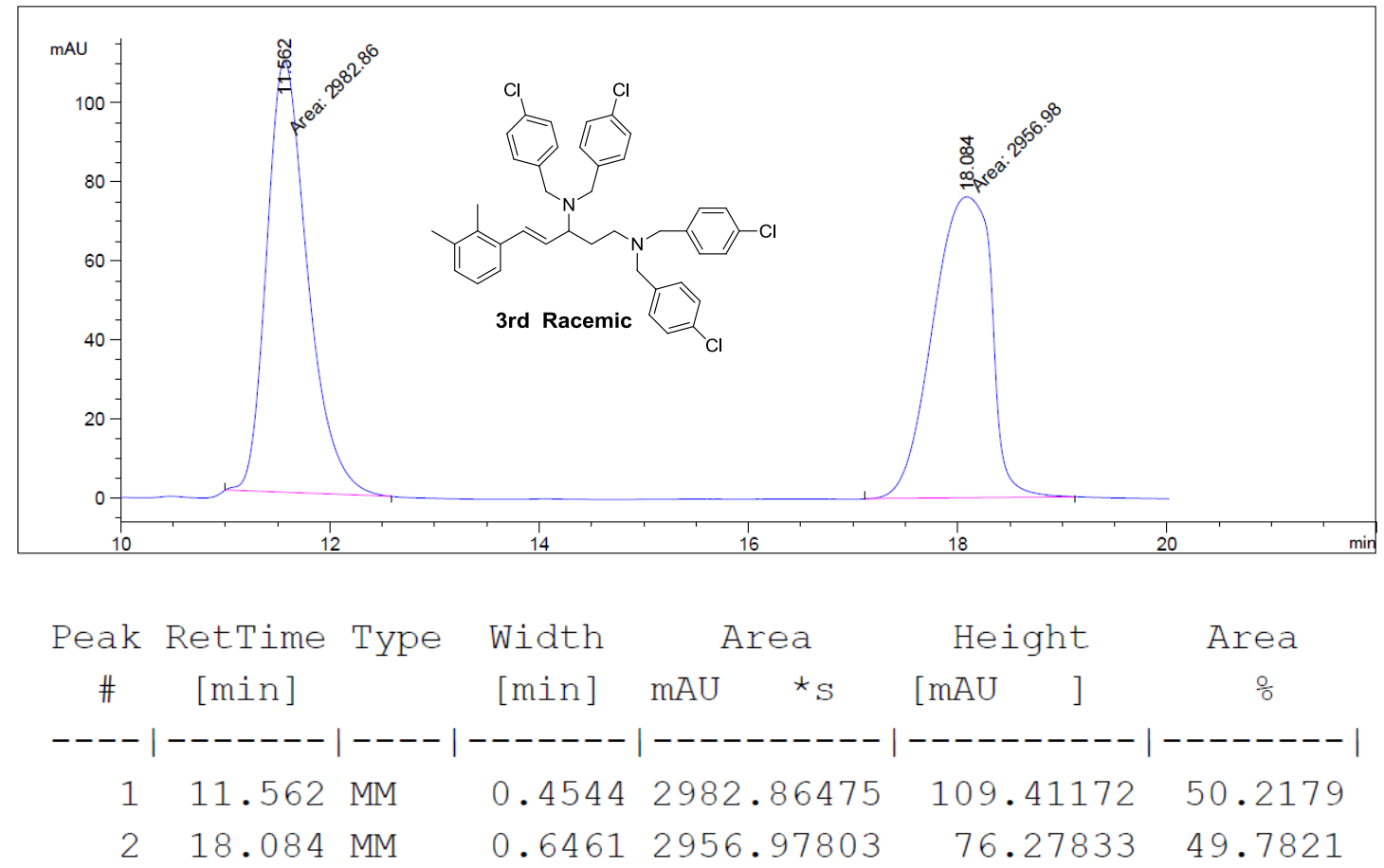

Totals :

$5939.84277 \quad 185.69005$

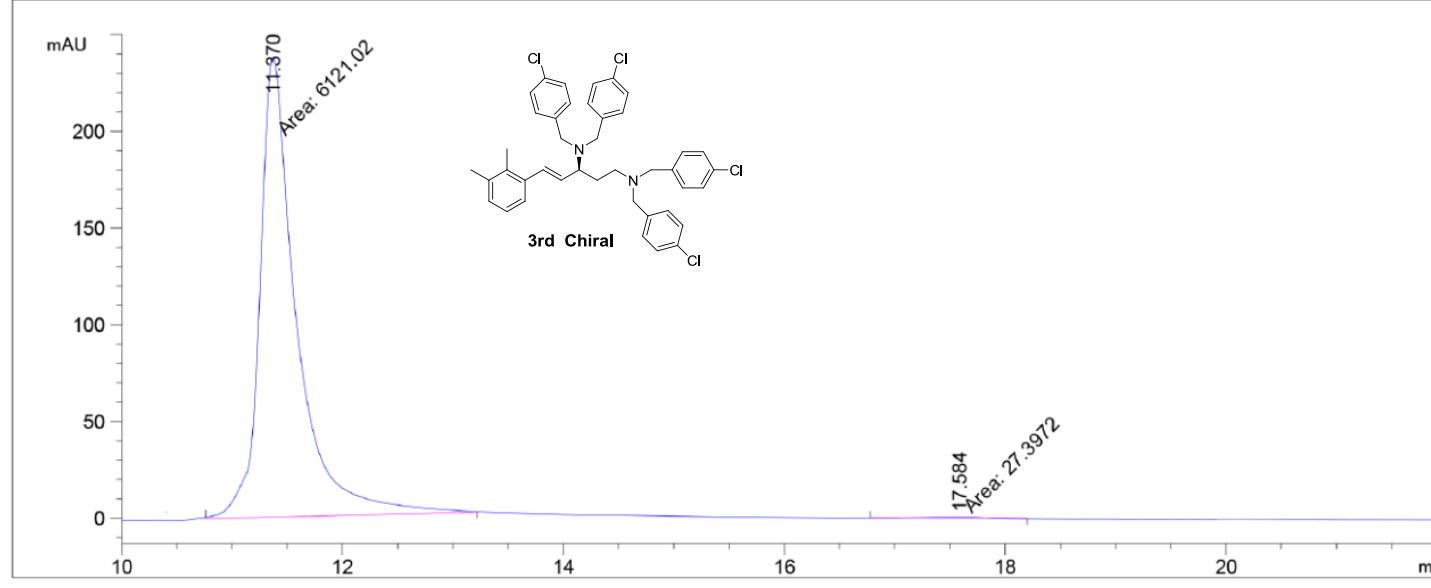

\begin{tabular}{|c|c|c|c|c|c|c|c|}
\hline \multirow{2}{*}{$\begin{array}{c}\text { Peak } \\
\#\end{array}$} & \multirow{2}{*}{$\begin{array}{c}\text { RetTime } \\
\text { [min] }\end{array}$} & \multirow{2}{*}{ Type } & \multirow{2}{*}{$\begin{array}{l}\text { Width } \\
\text { [min] }\end{array}$} & \multicolumn{2}{|c|}{ Area } & Height & \multirow{2}{*}{$\begin{array}{c}\text { Area } \\
\frac{\circ}{0}\end{array}$} \\
\hline & & & & mAU & $\star_{S}$ & {$[\mathrm{mAU}$} & \\
\hline & & & & & & & \\
\hline 1 & & & & 61 & & & \\
\hline 2 & & $M$ & & & 721 & 7.4 & 4456 \\
\hline
\end{tabular}

Totals :

$6148.41918 \quad 239.03048$ 

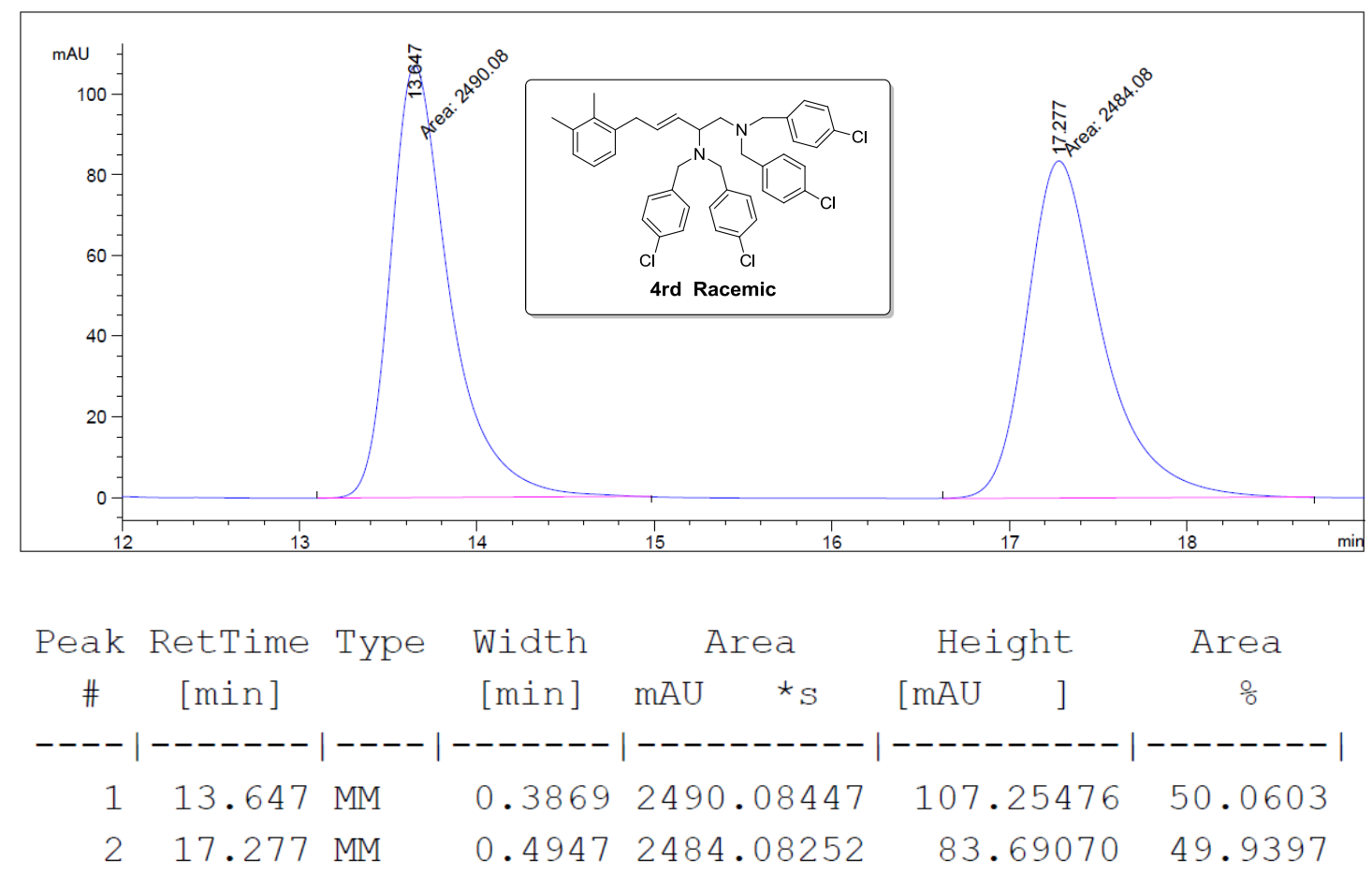

Totals :

$4974.16699 \quad 190.94547$

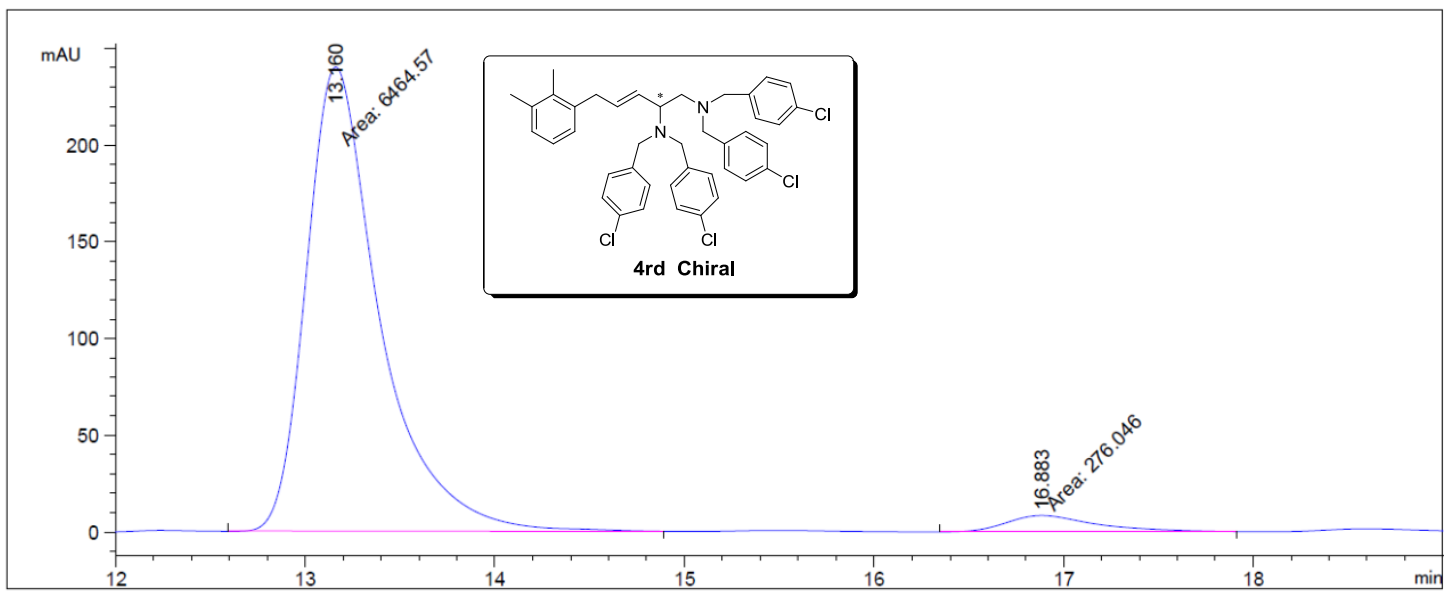

Peak RetTime Type Width Area Height Area

$\begin{array}{ccccccc}\# & {[\mathrm{~min}]} & {[\mathrm{min}]} & \mathrm{mAU} & { }_{\mathrm{S}} & {[\mathrm{mAU}]} & \frac{\circ}{0} \\ -1 & 13.160 & \mathrm{MM} & 0.4493 & 6464.56934 & 239.78325 & 95.9047 \\ 2 & 16.883 & \mathrm{MM} & 0.5458 & 276.04550 & 8.42890 & 4.0953\end{array}$

Totals :

$6740.61484 \quad 248.21215$ 


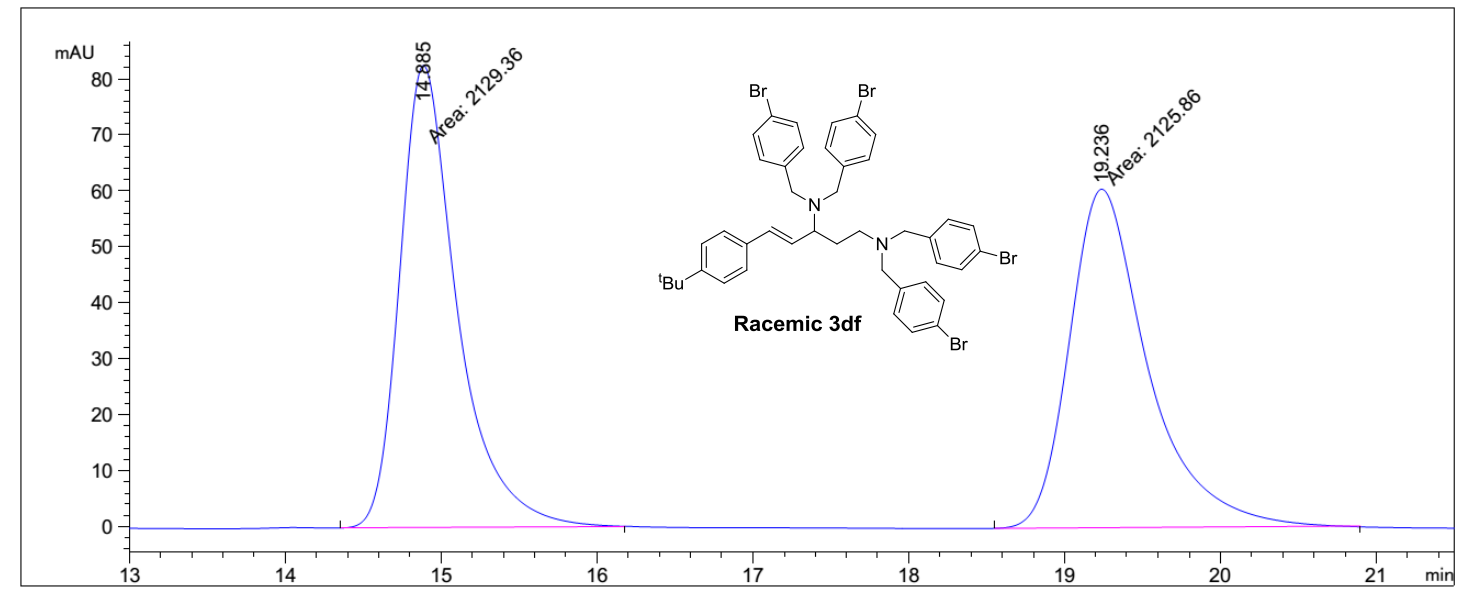

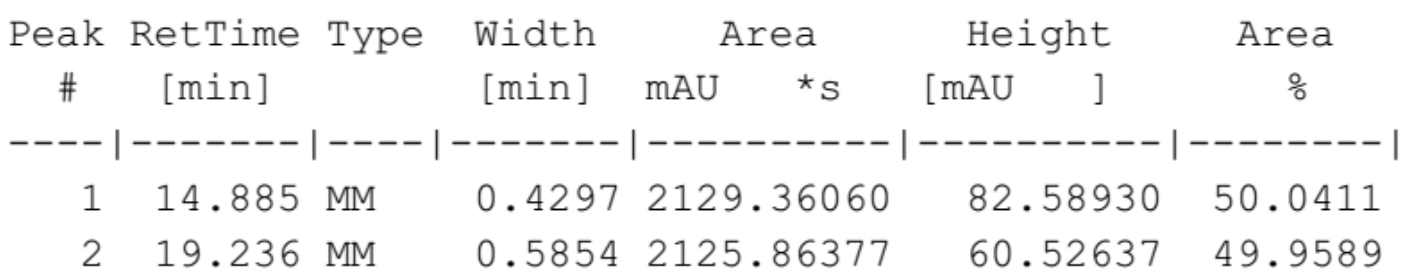

Totals :

$4255.22437 \quad 143.11567$
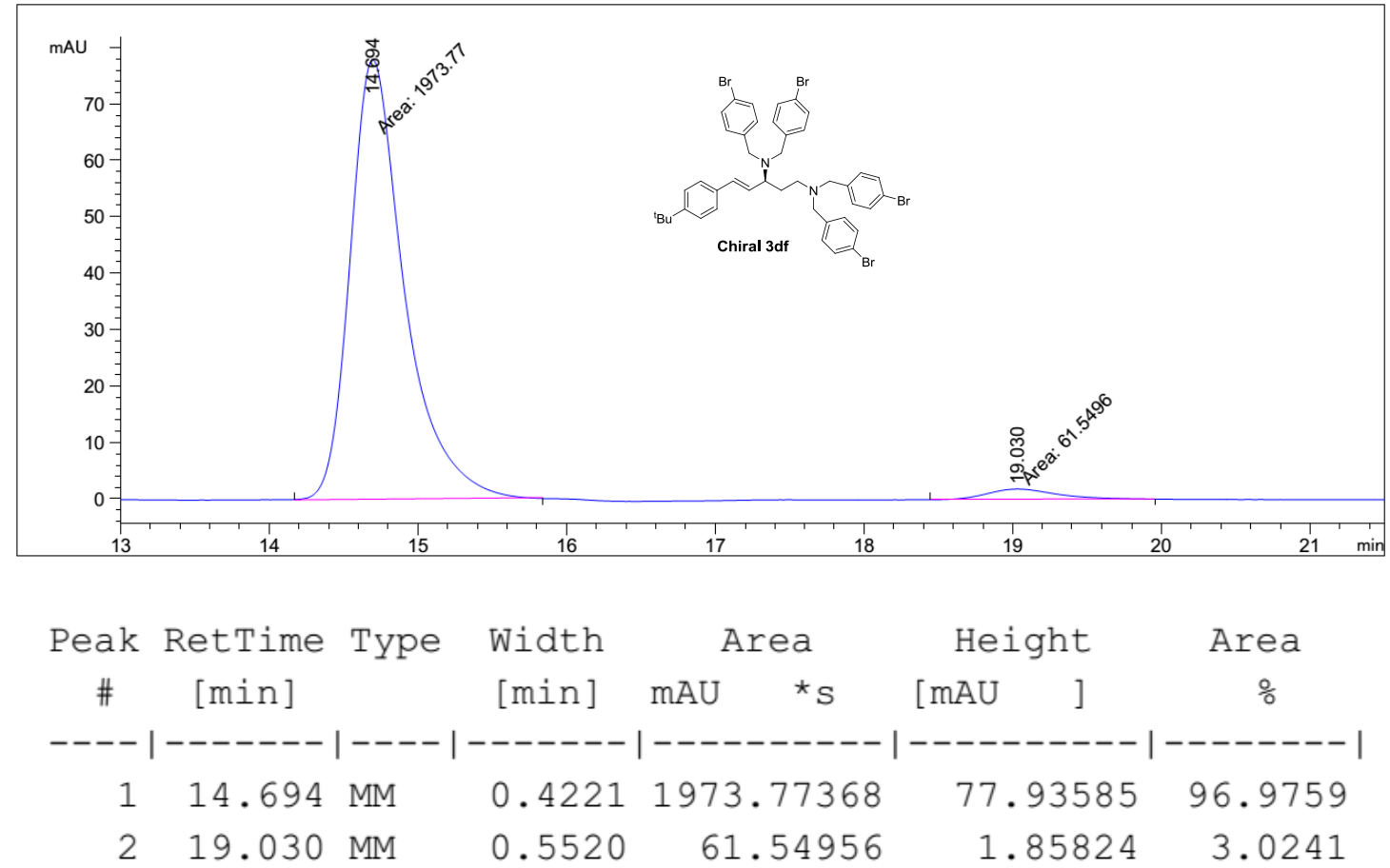

Totals :

$2035.32325 \quad 79.79410$ 


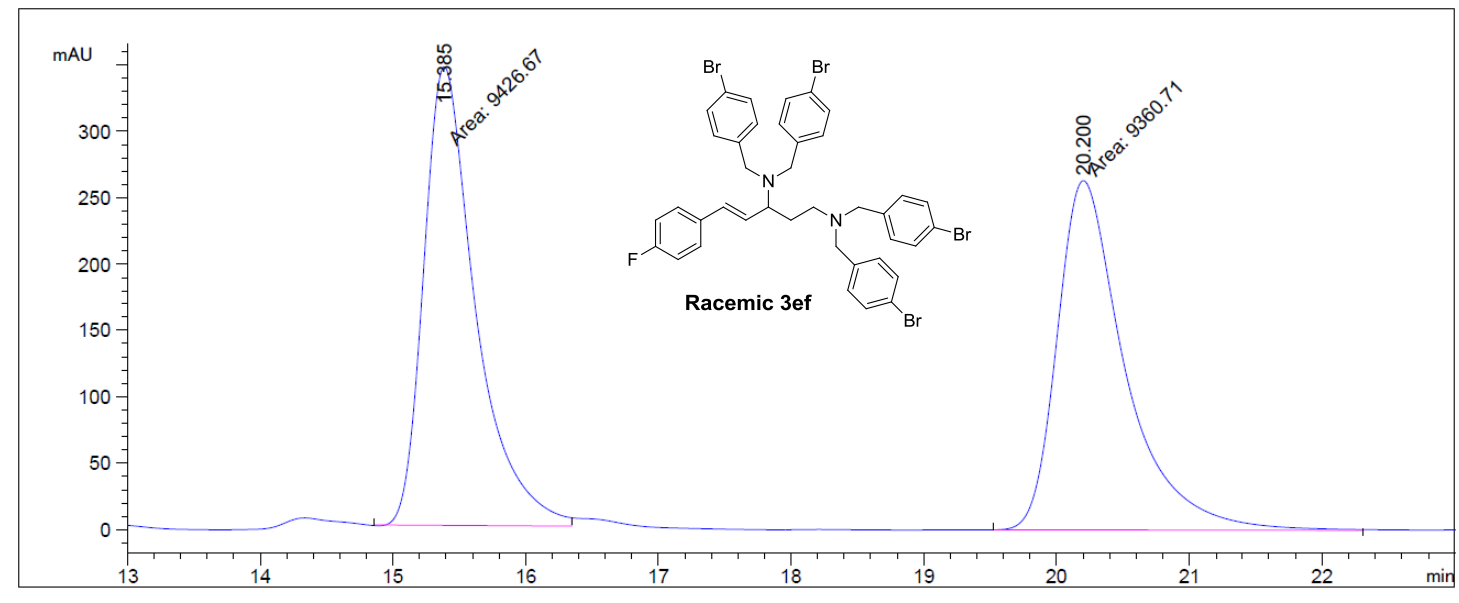

Peak RetTime Type Width Area Height Area

$\begin{array}{ccccccc}\# & {[\mathrm{~min}]} & {[\mathrm{min}]} & \mathrm{mAU} & * \mathrm{~S} & {[\mathrm{mAU}]} & \frac{\circ}{8} \\ ----\mid & -------\mid & ----\mid & -------\mid & ---------\mid & --------1 & -------1 \\ 1 & 15.385 & \mathrm{MM} & 0.4547 & 9426.66895 & 345.51541 & 50.1755 \\ 2 & 20.200 & \mathrm{MM} & 0.5936 & 9360.71289 & 262.81531 & 49.8245\end{array}$

Totals : $\quad 1.87874$ e4 608.33072

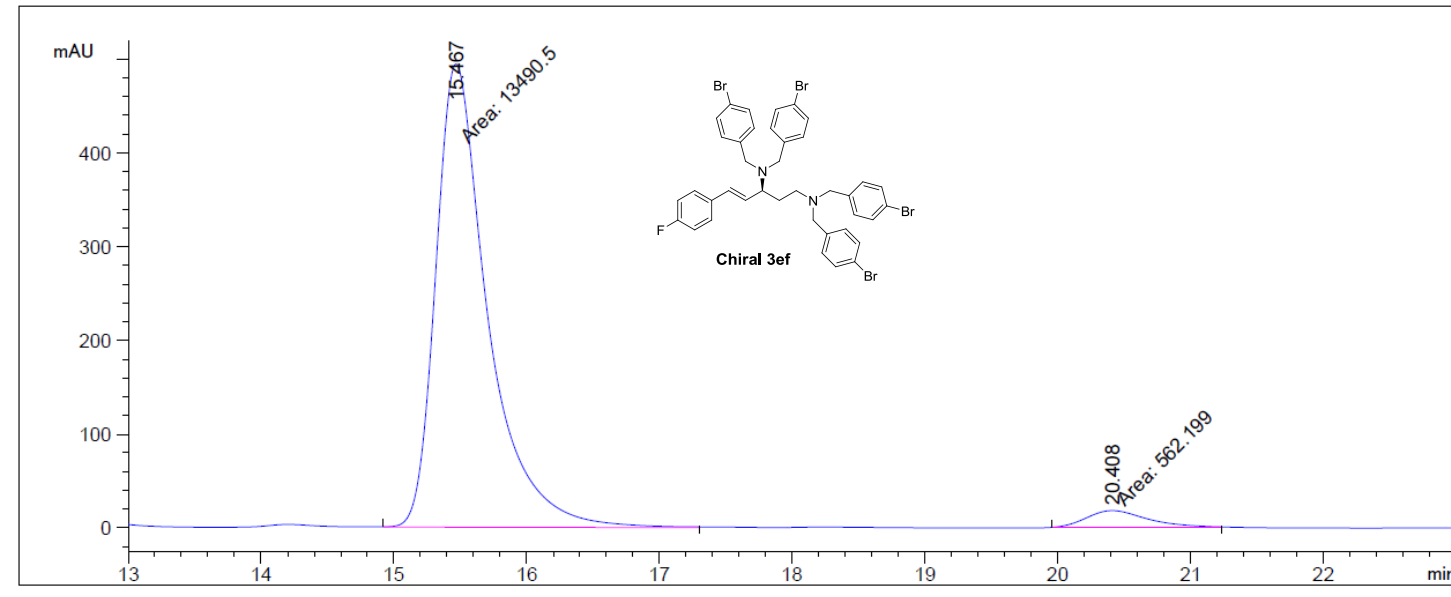

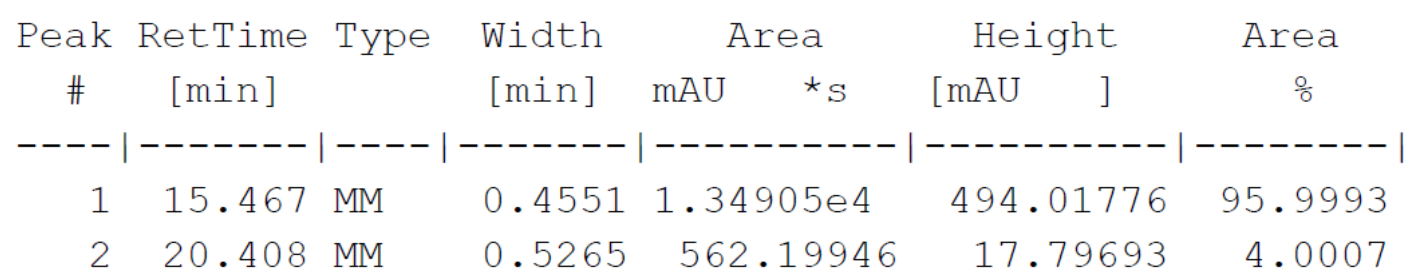

Totals :

$1.40527 e 4 \quad 511.81469$ 


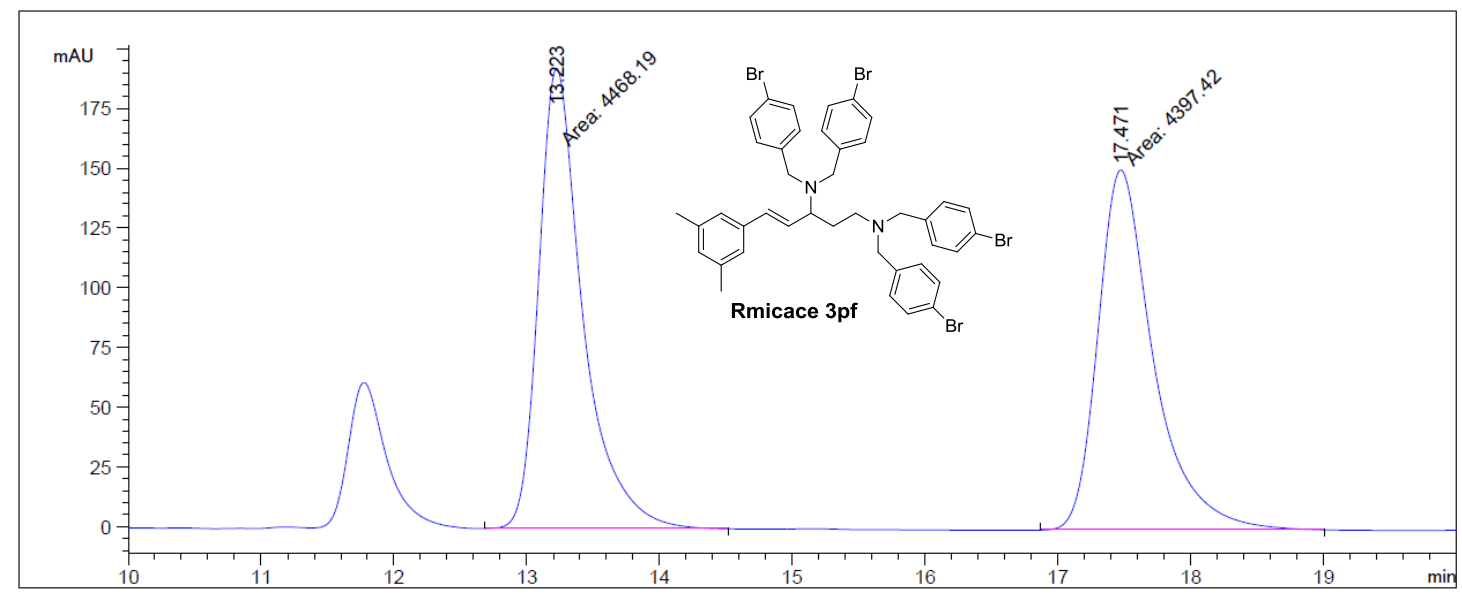

Peak RetTime Type Width Area Height Area $\# \quad[\mathrm{~min}] \quad[\mathrm{min}] \mathrm{mAU}{ }_{\mathrm{S}} \quad[\mathrm{mAU}] \quad$ \% ----|-------|----|-------|----------|----------|-------- |

$\begin{array}{llllllll}1 & 13.223 & \text { MM } & 0.3863 & 4468.18945 & 192.79187 & 50.3991\end{array}$

$\begin{array}{lllllll}2 & 17.471 & \mathrm{MM} & 0.4862 & 4397.42334 & 150.74707 & 49.6009\end{array}$

Totals : $\quad 8865.61279 \quad 343.53894$

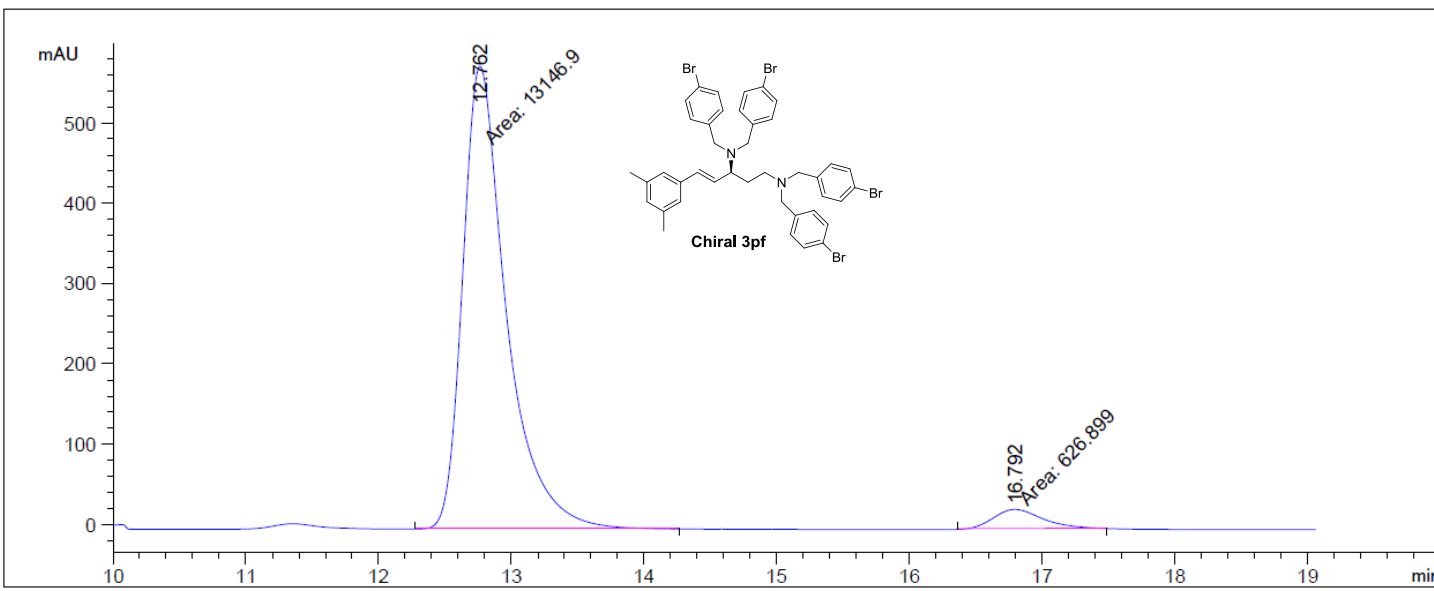

\begin{tabular}{|c|c|c|c|c|c|c|}
\hline \multirow{2}{*}{$\begin{array}{c}\text { Peak } \\
\#\end{array}$} & \multirow{2}{*}{$\begin{array}{c}\text { RetTime } \\
\text { [min] }\end{array}$} & \multirow{2}{*}{ Type } & \multirow{2}{*}{$\begin{array}{l}\text { Width } \\
\text { [min] }\end{array}$} & Area & Height & \multirow{2}{*}{$\begin{array}{c}\text { Area } \\
\frac{\circ}{0}\end{array}$} \\
\hline & & & & $\mathrm{mAU}$ & {$[\mathrm{mAU}$} & \\
\hline & & & & & & --- \\
\hline 1 & & 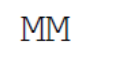 & & 1 & $57 !$ & 86 \\
\hline 2 & 16 & 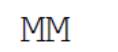 & & 626.89917 & 24.2 & 5514 \\
\hline
\end{tabular}

Totals :

$1.37738 e 4 \quad 599.64546$ 

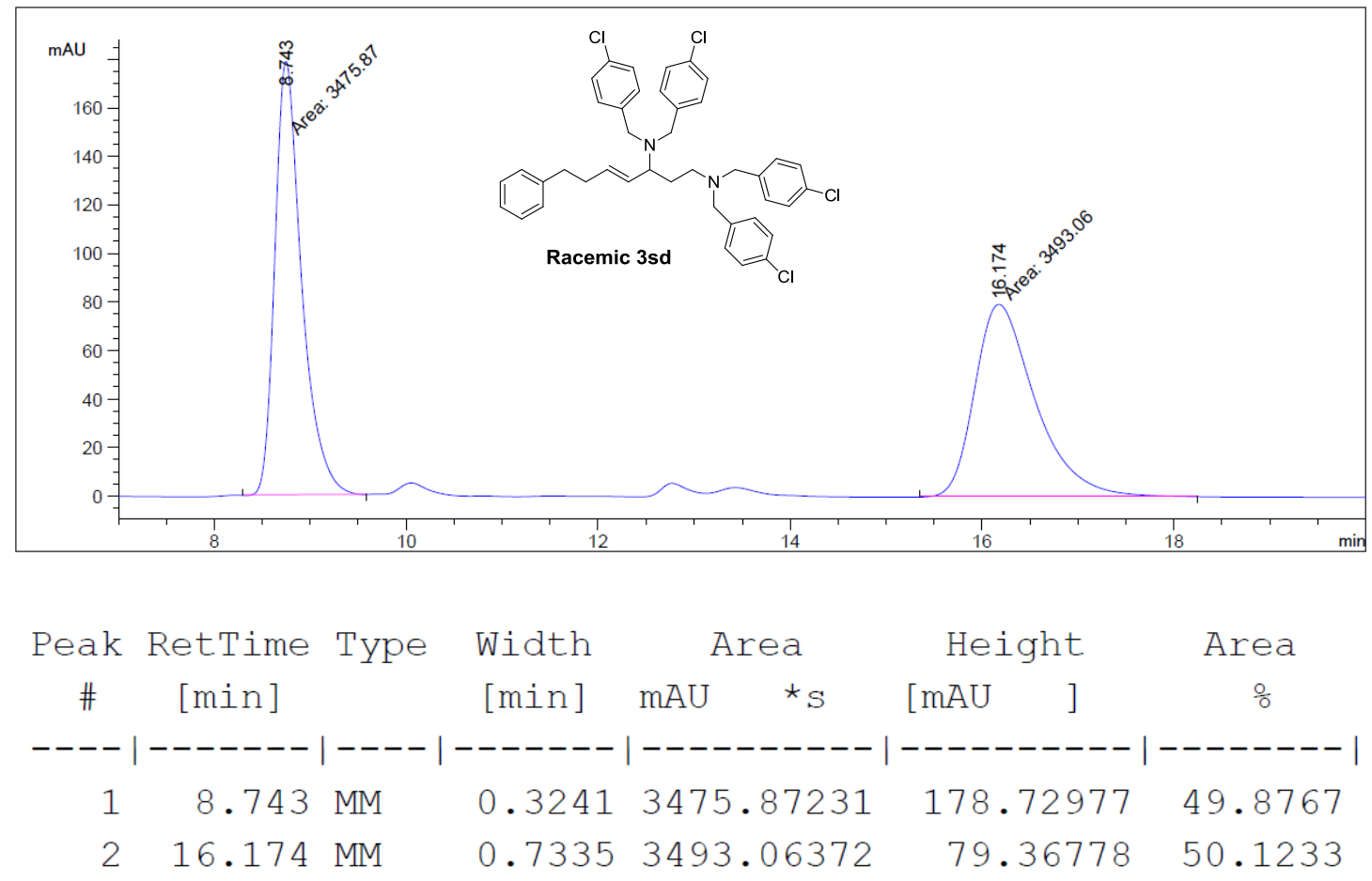

Totals :

$6968.93604 \quad 258.09755$

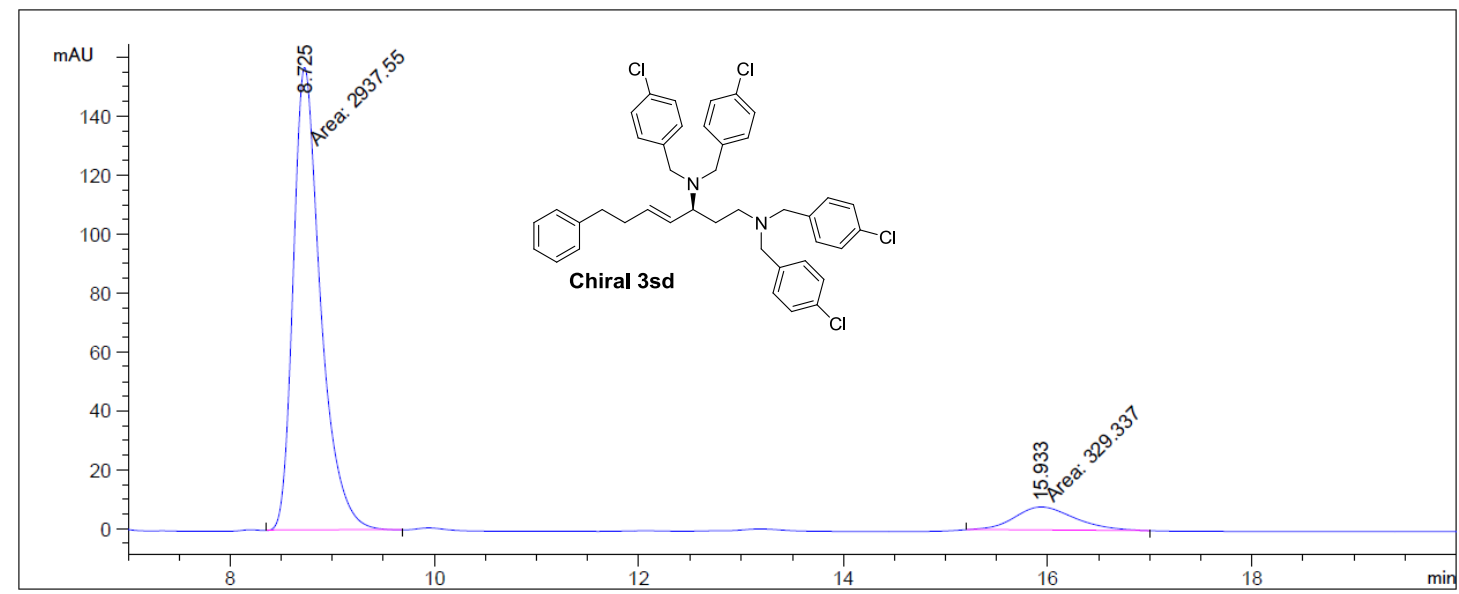

\begin{tabular}{|c|c|c|c|c|c|c|c|}
\hline \multirow{2}{*}{$\begin{array}{c}\text { Peak } \\
\text { \# }\end{array}$} & \multirow{2}{*}{$\begin{array}{c}\text { RetTime } \\
\text { [min] }\end{array}$} & \multirow[t]{2}{*}{ Type } & \multirow{2}{*}{$\begin{array}{l}\text { Width } \\
\text { [min] }\end{array}$} & \multicolumn{2}{|c|}{ Area } & Height & \multirow{2}{*}{$\begin{array}{c}\text { Area } \\
\quad \frac{\circ}{0}\end{array}$} \\
\hline & & & & $m A U$ & $\star_{\mathrm{S}}$ & {$[\mathrm{mAU}$} & \\
\hline & & & & & & & \\
\hline 1 & & & & 293 & 178 & $15^{\circ}$ & \\
\hline 2 & 33 & 1 & 41 & 32 & 33658 & 7.90796 & 081 \\
\hline
\end{tabular}

Totals :

$3266.88443 \quad 165.09263$ 


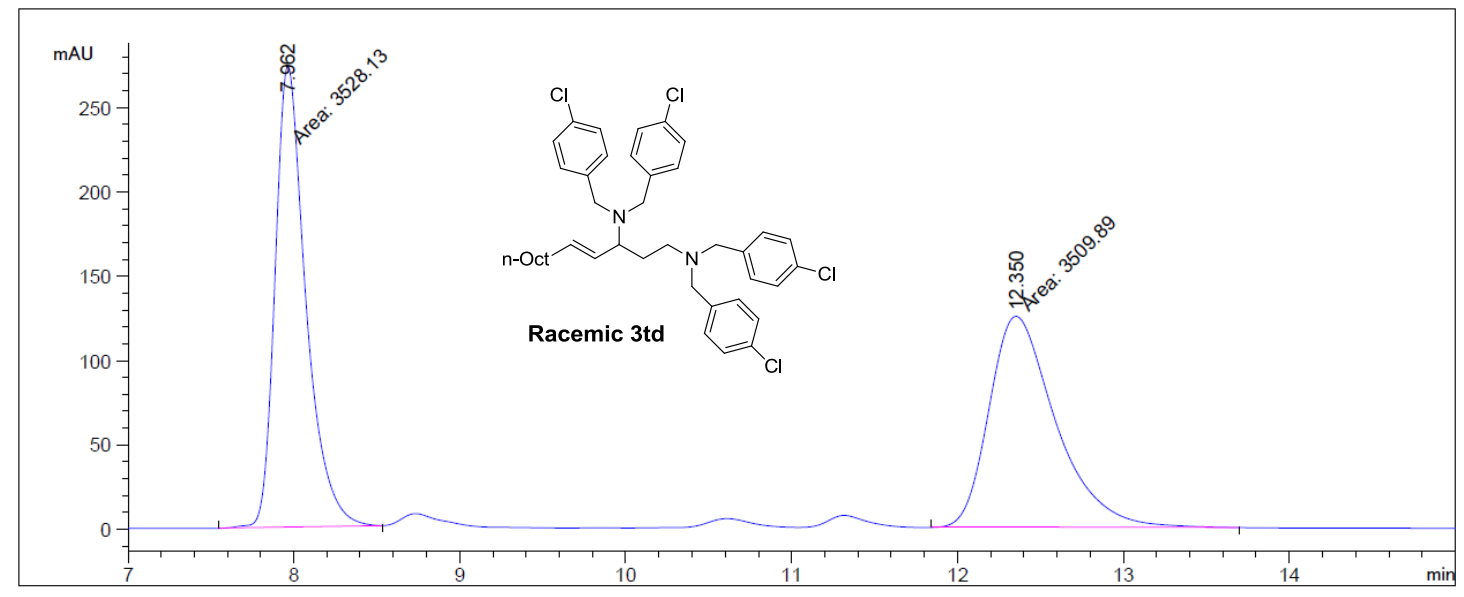

Peak RetTime Type Width Area Height Area

$\begin{array}{ccccccc}\# & {[\mathrm{~min}]} & {[\mathrm{min}]} & \mathrm{mAU} & { }_{\mathrm{S}} & {[\mathrm{mAU}]} & \text { о } \\ -1 & 7.962 & \mathrm{MM} & 0.2150 & 3528.12939 & 273.50446 & 50.1296 \\ 2 & 12.350 & \mathrm{MM} & 0.4672 & 3509.88525 & 125.19800 & 49.8704\end{array}$

Totals :

$7038.01465 \quad 398.70245$

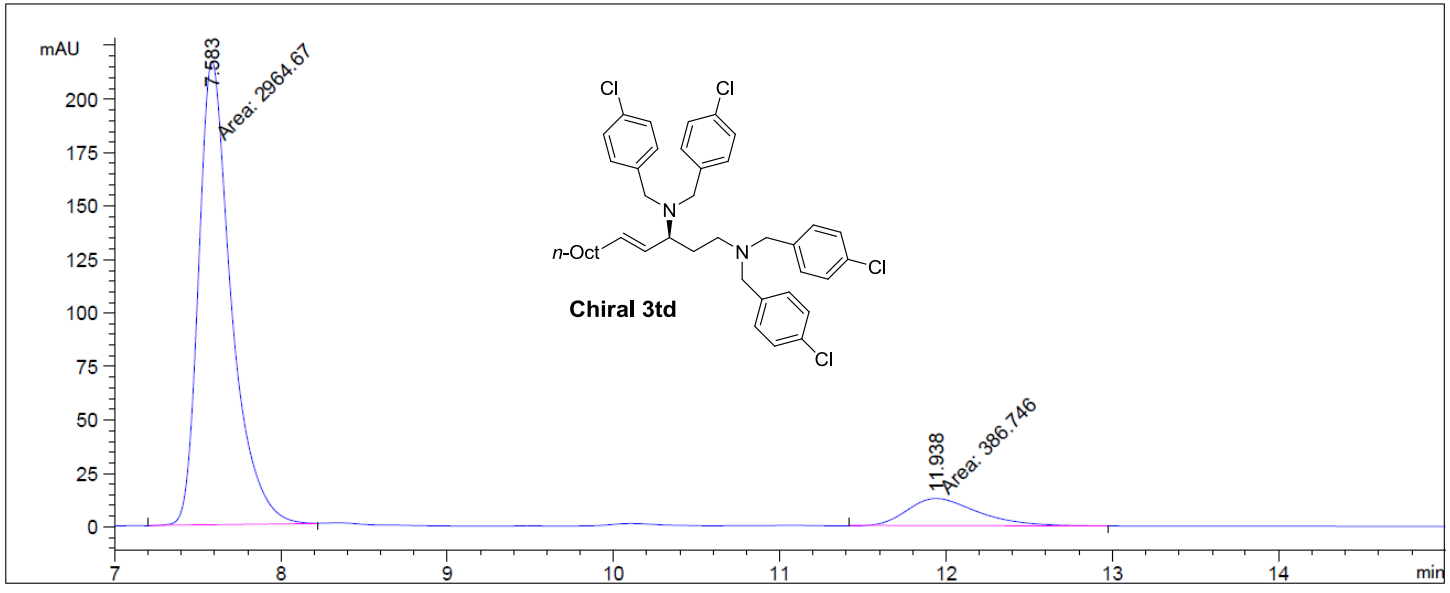

Peak RetTime Type Width Area Height Area

$\left.\begin{array}{llllll}\# & {[\mathrm{~min}]} & {[\mathrm{min}]} & \mathrm{mAU} & { }_{\mathrm{S}} & {[\mathrm{mAU}}\end{array}\right]$

---- $|-------|----|-------|----------|----------|--------\mid$

$\begin{array}{lllllll}1 & 7.583 & \mathrm{MM} & 0.2279 & 2964.66846 & 216.84094 & 88.4602\end{array}$

$\begin{array}{lllllll}2 & 11.938 & \text { MM } & 0.5019 & 386.74551 & 12.84191 & 11.5398\end{array}$

Totals :

$3351.41397 \quad 229.68285$ 

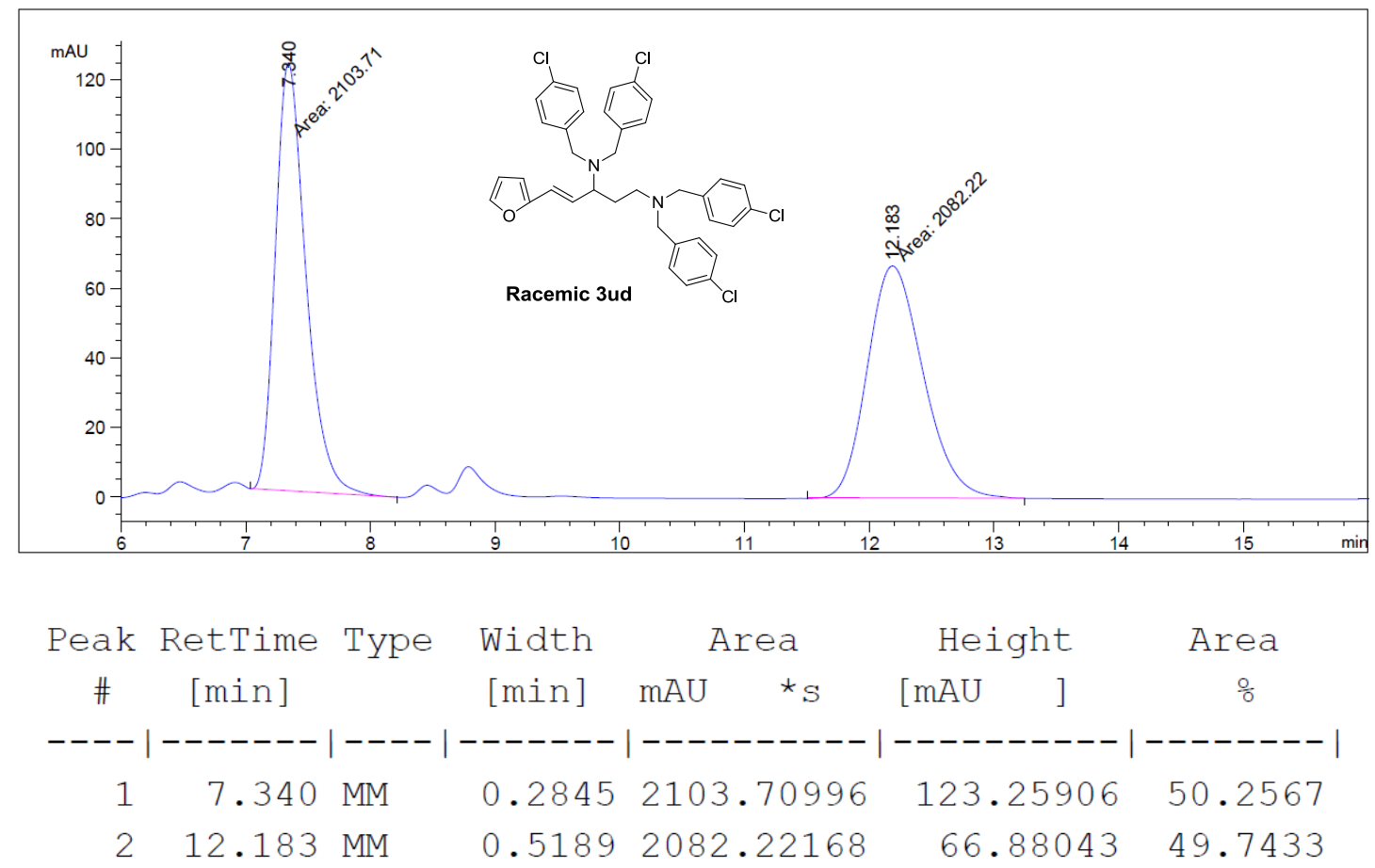

Totals :

$4185.93164 \quad 190.13950$

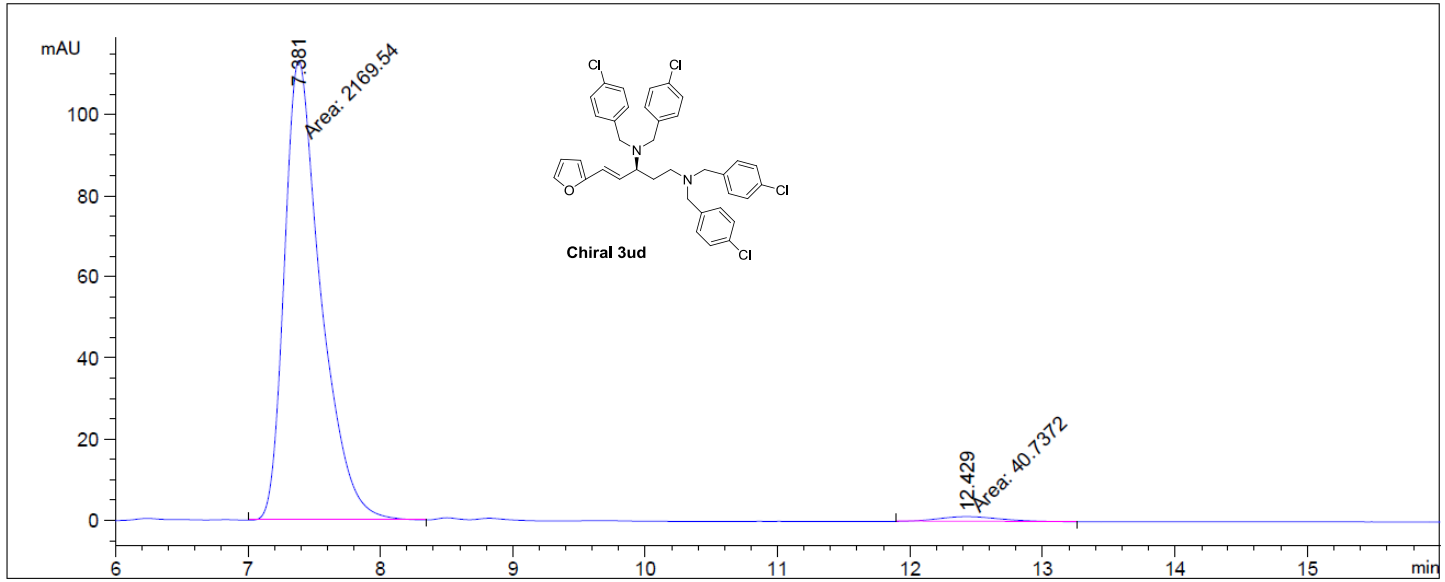

\begin{tabular}{|c|c|c|c|c|c|c|c|}
\hline \multirow{2}{*}{$\begin{array}{c}\text { Peak } \\
\#\end{array}$} & \multirow{2}{*}{$\begin{array}{c}\text { RetTime } \\
\text { [min] }\end{array}$} & \multirow{2}{*}{ Type } & \multirow{2}{*}{$\begin{array}{l}\text { Width } \\
\text { [min] }\end{array}$} & \multicolumn{2}{|c|}{ Area } & Height & \multirow{2}{*}{$\begin{array}{c}\text { Area } \\
\frac{\circ}{0}\end{array}$} \\
\hline & & & & $\mathrm{mAU}$ & $*_{\mathrm{S}}$ & {$[\mathrm{mAU}$} & \\
\hline & & & & & & - & \\
\hline 1 & & $\mathbb{M}$ & & 216 & 6 & 11 & 69 \\
\hline 2 & 12.429 & M & 80 & 4 & 73725 & & 8431 \\
\hline
\end{tabular}

Totals :

$2210.27240 \quad 114.43515$ 

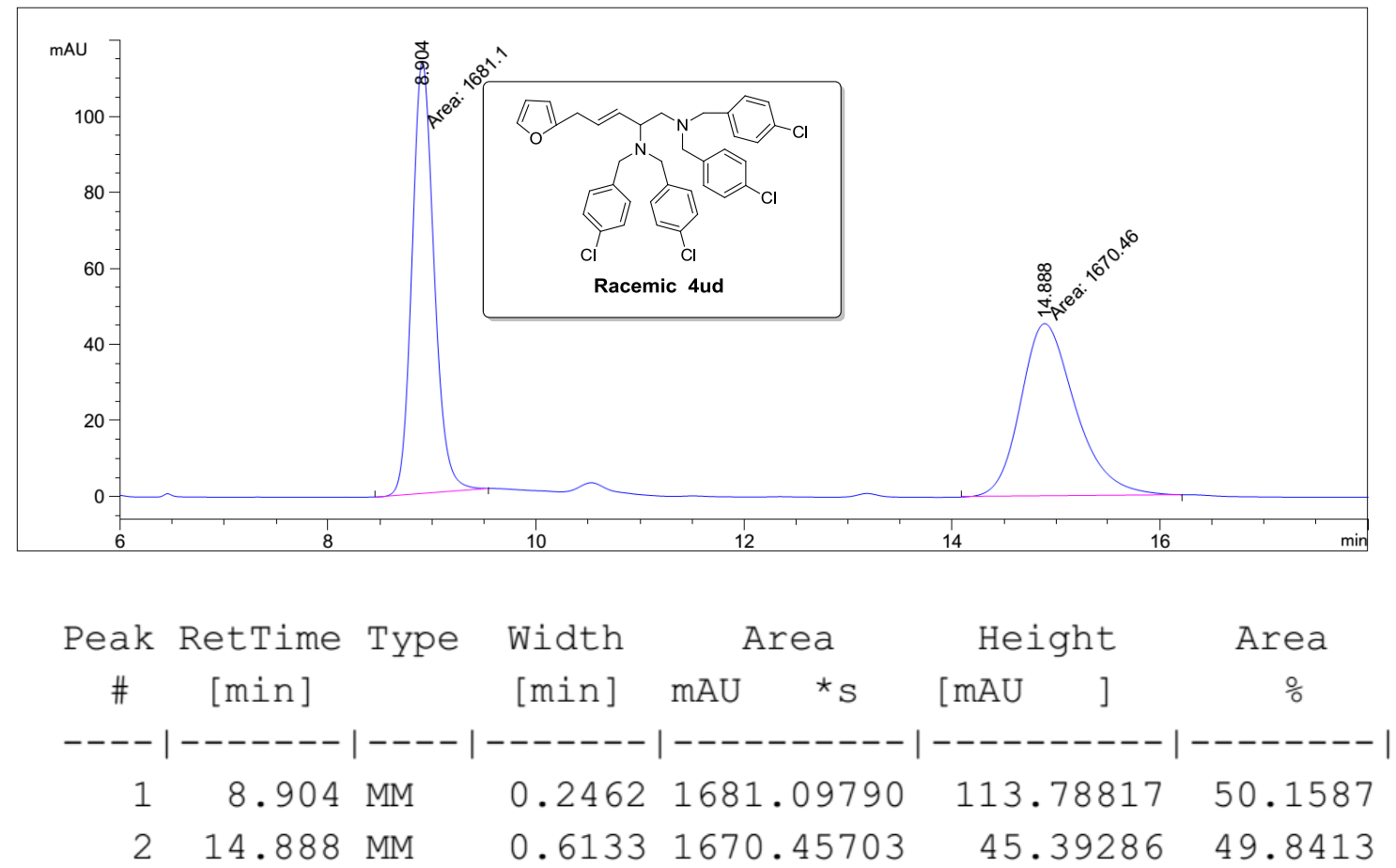

Totals :

$3351.55493 \quad 159.18103$

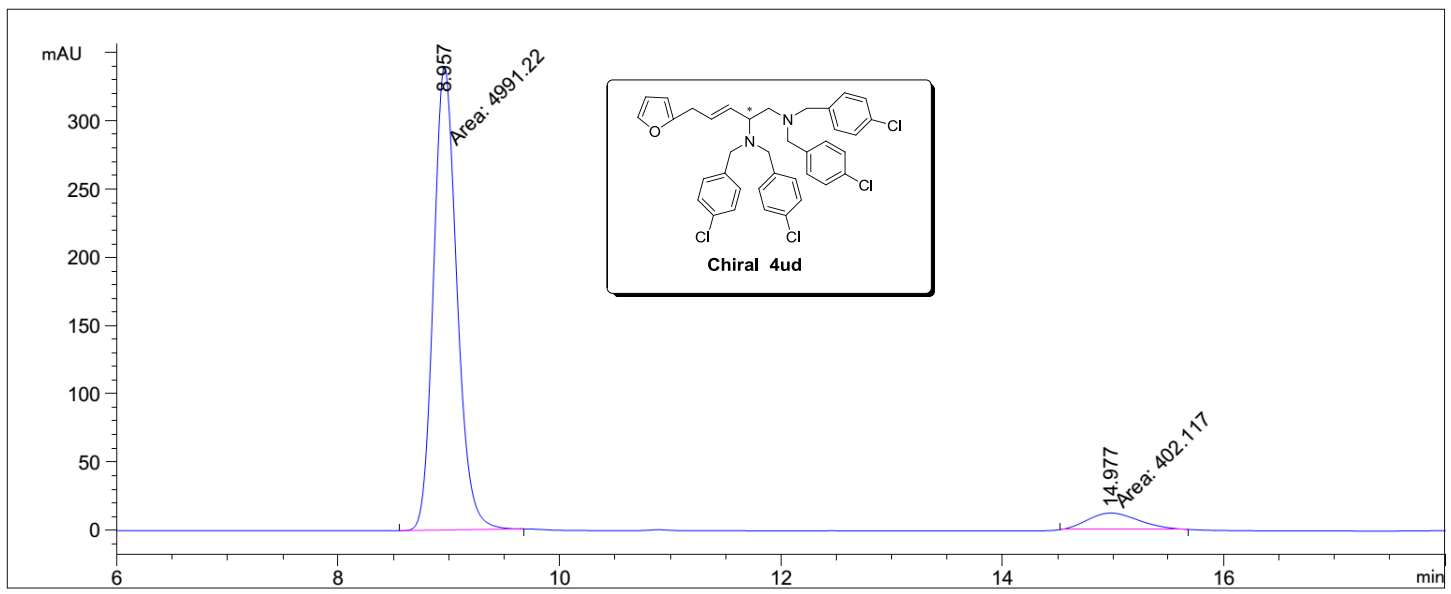

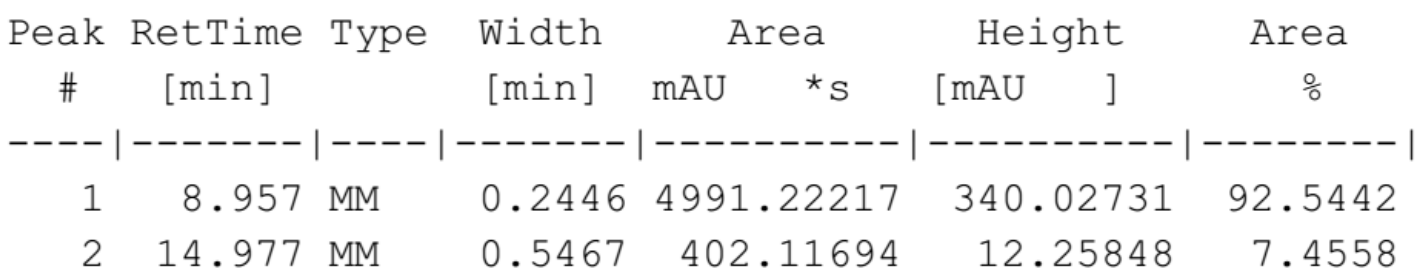

Totals :

$5393.33911 \quad 352.28580$ 

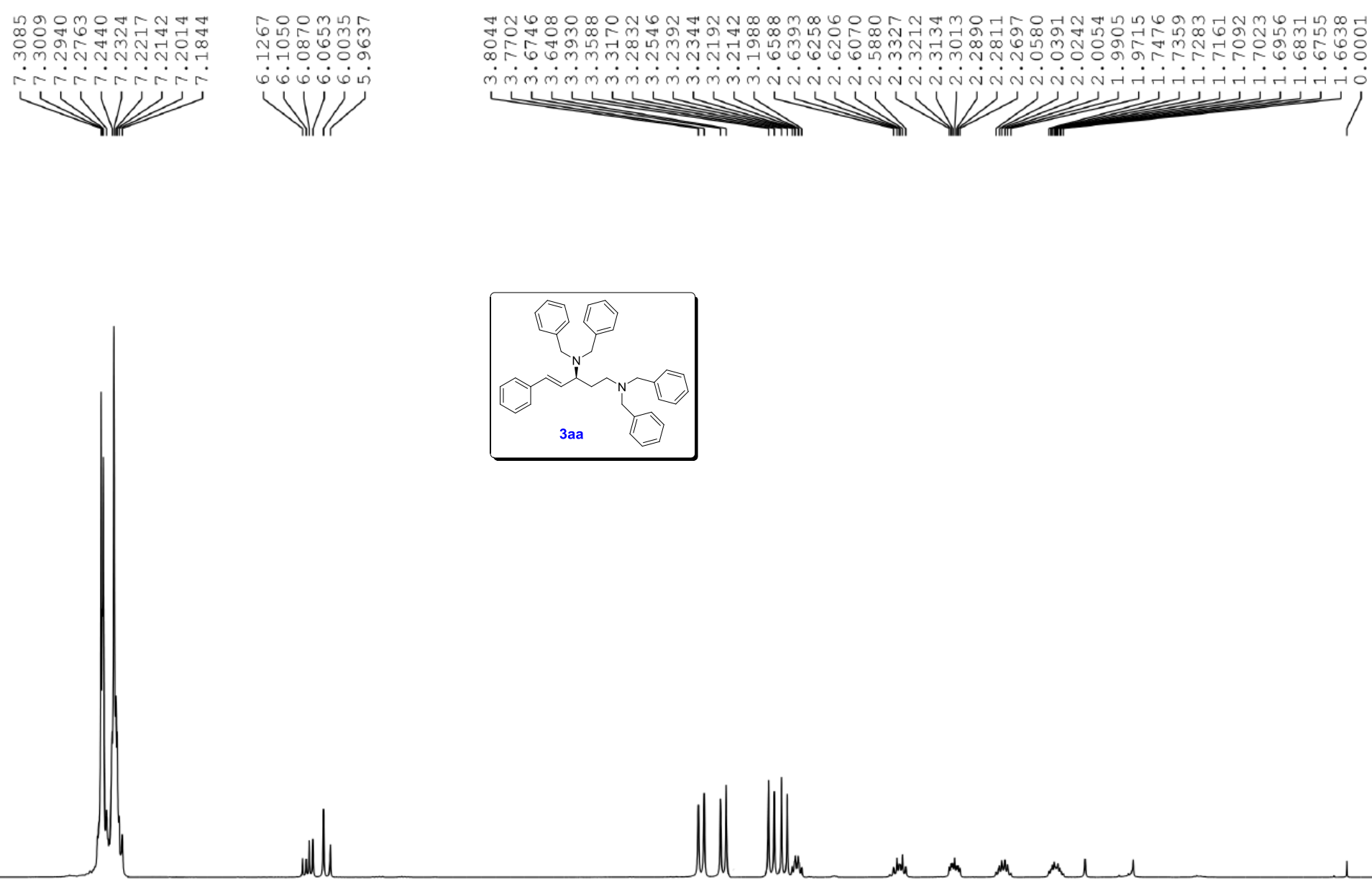

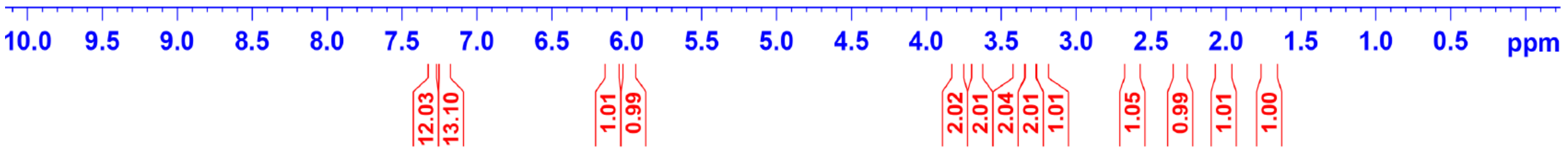



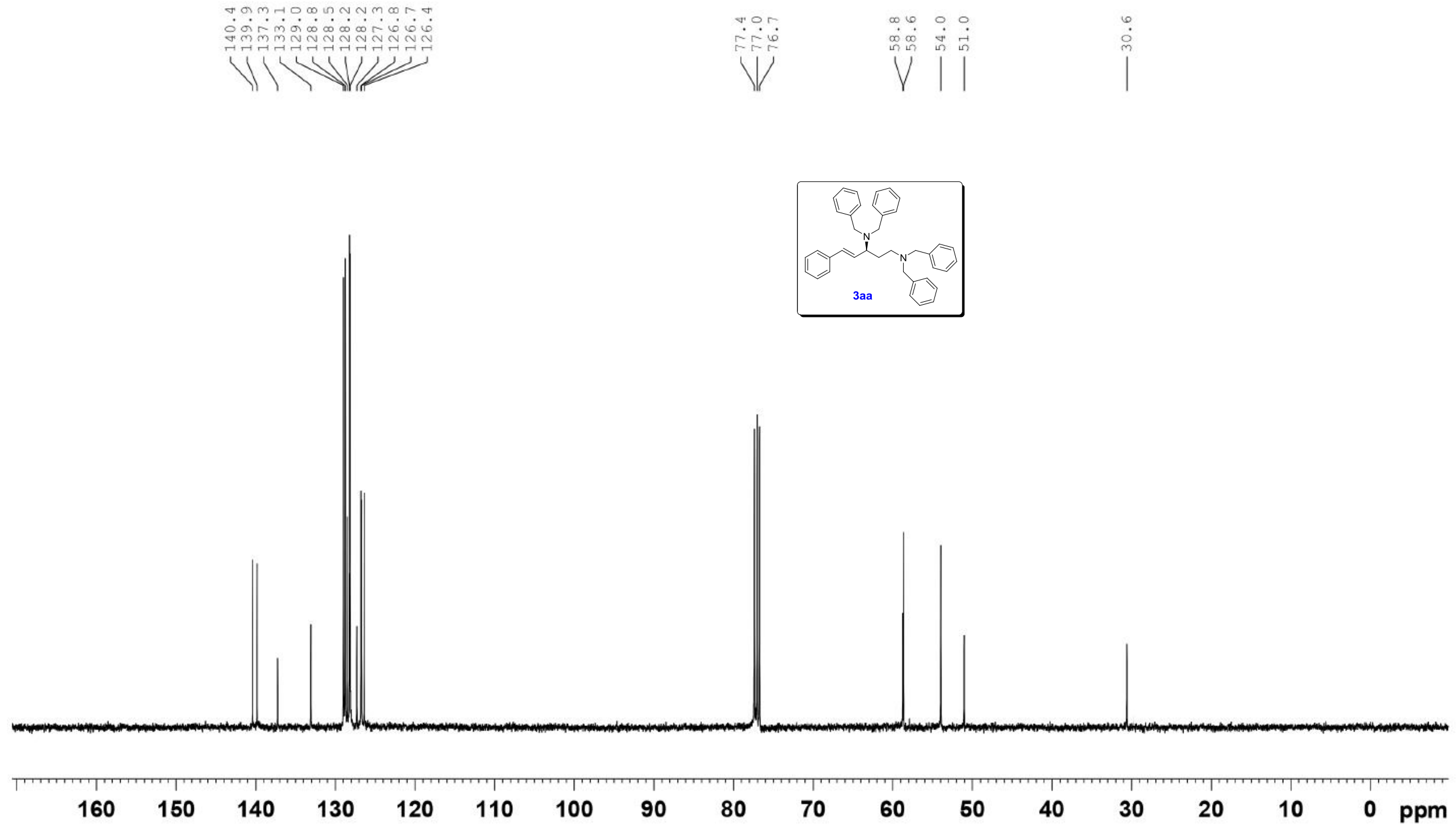

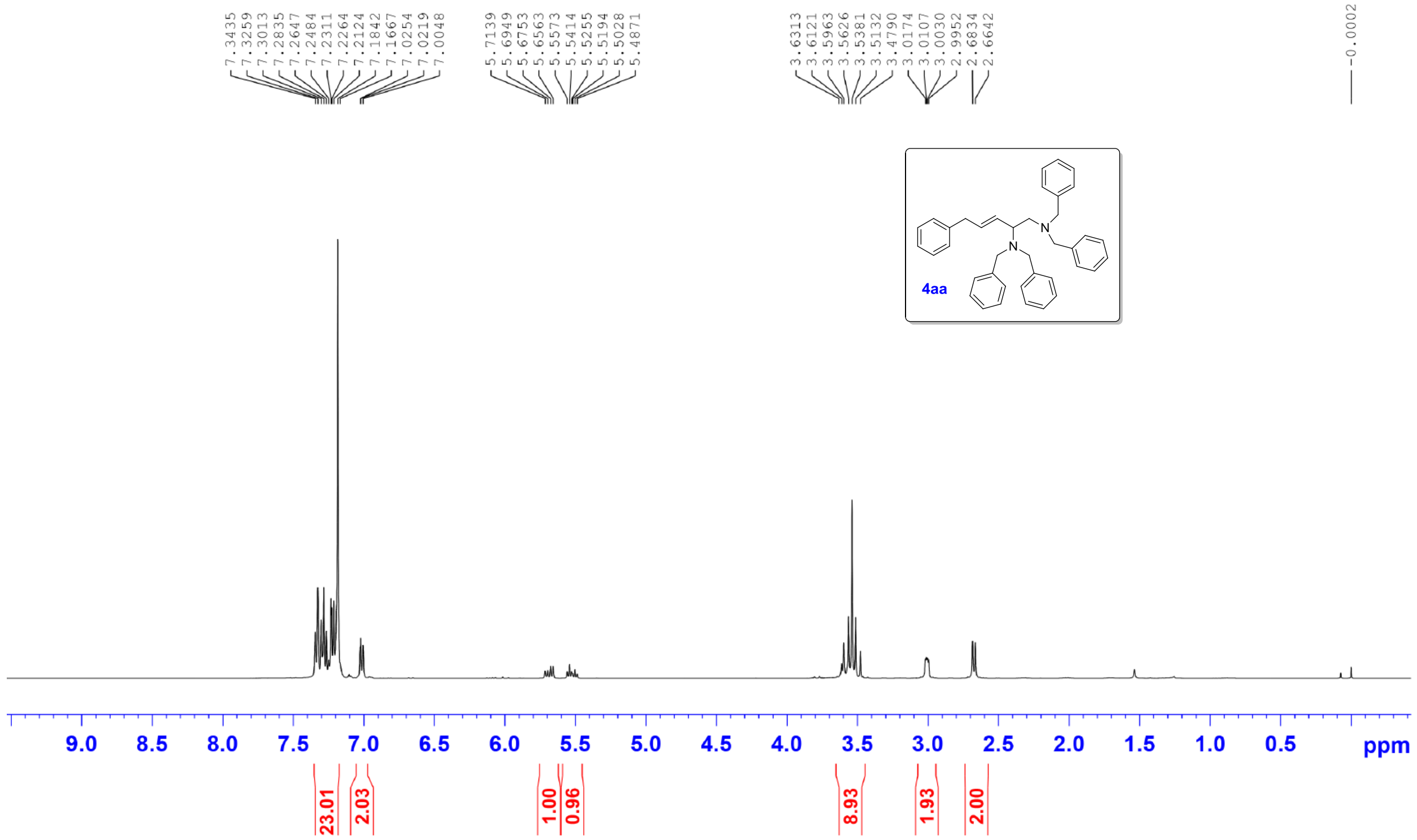

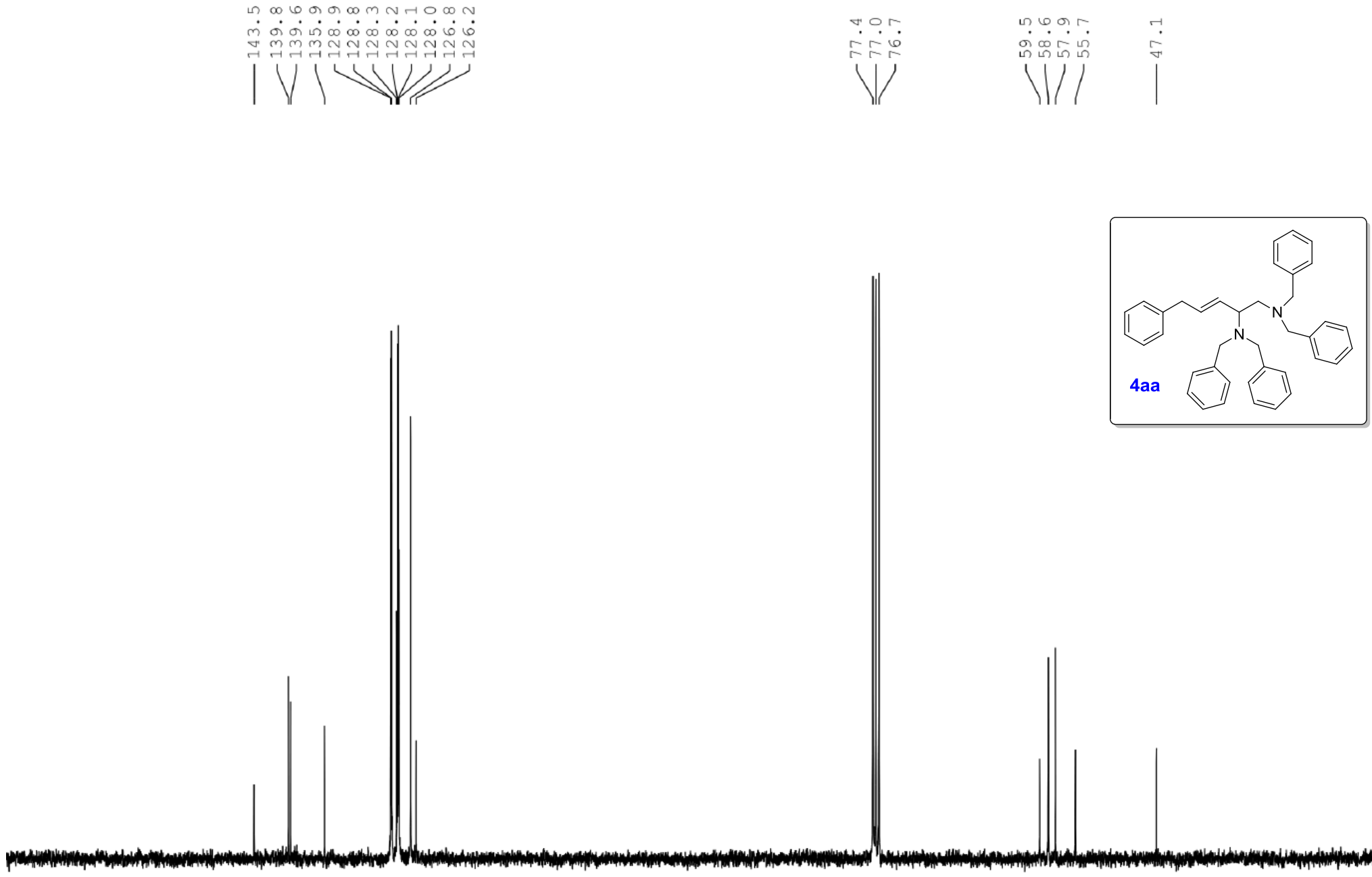

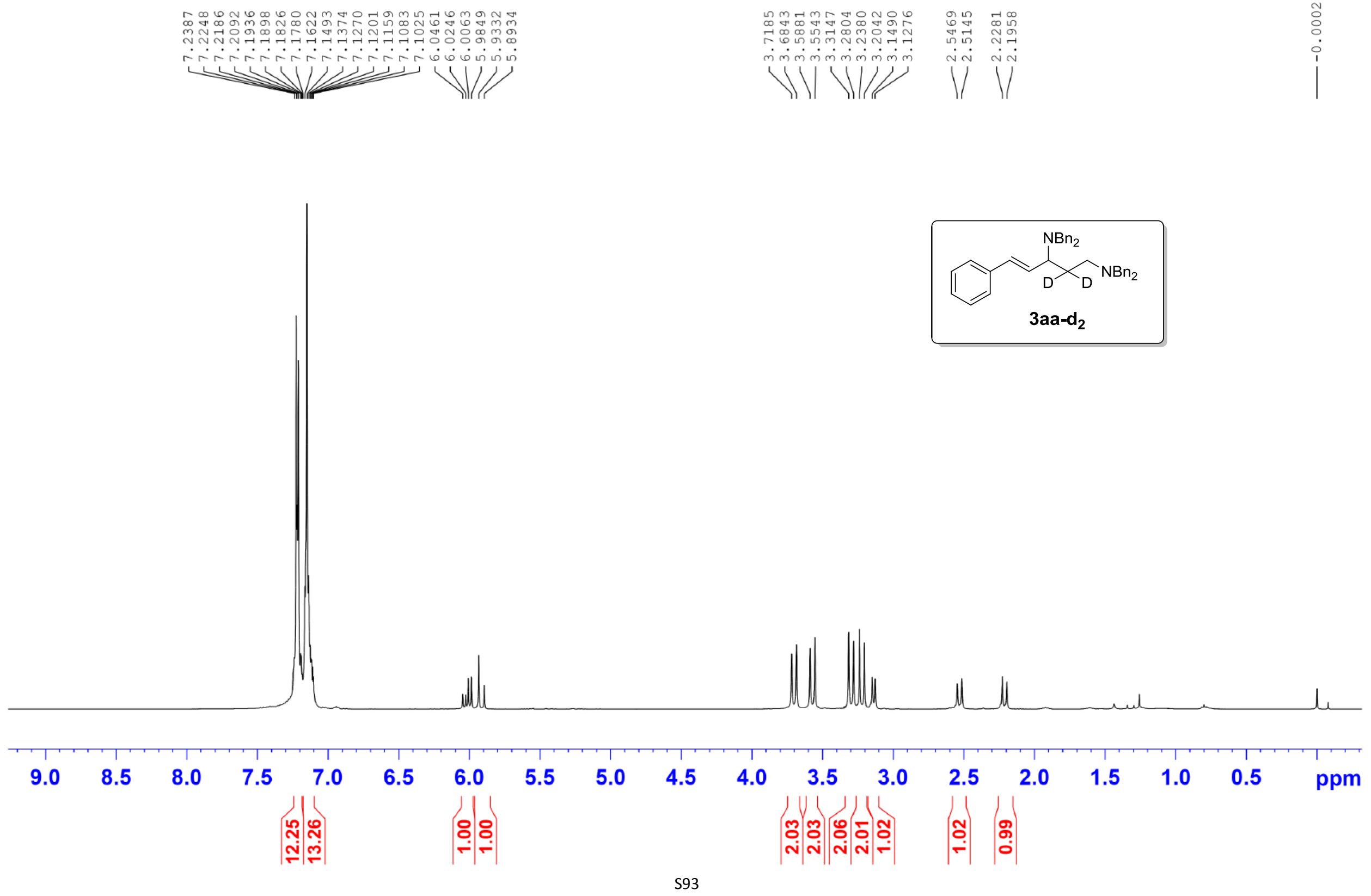

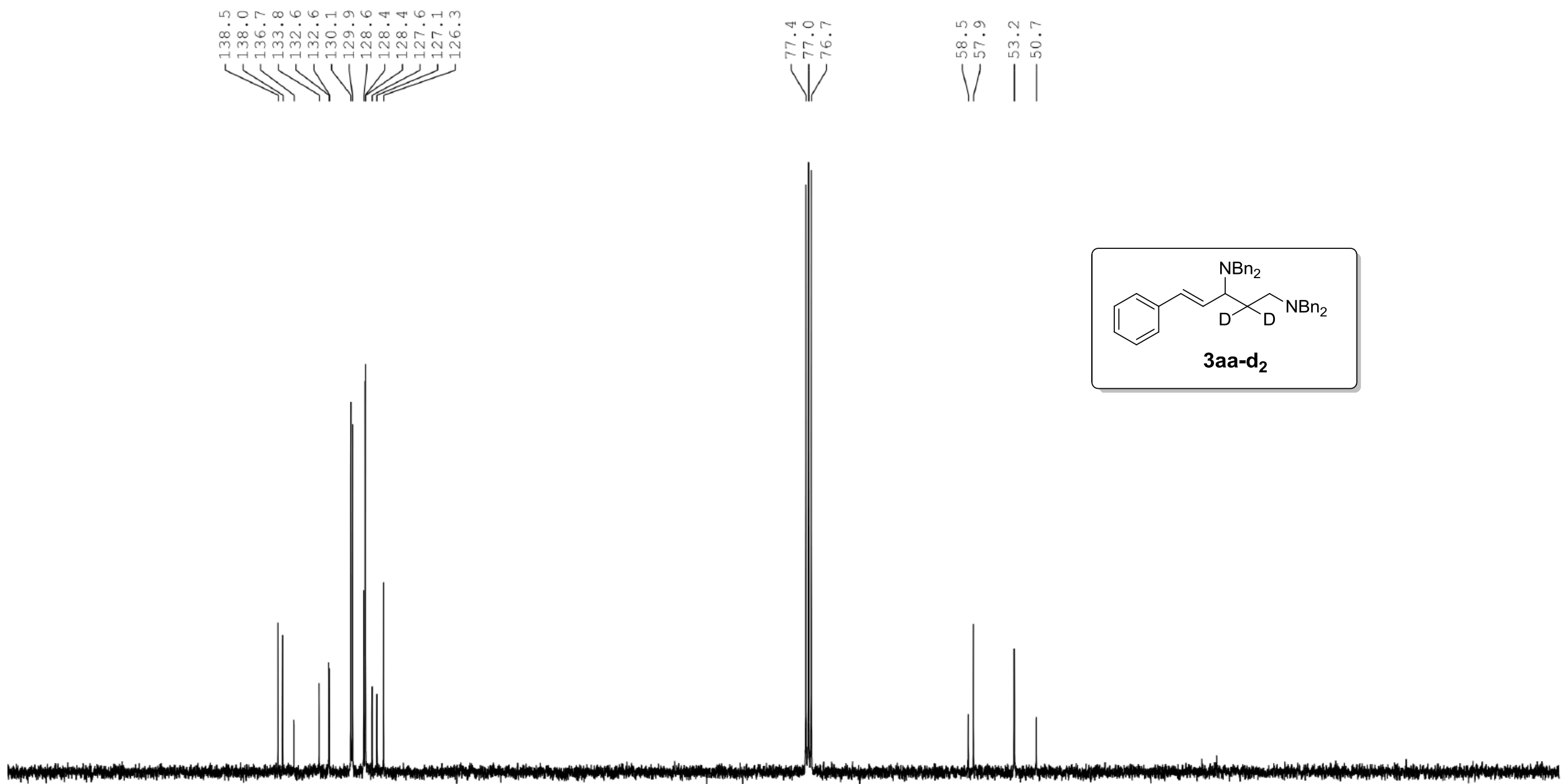
160
150
$140 \quad 130$
120
$110 \quad 100$
$90 \quad 80$
$70 \quad 60$
50
40
30
20
100 ppm 

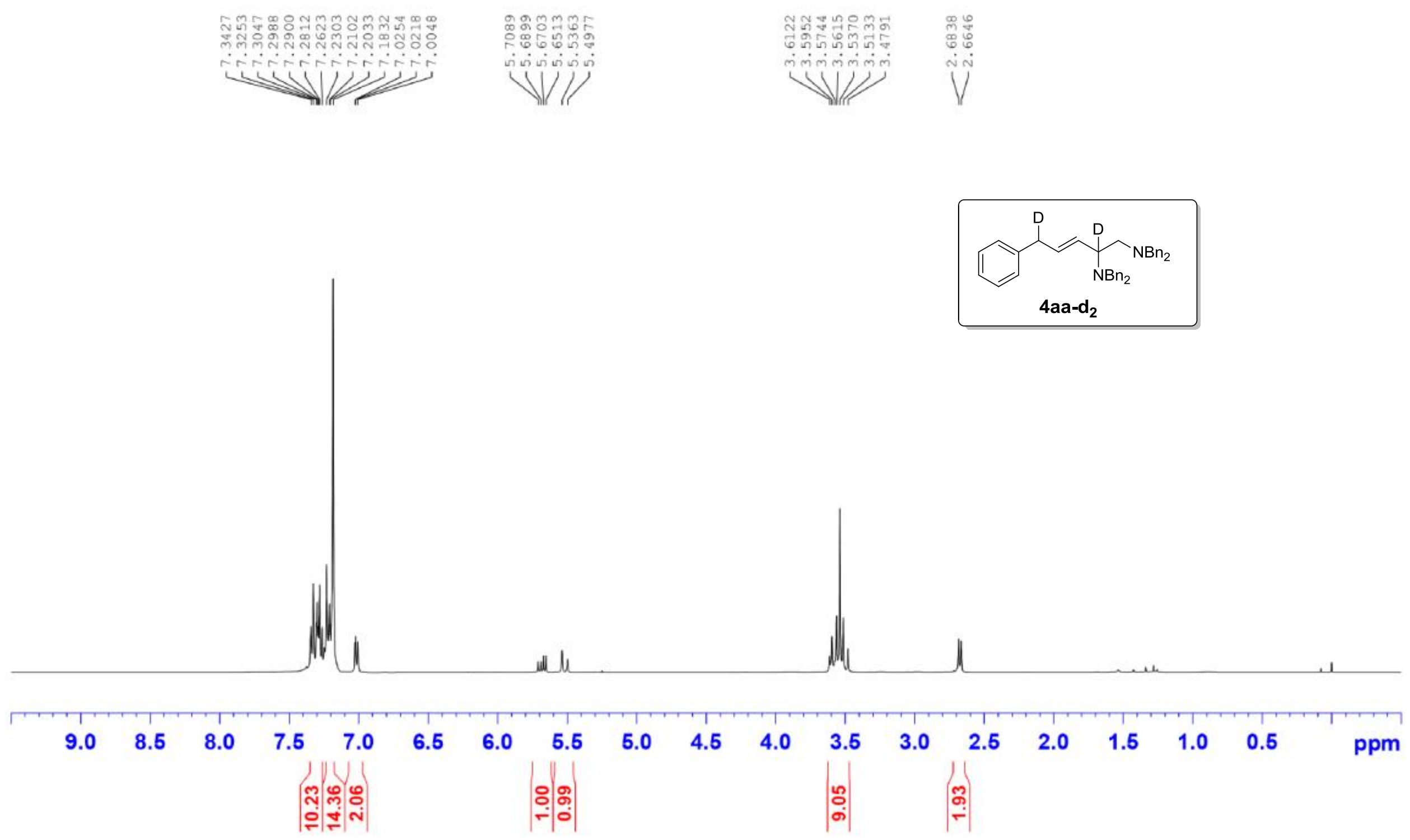

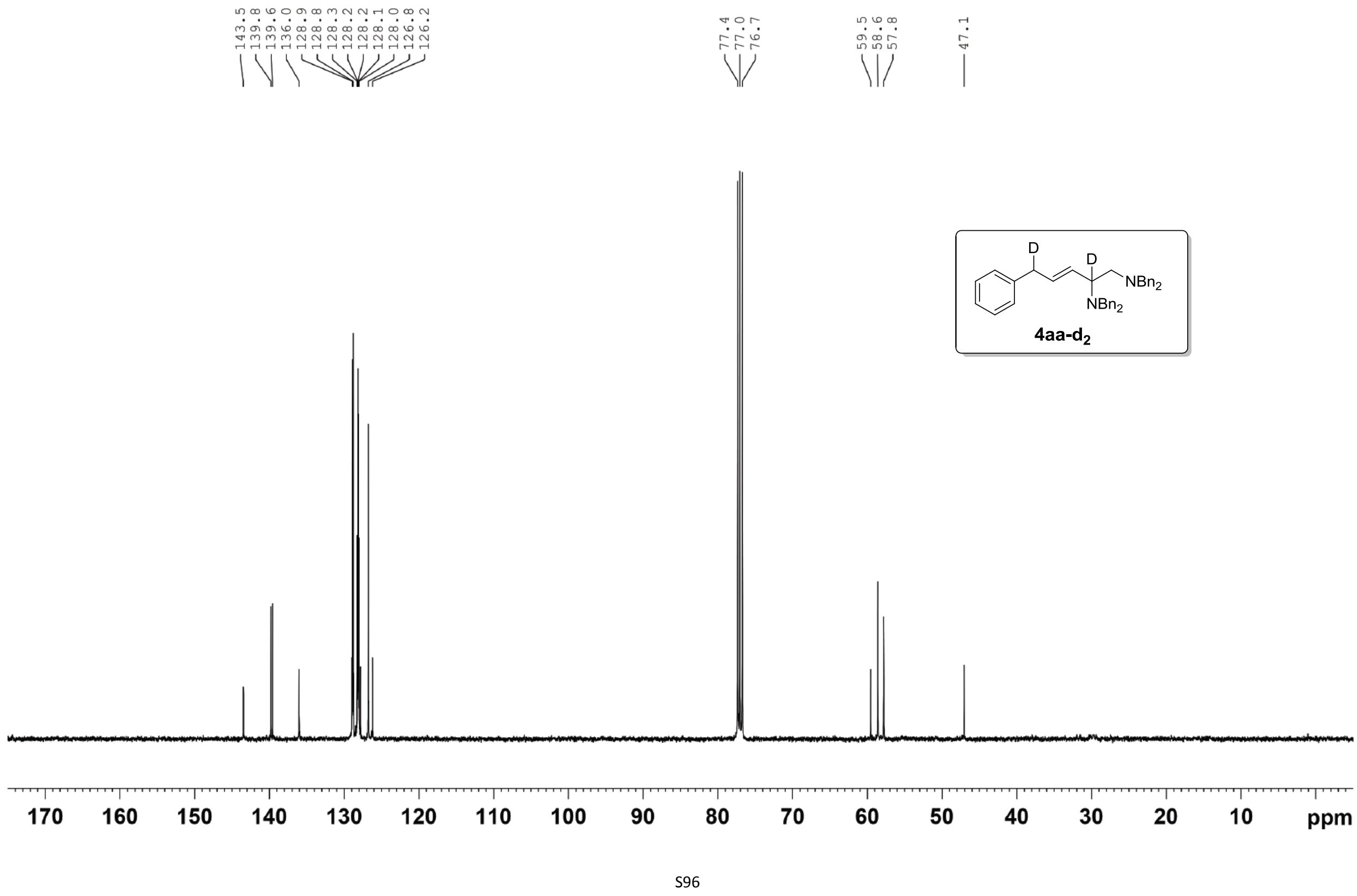
שกn

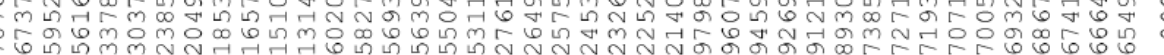
H nِ
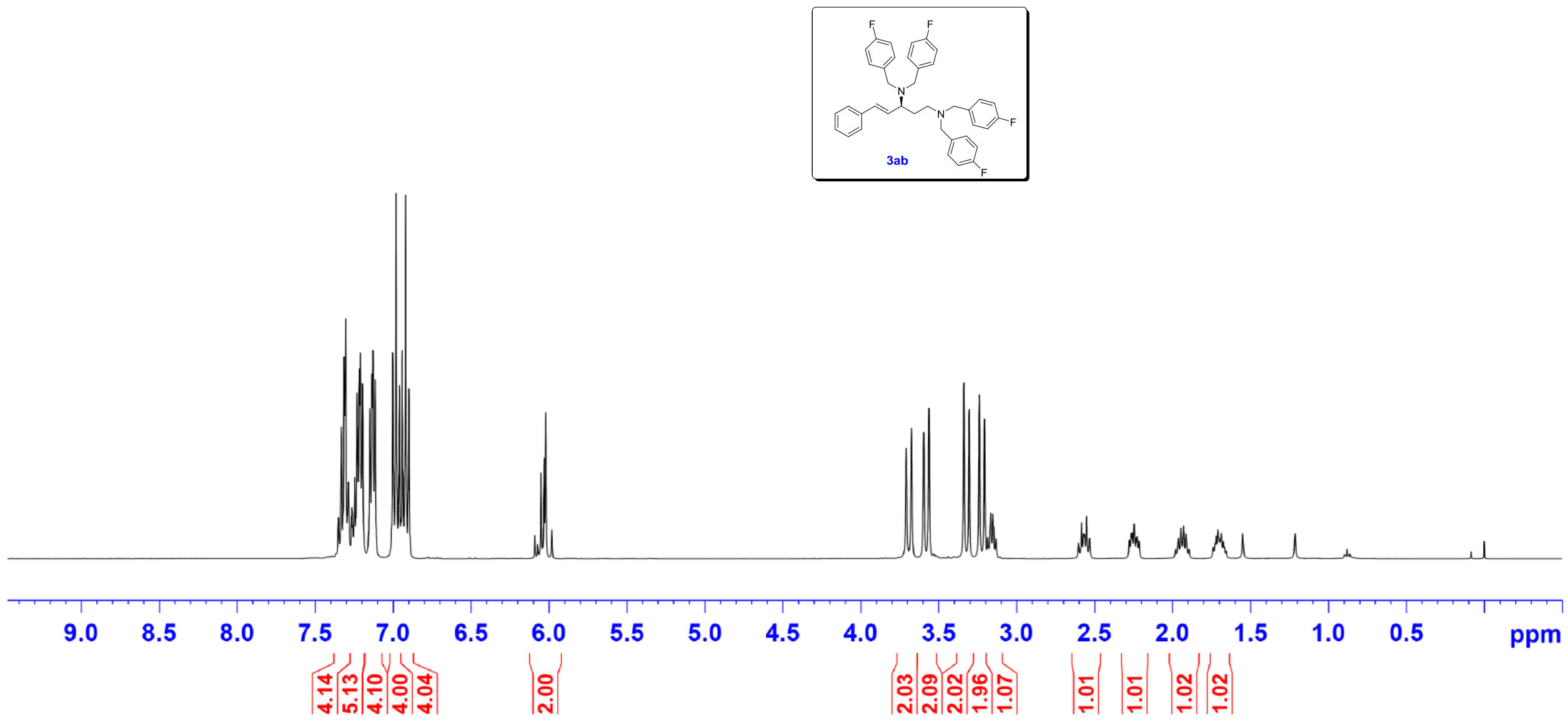

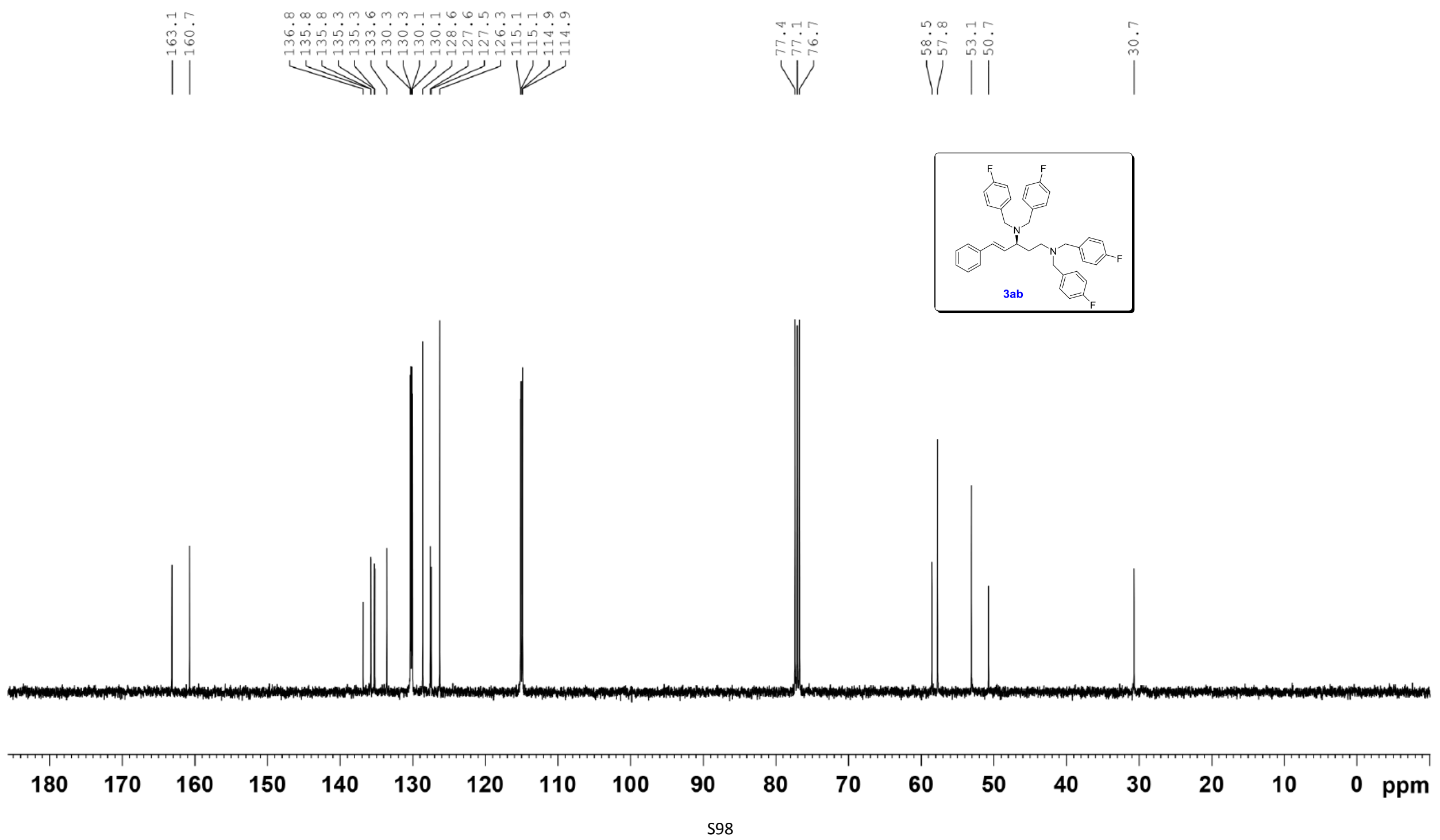
LY-X15Y10-1-FNMR
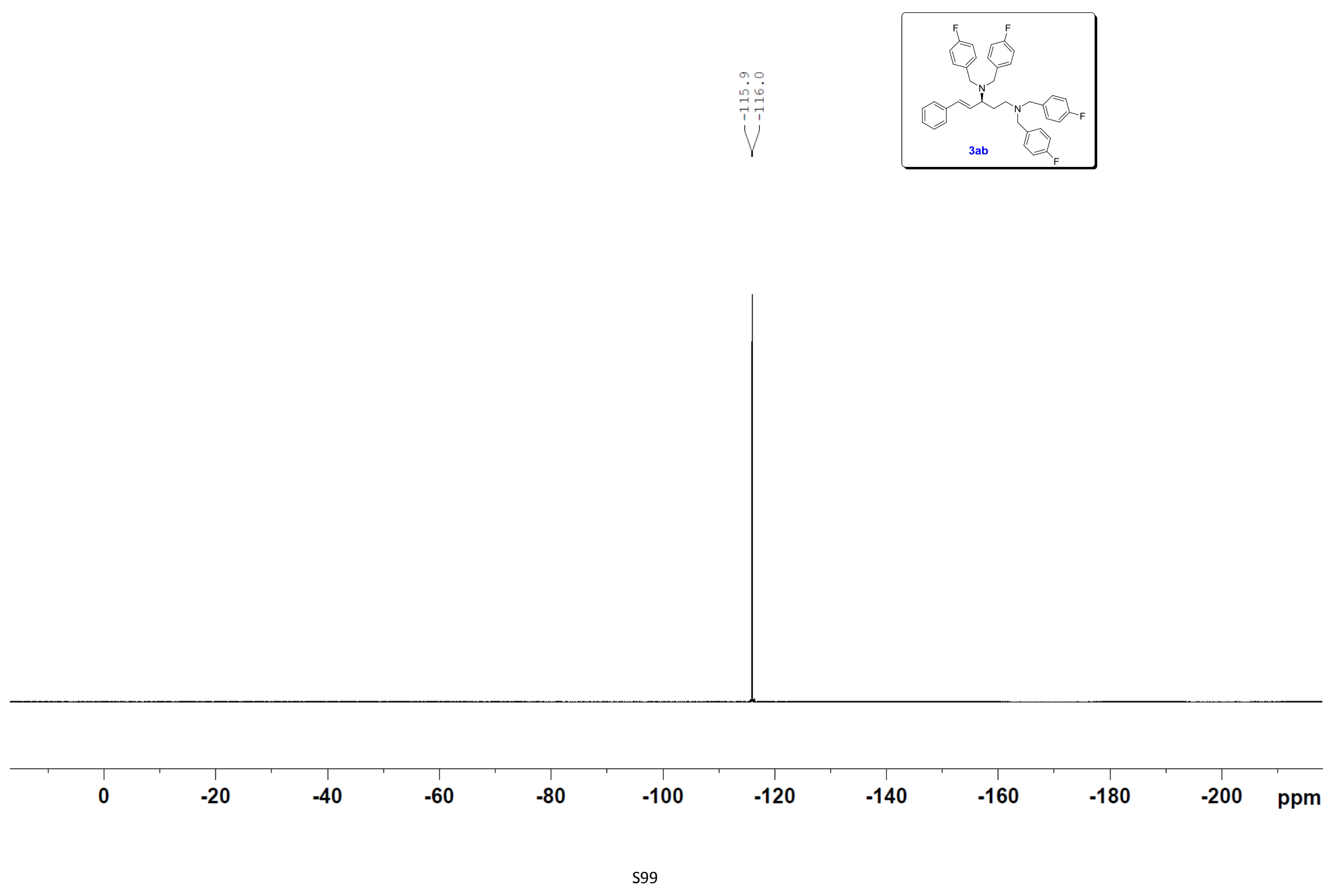

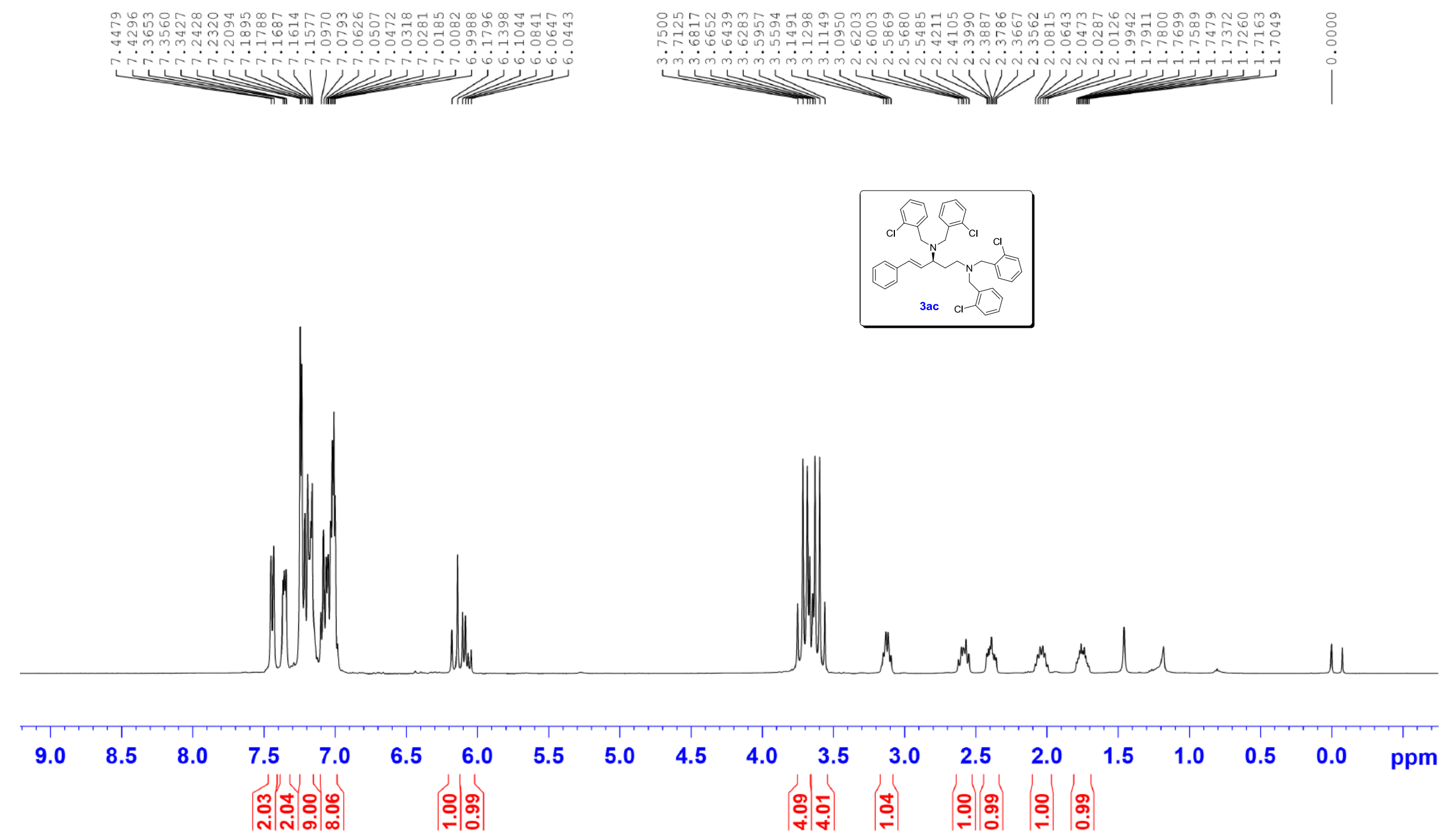

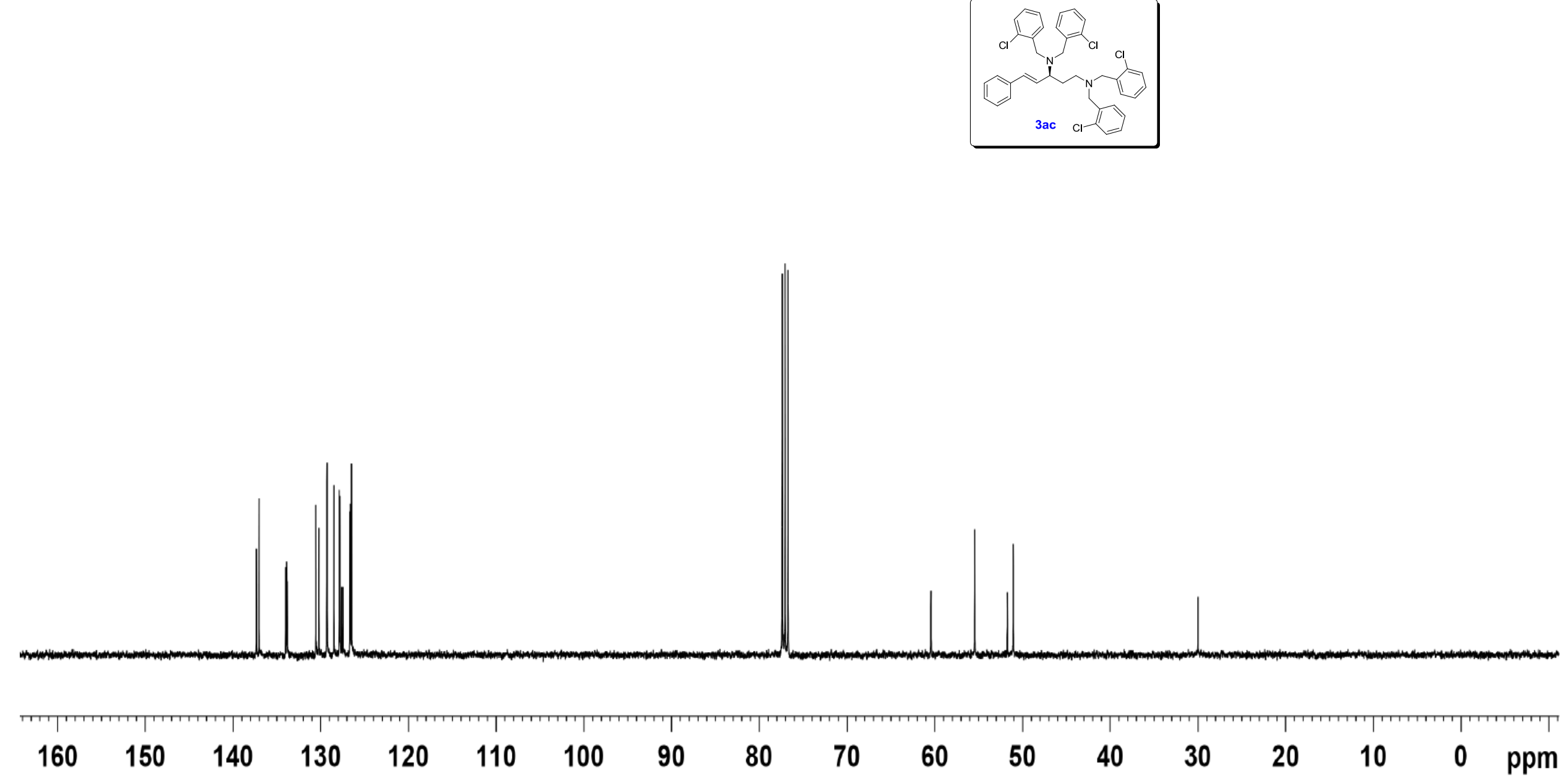

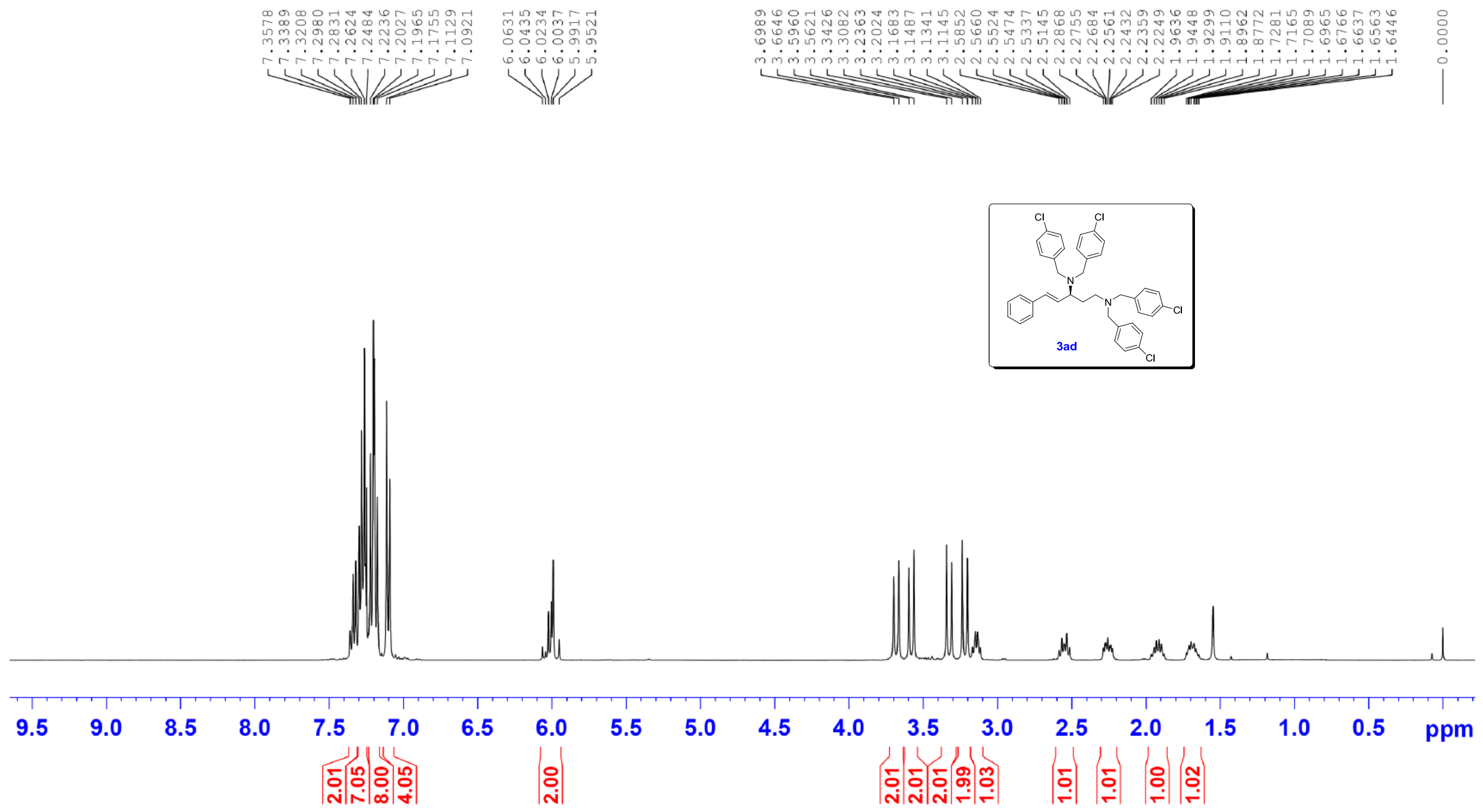

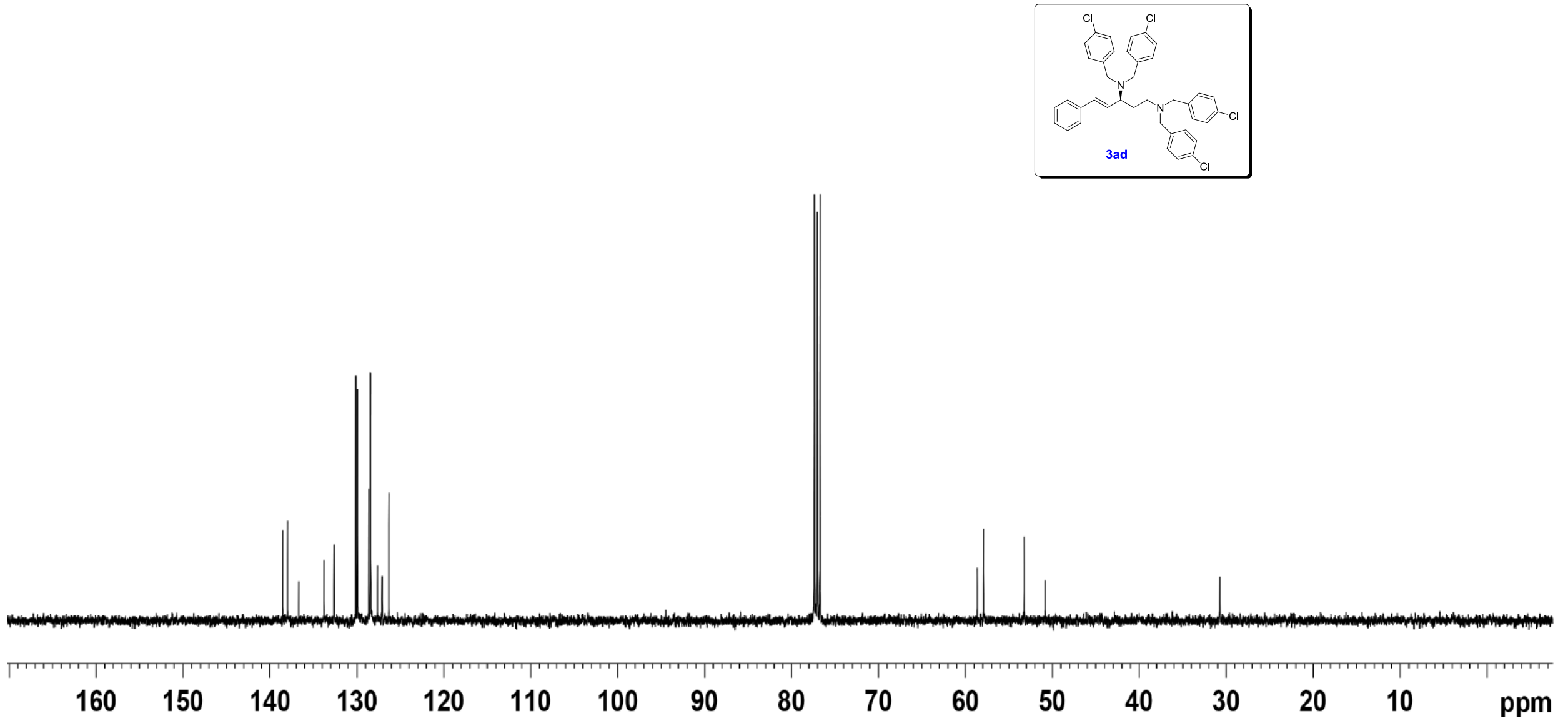

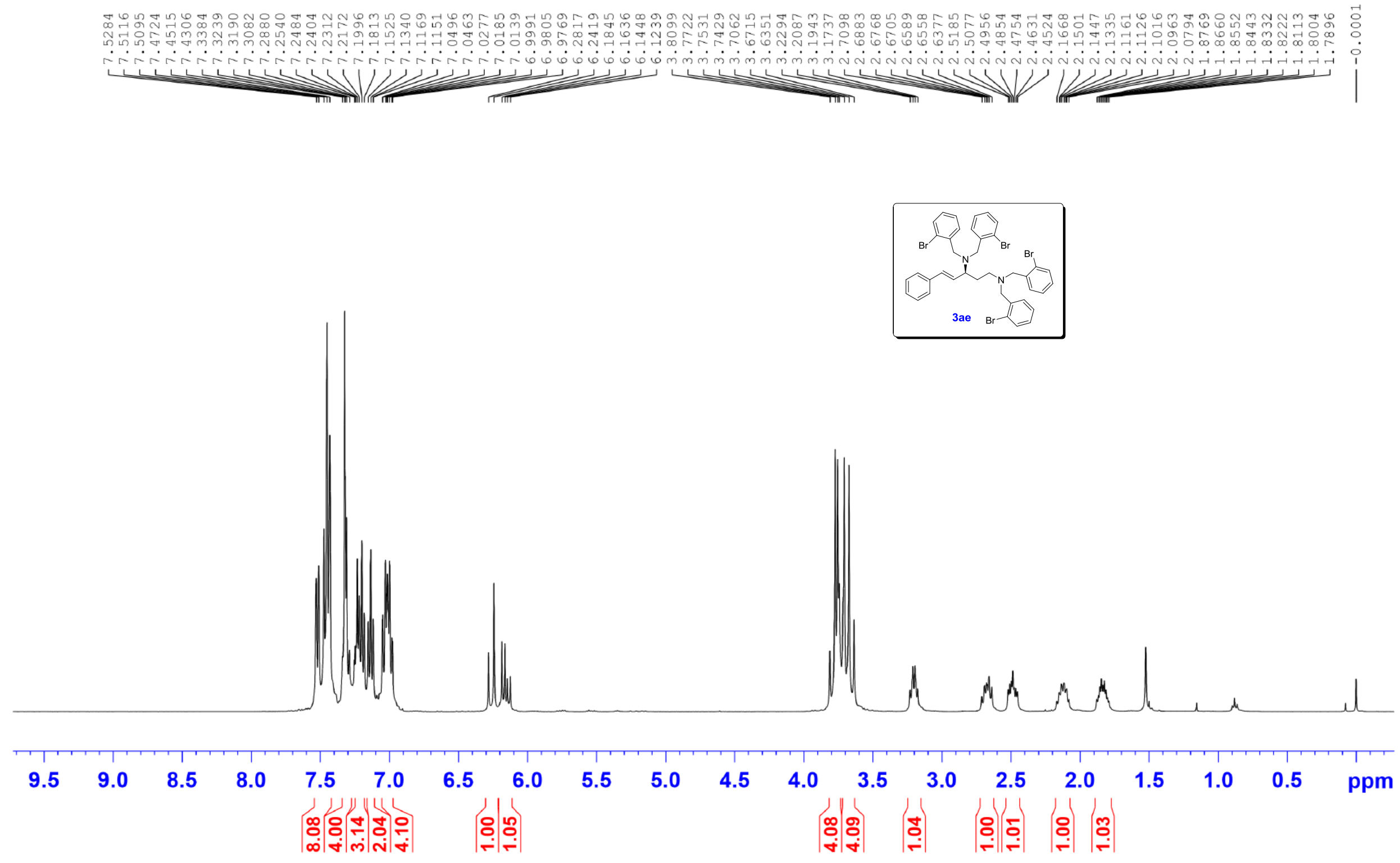


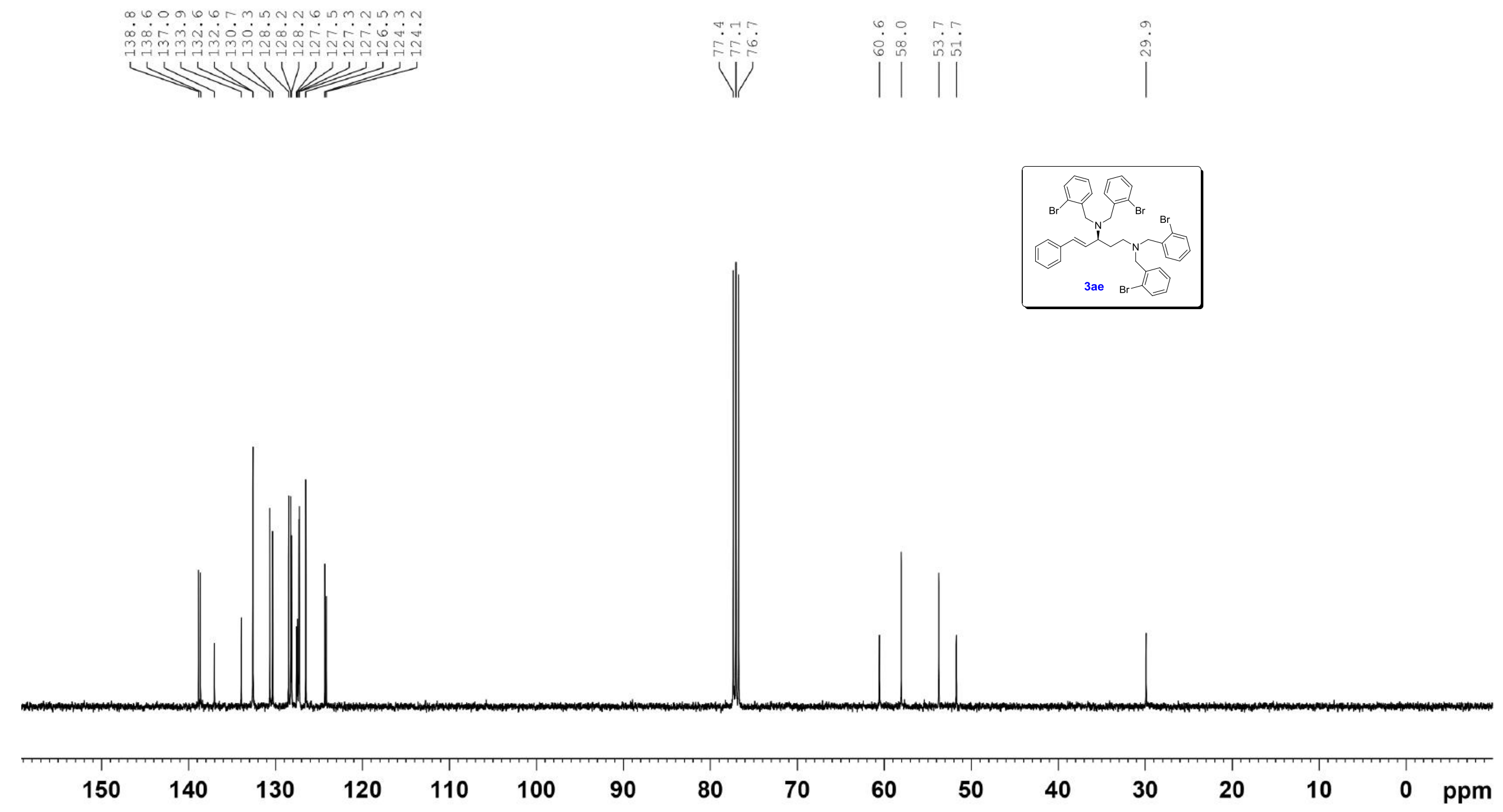



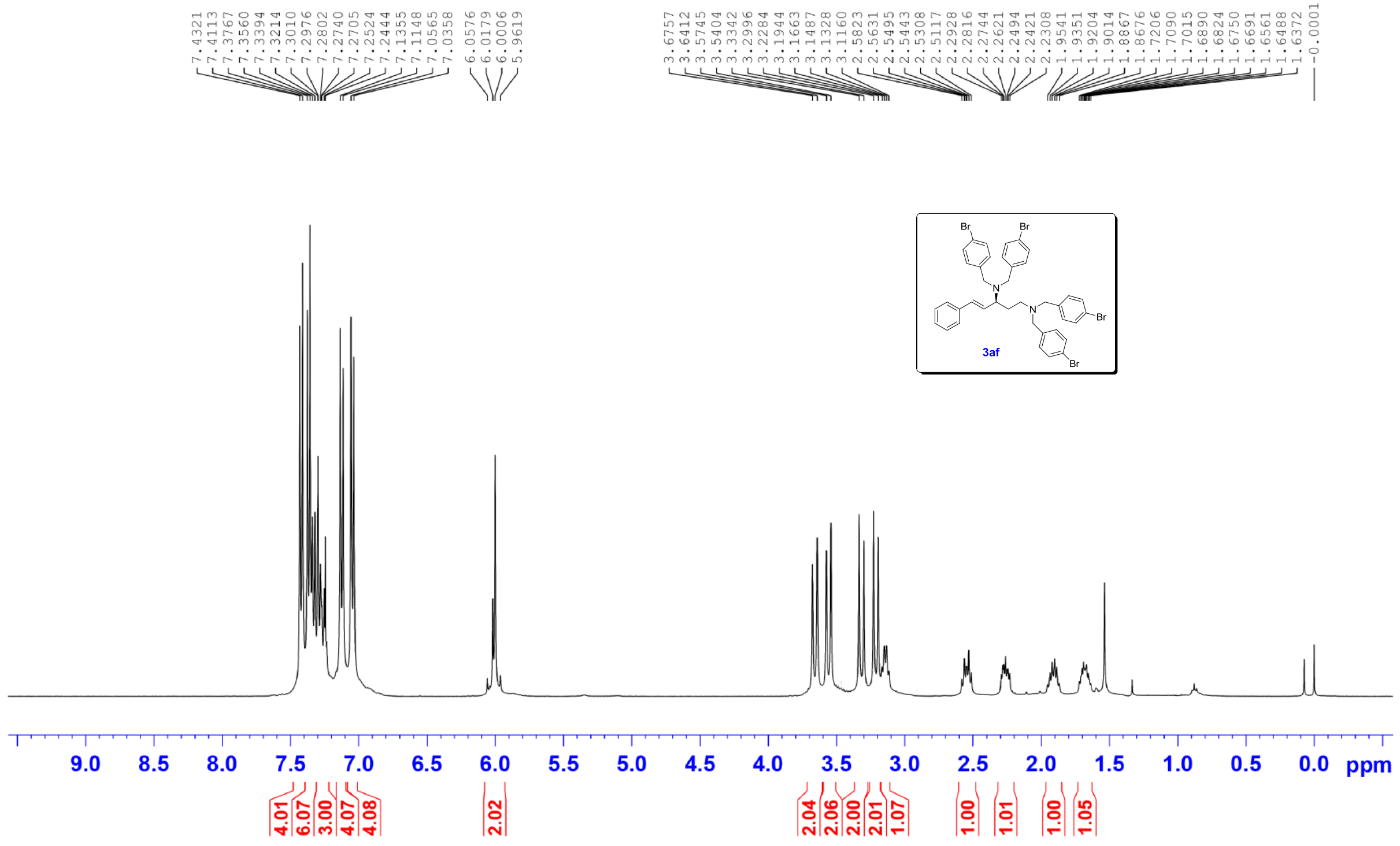


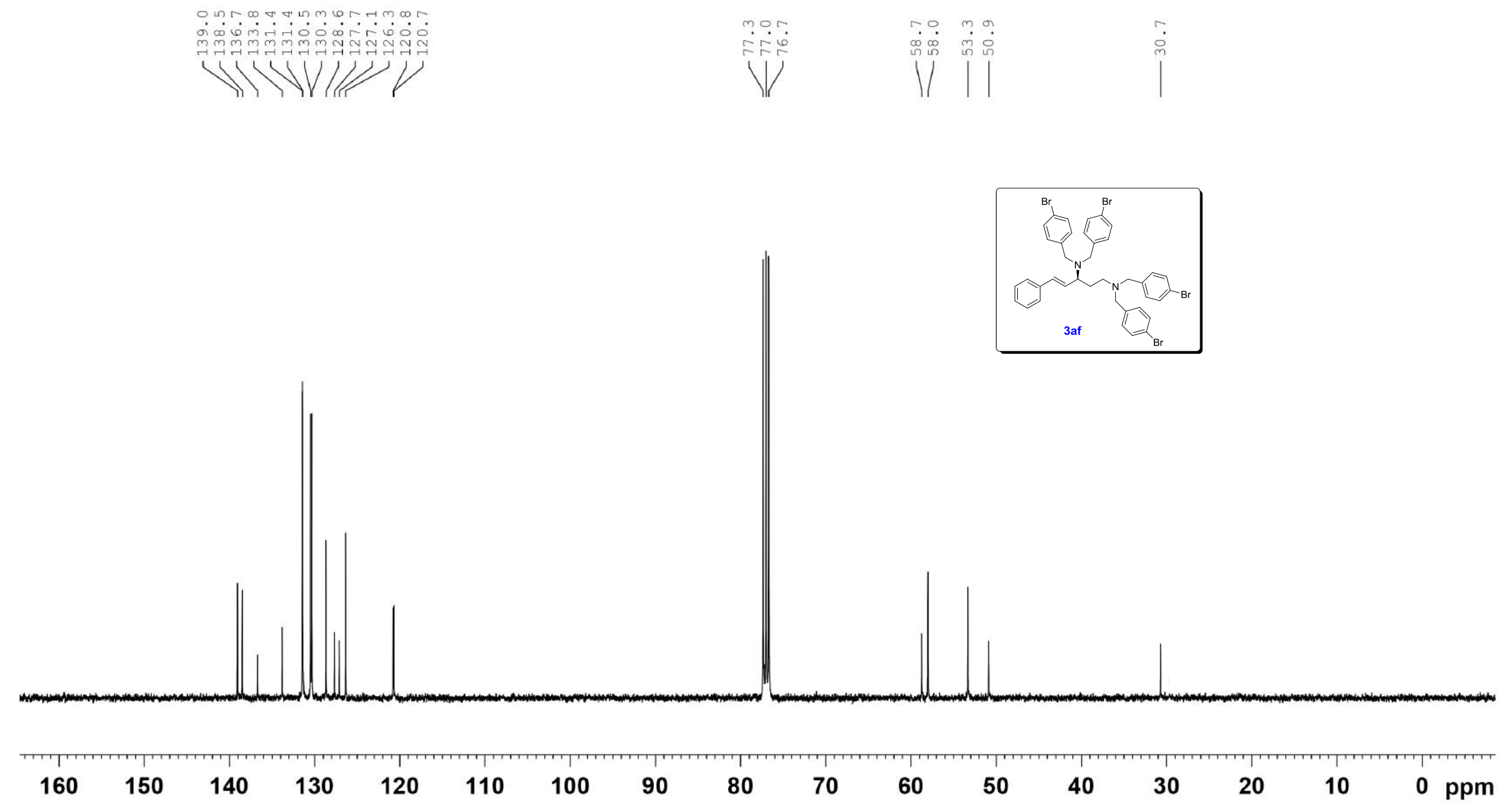




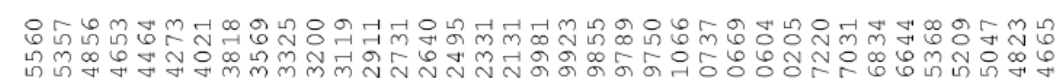
(1)

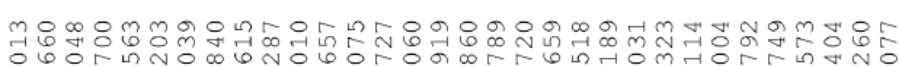

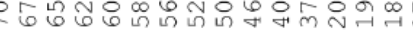

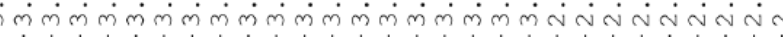

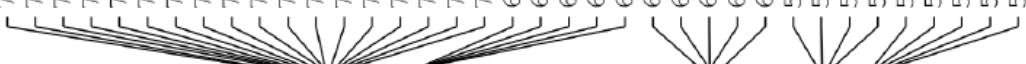
का

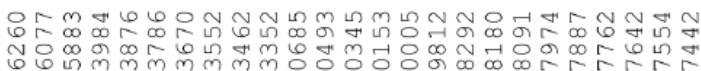

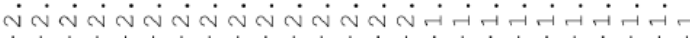

$+W$

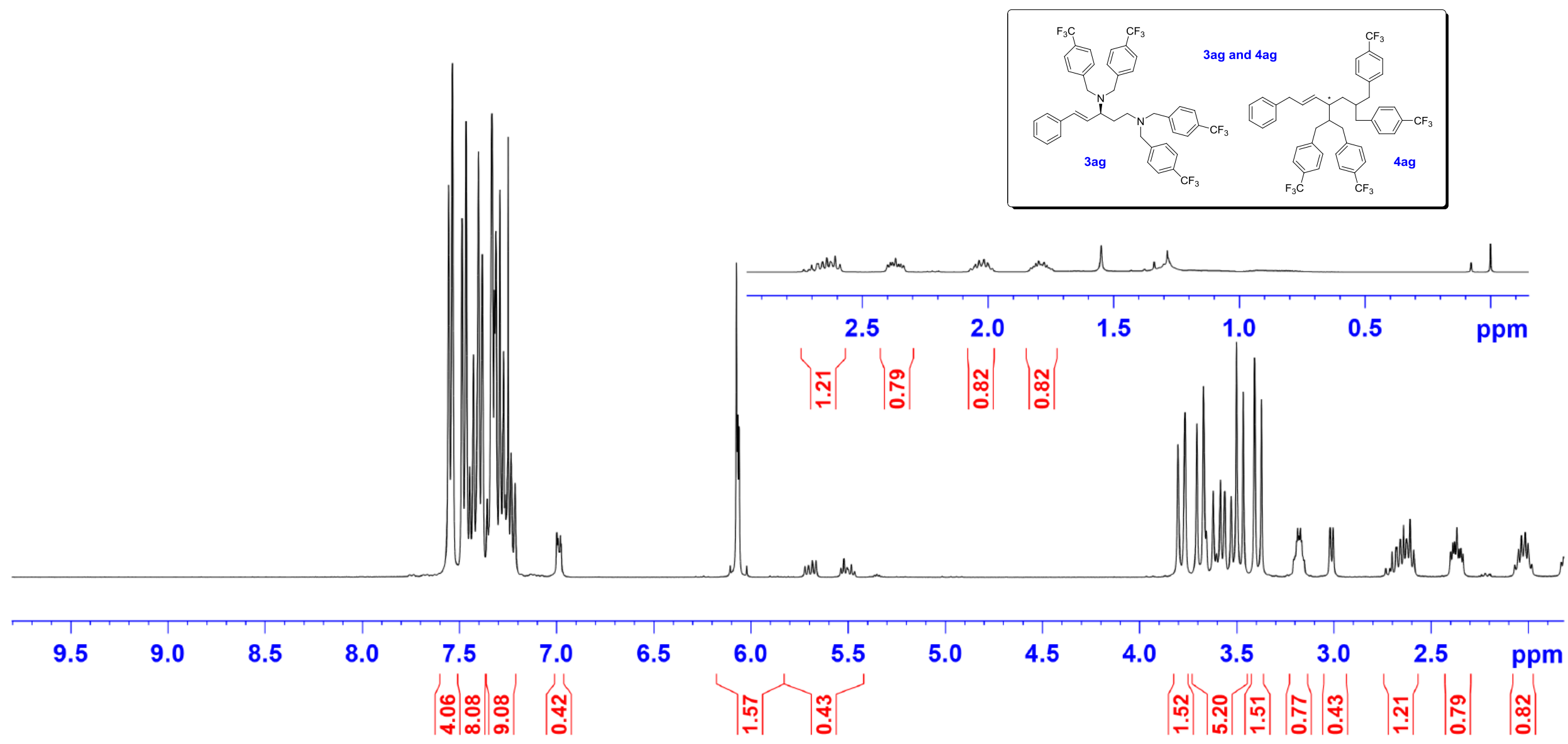



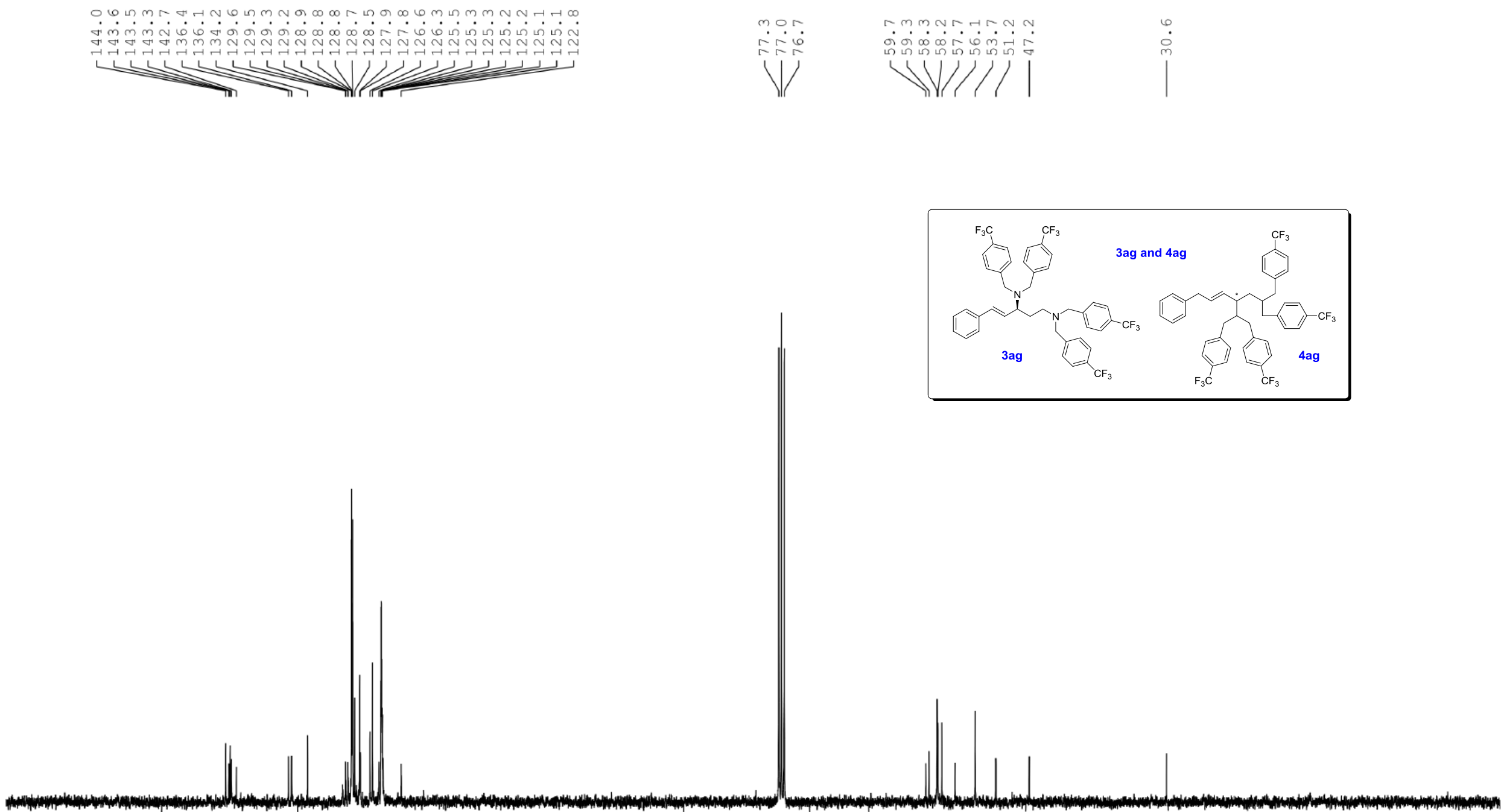

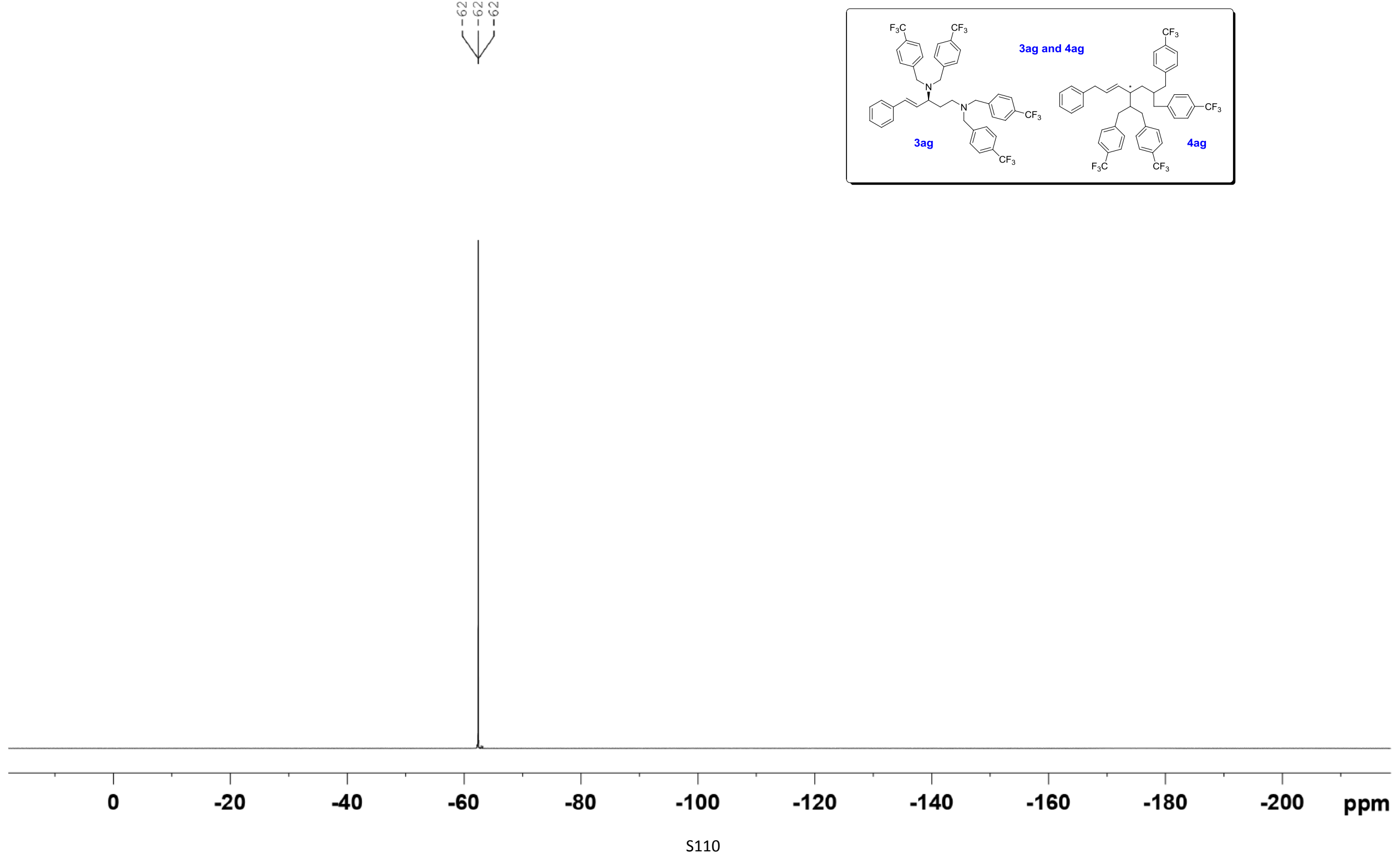

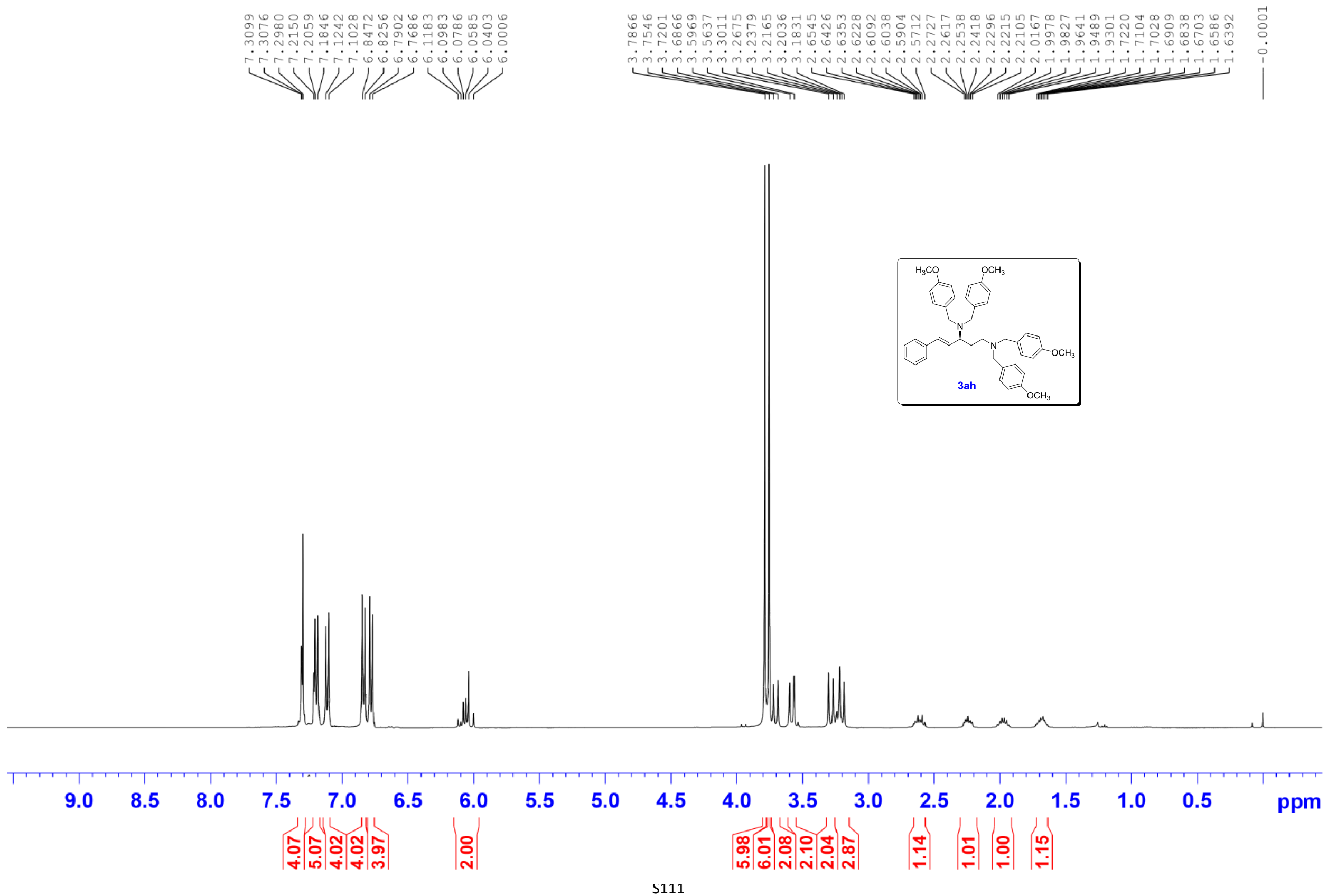

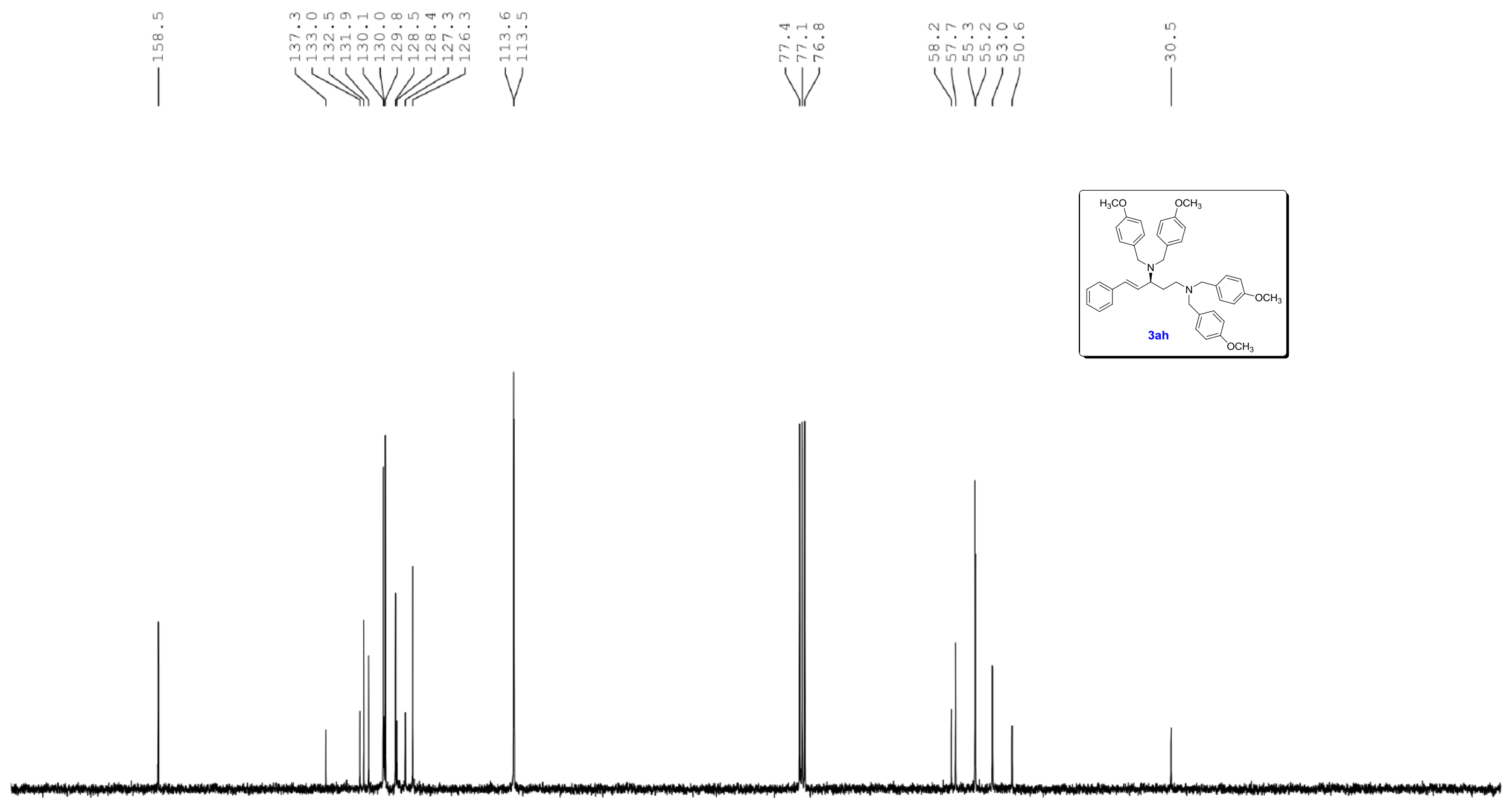

$\begin{array}{lllllllllllllllllll}170 & 160 & 150 & 140 & 130 & 120 & 110 & 100 & 90 & 80 & 70 & 60 & 50 & 40 & 30 & 20 & 10 & 0 & \text { ppm }\end{array}$



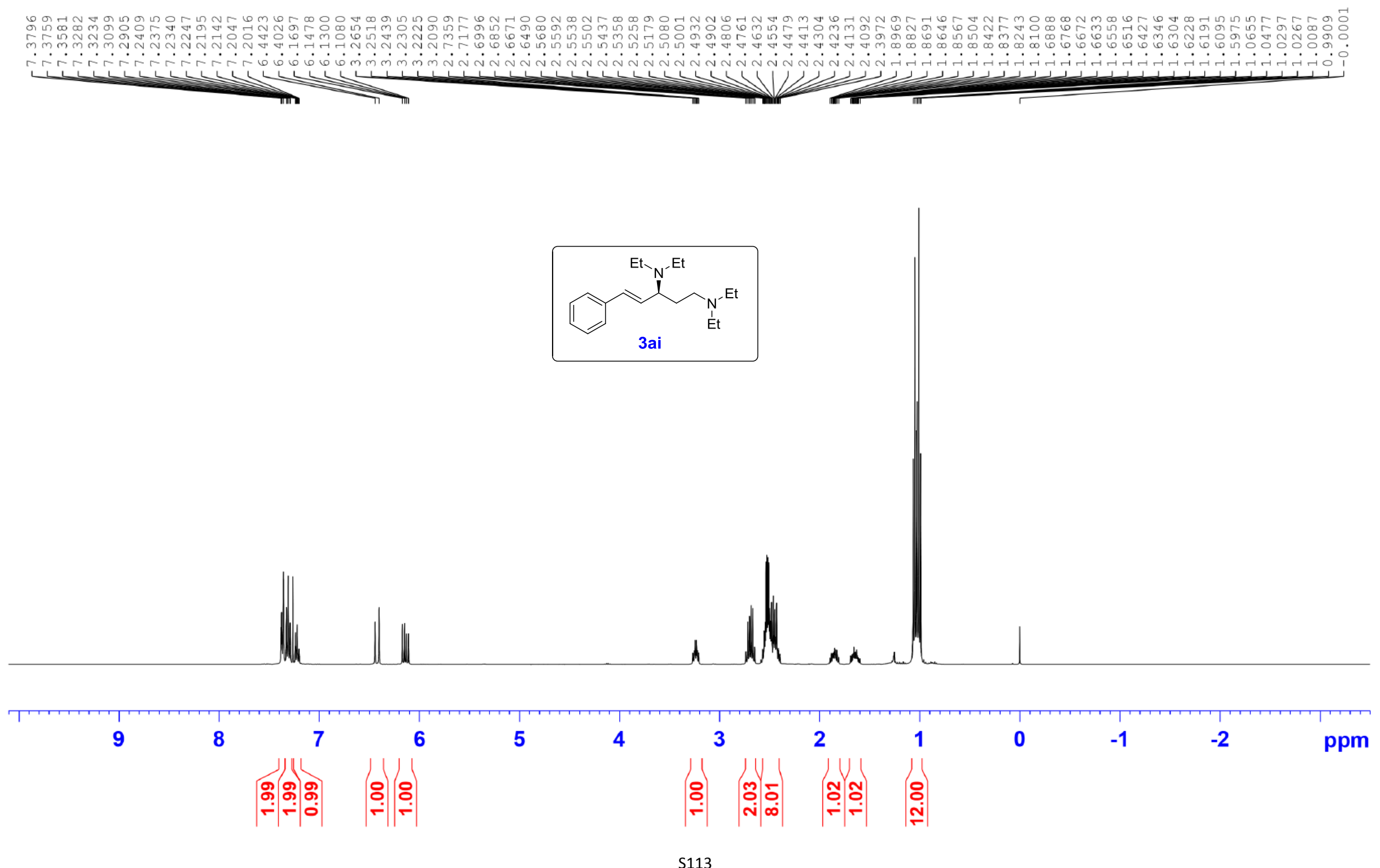

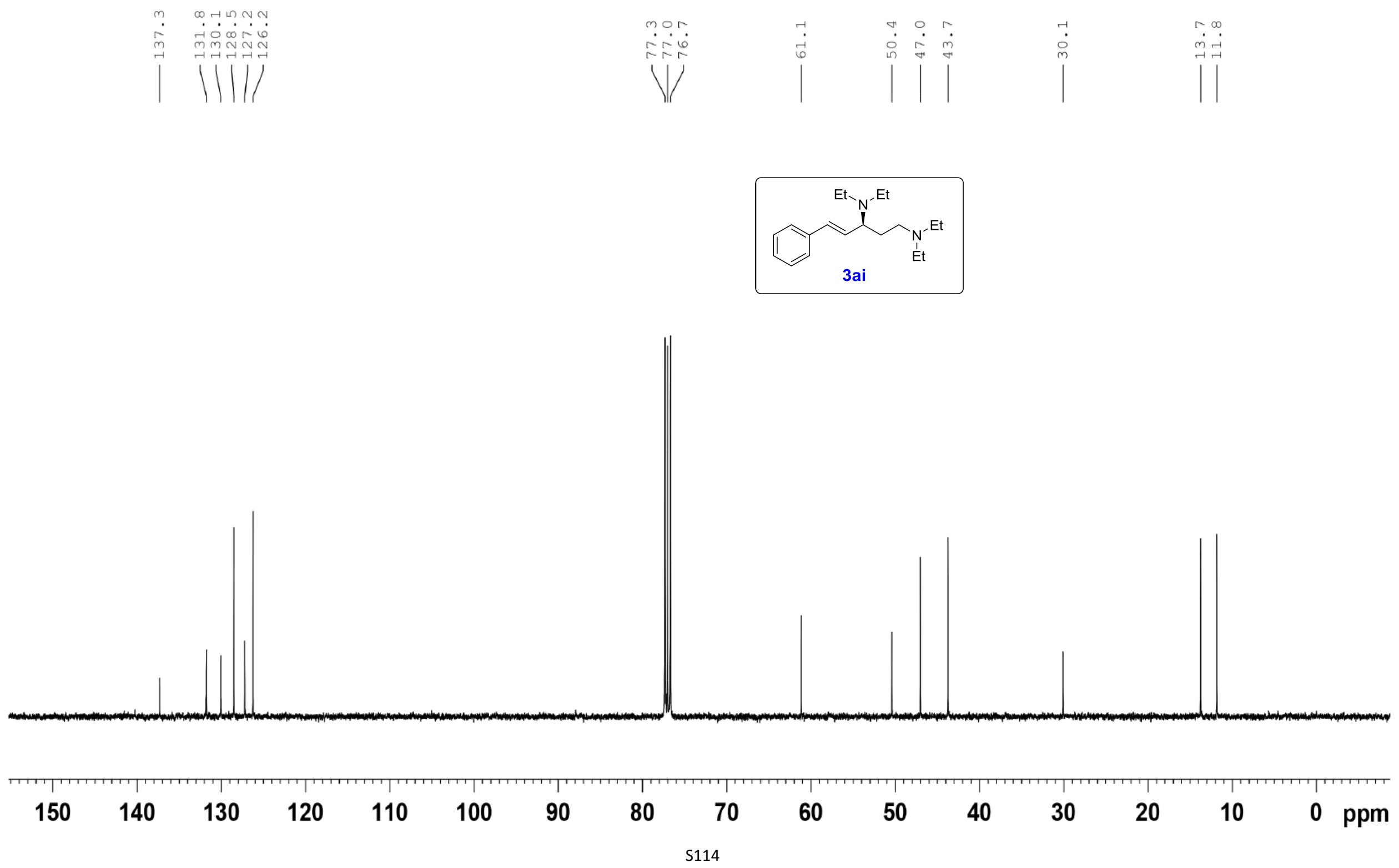
sen

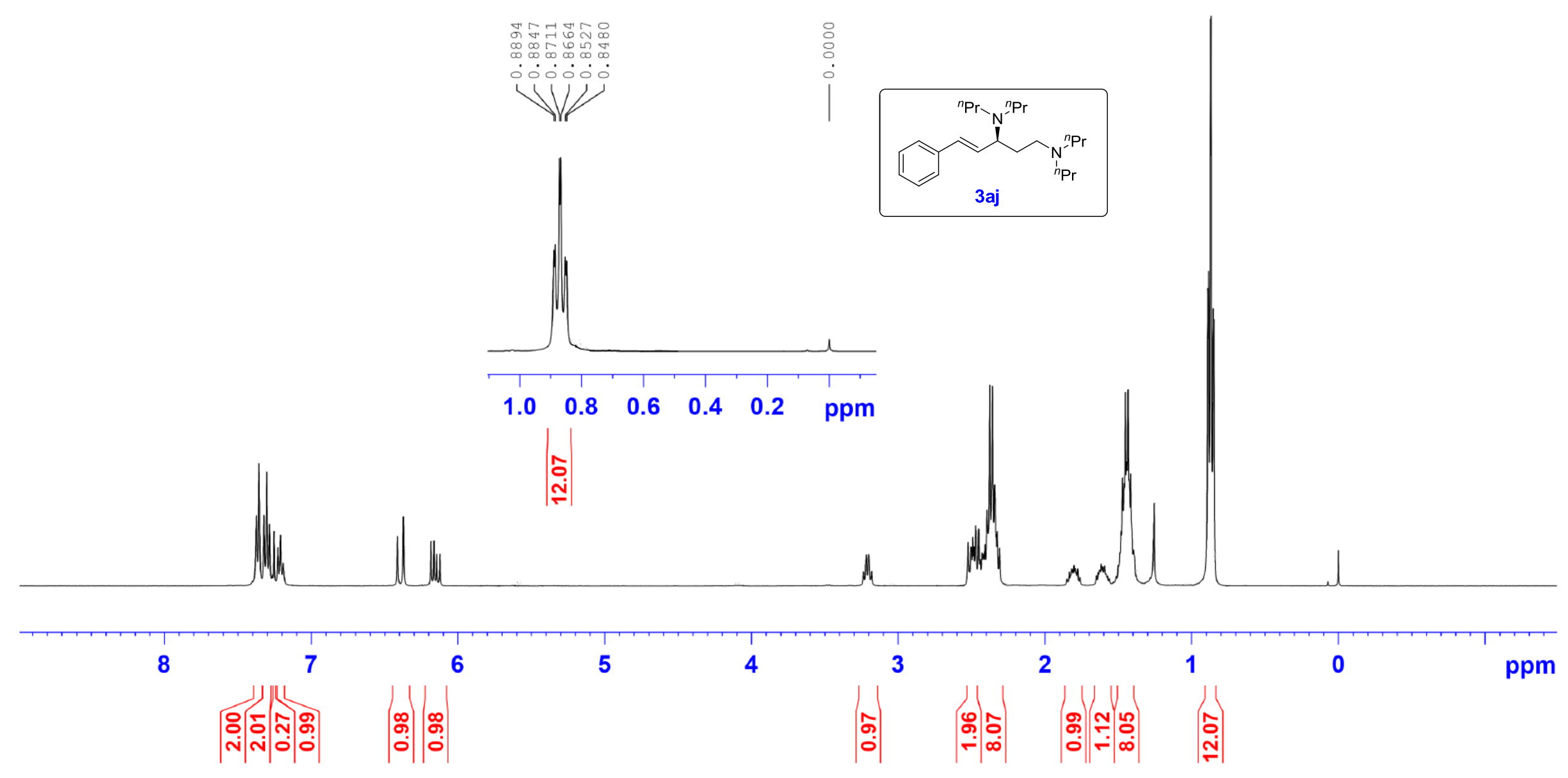



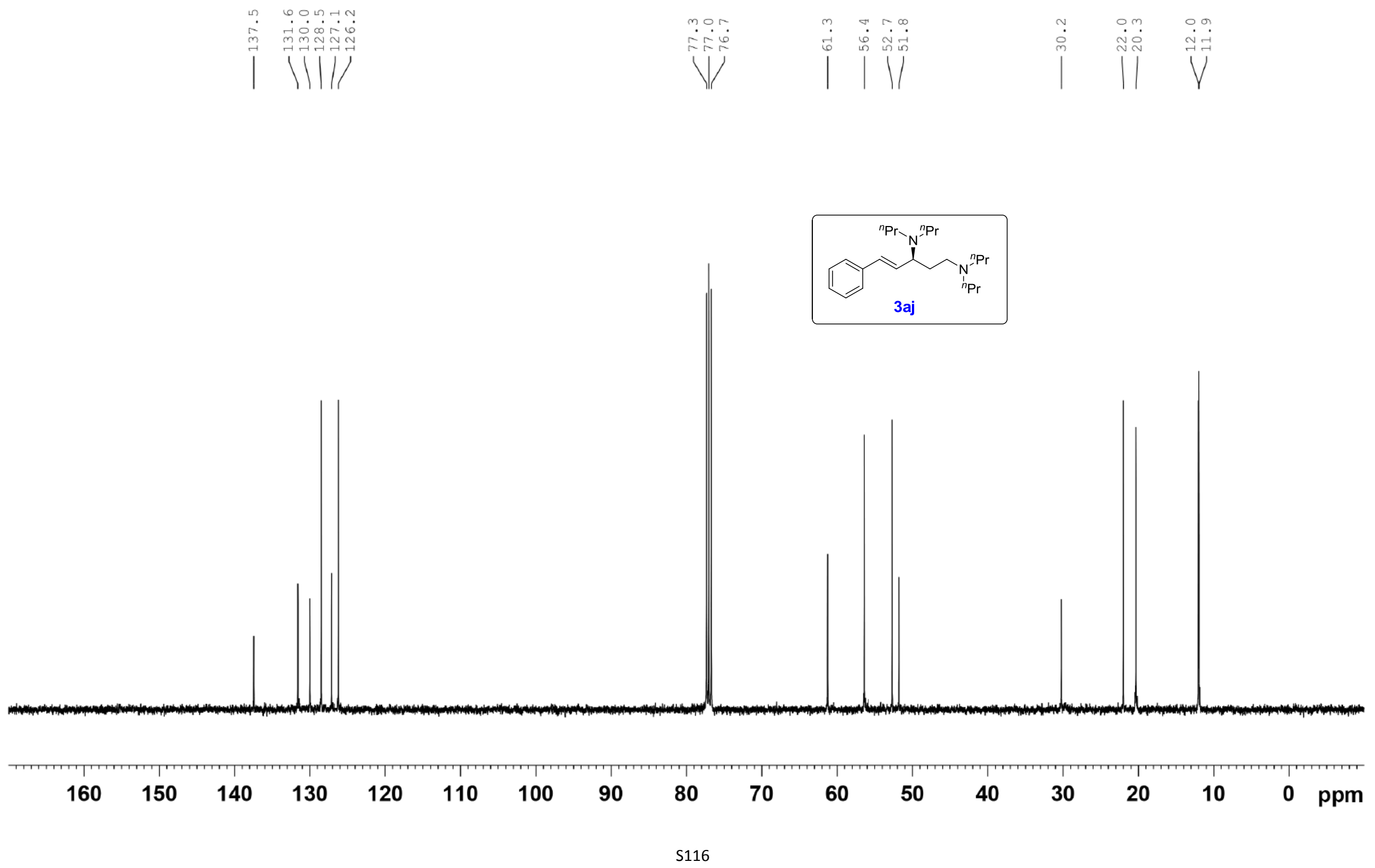


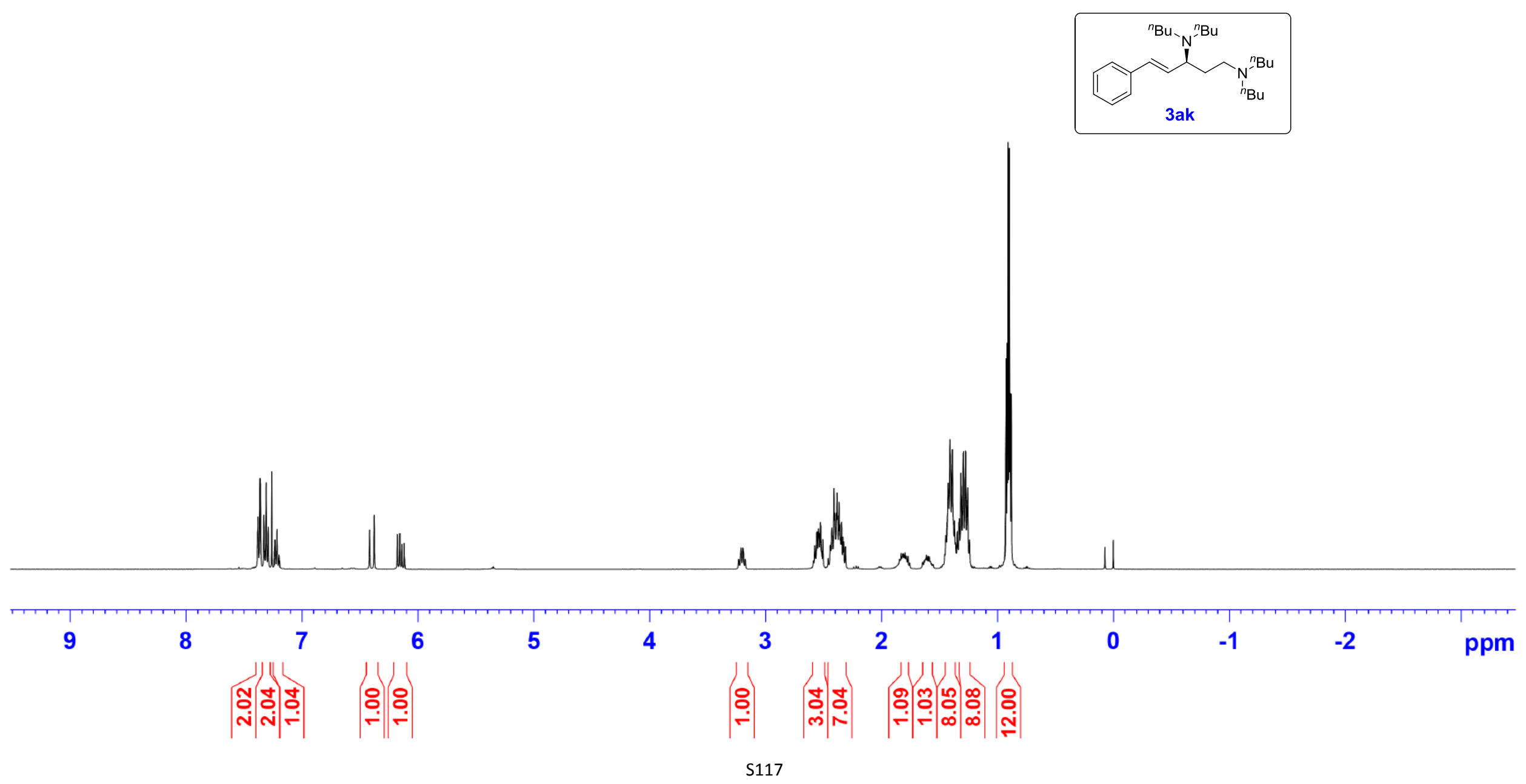



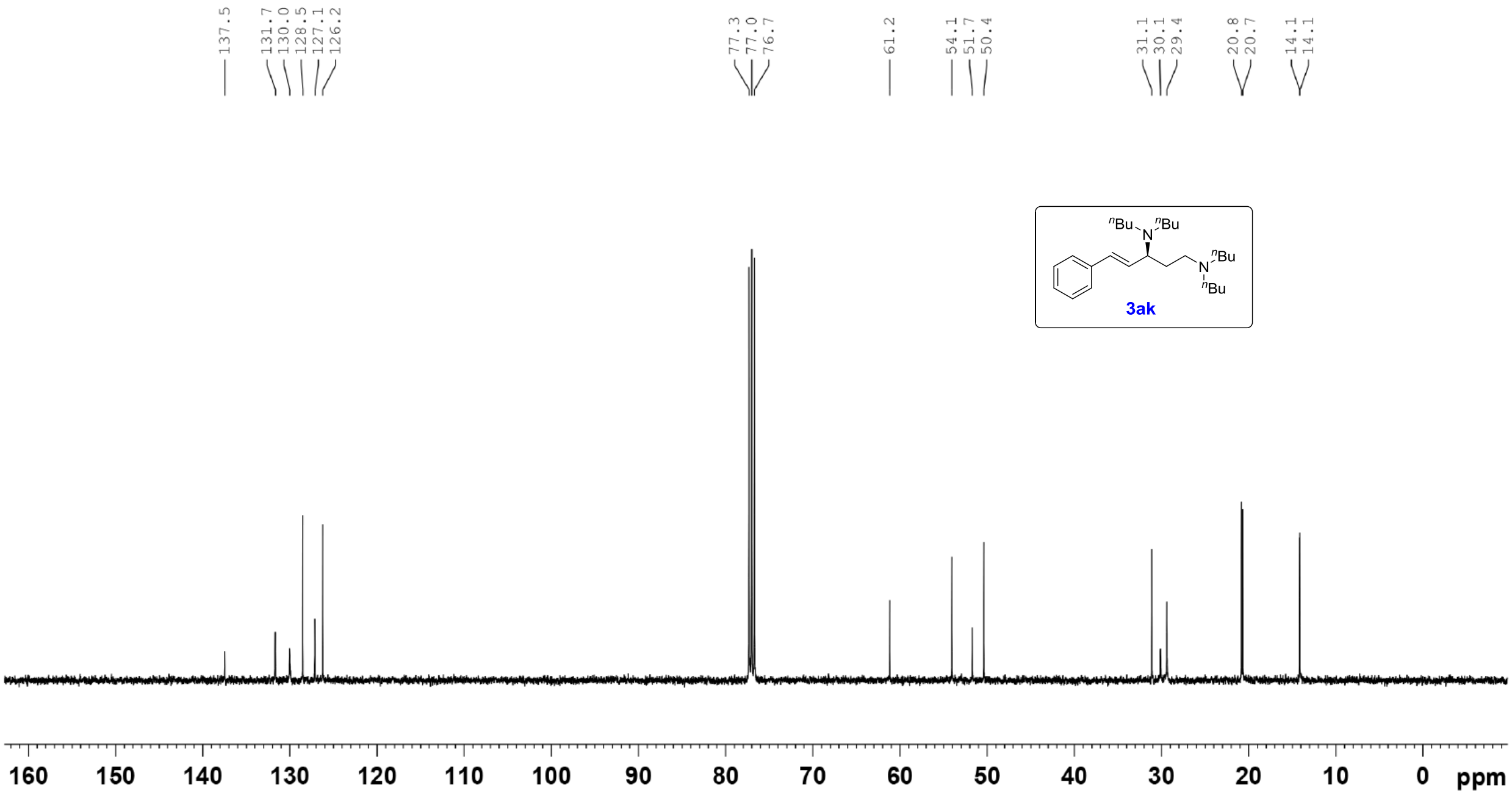

$\begin{array}{llllllllllll}00 & 90 & 80 & 70 & 60 & 50 & 40 & 30 & 20 & 10 & 0 & \text { ppm }\end{array}$




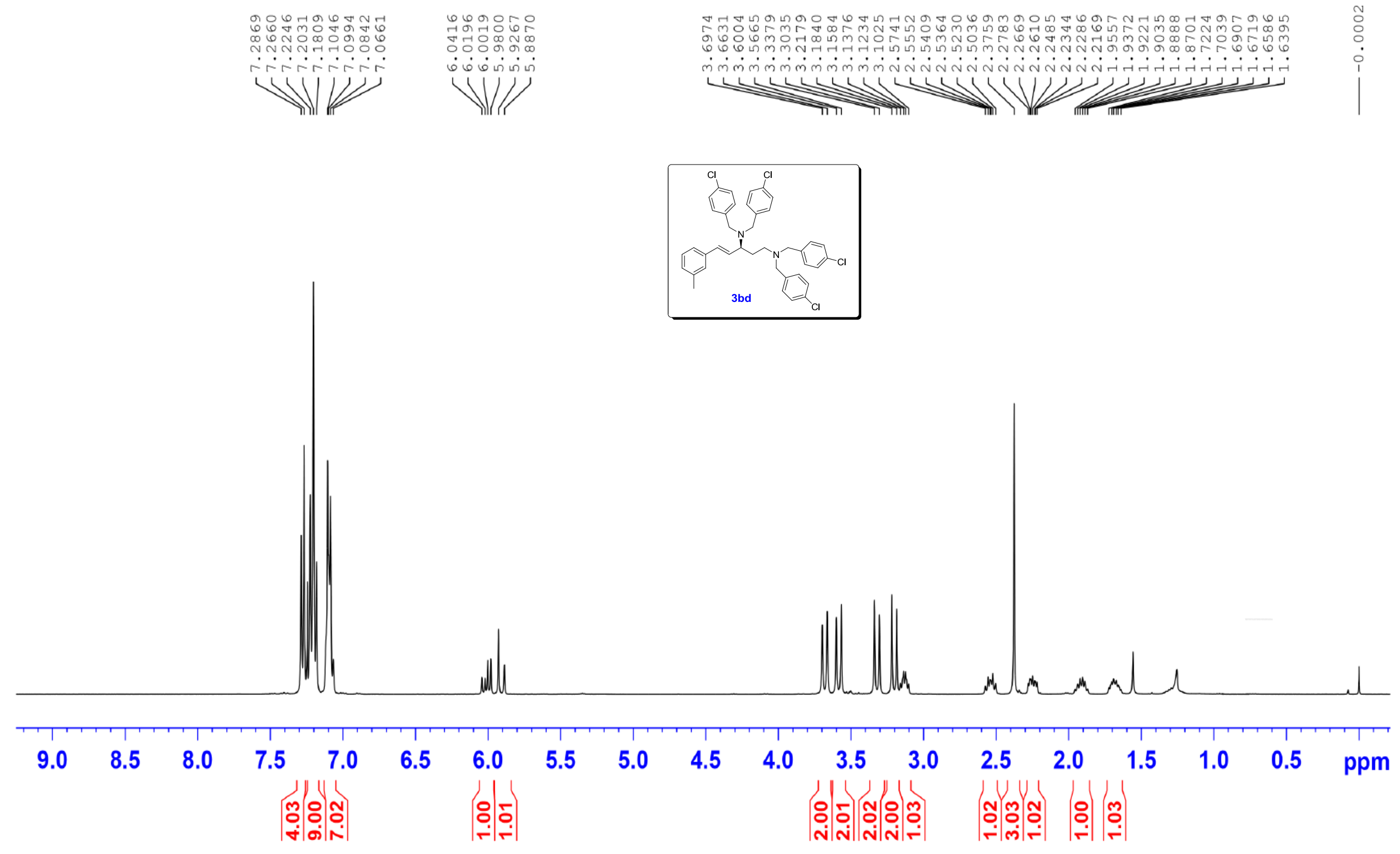




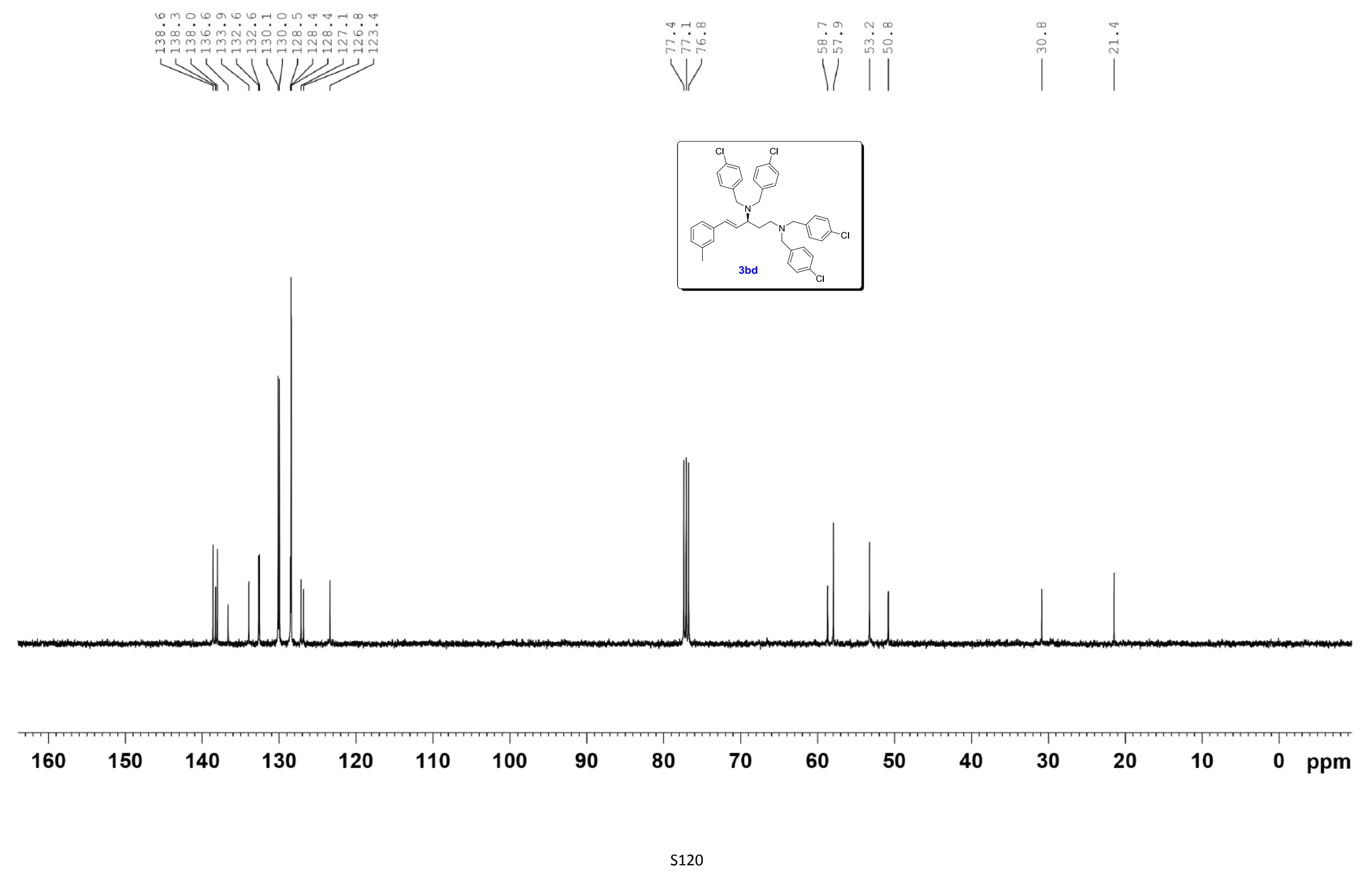



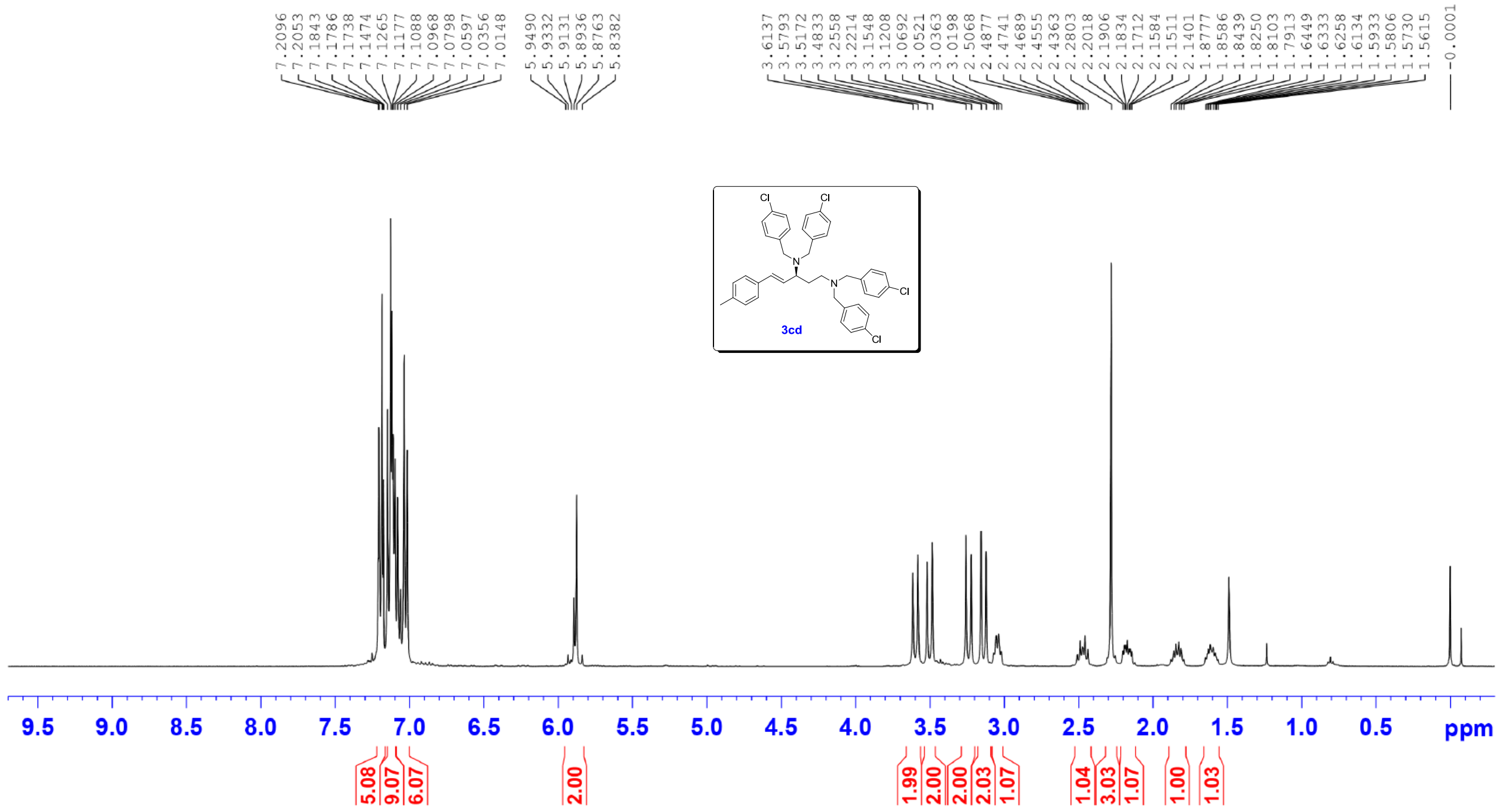

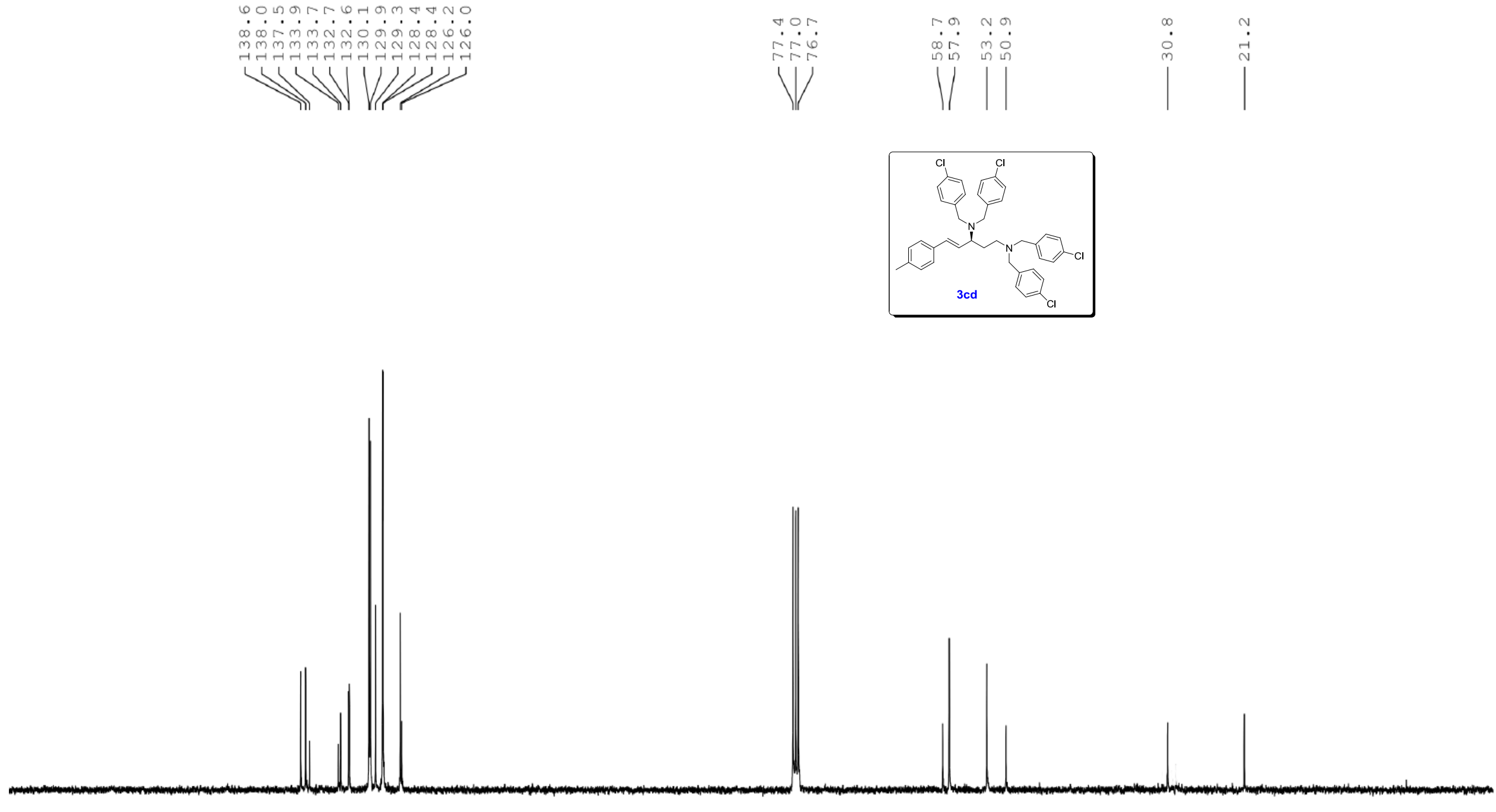
$170 \quad 160$
$150 \quad 140$
130
$120 \quad 11$
$100 \quad 90$
$80 \quad 70 \quad 60$
$\begin{array}{lll}60 & 50 \quad 40\end{array}$
$40 \quad 30$
$20 \quad 10 \quad 0 \quad \mathrm{ppm}$ 


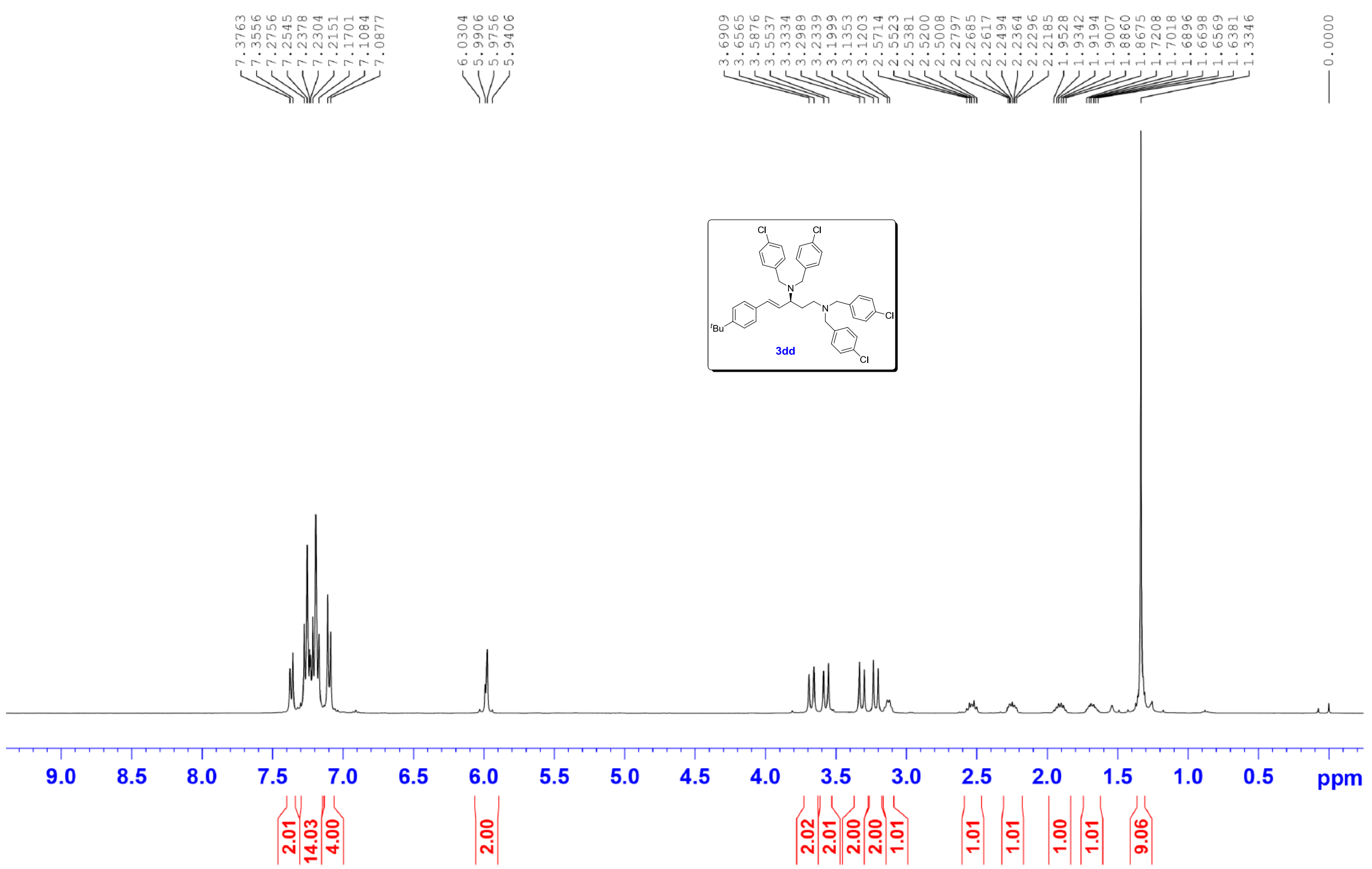



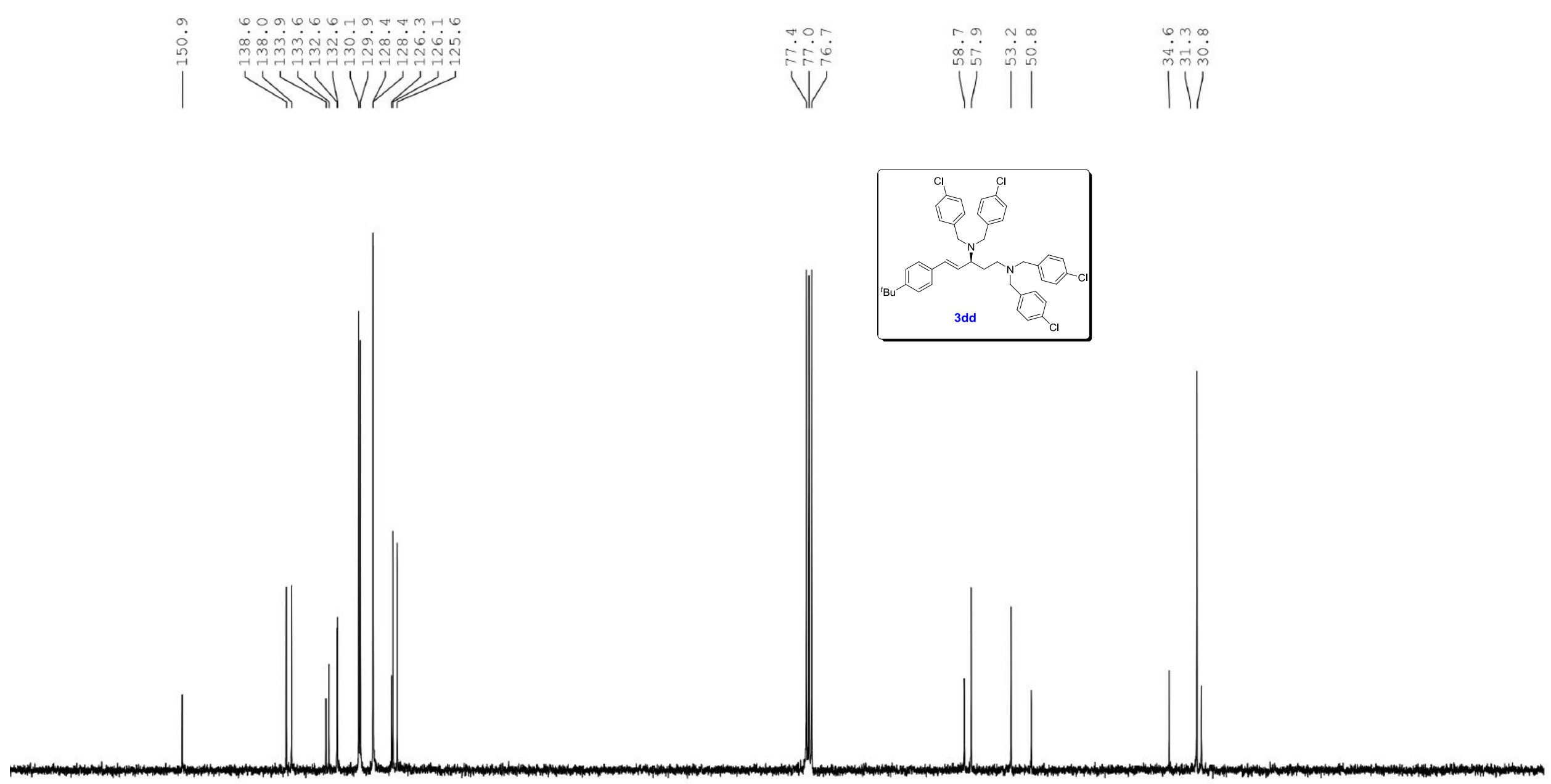
$160 \quad 150$
140
$130 \quad 120$
110100
90
80
70
60
50
$30 \quad 20$
$10 \quad 0$ ppm

40 

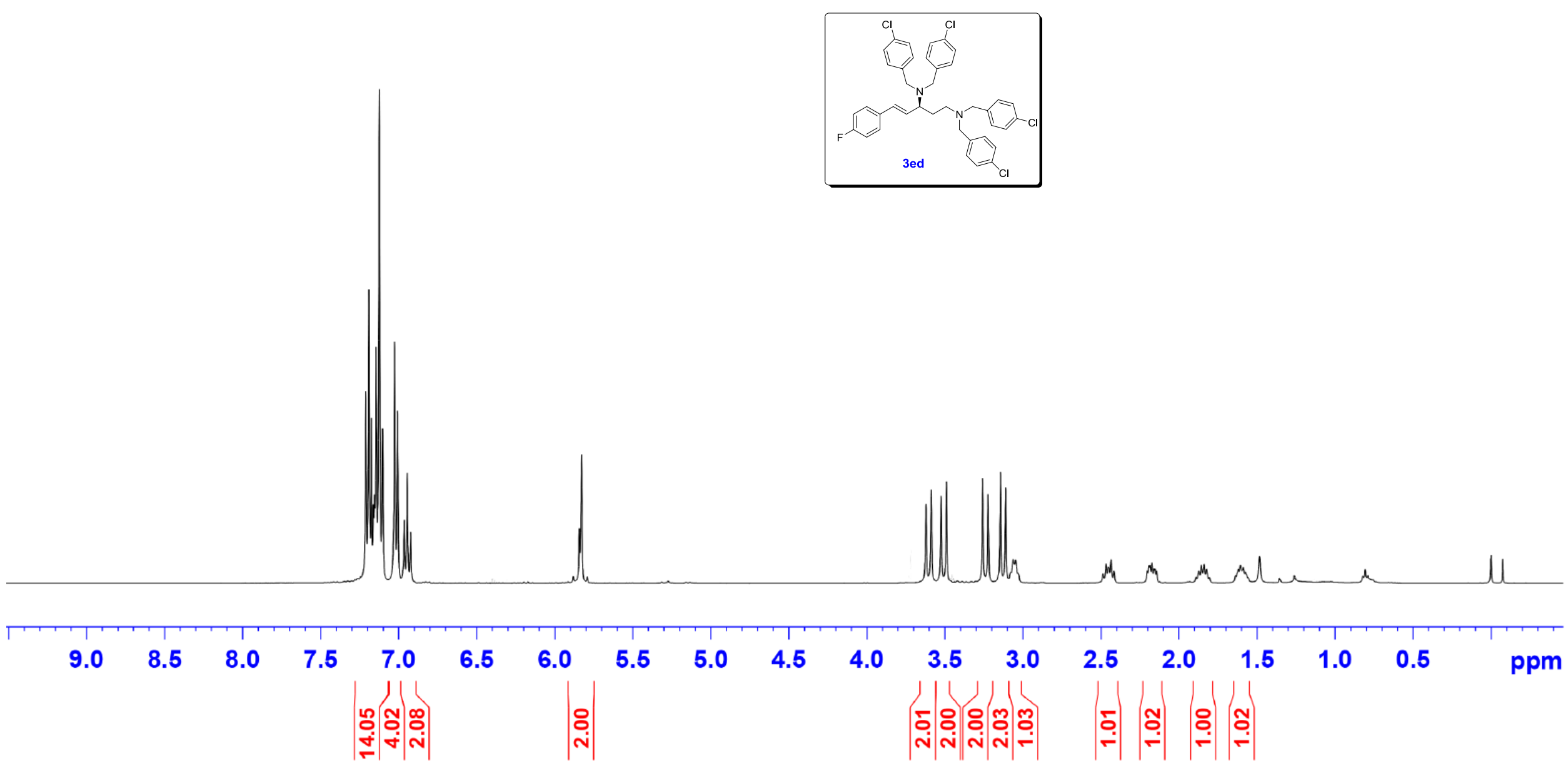


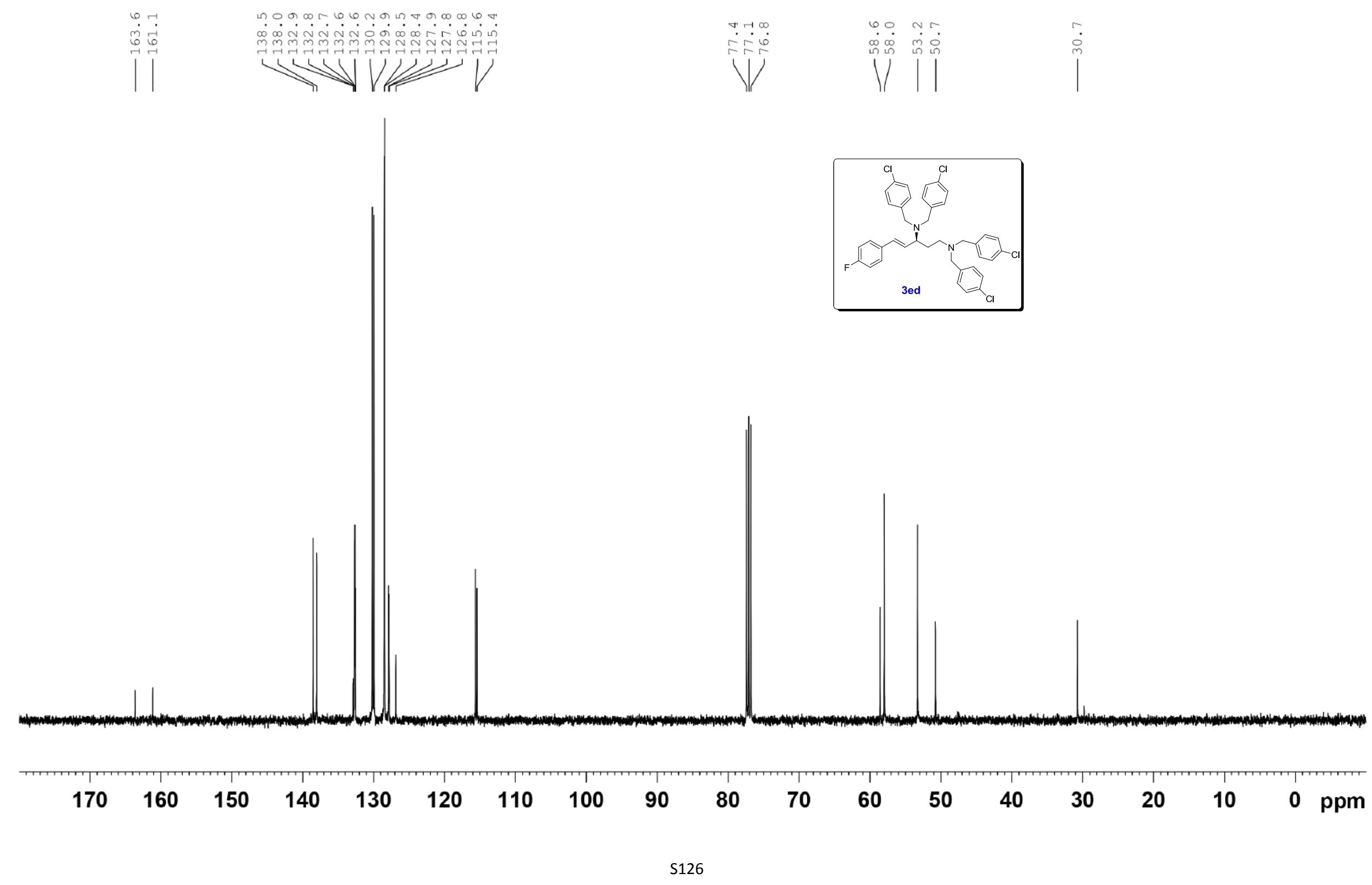




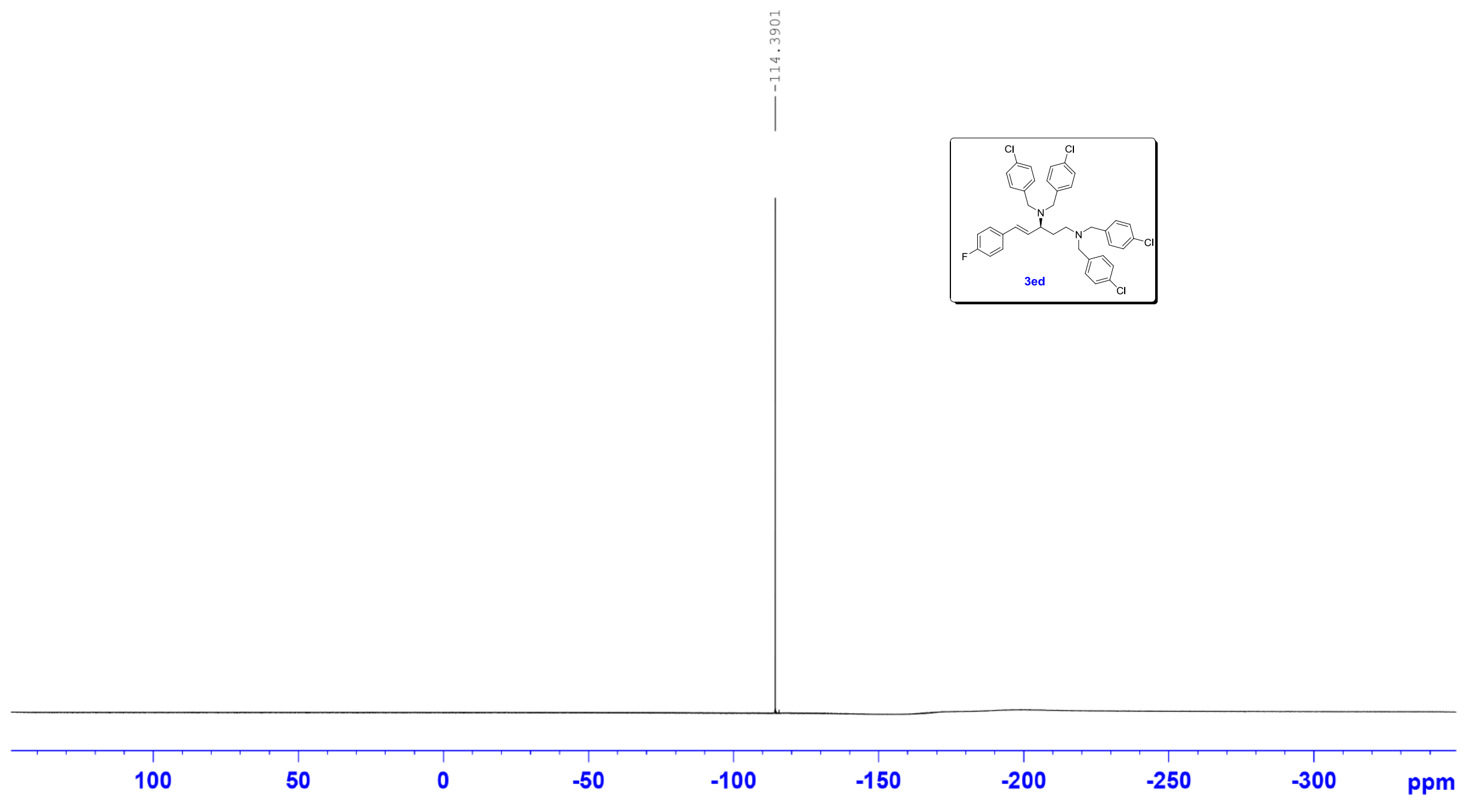



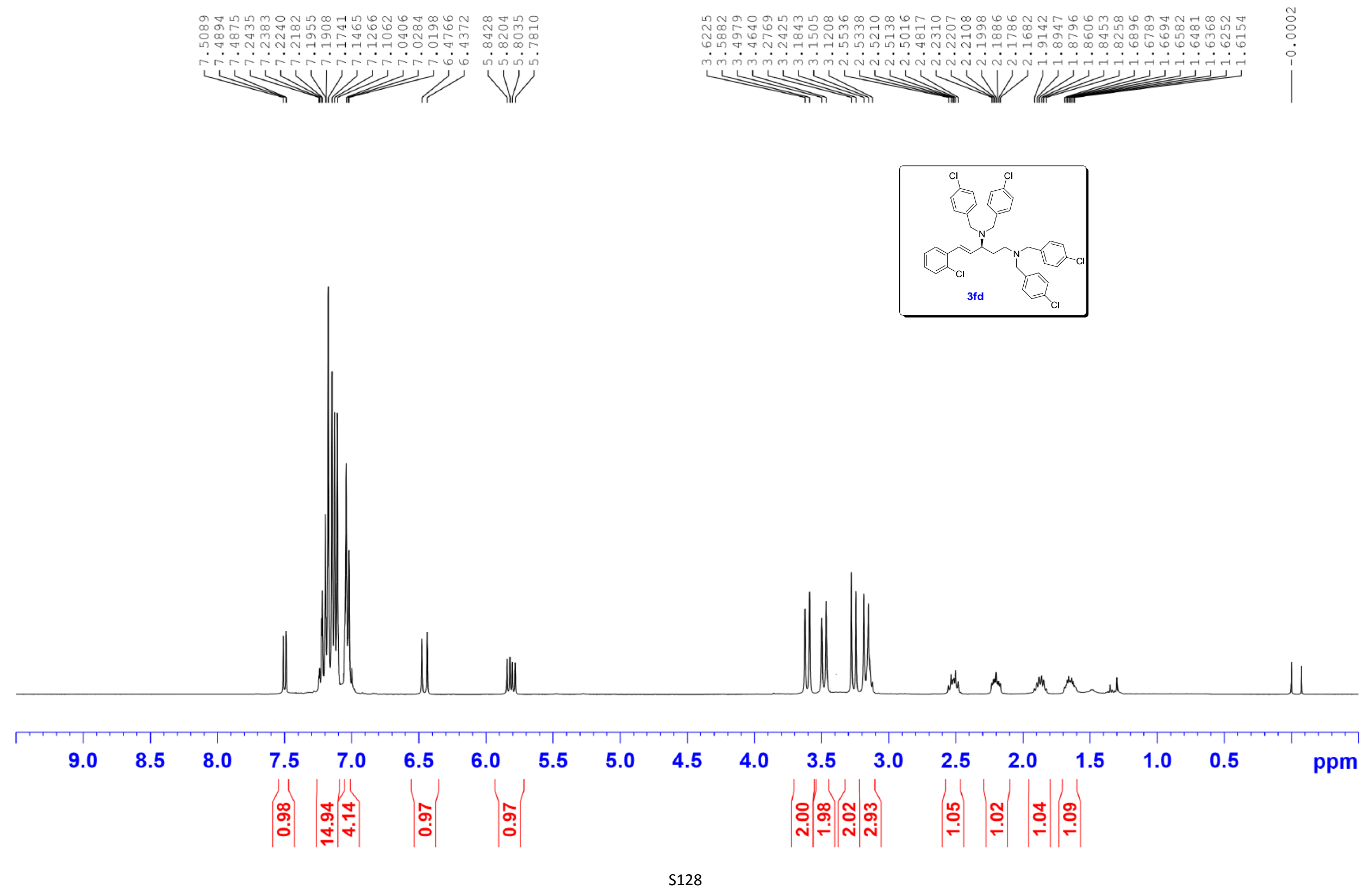

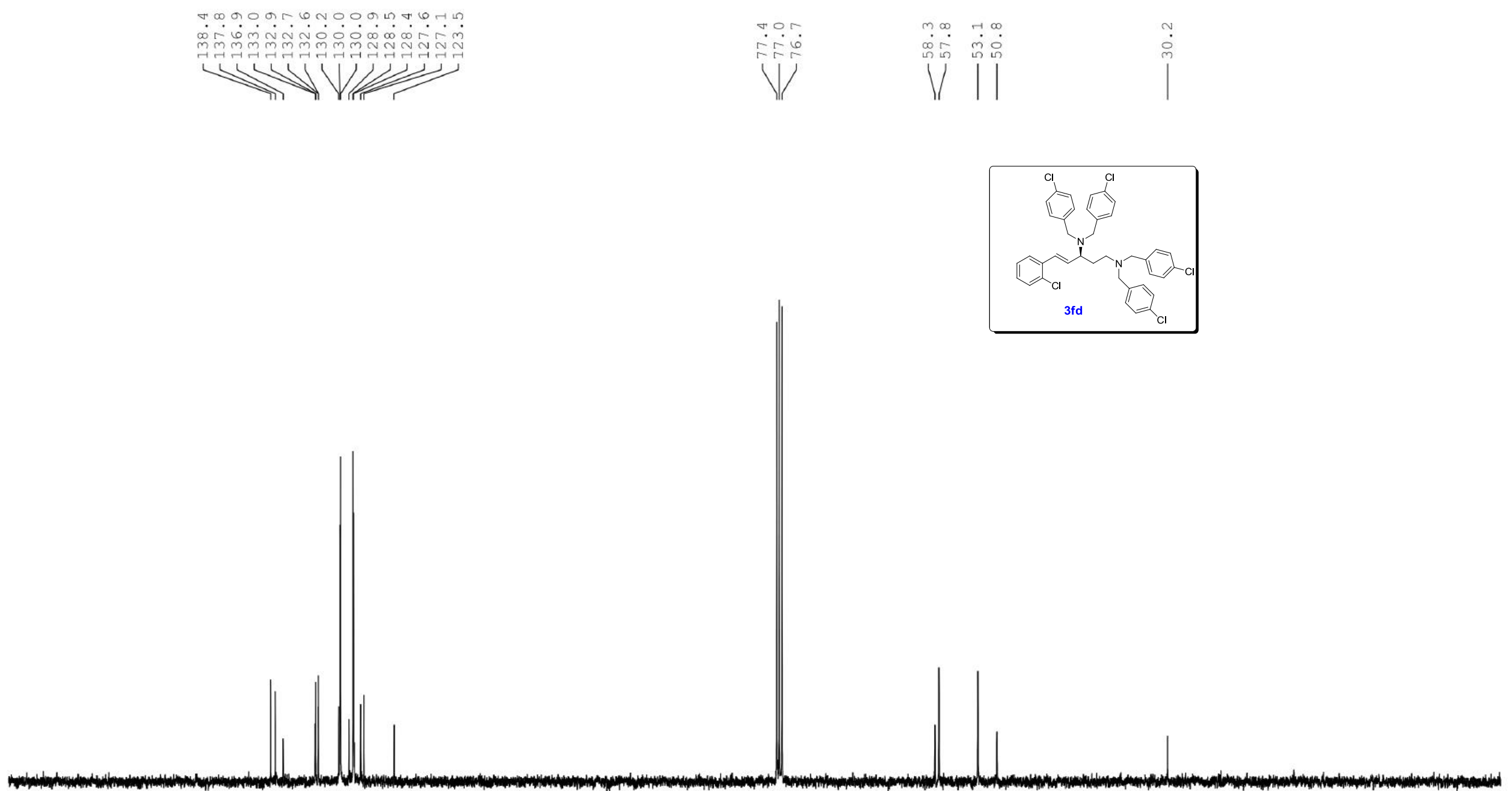

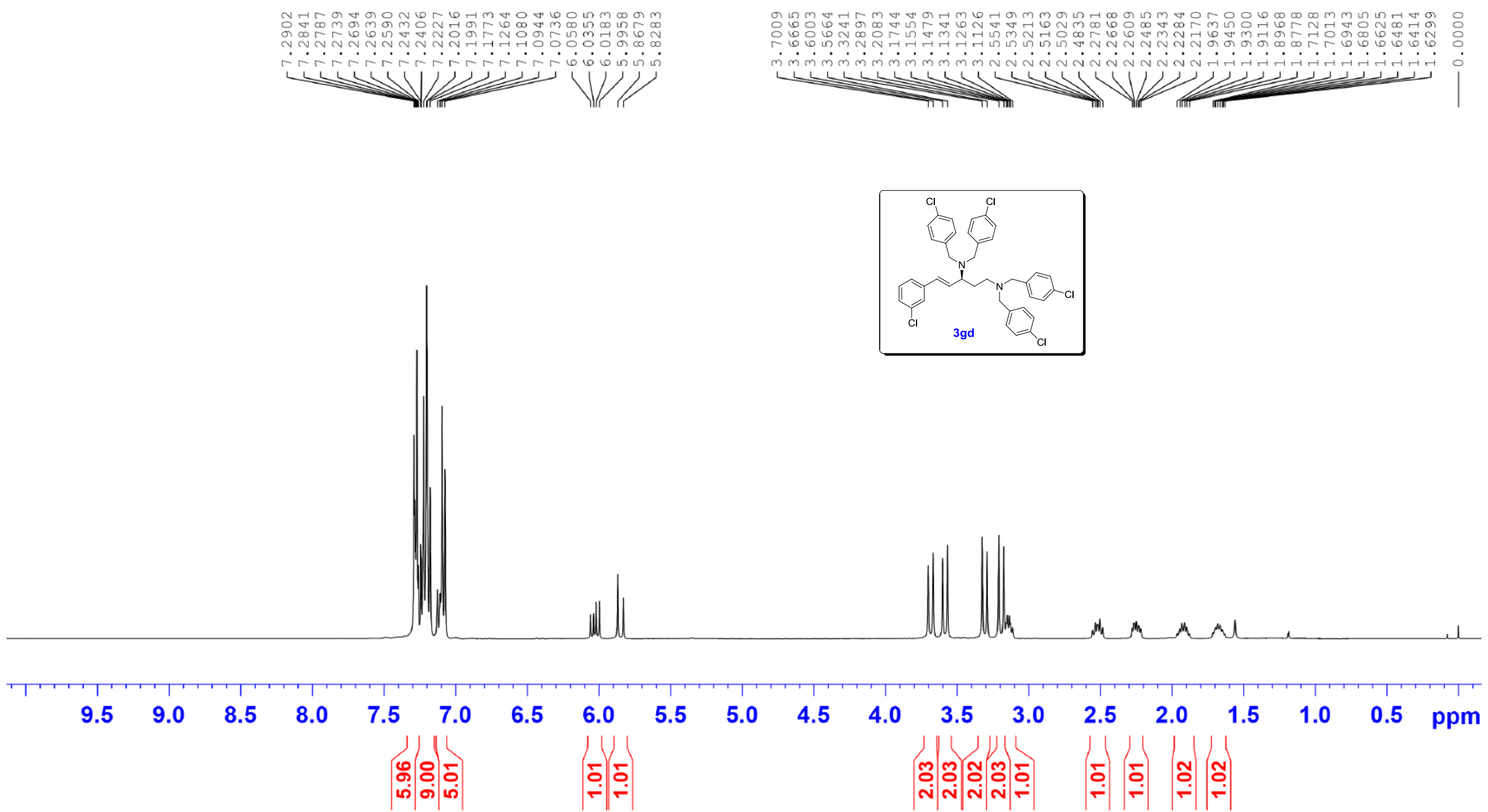

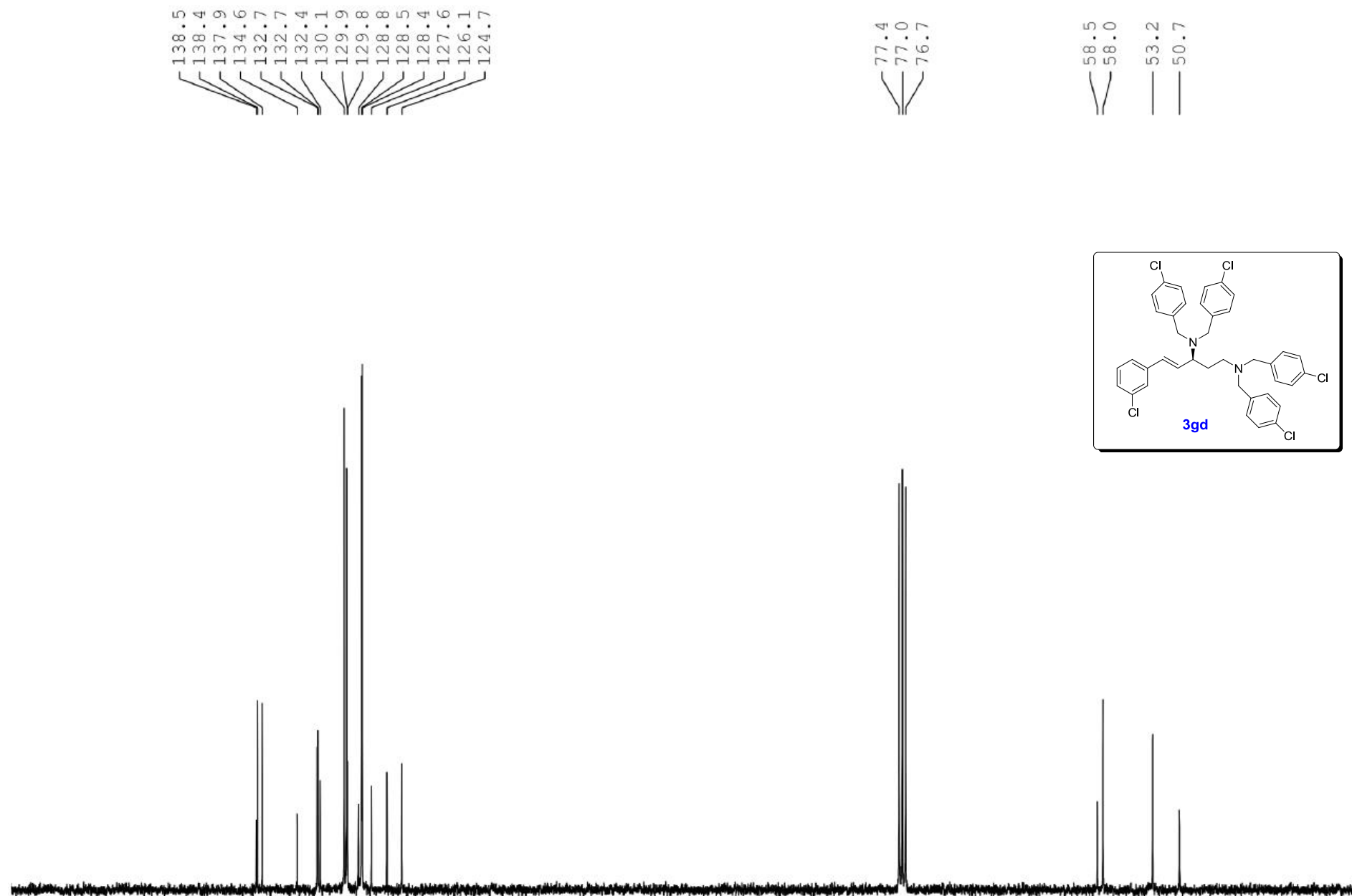


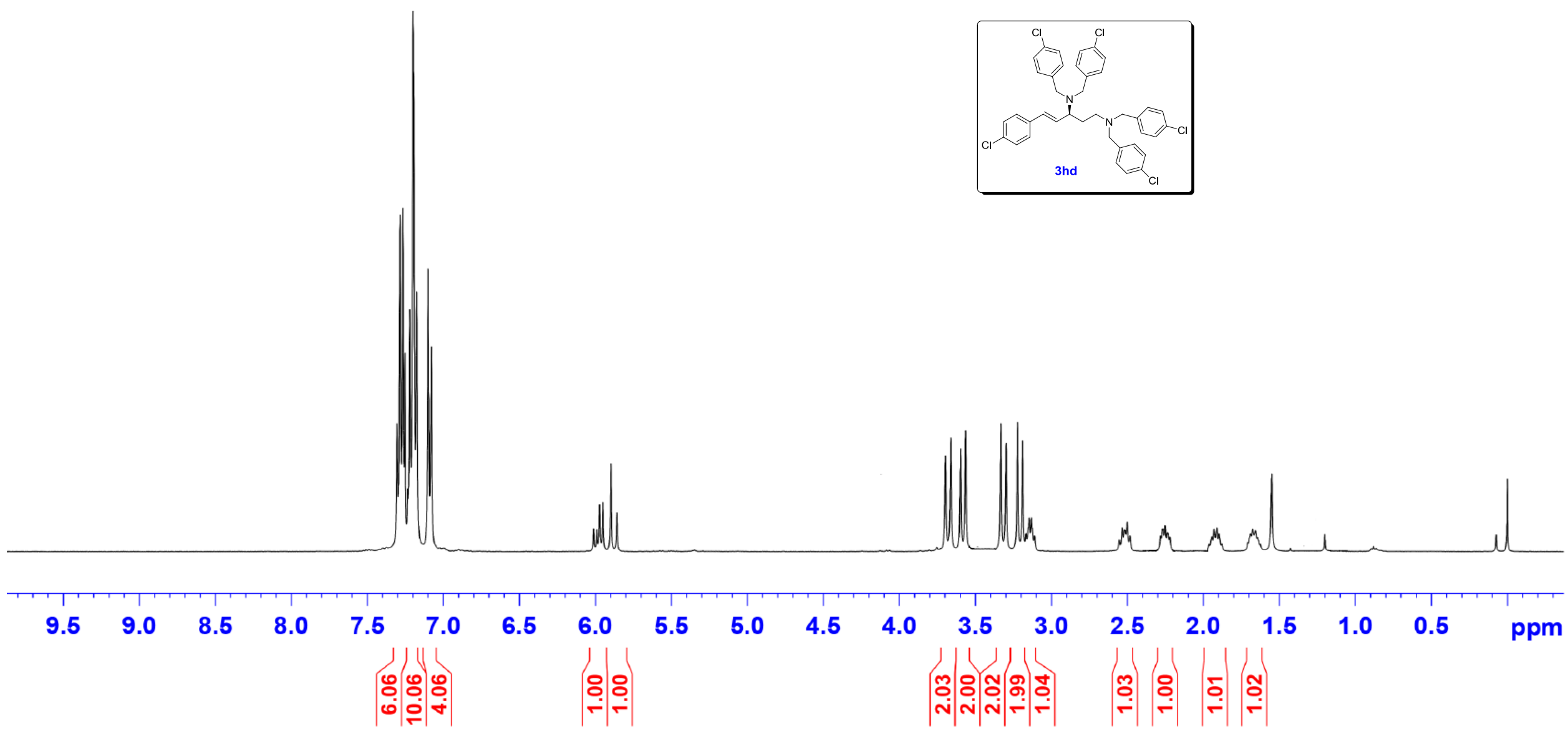




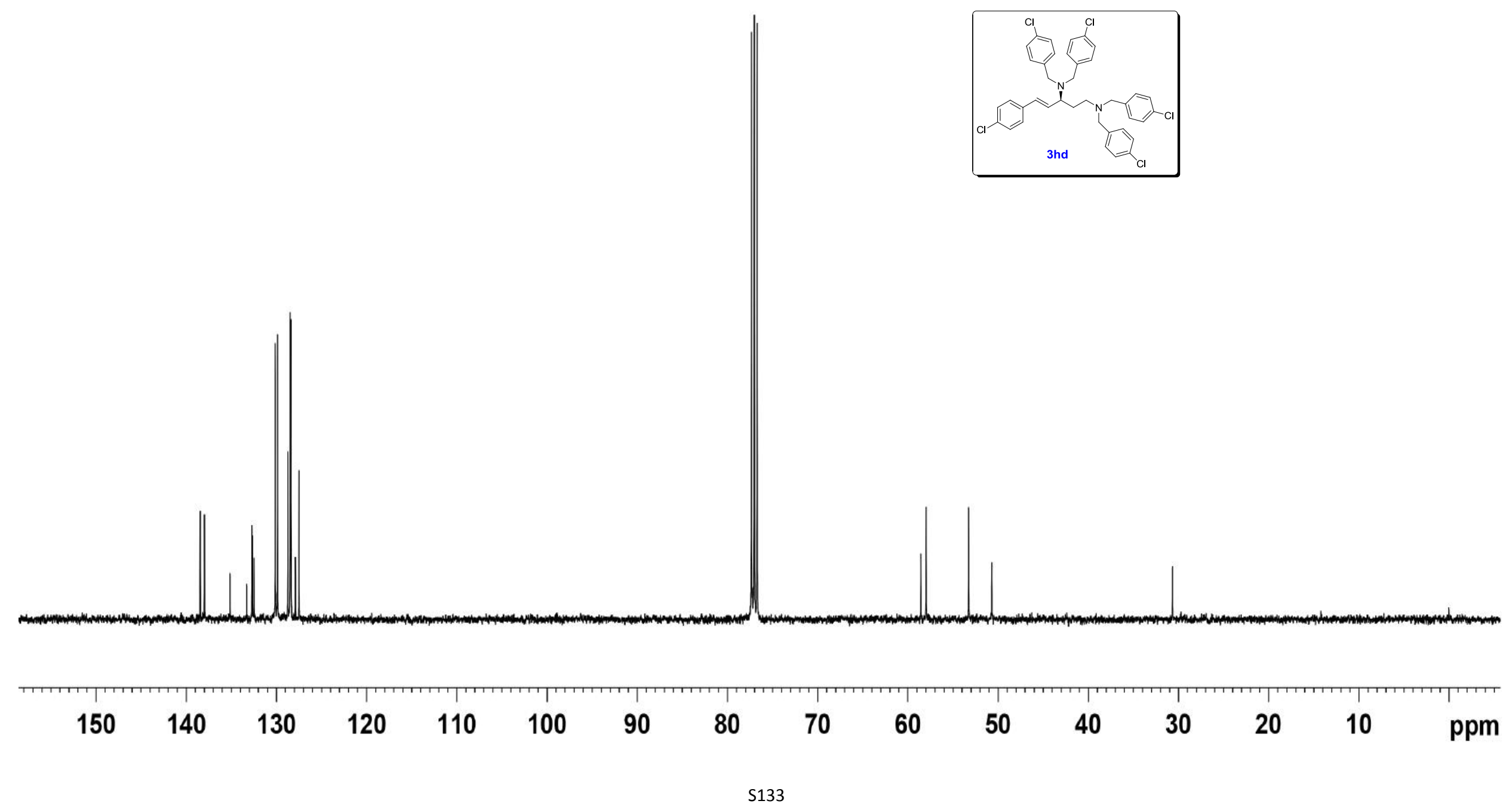



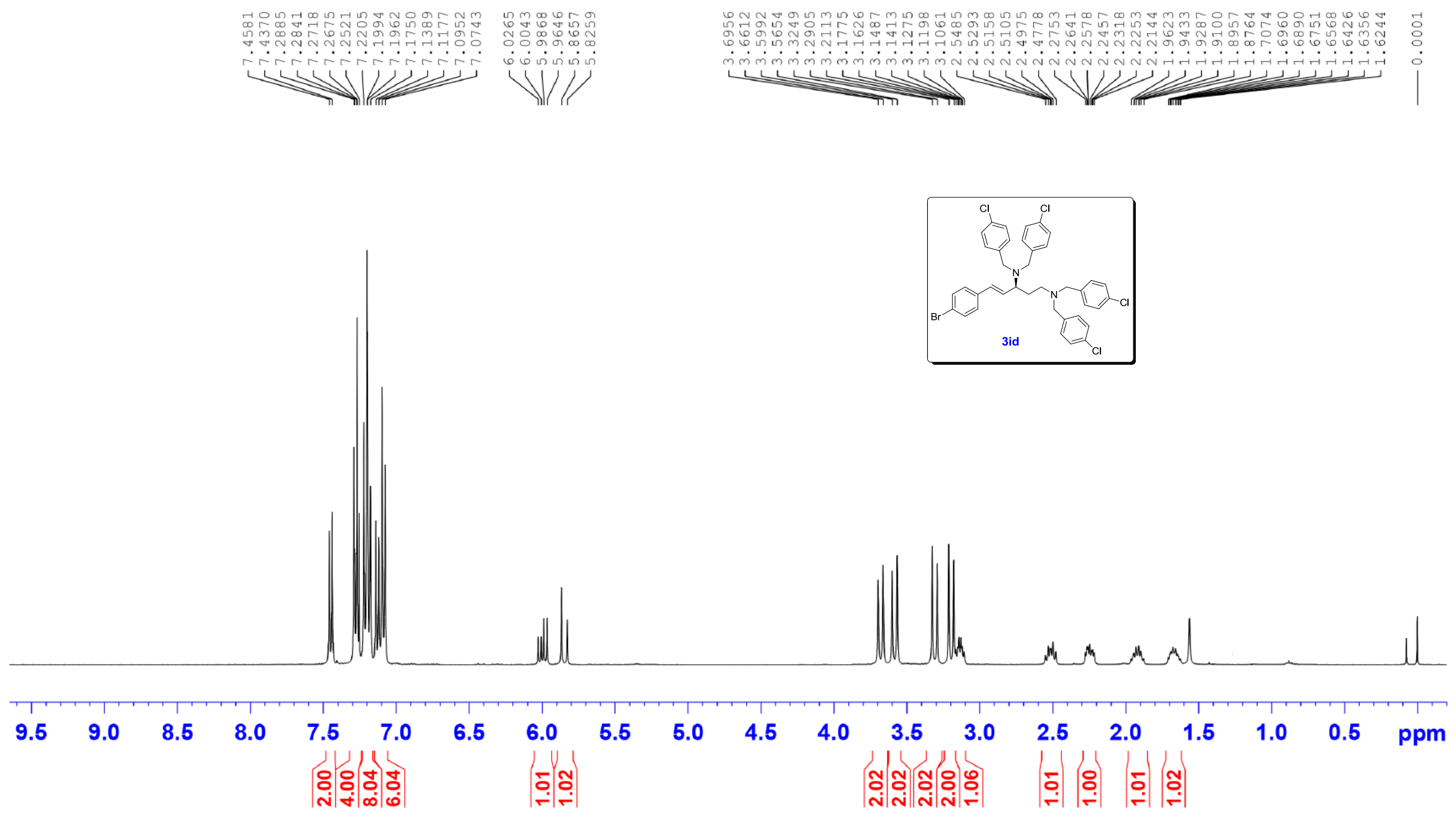


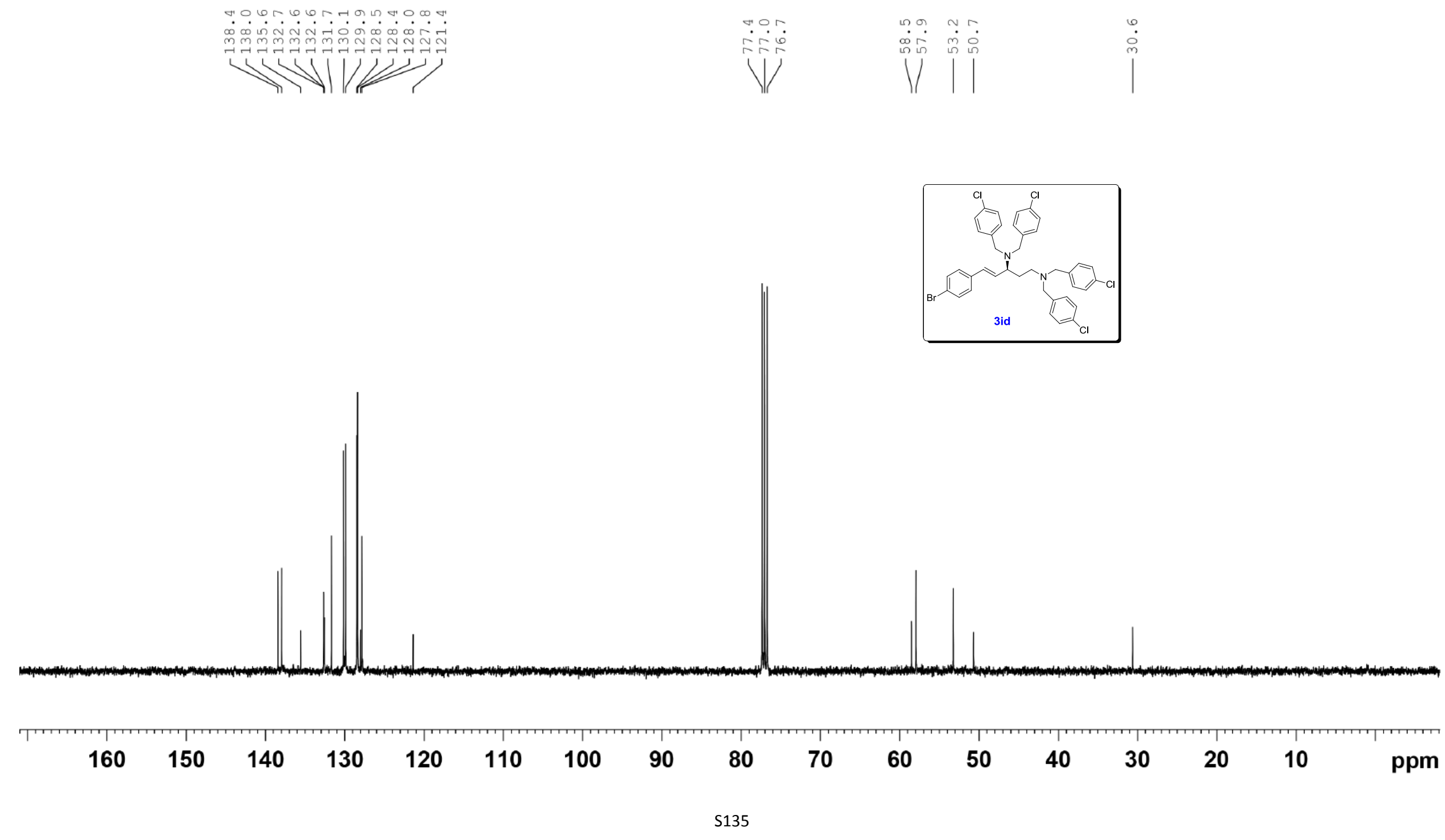




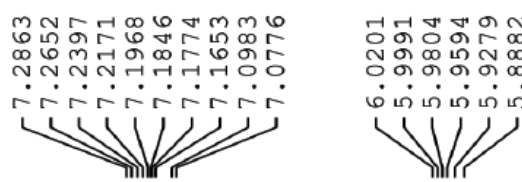

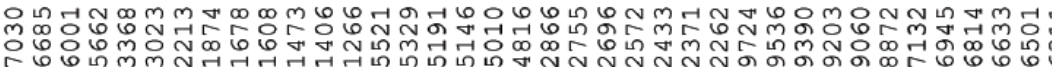
ๆ

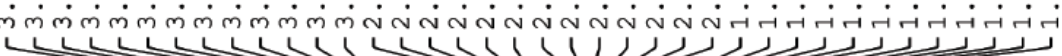
1 ता तो
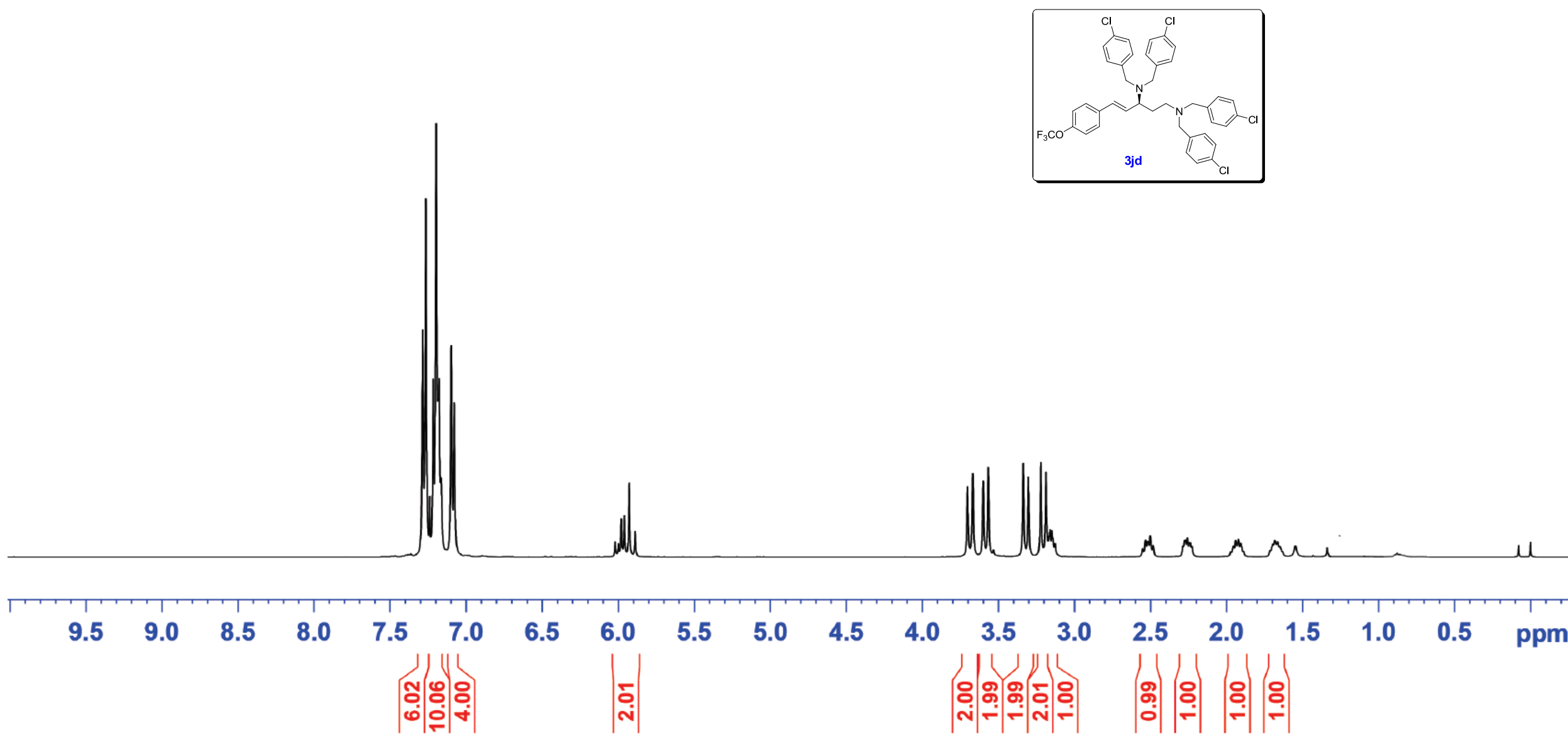

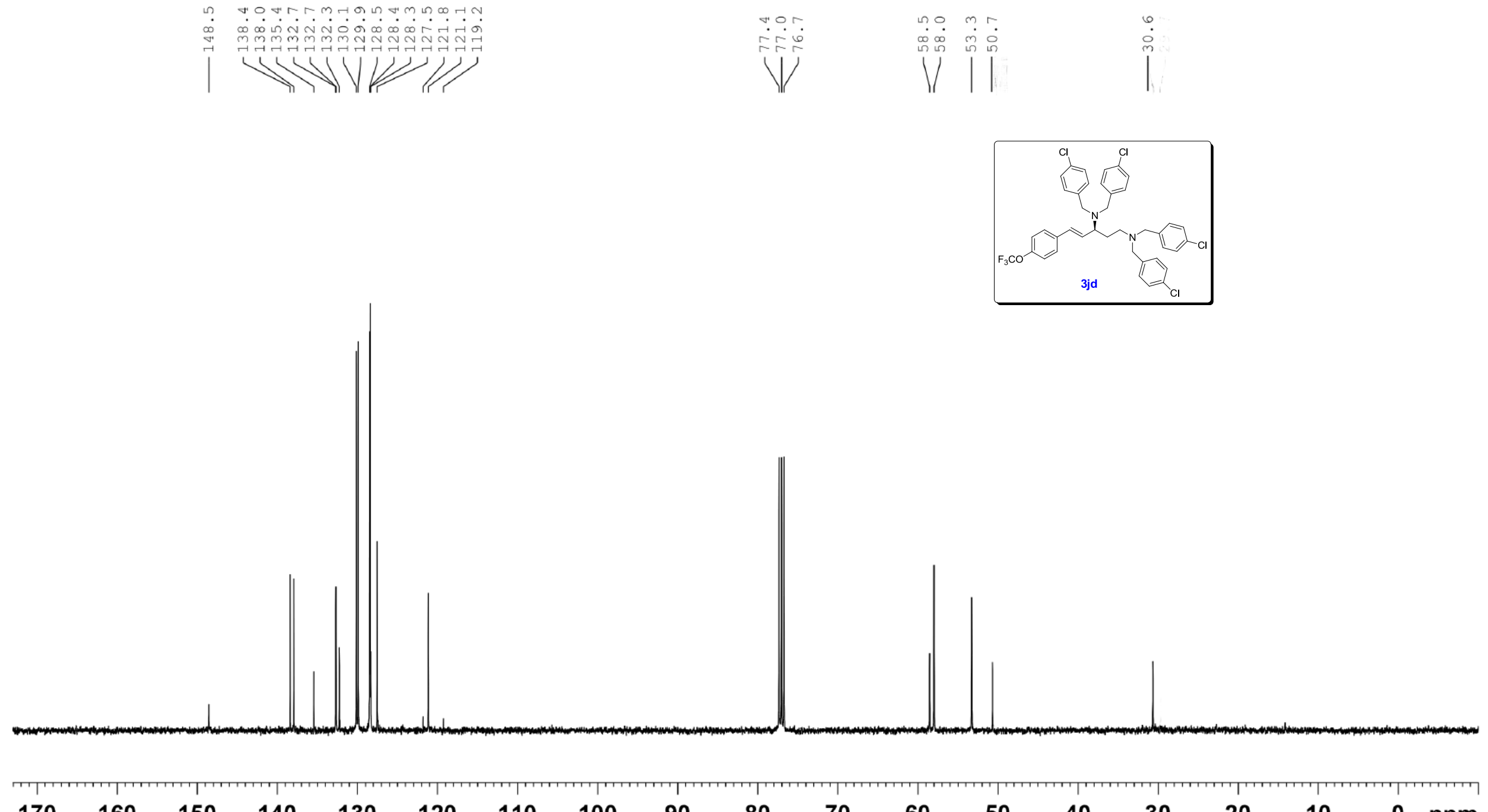

$170 \quad 160$

$150 \quad 140$

$130 \quad 120$

110100

90

80

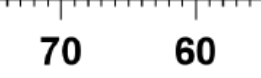

50

40

30

20 


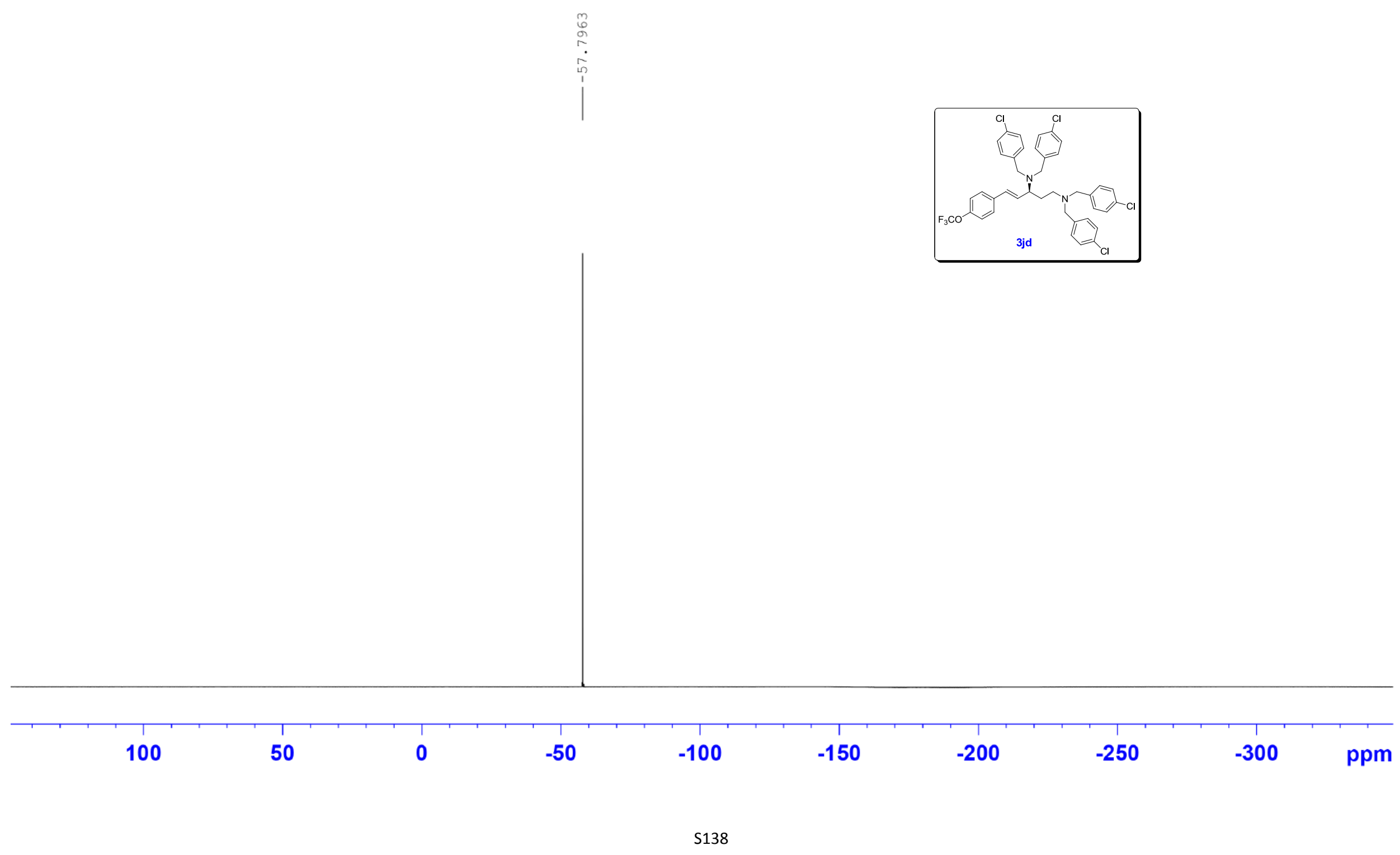




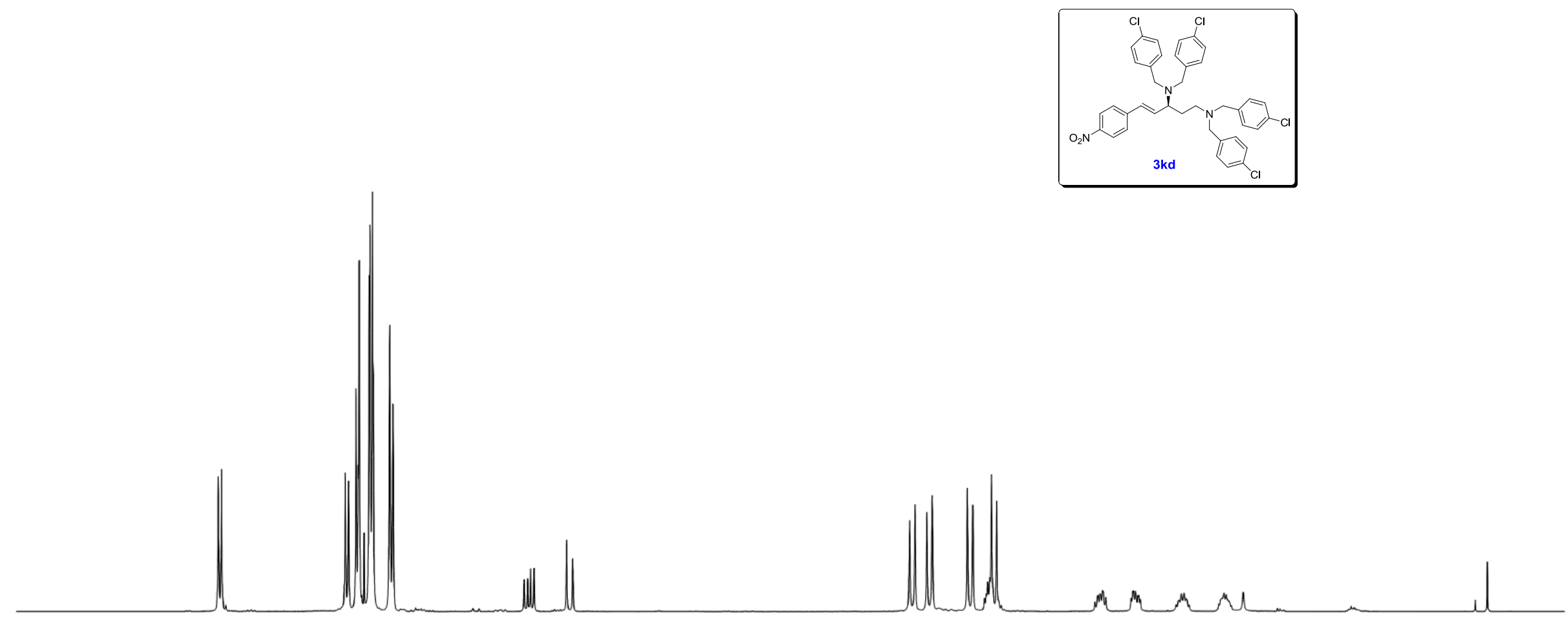

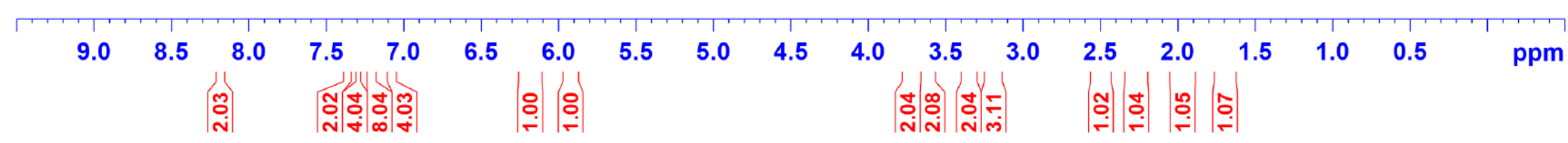



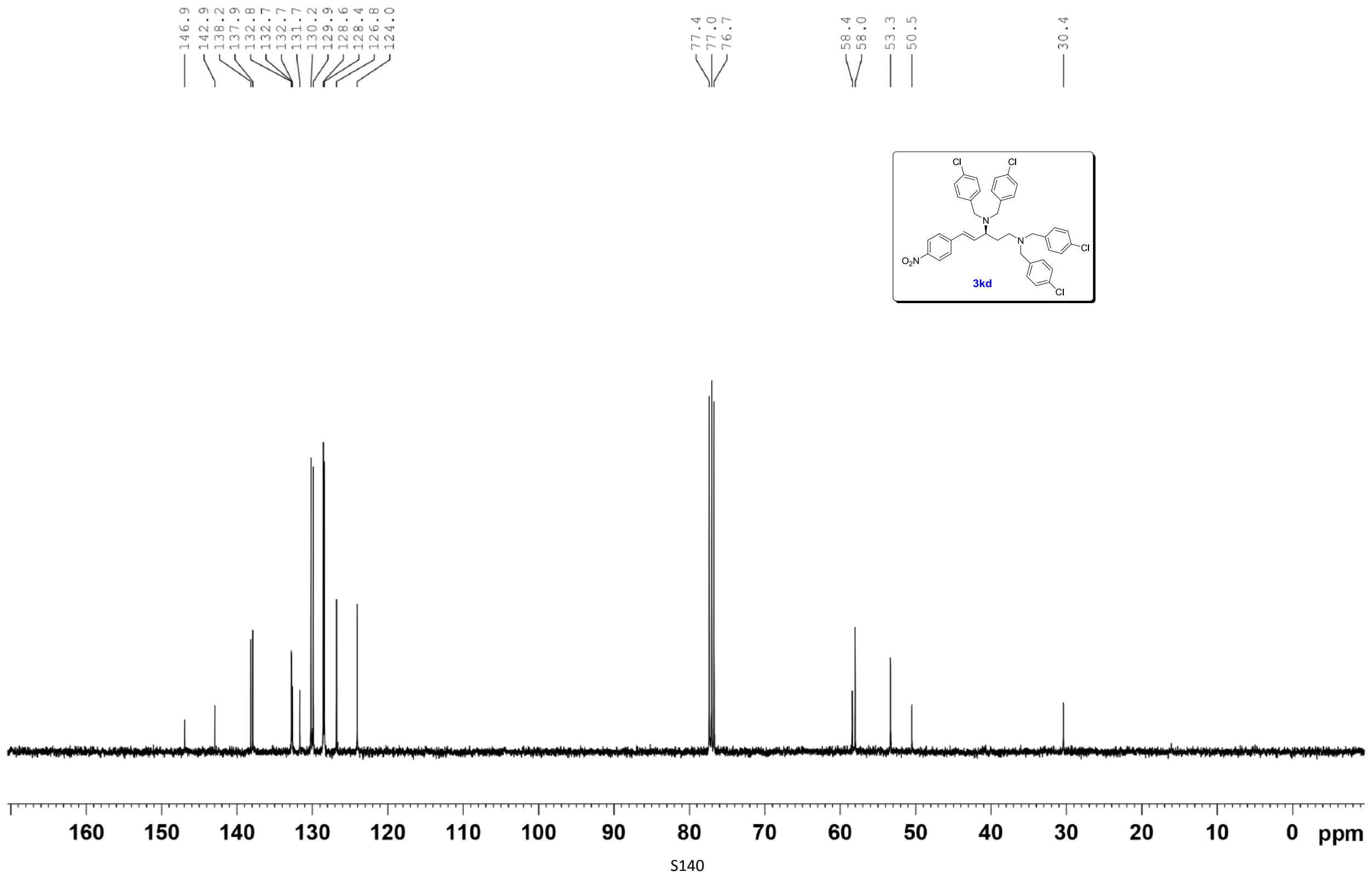

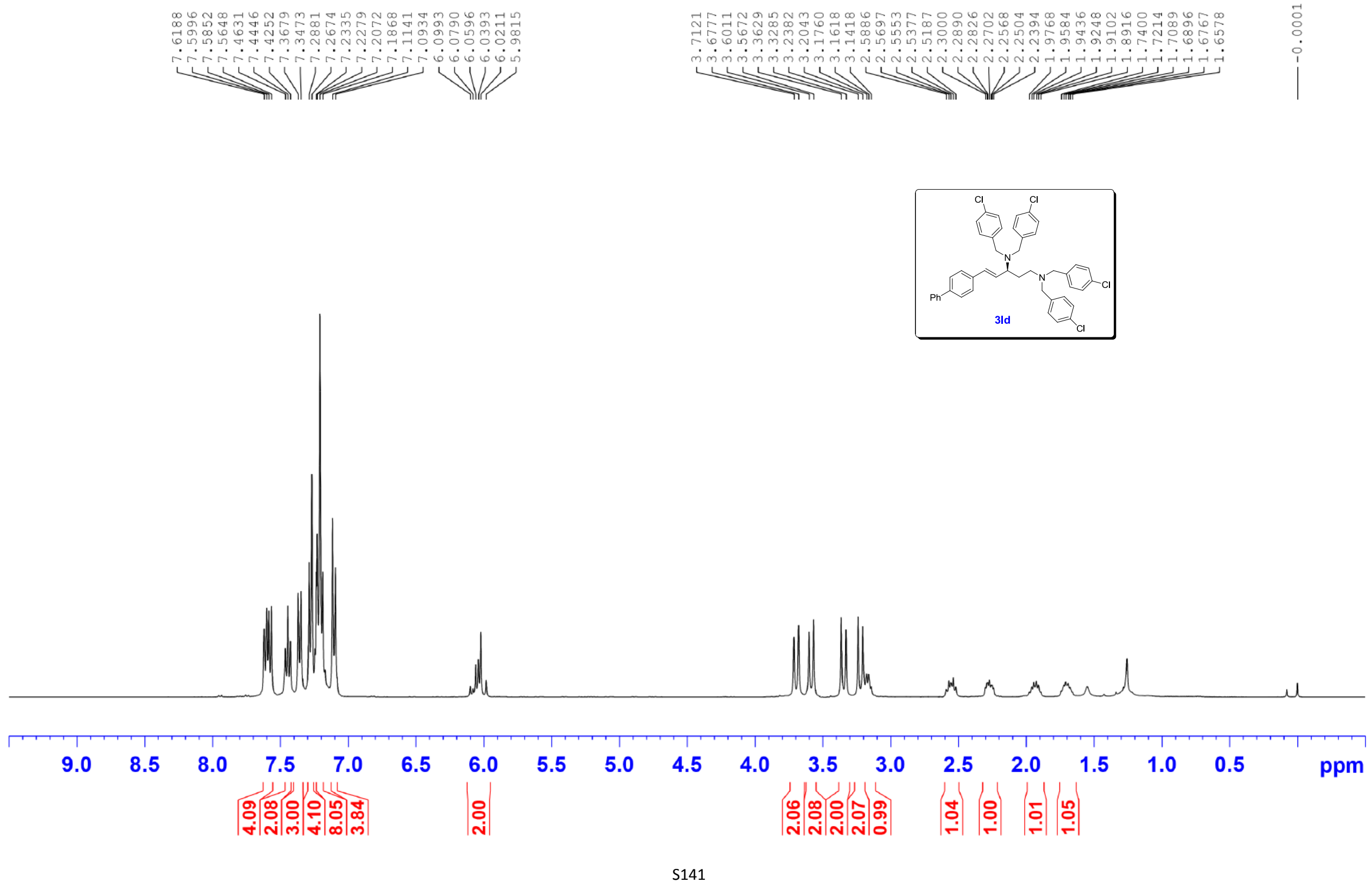

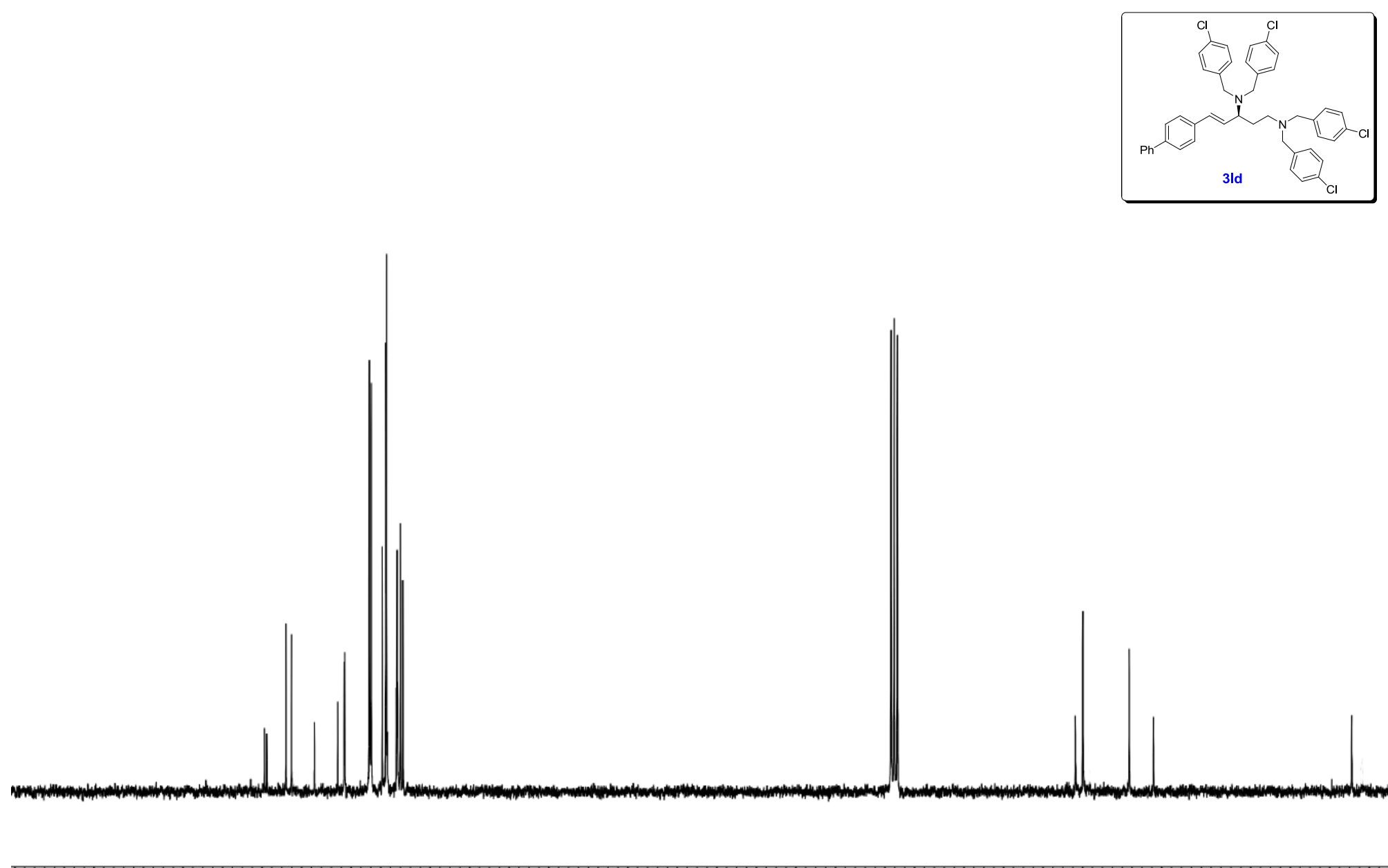

$160 \quad 150$
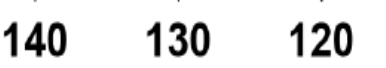

$110 \quad 100$

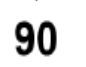

80

70

60

50

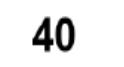

30

20

10

ppm 


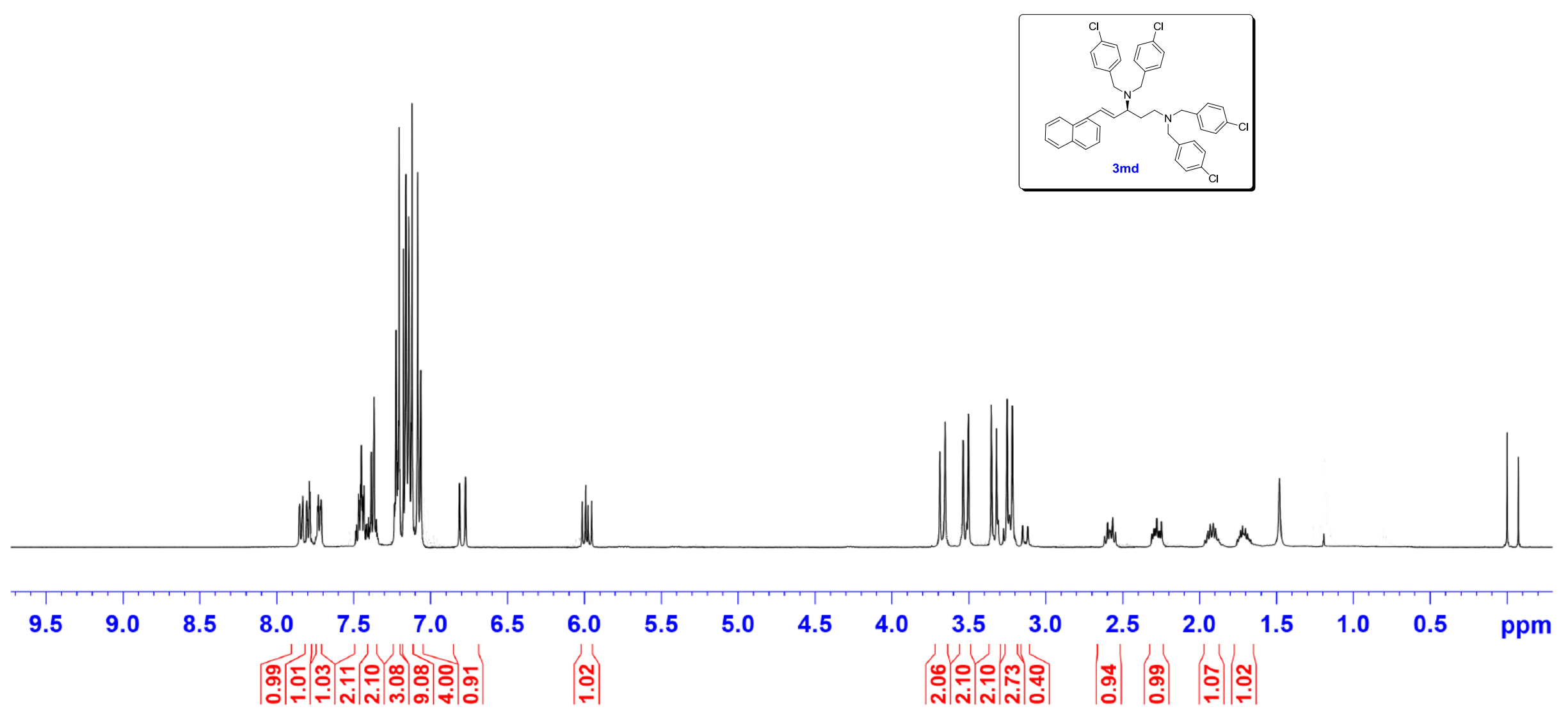



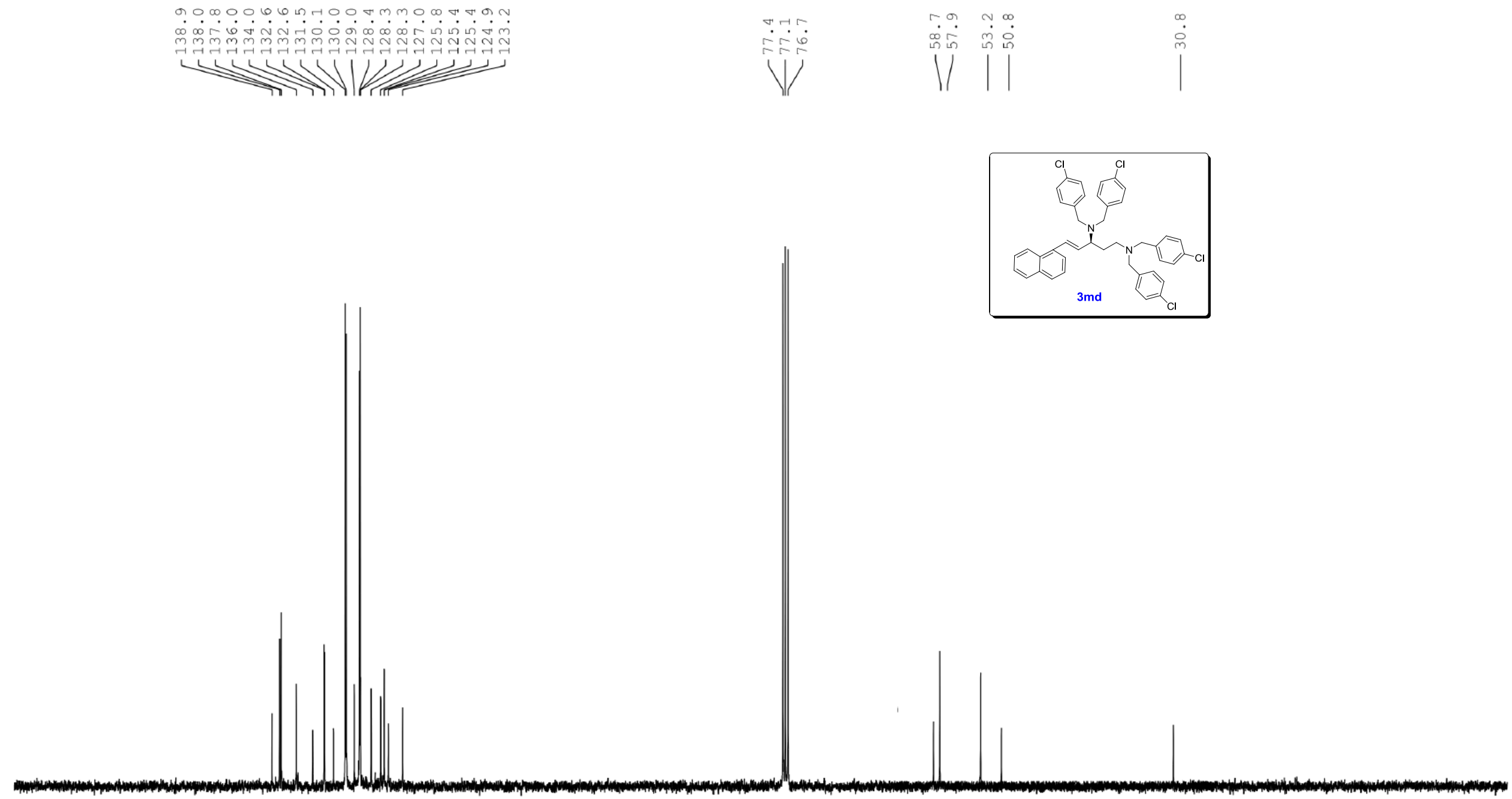

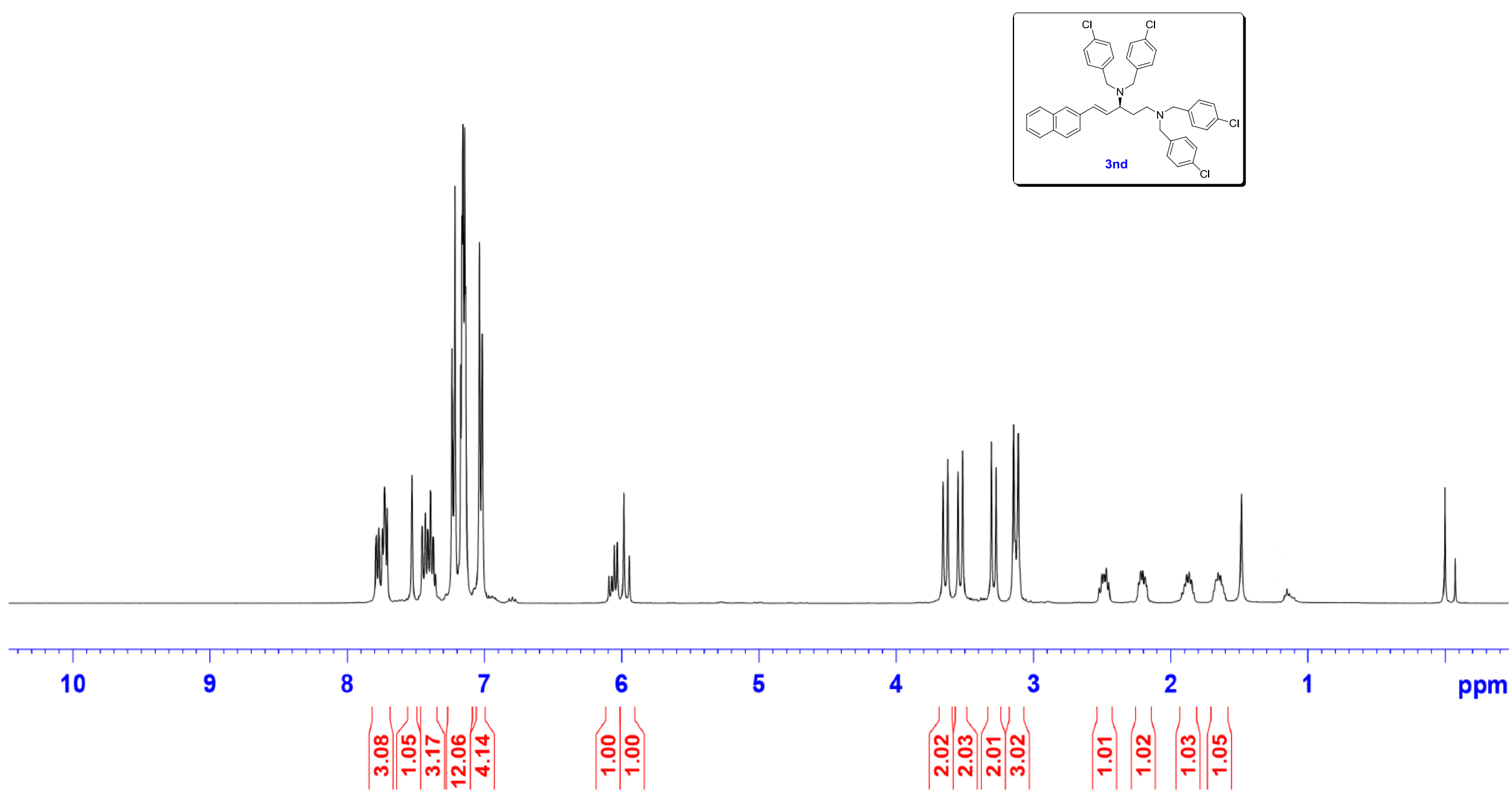

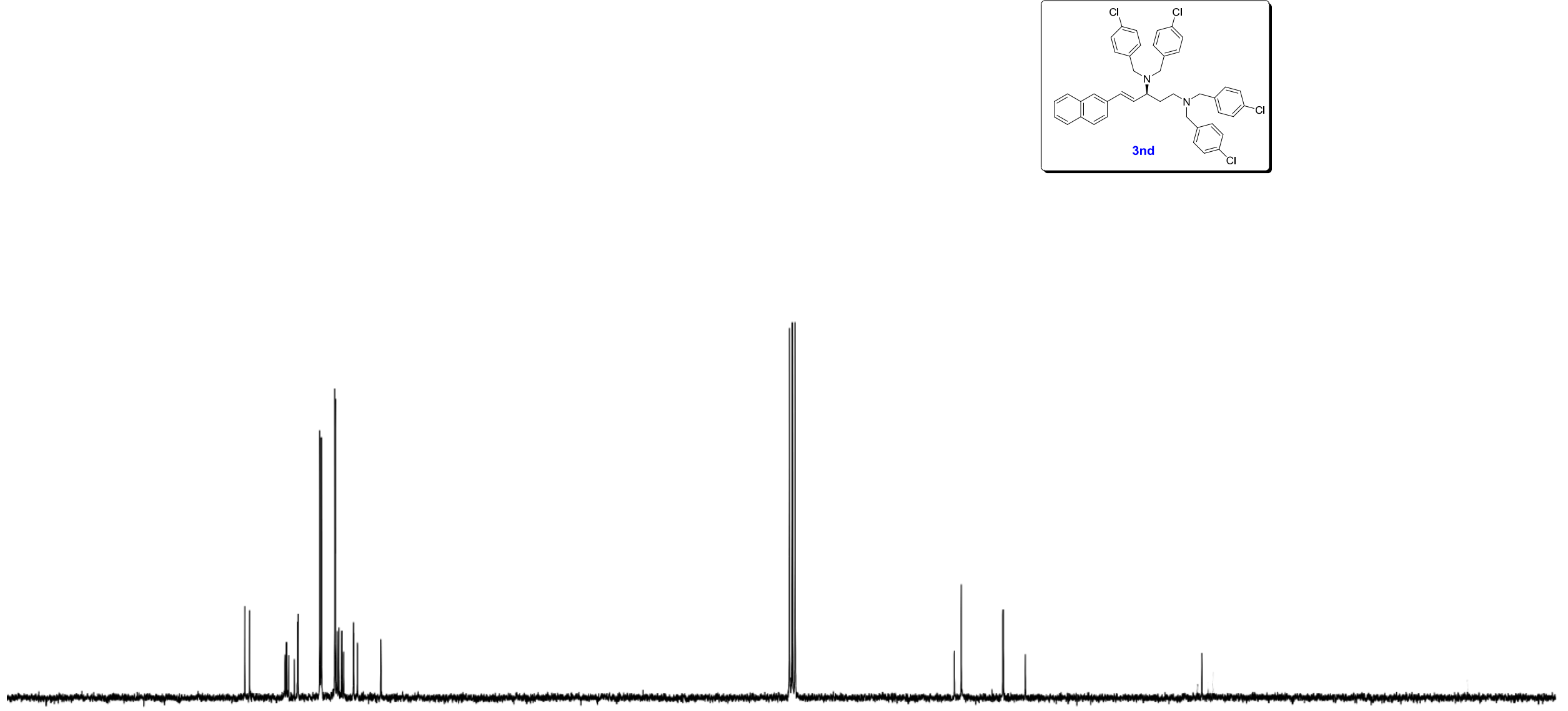

$\begin{array}{llll}160 & 150 & 140 & 130\end{array}$ 


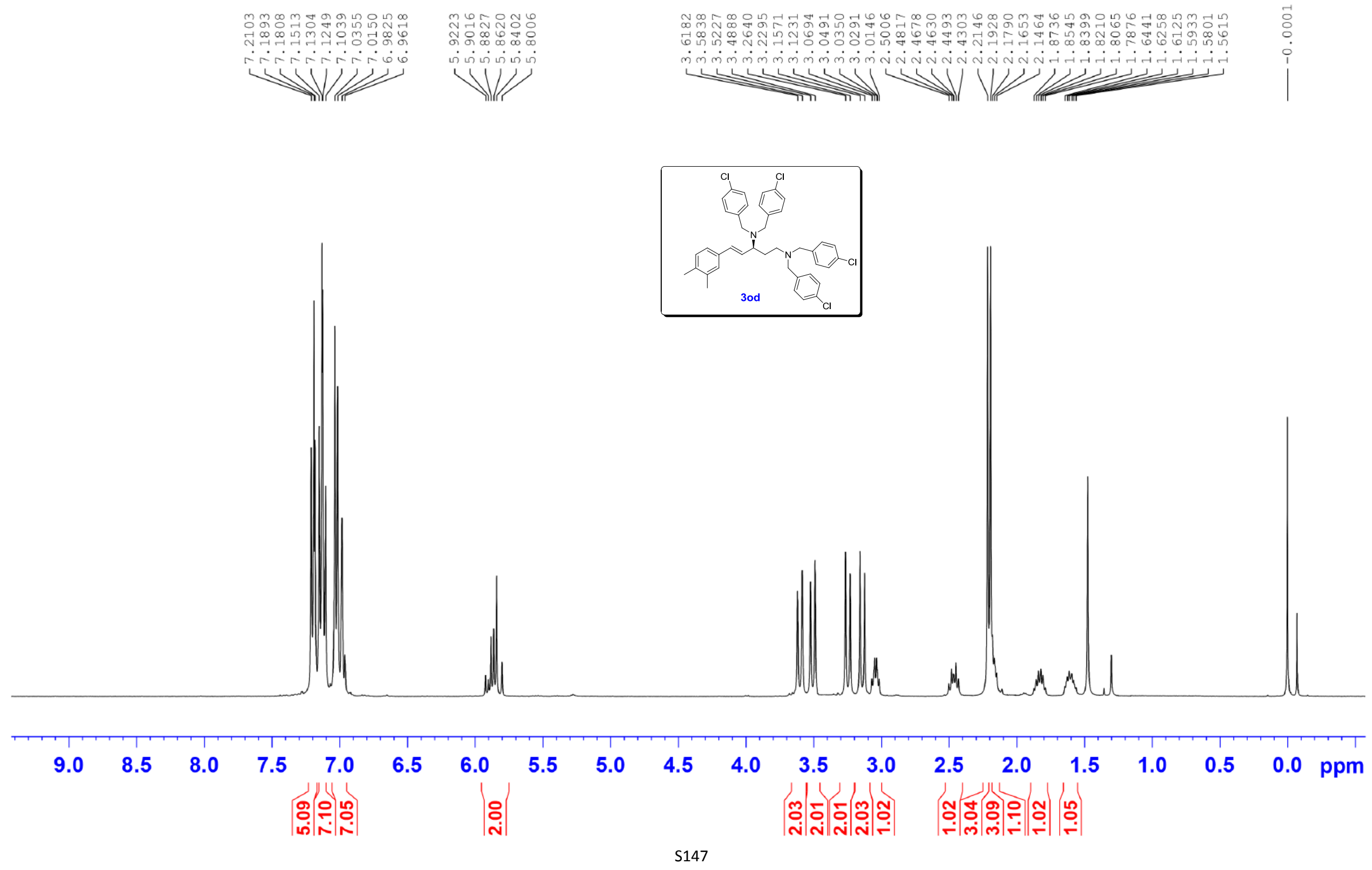




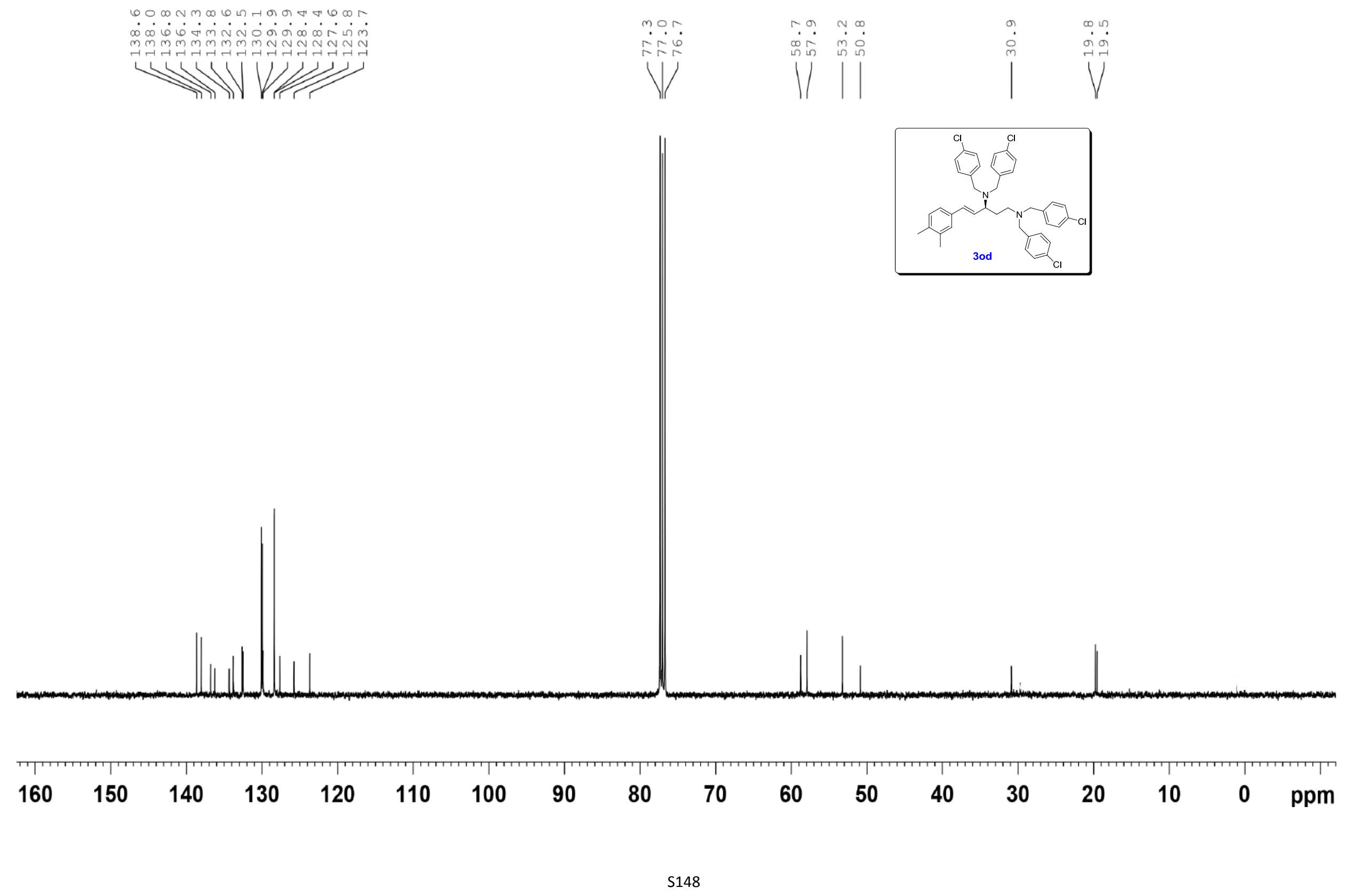



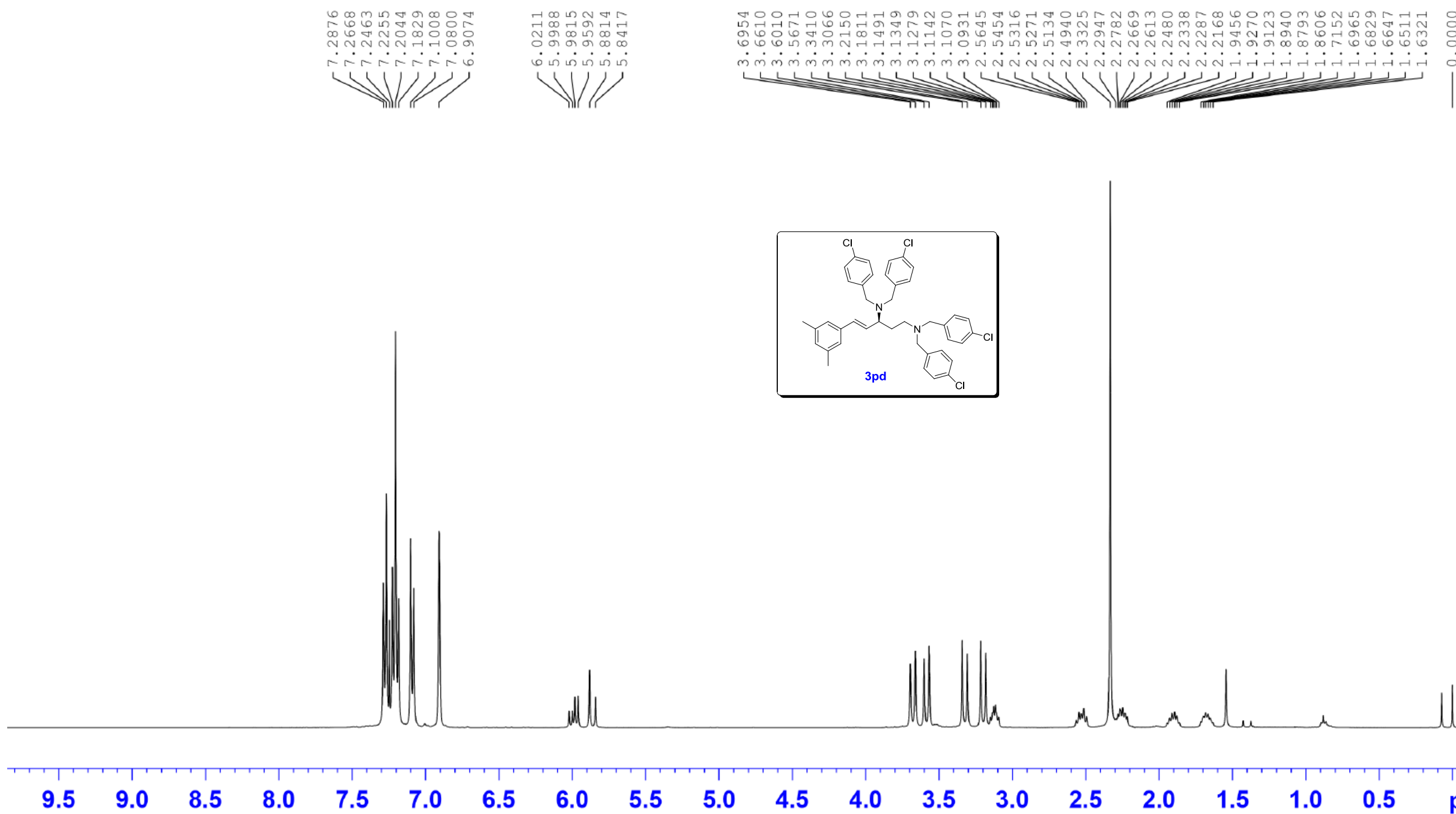

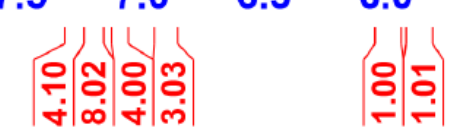
|)

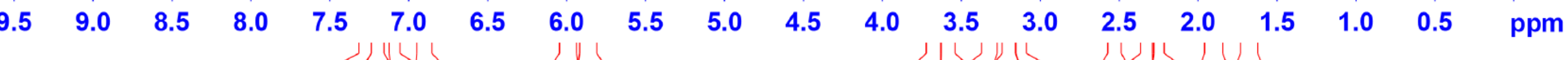

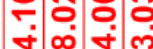 2)

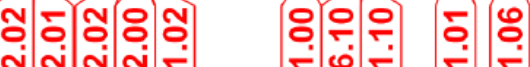

MII 


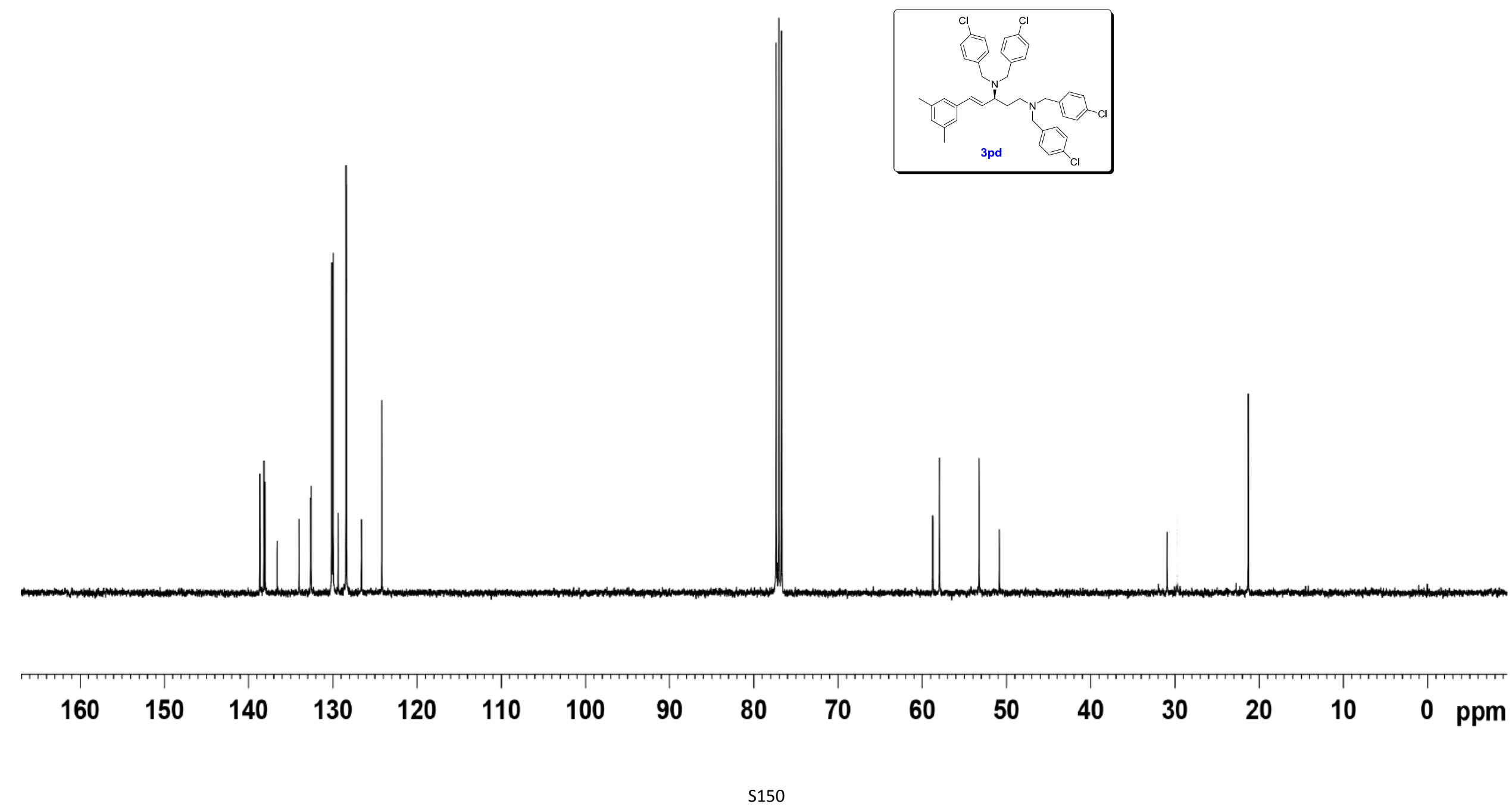




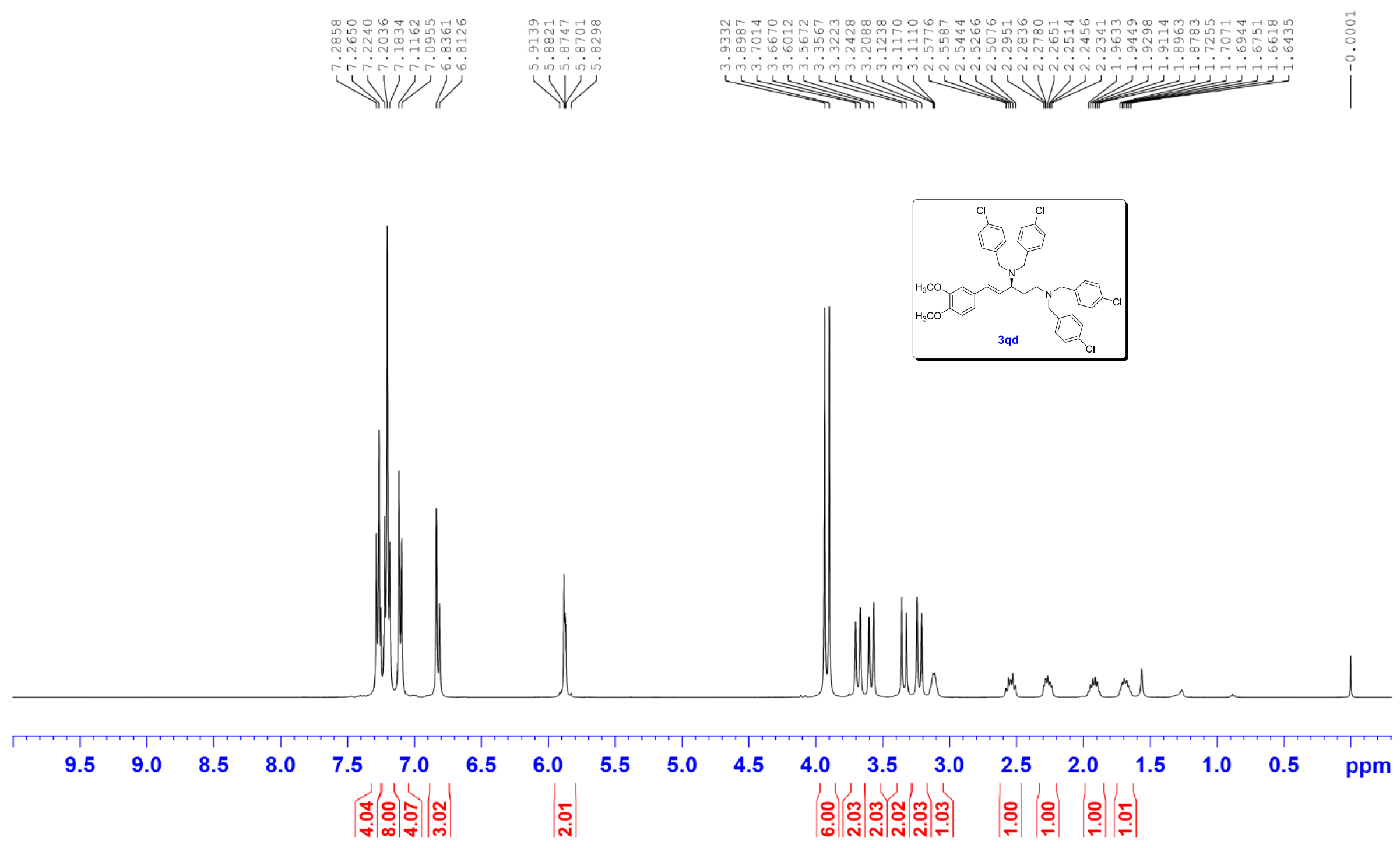



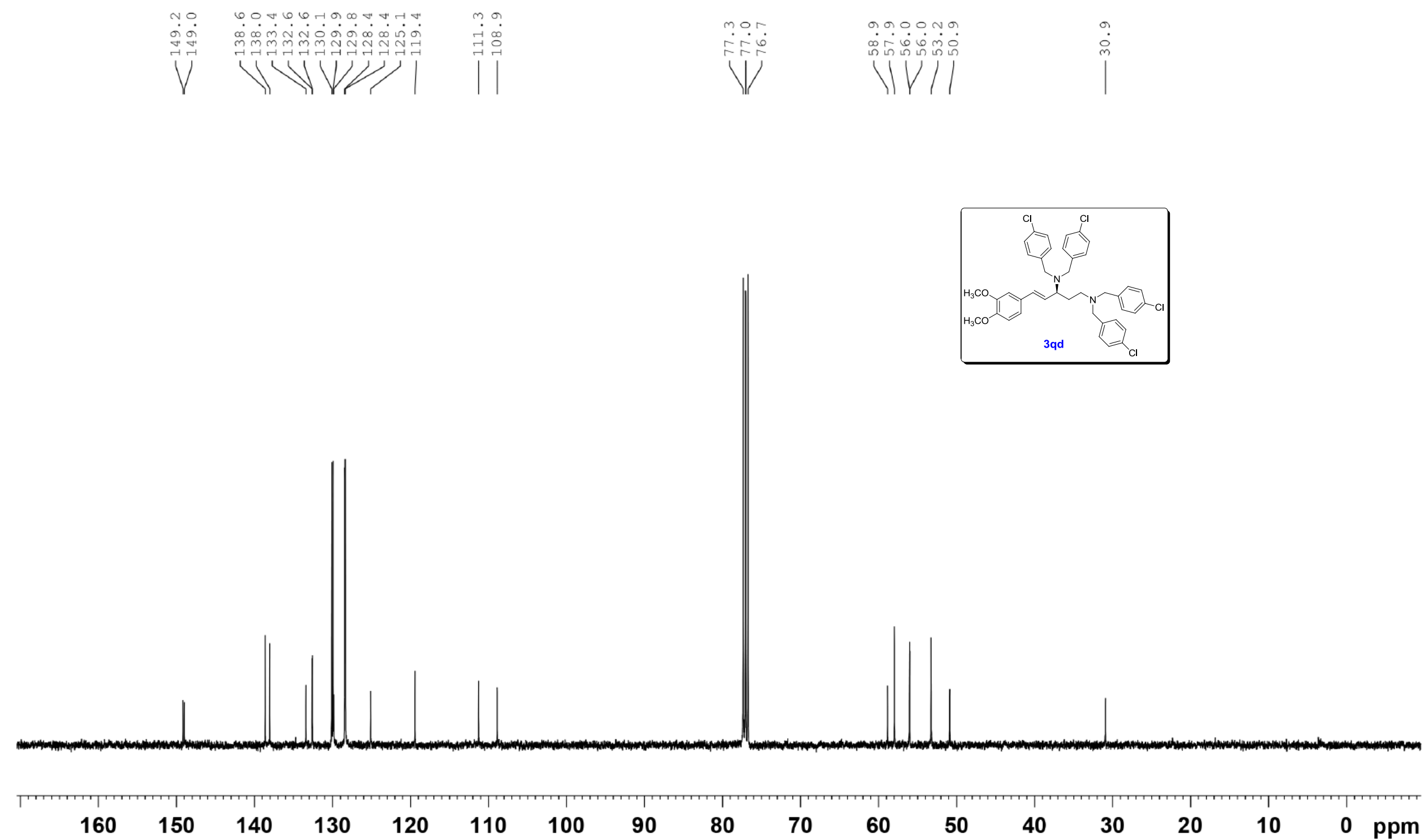

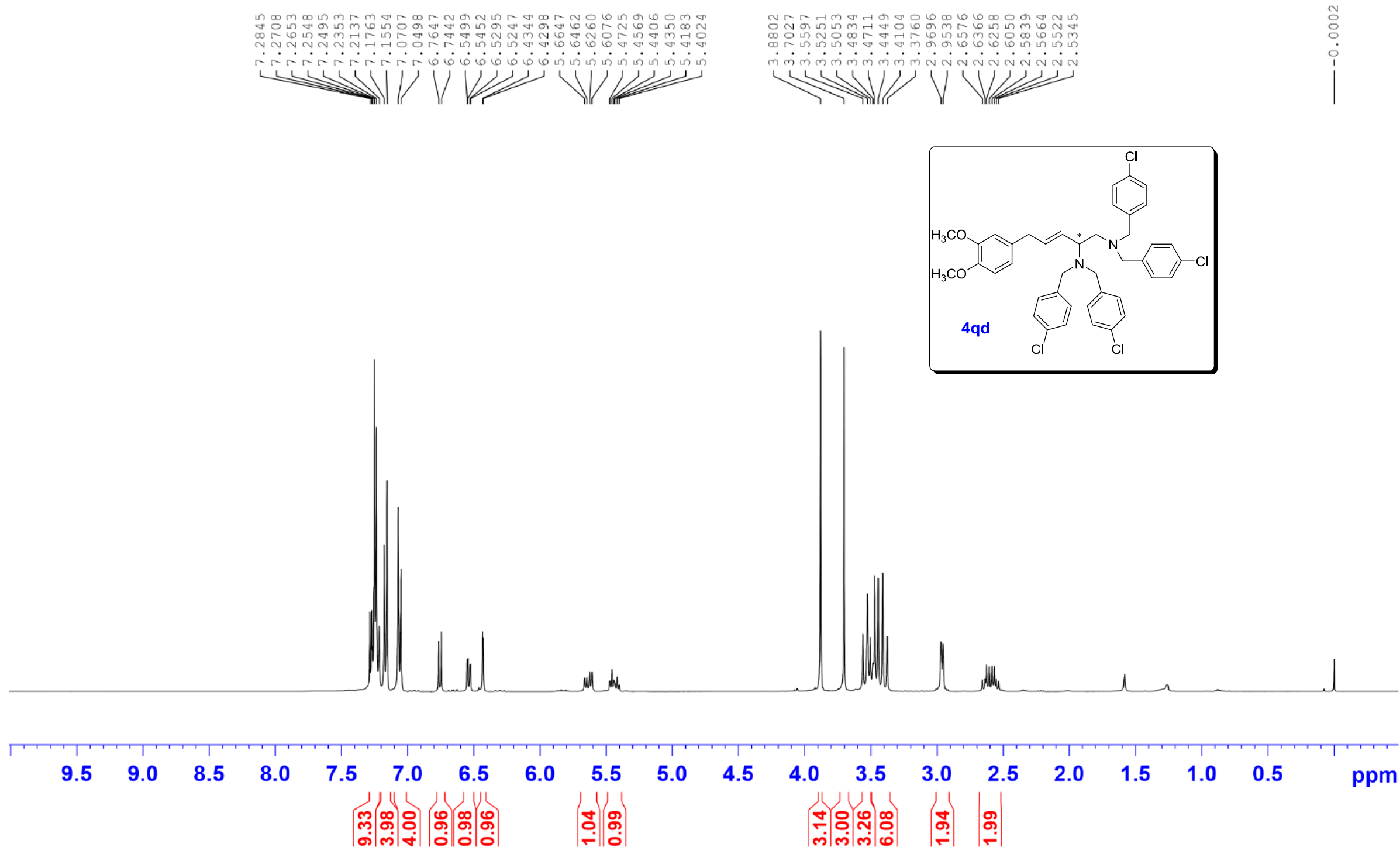

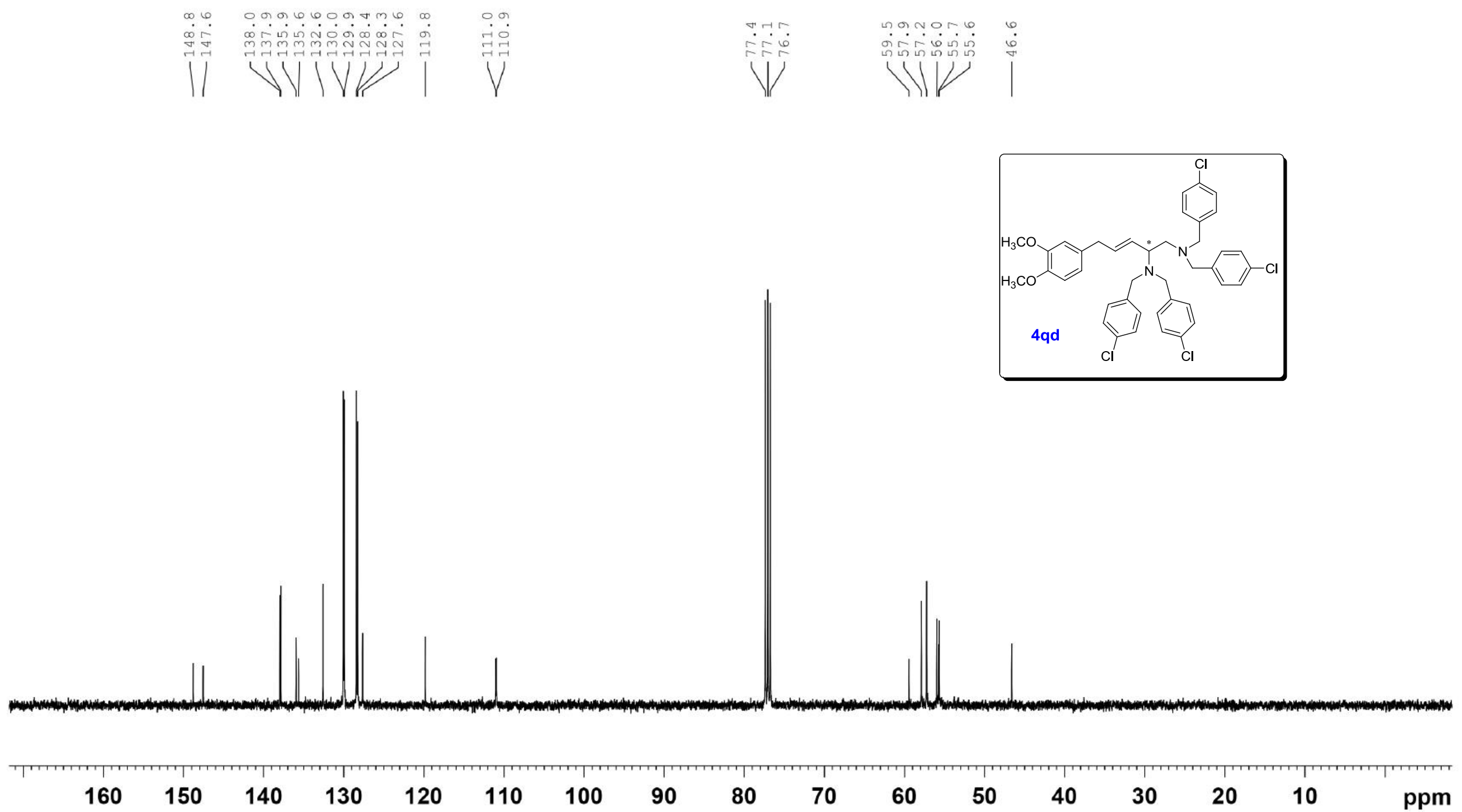

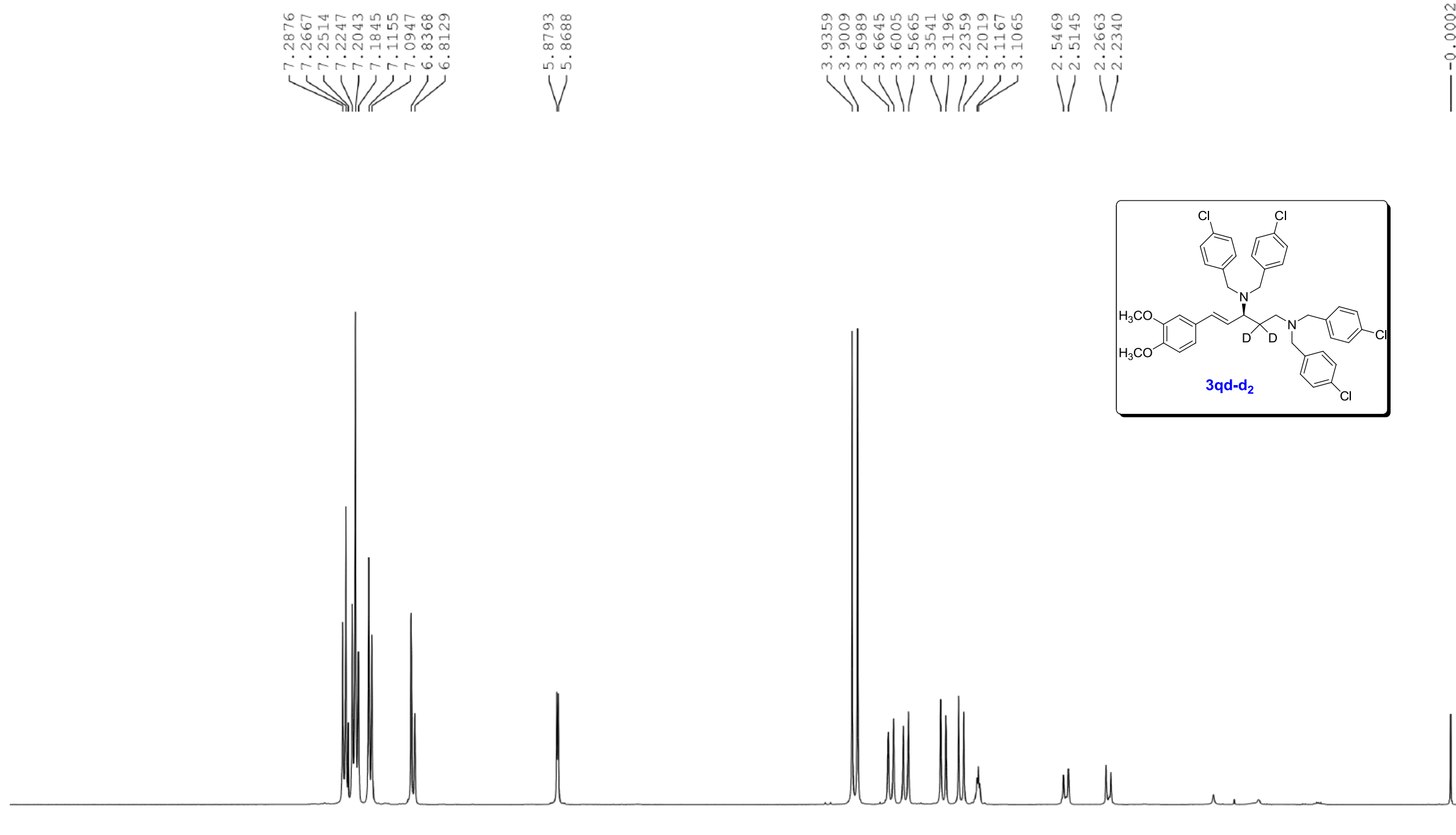

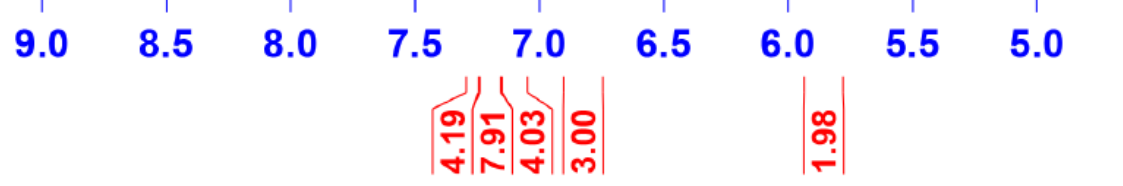

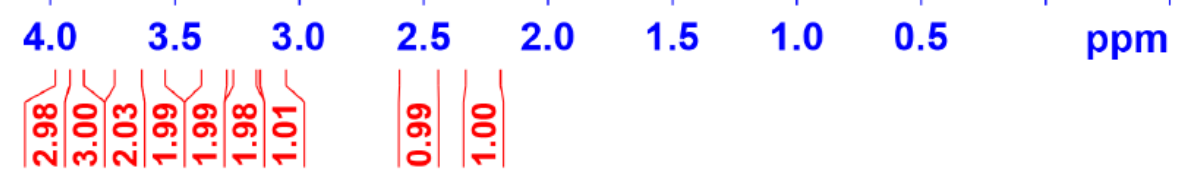



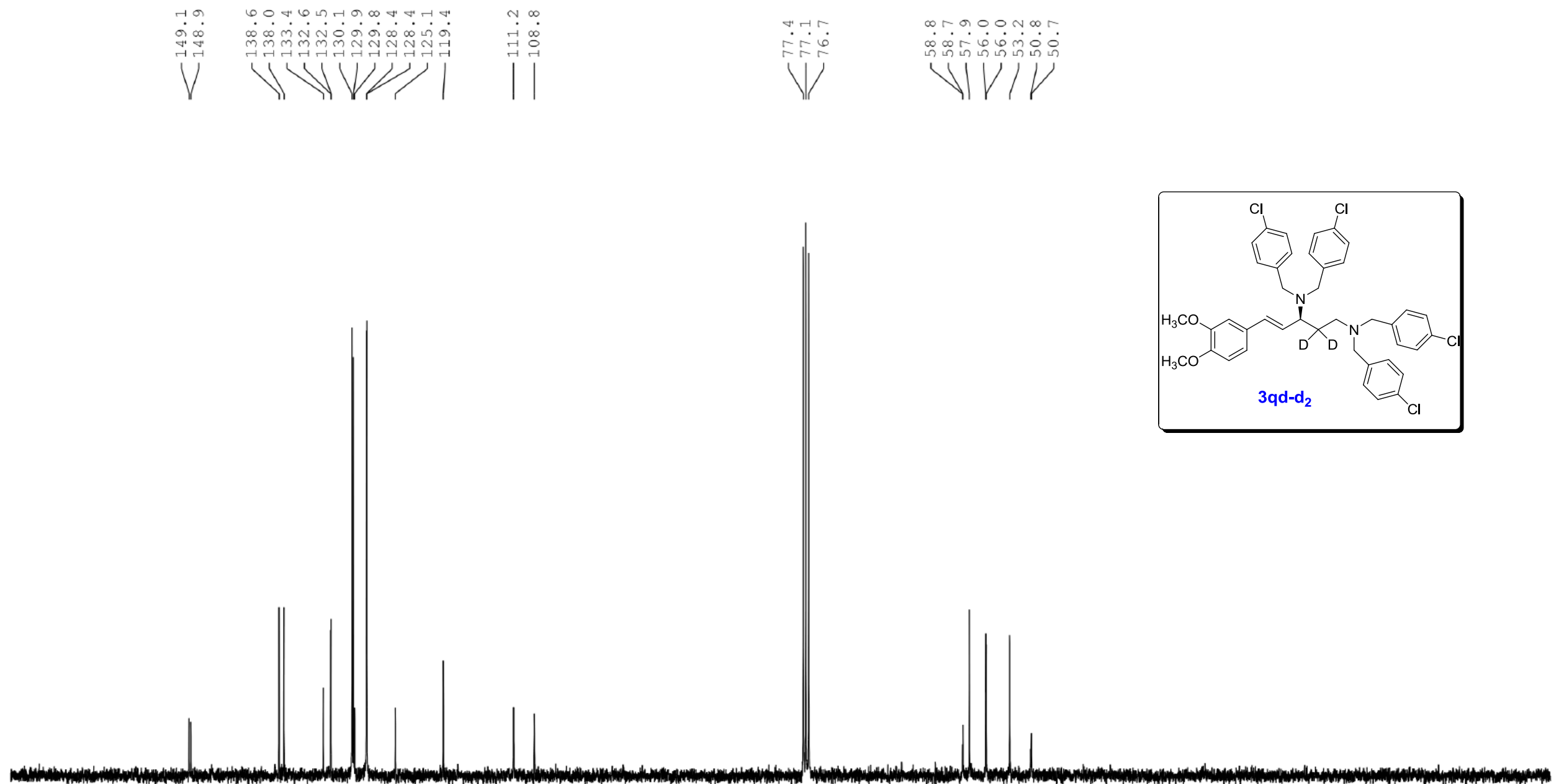


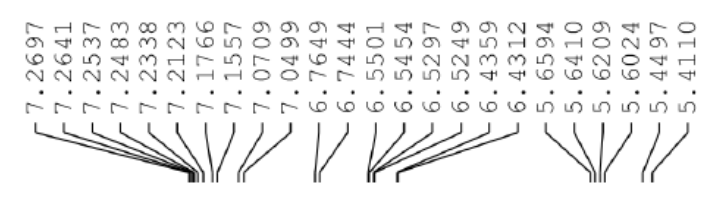

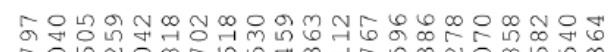

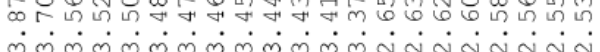

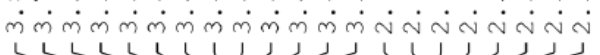
Now
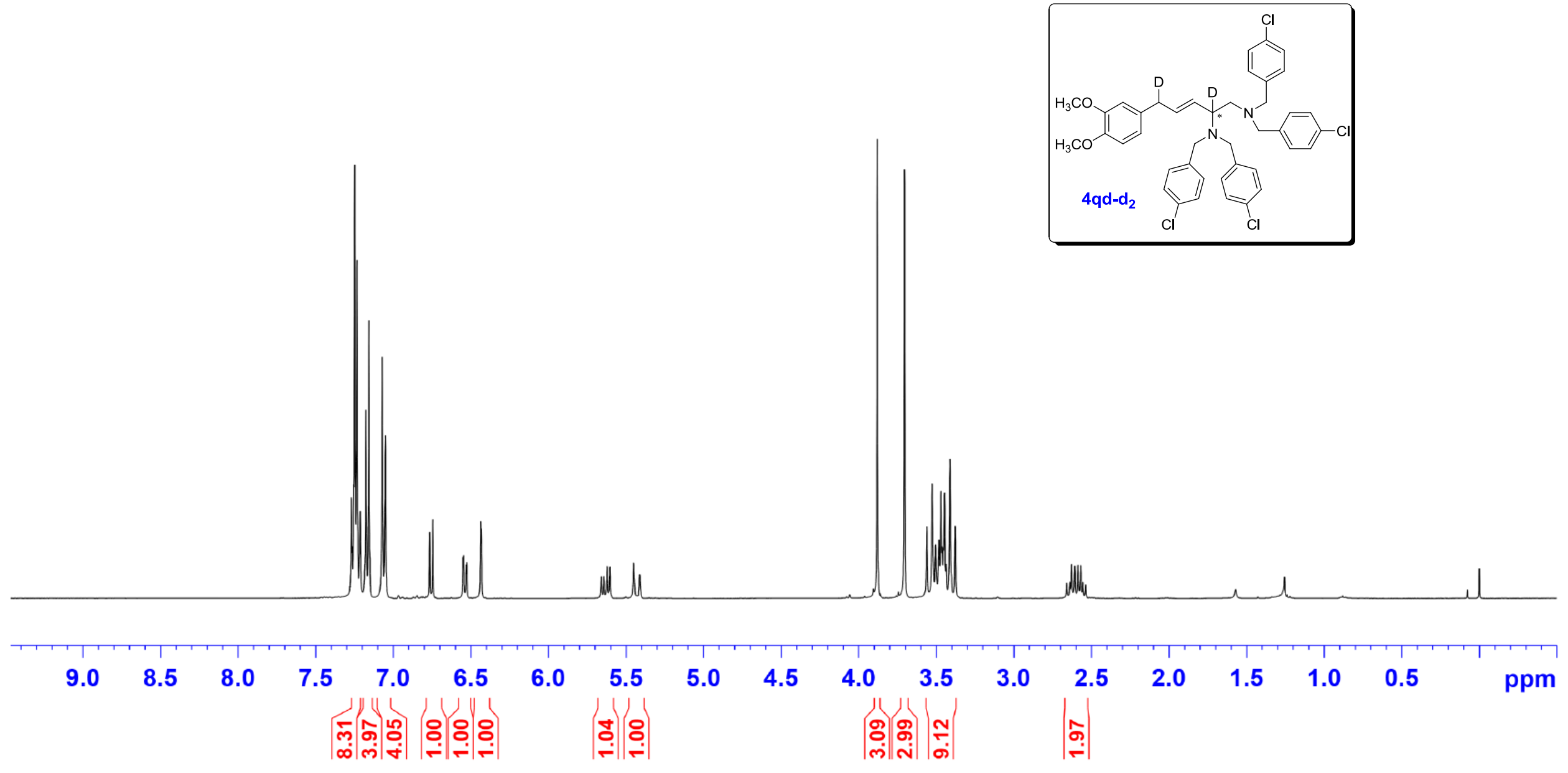

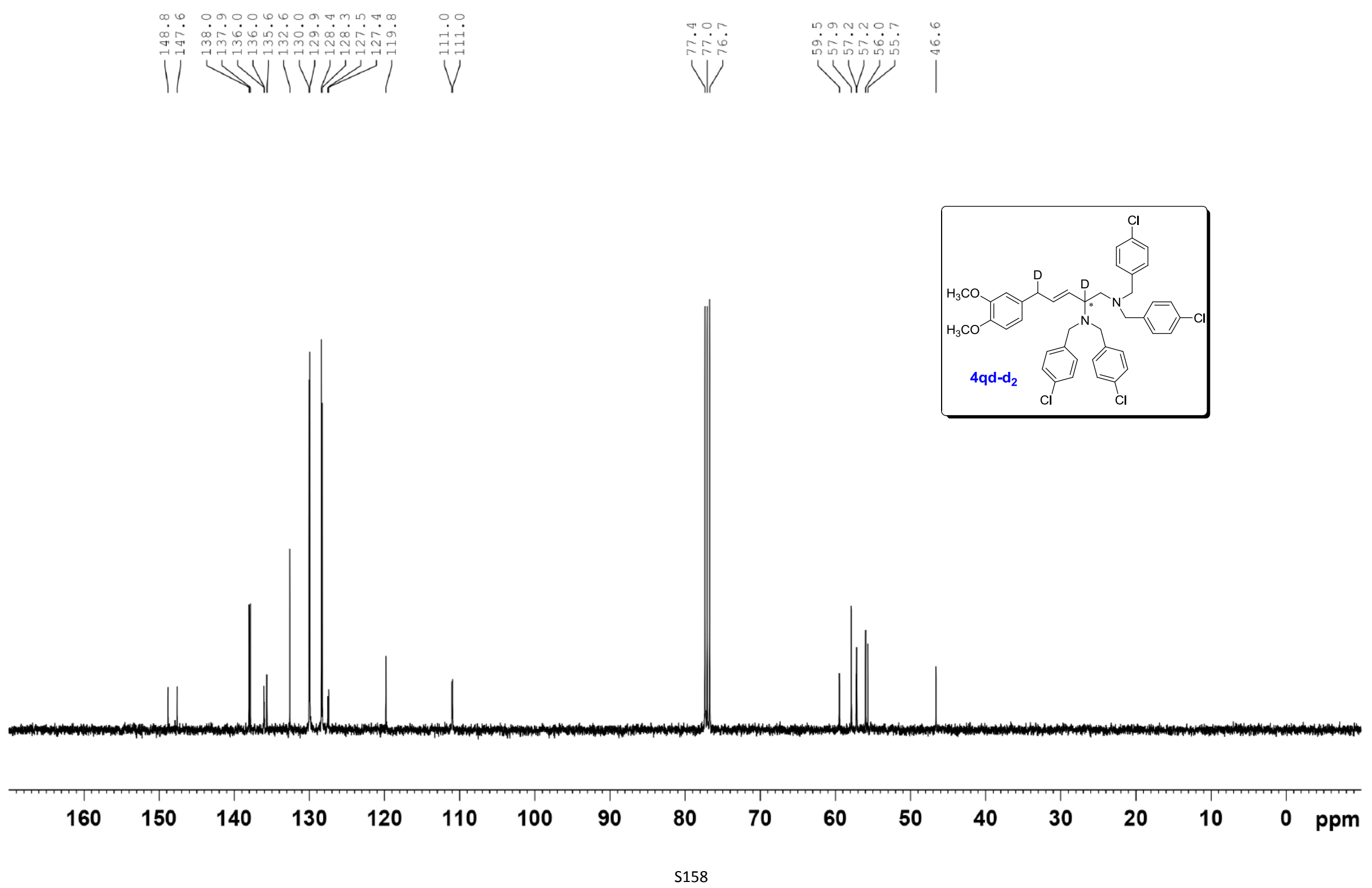

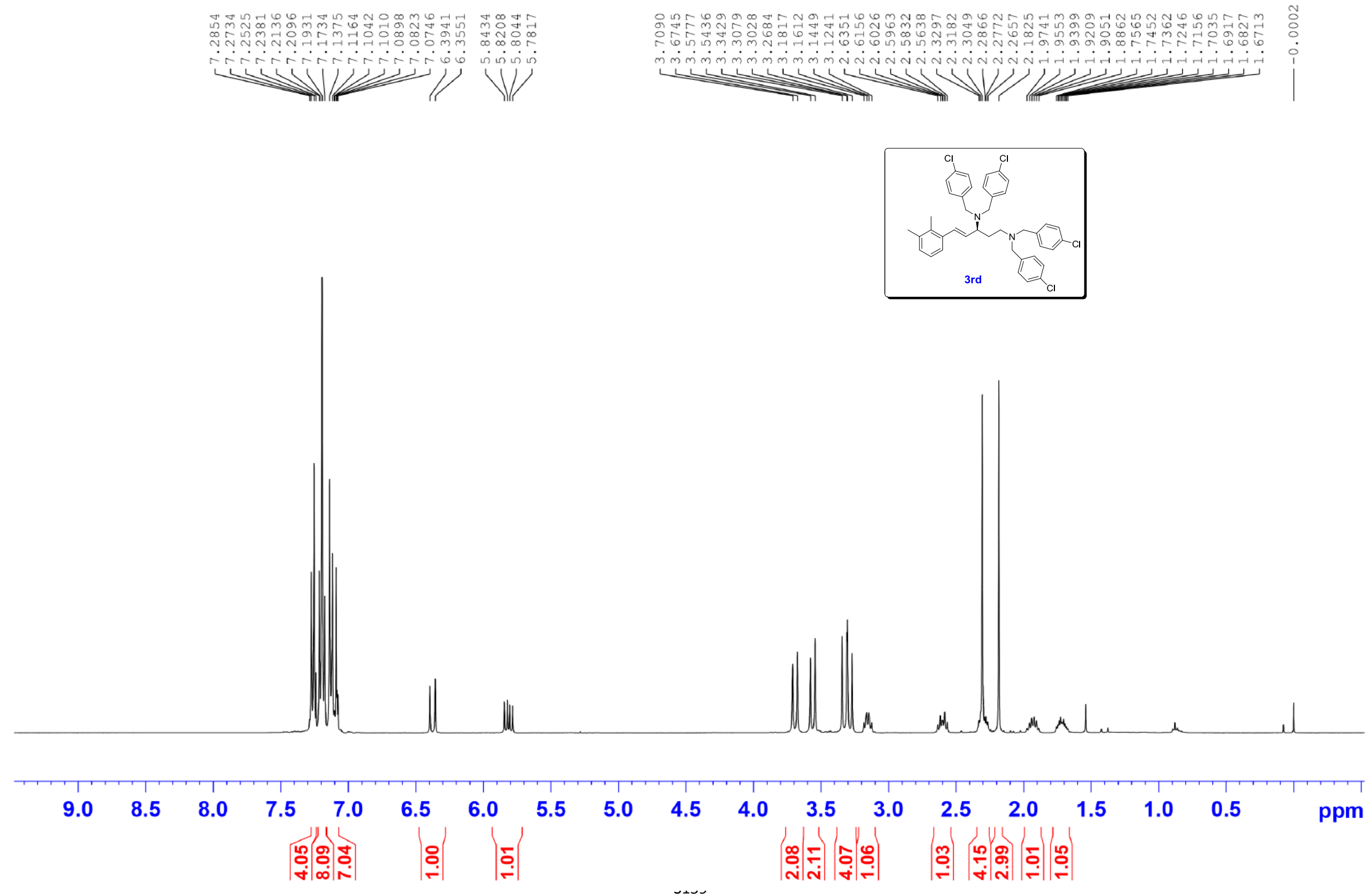

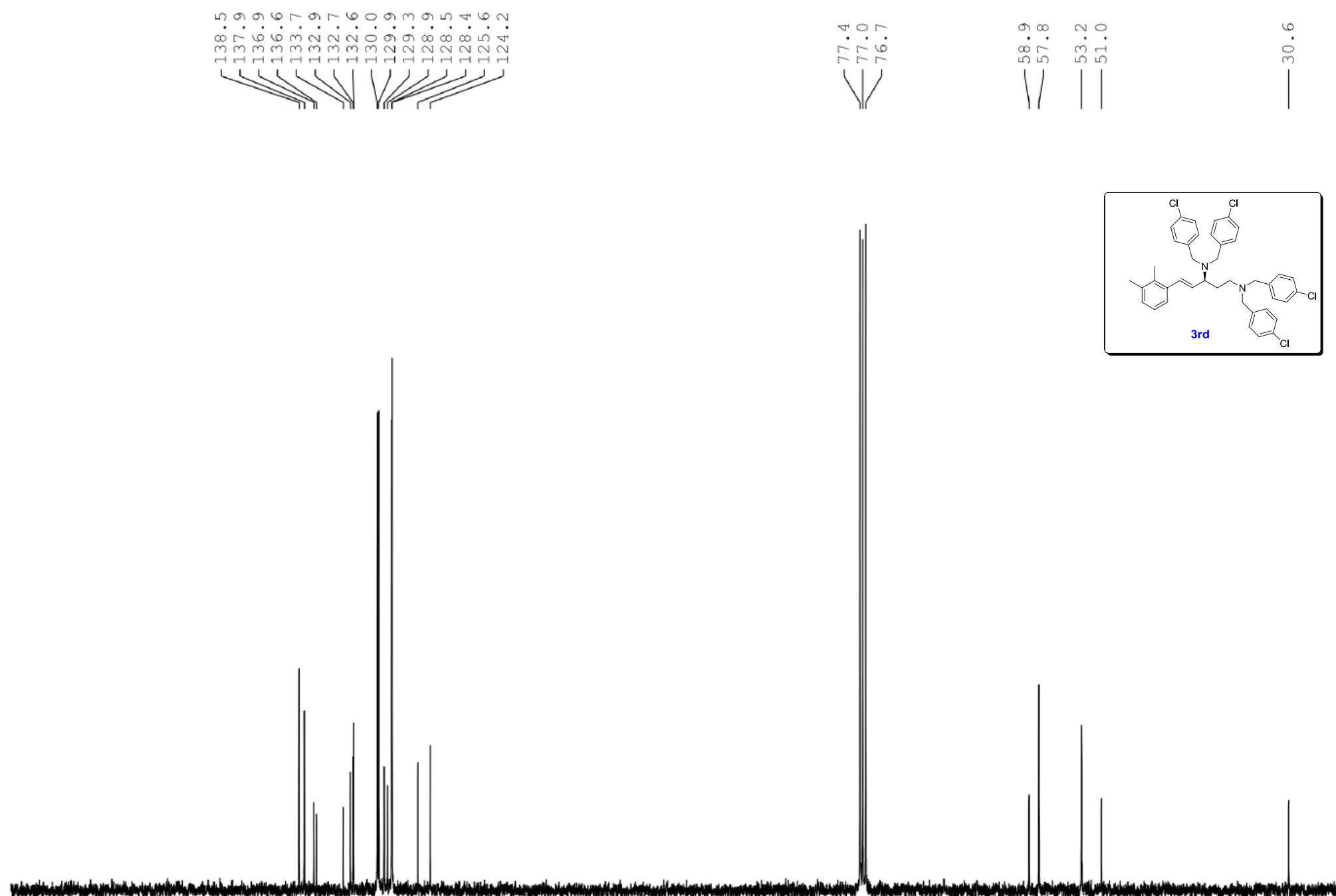

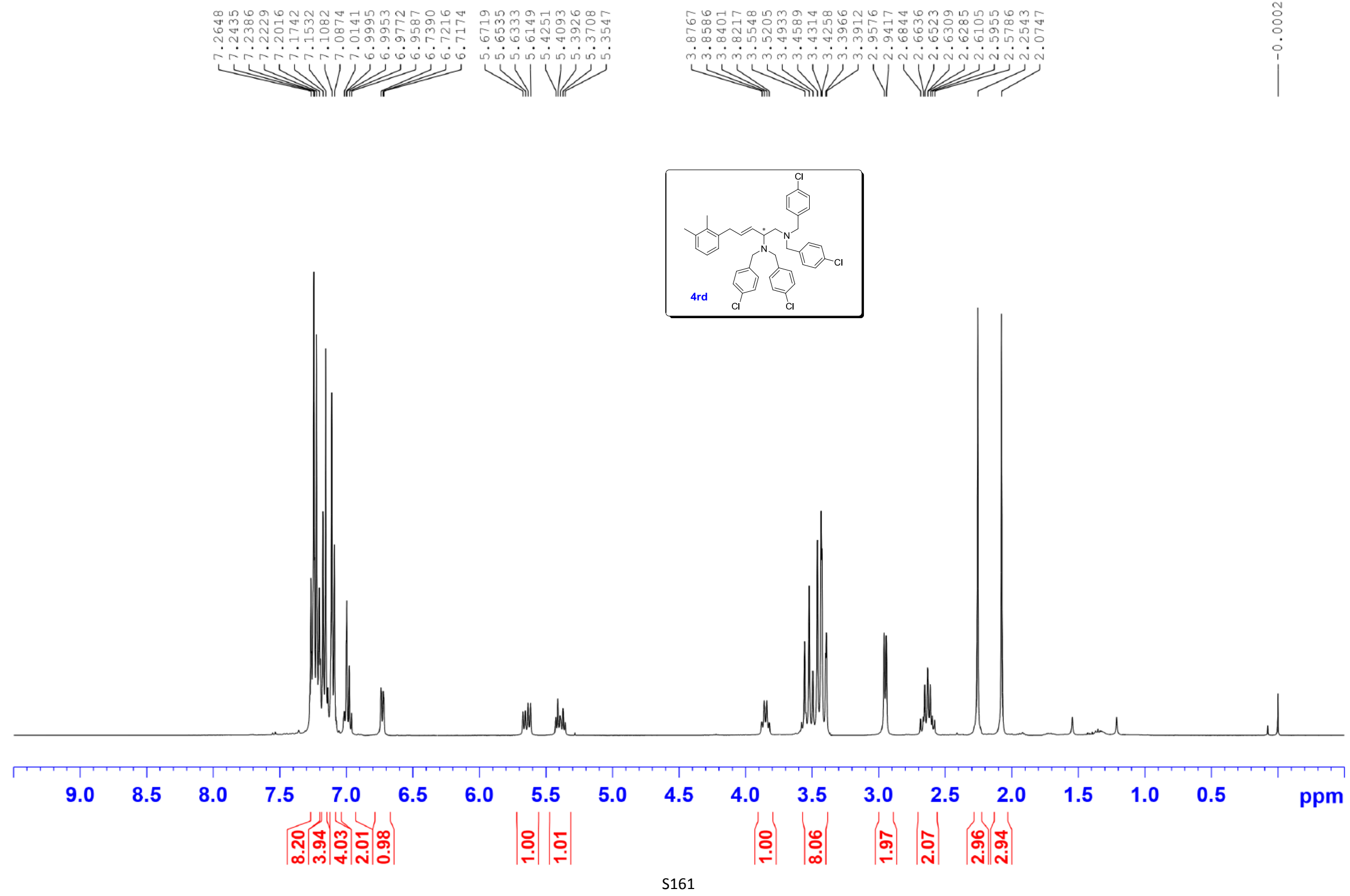

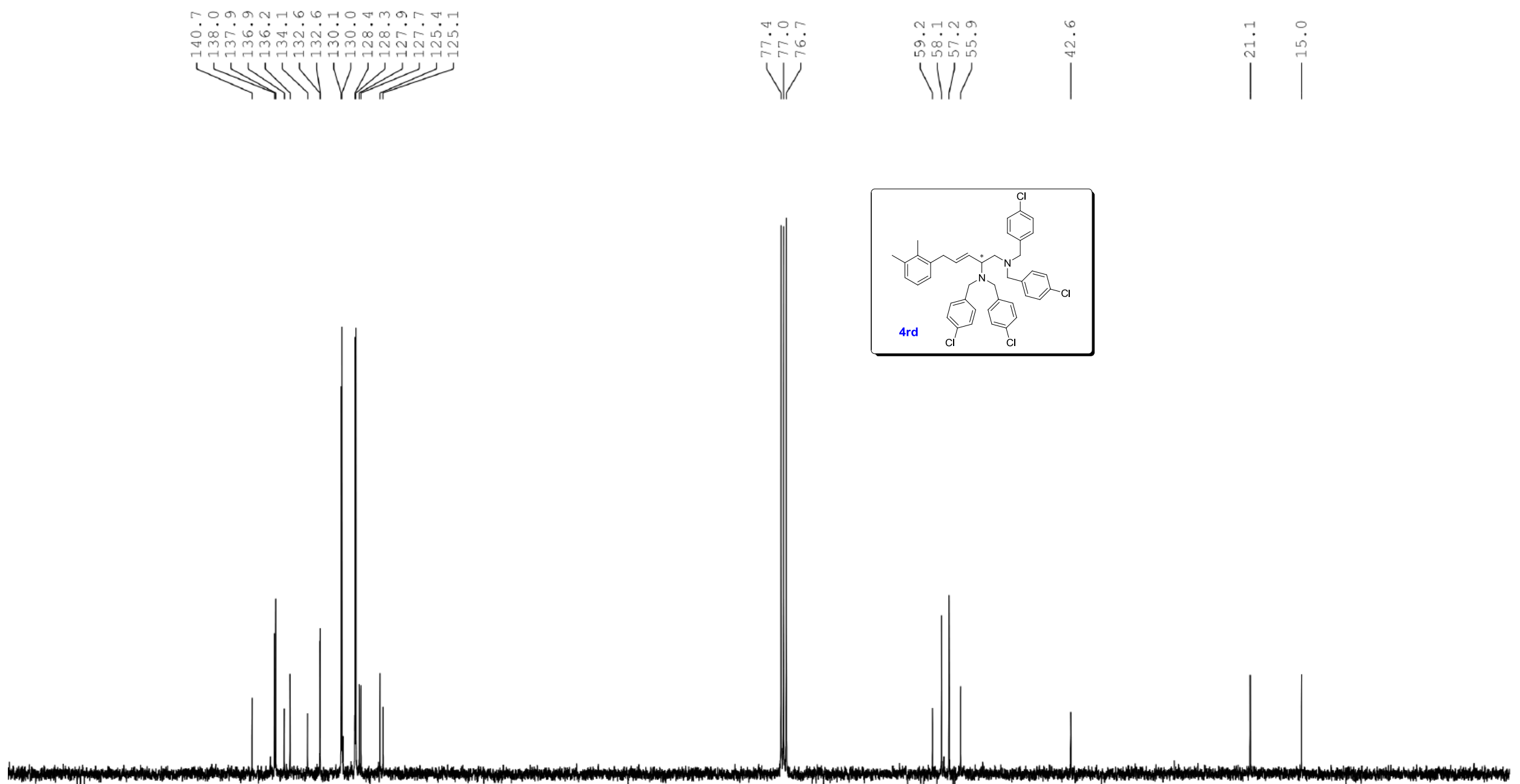

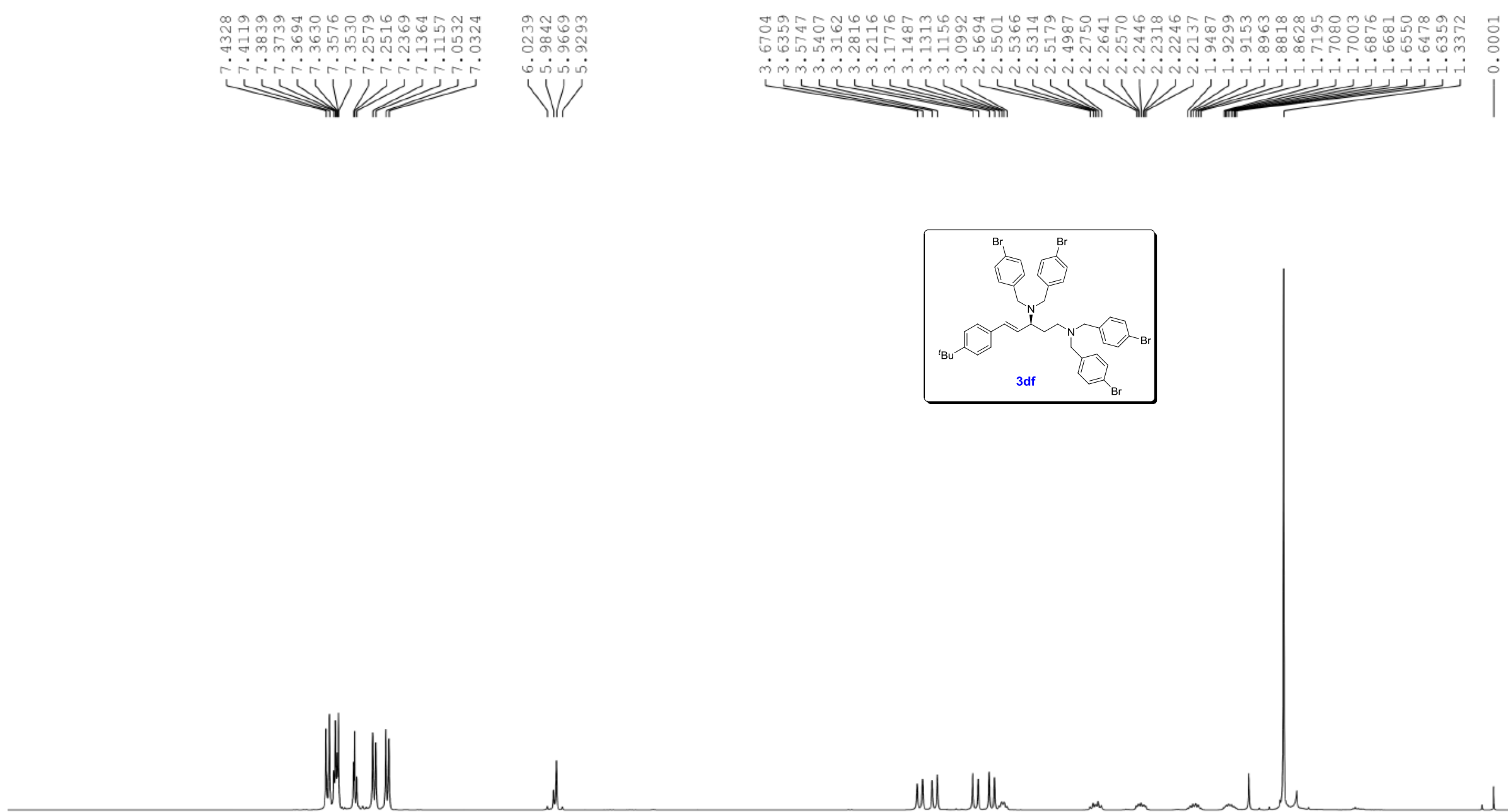

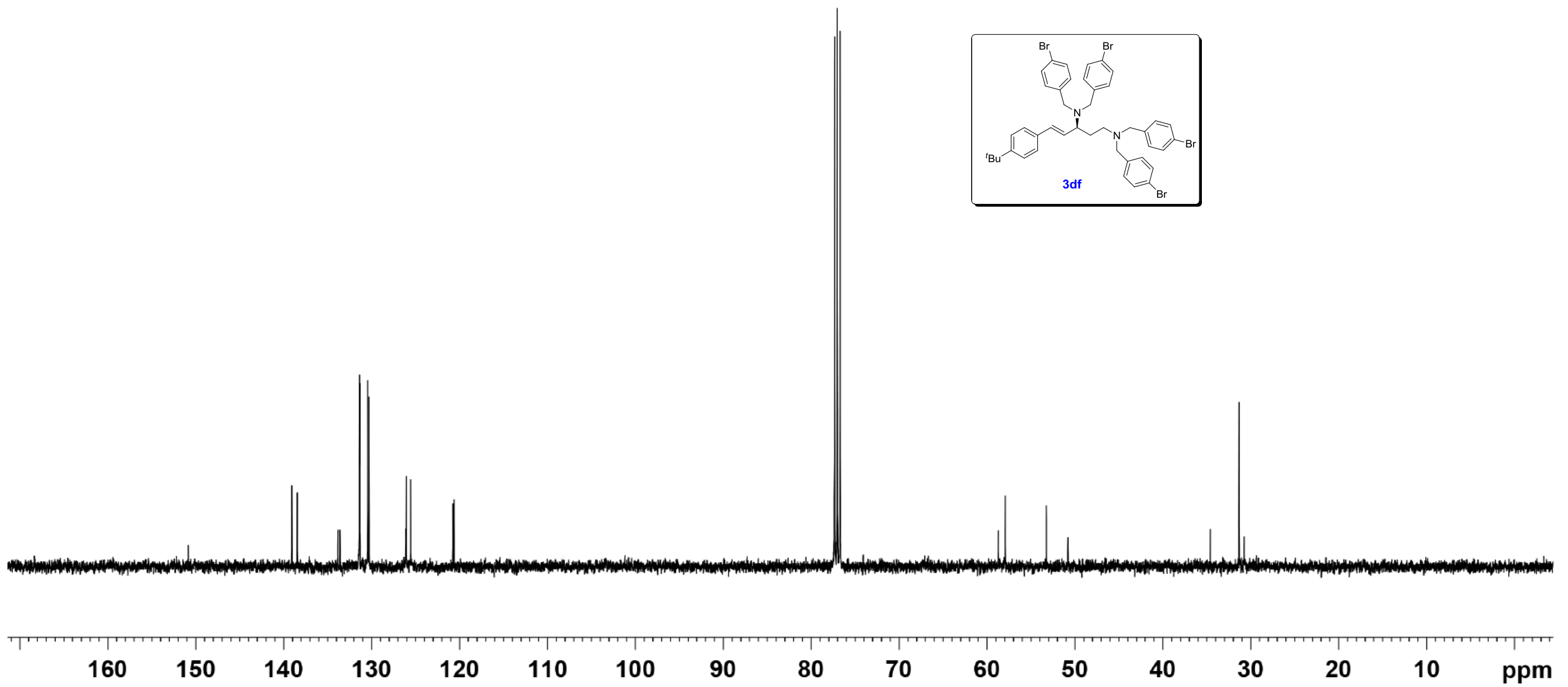


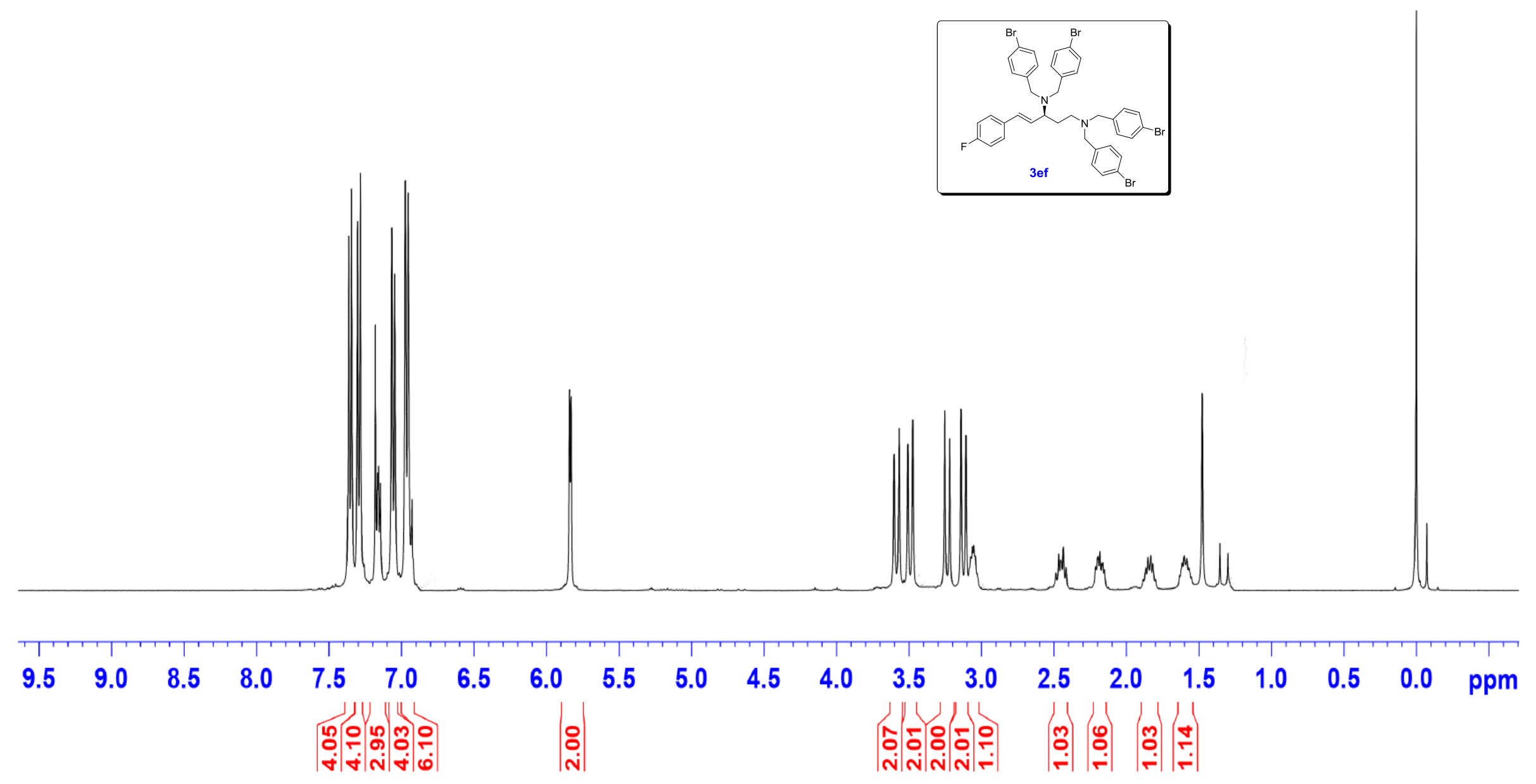




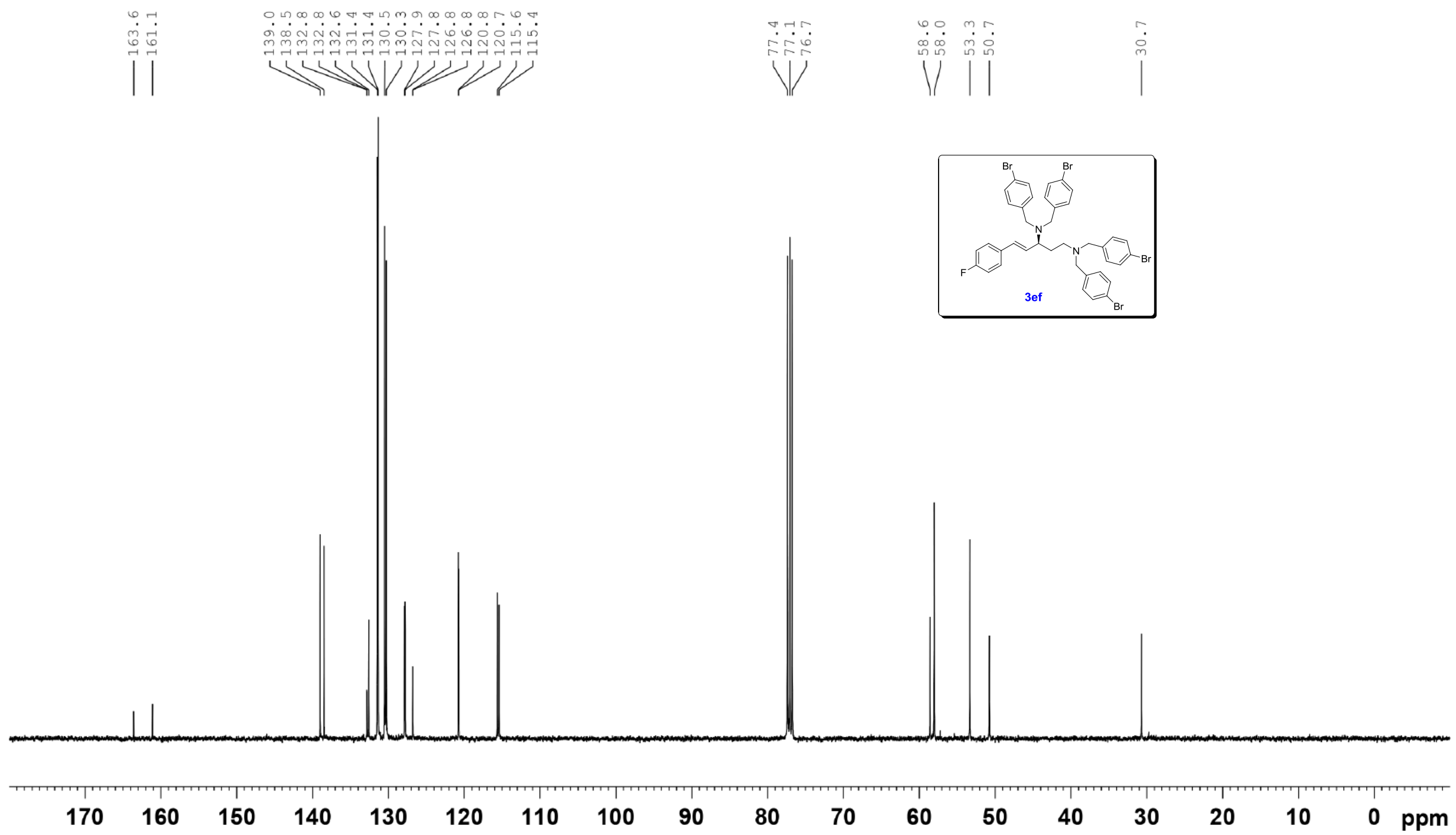



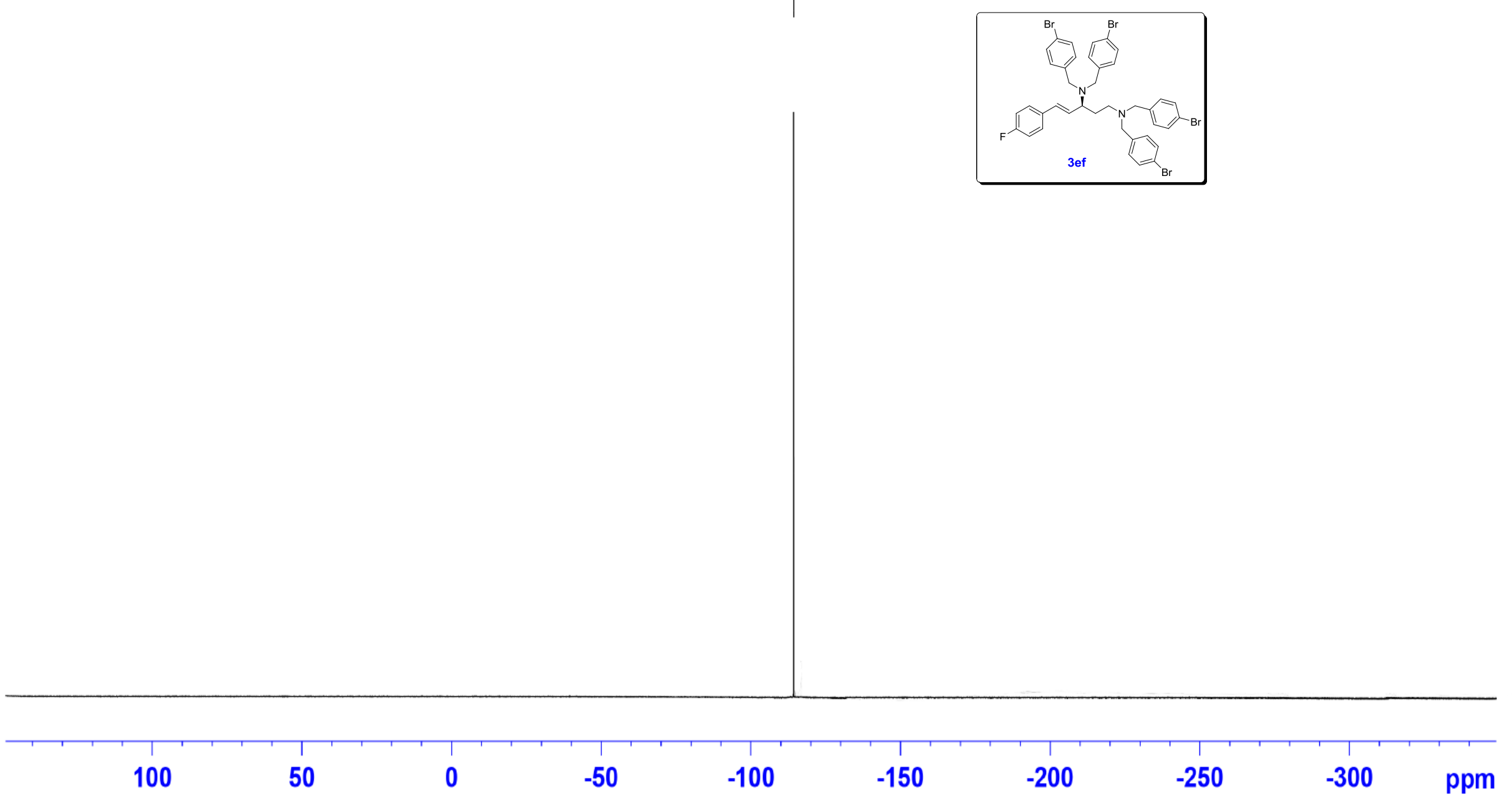

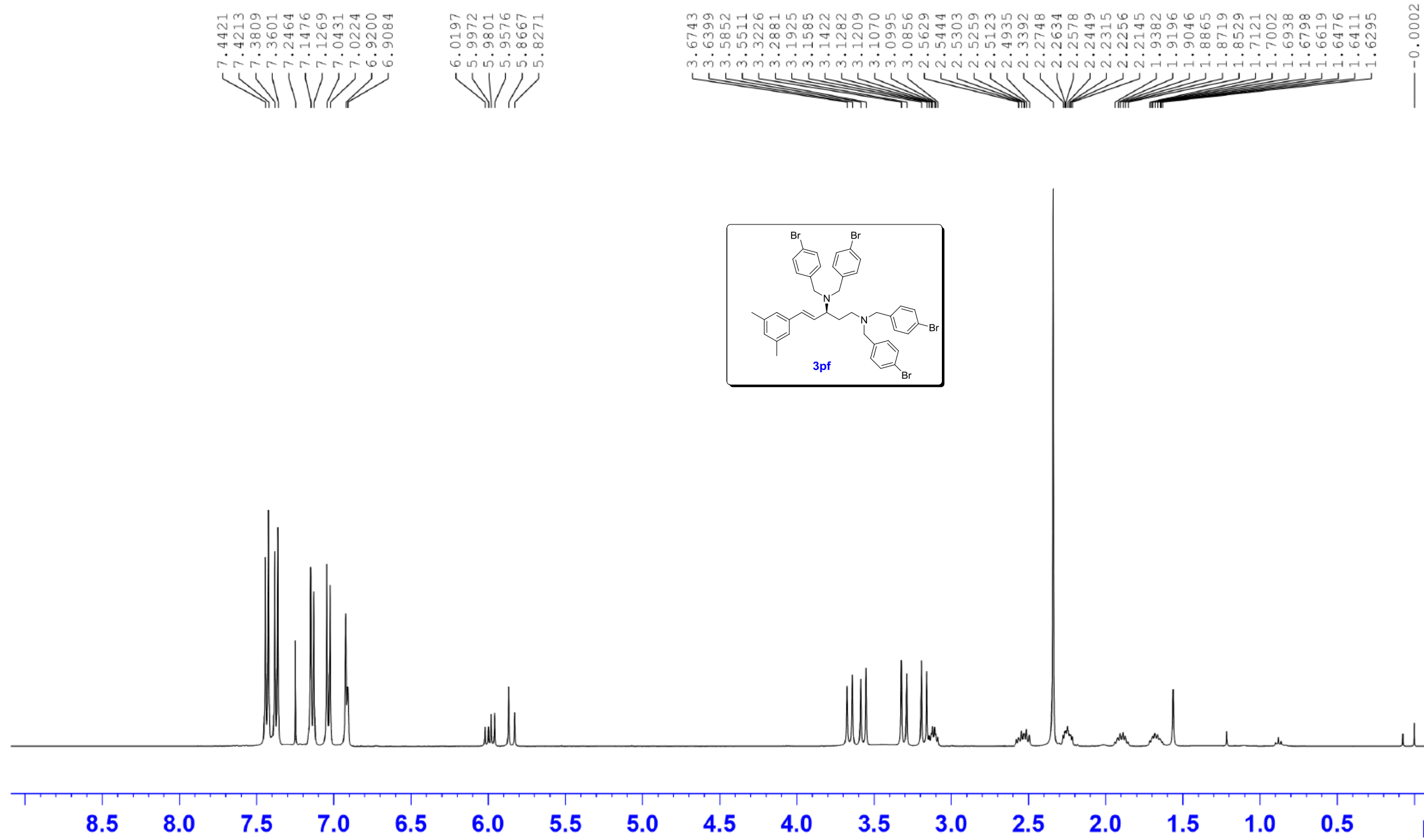

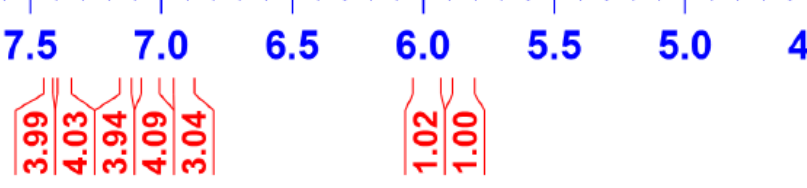
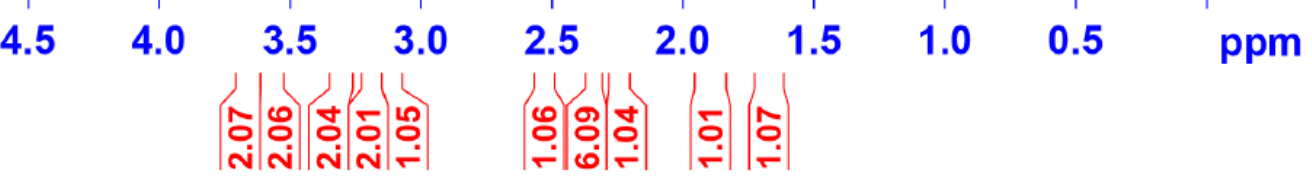


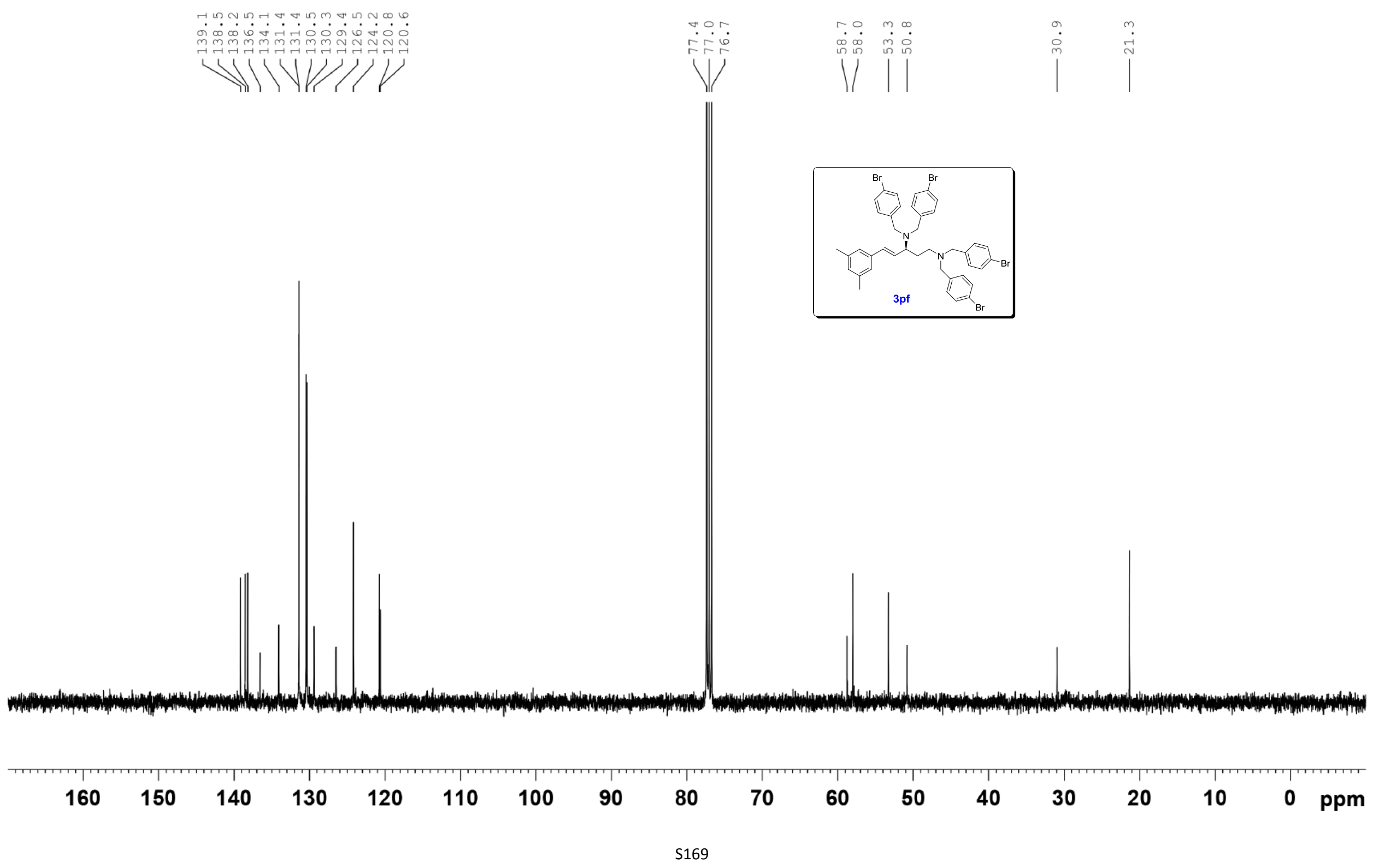




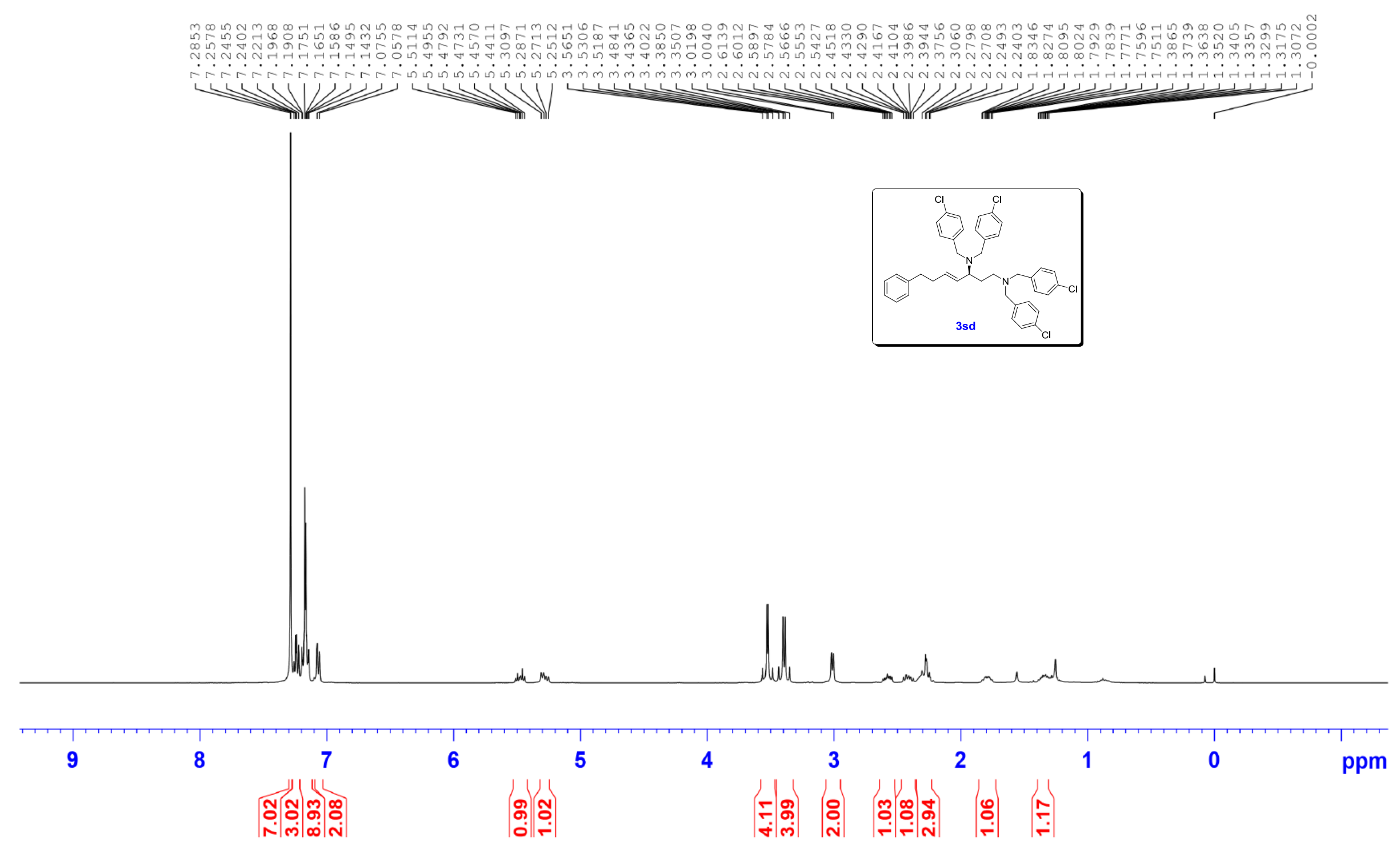




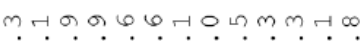

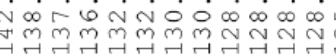

MVYH
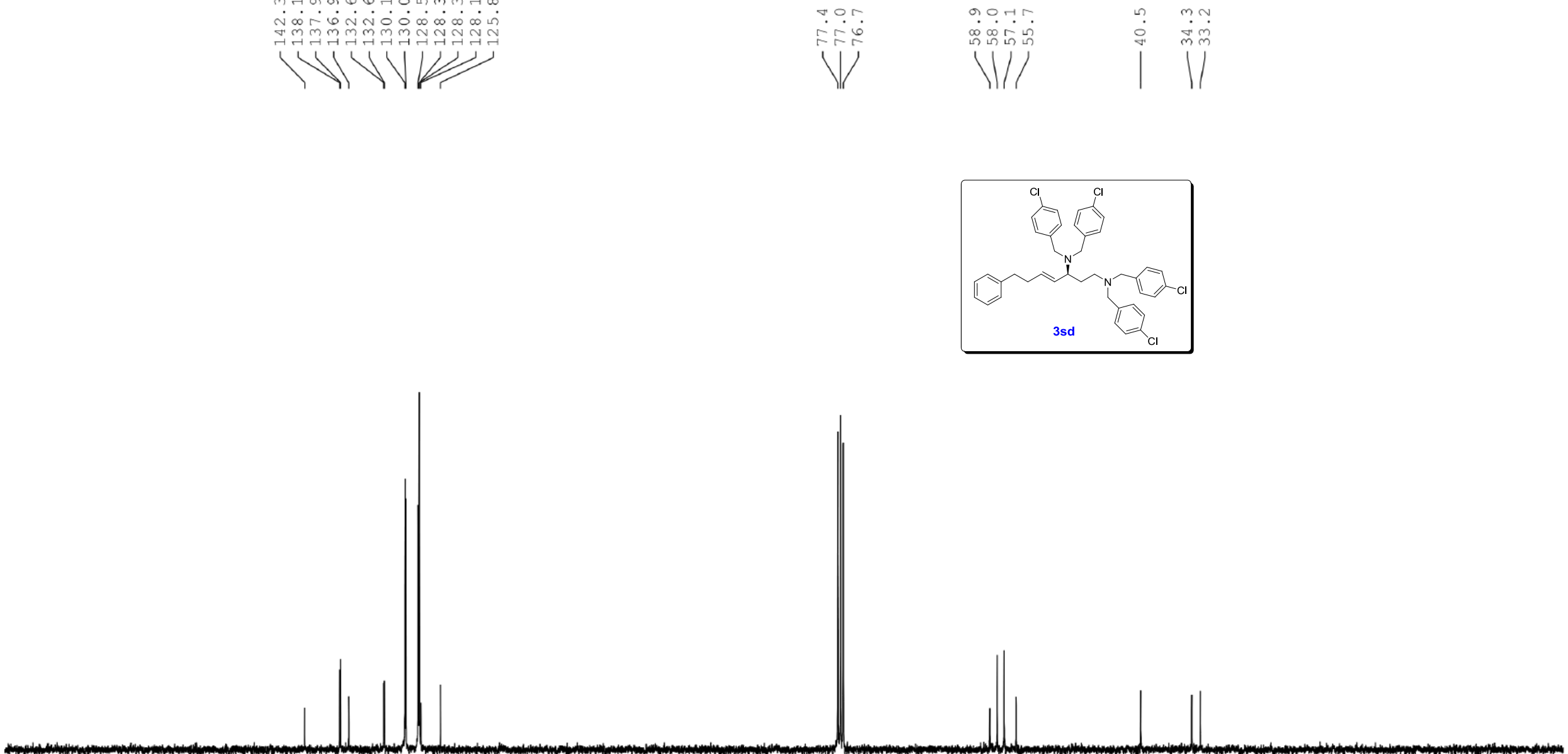


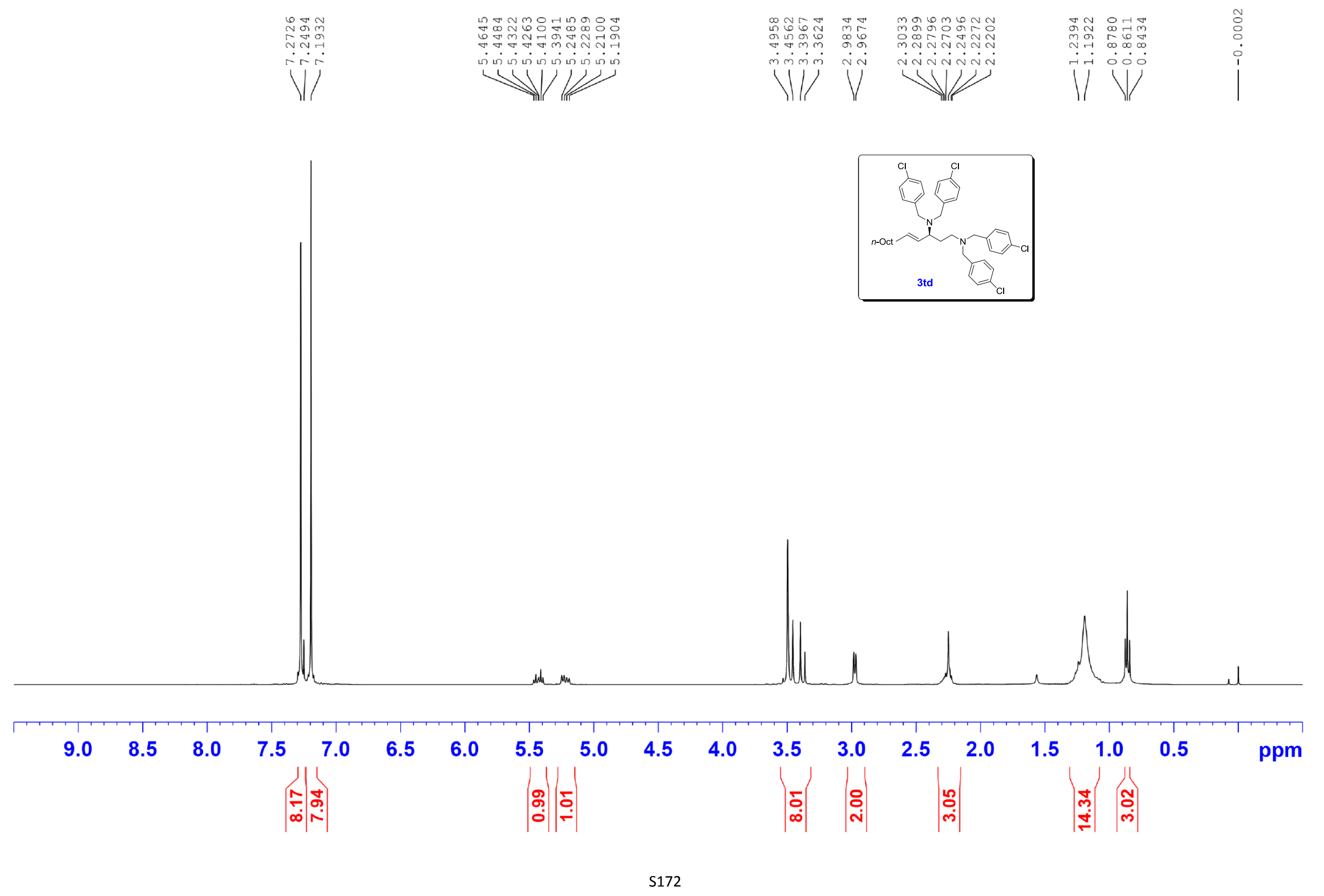



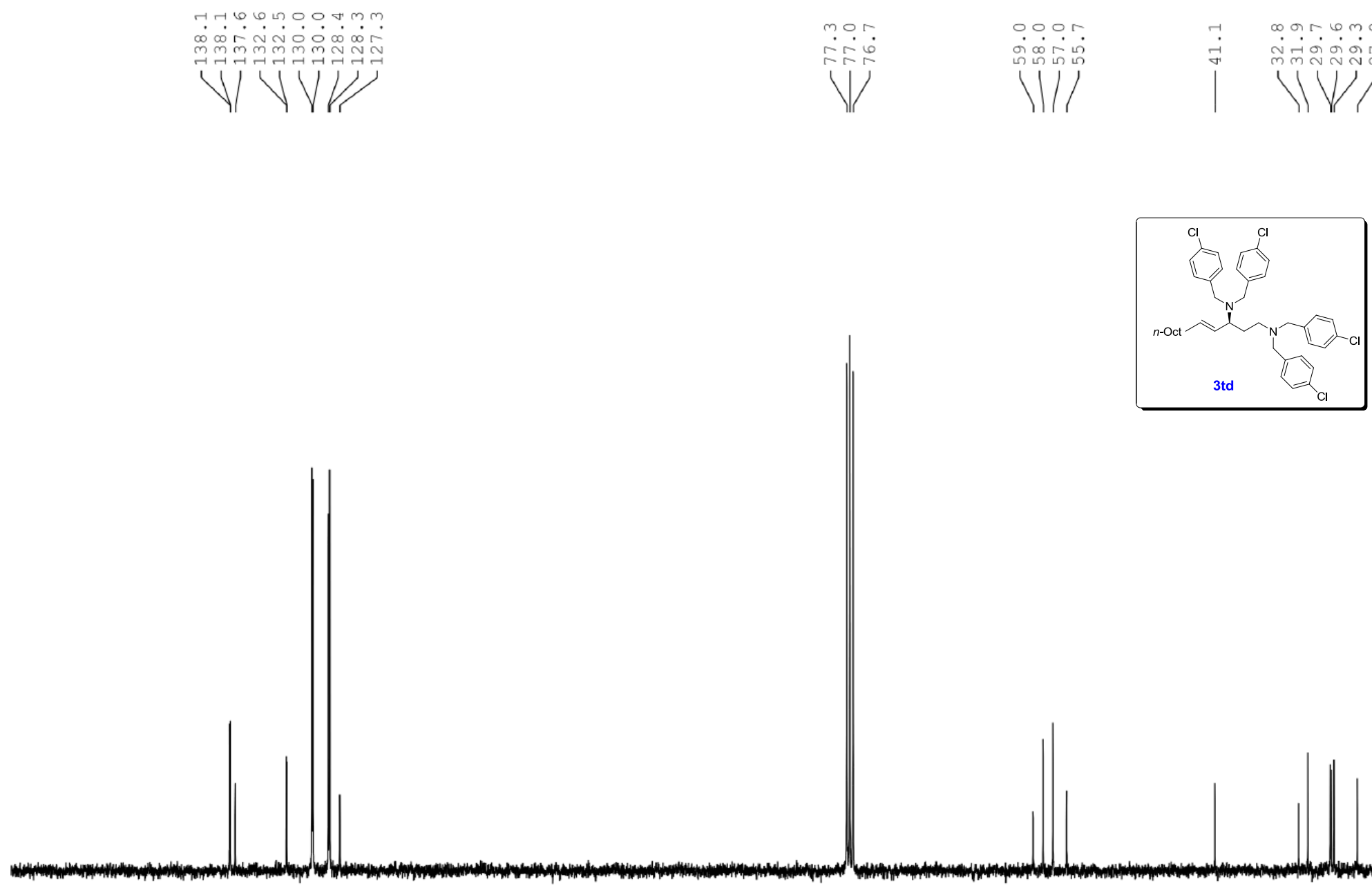

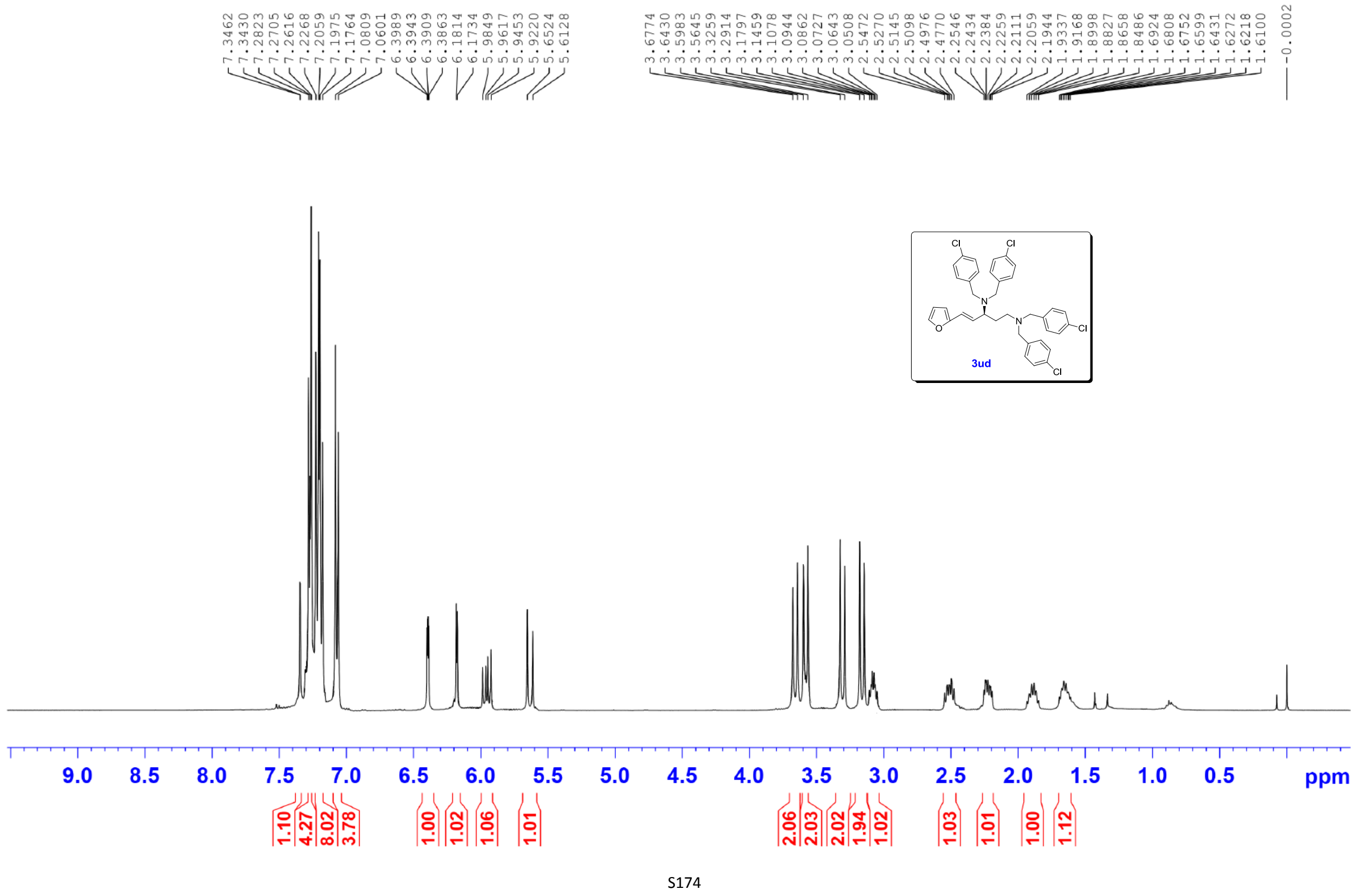


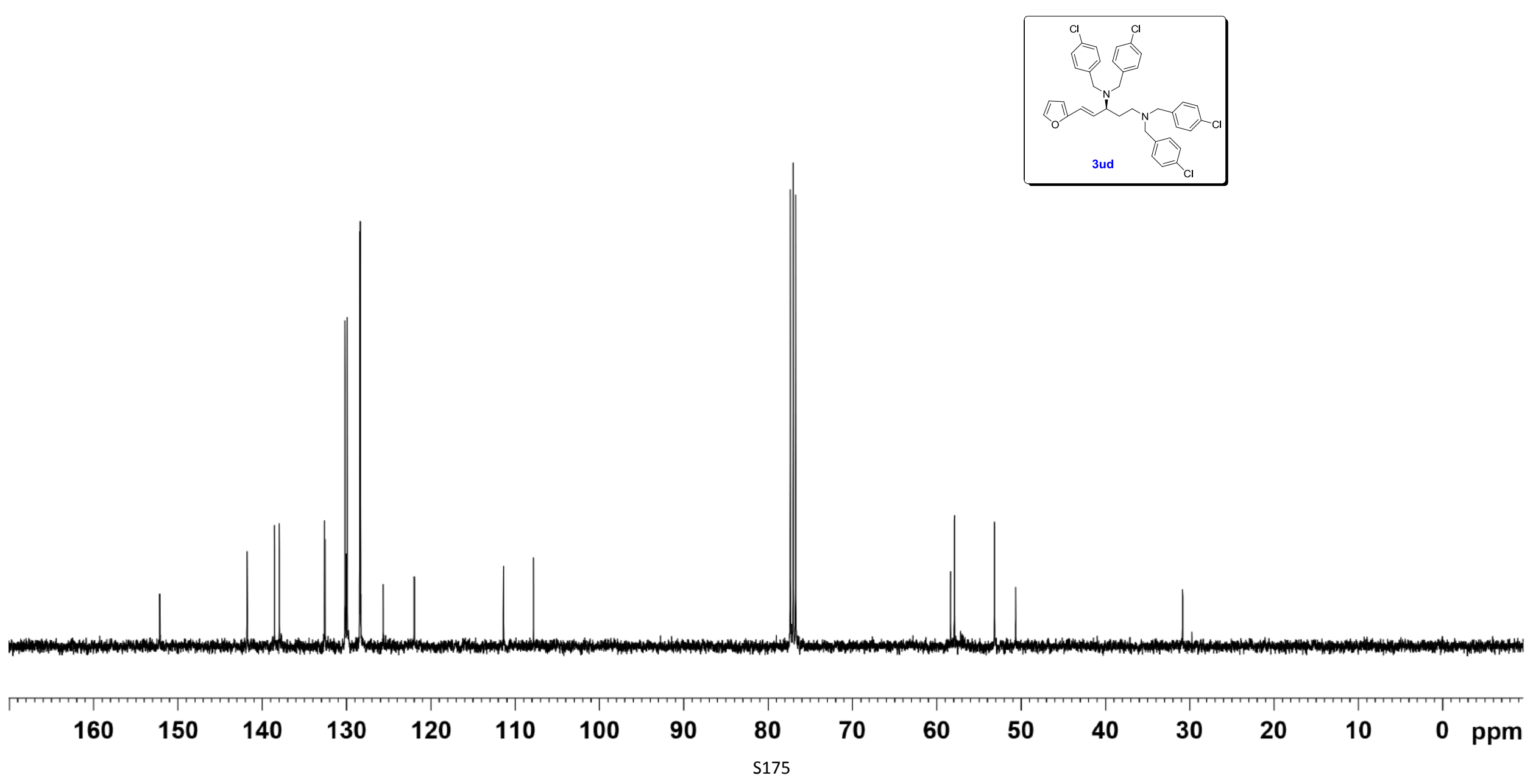




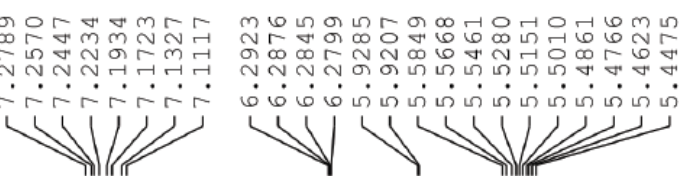

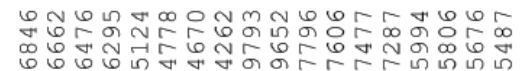

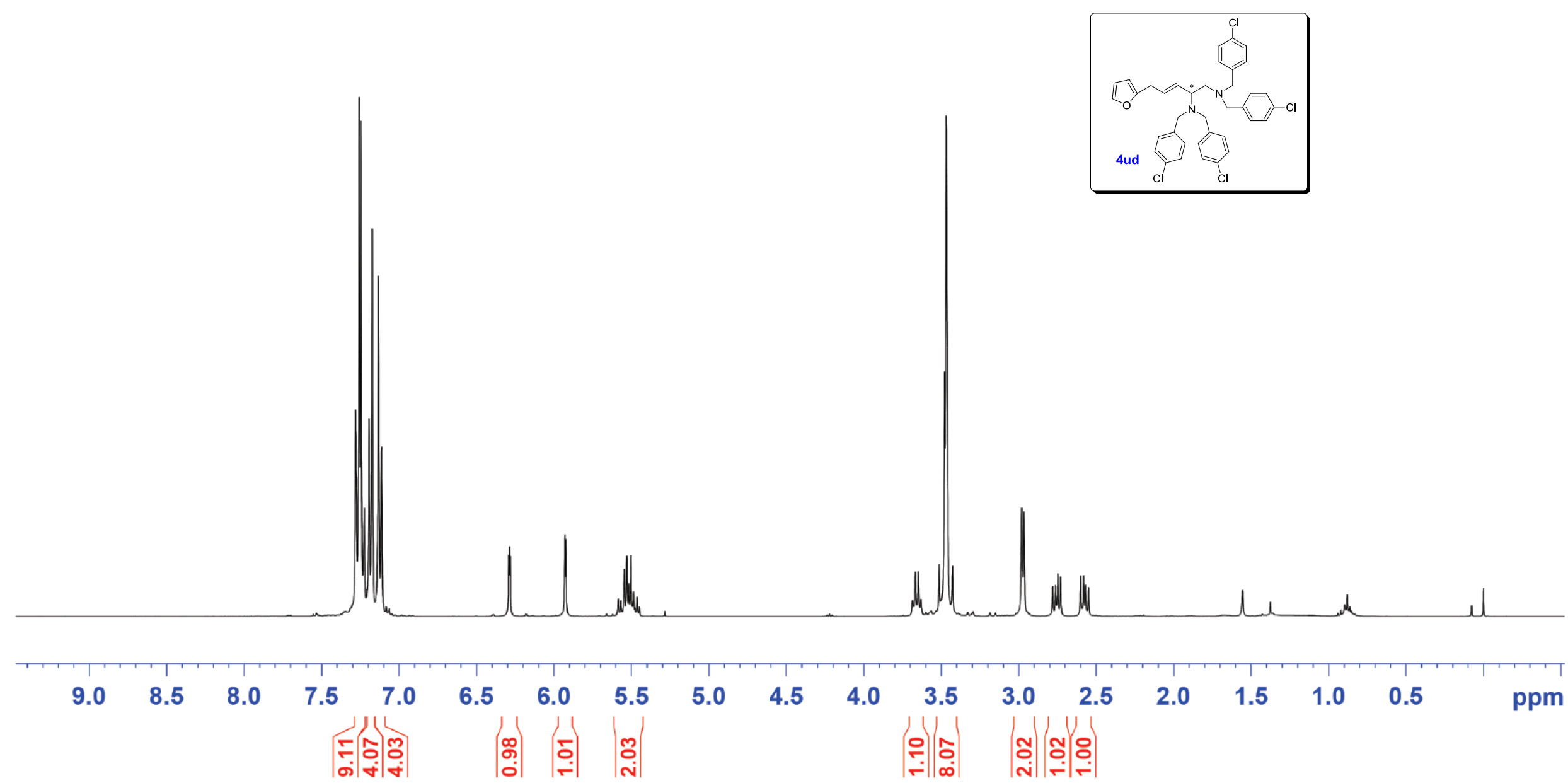



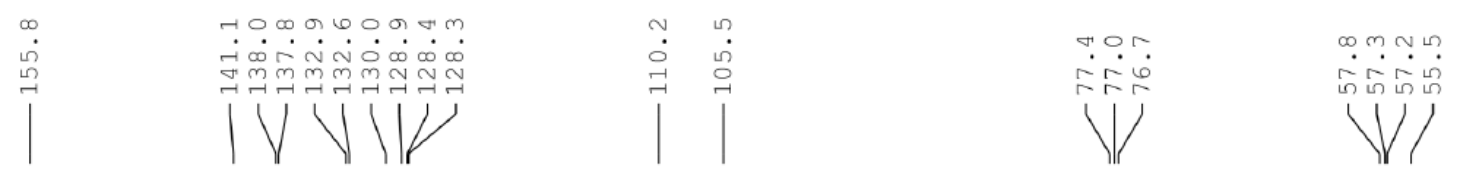

$\circ$
$\stackrel{\sigma}{+}$
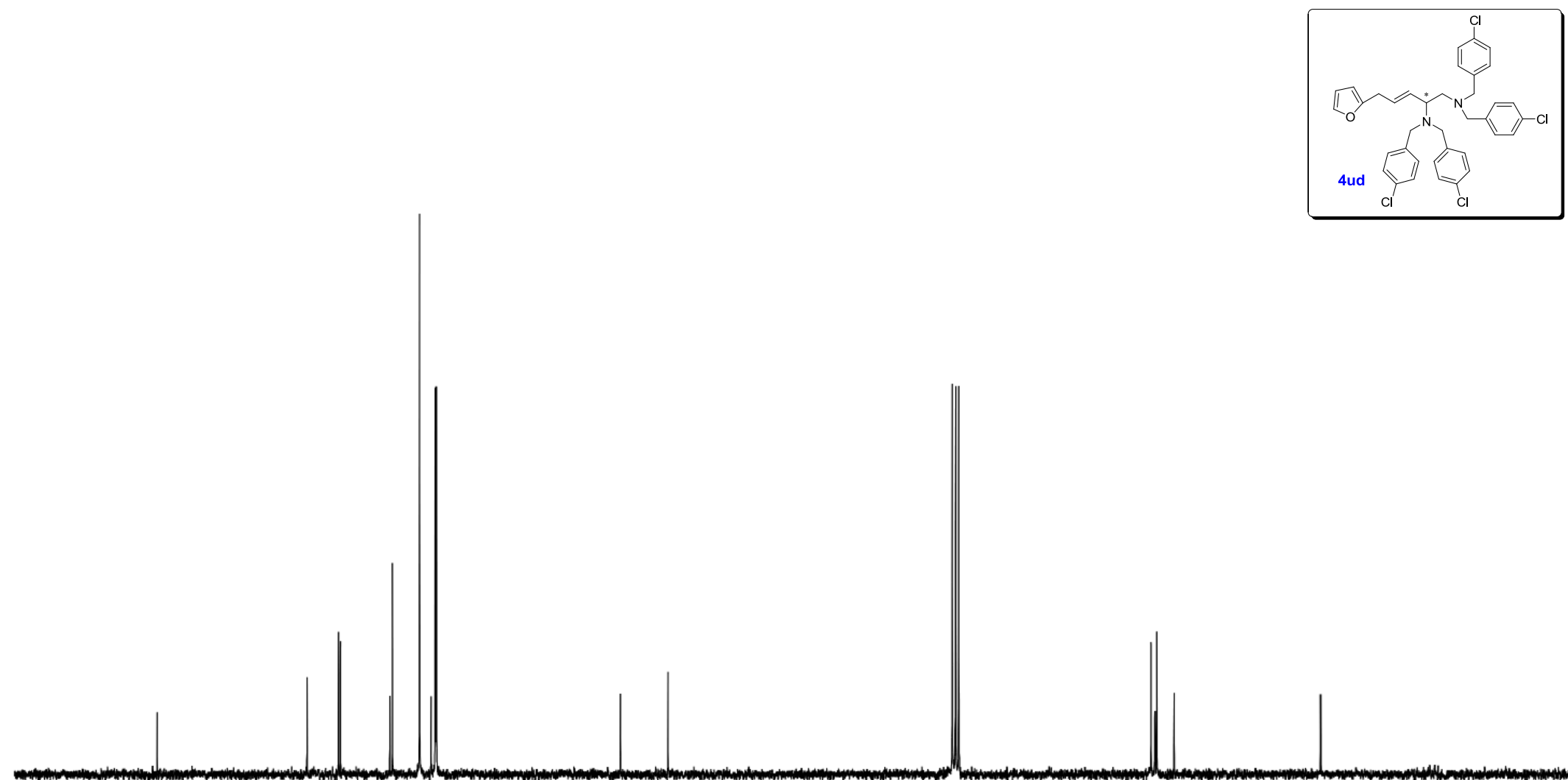

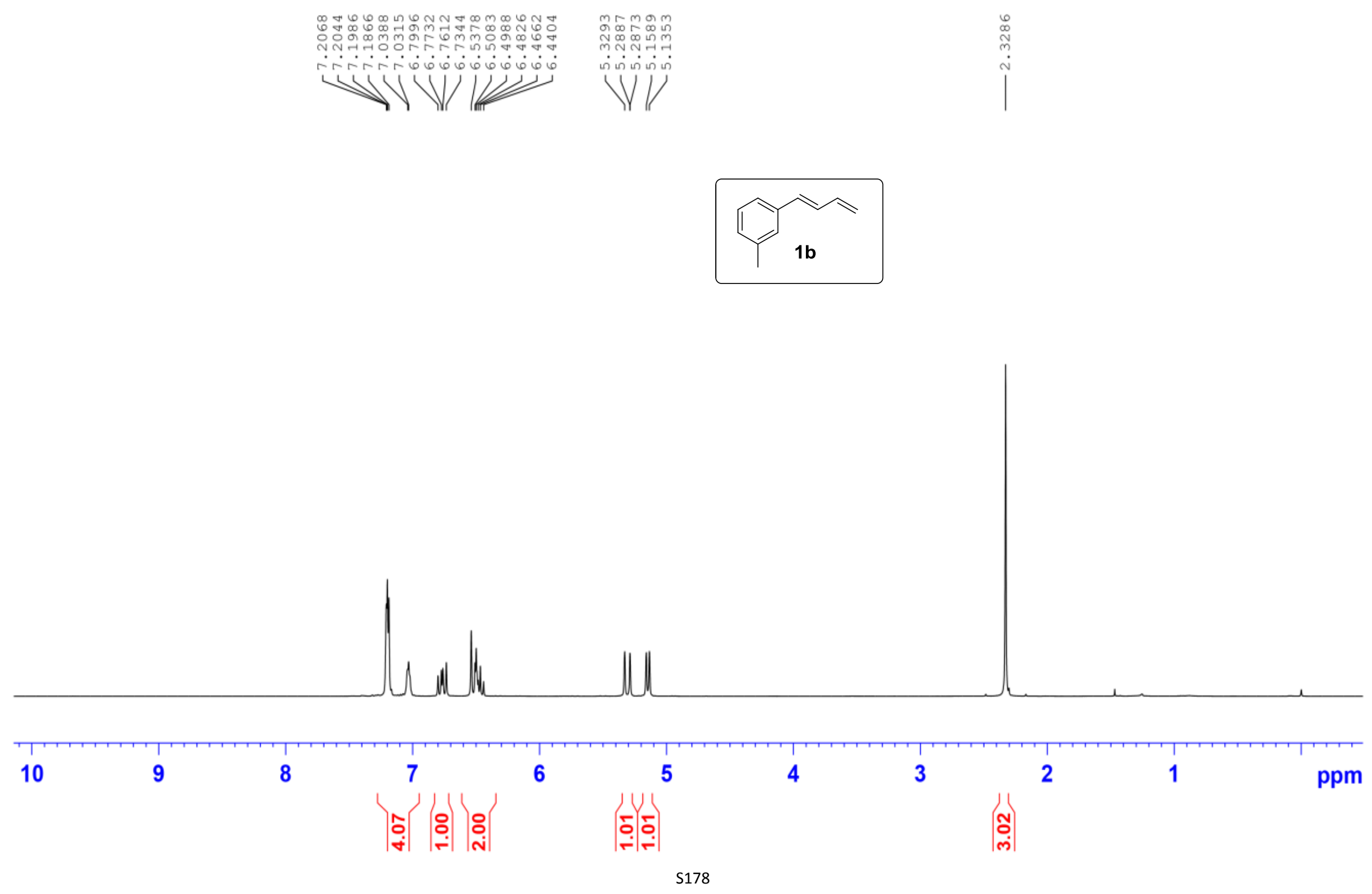

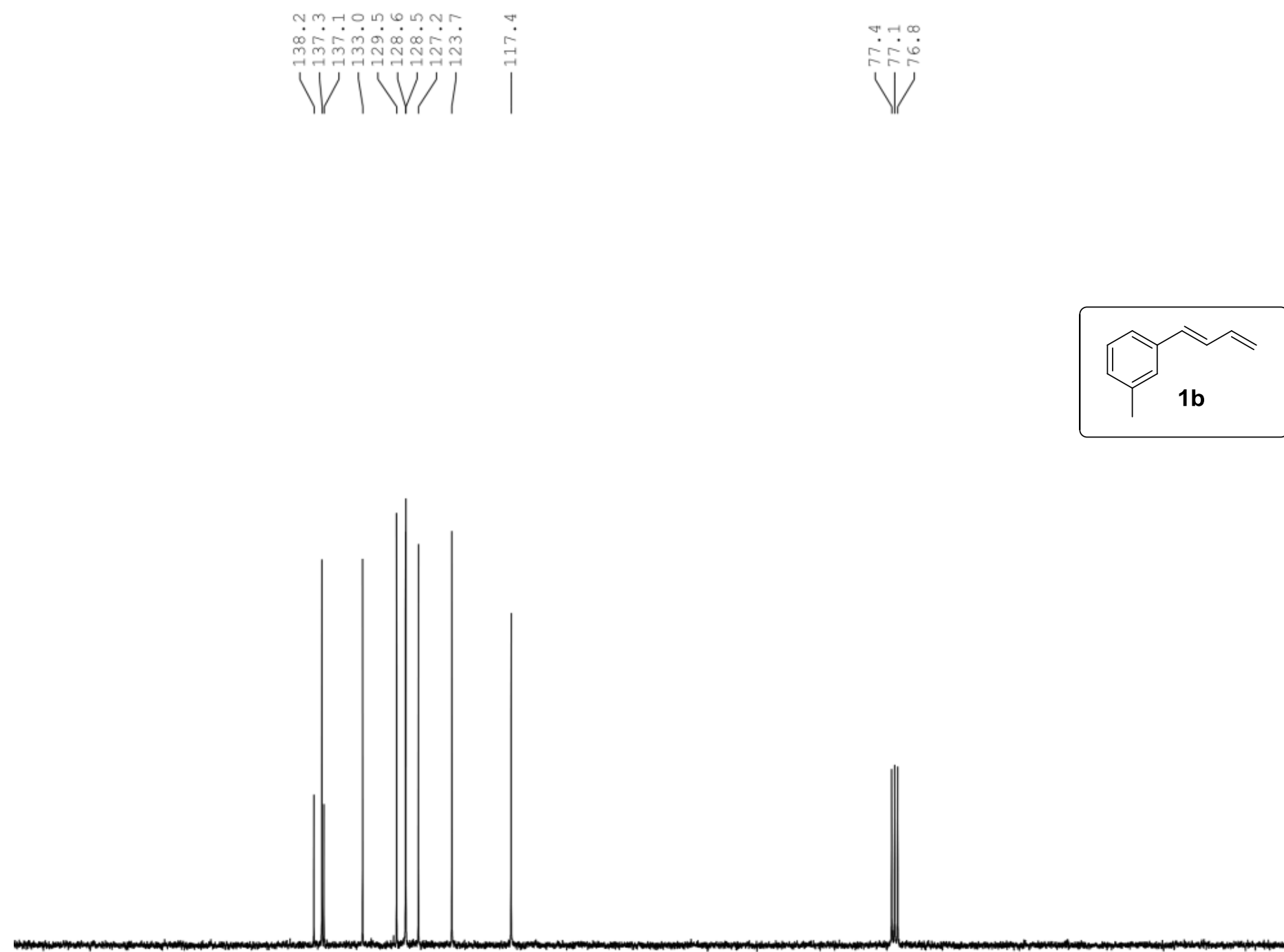

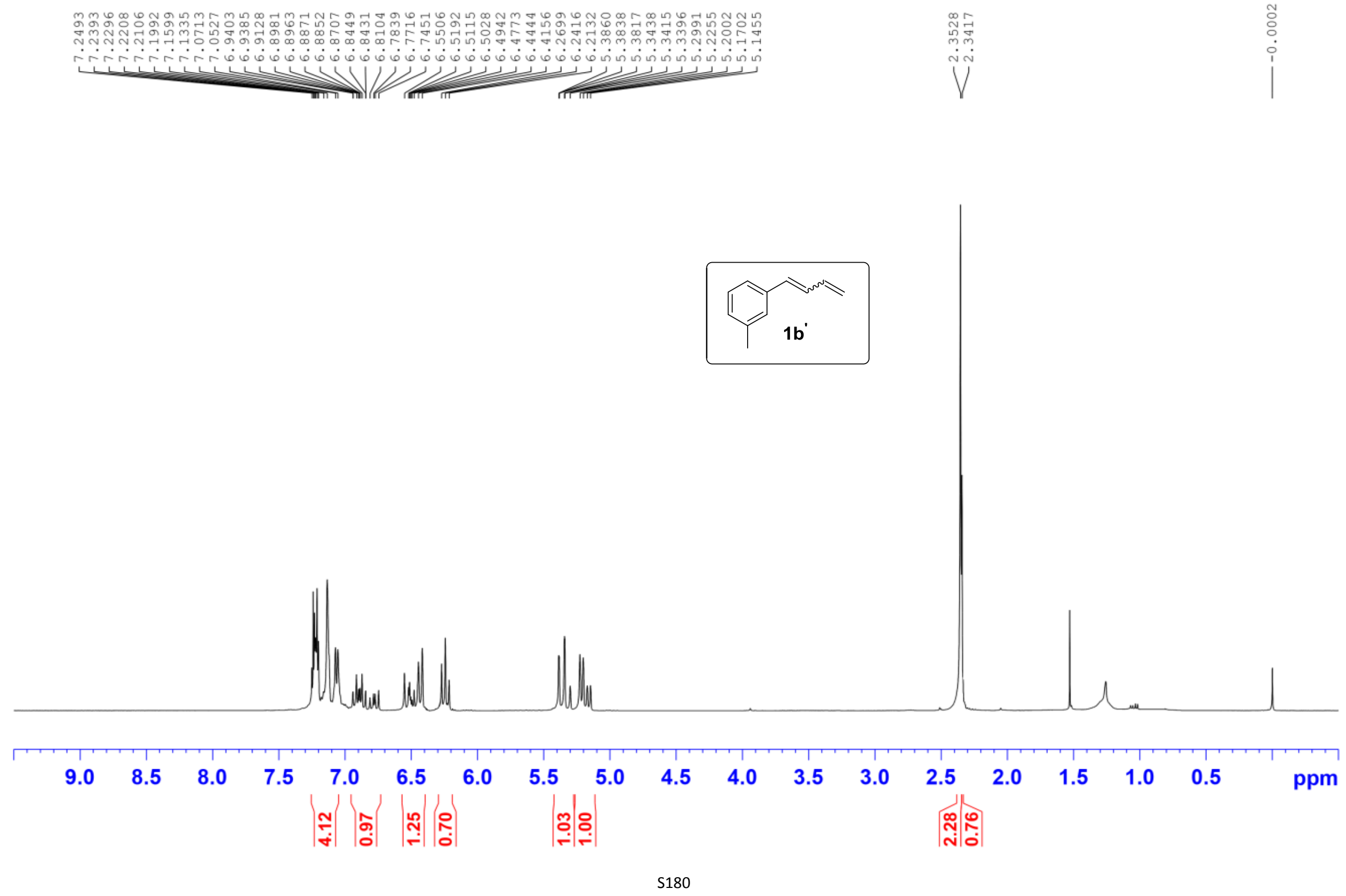

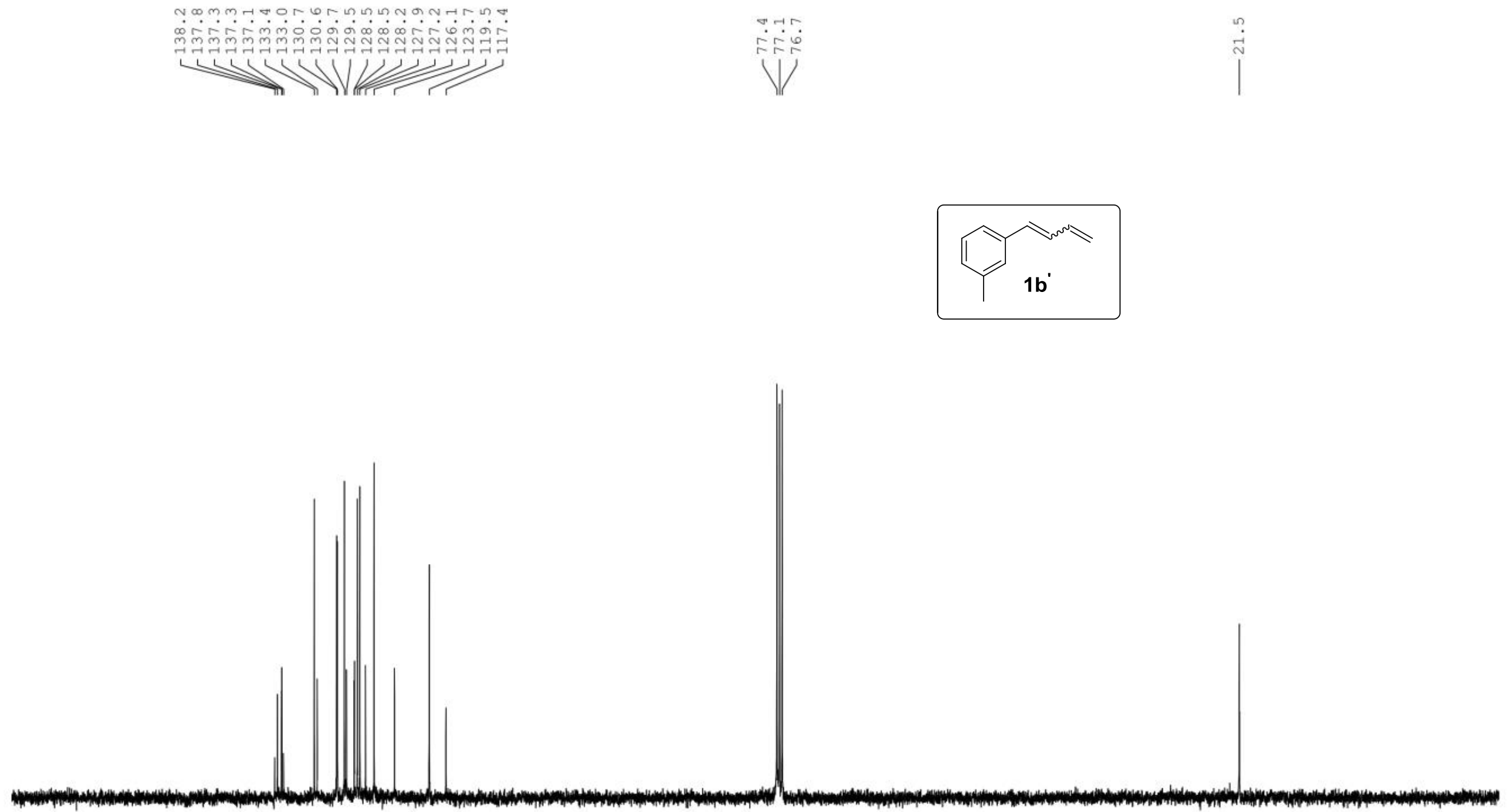

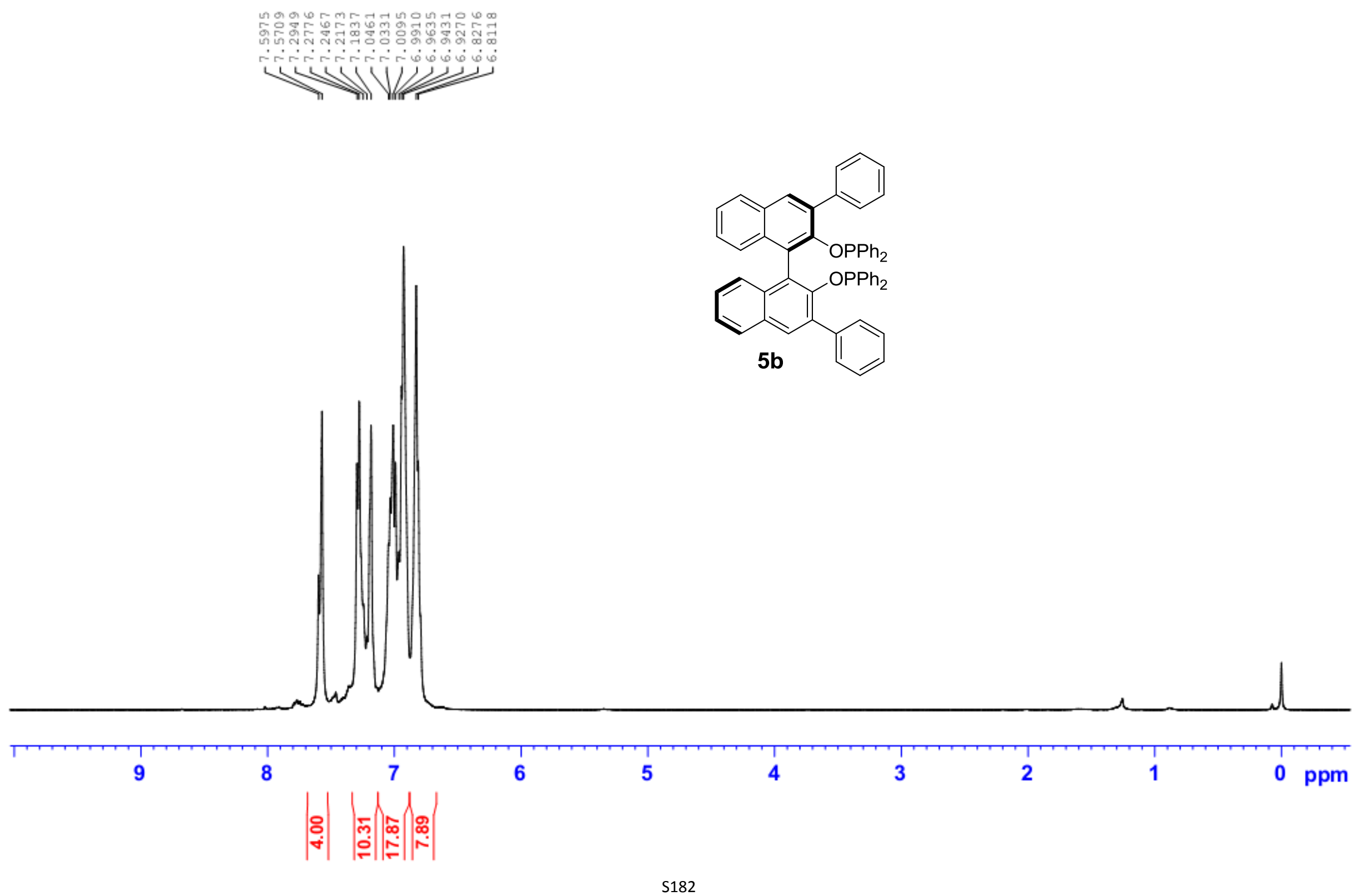


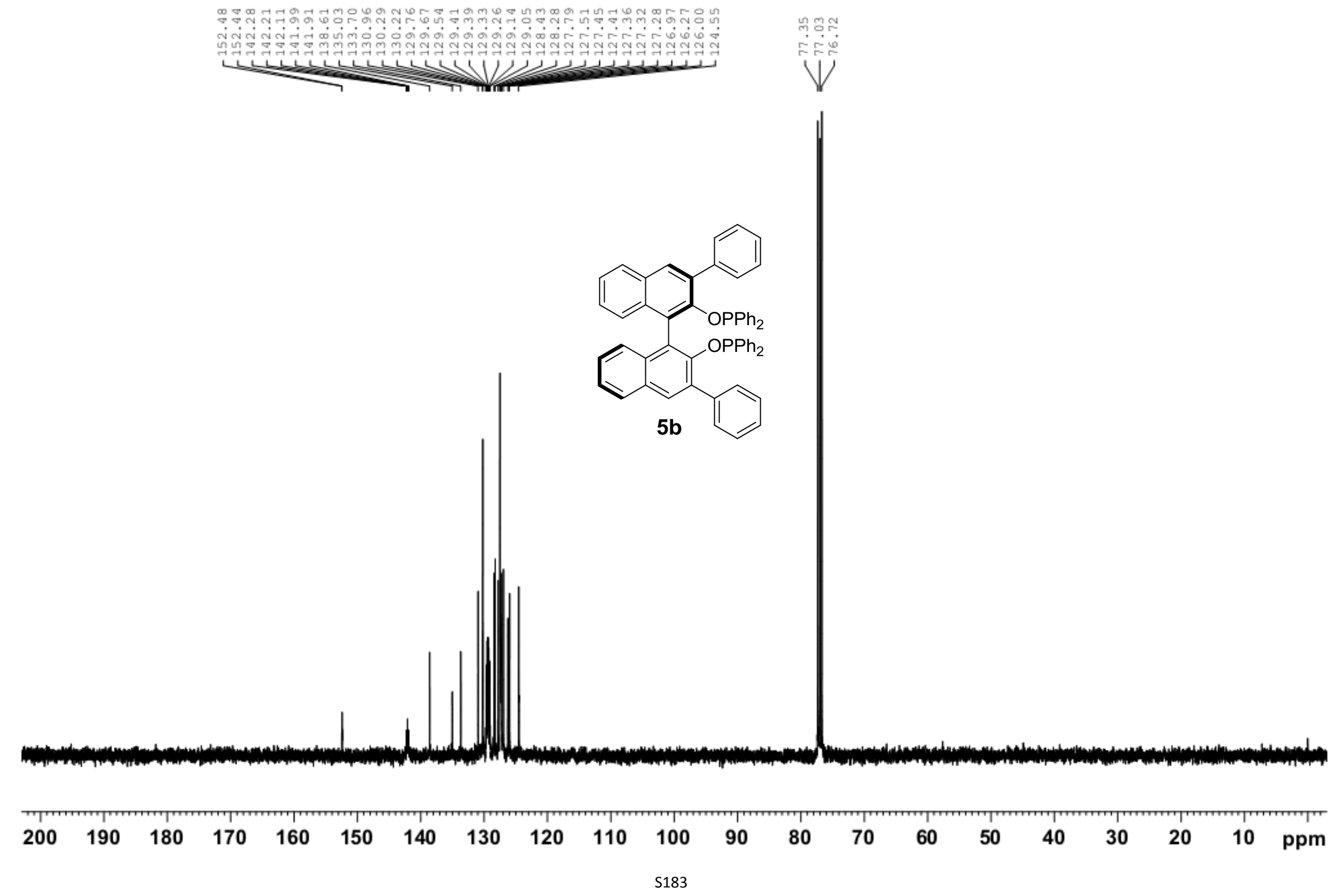




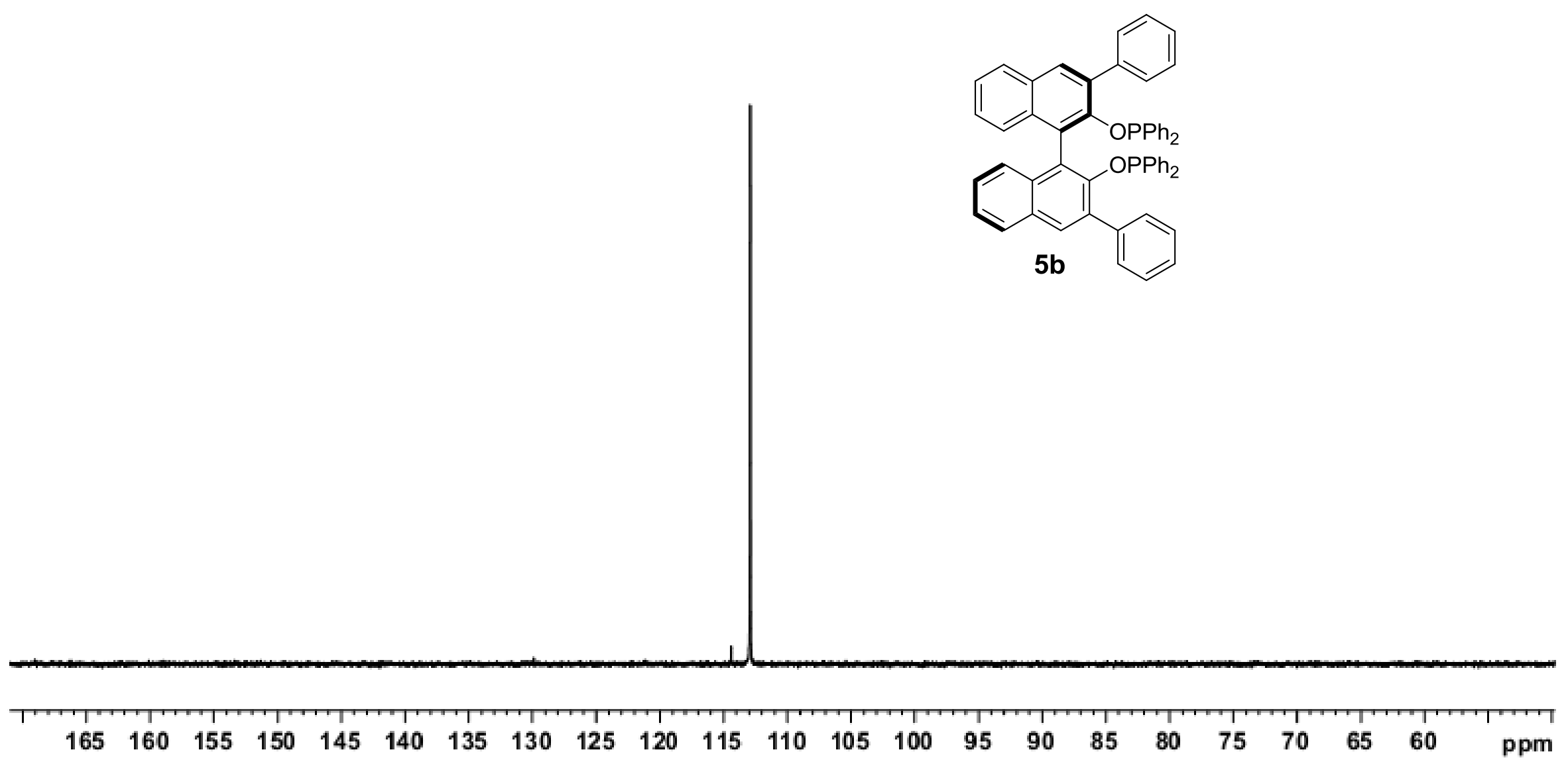




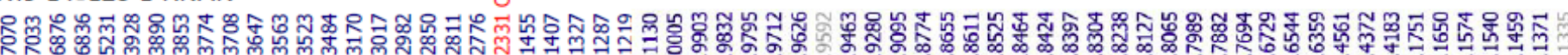

等

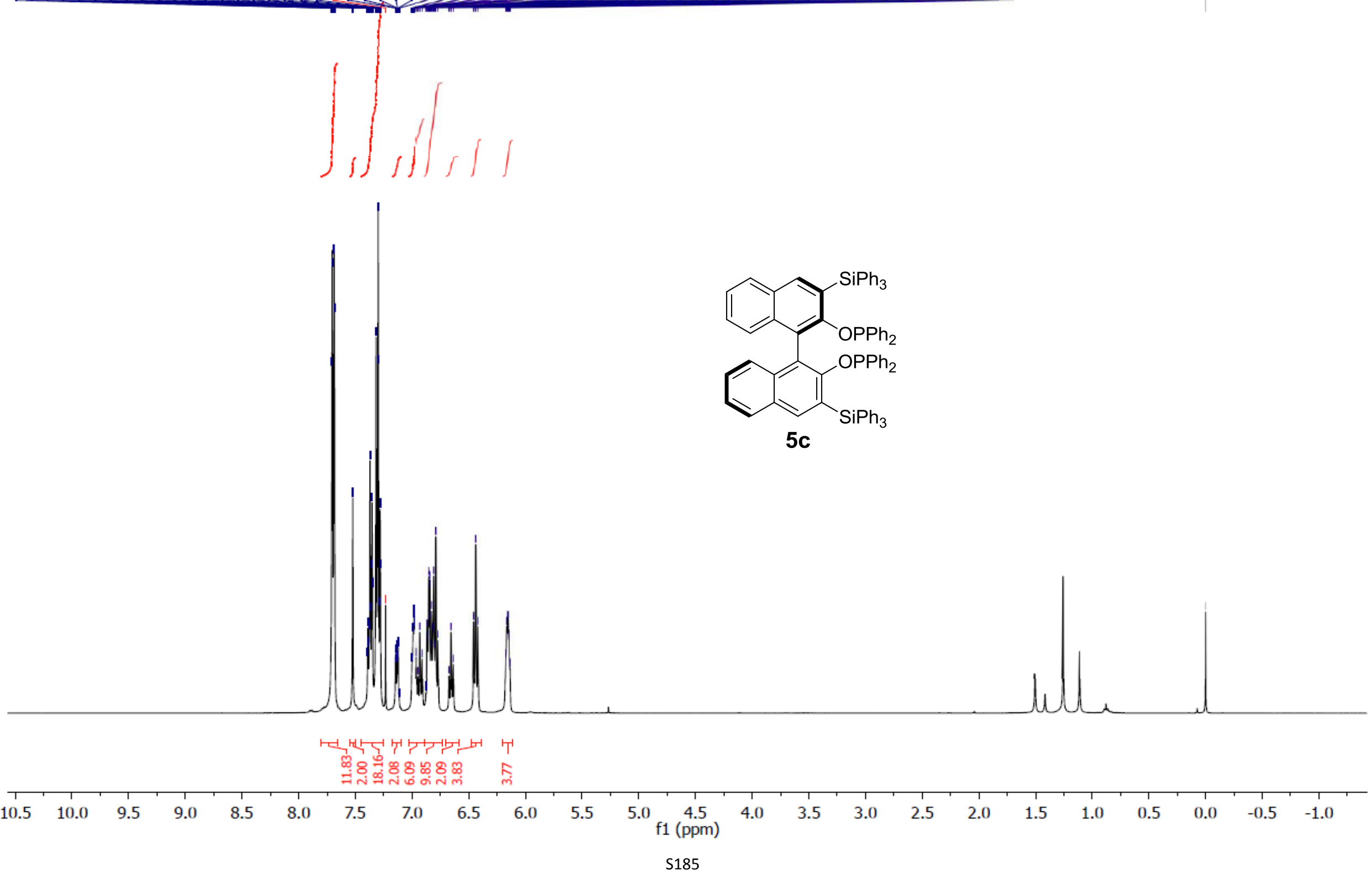



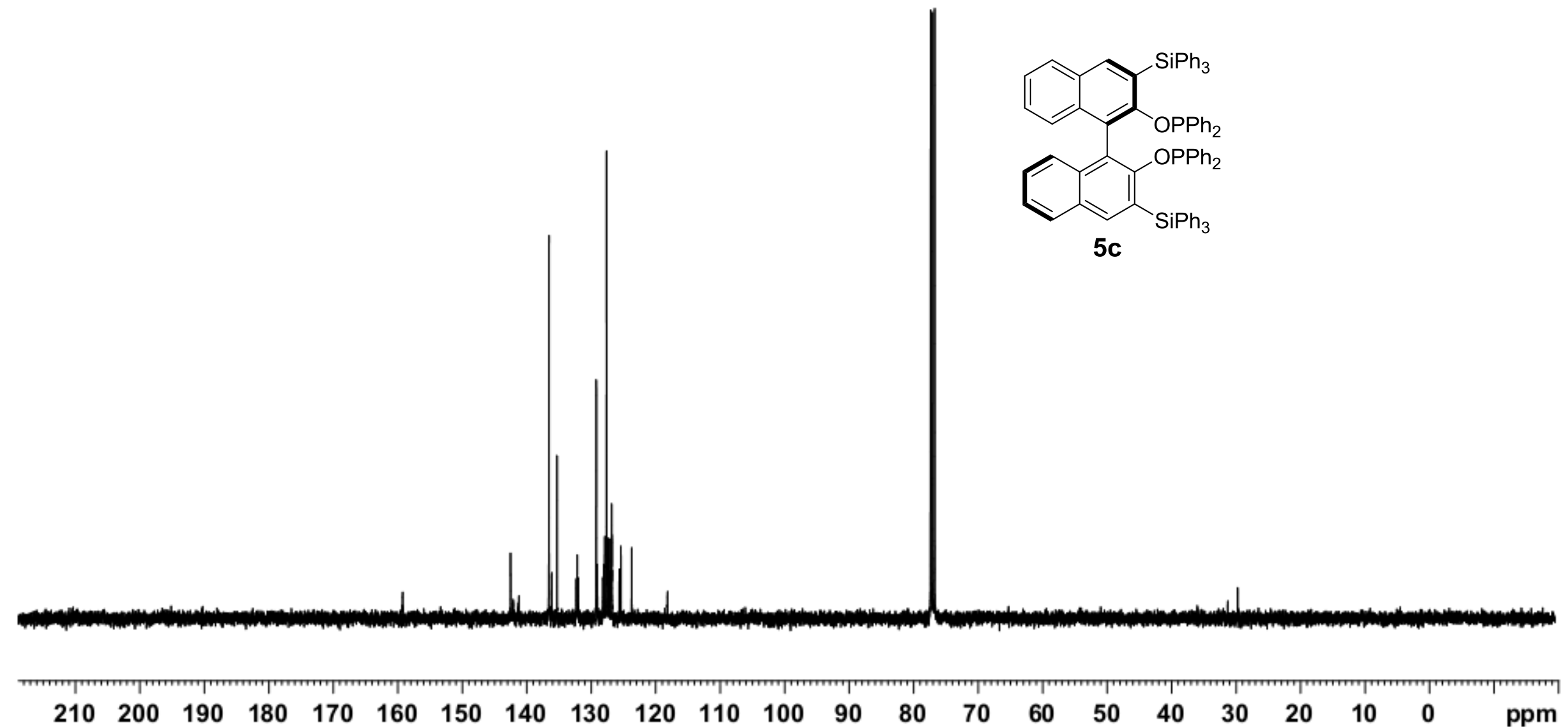


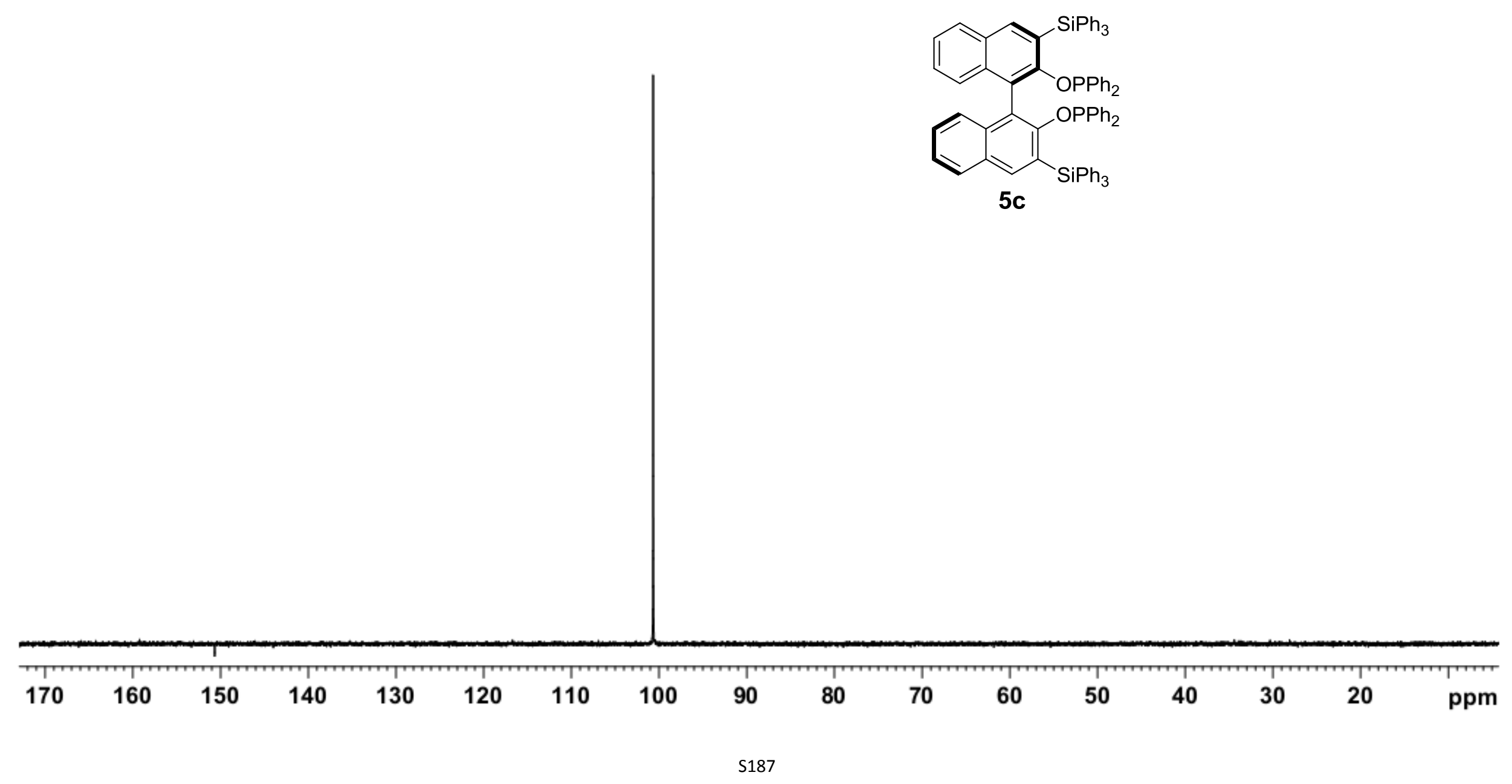




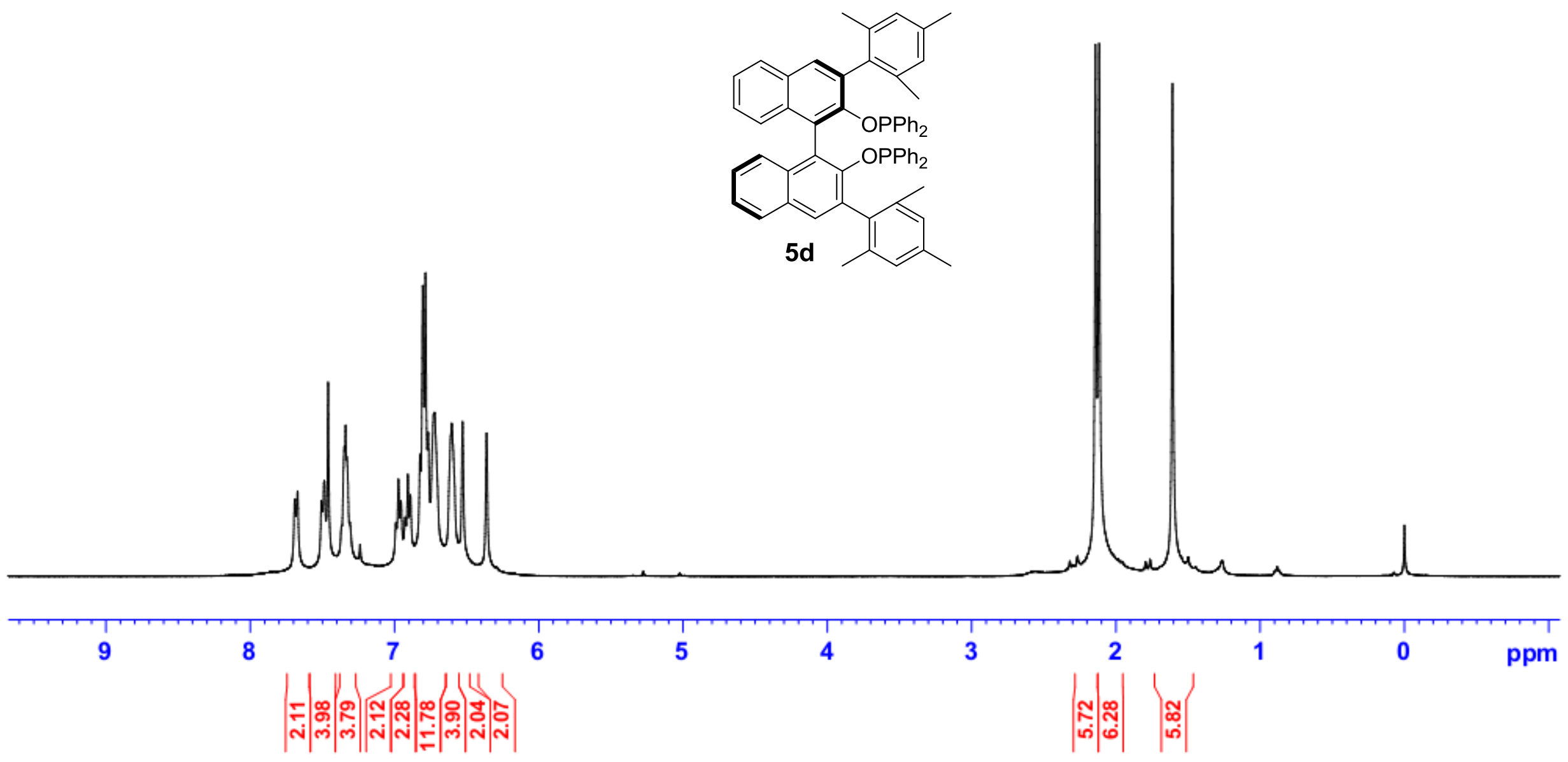




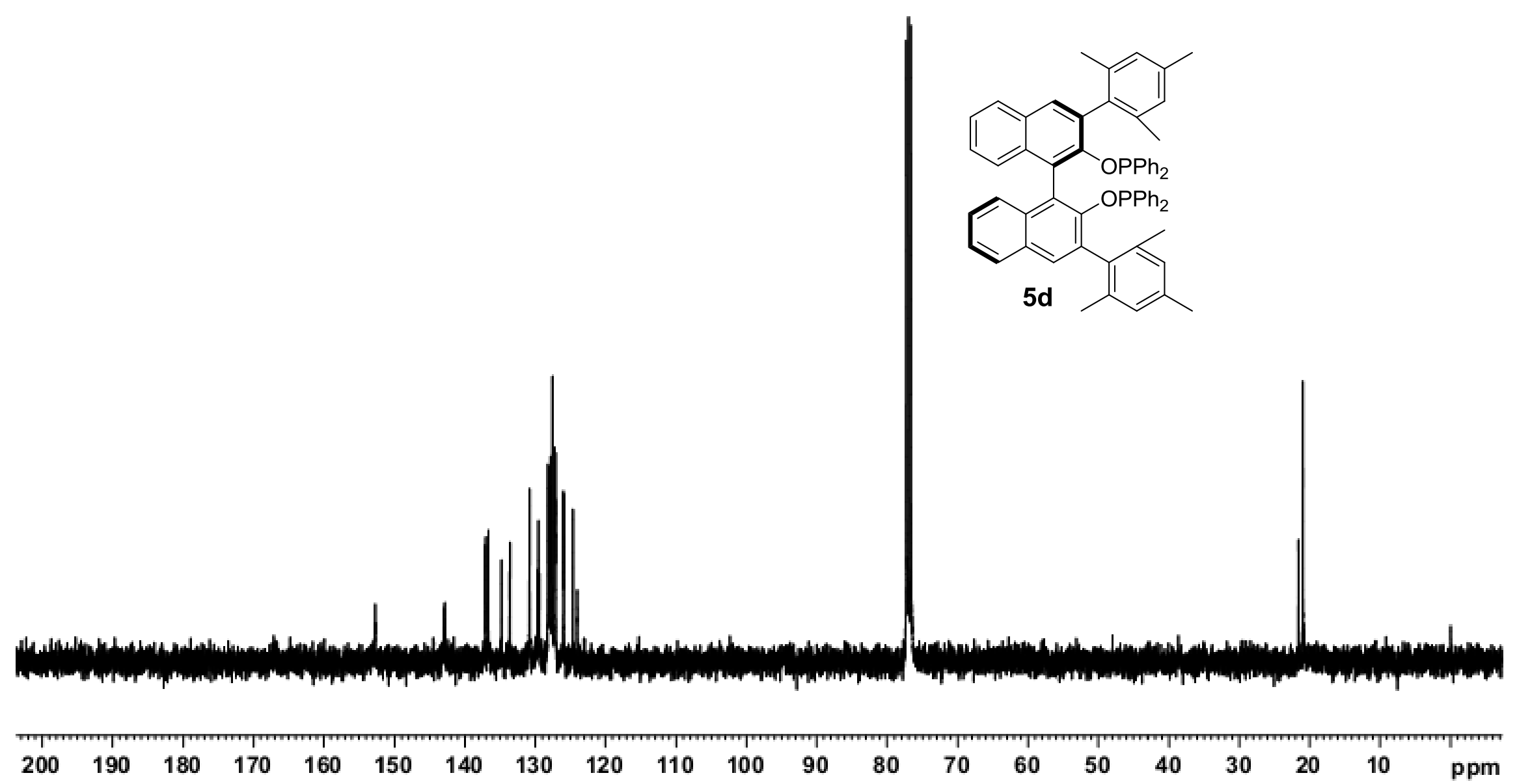




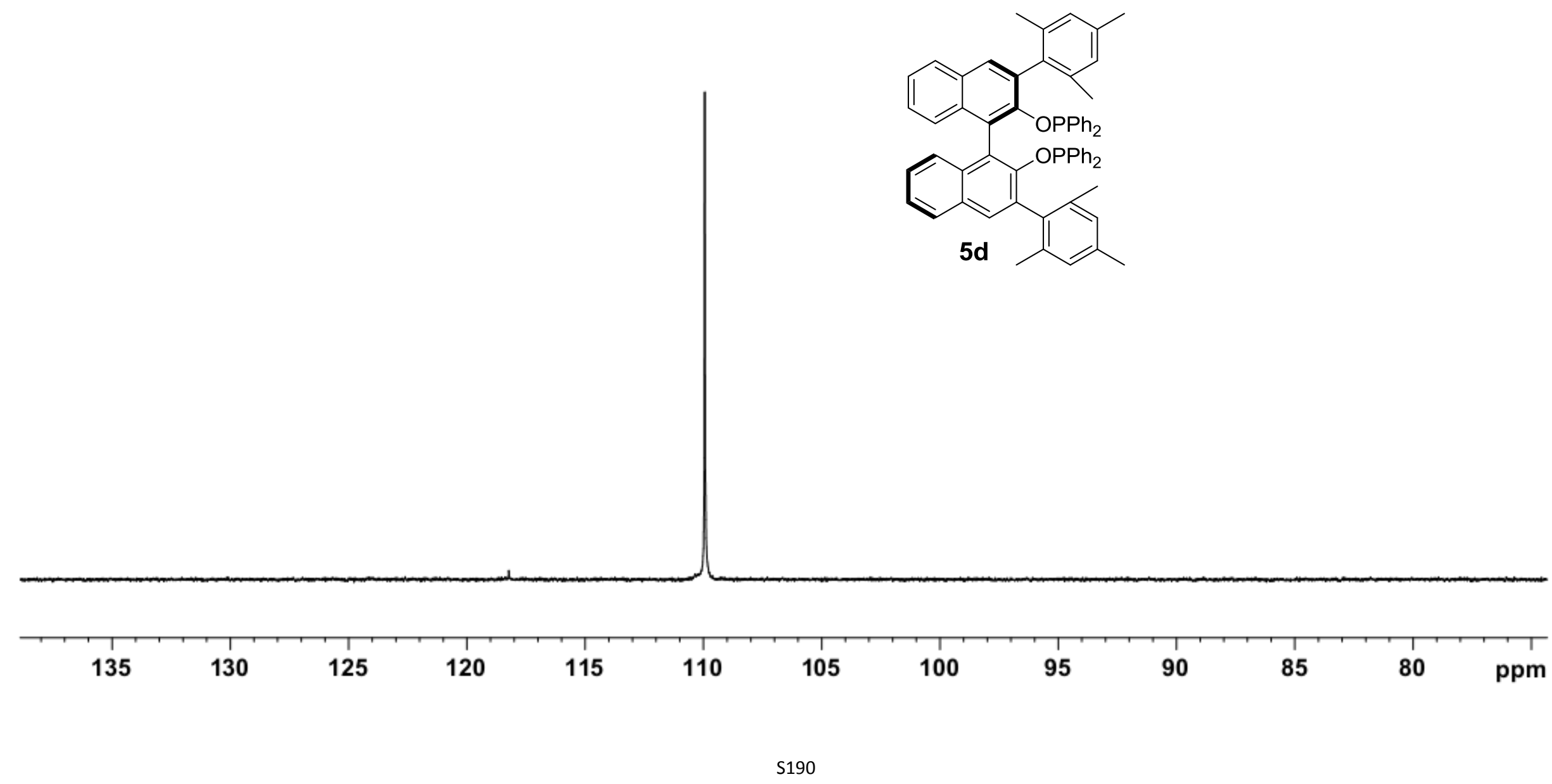



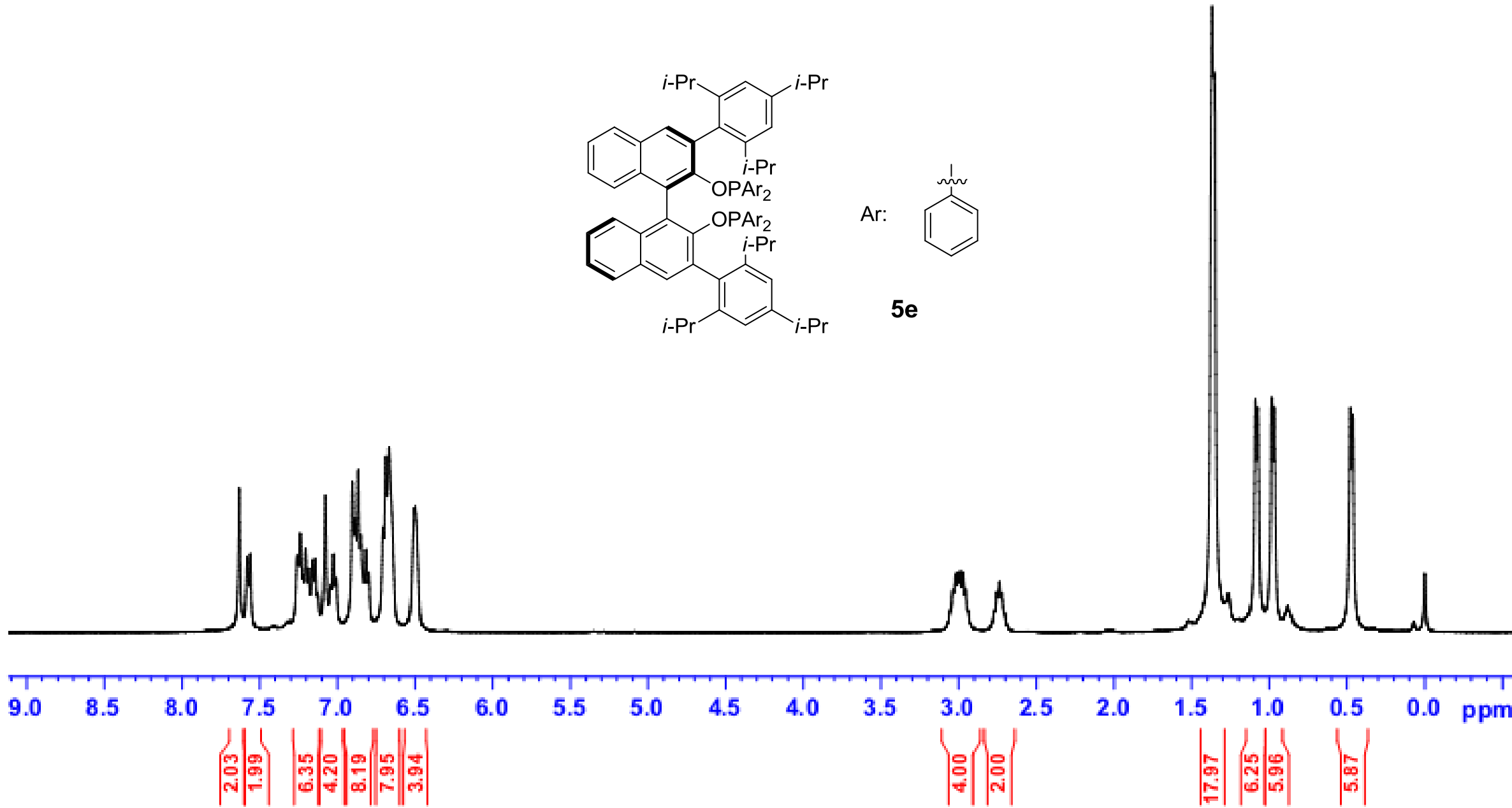

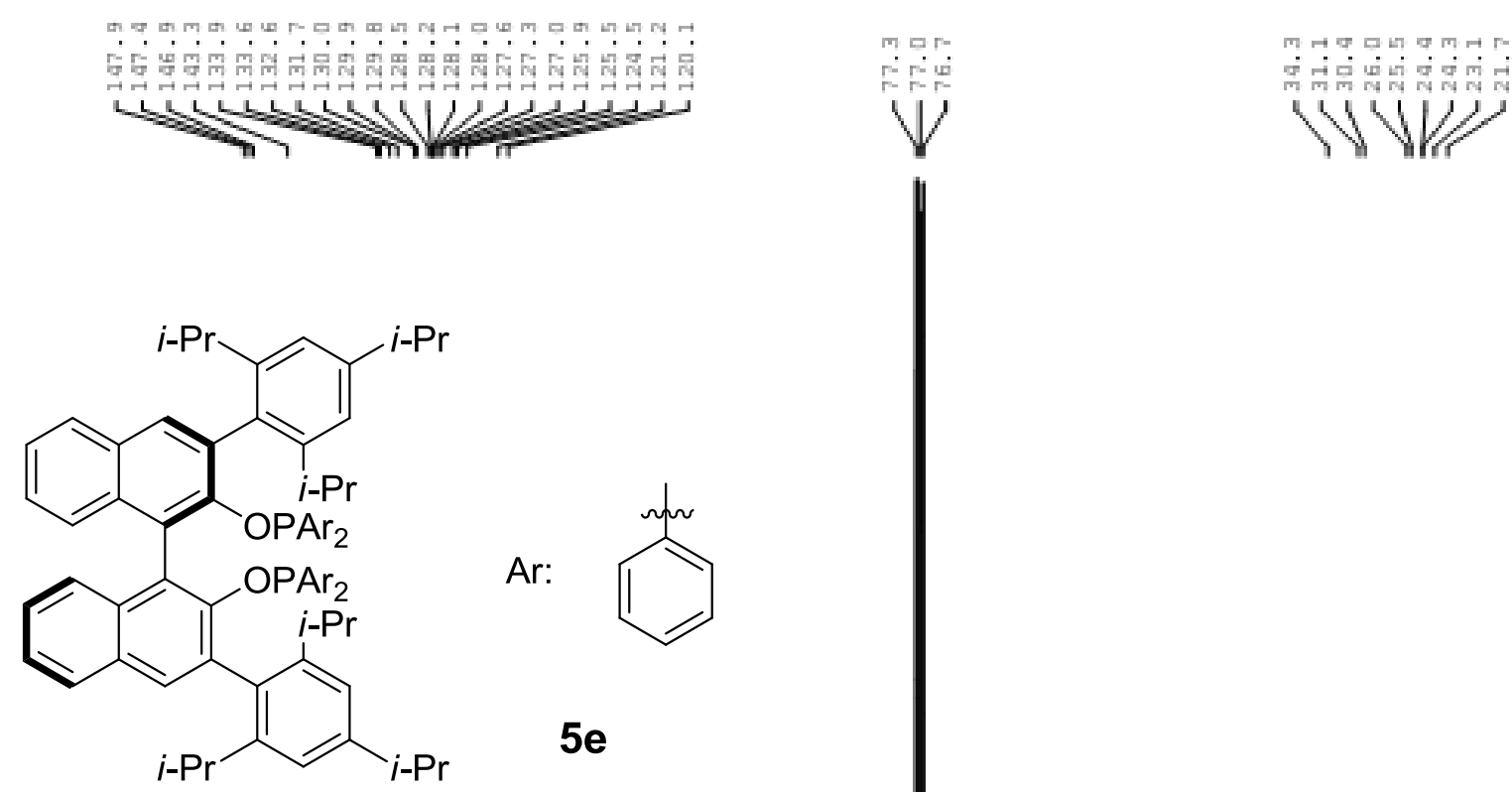

Ar:

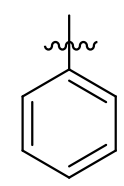

$5 e$

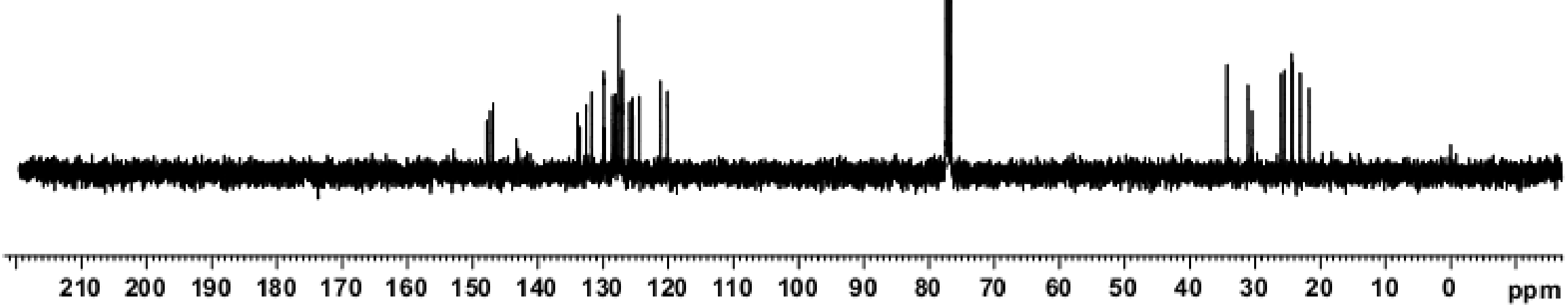




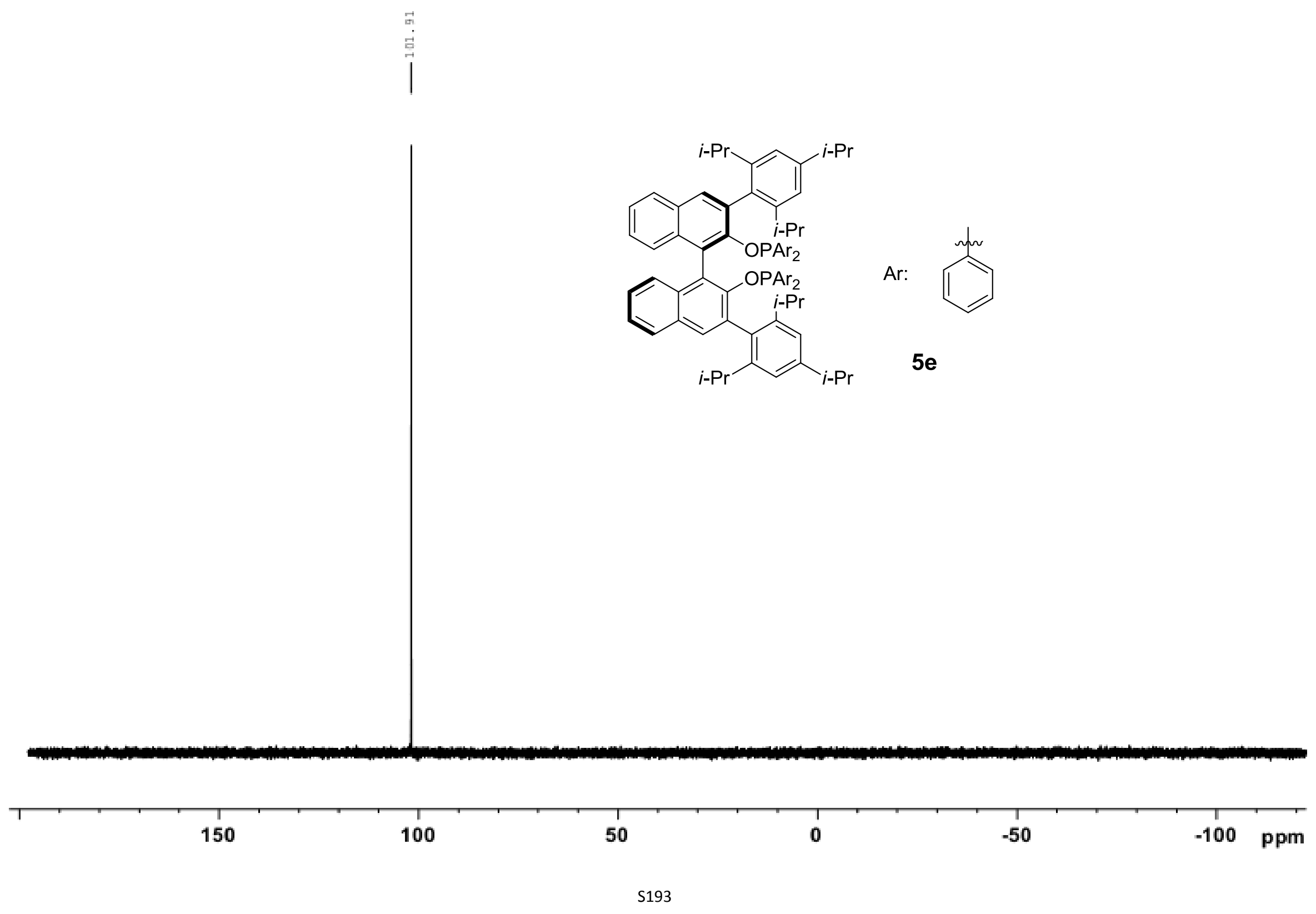



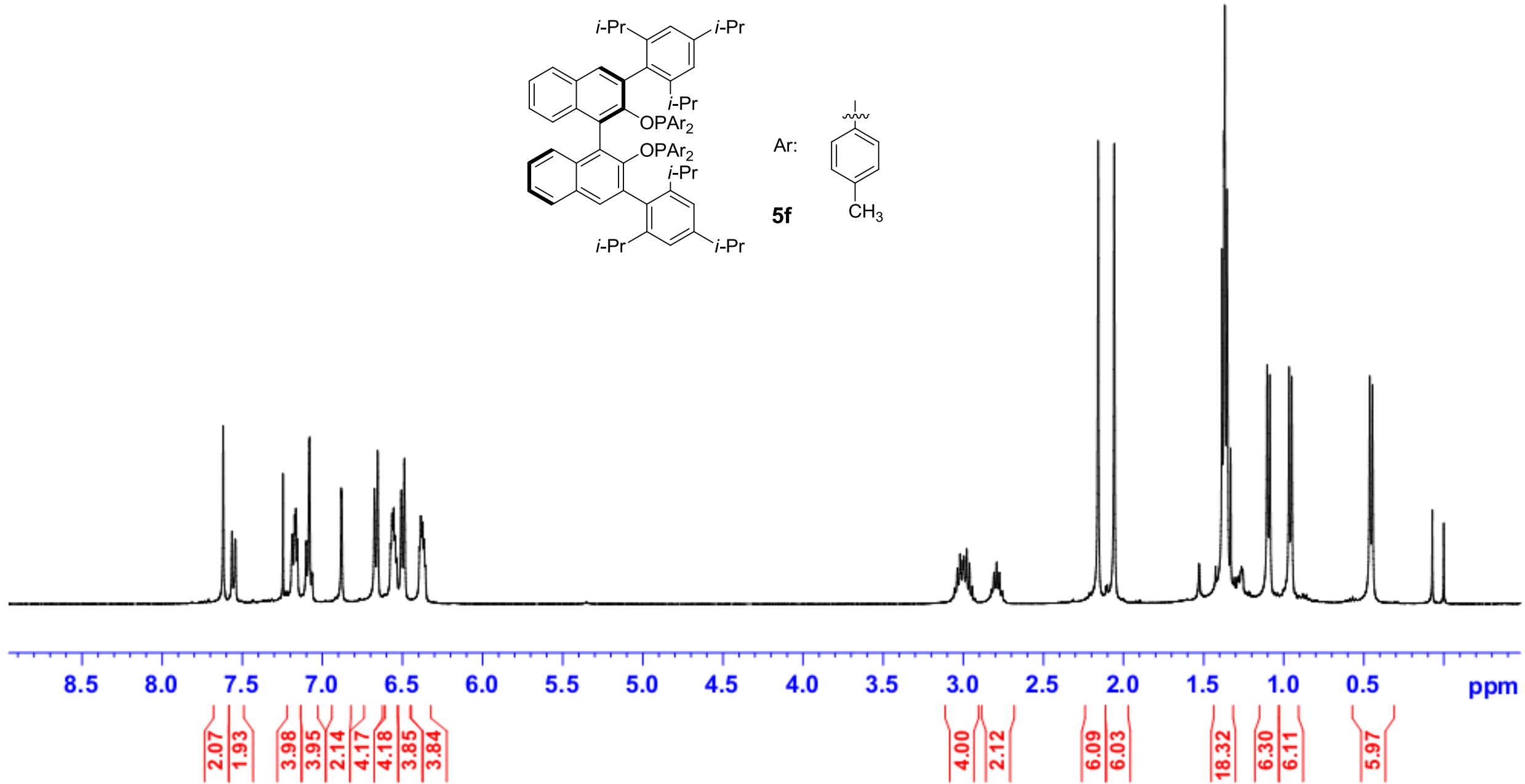


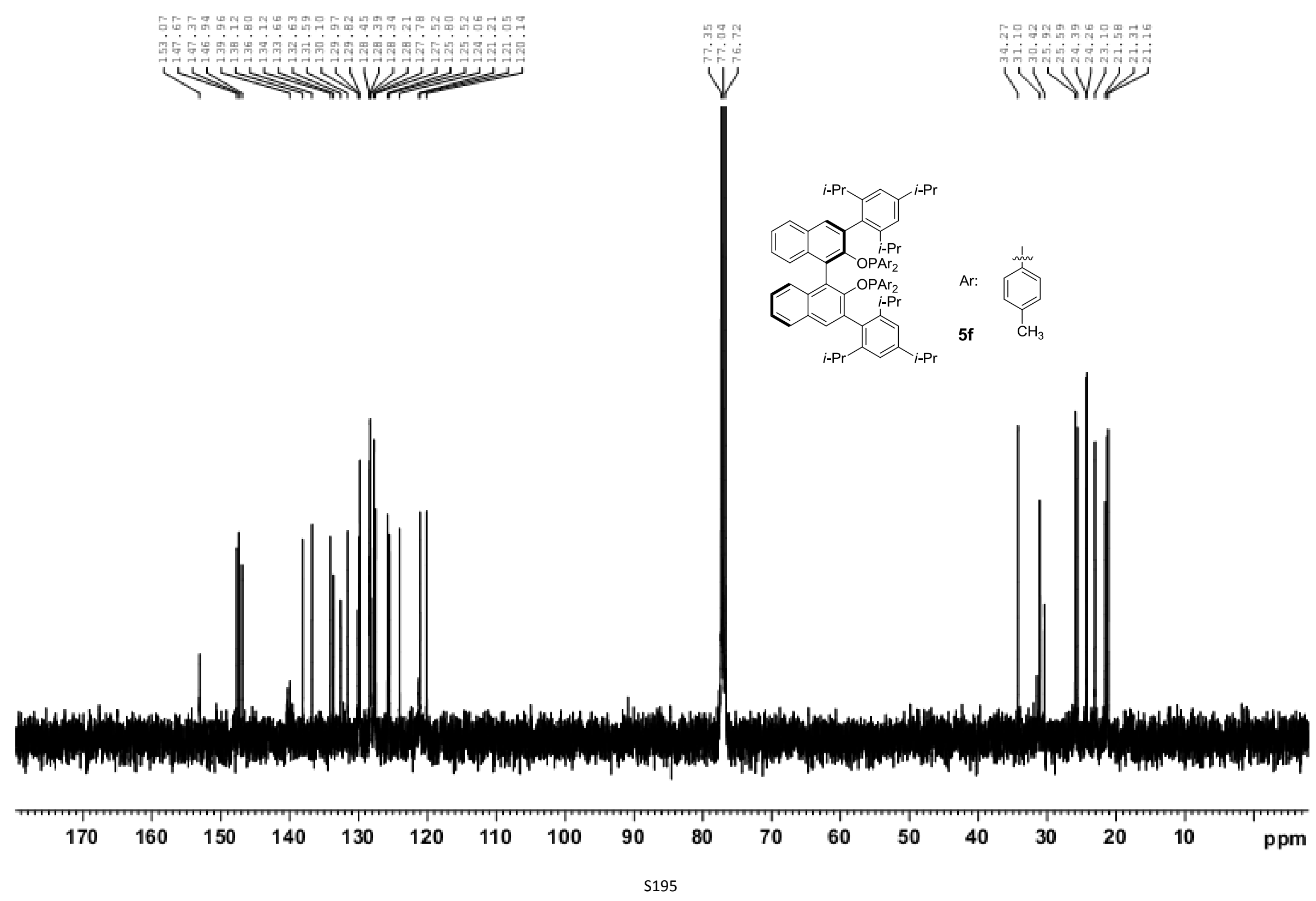




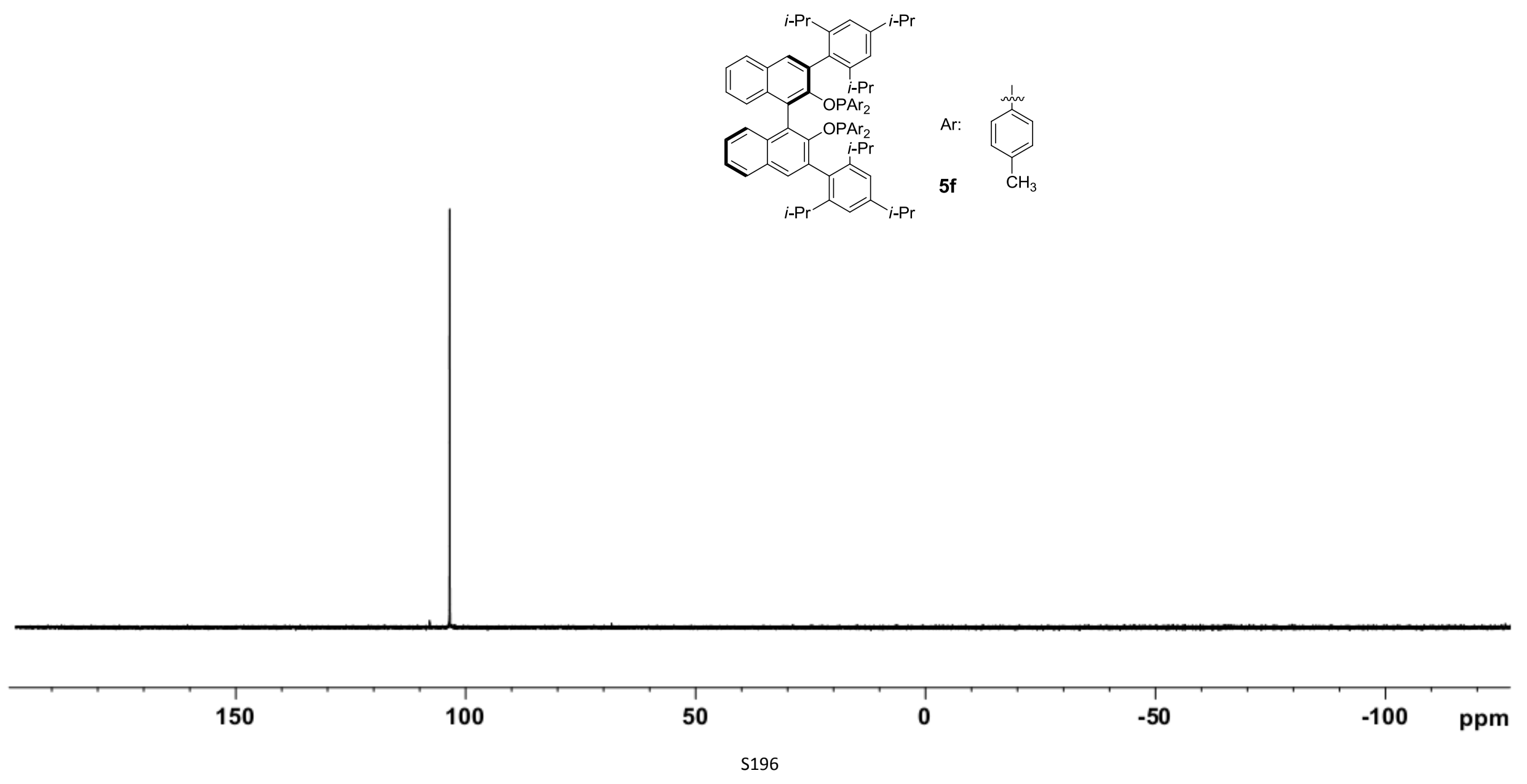




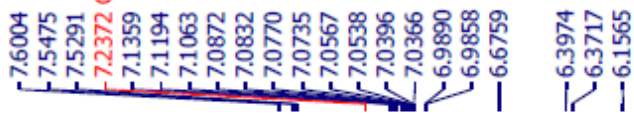
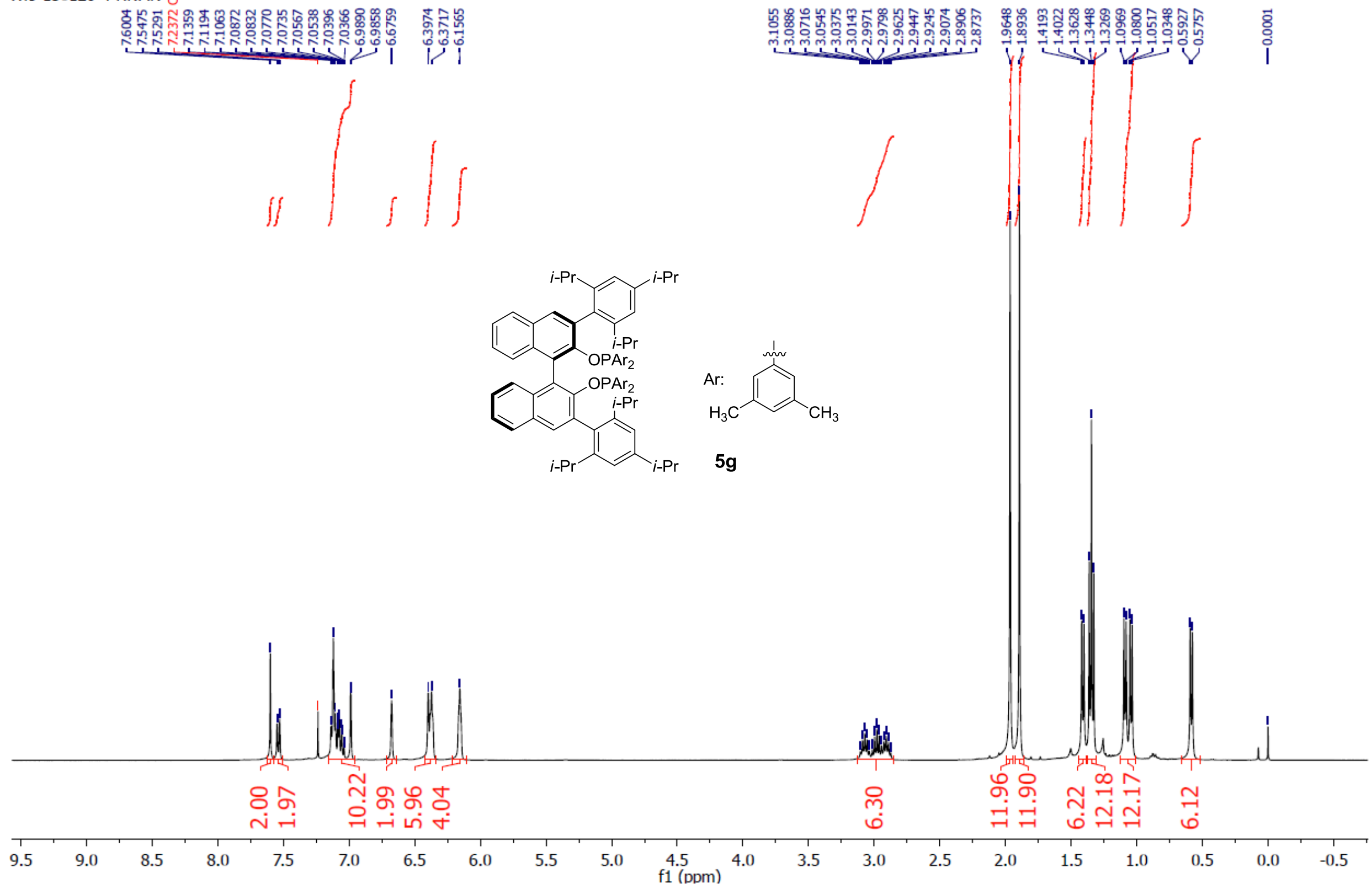


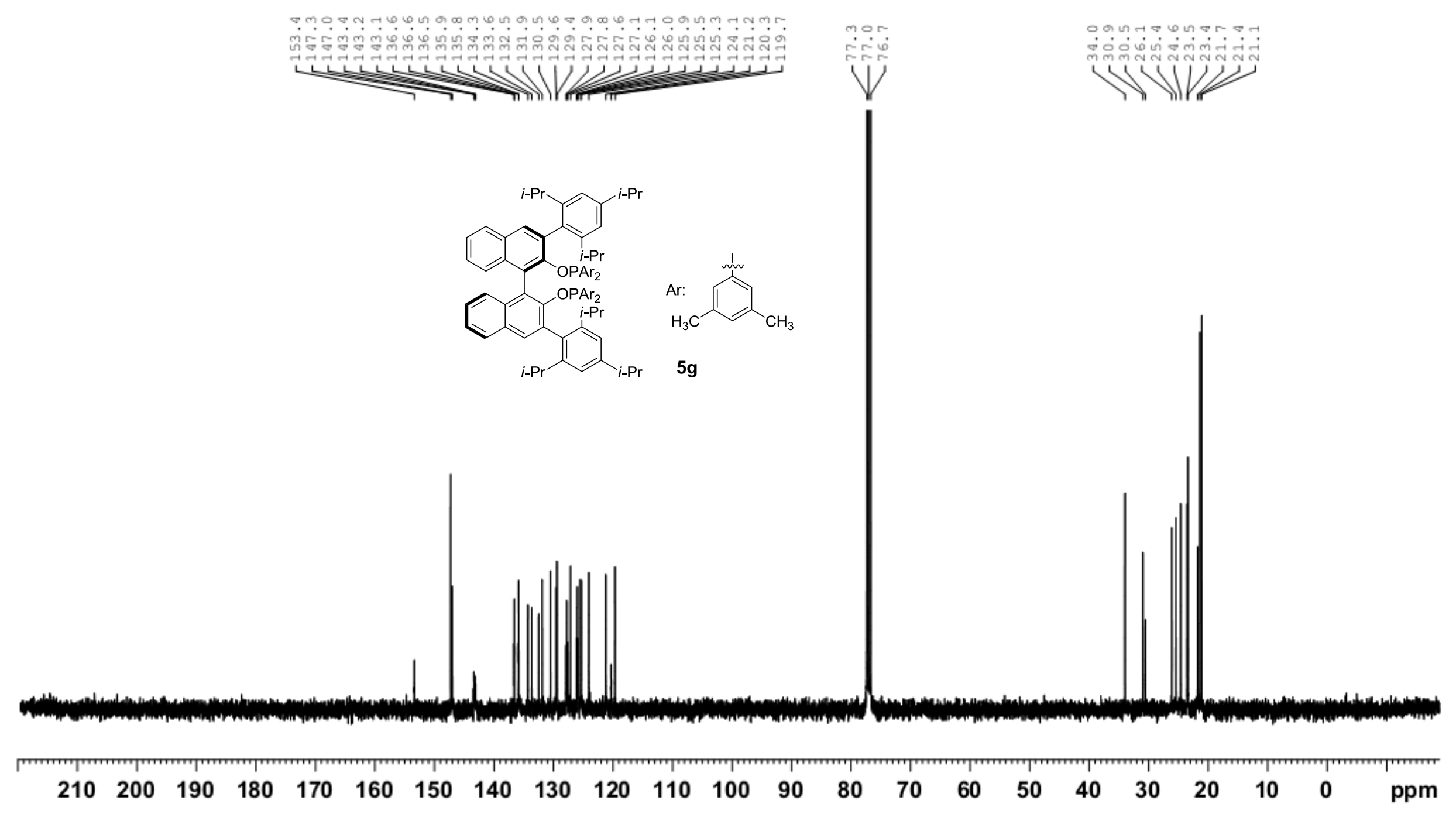



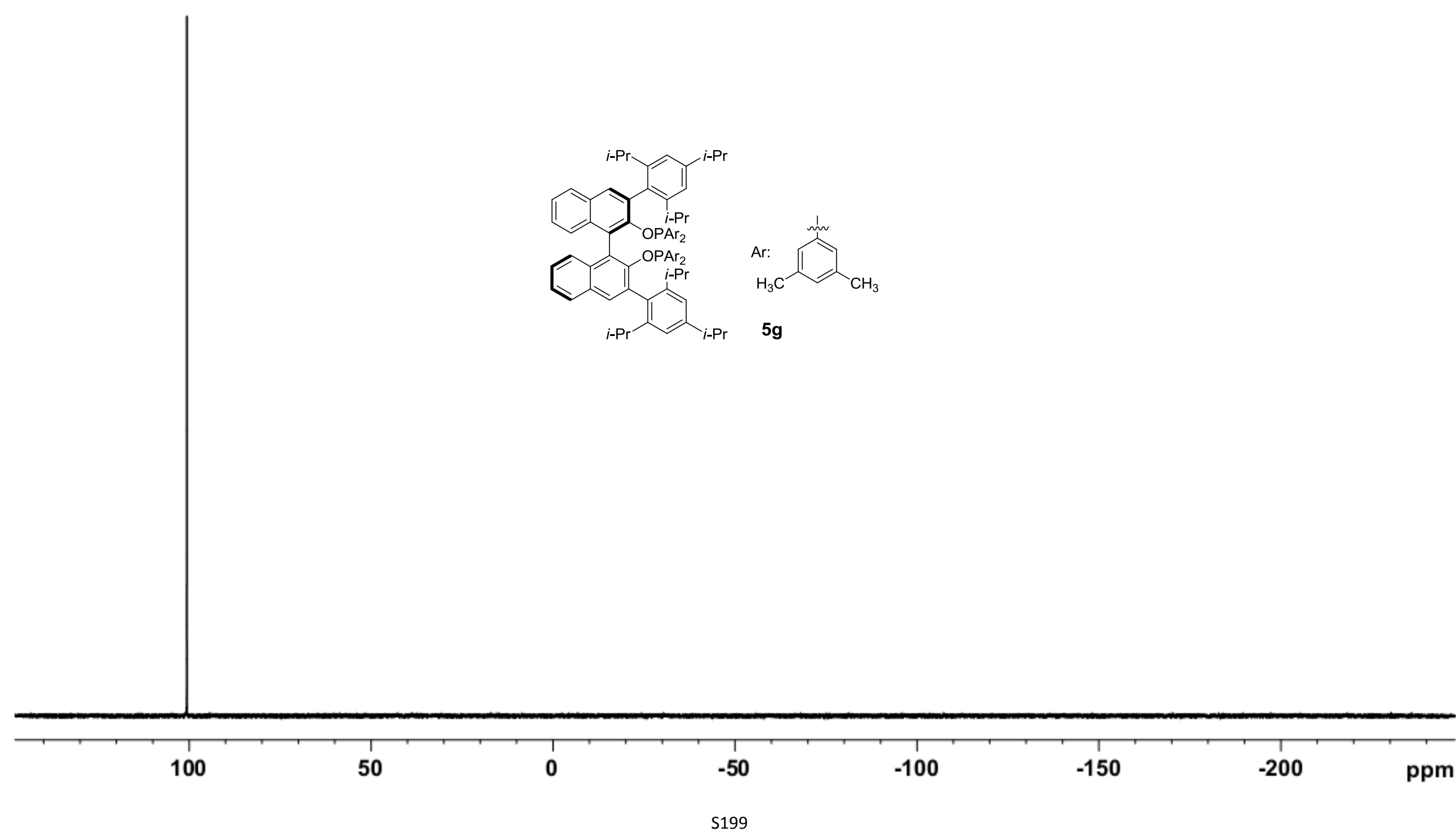


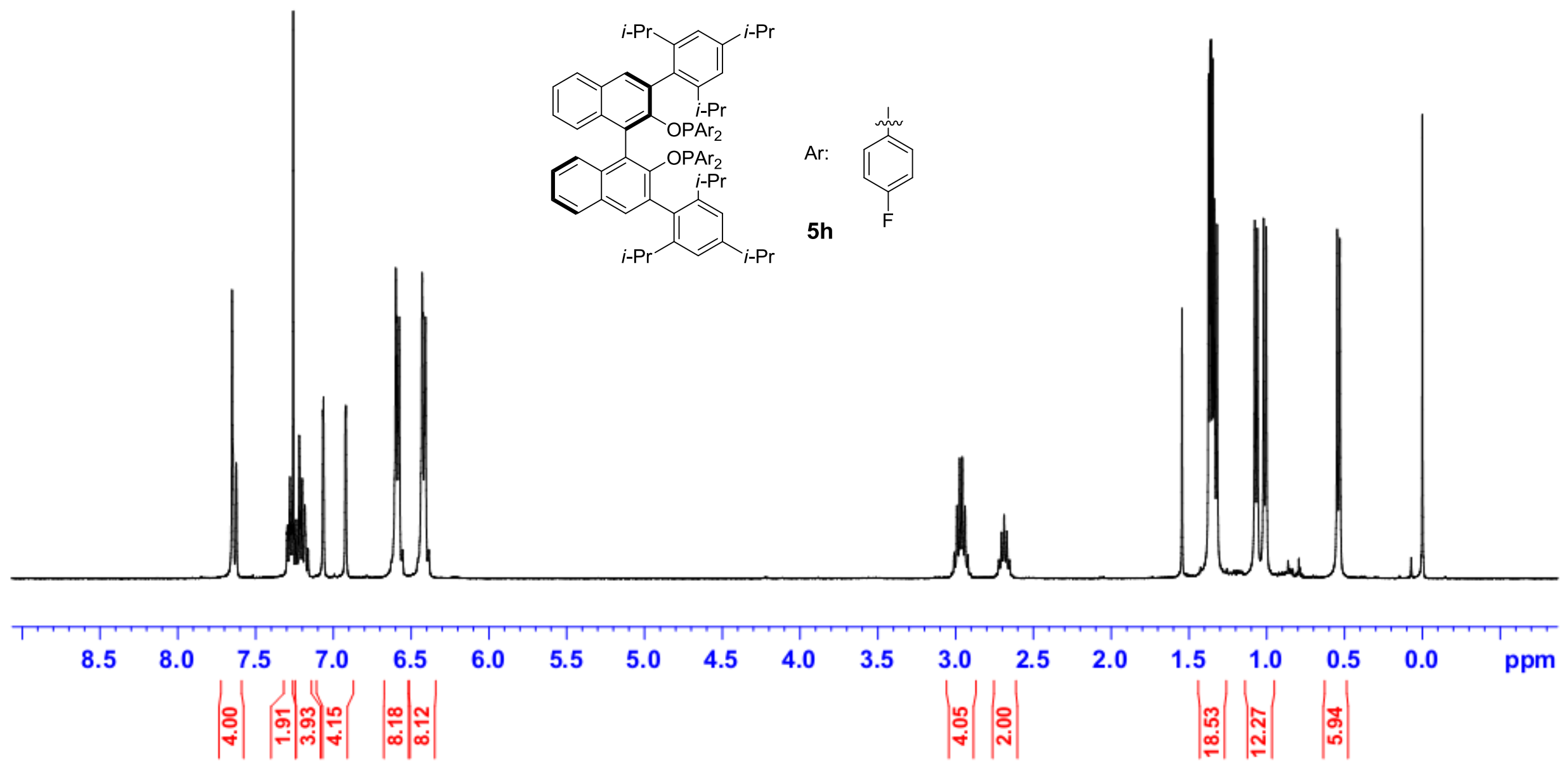



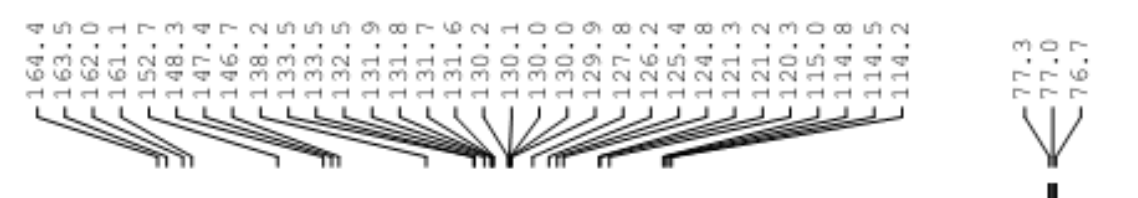

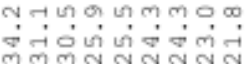

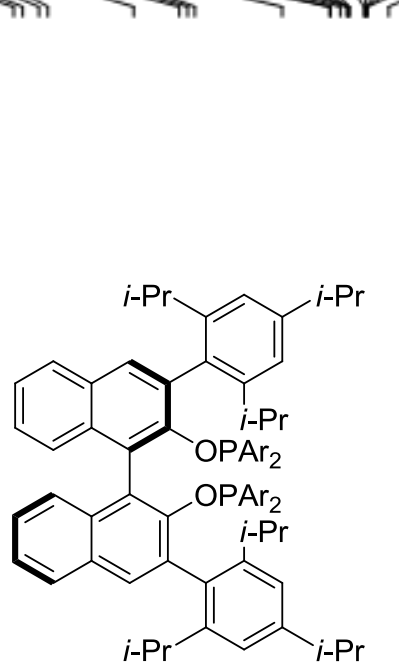

$\operatorname{Ar}$ :<smiles>Fc1ccccc1</smiles>

$5 \mathrm{~h}$

\section{Nin}

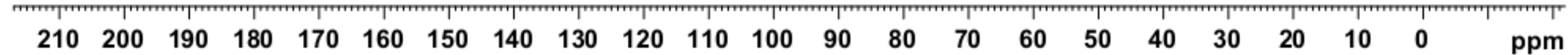




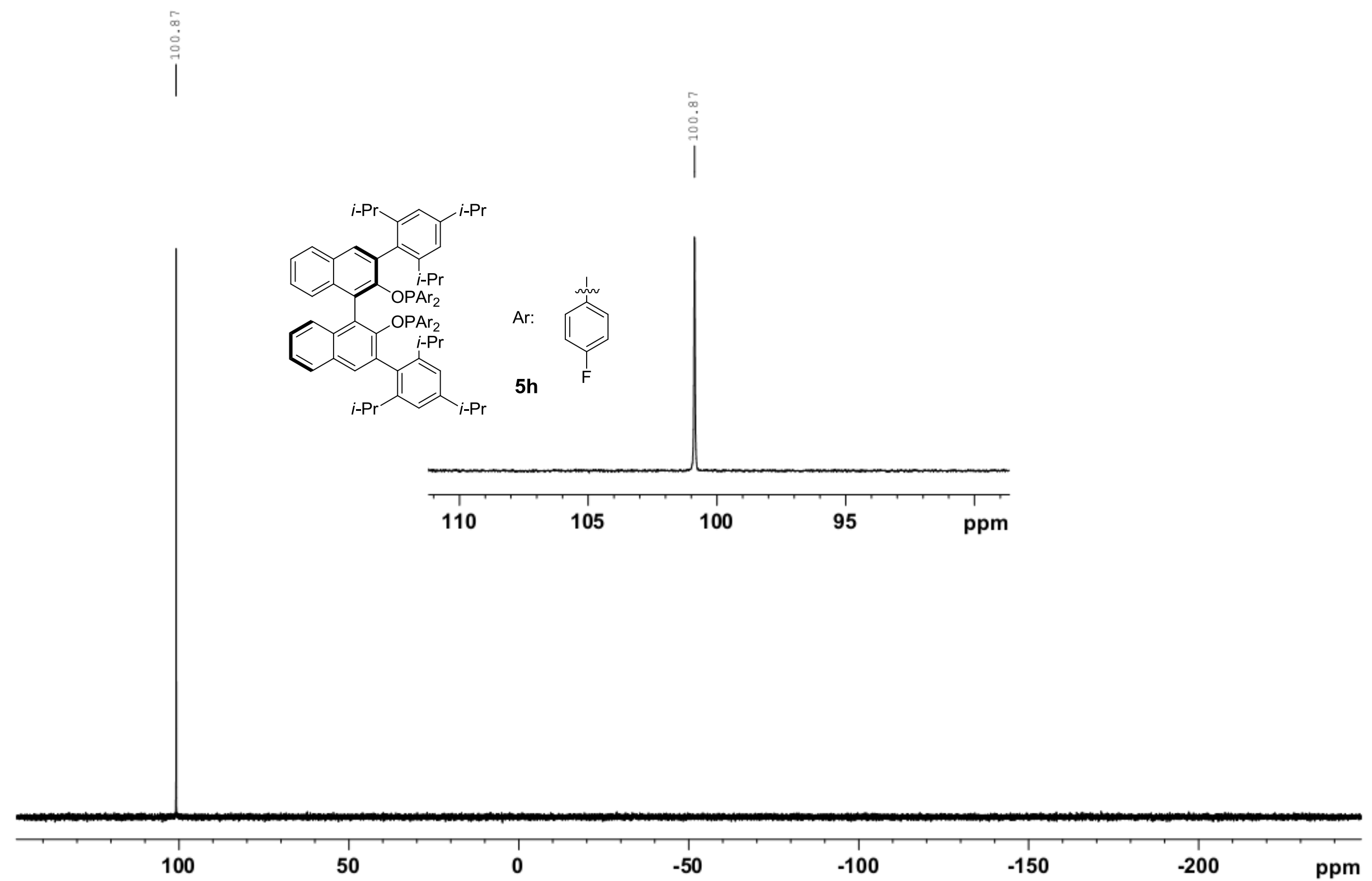




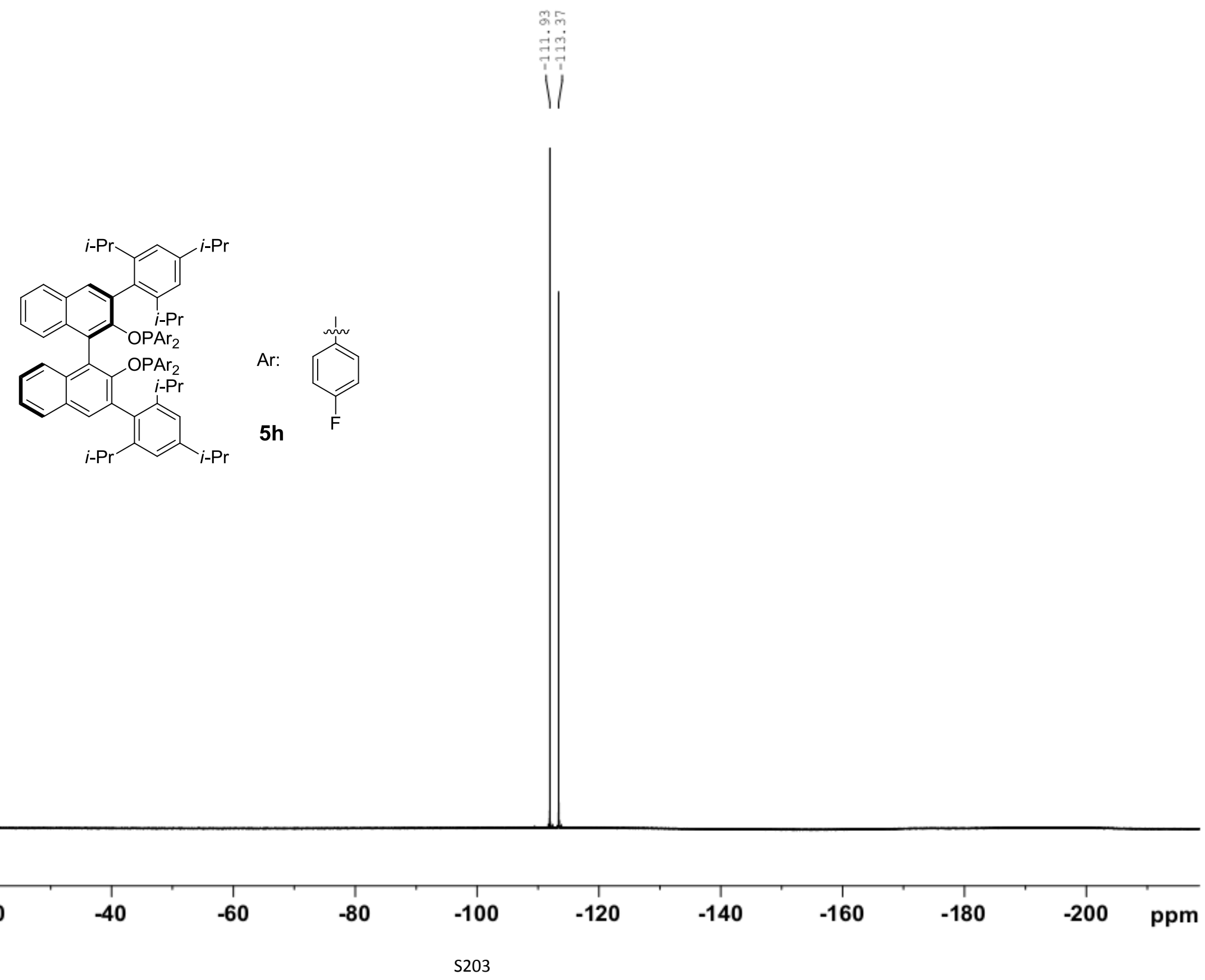


XYJ-14Z30-1-HNMR

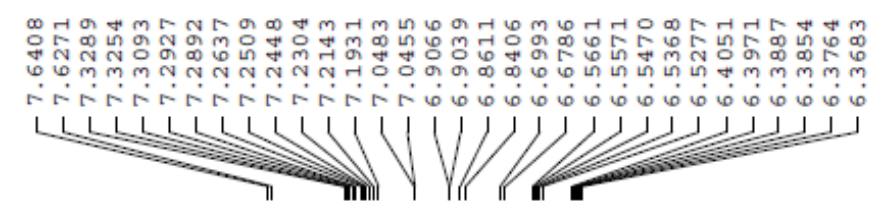

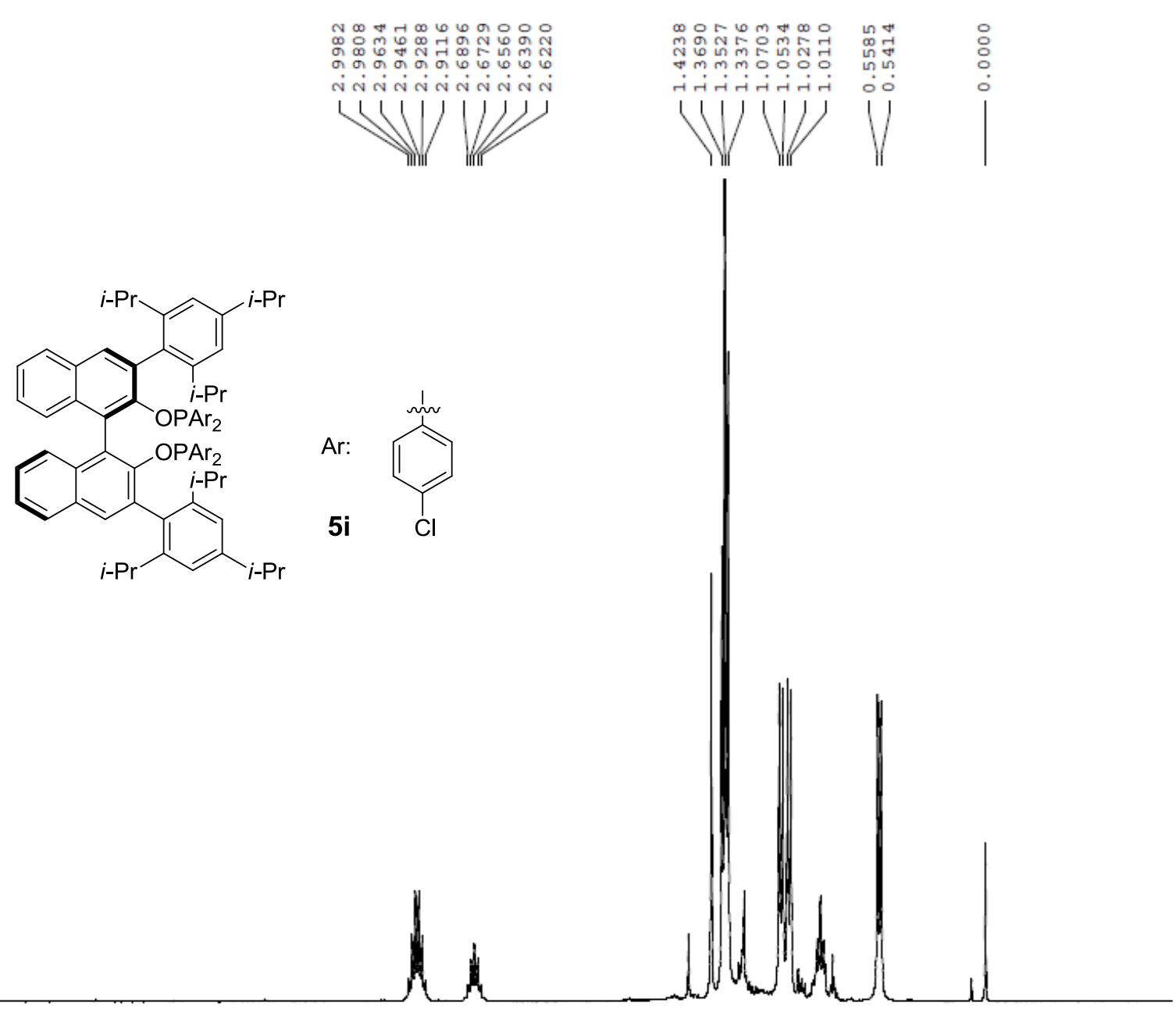
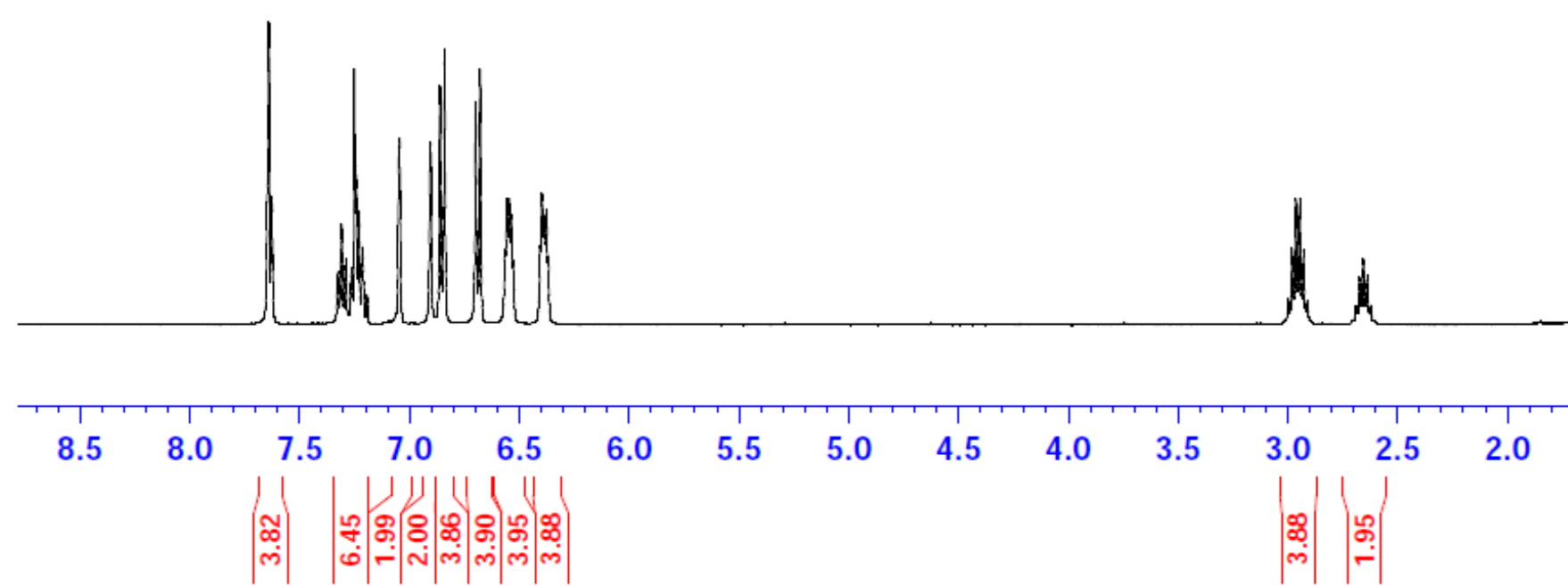

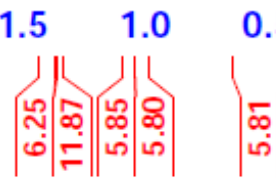




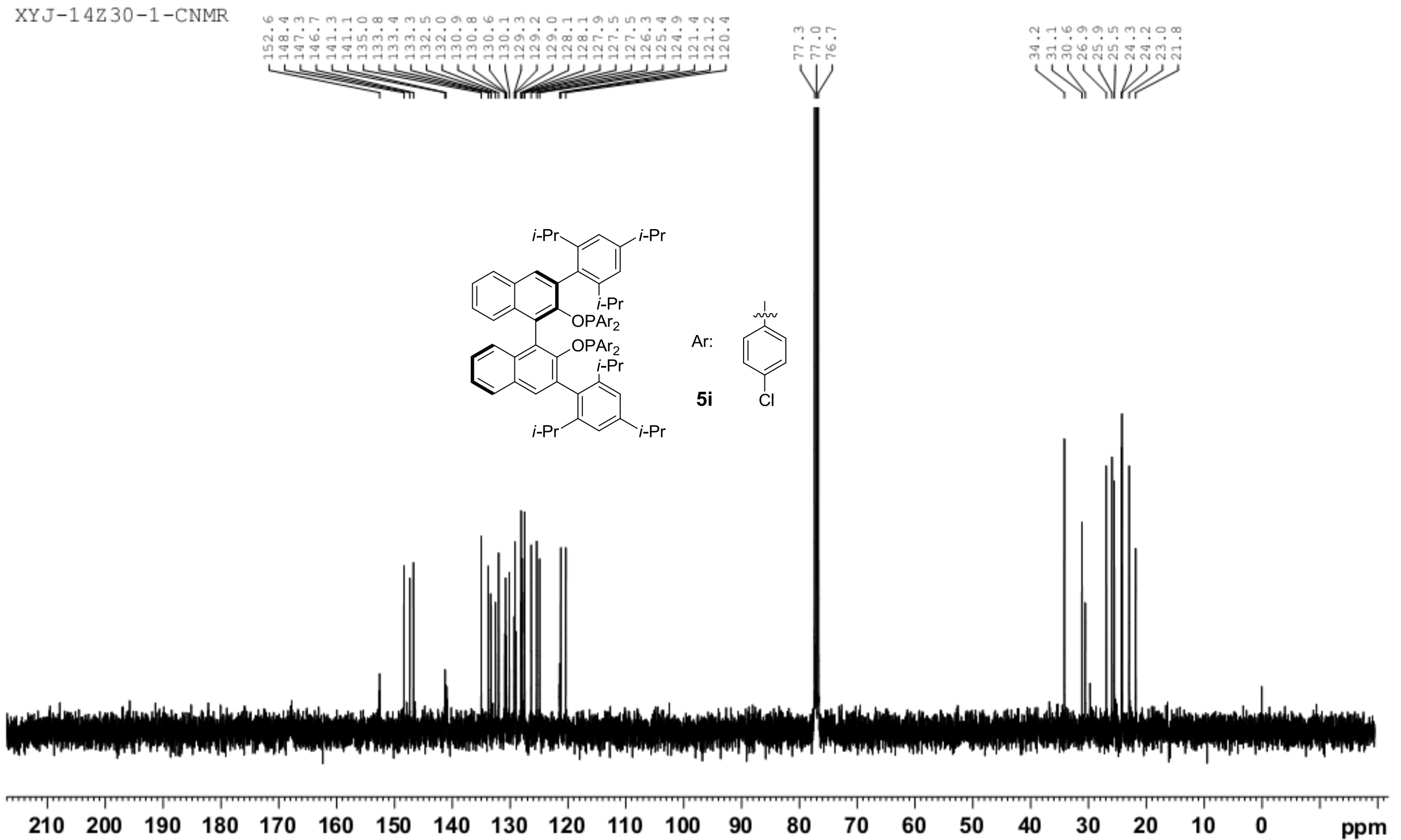




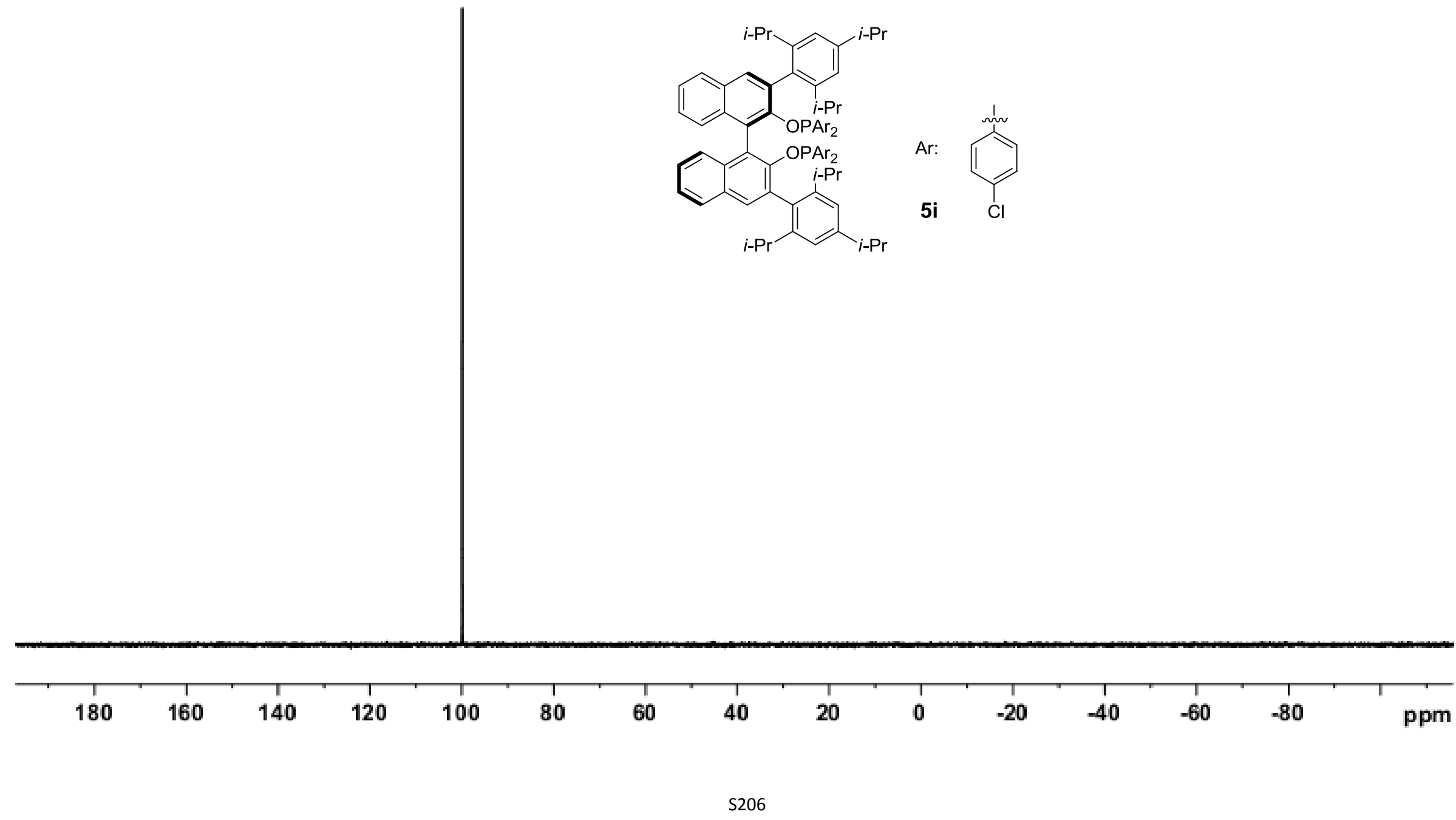


LY-150203-1-HNMR

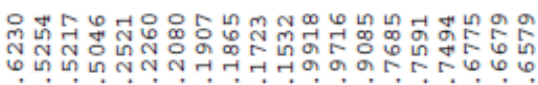

ans

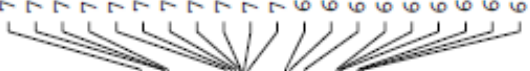

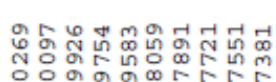

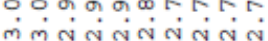

WV
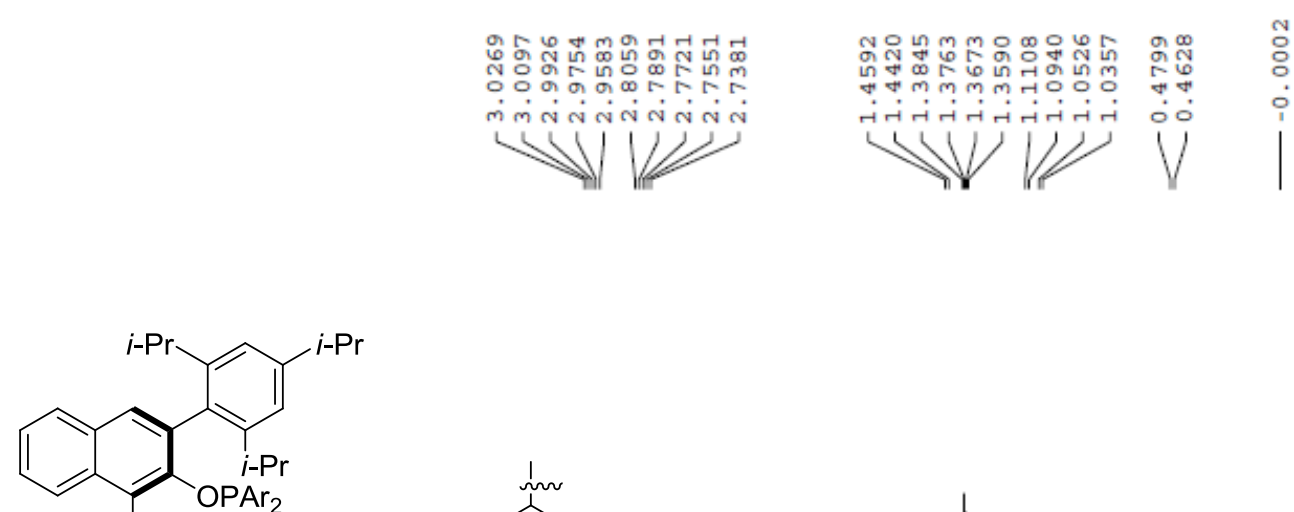

OPAr

i-Pr

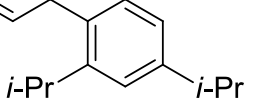

Ar:

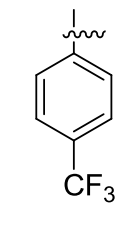

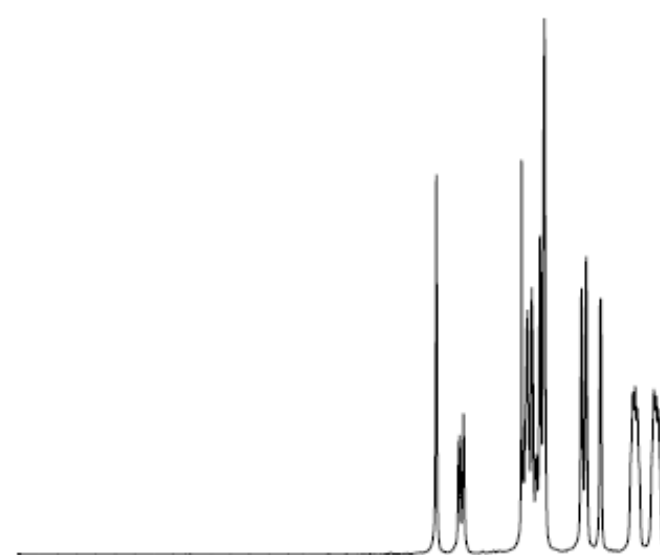
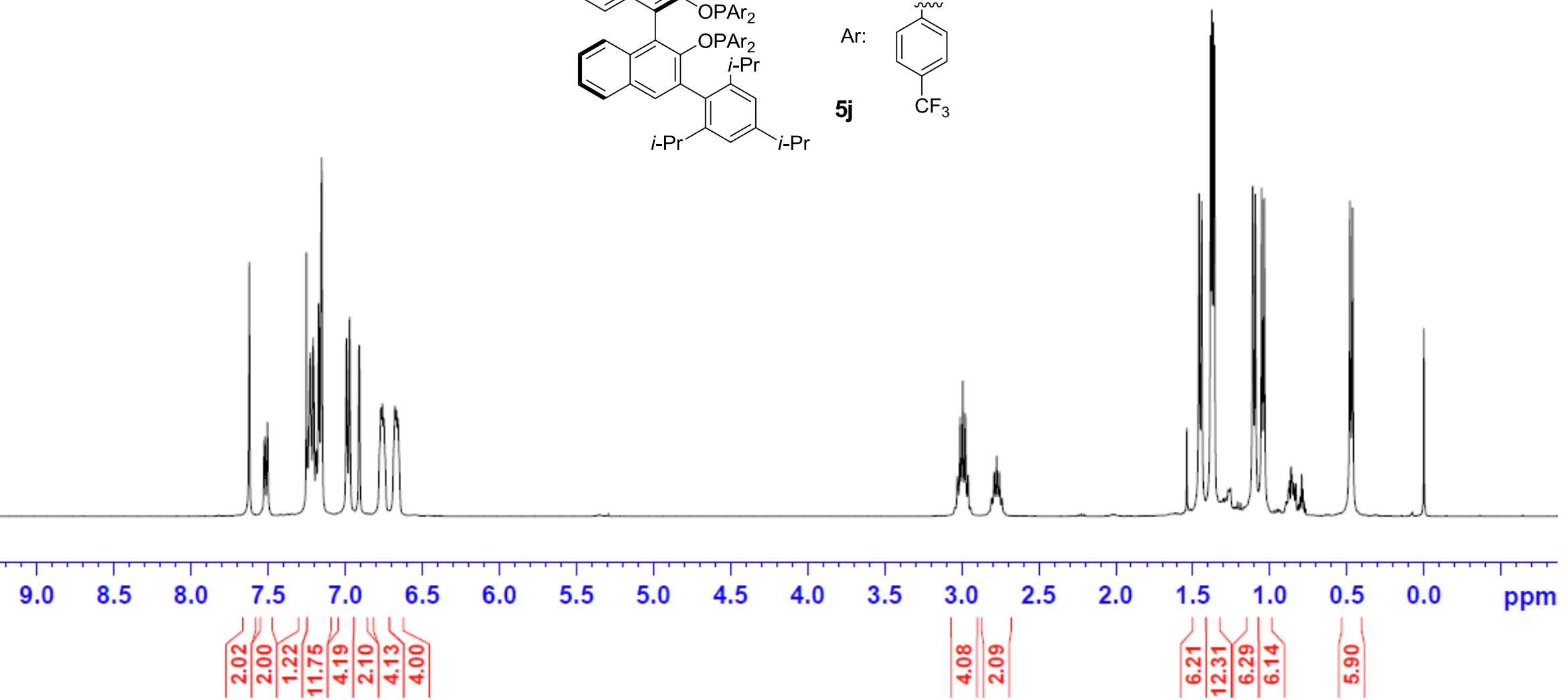


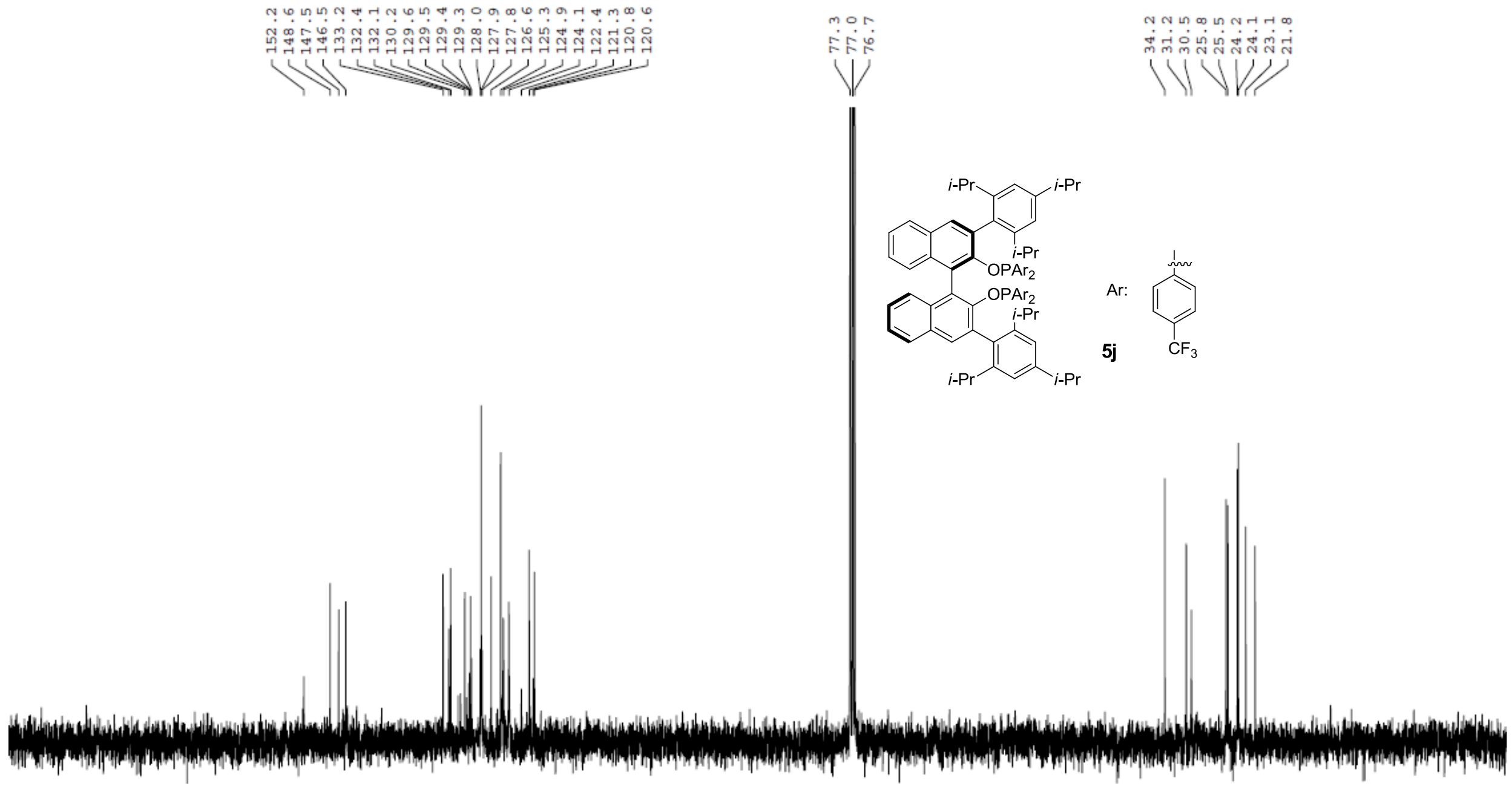


XYJ-14Z22-2-PNMR

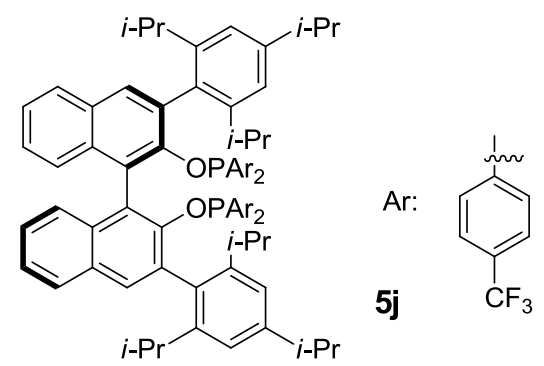

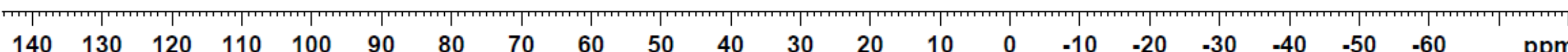


XYJ-14Z22-2 - FNMR

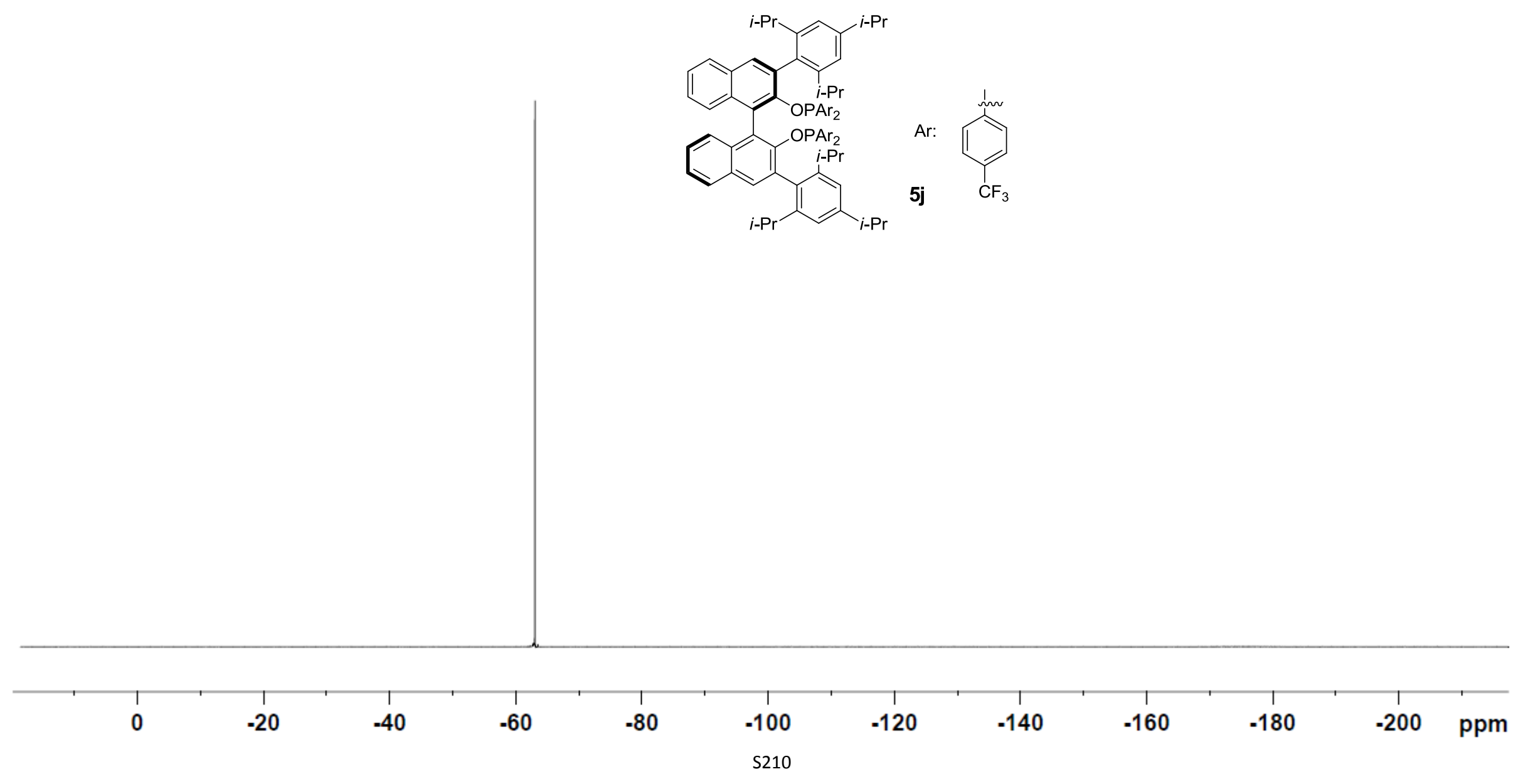




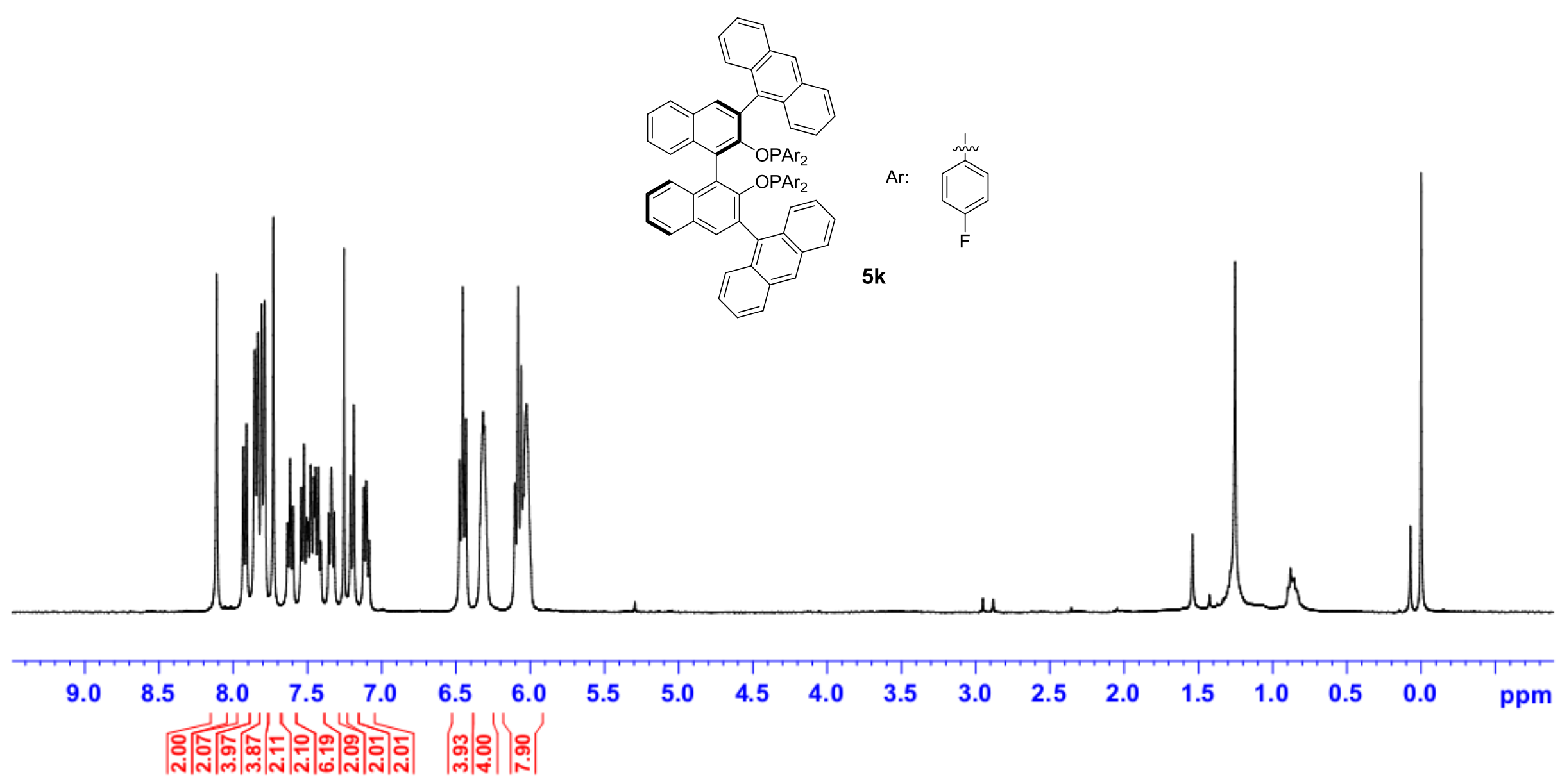



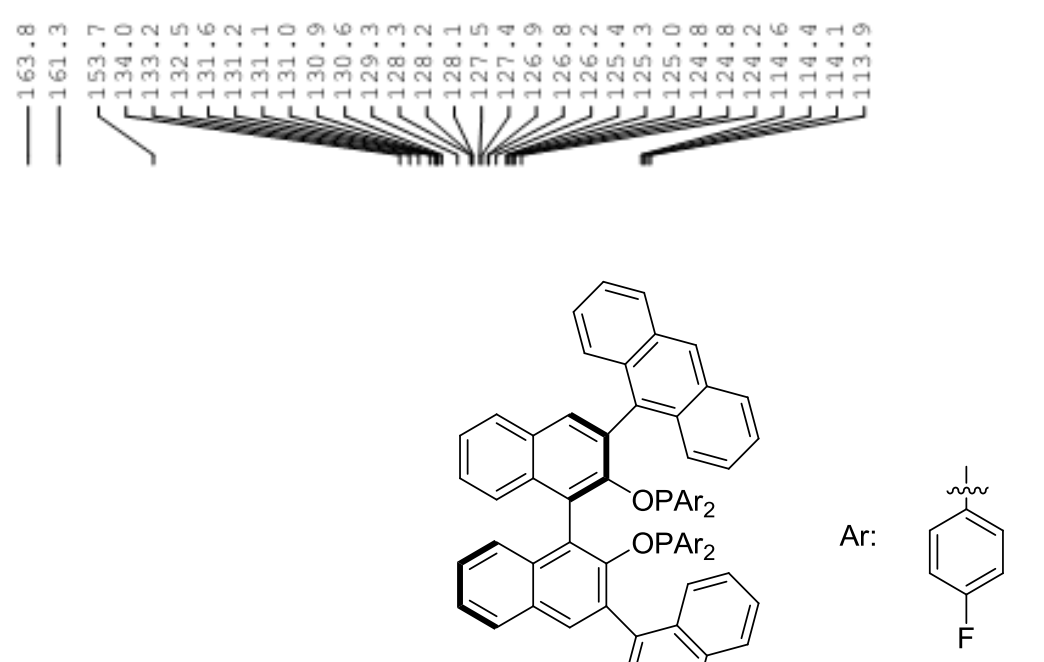

$5 k$
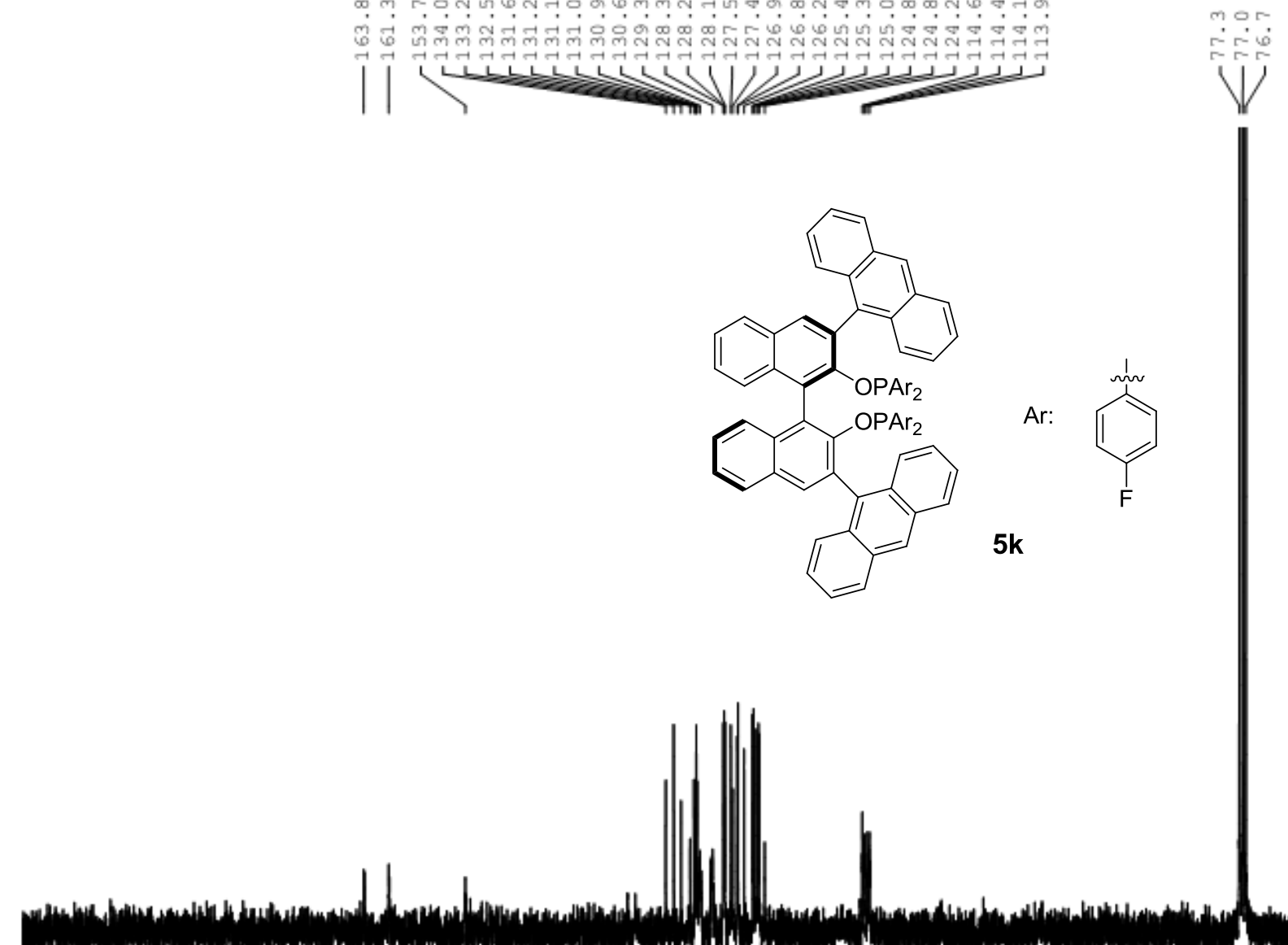

Cim

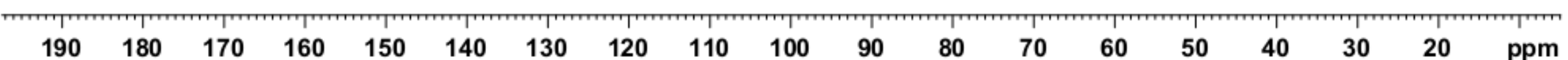




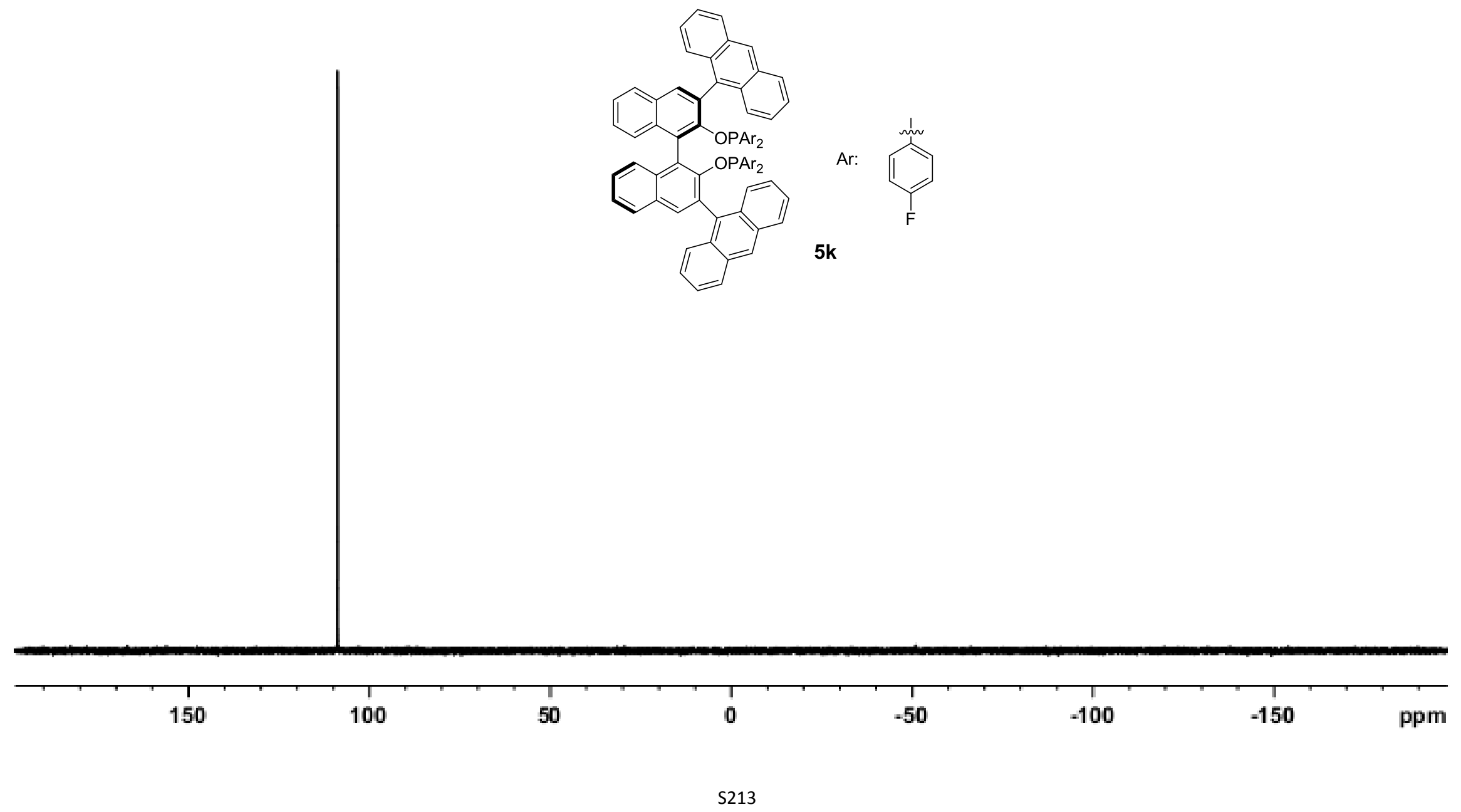



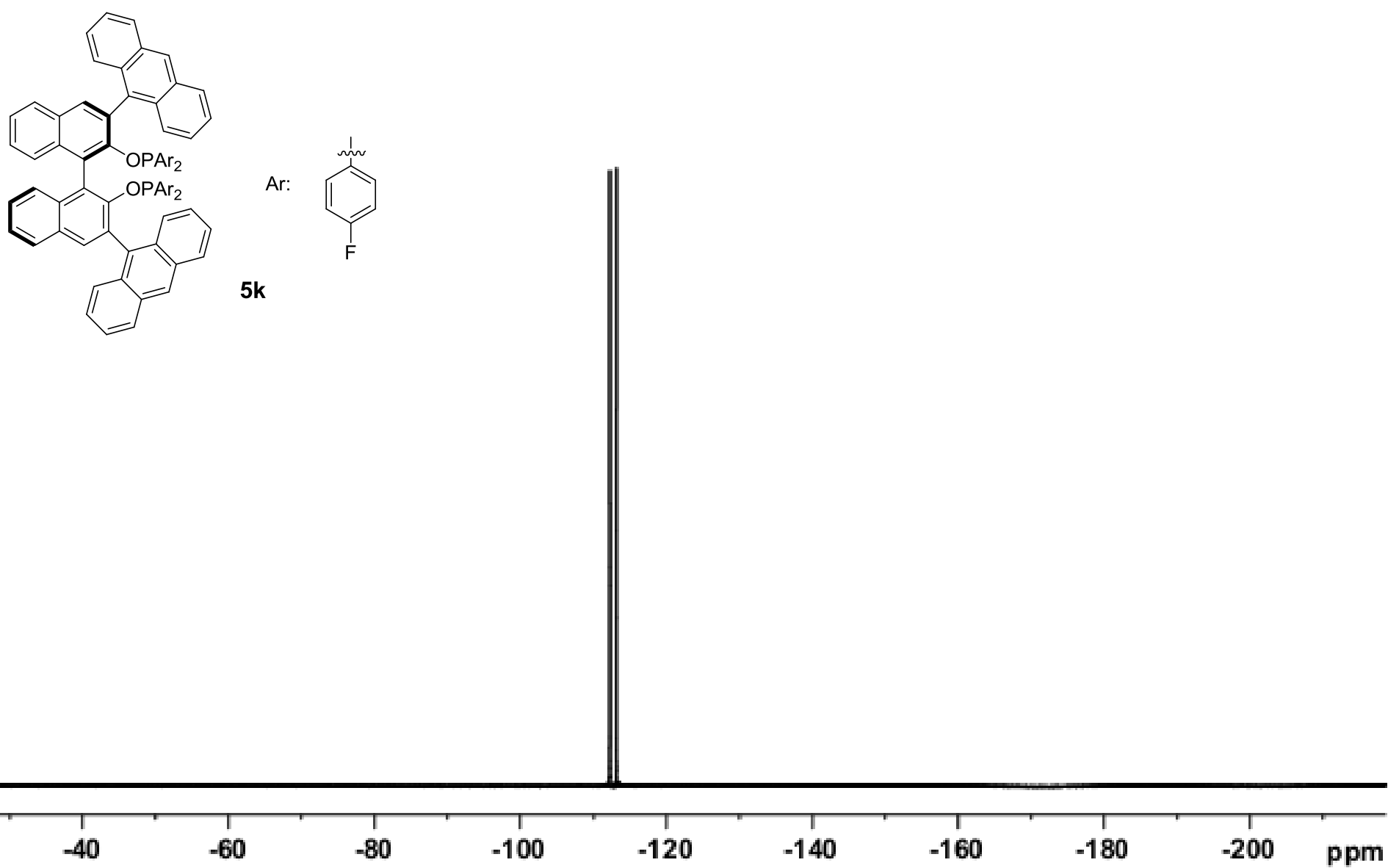


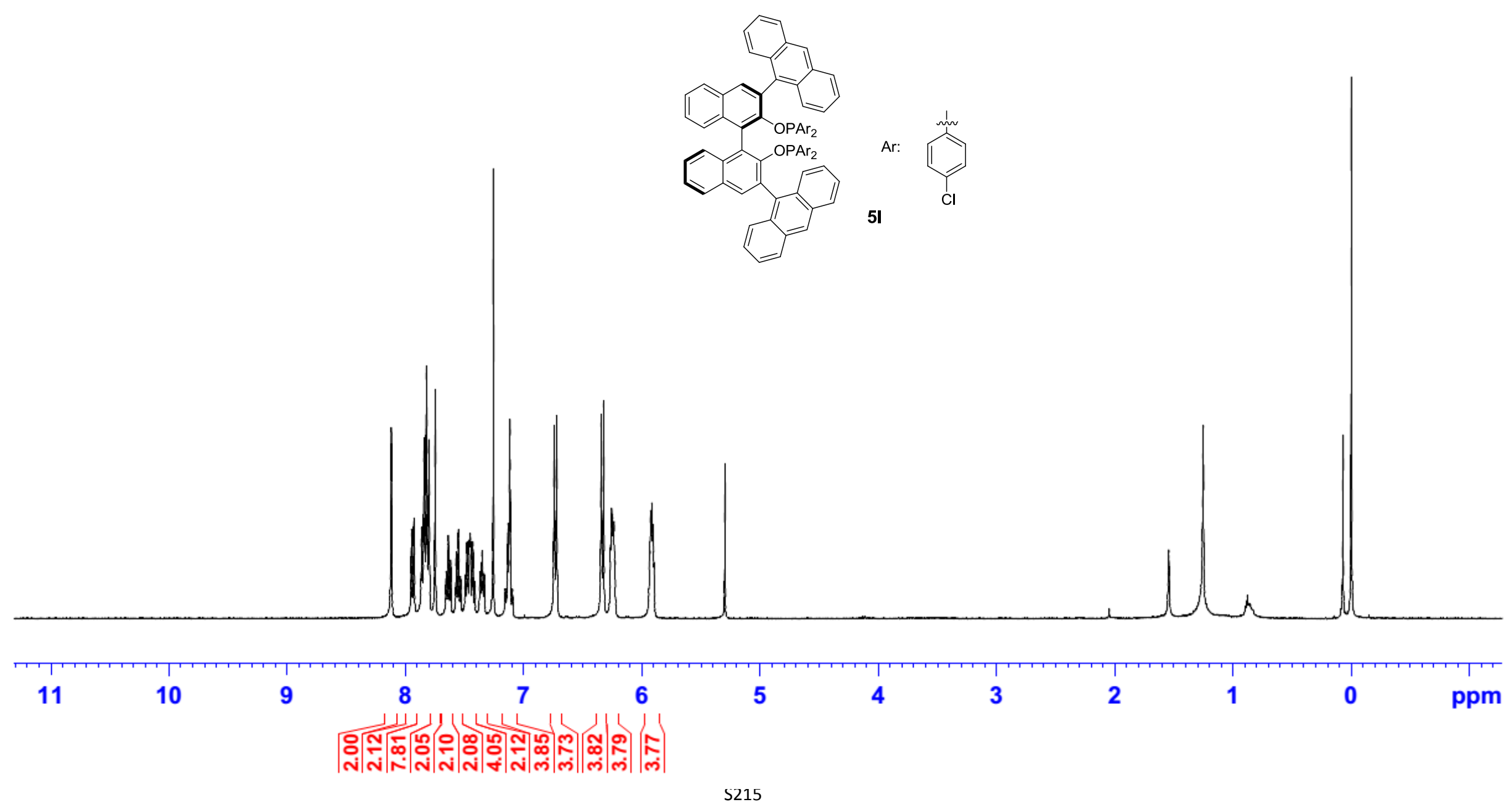




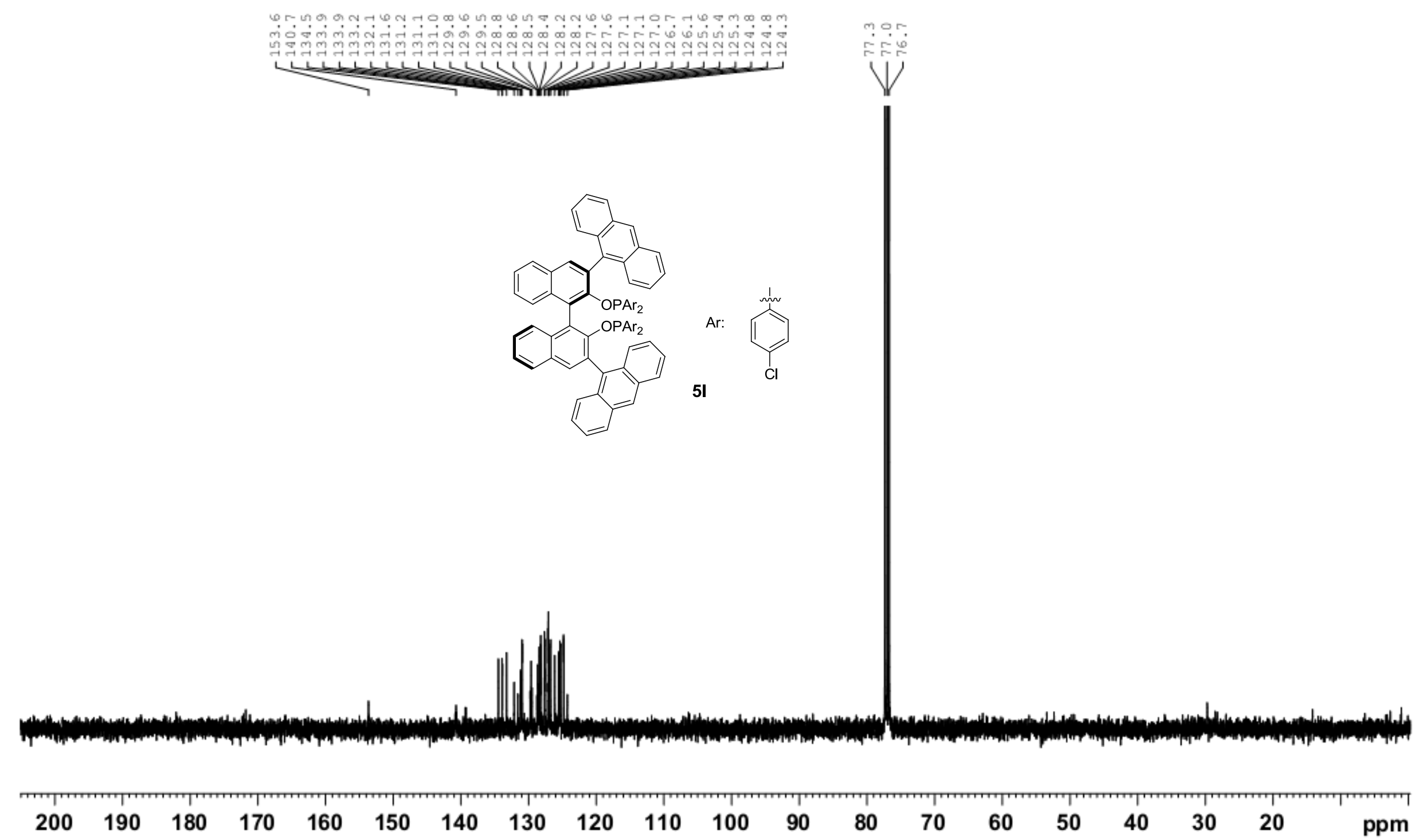




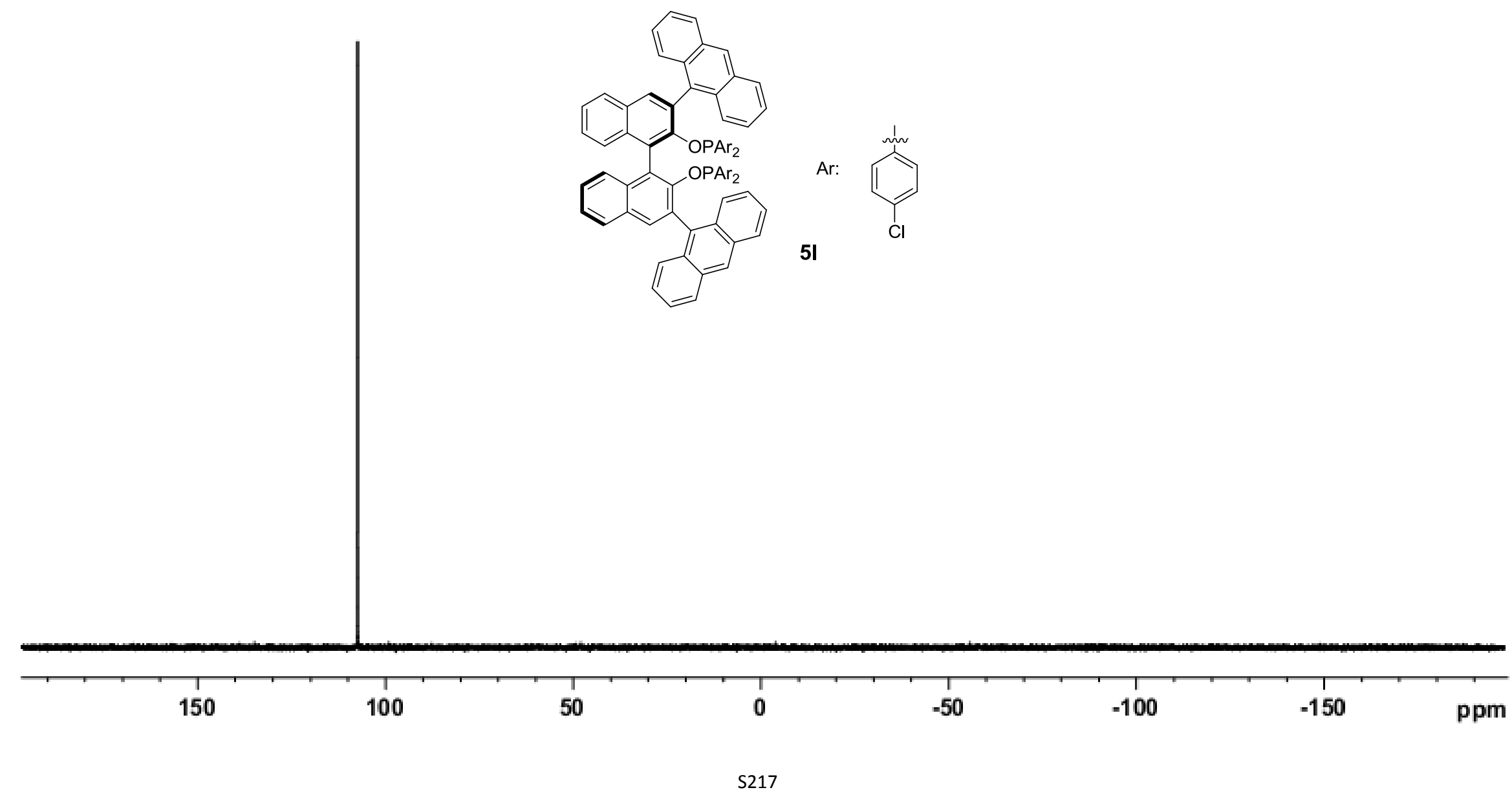




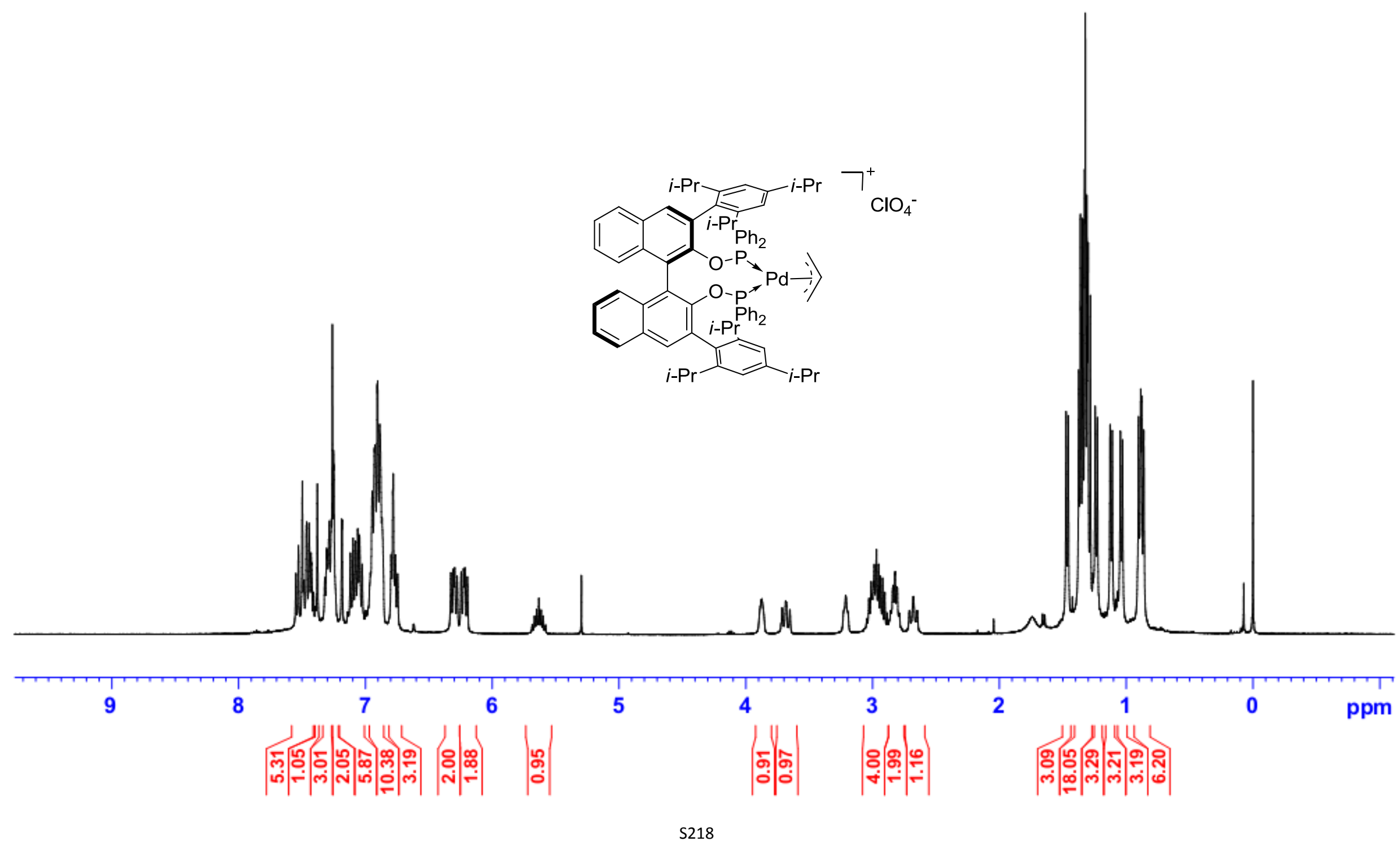




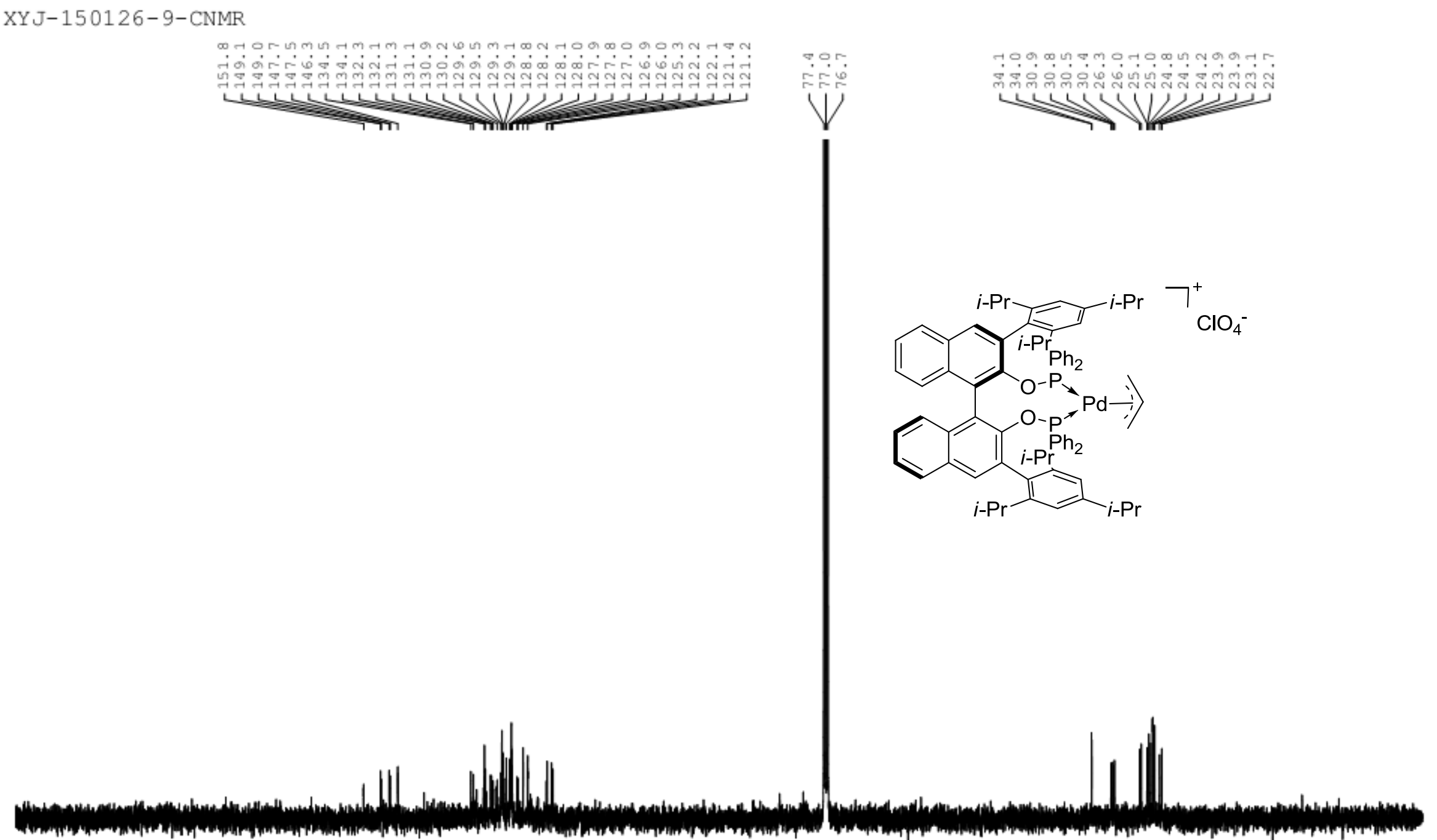

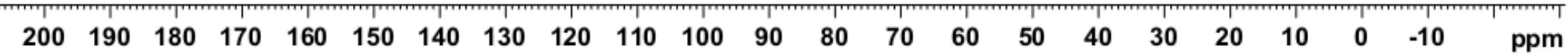




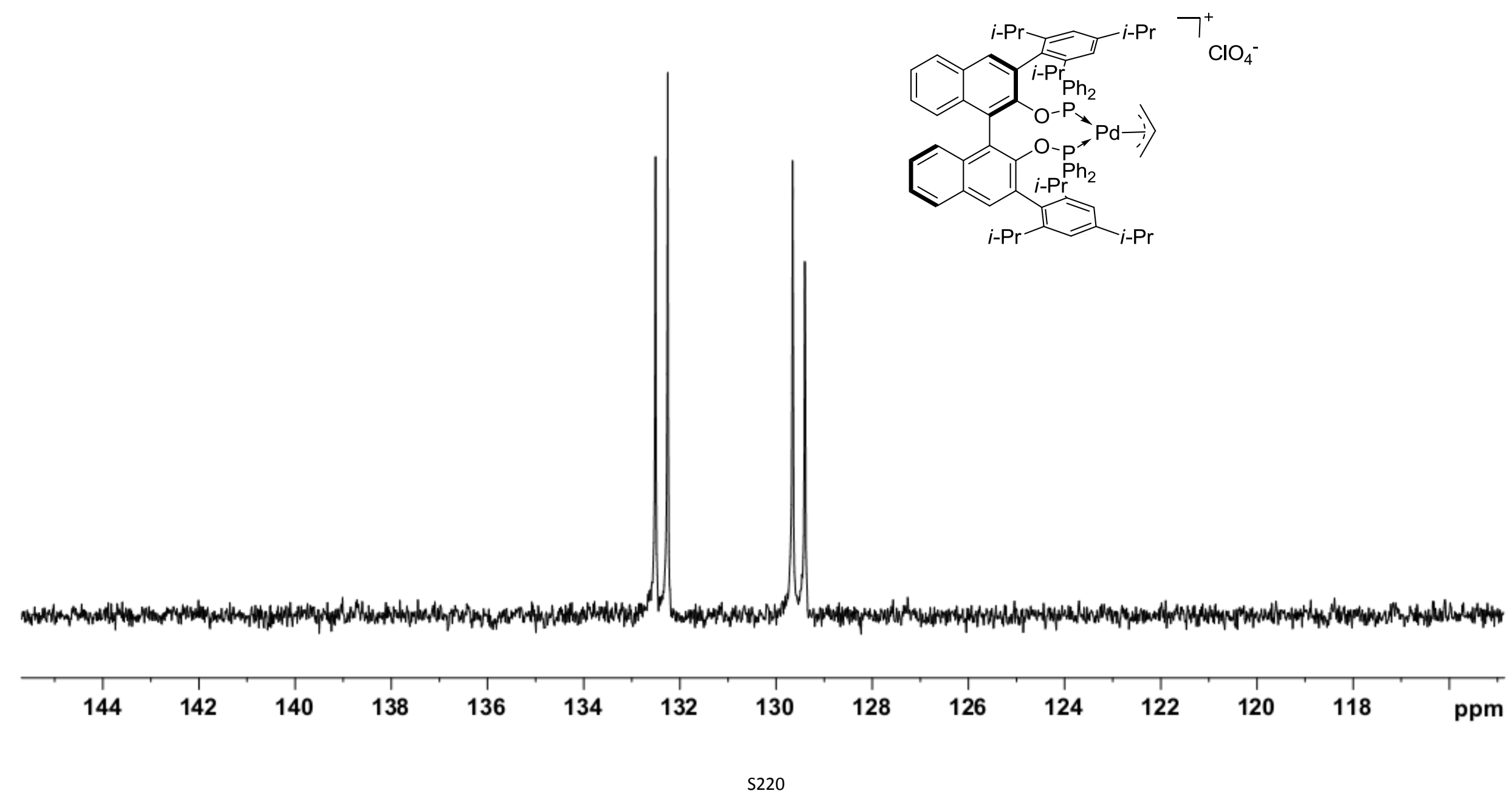

